


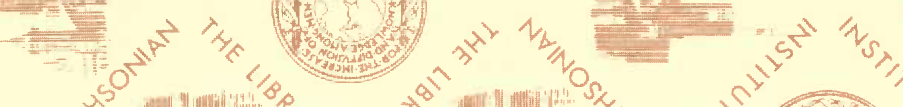

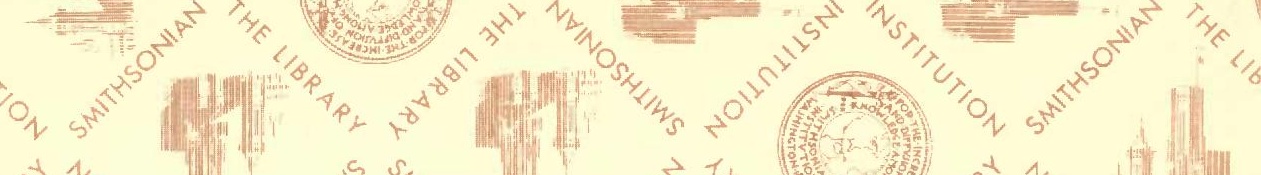
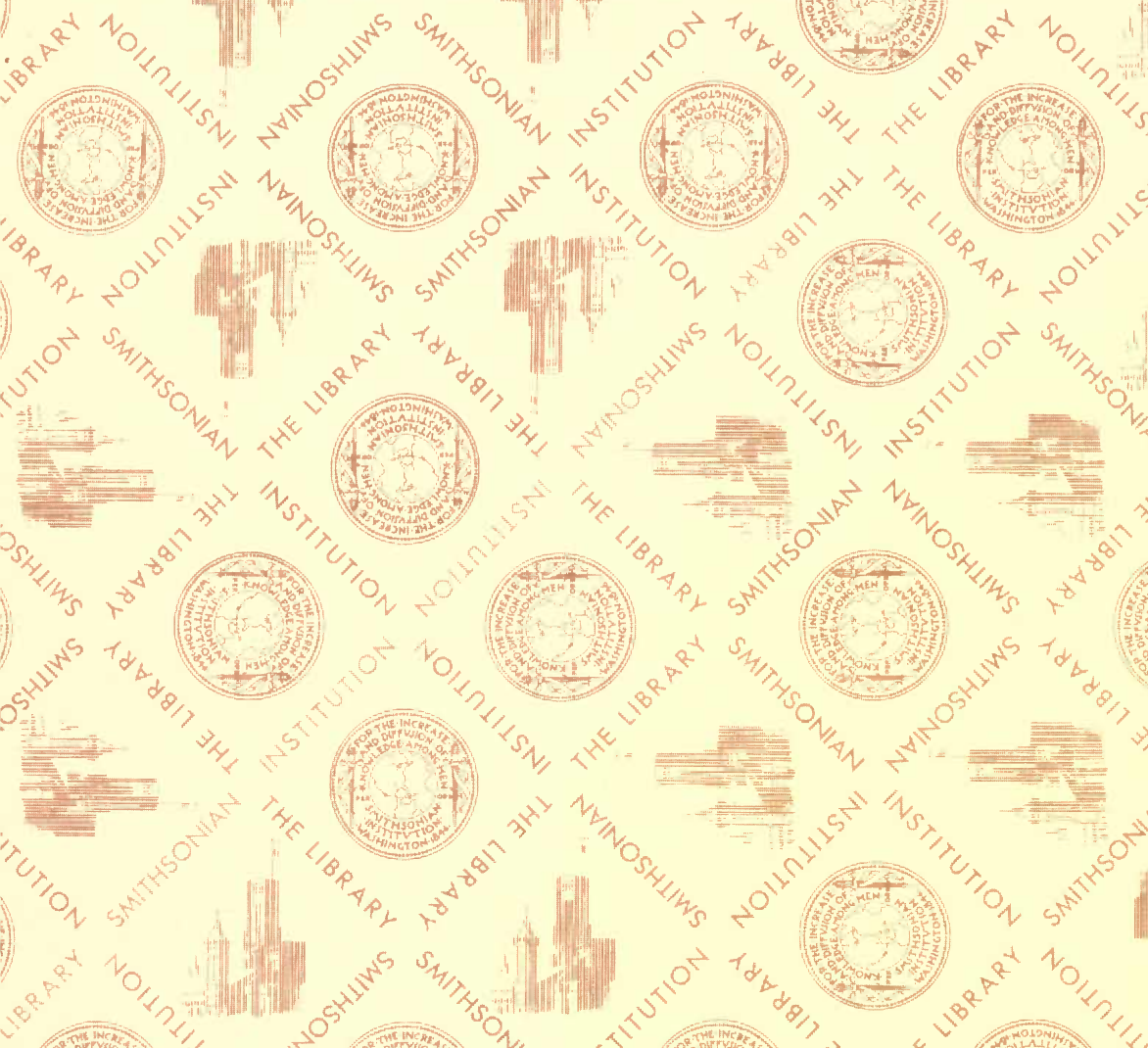

Int 


E 


\title{
CAT ${ }^{\wedge}$ LOGUS DIPTERORUM
}

\section{HUCUSQUE DESCRIPTORUM}

\author{
AUCTORE \\ D. C. KERTÉSZ.
}

\section{VOLUIMEN III.}

Strationyinda, Erinnidae, Coenomyiddae, Tabanidae, Pantophthalmidae, Rhagionidae.

\section{BUDA PESTINI.}

In commissione apud Guilelmum Engelmann, Lipsiae.

1908. 
Conclusum exitu anni 1905.

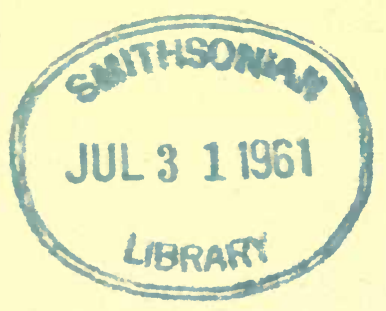




\title{
ORTHORRHAPHA BRACHYCERA.
}

\author{
PLATYGENYA.
}

\section{HOMOEODACTYLA.}

Notacantha.

STRATIOMYIIDAE.

\section{Pachygastrinae.}

\section{Neochauna}

Willist., Manual of N. Amer. Dipt., Ed. II., 48.

Chauna Lw., Stettin. Entom. Zeitg., VIII. 370. (1847). [pracocc.]

fermginea GERST. = variabilis Lw.

variabilis Lw., Stettin. Entom. Zeitg., VIII. 370. 1. tab. I. fig. 11-15. Cuba.

[Channa] (1847); Walk., List Dipt. Brit. Mus., V. suppl. 1., 102.

1. [Chauna] (1854); Lw., Berlin. Lntom. Zeitschr., II. 349.

[Chauna] (1858); Aldr., Catal. Nortl Amer. Dipt., 191. (1905).

ferruginea GERst., Linnaea Entomol., XI. 340. tab. III. fig. 7.

(1857).

\section{Blastocera}

Gerst., Linnalea Entomol., XI. 341. 8. (1857).

atra Wandoli., Wiener Entom. Zaitg., XVI. 216. (1897).

St. Cruz.

speciosa Gerst., Linnaea Entomol., XI. 342. tab. III. fig. 8. (1857);

Brasilia.

Willist., Trans. Amer. Entom. Soc. Philad., XV. 257. 32. (1888);

Hunter, Trans. Amor. Entom. Soc. Philad., XXVII. 127. (1901).

\section{Adraga}

Walk., Proc. Linn. Soc. London, III. 82. (1859).

univicta v. D. WULP = univitta WALK.

univitta WaLk., Proc. Linn. Soc. London, III. 82. 18. (1859) et VII. I.Aru, Mysol. 204. 10. (1864).

univicta v. D. Wulp, Catal. Dipt. South Asia, 57. (1896).

Kertész: Catalogus Dipterorum. III. 


\section{Panacris}

Gerst., Linnaea Entomol., XI. 346. 10. (1857).

lucida Gerst., Linnaea Entomol., XI. 347. tab. III. fig. 10, 10 a. Brasilia, (1857) ; Willist., 'Trans. Amer. Entom. Soc. Philad., XV. 257. 33. Cayenne. (1888); Hunter, Trans. Amer. Entom. Soc. Philad., XXVII. 127. (1901).

\section{Spyripoda}

Gerst., Limnaea Entomol., XI. 344. 9. (1857).

tarsalis Gerst., Limuaea Entomol., XI. 345. tab. III. fig. 9. (1857); Brasilia. Hunter, Trans. Amer. Entom. Soc. Philad., XXVII. 127. (1901).

\section{Caenacantha}

v. D. Wulp, Notes Leyden Mus., VII. 58. (1885).

bipartita v. D. Wulp, Notes Leyden Mus., VII. 59. 4. tab. V. fig. 1, Colımbia. 1a-b. (1885); Huntwe, Trans. Amer. Entom. Soc. Philad., XXVII. 127. (1901).

\section{Artemita}

WaLK., List Dipt. Brit. Mus., V. suppl. 1., 61. (1854). Acanthina Wied., Aussereurop. zweifl. Ins., II. 50. (1830) [nee Fisch., Moll., 1806].

Acanthinomyia Hunter, Trans. Amer. Entom. Soc. Philad., XXVII. 129. (1901).

Acanthinia MACQ., Suit. à Buffon, I. 240. 2. (1834) [lapsus].

Amenides Walk., List Dipt. Brit. Mus., III. 523. [Clitellaria] (1849) et V. suppl. 1., 61 et 63 et 305. nota. [Clitellaria] (1854).

argentea Ost.-SACK., Biolog. Centr. Amer., Dipt., I. 41.1. [Acanthina] (1886); Willist., Biolog. Centr. Amer., Dipt., I. 249. [Acanthina] (1901); Aldr., Catal. North Amer. Dipt., 191. [Acanthina] (1905). aurata BiG., Annal. Soc. Entom. France, sér. 5. IX. 207. [Acanthina] (1879).

aurata Macq., Dipt. exot., suppl. 1., 51. 2. tab. V. fig. 6. [Acanthina] (1846) ; Walk., List Dijut. Brit. Mus., V. suppl. 1., 25. 5. [Acanthina] (1854); Hunrer, Trans. Amer. Entom. Soc. Pliilad., XXVII. 129. [Acanthinomyia] (1901).

azurea Genst., Linnaea Entomol., XI. 335. 1. [Acanthina] (1857); ?Ost.-Sack., Berlin. Eutom. Zeitschr., XXVI. 99. [Acanthinc] (1882); v. D. Wurp, Catal. Dipt. South Asia, 56. [Acanthina] (1896).

Patriaignota.

America mer. et centr.

Columbia.

Columbia.

Asia mer., I.Philippinae, Arch.Inclicus, Nova Guinea.

?obesa Walk., Proc. Linn. Soc. London, V. 232. 13 et 271. 3. 
[Clitellaria] (1861) et VI. 20. 2. [Clitellaria] (1862); Ost.SAck., Annal. Mus. Civ. Genova, XVI.411. [Acanthina] (1882) et XVIII. 17. [Acanthina] (1883).

? Bellardii Gigt.-Tos., Bollet. Mus. Zool. ed Anat. comp. Torino, VI. Mexico. No. 102. 2. [? Acanthina] (1891) et Mem. R. Accad. Nat. Torino, ser. 2. XLIII. 110. 12. [? Acanthina] (1893); ALDr., Catal. North Amer. Dipt., 191. [? Acanthina] (1901).

convexa WaLk., List Dipt. Brit. Mus., V. suppl. 1., 304. [Acanthina] Br.ssilia. (1854).

elongata Wied., Analecta Entomol., 30.37. [Clitellaria] (1824) et Aussezeurop. zweifl. Ins., II. 52. 3. tab. VII. fig. $9 a-b$. [Acanthina] (1830); MAcQ., Suit. à Buffon, J. 240. 1. tab. VI. fig. 2. [Acanthinia] (1834); Walk., List Dipt. Brit. Mus., V. suppl. 1., 25. 2. [Acanthina] (1854); Schis., Novara Reise, Dipt., 52. 1. [Acanthina] (1868); Hunter, Trans. Amer. Entom. Soc. Philad., XXVII. 129. [Acanthinomyia] (1901).

Halala WaLK., List Dipt. Brit. Mus., III. 523. [Clitellaria] (1849) et V. suppl. 1., 60. 5 et 305. nota. [Clitellaria] (185t); Aldn., Catal. North Amer. Dipt., 189. [Clitcllaria] (1905).

hieroglyphica WiEd., Aussereurop. zweifl. Ins., II. 50. 1. [Acanthina] (1830) ; Walk., List Dipt. Brit. Mus., V. suppl. 1., 25. 3. [Acanthina] (1854); ?, Kansas Univ. Quart., I. - tab. VII. fig. 7. [Acanthina] (1892); Hunter, Trans. Amer. Entom. Soc. Philad., XXVII. 129. [Acanthinomyia] (1901).

inornata Willist., Biolog. Central. Amer., Dipt., I. 249. 2. tab. IV. fig. 17. [Acanthina] (1901); Aldor., Catal. North Amer. Dipt., 191. [Acanthina] (1905).

longa WIED., Aussereurop. zweifl. Ins., II. 51. 2. [Acanthina] (1830); WaLK., List Dipt. Brit. Mus., V. suppl. 1., 2`. 1. [Acanthina] (1854); Hunter, Trans. Amer. Entom. Soc. Philad., XXVII. 130. [Acanthinomyia] (1901).

longicornis WALK., Trans. Entom. Soc. London, n. ser. IV. 120. [Acanthina] (1857).

marmorata Gerst., Linnaea Entomol., XI. 337. 2. [Acanthina] (1857); Hunter, Trans. Amer. Entom. Soc. Philad., XXVII. 130. [Accunthinomyia] (1901).

nana BelL., Saggio di Ditterol. Messic., Append., 9. 2. [Acanthina] (1862); ALDr., Catal. North Amer. Dipt., 191. [Acanthina] (1905). obesa WALK. = ? azurea GERst.

ornata MAcQ., Dipt. exot., suppl. 1., 51. 1. tab. V. fig. 5. [Acanthina] (1846) ; WalK., List Dipt. Brit. Mus., V. suppl. 1., 25.4. [Acanthina] (1854); BeLl., Saggio di Ditterol. Messic., I. 28. 1. [Acanthina] (1859); Hunter, Trans. Amer. Entom. Soc. Philad., XXVII. 130. [Acanthinomyia] (1901); Aldr., Catal. North Amer. Dipt., 191. [Acanthina] (1905).

plana Walk., List Dipt. Brit. Mus., V. suppl. 1., 305. [Acanthina] Brasilia. (1854).

America mer.

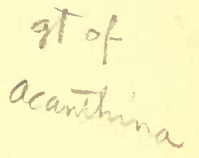

Honduras.

Brasilia.

Mexico.

Brasilia.

Amazonia.

Brasilia.

Mexico.

America c. et mer. 
porcata WaLk., Trans. Entom. Soc. London, n. sor., IV. 120. [Acanthina] (1857).

pulchripennis BraU., Denksehr. Akad. Wien, XLIV. 74. (1881).

\section{Evaza}

Walk., Proc. Limn. Soc. London, I. 109. (1857).

Evasa Auct. plur.

Nerna Watk., Proc. Linn. Soc. London, IV. 97. (1860).

Nerua Watk., Proc. Linn. Soc. London, III. 81. (1859).

argyroceps BıG., Annal. Soe. Entom. France, sér. 5. IX. 219. 2. Ins. Molueci. (1879) ; v. D. Wulp, Catal. Dipt. South Asia, 56. (1896).

bipars Walk., Proc. Linn. Soc. I sondon, I. 110. 25. tab. VI. fig. 2. Borneo.

(1857) ; v. D. Wulp, Catal. Dipt. South Asia, 56. (1896).

flavipes B1G., Annal. Soc. Entom. France, sér. 5. IX. 219. 1. (1879);

v. D. Wulr, Catal. Dipt. South Asia, 56. (1896) ot Természetrajzi Fïzetek, XXI. 416. 19. (1898).

fulviventris Big., Annal. Soc. Entom. France, sér. 5. IX. 220. 3. (1879); Ost.-SAck., Annal. Mus. Civ. Genova, XVI. 415. nota. (1882) ; v. D. Wulp, Catal. Dipt. South Asia; 56. (1896).

impendens Wark., Proe. Linn. Soc. London, IV. 97. 24. [Nerna] (1860) ; Ostr.-SAck., Annal. Múus. Civ. Genova, XVI. 414. [Nerua] (1882); v. D. Wulp, Notes Leyden Mus., VII. 57. 1. (1885) et Catal. Dipt. South Asia, 56. (1896).

mollis Ost.-Sack., Annal. Mus. Civ. Genova, XVI. 415. [Nerua] (1881); v. D. Wulr, Catal. Dipt. Soutb Asia, 56. (1896).

pallipes $\mathrm{Br}_{\mathrm{r}}=$ scenopinoides WALK.

pictipes Bı́., Annal. Soc. Entom. France, sér. 5. IX. 221. 5. (1879); v. D. Wulp, Catal. Dipt. South Asia, 56. (1896) et Természetrajzi Füzetek, XXI. 416. 20. (1898).

scenopinoides Walk., Proc. Linn. Soc. London, III. 81. 17. [Nerua] (1859) et VII. 223. 2, 232. 6. [Nerua] (1864); Ost.-SAck., Annal. Mus. Civ. Genova, XVI. 415. [Neria] (1882); v. D. Wulp, Notes Leydon Mus., VII. 57. 2. (1885), Catal. Dipt. South Asia, 56. (1896) et Természetrajzi Füzetek, XXI. 415. 18. (1898).

pallipes Big., Annal. Soc. Entom. France, sér. 5. IX. 220. 4. (1879).

\section{Culcua}

Walk., Proe. Linn. Soe. London, I. 109. (1857).

simulans Walk., Proc. Linn. Soc. London, I. 109. 24. tab. VI. fig. Borneo. 1. (1857) ; v. D. Wulp, Catal. Dipt. South Asia, 57. (1896). 


\section{Platyna}

Wred., Analecta Entomol., 12. (1824).

hastata FABr., Systema Antliat., 84. 24. [Stratiomys] (1805); WiEd., Analecta Entomol., 12. tab., fig. 2. (1824) et Aussereurop. zweifl. Ins., II. 53.1. tab. VIII. fig. 3. $a-c$. (1830) : MAcQ., Suit. à Buffon, I. 256. 1. tab. VI. fig. 8. (1834) et Dipt. exot., I. 1., 192. (1838); Walk., List Dipt. Brit. Mus., V. suppl. 1., 64. 1. (1854); Rö̀., Wien. Entom. Zeitg., XI. 271. (1892).

\section{Cynipimorpha}

Brav., Denkschr. Akad. Wien, XLIV. 75. (1881).

Bilimecki Brau., Denkschr. Akad. Wien, XLIV. 75. (1881); Aldr., Mexico. Catal. Nortl Amer. Dipt., 191. (1905).

minuta Willist,; Biolog. Central. Amer., Dipt., I. 252. 1. tab. IV. Mexico. fig. 19, 19a. (1901); Aldr., Catal. North Amer. Dipt., 191.(1905).

\section{Ptilocera}

\section{Wied., Nova Dipt. Gen., 7. (1820).}

amethystina Sxelt. v. Vollenh., Tijdschr. v. Entomol., I. 92. 2. Areh.Indicus, (1857) ; Ost.-SAck., Berlin. Entom. Zeitschr., XXVI. 100. (1882); I.Philippinae. v. D. Wulp, Catal. Dipt. South Asia, 56. (1896).

continua WALk., Iusect. Saunders., Dipt., I. 84. tab. III. fig. 2. (1851) Java. et List Dipt. Brit. Mus., V. suppl. 1., 5. 2. (1854); v. D. Wuls, Catal. Dipt. South Asia, 56. (1896).

fastuosa Gerst., Linnaea Entomol., XI. 332. 2. (1857); Schin., Novara Reise, Dipt., 52. 2. (1868) ; Rö̀., Entomol. Nachricht., XIX. 234. 2. (1893) ; v. D. Wulp, Catal. Dipt. South Asia, 56. (1896).

lateralis MACQ., Dipt. exot., suppl. 1., 48. 1. (1846); WaLk., List Patriaignota. Dipt. Brit. Mus., V. suppl. 1., 6. 4. (1854).

natalensis GERST. = quadrilineata FABR.

quadridentata FABR., Systema Antliat., 86. 33. [Stratiomys] (1805); Wren., Nova Dipt. Gen., 7. (1820) et Ausserenrop. zweifl. Ins., II. 59. tab. VIII. fig. 4. $a-d$. (1830); MACQ., Suit. à Buffon, I. 240. 1. tab. VI. fig. 1. (1834); WaLK., List Dipt. Irit. Mus., III. 525. (1849) et V. suppl. 1., 5. 3. (1854) ; Dor., Natuurkund. Tijdschr. Nederl. Indie, 11. ser. VII. (X). 407. 14. (1856); WaLk., Proc. Linn. Soc. London, I. 7. 9 et 108. 18. (1856); Gerst., Linnaea Entomol., XI. 332. 1. (1857); Snel.. v. Vollenh., 'Tijdselır. v. Entomol., I. 91.1. (1857); Dou., Natuurkund. Tijdschr. Nederl. Indie, ser. 4. III. (XVII.) 81. 8. (1858); WALK., Proc. Linn. Soc. London, III. 78. 6. (1859), V. 145. 6, 232. 11, 270. 1. (1861) et VI. 4. 2, 17. 1, 20. 1. (1862); v. D. Wulr, Tijdschr. v. Entomol.,

A sia merid,, Arch.Indicus, Nova Guinea. 
XXIII. 163. 10. (1880), Sumatra Exped., Dipt., 14. 1. (1881), Catal. Dipt. South Asia, 55. (1896) et Természetrajzi Füzetek, XXI. 415. 17. (1898); MEIJ., Bijdragen tot de Dierkunde, XVIII. 96. tab. VIII. fig. 12. (1904).

quadrilineata FABR., Mantissa Insect., II. 331. 16. [Strutiomys] (1787); Guel., Systema Naturae, V. 2835. 159. [Musca] (1792); FABr., Eutomol. System., IV. 268. 23. [Stratiomys] (1794) et Systema Antliat., 86. 34. [Stratiomys] (1805); S't. FArg. et Serv., Encyclop. Méthod., X. 803. 2. [Beris] (1825); Wred., Aussereurop. zweifl. Ins., II. 72. 19. [Stratiomys] (1830); WaLK., List Dipt. Brit. Mus., V. suppl. 1., 50. 93. [? Stratiomys] (1854); Lw., Öfvers. Kong]. Vet. Akad. Förhandl., XIII. 264. 14. (1856) et Dipt.-Fauna Siidafrika's, I. 13. 1. (1860).

natalensis Gerst., Linnaea Entomol., XI. 334. 3. (1857); ? WaLK.,

Trans. Entom. Soc. London, n. ser. V. 268. (1860).

smaragdifera WaLK., Proc. Linn. Soc. London, IV. 94. 12. (1860); Celebes.

v. D. Wul, Catal. Dipt. South Asia, 56. (1896).

smaragdina Snell. v. Volbenr., Tijdschr. v. Entomol., I. 92. 3. Arch.Indicus, (1857) ; Ost.-SAck., Annal. Mus. Civ. Genova, XVI. 412. (1882). Nova Guinea, smaragdina Walk., List Dipt. Brit. Mus., III. 525. (1849), V. suppl. Philippinae, 1., 5. 1 et 303. (1854) et Proc. Jinn. Soc. London, IV. 94. 11. Ceylon. (1860); v. D. Wulp, Catal. Dipt. South Asia, 55. (1896).

\section{Trichochaeta}

Bıg., Annal. Soc. Entom. France, sér. 5. VIII. Bull., XXII. (1878).

nemoteloides Big., Annal. Soc. Entom. France, sér. 5. VIII. Bull., Ternate. XXII. (1878) et sér. 5. IX. 191. (1879); v. D. Wulp, Catal. Dijt. South Asia, 55. (1896).

\section{Wallacea}

Dol., Natuurkund. Tijdschr. Nederl. Ind., ser. 4. III. (XVII). 82. (1858).

Gabaza Walk., Proc. Linn. Soc. London, III. 80. (1859).

argentea DoL., Natuurkund. Tijdschr. Nederl. Ind., ser.4 III. (XVII). Asia mer., 82. 11. (1858); v. D. Wulp, Catal. Dipt. South Asia, 57. (1896), Arch.Indicus, Természetrajzi Füzetek, XXI. 417. 23. (1898) et Tijdschr. v. NovaGuinea. Entomol., XLII. 53. (1899).

argentec WALK., Proc. Linn. Soc. London, III. 80. 13. [Gabaza] (1859) ; v. D. Wurp, Catal. Dipt. South Asia, 57. [Gabaza] (1896).

argentea $\mathrm{WALK}_{\mathrm{AL}}=$ argentea DoL. 


\section{Sternobrithes}

Lw., Dipt.-Fauna Südafrika's, I. 11. VIII. (1860).

Stenobrithes Lw., Öfv. Kongl. Vet. Akad. Förhandl., XIII. 264. 9. (1856) [lapsus].

tumidus Lw., Öfv. Kongl. Vet. Akad. Förhandl., XIII. 264. 13. Africa mer. [Stenobrithes] (1856) et Dipt.-Faura Südafrika's, I. 12. 1. tab. I. fig. 3-7. (1860) ; Johns., Proc. Acad. Nat. Sci. Philad., 1898. 157. (1898).

\section{Gobertina}

Bı̊., Annal. Soc. Entom. France, sér. 5. IX. 192 et Bull., LXVIII. (1879). argentata $\mathrm{BIG} .=$ picticornis $\mathrm{BIG}$ argentea $\mathrm{BIG} .=$ picticornis $\mathrm{BIG}$.

picticornis Big., Annal. Soc. Entom. France, sér. 5. IX. 192. 1. Sierra Leone. (1879).

argentata Big., Annal. Soc. Entom. France, sér. 5. IX. Bullet., LXVIII. (1879).

argentea Big., Annal. Soc. Entom. France, sér. 5. IX. 193. 2. (1879).

\section{Acraspidea}

Brau., Denkschr. Akad. Wien, XLIV. 75. (1881).

Felderi Brau., Denkschr. Akad. Wien, XLIV. 76. (1881); v. D. Wulp, Ceylon. Catal. Dipt. South Asia, 58. (1896).

\section{Tinda}

Walk., Proc. Linn. Soc. London, IV. 101. (1860).

Biastes Walk., Ins. Saund., Dipt., I. 80. (1851) [nec Panz. Hym., 1806].

Elasma Jaenn., Abhandl. Senckenb. Naturf. Ges., VI. 322. 10. (1867).

Phyllophora Macq., Mém. Soc. Scient. Lille, 1834. 507.(1835) [nec Thunb. Orthopt., 1812).

acanthinoidea Jakns., Abhandl. Senckenberg. Naturf. Ges., VI. 323. Java.

11. tab. XLIII. fig. 3. [Elasma] (1867); v. D. Wulp, Catal. Dipt.

South Asia, 57. (1896).

angusta WALK., Proc. Linn. Soc. London, I. 7. 15. [Phyllophora] Malacea.

(1856) ; v. D. Wulp, Catal. Dipt. Soutl Asia, 56. [Phyllophora]

(1896).

bispinosa THoMs. = indica WALK.

Cormasa $\mathrm{WALK}_{\mathrm{ALK}}=$ nigra $\mathrm{MACQ}$. 
indica WALk., Insecta Saunders., Dipt., I. 81. tab. III. fig. 3. [Biastes] (1851) et List Dipt. Brit. Mus., V. suppl. 1., 84. 1. [Biastes] (1854) ; Ost.-SAck., Annal. Mus. Civ. Genova, XVIII. 17. [Biastes] (1883) ; v. D. Wulp, Notes Leyden Mus., VII. 57. 3. (1885) et Catal. Dipt. South Asia, 57. (1896); MeıJ., Bijdragen tot de Dierkunde, XVIII. 96. (1904).

bispinosa Tuons., Eugenies Resa, Dipt., 454. 16. [Phyllophora] (1869).

modifera Walk., Proc. Linn. Soc. London, IV. 101. 30. (1860);

Ost.-SAck., Annal. Mus. Civ. Genova, XVI. 413. (1882).

modiferc WALK. = indica WALK.

nigra Macq., Mém. Soc. Scient. Lille, 1834. 508. tab. V. fig. 1-6.

Africa.

[Phyllophora] (1835) et Dipt. exot., I. 1., 178. 1. tab. XXII. fig.

1. [Phyllophora] (1838) ; Walk., List Dipt. Brit. Mus., V. suppl. 1., 24. 1. [Phyllophora] (1854).

Cormasa Walk., List Dipt. Brit. Mus., III. 524. [Clitellaria, ? Cyclogaster] (1849).

pallipes Bı̈., Annal. Soe. Entom. France, sér. 5. IX. 206. 1. [Biastes] (1879).

recedens WALK., Proc. Linn. Soe. London, V. 233. 15. (1861); v. D. NovaGuinea. Wulp, Catal. Dipt. South Asia, 57. (1896).

vicina Big., Annal. Soc. Entom. France, sér. 5. IX. 206. 2. [Biastes] Sierra Leonも. (1879).

\section{Rosapha}

Walk., Proc. Linn. Soc. London, IV. 100. (1860).

Calcochaetis Bıg., Annal. Soc. Entom. France, sér. 5. IX. 189. (1879).

Calochaetis Bıg., Annal. Soc. Entom. France, sér. 5. VII. Bull., LXXIV.

bicolor Big., Annal. Soc. Entom. France, sér. 5. VII. Bullet. LXXIV. (sine descr.) [Calochaetis] (1877) et sér. 5. IX. 189. [Calcochaetis] (1879); Osт.-SAck., Berlin. Entom. Zeitschr., XXVI. 99. (1882); v. D. Wulp, Catal. Dipt. South Asia, 58. (1896).

bimaculata v. D. WulP (descriptio) apud MeIJ., Bijdragen tot de Dier- Java. kunde, XVIII. 96. tab. VIII. fig. 13-14. (1904).

habilis Walk., Proc. Linn. Soc. London, IV. 100. 28. (1860); Ost.- I.Philippinae, SAck., Annal. Mus. Civ. Genova, XVI. 413. (1882); v. D. Wulp, Celebes.

Catal. Dipt. South Asia, 58. (1896). 


\section{Pachygaster}

MeIG. Illiger's Magaz. f. Ins., II. 266. 34. (18vi3).

Neopachygaster Ausr., Entom. Monthly Mag., XXXVII. 24i). (1901).

Vappo Latr., Hist. Nat. Crust. et Ins., XIV. 343. D. (1804). argentifer J JENN. = meromelas DuF.

atra Panz., Fauna Germ., LIV. tab. 5. [Nemotelus] (1798); LATr., Dict. Europa. d'Hist. Nat., XXIV. 193. 555. [Vappo] (1804); MeIG., Klassif., I. 146. 1. tab. VIII. fig. 19-24. (1804); Latr., Hist. Nat. d. Crust. et d'Ins., XIV. 343. [Vappo] (1804); FABr., System. Antliat., 254. 1. [Vappo] (1805) ; Latr., Gen. Crust. et Ins., IV. 278. [Vappo] (1809) et Consid. génér., 442. [Vappo] (1810); Lam., Hist. nat. anim. sans vertébr., III. 388. 3. [Oxyeera] (1816); Meiq., System. Beschreib., III. 102. 1. tab. XXIV. fig. 16-23. (1822); St. FArG. ET Serv., Encyclop. Méthod., X. 779. [Tappo] (1825); MacQ., Recueil. Soc. Sciec. Agricult. Lille, 433 tab. II. fig. 7. (1826); Schill., Entomol. Beitr., I. 94. tab. VIII. fig. 8. (1829) ; Meig., System. Beschreib., VI. 344. (1830); MAcQ., Suit. à Buff., I. 264. 1. tab. VI. fig. 14. [Vappo] (1834); Gut́r., Iconogr. d. Régne Anim., Ins., 545. tab. XCVIII. fig. 11. [Vappo] (1835); Duncan, Magaz. Zoolog. and Botany, I. 166. 1. fig. 1-3. (1837) ; MEIG., System. Beschreib., VII. 103. (1838); Lw., Programm Posen, 1840. 23. 1. (1840) et Isis, 1840. VII. 552. 1. (1840); Westw., Introd., II. 532. 127. 9. (1840) ; BuANch., Hist. Ins., IJI. 594.[ Tappo] (1840) ; ZETr., Dipt. Scand., I. 152. 1. (1842); WALK., List Dipt. Brit. Mus., III. 513. (1849) ; Schorrz, Breslau. Entom. Zeitschr., III. 19. (1849) ; Zetr., Dipt. Scand., VIII. 2961. 1. (1849); WaLk., Ins. Britann., Dipt., I. 27. 1. tab. I. fig. 7. (1851) ; Heeger, Sitzgsber. d. math.-naturwiss. Classe d. k. Akad. Wien, X. 176-178. tab. VI. fig. 1-8. (1853); WALk., List Dipt. Brit. Mus., V. suppl, 1., 101. 1. (1854); Schin., Verh. zool.-bot.Ver. Wien, V. 652. 1. et 673. (1855) et Fauna Austriaca, Dipt., I. 3. (1862); JAEnn., Berlin. Entom. Zeitschr., X. 218. 1. (1866) ; Lw., Zeitschr. f. d. ges. Naturwiss., N. F. I. (XXXV). 269. 1. (1870); v. D. Wulp, Diptera Neerlandica, I. 445. 1. tab. XIV. fig. 4-5. (1877); Becher, Denkschr. Akad. Wien, XLV. 140. tab. II. fig. 5. (1882); Neuhaus, Diptera Marchica, 77. 1. (1886); Brunerti, The Entomologist, XXII. 82. 1. (1889); Sсносн, Fauna Ins. Helvetiae, Dipt., 20. (1890) ; É. \& L. Coucke, Annal. Soc. Entom. Belgique, XXXVII. 436. (1893); Kert., Pótfüz. a Természettud. Közlönyhöz, XXXII. 122. (1900).

pachygaster FALL., Dipt. Suec., Stratiom., 13. 1. p. p. [Sargus] (1817).

flavipes O. G. Costa = Leachii CurT.

frontalis Ouiv. $=$ ? meromelas L. DuF. 
lativentris v. D. Wulr, Természetrajzi Füzetek, XXI. 416. 21. (1898) Nova Guinea. et Tijdschr. v. Entomol., XLII. 47. (1899).

Leachii Curt., Brit. Entomol., VIII. 42. tab. (1824); Duncan, Magaz. Europa. Zoolog. and Botany, I. 167. 2. (1837); WALK., List Dipt. Brit. Mus., III. 514. (1849), Insecta Britan., Dipt., I. 27. 2. (1851) et List Dipt. Brit. Mus., V. suppl. 1., 101. 3. (1854); Schin., Verh. zool.-bot. Ver. Wien, V. 653. 3 et 673. (1855) et Fauna Austriaca, Dipt., I. 3. (1862); Jaens., Berlin. Entom. Zeitschr., X. 221. 3. (1866) ; Lw., Zeitschr. f. d. ges. Naturwiss., N. F. I. (XXXV.) 270. 4. (1870); v. D. Wulp, Diptera Neerland., I. 446. 2. (1877); Neunaus, Diptera Marchica, 77. 2 (1886); Brunetri, The Entomologist, XXII. 82. 2. (1889) ; Schoch, Fauna Insect. Helvetiae, Dipt., 20. (1890) ; E. \& L. Coucke, Annal. Soc. Entom. Belgique, XXXVII. 436. (1893); Kent., Pótfüz. a Természettud. Közlönyhöz, XXXII. 121. (1900).

flavipes O. G. Costa, Atti Accad. Sci. Napoli, V. 2., 87. 5. tab. I. fig. 3. (1844).

pallidipennis Mera., System. Beschreib., VII. 104. 2. (1838). pallipennis Macq., Suit. à Buffon, I. 265. 2. (1834); Zuтт., Dipt. Scand., I. 153. 3. (1842) et VIII. 2961. 3. (1849); L. Duf., Mém. Acad. des Scienc., Matl. et Phys., XI. 171-360. tab. IV. fig. 45. [Vappo] (1850); WahlBG., Öfvers. Kongl. Vet. Akad. Förhland., XI. 212. 3. (1854) ; Zетт., Dipt. Scandinav., XII. 4557. 3. (1855).

limbipennis v. D. Wulp, Természetrajzi Füzetek, XXI. 417. 22. (1898) Nova Guinea. et Tijdschr. v. Entomol., XLII. 47. (1899).

maculicornis Hrne, Ohio Natural., II. 228. (1902); Aldr., Catal. Americasept. North Amer. Dipt., 192. (1905).

meromelaena Austen = meromelas L. Duf.

meromelas L. Duf., Annal. Scienc. Natur., sér. 2. XVI. 266. tab. Europa. XIV, A., fig. 17-19. (18t1); WaLK., List Dipt. Brit. Mus., V. suppl. 1., 101. 4. (1854); Schrn., Verh. zool.-bot. Ver. Wien, V. 652. 2. (1855) ; Lw., Zeitschr. f. d. ges. Naturwiss., N. F. I. (XXXV). 270. 3. (1870) et Beschreib. europ. Dipt., II. 57. Anmerk. (1871).

argentifer JAENn., Berlin. Entom. Zeitschr., X. 221. 4. (1866.) ? frontalis Ouıv., Encyclop. Méthod., VIII. 184. 7. [Nemotelus] (1811) ; MAcq., Suit. à Buffon, I. 266. 4. [Nemotelus] (1834); Meig , System. Beschreib., VII. 105. 10. [Nemotelus] (1838); Lw., Iinnaea Entomol., I. 459. 22. [Nemotelus] (1846); WaLK., List Dipt. Brit. Mus., V. suppl. 1., 82.8 et 317. [Nemotelus] (1854).

meromelaena Austen, Entom. Monthly Mag., XXXVII. 243. [Neopachygaster] (1901).

orbitalis WahlbG., Öfv. Kongl. Vet. Akad. Förhandl., XI. 212. 4. (1854) ; Zetr., Dipt. Scand., XII. 4557. 5. (1855); Sharr, Entom. Monthly Mag., ser. 2. XIV. (XXXIX). 222. (1903). 
pachygaster FALL., Dipt. Suec., Stratiom., 13. 1. p. p. [Sargus] (1817).

minutissima ZETT., Insecta Lappon., Dipt., 575. Obs. (1838), Iipt. Europac.ets. Scand., I. 153. 4. (1842), VIII. 2961. 4. (1849) et XI. 4261. 4. (1852) ; Walk., List Dipt. Brit. Mus., V. suppl. 1., 101. 5. (1854); Lw., Zeitschr. f. d. ges. Naturwiss., N. F. I. (XXXV). 271. 5. (1870) ; Мıк, Verh. zool.-bot. Ges. Wien, XXX. 590. 1. (1880) et Wien. Entom. Zeitg., IX. 290 et 291. (1890); Sharr, Entom. Monthly Mag., ser. 2. XIV. (XXXIX). 221. (1903).

pini Perr., Annal. Soc. Entom. France, sér. 4. X. 208. 14. tab. III. fig. $80-88$. (1870).

tenella JaEns., Berlin. Entom. Zeitschir., X. 22\%. 5. (1866).

var. unicolor JAEnN., Berlin. Entom. Zeitschr., X. 225. 5. var. Europacentr. a. (1866).

orbitalis $\mathrm{W}_{\text {AHLBG. }}=$ meromelas L. DuF.

pachygaster FALL. p. p. = atra PANz.

pachygaster FALL. p. p. = meromelas L. DuF.

pallidipennis MeIG. = Leachii CuRT.

pallipennis MaCQ. = Leachii CurT.

pini PerRIs = minutissima ZETT.

pulchra Lw., Berlin. Entom. Zeitschr., VII. 10. 16. (1863) ; Towns.,

America sept.

Trans. Amer. Entom. Soc. Philad., XXII. 55. 1. (1895); Aldr.,

Catal. North Amer. Dipt., 192. (1905).

pulchris Willist., Canad. Entomol., XVII. 128. (1885).

pulchris WiLLIST. = pulchra Lw.

vobusta JAENv. = tarsalis ZETT.

rufitarsis MAcQ., Dipt. exot., suppl. 1., 57. 1. tab. VI. fig. 3. (1846) ;

WALK., List Dipt. Brit. Mus., V. suppl. 1., 102. 6. (1854); v. D.

Wulp, Catal. Dipt. South Asia, 57. (1896).

tarsalis ZETT., Dipt. Scand., I. 152. 2. (1842) et VIII. 2961. 2. (1849); Europac.ets.

WaLk., List Dipt. Brit. Mus., V. suppl. 1., 101. 2. (1854); Scmin.,

Fauna Austriaca, Dipt., I. 3. (1862); Lw. Zeitschr. f. d. ges.

Naturwiss., N. F. I. (XXXV), 270. 2. (1870) ; BrunetTI, The

Entomologist, XXII. 82. 3. (1889) ; Sharp, Entom. Monthly Mag.,

ser. 2. XIV. (XXXIX). 222. (1903).

robusta JAENn., Berlin. Entom. Zeitschr., X. 219. 2. (1866). tenella $\mathrm{J}_{\mathrm{AENN}}=$ minutissima ZETT.

unicolor $\mathrm{J}_{\mathrm{AENN}}$ = = minutissima ZETT. var.

\section{Zabrachia}

Coquill., 47. Bull. New York State Mus., 1901. 585. (1901).

polita Coquill., 47. Bull. New York State Mus., 1901. 585. (1901); America sept. Aldr., Catal. North Amer. Dipt., 192. (1905). 


\section{Obrapa}

Walk., Proc. Linn. Soc. London, III. 82. (1859).

argentata v. D. Wulp, Természetrajzi Füzetek, XXI. 417. 24. tab. XX. fig. 5, 5a. (1898) et Tijdschr. v. Entomol., XLII. 47. (1899). celyphoides Walk., Proc. Linn. Soc. London, III. 83. 20. (1859) et V. 233. 18 et 273. 11. (1861); v. ग. Wulp, Catal. Dipt. South Asia, 57. (1896).

perilampoides WaLK., Proc. Linn. Soc. London, III. 82. 19. (1859), V. 233. 17, 273. 10, 300. 1. (1861) et VII. 204. 11. (1864); v. D. Wulp, Catal. Dipt. South Asia, 57. (1896).

\section{Lophoteles}

Lw., Berlin. Entom. Zeitschr., II. 110. II. (1858).

Lolphoteles WiLlist., Ent. News Philad., VII.185.(1896). [lapsus]. pallidipennis Willist., Biolog. Central. Amer., Dipt., I. 250. 1. tab. IV. Mexico. fig. 16. (1901); Aldr., Catal. North Amer. Dipt., 192. (1905). plumula Lw., Berlin. Entom. Zeitschr., II. 111. tab. I. fig. 16-18. Ins. Radak. (1858).

\section{Salduba}

WaLk., Proc. Linn. Soc. London, III. 79. (1859).

Enoplomyia Big., Annal. Soc. Entom. France, sér. 5. IX. 191. (1879).

Euplomyia Bıg., Annal. Snc. Entom. France, sér. 5. VIH. Bull., XXXV. (1878).

areolaris WALK., Proc. Linn. Soc. London, VII. 204. 9. (1864); v. D. Wulp, Catal. Dipt. South Asia, 51. (1896).

cothurnata BıG., Annal. Soc. Entom. France, sér. 5. VIII. Bull., Batjan. XXXV. [Euplomyia] (1878) et sér. 5. IX. 191. [Enoplomyia] (1879) ; v. D. Wulr, Catal. Dipt. South Asia, 58. [Enoplomyia] (1896).

diphysoides Walk., Proc. Linn. Soc. London, III. 79. 9. (1859); Ins. Aru. v. D. Wulp, Catal. Dipt. South Asia, 51. (1896).

exigua v. D. WulP, Természetrajzi Füzetek, XXI. 413. 13. tab. XX. Nova Guinea. fig. 3. (1898) et Tijdschr. v. Entomol., XLII. 47. (1899).

gradiens Walk., Proc. Linn. Soc. London, VII. 203. 8. (1864) ; Ost.SAck., Annal. Mus. Civ. Genova, XVI. 412. (1882); v. D. Wulp, Catal. Dipt. South Asia, 51. (1896) et Természetrajzi Fiizetek, XXI. 412. 11. tab. XX. fig. 1, 1a. (1898).

hilaris Walk., Proc. Linn. Soc. London, V. 271. 5. (1861); v. D. Wulp, Batjan. Catal. Dipt. South Asia, 51. (1896).

Nova Guinea, I. Mysol. 
lugubris Walk., Proc. Linn. Soc. London, V. 271. 6. (1861); v. D. Batjan.

Wulp, Catal. Dipt. South Asia,. 51. (1896).

signatipennis v. D. Wulp, Természetrajzi Fiizetek, XXI. 412. 12. tab. NovaGuinea.

XX. fig. 2, 2a. (1898) et Tijdschr. v. Entomol., XLII. 47. (1899).

singularis Walk., Proc. Linn. Soc. London, V. 272. 9. (1861); Ost.- Nova Guinea,

SAck., Annal. Mus. Civ. Genova, XVI. 412. (1882); r. D. Wut.p, Batjan.

Catal. Dipt. South Asia, 51. (1896).

\section{Clitellariinae.}

\section{Pycnomalla}

Gerst., Linnaea Entomol., XI. 319. (1857).

auriflua Erschs. in WAgNer: Reisen in den Regentsch. Algier, III. -193. 56. tab. IX. [Stratiomys] (1841); MaCQ., Explor. scient. de l'Algérie, Zool. III. 428. 39. [Stratiomys] (1819); WALK., List Dipt. Brit. Mus., V. suppl. 1., 311. [Stratiomys] (1854).

splendens FABr., Mantissa Insect., II. 330. 3. [Stratiomys] (1787); Europa mer. GmeL., Systema Naturae, V. 2833. 150. [Musca] (1792); FAbr., Entomol. System., IV. 264. 4. [Stratiomys] (1794); MeIG., Ǩlassif., I. 127. a. [Stratiomys] (1804); CoqueB., Illustr. Ieon. Insect., 116. tab. XXVI. fig. 2. [Stratiomys] (180t); Fabr., Systema Antliat., 78. 2. [Stratiomys] (1805); Wied. apud Muig., System. Beschreib., III. 142. 10. [Stratiomys] (1822); WaLk., I ist Dipt. Brit. Mus., III. 534. [Odontomyia] (1849) et V. suppl. 1., 30. 11. [Stratiomys] (1854); Gerst., Linnaea Entomol., XI. 319. (1857); Schis., Fauna Austriaca, Dipt., I. 13. (1862).

\section{Potamida}

Meig., Nouvelle Classification, 22. 30. (1800).

Clitellaria Meig., Illiger's Magaz. f. Ins., II. 265. 30. (1803). Ephippiomyia Bezzi, Zeitschr. f. syst. Hymen. u. Dipt., II. 191. 1. (1902).

Ephippium Latr., Hist. Nat. d. Crust. et d. Ins., XIV. 341. CCCCXCVIII. (1804).

angusta MACQ. = bilineata FABR.

bilineata FABr., Systema Antliat., 79. 5. [Stratiomys] (1805); MAcQ., Dipt. exot., I. 1., 191. [Ephippium] (1838); v. D. Wulf, Sumatra

Exped., Dipt., 14. 1. [Ephippium] (1881), Notes I.eyden Mus., Japonia, Asia mer., Arch.Indicus.

VII. 59. 5. [Negritomyia] (1885) et Catal. Dipt. South Asia, 5i3. [Ephippium] (1896).

angusta Mace., Suit. à Buffon, I. 252. 2. [Ephippium] (1834): 
Walk., List Dipt. Brit. Mus., V. suppl., 1., 62. 13. [Clitellaria] (1854).

bivittata Wien., Zoolog. Magaz., I. 3., 6. 9. [Clitellaria] (1819) et Aussereurop. zweifl. Ins., II. 46. 1. [Clitellaria] (1830); Walk., List Dipt. Brit. Mus., V. suppl. 1., 62. 11. [Clitellaria] (1854) et Proc. Linn. Soc. Iundon, I. 7. 11. [Clitellaria] (1856) ; Snell. v. Vollfinh., Tijdschr. v. Entomol., I. 93. [Clitellaria] (1857); Watк., Proc. Linn. Soc. London, III. 80. 12. [Clitellaria] (1859) et VI. 4. 4. [Clitellaria] (1862); BraU., Denkschr. Akad. Wien, XLIV. 76. [Engonia] (1881). spinigera Dol., Natuurkund. Tijdschr. Nederl. Indie, n. ser., VII. (X). 407. 13. tab. IX. fig 2. [Ephippium] (1856); BraU., Denkschr. Akad. Wien, XLIV. 76. [Engonia] (1881); Osт.SACK., Borlin. Entom. Zeitschr., XXVI. 373. [Ephippium] (1882) ; Brauer, Offenes Schreiben etc., 7. 20. [Ephippium] (1883).

spinithorax Macq., Dipt. exot., suppl. 3., 17. 3. tab. I. fig. 7. [Rhaphiocera] (1848); WaLk., List Dipt. Brit. Mus., V. suppl.

1., 79. 6. [?Rhaphiocera] (1854).

tenebrica WaLk., List Dipt. Brit. Mus., III. 522. [Clitellaria] (1849) et V. suppl. 1., 62. 10. [Clitellaria] (1854); Coqullu., Proc. Unit. Stat. Nat. Mus., XXI. 308. [Ephippium] (1898). bivittata WiED. = bilineata FABR.

cinerea DoL., Natuurkund. Tijdschr. Nederl. Indie, ser. 3. IV. 403. [Odontomyia] (1857); Ost.-SAck., Annal. Mus. Civ. Genova, XVI. 411. [? Nigritomyia] (1882) ; v. D. Wulp, Catal. Dipt. Sontlı Asia, 54. [Ephippium] (1896).

ephippium FaBr., Systema Entomol., 759. 2. [Stratiomys] (1775) et Amboina.

Spec. Ins., 417. 2. [Stratiomys] (1781); Hersst, Gemeinn. Naturg. d. Thierroich., VIII. tab. CCCXXXIX. fig. 3. [Musca] (1787) ; Fabr., Mantissa Insect., II. 330. 4. [Stratiomys] (1787); Rossi, Fauna Etrusca, II. 279. 1439. [Stratiomys] (17:0) ; Gner., Systema Naturae, V. 2834. 151. [Musca] (1792) ; Panz., Fauna Germ., VIII. tab. 23. [Stratiomys] (1793); FABr., Entomol. System., IV. 264. 6. [Stratiomys] (1794); Schrank, Fauna Boica, III. 94. 2379. [Stratiomys] (1803) ; Schellenb., Gattungen d. Fliegen, 36 et 37. tab. XXIV. fig. 1-2. [Stratiomys] (1803) ; MeIg., Klassif., I. 134. 1. tab. VII. fig. 27-30. [Clitellaria] (1804); FABr., Systema Antliat., 79. 4. [Stratiomys] (1805); Rossi, Fauna Etrusca, Ed. II., 431. 1439. [Stratiomys] (1807); LAM., Hist. nat. anim. sans vert., III. 387. 4. [Stratiomys] (1816); Cuv., Régne Animal., III. 623. [Oxycera] (1817); Meit., System. Beschreib., III. 122. 4. tab. XXV. fig. 25. [Clitellaria] (1822); MacQ., Recueil Soc. Sc. Agricult. Lille, 1826. 442. [Clitcllaria] (1826); Ros., Wiirttemberg. Correspondbl., I. 267. [Clitellaria] (1834); Duncan, Magaz. Zool. and Botany, I. 155. 1. fig. 1-3. [Clitellaria] (1837); Zeller, Isis, 1842. XI. 826. 9. tab. I. fig. 35-37. [Clitellaria] (1842); MärKEL, 
Germar's Zeitschr. f. Entomol., V. 266, 284 et 478. [Clitellaria] (1844); Walk., List Dipt. Brit. Mus., III. 521. [Clitellaria] (1849); Schoutz, Zeitschr. f. Entomol. Breslau, III. 20. [Clitellaria] (1849) ; Zeтt., Dipt. Scand., IX. 3710. 1. [Clitellaria] (1850); WaLK., Insecta Britann., Dipt., I. 24. 1. tab. I. fig. 5. [Clitellaria] (1851) et List Dipt. Brit. Mus., V. suppl. 1., 59. 1. [Clitellaria] (1854); Zeтt., Dipt. Scand., XII. 4555. 1. [Clitellaria] (1855); Rudow, Entomol. Naclıricht., XIV. 148. [Clitellaria] (1888); BezzI, Katal. d. paläark. Dipt., II. 4. [Ephippiomyia] (1903) ; Spels., Zeitschr. f. wissenscb. Insektenbiol., I. 406. 152. [Clitellaria] (1904).

inda Schrank, Enum. Ins. Austr., 438. 891. [Musca] (1781); Donov., Brit. Ins., XVI. 37. tab. 599. [Musca]'(1813); MıLL., Engrav. of Ins., tab. I. fig. 6 [Musca] (1759).

thoracica LAtr., Dict. d'Hist. Nat., XXIV. 192. 553. [Ephippium] (1804), Hist. Nat. d. Crust. et d. Ins., XIV. 341. 1. [Ephippium] (1804), Gen. Crust. et Ins., IV. 276. [Ephippium] (1809) et Consid. génér., 442. [Ephippium] (1810); MACQ., Suit. à Buffon, I. 252. 1. tab. VI. fig. T. [Ephippium] (1834); Guér., Iconogr. d. Régne Anim., Ins., 544. tab. XCVIII. fig. 7. [Ephippium] (1835); Westw., Introd., IJ. 533. fig. 127. [Ephippium] (1840); BцANch., Hist. Ins., III. 594. [Ephippium] (1840) ; Duf., Mém. Acad. d. Scienc., Matl. et Pys., XI. tab. IV. fig. 43. [Ephippium] (1850); Schin., Verl. zool.-bot. Ver. Wien, V. 638. 1. [Ephippium] (1855) et Fauna Austriaca, Dipt., I. 9. [Ephippium] (1862) ; JAEnv., Berlin. Entom. Zeitschr., X. 225. 17. [Ephippium] (1866); v. D. Wulp, Diptera Neerland,, I. 450. tab. XIV. fig. 10. [Ephippium] (1877); Neuraus, Diptera Marchica, 79. 1. [Ephippium] (1886); BrunetTi, The Entomologist, XXII. 82. 1. [Ephippium] (1889); Sсносн, Fauna Ins. Helvetiae, Dipt., 20. [Ephippium] (1890); É. \& L. Coucke, Annal. Soc. Entom. Belgique, XXXVII. 437. [Ephippium] (1893); StrobL, Mittheil. Naturwiss. Ver. Steiermark, XXIX. 1892. 8. [Ephippium] (1893); Kert., Pótfüiz. a Természettud. Közlönyhöz, XXXII. 124. [Ephippium] (1900).

gavisa Walk., Proc. Linn. Soc. London, IV. 95. 17. [Clitellaria] Celebes. (1860) ; v. D. Wurp, Catal. Dipt. South Asia, 54. [Ephippium] (1896).

inda SCHRANK = ephippium FABR.

nigerrima DoL., Natıurkund. Tijdschr. Nederl. Indie, ser. 4. III. (XVII). 81. 9. [Ephippium] (1858); v. D. Wulr, Catal. Dipt. South Asia, 54. [Ephippium] (1896).

responsalis W WLk., Proc. Linn. Soc. London, VIII. 106. 14. [Clitellaria] (1865); v. D. Wulp, Catal. Dipt. South Asia, 54. [Ephippium] (1896).

spinigera DoL. = bilineata $\mathrm{FABR}_{\mathrm{ABR}}$ 
spinithorax MACQ. = bilineata $\mathrm{FABR}_{\mathrm{AB}}$

tenebrica WALK. = bilineata FAPR.

thoracica LATR. = ephippium FABR.

\section{Negritomyia}

Big., Annal. Soc. Entom. France, sér. 5. IX. 190. (1879).

Engonia Brau., Denkschr. Akad. Wien, XLIV. 76. (1881).

Nigritomyia Big., Annal. Soc. Entom. France, sér. 5. VII.

Bull., LXXIV. (1877).

albitarsis Bra., Annal. Soc. Entom. France, sér. 5. IX. 207. 1 [Ephippium] (1879); ? Frogatr, Proc. Linn. Soc. New South Wales, XXI. 84. tab. IX. fig. 12-13. [Ephippium] (1896); v. D.

Wulp, Catal. Dipt. South Asia, 53. (1896).

aurata $\mathrm{BRAU} .=$ festinans WALK.

consobrina Bit., Annal. Soc. Entom. France, sér. 5. IX. 208. 2

[Ephippium] (1879); v. D. Wulp, Catal. Dipt. South Asia, 53.

(1896); Sharp in Willey : Zoological Results, IV. 389. [Engonia] (1900).

festinans Walk., Proc. Linn. Soc. London, IV. 95. 16. [Clitellaria] (1860) ; Ost.-SAck., Annal. Mus. Civ. Genova, XVI. 410. [Ephippium] (1882) et Berlin. Entom. Zeitschr., XXVI. 373. (1882); v. D. Wulp, Catal. Dipt. South Asia, 53. (1896).

aurata Brad., Denkschr. Akad. Wien, XLIV. 76. [Engonia] (1881).

maculipennis Lw., Öfvers. Kongl. Vet. Akad. Förhandl., XIII. 264.

12. [Ephippium] (1856) et Dipt.-Fauna Südafrika's, I. 4. nota. [Ephippium] (1860)'; Brau., Denkschr. Akad. Wien, XLIV. 76. [Engonia] (1881).

maculipennis MacQ., Dipt. exot., suppl. 4., 54. 3. [Ephippium] (1849) ; Schrn., Novara Reise, Dipt., 53. 3. [Ephippium] (1868); Big., Annal. Soc. Entom. France, sér. 5. VII. Bullet., LXXIV. [Nigritomyia] (1877) et sér. 5. IX. 190. (1879) ; Ost.-Sack., Annal. Mus. Civ. Genova, XVI. 410. [Ephippium] (1882) et Berlin. Entom. Zeitschr., XXVI. 99 et 373. (1882); v. D. Wulp, Catal. Dipt. South Asia, 52. [Odontomyia] et 53. (1896), Természetrajzi Fiizetek, XXI. 415. 15. [Nigritomyia] (1898) et Tijdschr. v. Entom., XIII. 52. (1899).

\section{Ampsalis}

Walk., Proc. Linn. Soc. Jondon, IV. 98. (1860).

geniata Wask., Proc. Linn. Soc. London, IV. 99. 26. (1860); v. n. Celebes.

Wulp, Catal. Dipt. South Asia, 48. (1896). 


\section{Octarthria}

BraU., Denkschr. Akad. Wien, XLIV. 67. (1881).

aberrans Schin., Novara Reise Dipt., 55. 11. [Clitellaria] (1868); Brau., Denkschr. Akad. Wien, XLIV. 67. (1881); Hutton, Catal. New Zealand Dipt. ete., 39. [Clitellaria] (1881) et Trans. New Zealand Instit., XXXIII. 10. [Clitellaria] (1901).

\section{Euparyphus}

Gerst., Linnaea Entomol., XI. 314. 3. (1857).

Euparhyphus Ost.-SAck., Biolog. Centr. Amer., Dipt., I. 39. (1886).

albipilosus Adams, Kansas Univ. Scienc. Bullet., II. 30. (1903); Aldr., Catal. North Amer. Dipt., 188. (1905).

amplus Coquill., Proc. Unit. Stat. Nat. Mus., XXV. 100. (1902); AdAms, Kansas Univ. Scienc. Bullet., II. 28. (1903) ; Aldr., Catal. North Amer. Dipt., 188. (1905).

apicalis Coquill., Proc. Unit. Stat. Nat. Mus., XXV. 99. (1902); Adams, Kansas Univ. Scienc. Bullet., II. 28. (1903); Aldr., Catal. North Amer. Dipt., 188. (1905).

atriventris Coquill., Proc. Unit. Stat. Nat. Mus., XXV. 100. (1902); Adams, Kansas Univ. Scienc. Bullet., II. 28. (1903); Aldr., Catal. North Amer. Dipt., 188. (1905).

aureovittatus Bı̈., Annal. Soc. Entom. France, sér. 5. IX. 203. 1. Patria ignota. (1879).

bellus Lw., Berlin. Entom. Zeitschr., X. 11. 18. (1866); WiLlist.,

Canad. Entomol., XVII. 127. (1885) ; Ost.-SAcK., Biolog. Centr. Amer., Dipt., I. 39. (1886) ; ADAMs, Kansas Univ. Scienc. Bullet., II. 28. (1903) ; Aldr., Catal. North Amer. Dipt., 188. (1905).

brevicornis Lw., Berlin. Entöm. Zeitschr., X. 10. 16. (1866); Ost.SAck., Biolog. Ceñtral. Amer.; Dipt., I. 39. (1886) ; Adans, Kansas Univ. Scienc. Bullet., II. 28. (1903); Aldr., Catal. North Amer. Dipt., 188. (1905).

carbonarius Gigl.-Tos, Bollet. Mus. Zool. ed Anat. comp. Torino, VI. No. 102., 2. (1901) et Mem. R. Accad. Scienc. Nat. Torino, ser. 2. XIIII. 108. 9. tab. I. fig. 5. (1893); AdAms, Kansas Univ. Scienc. Bullet., II. 28. (1903) ; ALDR., Catal. North Amer. Dipt., 188. (1905).

crucigerus Coquilu., Proc. Unit. Stat. Nat. Mus., XXV. 99. (1902); Adams, Kansas Univ. Scienc. Bullet., II. 28. (1903); Aldr., Catal. North Amer. Dipt., 188. (1905).

? decemmaculatus Ost.-Sıck., Biolog. Central. Amor., Dipt., I. 39. et 40. 2. [? Euparhyphus] (1886); Adams, Kansas Univ. Scienc. B.llet., II. 28. (1903); Aldr., Catal. North Amer. Dipt., 188. (1905).

elegans Wied., Aussereurop. zweifl. Ins., II. 58. 8. [Cyphomyia] Mexico.

Kertész: Catalogus Dipterorum. III.

America sept.

America sept.

America sept.

America sept.

America sept.

America sept.

Mexico.

America sept.

Mexico. 
(1830) ; Walk., List Dipt. Brit. Mus., V. suppl. 1., 15. 1. [Cyphomyia] (1854); Gerst., Linnaea Entomol., XI. 316. 1. (1857); Ost.-Sack., Biolog. Central. Amer., Dipt., I. 40. (1886); Adans, Kansas Univ. Scienc. Bullet., II. 28. (1903); ALDR., Catal. North Amer. Dipt., 188. (1905).

elongatulus Willist., Biolog. Central. Amer., Dipt., I. 249. 3. tab. Mexico.

IV. fig. 14. (1901); Adams, Kansas Univ. Seienc. Bullet., II. 28. (1903); ALdr., Catal. North Amer. Dipt., 188. (1905).

kabylinus Big., Annal. Soc. Entom. France, sér. 5. IX. 204. 2. Africa sept.

(1879) ; BezzI, Katal. d. palïarkt. Dipt., II. 5. [? Clitellaria] (1903). limbrocutris Adans. - Willist., Canad. Entomol., XVII. 126. [? $n$. sp. sine nom.] (1885). - Apaus, Kansas Univ. Science Bullet.,

II. 28 et 31. (1903); Aldr., Catal. North Amer. Dipt., 188. (1905). major Hine, Oliio Naturalist, I. 112. (1901).

mutabilis Adays, Kansas Univ. Scienc. Bullet., II. 29. (1903) ; ALDR., Catal. North Amer. Dipt., 188. (1905.)

niger Bı̈., Annal. Soc. Entom. France, sér. 5. IX. 204. 3. (1879); Adams, Kansas Univ. Scienc. Bullet., II. 29. (1903); ALdr., Catal. North Amer. Dipt., 188. (1905).

obliquus Hine, Canad. Entomol., XXXVI. 87. (1904).

ornatus Wiluist., Canad. Entomol., XVII. 126. (1885); Ost.-SAck., Biolog. Central. Amer., Dipt., J. 39. (1886) ; Adaus, Kansas Univ. Sci. Bullet., II. 28. (1903) ; Aldr., Catal. North Amer. Dipt., 188. (1905).

septemmaculatus Adams, Kansas Univ. Scienc. Bullet., II. 31. (1903) ; Aldr., Catal. North Amer. Dipt., 189. (1905).

stigmaticalis Lw., Berlin. Entom. Zeitschr., X. 10. 17. (1866); Ost.-Sack., Biolog. Central. Amer., Dipt., I. 39. (1886); Adaмs, Kansas Univ. Sci. Bullet., II. 28. (1903); ALDR., Catal. North Amer. Dipt., 189. (1905).

tahoensis Coquilu., Proe. Unit. Stat. Nat. Mus., XXV. 98. (1902); Adams, Kansas Univ. Sci. Bullet., II. 28. (1903); ALDR., Catal. North Amer. Dipt., 189. (1905).

tetraspilus Lw., Berlin. Entom. Zeitschr., X. 9. 15. (1866); Ost.SACK., Biolog. Central. Amer., Dipt., I. 39. (1886) ; Adays, Kansas Univ. Sci. Bullet, II. 28. (1903); Meland., Canad. Entomol., XXXVI. 20. (1904); Aldr., Catal. North Amer. Dipt., 189. (1905). tricolor Ost.-Sack., Biolog. Central. Amer., Dipt., I. 40. 1. (1886); Adaus, Kansas Univ. Sci. Bullet., II. 28. (1903); Aldr., Catal. North Amer. Dipt., 189. (1905).

\section{Euryneura}

Schin., Verh. zool.-bot. Ges. Wien, XVII. 308. (1867).

elegans Willist., Trans. Amer. Entom. Soc. Philad., XV. 252. 22. (1888) ; Hunter, Trans. Amer. Entom. Soc. Philad., XXVII. 130. (1901) ; Wiluist., Biolog. Central. Amer., Dipt., I. 250. (1901). 
fasciata $F_{A B R}=$ fascipennis FABR.

fascipennis FABR., Systema Antliat., 373 in erratis et pag. 21 in indice. [Strationys] (1805); WiED., Aussereurop. zweifl. Ins., II. 75. ¿5. [Stratiomys] (1830); Walk., List Dipt. Brit. Mus., V. suppl. 1., 44. 64. [Stratiomys] (1851); Schis., Novara Reise, Dipt., 57. 14. [nec tab. II. fig. 5.] (1868); Hunter, Trans. Amer. Entom. Soc. Philad., XXVII. 127. [Stratiomyia] et 130. (1901); Willist., Biolog. Central. Amer., Dipt., I. 250. (1901).

fasciata FABR., Systema Antliat., 84. 26. [Stratiomys] (1805). nasica Wiluss., Trans. Amer. Entom. Soc. Philad., XV. 252. 21. (1888) ; Hunter, Trans. Amer. Entom. Soc. Philad., XXVII. 130. (1901).

propinqua Schin., Novara Reise, Dipt., 57. 15. tab. II. fig. 5. (1868) ; Gigl.-Tos, Mem. R. Accad. Sci. Nat. Torino, ser. 2. XLIII. 111. 13. (1893) ; Hunter, Trans. Amer. Entom. Soc. Philad., XXVII. 130. (1901); Willist., Biolog. Central. Amer., Dipt., I. 250. (1901); Aldr., Catal. North Amer. Dipt., 189. (1905).

pygmaea Belu., Saggio di Ditterol. Messic., Append., 12. 1. tab. III. fig. 5. [Clitellaria] (1862); Schin., Novara Reise, Dipt., 57. (1868) ; Gigl.-Tos, Mem. R. Accad. Sci. Nat. Torino, ser. 2. XlIII. 111. 14. (1893) ; Aldr., Catal. North Amer. Dipt., 189. (1905).

\section{Adoxomyia}

Kert., Annal. Mus. Nat. Hung., V. 499. (1907).

Clitellaria Auct. PLur.

argentata WiLList., Canad. Entomol., XVII. 127. [Clitellaria] (1885); Aldr., Catal. North Amer. Dipt., 189. [Clitellaria] (1905).

Balius WALK. = Dahlii MEIG.

cinerascens Lw., Beschreib. europ. Dipt., III. 74. 38. [Clitellaria] (1873) ; Pleske, Sitzgsber. Naturf. Ges. Jurjeff, XIII. 50 et 53. 3. [Clitellaria] (1901).

Dahlii MeIG., System. Beschreib., VI. 346. 5. [Clitellaria] (1830); America sept. Americamer. et centr.

America mer.

Brasilia.

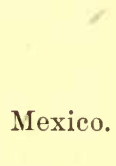

Balius WaLK., List Dipt. Brit. Mus., III. 533. [Odontomyia] (1849) et V. suppl. 1., 26. 1. [Stratiomys] (1854).

fenestrata Macq., Dipt. exot., suppl. 1., 54. 3. [Ephippium] (1846); Mexico.

Transcaspia, Turkestan, Persia.

Europa mer.

W'ALK., List Dipt. Brit. Mus., V. suppl. 1., 61. 6. [Clitellaria] (1854); ÁLdr., Catal. North Amer. Dipt., 189. [Clitellaria] (1905). 
flaviceps WatK., Proc. Linn. Soc. London, I. 7. 13 et 108. 20. Singapore, [Clitellaria] (1856); v. D. Wulp, Catal. Dipt. South Asia, 54. Borneo. [Clitellaria] (1896).

flavipes FaBr., Supplem. Entomol. System., 355. 9-10. [Stratiomys] (1798) et Systema Antliat., 80. 10. [Stratiomys] (1805); WIED., Aussereurop. zweifl. Ins., II. 47. 2. [Clitellaria] (1830); MAcQ., Dipt. exot., I. 1., 191. 1. [Ephippium] (1838); WaLK., List Dipt. Brit. Mus., V. suppl. 1., 61. 8. [Clitellaria] (1854).

heminopla Wied., Zoolog. Magaz., I. 3., 30. 45. [Clitellaria] (1819) et Aussereurop. zweifl. Ins., II. 48. 3. [Clitellaria] (1830) ; MACQ., Dipt. exot., I. 1., 191. [Ephippium] (1838); WALK., List Dipot. Brit. Mus., III. 522. [Clitellaria] (1849) et V. suppl. 1., 62. 12. [Clitellaria] (1854); v. D. Wus, Catal. Dipt. South Asia, 54. [Clitellaria] (1896).

lata Lw., Berlin. Entom. Zeitschr., XVI. 55. 9. [Clitellaria] (1872); Willist., Canad. Entomol., XVII. 127. [Clitellaria] (1885) ; Aldr., Catal. North Amer. Dipt., 189. [Clitellaria] (1905).

notabilis WALK., Proc. Linn. Soc. London, I. 108. 21. [Clitellaria] (1857) ; v. D. Wulp, Catal. Dipt. South Asia, 54. [Clitellaria] (1896). obesa WALK., Trans. Entom. Soc. London, n. ser., V. 270. [Clitellaria] (1860) ; Aldn., Catal. North Amer. Dipt., 189. [Clitellaricl] (1905). obscuripennis Lw., Beschreib. europ. Dipt., III. 72. 36. [Clitellaria] (1873) ; Pleske, Sitzgsber. Naturf. Ges. Jurjeff, XIII. 50 et 55. 5. [Clitellaria] (1901).

Portschinskii Pleske, Sitzgsber. Naturf. Ges. Jurjeff, XIII. 50 et 52. 2. [Clitellaria] (1901).

rufa Walk., List Dipt. Brit. Mus., IIT. 524. [Clitellaria, ? Cyclogaster] (1849) et V. suppl. 1., 64. 17. [Clitellaria] (1854).

ruficornis Lw., Beschreib. europ. Dipt., III. 74. 37. [Clitellaria] (1873) ; Pleske, Sitzgsber. Naturf. Ges. Jurjeff, XIII. 50 et 55. 6. [Clitellaria] (1901).

rufitarsis MACQ., Dipt. exot., I. 1., 192. 2. tab. XXII. fig. 5. [Ephippium] (1838) et Explor. scient. de l'Algérie, Zool., III. 429. 42. tab. I. fig. 9. [Ephippium] (1849) ; WaLk., List Dipt. Brit. Mus., V. suppl. 1., 62. 9. [Clitellaria] (1854).

rustica Ost.-SAck., Bullet. Unit. Stat. Geolog. Surv., III. 213. [Clitellaria] (1877); Beutenm., Bullet. Americ. Mus. Nat. Hist., XX. 87. [Clitellaria] (1904); Al.Dr., Catal. Nortlı Amer. Dipt., 189. [Clitellaria] (1905).

Sarudnyi Pleske, Sitzgsber. Naturf. Ges. Jurjeff, 50 et 53. 2. Persia. [Clitellaria] (1901).

scutellaris WALK., List Dipt. Brit. Mus., V.suppl.1., 313. [Clitellaria] Brasilia. (1854).

stigma Gigl.-Tos, Bollet. Mus. Zool. ed Anat. comp. Torino, VI. Mexico.

No. 102., 2. [Clitellaria] (1891) et Mem. R. Accad. Sci. Nat. Torino, ser. 2. XLIII. 109. 11. [Clitellaria] (1893); Aldr., Catal. North Amer. Dipt., 189. [Clitellaria] (1905). 
subulata Lw., Berlin. Entom. Zeitschr., IX. 147. 29. [Clitellaria] Virginia. (1865); Aldr., Catal. North Amer. Dipt., 189. [Clitellaria] (1905). tibialis Walk., Proc. Linn. Soc. London, V. 258. 1. [Clitellaria] (1861) ; v. D. Wusp, Catal. Dipt. South Asia, 54. [Clitellaria] (1896). unicolor WaLk., List Dipt. Brit. Mus., V. suppl. 1., 61. 7 et 312 . Brasilia. [Clitellaria] (1854).

varia Walk., List Dipt. Brit. Mus., V. suppl. 1., 63. 14 et 305 . note. [Clitelluria] (1854) et Proc. Linn. Soc. London, I. 7. 12 et 108. 19. [Clitellaria] (1856); v. D. Wulp, Catal. Dipt. South Asia, 54. [Clitellaria] (1896).

\section{Musama}

Walk., Proc. Linn. Soc. London, VII. 205. (1864).

paupera WaLK., Proc. Linn. Soc. London, VII. 205. 13. (1864); Ost.-SAck., Berlin. Entom. Zeitschr., XXVI. 100. (1882); v. D. Wulp, Catal. Dipt. South Asia, 55. (1896).

\section{Nemotelus}

Geofrr., Hist. Nat. d'Ins., II. 542. (1764).

Nematotehus Ost.-Sack., Catal. Dipt. N. Amer. Ed. II. 50. nota. (1878).

abdominalis AdAms, Kansas Univ. Sci. Bullet., II. 221. (1903); Meland., Psyche, XI. 33. (1901); Aldr., Catal. North Amer. Dipt., 189. (1905).

acutirostris Lw., Berlin. Entom. Zeitschr., VII. 8. 13. (1863);

Coquill., Proc. Washington Acad. Sci., III. 373. (1901) ; Meland., Psyche, X. 181. 15. (1903); Aldr., Catal. North Amer. Dipt., 189. (1905).

advena WALK., Ins. Saund., Dipt., I. 80. (1851) et List Dipt. Brit. Patriaignota. Mus., V. suppl. 1., 84. 18. (1854).

aemulus Lw., Beschroib. ourop. Dipt., II. 54. 32. (1871). Hispania. acrosus Gıму. $=$ ? Lomnickii Мıк.

albifacies Beck., Mitteil. Zoolog. Mus. Berlin, IJ. 9. 3. (1902).

Aegyptus.

albirostris MAcQ., Dipt. exot., suppl. 4., 55. 3. tab. III. fig. 8. (1849) ; Meland., Psyche, X. 182. 17. tab. IV. (1903); Aldr., Catal. North Amer. Dipt., 189. (1905).

albiventris Troms., Eugenies Resa, Dipt., 462. 28. (1869); v. D. Wulp, Catal. Dipt. South Asia, 55. (1896); ? Coquill., Proc. I.Philippinae, Ins. Mysol.

Cuba,

I. Galapagos.

America sept.

Washingt. Acad. Sci., III. 373. (1901).

algericus $\mathrm{J}_{\mathrm{AENN}}=$ ? longirostris WIED.

anchora Lw., Linnaea Entomol., I. 429. 3. (1846); WALk., List Europa mer.

Dipt. Brit. Mus., V. suppl. 1., 314. (1854); Jatenn., Berlin. Entom. Zeitschr., X. 225. 12. (1866); Lw., Beschr. europ. Dipt., II. 45. (1871).

Manilla, ?I. Galapag. 
siculus JaEnn., Berlin. Entom. Zeitschr., X. 224. 10. (1866). angulatus Dur. $=$ cingulatus DuF. annulatus Lw. = cingulatus DuF.

arator Meland., Psyche, X. 179. 10. tab. IV. (1903); Aldr., Catal. America sept. North Amer. Dipt., 189. (1905).

argentifer Lw., Linnaea Entomol., I. 453. 14. (1846); WALK., List Dipt. Brit. Mus., V. suppl. 1., 316. (1854); JAEns., Berlin. Entom. Zeitschr., X. 225. 16. (1866).

atriceps Lw. in Rosenhauer, Die Thiere Andalusiens, 384. (1856) Hispania.

et Beschreib. europ. Dipt., II. 55. 33. (1871).

bellulus Meland., Psyche, X. 183. 19. tab. IV. (1903) et XI. 33. Americasept.

(1904) ; Aldr, Catal. North Amer. Dipt., 189. (1905).

bifasciatus MeIG. = uliginosus L. var.

bipunctatus Lw., Linnaea Entomol., I. 451. 12. (1846); WALK., List Asia min.

Dipt. Brit. Mus., V. suppl. 1., 316. (1854).

brachystomus Lw., Linnaea Entomol., I. 443. 8. (1846) ; WaLk., List

Dipt. Brit. Mus., V. suppl. 1., 315. (1854) ; Schin., Verh. zool.bot. Ver. Wien, V. 642. 10. (1855) et Fauna Austriaca, Dipt., I. 4. 4. (1862) ; Кеrт., Pótfüz. a Természettud. Közlönyhöz, XXXII. 122 et 123. (1900).

brevirostris MeIG., System. Beschreib., III. 117. 6. (1822); Macq., Suit. à Buffon, I. 266. 6. (1834); Duncan, Magaz. Zool. and Botany, I. 160. 4. (1837); Curt., Brit. Entomol., VIII. 729., 2. 4. (1839) ; Lw., Linnaea Entomol., I. 457. 19. (1846); WaLK., Insecta Britann., Dipt., I. 26. 3. (1851) et List Dipt. Brit. Mus., V. suppl. 1., 81. 7, 315 et 317. (1854) ; Schin., Verh. zool.-bot. Ver. Wion, V. 642. 9 et 672 . (1855) et Fauna Austriaca, Dipt., I. 4.4 et 6 . 12. (1862) ; Kert., Pótfüz. a Természettud. Közlönyhöz, XXXII. 122 et 123. (1900).

Bruesii Meland., Psyche, X. 179. 11. tab. IV. (1903); Aldr., Catal. Nortlı Amer. Dipt., 189. (1905).

canadensis Lw., Berlin. Entom. Zeitschr., VII. 7. 12.(1863) ; Meland., Psyche, X. 175. 2. tab. IV. (1903); Aldr., Catal. North Amer. Dipt., 189. (1905).

capensis Walk., Insecta Saunders., Dipt., I. 80. (1851) et List Dipt. Brit. Mus., V. suppl. 1., 83. 15. (1854).

carbonarius Lw., Berlin Entom. Zeitschr., XIII. 5. 6. (1869); Meland., Psyche, X. 177. 4. (1903); Aldr., Catal. North Amer. Dipt., 190. (1905).

Cardinalii Bezzr, Bull. Soc. Entom. Ital., XXX. 25. 19. (1898). carneus Walk., List Dipt. Brit. Mus., III. 521. (1849) et V. suppl. 1., 83. 12. (1854); Meland., Psyche, X. 178. 7. (1903); Aldr., Catal. North Amer. Dipt., 190. (1905).

cingulatus Duf., Annal. Soc. Entom. France, sér. 2. X. 5. 1. tab. I. Hispania. No. I. fig. 1-5. (1852) ; Walk., List Dipt. Brit. Mus., V. suppl. 1., 318. (1854).

angulatus Duf., Annal. Soc. Entom. France, sẻr. 2. X. tab. I. No. I. [lapus] (1852).

Europa centr. et mer., Asia min.

Europa centr. et mer.

America sept.

America sept.

Prom. bon.sp.

America sept.

Europa mer.

America sept. 
annulutus Isw., Beschreib. europ. Dipt., II. 55. Anmerk. (1871). cothurnatus Bı́., Annal. Soc. Entom. France, sér. 5. IX. 232. 1. (1879). crassus Lw., Berlin. Entom. Zeitschr., VII. 7. 10. (1863); WıLlist., Canad. Entomol., XVII. 128. (1885); Meland., Psyche, X. 175. 1. tab. IV. (1903); Aldr., Catal. North Amer. Dipt., 190. (1905). crenatus EgG., Verh. zool.-bot. Ges. Wien, IX. 395. (1859); Scnis., Europa mer. Fauna Austriaca, Dipt., I. 6. 13. (1862).

dentatus Beck., Mitteil. Zoolog. Mus. Berlin, II. 7. 1. (1902).

dissimilis Lw., Öfvers. Kongl. Vet. Akad. Förhandl., XIII. 264. 9. Caffraria. (1856) et Dipt.-Fauna Südafrika's, I. 10. 1. (1860).

fasciatus OuIv. = niloticus OLIv.

fasciatifrons E. L. Arrib., Stettin. Entom. Zeitg., XLII. 190. 2. Argentinia. (1881), Exped. Rio Negro, Zool., 89. (1881) et Bolet Acad. Nat. Cienc. Córdoba, IV. 130. 52. [Nematotelus] (1882); Hunter, Trans. Amer. Entom. Soc. Philad., XXVII. 130. (1901).

flavicornis Jouns., Proc. Acad. Nat. Scienc. Philad., 1894.272. (1894); Meland., Psyche, X. 180. 13. (1903); Aldr., Catal. North Amer. Dipt., 190. (1905).

fraternus Lw., Linnaea Entomol., I. 448. 10. (1846); WALK., List Dipt. Brit. Mus., V. suppl. 1., 315. (185t) ; Schis., Fauna Austriaca, Dipt., I. 7. (1862) ; JAEnN., Berlin. Entom. Zeitschr., X. 223. 7. (1866).

fuscinervis Lxw., Beschreib. europ. Dipt., II. 46. 27. (1871).

? punctatus MeIG. (nee FABr.), Klassif., I. 140.2. (1804) et System.

Beschreib., III. 116. 4. tab. XXV. fig. 17-18. (1822); MACQ., Suit. à Buffon, I. 266. 3. (183£); Lw., Linnaea Entomol., I. 456. 17. (1846) et Beschreib. europ. Dipt., II. 47. nota. (1871). gadensis Scurn., Novara Reise, Dipt., 56. 13. (1868).

glaber Lw., Berlin. Entom. Zeitschr., XVI. 56. 10. (1872) ; Meland., Psyche, X. 177. 6. (1903); Aldr., Catal. North Amer. Dipt., 190. (1905).

globuliceps Lw., Linnaea Entomol., I. 441. 7. (1846); Schis., Verh. zool.-bot. Ver. Wien, V. 642. 8, 672 et 673 . (1855) et Fauna Austriaca, Dipt., I. 4.5 et 6. 14. (1862); Kent., Pótfüz. a Természe:tud. Közlönyhöz, XXXII. 122 et 123. (1900).

uliginosus Lw. ol. (nec L.), Programm Posen, 1840. 24. 2. (1840) et Isis, 1840. VII. 554. 2. (1840).

gracilis Lw., Linnaea Entomol., I. 449. 11. (1846); WaLK., List Dipt. Brit. Mus., V. suppl. 1., 316. (1854) ; Rö̀., Borlin. Entom. Zeitschr., XXXI. 73. (1887).

haemorrhous Lww., Öfvers. Kongl. Vet. Akad. Förhandl., 264. 10. (1856) et Dipt.-Fauna Südafrika's, I. 10.2. (1860); Schiv., Novara Reise, Dipt., 56. 12. (1868).

hirtulus Big., Annal. Soc. Entom. France, sér. 5. IX. 233. 2. (1879). immaculatus Johns., Proc. Acad. Nat. Sci. Philad., 1895. 304. (1905) ; Meland., Psyche, X. 181. 16. (1903) ; Aldr., Catal. North Amer. Dipt., 190. (1905).

Hispania.

Amorica sept.

Aegyptus.

Jamaica.

Europa contr.

Europa mer.

Hispania.

America sept.

Europa centr. et mer.

Asia min., Creta.

Africa mer.

Natal.

America sept. 
kansensis ADAMs, Kansas Univ. Scienc. Bullet., II. 221. (1903); Meland., Psyche, XI. 34. (1904); Aldr., Catal. North Amer. Dipt., 190. (1905).

lasiops Lw., Linnaea Entomol., I. 426. 2.(1846); Walk., List Dipt. Sicilia. Brit. Mus., V. suppl. 1., 314. (1854).

lateralis L. Duf., Annal. Soc. Entom. France, sér. 2. X. 6. 2. tab. 1. No. I. fig. 6. (1852); Walk., List Dipt. Brit. Mus., V. suppl. 1., 318. (1854).

latiusculus Lw., Beschreib. europ. Dipt., II. 49. 29. (1871) ; SтroвL, Wien. Entom. Zeitg., XVII. 295. 1. (1898).

leucocephalus Beck. = leucorrhynchus A. Costa.

leucorrhynchus A. Costa, Atti R. Accad. Sci. Napoli, ser. 2. I. 61.

1. (1884) et Bullet. Soc. Entom. Ital., XVII. 252. (1885).

leucocephalus Beck., Mitteil. Zoolog. Mus. Berlin, II. 6. (1902).

limbatus EgG., Verh. zool.-bot. Ges. Wien, IX. 396. (1859); Lw., Europa mer.

Beschreib. europ. Dipt., II. 45. (1871).

Lomnickii Mıx, Verh. zool.-bot. Ges. Wien, XVII. 413. tab. X. fig. 6. (1867) ; Lw., Beschreib. europ. Dipt., II. 46. (1871) ; Ost.SAck., Wion. Entom. Zeitg., I. 150. (1882).

? acrosus Gimm., Progr. z. Feier s. 50. jälır. Doctor Jubiläums v. Fischer v. Waldheim, 10. (1847) et Bull. Soc. Imp. Nat. Moscou, XX. 2., 167. 2. (1847); Walk., List Dipt. Brit. Mus., V. suppl. 1., 82. 11. (1854); Fedtsch., Acta Soc. Amic. Nat. Moscou, VI. 59. 3. (1868); Jaroscheff., Trudy Kharkow, XI. 332. (1877).

longirostris WIED., Analecta Entomol., 30. 38. (1824) et Ausserourop. zweifl. Ins., II. 45. 1. tab. VII. fig. 8. (1830); MACQ., Suit. à Buffon, I. 267. 8. (1834); Lw., Linnaea Entomol., I. 455. 16. (1846) ; Macq., Explor. scient. de l'Algérie, Zool., III. 430. 45. (1849) ; Walk., List Dipt. Brit. Mus., V. suppl. 1., 83.16 et 317. (1854); Lw., Beschreib. europ. Dipt., II. 45. (1871).

? algericus Jaenn., Berlin. Entom. Zeitsclur., X. 225. 11. (1866). luteicornis EgG., Verh. zool.-bot. Ges. Wien, IX. 394. (1859) ; Schin., Fauna Austriaca, Dipt., I. 5. 6. (1862); Kent., Pótfüz. a Természettud. Közlönyhöz, XXXII. 122 et 123. (1900).

maculiventris Big., Annal. Soc. Entom. France, sér. 3. VIII. 770. Sicilia. (1860).

marginatus $\mathrm{FABR}_{\mathrm{AB}}=$ pantherinus $\mathrm{L}$.

marginellus FALL. = pantherinus $\mathrm{L}$.

margincllus GmeL. = pantherinus $\mathrm{L}$.

marinus Beck., Mitteil. Zoolog. Mus. Berlin, II. 9. 4. (1902).

modestus Lw., Beschreib. europ. Dipt., II. 51. 30. (1871).

Aegyptus.

muticus $\mathrm{FABR}_{\mathrm{AB}}=$ uliginosus $\mathrm{L}$.

muticus Schrank = pantherinus $\mathrm{L}$.

niger Big., Annal. Soc. Entom. France, sér. 5. IX. 233. 3. (1879);

Europacentr. et or.

Africa sept.

Europa centr. et mer.

Ins. Naxos.

Chile.

Hunter, Trans. Amer. Entom. Soc. Philad., XXVII. 130, (1901). 
nigrifrons Lw., Iinnaea Entomol., I. 452. 13. (1846); Wal.k., List Sicilia. Dipt. Brit. Mus., V. suppl. 1., 316. (18j4).

nigrinus Fall., Dipt. Suee. Stratiom., 6. 3. (1817); Meig., System. Europa, Beschreib., III. 117. 5. (1822); MacQ., Recueil Soc. Sci. Agric!lt. ?America s. Lille, 1826. 437. (1826) et Suit. à Buffon, I. 266. 5. (18;); Duncan, Magaz. Zoolog. and Botany, I. 159. 3. (1837); Zе:TT., Insecta Lappon., Dipt., 575. 1. (1838); CurT., Brit. Entomol., VIII. 729. tab. (1839); Lw., Programm Posen, 1840. 24. 1. (1840) et Isis, 1840. VII. 554. 1. (1840); Zетт., Dipt. Scand., I. 151. 5. (1842) ; Lw., Linnaea Entomol., I. 454. 15. (1846); WaLK., List Dipt. Brit. Mus., III. 520. (1849); ZeTr., Dijt. Scand., VIII. 2961. 5. (1849); WaLk., Insecta Britann., Dipt., I. 26. 4. (1851) et List Dipt. Brit. Mus., V. suppl. 1., 81.6 et 316. (185t); Scnin., Verh. zool.-bot. Ver. Wien, V. 643. 17 et 672. (1855); Bonsd., Finland tvaving. Ins., I. 118. 3. (1861) ; Scmix., Fauna Austriaca, Dipt., I. 5. 7. (1862) ; JaEns., Berlin. Entom. Zeitschr., X. 223. 9. (1866); v. D. Wulp, Diptera Neerland., I. 447. 1. (1877); JAroschefr., Trudy Kharkow, XI. 331. 2. (1877); Neuhaus, Diptera Marchica, 79. 2. (1886); Beck., Berlin. Entom. Zeitschr., XXXI. 102. 10. (1887) ; Brunettr, The Entomologist, XXII. 83. 3. (1889) ; 今сносн, Fauna Insect. Holvetiae, Dipt., 20. (1890); É. \& L. Coucke, Annal. Soc. Entom. Belgique, XXXVII. 436. (1893); Kent., Pótfïz. a Természettud. Közlönyhöz, XXXII. 122 et 123. (1900); ALDr., Catal. North Amer. Dipt., 190. (1905).

nigritus Paxz., Fauna Germ., CVII. tab. 17. (? 1820).

nigrinus v. D. Wulp (nec FALL.) = unicolor Lw.

nigripennis Bra., Aunal. Soc. Entom. France, sér. 6. VII. 29. 1. Prom.bon.sp. (1887).

nigritus Merg., Klassif., I. 140. 3. (1804) et System. Beschreib., III. 116. 3. (1822); Lw., Linnaea Entomol., I. 457. 18. (1846); WaLK., List Dipt. Brit. Mus., V. suppl. 1., 81. 4 et 317. (1854).

nigritus $\mathrm{PANZ}_{\mathrm{A}}=$ nigrinus $\mathrm{FALL}$.

nigroaeneus VerH., Entomol. Nachricht., XVII. 3. (1891).

niloticus Ourv., Encyclop. Méthod., VIII. 183. 3. (1811); Macq.,

Dipt. oxot., I. 1., 205. 1. tab. XXV. fig. 3. (1838) et I. 2., 191. (1839); Lw., Linnaea Entomol., I. 459. 23. (1846); WALK., List Dipt. Brit. Mus., V. suppl. 1., 84. 17 et 317. (1854).

fasciatus Oriv., Encyclop. Méthod., VIII. 183. 4. (1811); Macq., Suit. à Buffon, I. 267. 7. (1834).

notatus Zetr., Dipt. Seand., I. 148. 3. (1842); Heydex, Stettin. Europa orc.

Entom. Zeitg., V. 203. (1844); Lw., Linnaea Entomol., I. 437. 6. (1846) ; ZETT., Dipt. Scand., VIII. 2960. 3. (1849); WaLk., List Dipt. Brit. Mus., V. suppl. 1., 82. 9 et 315. (1854); Scmin., Verlı. zool.-bot. Ges. Wien, V. 642. 7. (1855) ot Fauna Austriaca, Dipt., I. 5.6 et 6. 15. (1862); JaEnn., Berlin. Entom. Zeitschr., X. 225. 15. (1866); v. D. Wulp, Diptera Neerland., I. 449. 4. (1877); Brunetti, The Entomologist, XXII. 83. 4. (1889); É. \& L. Coucke,

Europa sept. Aegyptus.

Europa. 
Annal. Soc. Entom. Belgique, XXXVII. 437. (1893); Ker'r., Pótfüz. a Természettud. Közlönyliöz, XXXII. 122 et 123. (1900).

var. ventralis Zетт. (nee Meig.), Dipt. Scand., I. 149. (1812); Europa sept. Walk., List Dipt. Brit. Mus., V. suppl. 1., 82 et 317. (1854).

obscuripes I.w., Beschreib. europ. Dipt., II. 48. 28. (1871).

pallipes SAY, Journ. Acad. Nat. Sci. Philad., III. 29. (1823) et Compl. Writt., II. 52. (1859) ; WiEd., Aussereurop. zweifl. Ins., II. 45. 2. (1830) ; WaLk., List Dipt. Brit. Mus., V. suppl. 1., 83. 13. (1854); Hine, Ohio Natural., II. 228. (1902); Meland., Psyche, X. 178. 8. (1903) ; Aldr., Catal. North Amer. Dipt., 190. (1905).

paludosus Meig., System. Beschreib., VI. 345. 8. (1830) ; Lw., Linnaea Entomol., I. 458. 21. (1816) ; Walk., List Dipt. Brit. Mus., V. suppl. 1., 317. (1854); Lw., Beschreib. europ. Dipt., II. 45. (1871). pantherinus L., Systema Naturae, Ed. X. 590. 6. [Musca] (1758); Geofrr., Hist. Nat. Ins., II. 543. 1. tab. XVIII. fig. 1. [-] (1762); O. F. Müller, Fauna Insect. Fridrichsdal., 80. 705. [Musca] (1764) ; I., Systoma Naturae, Ed. XII., II. 980. 8. [Musca] (1767); Guel., Systema Naturae, V. 2830. 8. [Musca] (1792); Meig., System. Besclireib., III. 115. 2. tab. XXV. fig. 20. (1822) ; Dunér., Consid. génér., tab. XLVIII. fig. 9. (1823); MAcQ., Recueil Soc. Sci. Agricult. Lille, 1826. 436. 2. tab. II. fig. 8. (1826) et Suit. à Buffon, I. 265. 19. (1834); Duncss, Magaz. Zoolog. and Botany, I. 159. 2. (1837) ; Curt., Brit. Eutomol., VIII. 729., 2. 2. (1839); Blanch., Hist. d'Ins., III. 594. (1840) ; Lw., Programm Posen, 1840. 24. 3. (1840) et Isis, 1840. VII. 554. 3. (1840) ; Zeтt., Dipt. Scand., I. 150.4. (1842) ; Lw., Linnaea Entomol., I. 445. 9. (1846); Walk., List Dipt. Brit. Mus., III. 520. (1849) ; ZeтT., Dipt. Scand., VIII. 2960. 4. (1849); MACQ., Explor. scient. de l'Algérie, Zool., III. ‘31. 46. (1849); WALK., Insecta Britann., Dipt., I. 25.2. (1851) ; Halid., Stettin. Entom. Zeitg., XII. 136. (1851) ; ZeтT., Dipt. Scand., XI. 4261. 4. (1852) ; Walk., List Dipt. Brit. Mus., V. suppl. 1.„80. 3 et 315. (1854); Schin., Verh. zool.-bot. Ges. Wien, V. 642. 11 et 672. (1855) et Fauna Austriaca, Dipt., I. 4.3 et 5. 9. (1862); Jaenn., Berlin. Entom. Zeitschr., X. 223. 6. (1866) ; v. D. Wul., Diptera Neerland., I. 447. 2. (1877) ; JАRoscherf., Trudy Kharkow, XI. 331. 1. (1877); Neunaus, Diptera Marchica, 78. 1. (1886); BRunetri, The Entomologist, XXII. 83. 2. (1889) ; Sсносн, Fauna Insect. Helvetiae, Dipt., 20. (1890); É. \& L. Coucke, Annal. Soc. Entom. Belgique, XXXVII. 436. (1893) ; Kert., Pótfüz. a Természottud. Közlönyliöz, XXXII. 122 et $123 .(1900)$.

marginatus FABR., Systema Entomol., 761. 10. [Stratiomys] (1775), Spec. Insect., II. 419. 13. [Stratiomys] (1781), Mantissa Insect., II. 332. 19. [Stratiomys] (1787) et Entomol. System., IV. 270. 3. (1794); PANz., Fauna Germ., XLVI. tab. 22. (1797) ; FABr., Systema Antliat., 88. 3. (1805); Ourv., Encyclop. Méthod, VIII. 183, 2. (1811). 
marginellus FaLL., Dipt. Suec., Stratiom., 5. 2. (1817). marginellus Gmel., Systema Naturae, V. 2836. 163. [Musca] (1792).

muticus Schrank, Fauna Boica, III. 97. 2389. [Stratiomys] (180 i). uliginosus Panz., Fauna Germ., XLVI. tab.21.(1797) ; Schell., Gattungen der Fliegen, 38 et 39. tab. XXV. fig. 1. (1803); Meig., Klassif., I. 139. 1. tab. VIII. fig. 5-9. (1804) ; Latr., Hist. Nat. d. Crust. et d. Ins., XIV. 344. tab. CXI. fig. 8. (1804), Gen. Crust. et Ins., IV. 276. (1809) et Consid. génér., 442. (1810).

Pica Lw. = uliginosus L. var.

pilosus Lw., Beschreib. europ. Dipt., II. 43. 26. (1871).

plagiatus Scrin., Verh. zool.-bot. Ges. Wien, VII. 19. 3. (1857) et

IIispania.

Fauna Austriaca, Dipt., I. 5. 6 et 6. 10. (1862); Kent., Pótfüz.

a Természettud. Közlönyhöz, XXXII. 122 et 123. (1900).

polyposus SAy, Journ. Acad. Nat. Sci. Philad., VI. 160. (1829) et

Compl. Writt., II. 356. (1859); WaLK., List Dipt. Brit. Mus., V. suppl. 1., 115. (1854); Wilust., Biolog. Central. Amer., Dipt., T. 251. 1. (1901); Meland., Psyche, X. 178. 9. (1903); Aldr., Catal. North Amer. Dipt., 190. (1905).

proboscideus Lw., Linnaea Entomol., I. 423. 1.(1846); WaLk., List

Dipt. Brit. Mus., V. suppl. 1., 314. (1854); JaEns., Berlin. Entom.

Zeitschr., X. 225. 14. (1866); A. CostA, Atti R. Accad. Sci. Napoli, ser. 2. V. No. 14., 21. 1. (1893).

pulcher Lw., Beschreib. europ. Dipt., II. 51. 31. (1871).

pullus Lw., Beschreib. europ. Dipt., II. 57. 34. (1871).

punctatus FABR., Entomol. System., IV. 271. 4. (1794) ; Coqueb.,

Illustr. Icon. Insect., 101. tab. XXIII. fig. 6. (1804); FABr., Systema Antliat., 88. 4. (1805) ; LATr., Gen. Crust. et Ins., IV. 277. (1809) ; Ourv., Encyclop. Méthod., VIII. 183. 5. (1811) ; Lam., Hist. nat. anim. sans vestébr., III. 389. 2. (1816) ; WALK., List Dipt. Brit. Mus., V. suppl. 1., 81. 5 et 317. (1854); Lw., Beschreib. europ. Dipt., II. 45. Anmerk. (1871).

punctatus MeIG. (nec FABR.) = ? fuscinervis Lw.

punctiventris Beck., Mitteil. Zoolog. Mus. Berlin, II. 8. 2. (1902). ruficornis Big., Annal. Soc. Entom. France, sér. 5. IX. 234. 4. (1879); Hunter, Trans. Amer. Entom. Soc. Philad., XXVII. 130. (1901). rufiventris Portsch., Horae Soc. Entom. Rossicae, XXI. 177. (1887). serbicus Strobl, Glasnik Zem. Mus. Bosni i Herzegov., XIV. 462. (1902) et Wissensch. Mitteil. Bosnien u. Herzegov., IX. 520.(1904). siculus $\mathrm{J}_{\mathrm{AENN}}$. = anchora $\mathrm{Lw}$.

signatus Friv. apud. Schin., Verh. zool.-bot. Ver. Wien, V. 81. tab., fig. 9-10. (1855) ; Schin., Verh. zool.-bot. Ver. Wien, V. Sitzgsber., 67 et Abhandl., 640. 1 et 672. (1855) et Fauna Austriaca, Dipt., I. 6. 11. (1862); Becher, Denkschr. Akad. Wien, XLV. 140. tab. II. fig. 8. (1882); Kert., Pótfiiz. a Természettud. Közlönyhöz, XXXII. 122 et 123. (1900).

Europa centr. et mer.

Mexico.

Europa mer., Africa sept.

Hispania. Hispania. Africa sept.

Aegyptus. Chile.

Armenia.

Europa mer.

Europacentr. et mer. 
singularis MACQ., Dipt, exot., suppl. 1., 57. 2. tab. VI. fig. 4.(1816); WaLK., List Dipt. Brit. Mus., V. suppl. 1., 83.14. (1854); Hunter, Trans. Amer. Entom. Soc. Philad., XXVII. 130. (1901).

Slossonae Johns., Proc. Acad. Nat. Sci. Philad., 1895. 304. (1895); Meland., Psyche, X. 181. 14. (1903); Aldr., Catal. North Amer. Dipt., 190. (1905).

trinotatus Meland., Psyche, X. 180. 12. tab. IV. (1903) et XI. 34. (1904) ; Aldr., Catal. North Amer. Dipt., 190. (1905).

tristis Big., Annal. Soc. Entom. France, sér. 6. VII. 30. 2. (1887); Meland., Psyche, X. 177. 5. (1893); Aldr., Catal. North Amer. Dipt., 190. (1905).

uliginosus L., Systema Naturae, Ed. XII., II. 983. 22. [Musca] (1767); Guel., Systema Naturae, V. 2836. 22. [Musea] (1792); FABr., Entomol. System., IV. 269. 1. (1794) et Systema Antliat., 87. 1. (1805) ; Donov., Brit. Ins., XV. 31. tab. 519. [Musca] (1811); Oliv., Encyclop. Mèthod., VIII. 183. 1. (1811); Lam., Hist nat. anim. sans vestébr., III. 389. 1. (1816); Fall., Dipt. Suec., Stratiom., 5. 1. (1817); MeIG., System. Beschreib., III. 114. 1. tab. XXV. fig. 19. (1822); MAcQ., Recueil Soc. Sci. Agricult. Lille, 1826. 435. 1. (1826); ? Meia., system. Beschreib., VI. 345. (1830) ; Macq., Suit. à Buffon, I. 265. 2. tab. VI. fig. 15. (1834) ; Duncan, Magaz. Zoolog. and Botany, I. 158. 1. fig. 1-2. (1837); Curt., Brit. Entomol., VIII. 729., 2. 1. (1839) ; ZETT., Dipt. Scand., I. 146. 1. (1842); Lw., Linnaea Entomol., I. 432. 4. (1846); MAcQ., Explor. Scient. de l'Algérie, Zool., III. 431. 48. (1849); Walk., List Dipt. Brit. Mus., III. 520. (1849) ; Zetr., Dipt. Scand., VIII. 2959. 1. (1849); WaLK., Insecta Britannica, Dipt., I. 25.1. tab. I. fig. 6. (1851) et List Dipt. Brit. Mus., V. suppl. 1., 80. 1 ct 314. (1854) ; Schin., Verh. zool.-bot. Ver. Wien, V. 641. 5, 672 et 673. (1855) ; Zetr., Dipt. Scand., XII. 4556. 1. (1855); Halid., Nat. Hist. Review, IV. 193. tab. XI. fig. 5-22. (1857) ; Borsd., Finl. tvaving. Ins., I. 117. 1. (1861); Schin., Fauna Austriaca, Dipt., I. 5 et 6. 10. (1862) ; JaExs., Berlin. Entom. Zeitschr., X. 223. 8. (1866) ; v. D. Wulp, Diptera Neerland., I. 448. 3. (1877); Brunetri, The Entomologist, XXII. 83. 1. (1889); Schocr, Fauna Insect. Helvetiae, Dipt., 20. (1890); É. \& L. Coucke, Annal. Soc. Entom. Belgique, XXXVII. 437. (1893); Kent., Pótfüz. a Természettud. Közlönyhöz, XXXII. 122 et 123. (1900).

muticus FABR, Species Insect., II. 419. 14. [Stratiomys] (1781)

et Mantissa Insect., II. 332. 20. [Stratiomys] (1787).

var. bifasciatus MeIG., System. Beschreib., VII. 104. 9. (1838); ZetT., Dipt. Scand., I. 148. 2. (1842); Macq. in Lucas: Explor. scient. de l'Algérie, Zool., III. 431. 47. (1849); Zетт., Dipt. Scand., VIII. 2960. 2. (1849); WaLK., List Dipt. Brit. Mus., V. suppl 1., 80. 2. (1854); Bonsd., Finl. tvaving. Ins., I. 118. 2. (1861); Lw. Beschreib. europ. Dipt., II. 57. (1871).

var. Pica Lw., Programm Posen, 1840. 24. 4. (1810), Isis, 1840. Europa.
Europa,

Africa sept

Europa,

Africa sept. 
VII. 554. 4. (1840) et Linnaea Entomol,, I. 436. 5. (1846); Giмм., Bullet. Soc. Imp. Nat. Moscou, XX. 2., 167. 1. (1847); ZETT., Dipt. Scand., VIII. 2960. Obs. 2. (1849); WALK., List Dipt. Brit. Mus., V. s'ippl. 1., 82.10 et 314. (1854); Schiv., Fauna Austıiaca, Dipt., I. 6. (1862) ; Jaenn., Berlin. Entom. Zeitschr., X. 225. 13. (1866) ; Jw., Beschreib. europ. Dipt., II. 57. (1871).

uliginosus Lw. olim (nee L.) = globuliceps L.

uliginosus Paxz. (nee L.) = pantherinus L.

unicolor Lw., Berlin. Entom. Zeitschr., VII. 7. 11. (1863); Willist,

Canad. Entomol., XVII. 128. (1885) ; HART, Bull. Illinois State Laborat. of Nat. Hist., IV.266.(1895) ; WiLlist., Biolog. Central.A mer., Dipt., I. 251. 2. (1901); Meland., Psyche, X. 176. 3. tab. IV. (1903) ; Aldr., Catal. North Amer. Dipt., 190. (1905).

nigrinus v D. Wulp. (nee Fall.), Tijdschr. v. Entomol., ser. 2.

II. (X). 1867. 126. (1868).

varius Lw., Beschreib. europ. Dipt., II. 58. 35. (1871).

ventralis Meig., System. Beschreib., VI. 345. 7. (1830); Lw., Linnaea

Entomol., I. 458. 20. (1846).

ventralis ZETT. (nee MEIG.) = notatus ZETT. var.

Wheeleri Meland., Psyche, X. 182. 18. tab. IV. (1903); Aldr., Catal. Americasept. North Amer. Dipt., 190. (1905).

Zichyi Kert., Zoolog. Ergebn. d. III. asiat. Forschungsreise d. Sibiria.

Grafen E. Zichy, II. 190. 16. fig. 4. (1901).

\section{Akronia}

HrNץ, Ohio Natural., I. 113. (1901).

frontosa Hine, Ohio Natural., I. 113. fig. 1. (1901).

America sept.

\section{Lasiopa}

Brullè, Expéd. scient. de Morée, III. 1., 307. (1832).

Cyclogaster MACQ., Suit. à Buffon, I. 256. 12. (1834).

? Inermia Bıg., Annal. Soc. Entom. France, sér. 3. VII. 210. (1859).

? Inermyia Bı́., Annal. Soc. Entom. France, sér. 3. IV. 82. (1856).

atrata FABr., Systema Antliat., 83. 23. [Stratiomys] (1805); WIED., Analecta Entomol., 14. [Clitellaria] (1824) et Aussereurop. zweifl. Ins., II. 49. 5. tab. VII. fig. 10. [Clitellaria] (1830); Macq., Suit. à Buffon, I. 257. 2. [Cyclogaster] (1834) et Dipt. exot., I. 1., 193. [Cyclogaster] (1838) ; WALK., List Dipt. Brit. Mus., V. suppl. 1., 65. 4 et 313 [Cyclogaster] (1854); Huxter, Trans. Amer. Entom. Soc. Philad., XXVII. 127. [Lasiopa] et 130. [Clitellaria] (1901). 
calva Wied. apud Meia., System. Beschreib., III. 121. 2. [Clitellaria] (1822); Walk., List Dipt. Brit. Mus., V. suppl. 1., 65. 2. [Cyclogaster] (1854); Schis., Verh. zool.-bot. Ver. Wien, V. 640. 2 et 671. [Cyclogaster] (1855) et Fauna Austriaca, Dipt., I. 8. (1862) ; Engel, Entomol. Nachricht., XI. 177. (1885); Kert., Pótfüz. a Természettud. Közlönyhöz, XXXII. 123. (1900) ; PLEske, Sitzgsber. Naturf. Ges. Jurjeff, XII. 1900. 338. 4. [Cyclogaster] (1901) ; Lichтw., Zeitschr. f. syst. Hymen. u. Dipt., I. 69. (1901). caucasica Pleske, Sitzgsber. Naturf. Ges. Jurjeff, XII. 1900. 336. 2. [Cyclogaster] (1901); Licurw., Zeitschr. f. syst. Hymen. u. Dipt., I. 272. (1901).

Königi Lichтw., Zeitschr. f. syst. Iymen. u. Dipt., I. 68. (1901).

detracta WALK., Proc. Linn. Soc. London, I. 108. 22. [Cyclogaster] (1857) ; v. D. Wulp, Catal. Dipt. South Asia, 55. (1896).

edentula WrED., Analecta Entomol., 29. 35. [Stratiomys] (1824) et Aussereurop. zweifl. Ins., II. 70.17. [Stratiomys] (1830); WALK., List Dipt. Brit. Mus., V. suppl. 1., 50. 89. [Stratiomys] (1854); Gerst., Linnaea Entomol., XI. 322. [Cyclogaster] (1857); BIG., Annal. Soc. Entom. France, sér. 3. IV. 63. [Inermyia] (1856); Isw., Dipt.-Fauna Südafrika's, I. 7. [Cyclogaster] (1860).

infera Walk., Proc. Linn. Soc. London, I. 109. 23. [Cyclogaster] (1857); v. D. Wulp, Catal. Dipt. South Asia, 55. (1896).

Königi Lichitw. $=$ caucasica PLeske.

Mannii Mrк, Verh. zool.-bot. Ges. Wien, XXXI. 315. (1881) ; Pleske, Sitzgsber. Naturf. Ges. Jurjeff, XII. 1900. 339. 6. [Cyclogaster] (1901) ; Lichтw., Zeitschr. f. system. Hymen. u. Dípt., I. 69. (1901).

Paulseni Phil., Verh. zool.-bot. Ges. Wien, XV. 732. 1. [Cyclogaster] (1865) ; Hunter, Trans. Amer. Entom. Soc. Philad., XXVII. 127. (1901).

Peleteria Brullé, Expéd. scient. de Morée, III. 1., 308. 664. tab. XLVII. fig. 6. (1832); Watk., List Dipt. Brit. Mus., VII. suppl. 3., Addenda. (1855) ; Scurv., Wien. Entomol. Monatschr., IV. 48. (1860) ; Lw., Berlin. Entom. Zeitsehr., VI. 75. 4. [Cyclogaster] (1862); Mık, Verh. zool.-bot. Ges. Wien, XXXI. 316. Anmerk. (1881); Pleske, Sitzgsber. Naturf. Ges. Jurjeff, XII. 1900. 339. 5. [Cyclogaster] (1901); Lichтw., Zeitschr. f. system. Hymen. u. Dipterol., I. 68. (1901).

peregrina Hutron, Trans. New Zeeland Ins!it., XXXIII. 10. [Cyclogaster] (1901).

radians WALK., Proc. Linn. Sje. London, I. 7. 14. [Cyclogaster] (1856) ; v. D. Wulp, Catal. Dipt. South $\Lambda$ sia, 55. (1896).

rubriceps Pirl., Verb. zool.-bot. Ges. Wien, XV. 732. 2. [Cyclogaster] (1865) ; Bı́., Annal. Soc. Entom. France, sér. 5. IX. 186. (1879); Hunter, Trans. Amer. Entom. Soe. Philad., XXVII. 127. (1901).

tenuirostris Lw., Neue Beitr., II. 16. 31. [Cyclogaster] (1854); Europa mer.
Europacentr. et mer., Africa sept.

Caucasus.

Borneo.

Prom.bon.sp.

Borneo.

Europa mer., Asia min.

Chile.

Graecia.

N. Selandia.

Singapore.

Chile. 
Schrn., Verh. zool.-bot. Ver. Wien, V. 640. 3 et 671. [Cyclogaster] (1855) et Fauna Austriaca, Dipt., I. 8. (1862); Lw., Wien. Entom. Monatschr., VII. 33. 3. [Cyclogaster] (1863); JAEnv., Berlin. Entom. Zeitschr., X. 225. 16 b. (1866); Kent., Pótfüz. a Természettud. Közlönylıöz, XXXII. 124. (1900) ; PLEske, Sitzgsber. Naturf. Ges. Jurjeff, XII. 1900. 338. 3. [Cyclogaster] (1901); Lichтw., Zeitschr. f. system. Hymen. u. Dipt., I. 69. (1901). villosa Fabr., Entomol. System., IV. 270. 2. [Nemotelus] (1794); Panz., Fauna Germ., LVIII. tab. 16. [Nemotelus] (1798); FAbr., Supplem. Entomol. Sy stem., 556. 26. [Stratiomys] (1798) ; Coqueb., Illustr. Icon. Insect., 100. tab. XXIII. fig. 3. [Stratiomys] (1804); FABr., Systema Antliat., 88. 2. [Nemotelus] (1805); Oliv., Encyclop. Méthod., VIII. 434.12. [Odontomyia](1811); Meig., Syst. Beschreib., III. 120. 1. tab. XXV. fig. 24. [Clitellaria] (1822); MacQ., Suit. ̀̀ Buffon, I. 257. 1. tab. VI. fig. 9. [Cyclogaster] (1834); Meig., System. Beschreib., VII. 105. [Clitellaria] (1838); Blanch., Hist. d'Ins., III. 594. [Cyclogaster] (1840); MAcQ., Explor. Scient. de l'Algérie,'Zool., III. 430. 43. [Cyclogaster] (1849); WALK., List Dipt. Brit. Mus., V. suppl. 1., 64. 1. [Cyelogaster] (1854) ; Scнin., Verh. zool.-bot. Ver. Wien, V. 639. 1 et 671. [Cyclogaster] (1855) et Fauna Austriaca, Dipt., I. 7. 1. (1862); v. D. Wulp, Diptera Neerland., I. 449. nota, tab. XIV. fig. 8-9. (1877) ; Błcher, Denkschr. Akad. Wien, XLV. 140. tab. II. fig. 6. (1882); Mıк in BeCKer : Fauna v. Hernstein, II. 2., 53. (1885); Sсносн, Fauna Insect. Helvetiae, Dipt., 20. (1890); StroвL, Mittheil. Naturwiss. Ver. Steiermark, XXIX. 1892. 8. (1893); KER'T., Pótfüz. a Természettud. Közlönyhöz, XXXII. 123. (1900); PLEske, Sitzgsber. Naturf. Ges. Jurjeff, XII. 1900. 336. 1. [Cyclogaster] (1901); Licutw., Zeitschr. f. syst. Hymen. u. Dipt., I. 68. (1901).

vittata v. D. WulP, Tijdschr. v. Entomol., XXIV. 155. 7. (1881); Hunter, Trans. Amer. Entom. Soe. Philad., XXVII. 127. (1901).

\section{Ruba}

WatK., Proc. Linn. Soc. London, IV. 100. (1860).

Thylacosoma Brau., Denkschr. Akad. Wien, XLIV. 7T. (1881). amboinensis BraU. = ? inflata WALK.

inflata WaLk., Proc. Linn. Soc. London, IV. 101. 29. (1860); v. D. Wulp, Catal. Dipt. South Asia, 54. (1896).

? amboinensis Brav., Denkschr. Akad. Wien, XLIV. 77. [Thylacosoma] (1881).

opponens WALK., Proc. Linn. Soc. London, VIII. 107. 18. (1865) ; v. D. Wulp, Catal. Dipt. South $\Lambda$ sia, 54. (1896) et Természetrajzi Füzetek, XXI. 415. 16. (1898).
Europa centr. et mer.

Africa sept.

Argentinia.

$$
\begin{aligned}
& \text { Celebes, } \\
& \text { ? Amboina. }
\end{aligned}
$$

Nova Guinea. 


\section{Aulana}

Walk., Proc. Linn. Soc. London, VII. 204. (1864).

confirmata Walk, Proc. Linn. Soc. London, VII. 204. 12. (1864); Ins. Mysol. v. D. Wurp, Catal. Dipt. South Asia, 55. (1896).

\section{Brachycara}

Thoms., Eugenies Resa, Dipt., 460. (1869).

ventralis Thoss., Eugenies Resa, Dipt., 461. 26. tab. IX. fig. 4. Ins. Rossi, (1869) ; v. D. Wurp, Catal. Dipt. South Asia, 50. (1896) et Ter- Nova Guinea. mészetrajzi Fiizetek, XXI. 412. 10. (1898).

\section{Saruga}

Walk., Proc. Limn. Soc. London, IV. 101. (1860).

conifera Walk., Proc. Linn. Soc. London, IV. 102. 31. (1860) et V. NovaGuinea, 233. 16. (1861); v. D. Wrup, Catal. Dipt. South Asia, 54. (1896). Celebes.

\section{Hermione}

Meig., Nouvelle Classification, 22. 31. (1800).

Heteroxycera Big., Annal. Soc. Entom. France, sér. 3. IV. 63 et $85 .(1856)$.

Hypoleon Dur., Exposit. d'une méthod. natur., p.? (? 1801).

Oxycera MEIG., Illiger's Magaz. f. Ins., II. 265. 31. (1803).

aemula Lw., Beschreib. europ. Dipt., III. 81. 43. [Oxycera] (1873). Turkestan. affinis CuRT. = pygmaea FALL.

? alpina Lror, Atti Istit. Veneto, ser. 3. IX. 588. [Pachygaster] (1864). Italia.

amoena Lw., Berlin. Entom. Zeitschr., J. 33. [Oxycera] (1857); Europacontr.

Schin., Verh. zool.-bot. Ges. Wien, VIII. 35. [Oxycera] (1858) et et sept.

Fauna Austr., Dipt., I. 11. 11. [Oxycera] (1862); Jaenn., Berlin.

Entom. Zeitschr., X. 227. 22. [Oxycera] (1866); Beck., Berlin.

Entom. Zeitschr., XXXI. 98. 4. [Oxycera] (1887); Sсносн, Fauna

Ins. Helvetiae, Dipt., 22. [Oxycera] (1890); SтroвL, Mittheil.

Naturwiss. Ver. Steiermark, XXIX. 1892. 8. [Oxycera] (1893). pardalina Zетt. (nec Meig.), Dipt. Scand., I. 143. 3 [Oxycera]

(1842) et VIII. 2959. 3. [Oxycera] (1849) ; Schis., Verh. zool.bot. Ver. Wien, V. 635. 6 et 671 [Oxycera] (1855).

analis Wied. ap. Mrig., System. Beschr., III. 130. 10. [Oxycera] Europa.

(1822) ; Curt., Brit. Entomol., VIII. 441., 2. 9. [Oxyeera] (1833);

Dencan, Magaz. Zoolog. and Botany, I. 158. 6. [Oxycera] (1837); Meia., System. Beschr., VII. 105. 10. [Oxycera] (1838); DaLe, 
Annal. Magaz. Nat. Hist., VIII. 432. 8. [Oxycera] (1841); WaLK., List Dipt. Brit. Mus., III. 527. [Oxycera] (1849), Ins. Britann., Dipt., I. 23. 10. [Oxycera] (1851) et List Dipt. Brit. Mus., V. suppl. 1., 73. 12. [Oxycera] (1854); Schin., Verh. zool.-bot. Ver. Wien, V. 636. 12 et 670. [Oxycera] (1855); Lw., Berlin. Entom. Zeitschr., I. 26. [Oxycera] (1857); Schin., Fauna Austr., Dipt., I. 9. 2. [Oxycera] (1862); JAENN., Berlin. Entom. Zeitschr., X. 226. 19. [Oxycera] (1866); Brunetti, The Entomologist, XXII. 84. 2. [Oxycera] (1889); Зсносн, Fauna Ins. Helvetiae, Dipt., 21. [Oxycera] (1890).

atra Lw., Beschreib. europ. Dipt., III. 77. 40. [Oxycera] (1873).

bipunctata Lw., Beschreib. europ. Dipt., III. 88. 47. [Oxycera]

Turkestan.

Turkestan. (1873).

calceata Lw., Beschreib. europ. Dipt., II. 41. 24. [Oxycera] (1871). centralis Lw., Berlin. Entom. Zeitschr., VII. 8. 14. [Oxycera] (1863) ; Aldr., Catal. North Amer. Dipt., 190. [Oxyecra] (1905). collaris BRUNETTI = trilineata $\mathrm{L}$. var.

Crotchi Ost.-SAck., Bull. Unit. Stat. Geolog. Surv., III. 212. [Oxycera] (1877) ; Aldr., Catal. North Amer. Dipt., 190. [Oxyeera] (1905). decipiens Guḱr., Voyage de la Coquille, Zool. II. P. 2., 291. [Oxycera] (1830).

dives Lw., Dipterol. Beitr., I. 15. 4. tab., fig. 7-8. [Oxycera]; WALK., Insecta Britann., Dipt., I. 21. 3. [Oxycera] (1851) et ? List Dipt. Brit. Mus., V. suppl. 1., 71. 4. [Oxycera] (1854); Scuıs., Verh. zool.-bot. Ver. Wien, V. 634. 4 et 670. [Oxycera] (1855); Lw., Berlin. Entom. Zeitschr., I. 25. [Oxycera] (1857); Scurn., Fauna Austriaca, Dipt., I. 12. 14. [Oxycera] (1862); JAEnn., Berlin. Entom.Zeitschr., X. 228. 26. [Oxycera] (1866); Beck., Berlin. Entom. Zeitschr., XXXI. 99. 7. [Oxycera] (1887); Sтroвц, Mittheil. Naturwiss. Ver. Steiermark, XXIX. 1892. 9. [Oxycera] (1893); Krrt., Pótfüz. a Természettud. Közlönyhöz, XXXII. 126. [Oxycera] (1900) ; Carter, Entom. Monthly Mag., ser. 2. XV. (XL). 16. [Oxycera] (1904).

?hypoleon Schrank (nce L.), Fauna Boica, III. 96. 2385. [Stratiomys] (1803).

engadinica J Aenn., Berlin. Entom. Zeitschr., X. 227. 23. [Oxycera] (1866) ; Beck., Berlin. Entom. Zeitschr., XXXI. 98. 5. [Oxycera] (1887); Sс́носн, Fauna Ins. Helvetiae, Dipt., 22. [Oxycera] (1890).

Falleni Staeg., Stettin. Entom. Zeitg., V. 410. 3. [Oxycera] (1814); Lw., Dipterol. Beitr., I. 13. 2. tab., fig. 3-4. [Oxycera] (1845); WALK., Insecta Britann., Dipt., I. 20. 2. [Oxycera] (1851) et List Dipt. Brit. Mus., V. suppl. 1., 71. 2. [Oxycera] (1854); Schin., Verh. zool.-bot. Ver. Wien, V. 633. 2 et 6r1. [Oxycera] (1855) et Fauna Austriaca, Dipt., I. 12. 13. [Oxycera] (1862); StronL, Mittleil. Naturwiss. Ver. Steiermark, XXIX. 1892. 9. [Oxycera] (1893) ; Kert., Pótfïz. a Természettud. Közlönyhöz, XXXII. 126. [Oxycera] (1900).

Europa centr. America e.

America sept.

Australia.

Europa centr.

Europa centr.

Europa centr. et sept.

Kertész: Catalogus Dipterorum. III. 
hypoleon FALL. (nec L.), Dipt. Suec., Stratiom., 10. 7. [Stratiomys] (1817) ; Donov., Brit. Ins., tab. CXLVI. fig. 2. [Musca] (1811) ; Zетт., Dipt. Scand., I. 142. 1. p. p. [O.xycera] (1842) et VIII. 2955. 1. [O.xycera] (1849).

fasciventris Lw., Beschreib. europ. Dipt., III. 86. 46. [Oxycera] Turkestan. (1873).

flavipes $\mathrm{Lw}_{\mathrm{w}}=$ muscaria $\mathrm{F}_{\mathrm{ABR}}$.

formosa MacQ. (nec Wied.) = pardalina MeIG.

formosa Wied. apud Meig., System. Beschreib., III. 127. 5. [Oxycera] Eu opa. (1822) et VI. 346. [Oxycera] (1830) ; CurT., Brit. Entomol., VIII. 441., 2. 3. [Oxycera] (1833); Duncan, Magaz. Zool. and Botany, I. 157. 4. [Oxycesa] (1837); Dale, Annal. Mag. Nat. Hist., VIII. 43-2. 4. [Oxycera] (1841) ; ZETT., Dipt. Scand., I. 145. 5. [Oxycera] (1842) ; Walk., List Dipt. Brit. Mus., III. 527. [Oxycera] (1849) ; ZETT., Dipt. Scand., VIII. 2959. 5. [Oxycera] (1849); ? WaLK., Insecta Britann., Dipt., I. 22. 6. [Oxycera] (1851) et List Dipt. Brit. Mus., V. suppl. 1., 71. 5. [Oxycera] (1854); Schin., Verh. zool.-bot. Ver. Wien, V. 635. 8 et 671. [Oxycera] (1855); I w., Berlin. Entom. Zeitschr., I. 26. [Oxycera] (1857); Schns., Fauna Austriaca, Dipt., I. 10. 9. [Oxycera] (1862); JAENN., Berlin. Entom. Zeitschr., X. 227. 21. [Oxycera] ; v. D. WulP, Diptera Neerland., I. 458. [Oxycera] (1877); Neuhaus, Diptera Marchica, 80. 3. [Oxycera] (1886); BRunetri, The Entomologist, XXII. 85. 6. [Oxyсега] (1889); Sсносн, Fauna Ins. Helvetiae, Dipt., 21. [Oxycera] (1890) ; É. \& L. Coucke, Annal. Soc. Entom. Belgique, XXXVII. 438. [Oxycera] (1893); Gazagn., Annal. Soc. Entom. France, sér. 7. III. Bull. CCXLII. [Oxycera] (1893).

muscaria Panz. (nec Fabr.), Fauna Germ., CVIII. tab. 15. (ठ') et 16. (q) [Stratiomys] (1809); MeIG., System. Beschreib., III. 126. 4. [Oxycera] (1822); MACQ., Sui ${ }^{+}$à Buffon, I. 251. 6. tab. VI. fig. 6. [Oxycera] (1834); WaLK., List Dipt. Brit. Mus., III. 527. [Oxycera] (1849).

nigricornis IATTR., Encyclop. méthod., VIII. 601. 5. [Oxycera] (1811); Macq., Recueil Soc. Sci. Agricult. Lille, 1826. 440. 4. [Oxycera] (1826); Meig., System. Beschreib., VII. 105. 11. [O.xycera] (1838); WaLK., List Dipt. Brit. Mus., V. suppl. 1., 73. 14. [Oxycera] (1854); Lw., Berlin. Entom. Zeitschr., I. 26. [Oxycera] (1857).

fraterna Lw., Beschreib. europ. Dipt., III. 95. 51. [Oxycera] (1873). grata Lw., Beschreib. europ. Dipt., I. 24. 24. [Oxycera] (1869).

Turkestan. Graecia. hirticeps Lw., Beschreib. europ. Dipt., III. 96. 52. [Oxycera] (1873). Turkestan. hybrida Lw., Beschreib. europ. Dipt., III. 89. 49. [Oxycera] (1873). Turcomania. Hydroleon HaRRIs = trilineata $\mathrm{L}$.

hypoleon FaBR. (nec L.) = Meigenii StTaEg. hypoleon FaLL. (nec L.) = Falléni StaEG. hypoleon L. = trilineata L. var. hypoleon Meig. 1804 (nec L.) = pulchella Mria. 
hypoleon Staeg. (nec L.) = pulchella Meig.

hypoleon Schrank (nec L.) = ? dives Lw.

leonina PANz., Fauna Germ., LVIII. tab. 21. [Strationys] (1798); Europa.

Meig., Klassif., I. 138. a. [Oxycera] (1804); ? Latr., Gen. Crust. et Ins., IV. 275. [Odontomyia] (1809) et Encyclop. Méthod., VIII. 601. 6. [Oxycera] (1811); MeIG., System. Beschreib., III. 129. 8. [Oxycera] (1822); MacQ., Recueil Soc. Sci. Agricult. Lille, 1826. 445. 1. [Oxycera] (1826); CurT., Brit. Entomol., VIII. 441., 2. 8. [Oxycera] (1833); MAcQ., Suit. à Buffon, I. 251. 7. [Oxycera] (1834); MeIG., System. Beschreib., VII. 105. 8. [Oxycera] (1838); Lw., Programm Posen, 1840. 25. 2. [Oxycera] (1840) et Isis, 1840. VII. 556. 2. [Oxycera] (1840); Dale, Annal. Mag. Nat. Hist., VIII. 432. 9. [Oxycera] (1841); Jacobsen, Naturhist. Tiddskr., ser. 2. I. 40. [Oxycera] (1844); Walk., List Dipt. Brit. Mus., V. suppl. 1., 72. 7. [Oxycera] (1854); Schin., Verh. zool.-bot. Ver. Wien, V. 634. 5 et 670. [Oxycera] (1855); Lw., Berlin. Entom. Zeitschr., I. 25. [Oxycera] (1857); Scims., Fauna Austriaca, Dipt., I. 10. 4. [Oxycera] (1862); v. D. Wulp, Diptera Neerland., I. 458. 2. [Oxycera] (1877) ; Neuhaus, Diptera Marchica, 80. 2. [Oxycera] (1886); Sсносн, Fauna Insect. Helvetiae, Dipt., 21. [Oxycera] (1890); É. \& L. Coucke, Annal. Soc. Entom. Belgique, XXXVII. 437. [Oxycera] (1893) ; Strobl, Mittheil. Naturwiss. Ver. Steiermark, XXIX. 1892. 8. [Oxycera] (1893); Kınт., Pótfüz. a Természettud. Közlönyhöz, XXXII. 125. [Oxycera] (1900).

Liburna Walk., List Dipt. Brit. Mus., III. 528. [Oxycera] (1849) et V. suppl. 1., 75. 20. [Oxycera] (1854); Aldr., Catal. North Amer. Dipt., 190. [Oxycera] (1905).

limbata Lw., Berlin. Entom. Zeitschr., VI. 73. 4. [Oxycera] (1862); Kent., Pótfüz. a Természettud. Közlönyhöz, XXXII. 125. [Oxycera] (1900).

Jocuples Lw., Berlin. Entom. Zeitschr.,. I. 23. [Oxycera] (1857); Schin., Fauna Austriaca, Dipt., I. 12. 14. [Oxycera] (1862); J AENN., Berlin. Entom. Zeitschr., X. 228. 25. b. [Oxycera] (1866); BEcK., Berlin. Entom. Zeitschr., XXXI. 97. 3. [Oxycera] (1887); Sсносн, Fauna Insect. Helvetiae, Dipt., 22. [Oxycera] (1890). longicornis $\mathrm{DALE}=$ tenuicornis MACQ.

maculata LAtr., Encyclop. Méthod., VIII. 600. 4. [Oxycera] (1811); Macq., Dipt. exot., I. 2., 190. [Oxycera] (1838); Walk., List Dipt. Brit. Mus., V. suppl. 1., 75. 19. [Oxycera] (1854); Meland., Canad. Entomol., XXXVI. 20. 1. [Oxycera] (1904); Aldr., Catal. North Amer. Dipt., 191. [Oxycera] (1905).

maculata MeIG. (nee LAtri.) = pulchella MeIG.

maculata ZетT. (nec LATR.), Insecta Lappon., Dipt., 576. 1. [Oxycera] Jamaica.

Europa centr. et mer.

Europa centr.

America sept.

(1838), Dipt. Scand., I. 144. 4. [Oxycera] (1842) et VIII. 2959. 4. [Oxycera] (1819); Walk., List Dipt. Brit. Mus., V. suppl. 1., 72. 6. [Oxycera] (1854); Schin., Verh. zool.-bot. Ver. Wien, V. 635. 7. [Oxycera] (1855) ; Lw., Berlin. Entom. Zeitschr., I. 32. [Oxycera] (1857). 
manens Walk., Proc. Linn. Soc. London, IV. 96. 18. [Oxycera] (1860) ; v. D. Wulp, Catal. Dipt. South A sia, 54. [Oxyeera] (1896).

marginata Lw., Wien. Entom. Monatschr., III. 211. (recte 221.) Sicilia. 1. [Oxycera] (1859).

Meigenii Stakg., Stettin. Entom. Zeitg., V.410. 2. [Oxycera] (1844); Europa. Lw., Dipt. Beitr., I. 11. 1. tab., 6g. 1-2. [Oxycera] (1845); Grmm., Bull. Soc. Imp. Nat. Moscou, XX. 2., 168. 1. [Oxycera] (1847) ; Zетт., Dipt. Scand., VIII. 2957. 1-2. [Oxycera] (1849); Walk., List Dipt. Brit. Mus., V. suppl. 1., 70. 1. [Oxycera] (1854); Schin., Verh. zool.-bot. Ver. Wien, V. 632. 1 et 671. [Oxycera] (1855) ; HeEG., Sitzgsber. math.-naturw. Cl. d. k. Akad. Wien, XX. 345. tab. III. fig. 1-13. [Oxycera] (1856); Schix., Fauna Austriaca, Dipt., I. 12. 13. [Oxyeera] (1862); JAENN., Berlin. Entom. Zeitschr., X. 228. 25. a. [Oxycera] (1866) ; ВеснеR, Denkschr. Akad. Wien, XIJ. 140. tab. II. fig. 9. [Oxycera] (1882) ; Schосн, Fauna Ins. Helvetiae, Dipt., 22. [Oxyeera] (1890); E. \& L. Coucke, Annal. Soc. Entom. Belgique, XXXVII. 438. [Oxycera] (1893) ; Кenт., Pótfüz. a Természettud. Közlönyhöz, 126. [Oxycera] (1900).

hypoleon FABr. (nec L.) Systema Entomolog., 760. 8. [Stratiomys] (1775), Spec. Insect., II. 418. 10. [Stratiomys] (1781) et Mantissa Insect., II. 331. 15. [Stratiomys] (1787); Preissi., Verzeichn., I. 81. 75. [Stratiomys] (1790); Guel., Systema Naturae, V. 2835. 7. [Musea] (1792) PAnz., Fauna Germ., I. tab. 14. [Stratiomys] (1793); Fabr., Entomol. System., IV. 267. 20. [Stratiomys] (1794); Latr., Hist. Nat. d. Crust et d. Ins., XIV. 342. 2. [Sargus] (1804) ; FABr., Systema Antliat., 85. 29. [Stratiomys] (1805); LAtr., Gen. Crust. et Ins., IV. 277. [Oxycera] (1809) et Consid. génér., 442. [Oxycera] (1810) et Encyclop. Méthod., VIII. 600. 1. [Oxycera] (1811); Lav., Hist. nat. anim. sans vertebr., III. 388. 1. [Oxycera] (1816); Cuv., Régne Animal., III. 624. [Oxycera] (1817); Mrig., System. Beschreib., III. 124. 1. [Oxycera] (1822); Macq., Recueil Soc. Sci. Agricult. Lille, 1826. 438. 1. [Oxycera] (1826) et Suit à Buffon, I. 250. 2. [Oxycera] (1834); Lw., Programm Posen, 1840. 25. 1. [Oxycera] (1840) et Isis, 1840. VII. 555. 1. [Oxycera] (1840); DALe, Annal. Mag. Nat. Hist., VIII. 432. 1. [Oxycera] (1841); Zetr., Dipt. Scand., I. 142. 1. p. p. [Oxycera] (1842); JAcoBsen, Naturh st. Tidsskr., ser. 2. I. 40. [Oxycera] (1844); ? Gimm., Bull. Soc. Imp. Nat. Moseou, XX. 2., 168. 2. [Oxycera] (1847).

melanodactyla L.w., Beschreib. europ. Dipt., III. 80. 42. [Oxycera] Turkestan. (1873).

metallica*) Wred., Aussereurop. zweifl. Ins., II. 60. [Oxycera] I.St.Thomas. (1830) ; MACQ., Dipt. exot., I. 1., 194. [Oxycera] (1838); WALk,

*) ? Nothomyia. 
List Dipt. Brit. Mus., V. suppl. 1., 75. 21. [Oxycera] (1854); Aldr., Catal. North Amer. Dipt., 191. [Oxycer] (1a905).

Morrisii Curt., Brit. Entomol., VIII. 441. tab. [Oxyeera] (1833); Dale, Annal. Mag. Nat. Hist., VIII. 432. 6. [Oxycera] (1841); WALK., Insecta Britann., Dipt., I. 22. 8. [Oxycera] (1851) et List Dipt. Brit. Mus., V. suppl. 1, 73. 10. [Oxycera] (1854); Lw., Berlin. Entom. Zeitschr., I. 32. [Oxycera] (1857); ? HaLid., Nat. Hist. Review, IV. 193. tab. XI. [Oxycera] (1857); Brunetri, Tho Entomologist, XXII. 85. 10. [Oxycera] (1889).

muscaria FABR., Entomol. System., IV. 268. 21. [Stratiomys] (1794) et Systema Antliat., 86. 31. [Stratiomys] (1805); Cunt., Brit. Entomol., VIII. 441., 2. 6. [Oxycera] (1833); Duncan, Magaz. Zoolog. and Bolany, I. 157. 3. [O.xycera] (1837); DaLe, Annal. Magaz. Nat. Hist., VIII. 432. 5. [Oxycera] (1841); WALK., List Dipt. Brit. Mus., V. suppl. 1., 72. 8. p. p. [Oxycera] (1854); Scrus., Verh. zool.-bot. Ver. Wien, V. 635. 9 et 671. [Oxycera] (1855), VII. 12. [Oxycera] (1857), VIII. 36. [Oxycera] (1858) et Fauna Austriaca, Dipt., I. 11. 10. [Oxycera] (1862); Kent., Pótfüz. a Természettud. Közlönyhöz, XXXII. 125. [Oxycera] (1900).

flavipes Lw., Berlin. Entom. Zeitschr., I. 29. [Oxycera] (1857). muscaria PANZ. (nec FABR.) = formosa WIED. muscaria WALK. (nec FABR.) = pygmaea FALL.

nana Lw., Beschreib. europ. Dipt., III. 83. 44. [Oxycera] (1873). nigra Macq., Suit. à Buffon, I. 251. 8. [Oxycera] (1834); Meig., System. Beschreib., VIl. 106. 13. [Oxycera] (1838); WaLk., List Dipt. Brit. Mus., V. suppl. 1., 74. 15. [Oxycera] (1854); Lw., Berlin. Entom. Zeitschr., 1. 33. [Oxycera] (1857). nigricornis $\mathrm{LATR}$. = formosa WIED.

nigripes Verrall, Entom. Monthly Mag., ? et List Brit. Dipt., 2nd

Edit., 15. [Oxycera] (1901); BrunetTI, The Entomologist, XXII.

85. 8. [Oxyeera] (1889).

pardalina WaLK. (nec MeiG.), Insecta Britann., Dipt., I. 21. 5. [Oxycera] (1851) et List Dipt. Brit. Mus., V. suppl. 1., 72. 9. [Oxycera] (1854).

nigriventris Lw., Beschreib. europ. Dipt., III. 91. 49. [Oxycera] Turkestan. (1873).

notata Lw., Beschreib. europ. Dipt., III. 92. 50. [Oxycera] (1873). nubifera Lw., Öfvers. Kongl. Vet. Akad. Förhandl., XIII. 264. 11. Turkestan. Caffraria. [Oxycera] (1856) et Dipt.-Fauna Sïdafrika's, I. 11. 1. tab. I. fig. 1-2. [Oxycera] (1860).

octomaculata JARosch., Trudy Kharkoff, XI. 336. 3. [Oxycera] Turkestan. Europa occid.

Britannia. (1877) ; Ost.-SAck., Wien. Entom. Zeitg., I. 150. [Oxycera] (1882). pantherina $\mathrm{L} .=$ trilineata $\mathrm{L}$.

pardalina MeIG., System. Beschreib., III. 128. 6. tab. XXV. fig. Europacentr. 30-31. [Oxycera] (1822); CurT., Brit. Entomol., VIII. 441., 2. 4. [Oxycera] (1833); Dale, Annal. Mag. Nat. Hist., VIII. 432. 7. [Oxycera] (1841); Lw., Berlin. Entom Zeitschr., I. 27. [Oxycera] 
(1857) ; Schin., Famna Austriaca, Dipt., I. 11. [Oxycera] (1862) ; v. D. Wulu, Diptera Neerland., I. 459. [Oxycera] (1877); Brunetri, The Entomologist, XXII. 84. 5. [Oxyeera] (1889); Scносн, Fauna Insect. Helvetiae, Dipt., 21. [Oxycera] (1890); Kent., Pótfüiz. a Természettud. Közlönyhöz, XXXII. 125. [Oxycera] (1900).

formosa Macq., Suit. à Buffon, I. 250. 4. [Oxyeera] (1834). pardalina WALK. (nee MeIG.) = nigripes Verr. pardalina ZETT. (nee MEIG.) = amoena Lw.

picta v. D. Wulp, Tijdschr. v. Entomol., ser. 2. II. (X). 1867. 133. 6. [Oxycera] (1868); Lw., Zeitschr. f. d. ges. Naturwiss., N. F. II. (XXXVI). 117. [Oxycera] (1870); Aldor., Catal. North Amer. Dipt., 191. [Oxycera] (1905).

proxima Lw., Beschreib. europ. Dipt., III. 85. 45. [Oxycera] (1873). pulchella Meig., System. Beschreib., III. 125. 2. tab. XXV. fig. 29. [Oxycera] (1822); MAcq., Recueil Soc. Sci. Agricult. Lille, 1826. 439. 2. tab. III. fig. 1. [Oxycera] (1826) ; Curt., Brit. Entomol., VIII. 441., 2. 1. [Oxycera] (1833) ; MACQ., Suit. à Buffon, I. 249. 1. [Oxyecra] (1834); Duncan, Magaz. Zoolog. and Botany, I. 156. 1. fig. 1-2. [Oxycera] (1837) ; Blanch., Hist. d'Ins., III. 593. tab. II. fig. 8. [Oxycera] (1840); DALe, Annal. Mag. Nat. Hist., VIII. 432. 2. [Oxycera] (1841); Iw., Neue Beitr., I. 14. 3. tab., fig. 5-6. [Oxycera] (1845); Schin., Verh. zool.-bot. Ver. Wien, V. 633. 3 et 671. [Oxyeera] (1855); Lw., Berlin. Entom. Zeitschr., I. 22. [Oxycera] (1857); Scmin., Fauna Austriaca, Dipt., I. 11. 12. [Oxycera] (1862); JaEns., Berlin. Entom. Zeitschr., X. 228. 24. [Oxycera] (1866); v. D. Wuup, Diptera Neerland., I. 458. 3. [Oxycera] (1877); Brunetti, The Entomologist, XXII. 85. 9. [Oxycera] (1889); Хсносн, Fauna Insect. Helvetiae, Dipt., 22. [Oxycera] (1890) ; É. \& L. Coucke, Annal. Soc. Entom. Belgique, XXXVII. 438. [Oxycera] (1893); Strobl, Mittheil. Naturwiss. Ver. Steiermark, XXIX. 1892. 8. [Oxycera] (1893) ; Kent., Pótfüz. a Természettud. Közlönyhöz, XXXII. 125. [Oxycera] (1900).

hypoleon MeIG., Klassif., I. 137. 1. tab VIII. fig. 3. [Oxycera] (1804).

hypoleon Staeg., Stettin. Entom. Zeitg., V. 409. 1. [O.xycera] (1844); Walk., List Dipt. Brit. Mus., III. 526. [Oxycera] (1849).

maculata Meia., System. Beschreib., III. 125. [Oxycera] (1822). ? rara Scor., Entomol. Carniol, 933. 912. [Musca] (1763); WALK.,

Insecta Britann., Dipt., I. 20. 1. tab. I. fig. 4. [Oxyecra] (1851) et List Dipt. Brit. Mus., V. suppl. 1., 71. 3. [Oxycera] (1854); Scmin., Verh. zool.-bot. Ver. Wien, VI. 414. 912. [Oxycera] (1856).

? tardigrada Harris, Expos. Engl. Ins., 46. tab. XI. fig. 6. [Musca] (1782).

pygmaea Fall., Dipt. Suec., Stratiom., 11. 9. [Stratiomys] (1817); Meig., System. Beschreib., III. 129. 7. [Oxycera] (1822); DaLe,

America sept.

Europa centr. Europa. 
Annal. Mag. Nat. Hist, VIII. 432. 5. [Oxycera] (1841); Zетт., Dipt. Scand., I. 145. 6. [Oxycera] (1842); ? WALK., List Dipt. Brit. Mus., III. 527. [Oxycera] (1849); Zetт., Dipt. Scand., VHI. 2959. 6. [Oxycera] (1849) et XI. 4261. 6. [Oxycera] (1852); ? WaLK., List Dipt. Brit. Mus., V. suppl. 1., 72. [Oxycera] (1854); Schin., Verh. zool.-bot. Ver. Wien, V. 636. 13 et 670. [Oxycera] (1855); Lw., Borlin. Entom. Zeitschr., I. 31. [Oxycera] (1857); Schin., Fauna Austriaca, Dipt., I. 10. 5. [Oxycera] (1862); JaEnn., Berlin. Entom. Zeitschr., X. 226. 20. [Oxycera] (1866); v. D. Wulp, Diptera Neerland., I. 459. [Oxycera] (1877); Beck., Berlin. Entom. Zeitschr., XXXI. 99. 6. [Oxycera] (1887); Brunett, The Entomologist, XXII. 85. 7. [Oxycera] (1889); Sспосн, Fanna Insect. Helvetiae, Dipt., 21. [Oxycera] (1890); É. \& L. Coucke, Annal. Soc. Entom. Belgique, XXXVII. 438. [Oxycera] (1893).

affinis CurT., Brit. Entomol., VIII. 441., 2. 7. [Oxycera] (1833). muscaria WaLK., Insecta Britann., Dipt., I. 22. 7. [Oxycera] (1851) et List Dipt. Brit. Mus., V. suppl. 1., 72. 8. p. p. [Oxycera] (185!).

Ranzonii Schin., Verh. zool.-bot. Ver. Wien, VII. 18. 1. [Oxycera] (1857); Lw., Berlin. Entom. Zeitschr., I. 29. [Oxycera] (1857); Schin., Fauna Austriaca, Dipt., I. 11. 10. [Oxyceru] (1862); Kert., Pótfüz. a Természettud. Közlönyhöz, XXXII. 125. [Oxycera] (1900).

rara Scop. = ? pulchella MeIG.

rufifrons I.w., Beschreib. europ. Dipt., III. 78. 41. [Oxycera] (1873). Turkestan. tardigrada $\mathrm{H}_{\mathrm{ARRIS}}=$ pulchella MeIG.

tenuicornis Macq., Suit. à Buffon, I. 251.5. [Oxycera] (1834) ; Meı., System. Bescnreib., VII. 105. 12. [Oxycera] (1838); WaLk., List Dipt. Brit. Mus., V. suppl. 1., 74. 16. [Oxycera] (1854); Lw., Berlin. Entom. Zeitschr., I. 31. [Oxycera] (1857); Verr., List Brit. Dipt., Ed. II. 15. [Oxycera] (1901).

longicornis DALE, Annal. Mag. Nat. Hist., VIII. 431. [Oxycera] (1841) ; WaLK., Insecta Britann., Dipt., I. 23. 11. [Oxyeera] (1851) et List Dipt. Brit. Mus., V. suppl. 1., 73. 13. [Oxycera] (1854) ; Lw., Berlin. Entom. Zeitsehr., I. 30. [Oxycera] (1857); Brunetri, The Entomologist, XXII. 84. 3. [Oxycera] (1889). terminata WaLK. (nee Wied.), Insecta Britann., Dipt., I. 23. 9. [Oxycera] (1851) et List Dipt. Brit. Mus., V. suppl. 1., 73. 11. [Oxycera] (1854).

terminalis DALE $=$ terminata WIED.

terminata WALK. (nee WiED.) = tenuicornis MACQ.

terminata WIED. apud Meig., System. Beschreib., III. 130. 9. [Oxycera] (1822) ; CurT., Brit. Entomol., VIII. 441., 2. 10. [Oxycera] (1833) ; Duncan, Magaz. Zoolog. and Botany, I. 157. 5. [Oxycera] (1837) ; ?? WaLk., List Dipt. Brit. Mus., IUI. 527. [Oxycera] (1849); Schin., Verh. zool.-bot. Ver. Wien, V. 635. 10 et 670. [Oxycera] (1855) ; Lw., Berlin. Entom. Zeitschr., I. 25. [Oxycera]

Europacentr. et sept.

Europa centr. 
(1857) ; Schin., Fauna Austriaca, Dipt., I. 10. 4. [Oxycera] (1862); Brunettr, The Entomologist, XXII. 84. 4. [Oxycera] (1889); Kert., Pótfüiz. a Természettud. Közlönyhöz, XXXII. 125. [Oxycera] (1900).

terminalis Dare, Annal. Mag. Nat. Hist., VIII. 432. 10. [Oxycera] (1841).

tricolor Lw., Beschreib. europ. Dipt., III. 76. 39. [Oxycera] (1873). trilineata L., Systema Naturae, Ed. XII., II. 980. 6. [Musca] (1767);

Turkestan. Donov., Brit. Ins., V. 425. tab. CLI. fig. 5. [Musca] (1796) ; FABr., Systema Entomolog., 760. 7. [Stratiomys] (1775) ; O. F. Múller, Zoolog. Dan. Prodr., 177. 2085. [Stratiomys] (1776) ; FarR., Species Insect., II. 418. 9. [Stratiomys] (1781) et Mantissa Insect., II. 331. 14. [Stratiomys] (1787) ; Gmes., Systema Naturae, V. 2835. 6. [Musca] (1792); PAnz., Fauna Germ., I. tab. 13. [Stratiomys] (1793) ; Faur., Entomol. System., IV. 267. 19. [Stratiomys] (1794); Schrank, Fauna Boica, III. 97. 2386. [Stratiomys] (1803) ; MeIG., Klassif., I. 137. 2. tab.VIII. fig. 2. /Oxycera] (1804); LAtr., Hist. Nat. d. Crust. et d. Ins., XIV. 342. 1. [Sargus] (1804); FABr., Systema Antliat., 85. 28. [Stratiomys] (1805); Latr., Gen. Crust. et Ins., IV. 278. [Oxycera] (1809) et Encyclop. Méthod., VIII. 600. 2. [O.xycera] (1811); Fall., Dipt. Suec., Stratiom., 11. 8. [Stratiomys] (1817); MeIg., System. Boschreib., III. 126. 3. [Oxycera] (1822); Macq., Recueil Soc. Sci. Agricult. Lille, 1826. 439. 3. [Oxycera] (1826); Curt., Brit. Entomol., VIII. 441., 2. 2. [Oxycera] (1833); Macq., Suit. à Buffon, I. 250. 3. [Oxycera] (1834) ; Guér., Iconogr. d. Régne Anim., Ins., 554. tab. XCVIII. fig. 8. [Oxycera] (1835); Duncan, Magaz. Zoolog. and Botany, I. 156. 2. [Oxycera] (1837); Lw., Programm Posen, 1840. 25. 3. [Oxyecra] (1840) et Isis, 1840. VII. 556. 3. [Oxycera] (1840); Dale, Annal. Mag. Nat. Hist., VIII. 432. 3. [Oxycera] (1841); Zeтт., Dipt. Scand., I. 142. 2. [Oxycera] (1842) ; Lw., Dipt. Beitr., I. 17. tab., fig. 9. [Oxycera] (1845) ; WALK., List Dipt. Brit. Mus., III. 526. [Oxycera] (1849); Zeтt., Dipt. Scand., VIII. 2958. 2. [Oxycera] (1849) ; Halm., Stettin. Entom. Zeitg., XII. 137. [Oxycera] (1851); WALK., Insecta Britann., Dipt., I. 21. 4. [Oxycera] (1851) ; ZetT., Dipt. Scand., XI. 4261. 2. [Oxycera] (1852) ; WaLK., List Dipt. Brit. Mus., V. suppl. 1., 74. 17. [Oxycera] (1854); Schin., Verh. zool.-bot. Ver. Wien, V. 636. 14 et 670. [Oxycera] (1855) ; HeEg., Sitzgsber. math.-naturw. Cl. d. i. Akad. Wien, XX. 345. tab. IV. fig. 1-12. [Oxycera] (1856); Lw., Zeitschr. f. d. ges. Naturwiss., X. 99. 8. [Oxycera] (1857) ; Schin., Verh. zool.bot. Ver. Wien, VII. 13. [Oxycera] (1857); Lw., Borlin. Entom. Zeitschr., I. 21. [Oxycera] (1857) ; Scurn., Fauna Austriaca, Dipt., I. 9. 1. [Oxycera] (1862); Lw., Zeitschr. f. d. ges. Naturwiss., XXIV. 389. 39. [Oxycera] (1864) ; JAENn., Berlin. Entom. Zeitschr., X. 226. 18. [Oxycera] (1866) ; v. D. Wulp, Diptera Neerland., I. 457. 1. [Oxycera] (1877); Nevhaus, Diptera Marehica, 80. 1. 
[Oxycera] (1886); BRunetri, The Entomologist, XXII. 84. 1.

[O.xycera] (1889); Schocr, Fauna Insect. Helvetiae, Dipt., 21. [Oxycera] (1890) ; É. \& L. Coucke, Annal. Soc. Entom. Belgique, XXXVII. 437. [O.xycera] (1893); Kent., Pótfüz. a Természettud. Közlönyhöz, XXXII. 125. [Oxycera] (1900).

Hydroleon Harris, Expos. Engl. Ins., 46. tab. XI. fig. 4. [Musca] (1782).

pantherina L., Fauna Suec., Ed. Alt., 440. 1783. [Musca] (1761). var. collaris BRunetri, The Entomologist, XXII. 84. 1. [Oxy- Europacentr. cera] (1889).

var. hypoleon L., Systema Naturae, Ed XII., II. 980. 7. Europacentr. [Musca] (1767); Schin., Verh. zool.-bot. Ver. Wien, V. 637. 15. [Oxycera] (1855).

unifasciata Lw., Berlin. Entom. Zeitschr., VII. 9. 15. [Oxycera] Americasept.

(1863); Meland., Canad. Entomol., XXXVI. 20. 2. [Oxycera] (1904); Aldr., Catal. North Amer. Dipt., 191. [Oxycera] (1905). variegata LAtr., Encyclop. Méthod., VIII. 600. 3. [Oxycera] (1811); Macq., Dipt. exot., I. 2., 191. [Oxycera] (1838); WaLK., List Dipt. Brit. Mus., V. suppl. 1., 74. 18. [Oxycera] (1854); Aldr., Catal. North Amer. Dipt., 191. [Oxycera] (1905).

varipes Isw. in HeydeN: Entomol. Reise n. d. südl. Spanien, 211. Lusitania.

1. [Oxycera] (1870) et Beschreib. europ. Dipt., II. 42. 25. [Oxycera] (1871).

\section{Aochletus}

Ost.-SAck., Biolog. Centr. Amer., Dipt., I. 38. (1886).

bistriatus Wulist., Trans. Entom. Soc. London, 1896. 300. 1. tab. X. fig. 76. (1896); Hunter, Trans. Amer. Entom. Soc. Philad., XXVII. 128. (1901); Aldr., Catal. North Amer. Dipt., 191. (1905). cinctus Osr.-SAck., Biolog. Centr. Amer., Dipt., I. 38. 1. (1886) ; Mexico. Aldr., Catal. North Amer. Dipt., 191. (1905).

obscurus Coquil., Proc. Unit. Stat. Nat. Mus., XXV. 98. (1902); Americasept. Aldr., Catal. North Amer. Dipt., 191. (1905).

\section{Stratiomyiinae.}

\section{Chordonota}

Gerst., Linnaea Entomol., XI. 311. 2. (1857).

carbonaria Bel.., Saggio di Ditterolog. Messic., Append., 11. 2. Mexico. (1862) ; Aldr., Catal. North Amer. Dipt., 181. (1905).

? Coprates WALK., List Dipt. Brit. Mus., III. 523. [Clitellaria, Patriaignota. ? Cyelogaster] (1849) et V. suppl. 1., 16. 3. [Cyphomyia] (1854); Gerst., Linnaea Entomol., XI. 313. [? Chordonata] (1857). 
fuscipennis BelL., Saggio di Ditterol. Messic., Append., 11. 1. tab. Mexico.

III. fig. 6. (1862); Aldr., Catal. North Amer. Dipt., 181. (1905). inermis WiEd., Aussereurop. zweifl. Ins., II. 55. 3. [Cyphomyia]

(1830) ; MAcQ., Suit. à Buffon, I. 242. 3. [Cyphomyia] (1834); WAlk., List Dipt. Brit. Mus., V. suppl. 1., 16. 6. [Cyphomyia] (1854) ; Gerst., Linnaea Entomol., XI. 312. 1. (1857); Hunter, Trans. Amer. Entom. Soc. Philad., XXVII. 130. (1901).

leiophthalma Willist., Trans. Entom. Soc. London, 1896. 302. 1.

I. St. Vincent.

(1896) ; Hunter, Trans. Amer. Entom. Soc. Philad., XXVII. 130.

(1901) ; Aldr., Catal. North Amer. Dipt., 181. (1905).

nigra Wilusst., 'Trans. Amer. Entom. Soc. Philad., XV. 255. 27. Brasilia.

(1888) ; Hunter, Trans. Amer. Entom. Soc. Philad., XXVII. 130. (1901).

\section{Cyphomyia}

W1ed., Zoolog. Magaz., I. 3., 55. (1819).

affinis Gerst., Linnaea Entomol., XI. 289. 7. (1857); Hunter, Trans. Venesuela. Amer. Entom. Soc. Philad., XXVII. 128. (1901).

albicaput WALK. = leucocephala WIED.

albitarsis FABR., Systema Antliat., 80. 12. [Stratiomys] (1805); Gerst., Linnaea Entomol., XI. 300. 18. (1857); Bellardi, Saggio di Ditterol. Messic., I. 22. 2. (1859) ; Schin., Novara Reise, Dipt., 53. 4. (1868) ; Ost.-SACK., Biolog. Centr. Amer., Dipt., I. 35.3. (1886) ; Gigl.-Tos, Mem. R. Accad. Sci. Nat. Torino, ser. 2. XLIII. 105. 5. (1893) ; Willist., Biolog. Centr. Amer., Dipt., I. 243. (1900) ; Hunter, Trans. Amer. Entom. Soc. Pliilad., XXVII. 128. (1901); Aldr., Catal. North Amer. Dipt., 181. (1905).

fenestrata MaCQ., Dipt. exot., suppl. 1., 48. 3. (1846); WaLK.,

List Dipt. Brit. Mus., V. suppl. 1., 16. 5. (1854).

albitarsis WIED. (nee FABR.) = Wiedemanni GERST.

androgyna Ost.-SAck., Biolog. Centr. Amer., Dipt., I. 34. 2. (1886); Panama.

Aldr., Catal. North Amer. Dipt., 181. (1905).

auriflamma W1ed., Zoolog. Magaz., I. 3., 54. 21. (1819); Sт. FARG. ET Serv., Encyclop. Méthod., X. 804. 3. (1825) ; Wien., Aussereurop. zweifl. Ins., II. 54. 1. (excl. Q.) tab. VIII. fig. 1a. (1830); Macq., Suit. à Buffon, I. 242.2 (1834) ; Guḱr., Iconogr. d. Régne Anim., Ins., 544. tab. XCVIII. fig. 5. (1835); WALK., List Dipt. Brit. Mus., III. 525. (1849); Cuvier, Régne animal, nouv. edit. (Masson), Ins. tab. CLXXII bis, fig. 5. (1849); WaLk., List Dipt. Brit. Mus., V. suppl. 1., 18. 11. (1854) ; Gerst., Linnaea Entomol., XI. 276. 2. (1857); Bell., Saggio di Ditterol. Messic., I. 21. 1. (1859) ; E. L. Arrib., Bolet. Acad. Nat. Cienc. Córdoba, IV. 129. 50. (1882) ; Willist., Trans. Amer. Entom. Soc. Philad., XV. 257. 34. (1881) ; Hunter, Trans. Amer. Entom. Soc. Philad., XXVII. 128. (1901); Aldr., Catal. Nortlı Amer. Dipt., 181. (1905). chrysodota Perty, Delect. anim, articul. Brasil., 184. tab.

America mer. et centr.

America mer. et centr. 
XXXVI. fig. 13. (1830-34); WaLk., List Dipt. Brit. Mus., V. suppl. 1., 18. 12. (1854).

coerulea MAcq., Suit. à Buffon, Expl. d. Planch., 4., tab. VI.

fig. 3. (1834).

cyanea MACQ. (nec FABr.), Suit. à Buffon, I. 242. 1. (1834).

auriflamma WIED. p. p. = aurifrons WIED.

aurifrons Wied., Aussereurop. zweifl. Ins., II. 56. 4. (1830) ; WALK.,

List Dipt. Brit. Mus., V. suppl. 1., 18. 13. (1854); Gerst., Linnaea

Entomol., XI. 280. 3. (1857); Hunter, Trans. Amer. Entom. Soc.

Philad., XXVII. 129. (1901).

auriflamma W1ED., Analecta Entomol., 13. tab., fig. 4. (1824) et Ausereurop. zweifl. Ins., II. 54. 1. (excl. ơ) tab. VIII. fig. $1 b$. (1830).

bicarinata WiLl!st., Biolog. Centr. Amer., Dipt., I. 244. 7. (1900) ; Aldr., Catal. North Amer. Dipt., 181. (1905).

chrysodota PERTY = auriflamma WIED.

claripennis Macq., Dipt. exot., suppl. 2., 29.4. (1847); WALK., List

Dipt. Brit. Mus., V. suppl. 1., 17. 9. (1854); Gerst., Linnaea Entomol., XI. 299.15. (1857) ; Hunter, Trans. Amer. Entom. Soc. Philad., XXVII. 129. (1901).

coerulea $\mathrm{MACQ}_{\mathrm{A}}=$ auriflamma WIED.

costalis WaLK., Trans. Linn. Soc. London, XVII. 341. 28. (1837) et

List Dipt. Brit. Mus., V. suppl. 1., 18. 10. (1854); Gerst., Linnaea Entomol., XI. 299. 16. (1857); Hunter, Trans. Amer. Entom. Soc. Philad., XXVII. 129. (1901).

cyanea FABr., Entomol. System., IV. 265. 11. [Stratiomys] (1794); Coques., Illustr. Icon. Ins., 100. tab. XXIII. fig. 4. [Stratiomys] (1804); FABr., Systema Antliat., 80. 11. [Stratiomys] (1805); OLiv., Encyclop. Méthod., VIII. 432. 5. [Odontomyia] (1811); W1ed., Analecta Entomol., 13. (1824); St.-FArg. et Serv., Encyclop. Méthod., X. 804. 1. (1825); WIED., Aussereurop. zweifl.

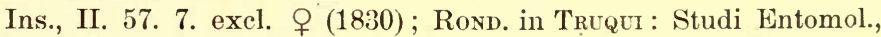
I. 100. 68. (1848); WALK., List Dipt. Brit. Mus., III. 525. (1849) et V. suppl. 1., 19. 15. (1851); Gerst., Linnaea Entomol., XI. 284. 5. (1857) ; Hunter, Trans. Amer. Entom. Soc. Philad., XXVII. 129. (1901).

cyanea MACQ. = auriflamma Wied.

cyanea Wied. p. p. (nec FABr.) = verticalis Gerst.

cyanispinis BIG., Annal. Soc. Entom. France, sér. 5. V.487. 1. (1875);

Hunter, Trans. Amer. Entom. Soc. Philad., XXVII. 129. (1901).

cyanocephala Gerst., Linnaea Entomol., XI. 274. 1. (1857) ; Hunter,

Trans. Amer. Entom. Soc. Philad., XXVII. 129. (1901).

dispar Schin., Novara Reise, Dipt., 54. 9. (1868); Hunter, Trans. Americamer. Amer. Entom. Soc. Philad., XXVII. 129. (1901).

fascipes WaLk., List Dipt. Brit. Mus., V. suppl. 1., 17. 7 et 303. Brasilia. (1851) ; Gerst., Linnaea Entomol., XI. 310. 24. (1857); Hunter, Trans. Amer. Entom. Soc. Philad., XXVII. 129. (1901). 
fenestrata MACQ. $=$ albitarsis FABR.

flavimana Gerst., Linnaea Entomol., XI. 291. 10. (1857); Huster, Brasilia.

Trans. Amer. Entom. Soc. Philad., XXVII. 129. (1901).

flavispinis MACQ. $=$ ? Wiedemanni Gerst.

geniculata Gerst., Linnaea Entomol., XI. 290. 9. (1857); Hunter, N. Granada.

Trans. Amer. Entom. Soc. Philad., XXVII. 129. (1901).

gracilicornis Gerst., Linnaea Entomol., XI. 295. 13. (1857); Hunter, Brasilia.

Trans. Amer. Entom. Soc. Philad., XXVII. 129. (1901).

hybrida Gerst., Linnaea Entomol., XI. 307. 21. (1857); Hunter, R. de Janeiro.

Trans. Amer. Entom. Soc. Philad., XXVII. 129. (1901).

lanuginosa Gerst., Linnaea Entomol., XI. 297. 14. (1857); Hunter, Brasiliil.

Trans. Amer. Entom. Soc. Philad., XXVII. 129. (1901).

Iasiophthalma Willist., Trans. Entom. Soc. London, 1896. 301. 1. I.St. Vincent.

(1896) ; Hunter, Trans. Amer. Entom. Soc. Philad.. XXVII. 129.

(1901); Aldr., Catal. North Amer. Dipt., 181. (1905).

lasiophthalma Willist., Biolog. Central. Amer., Dipt., I. 244. 6. tab.

IV. fig. 15. (1900) ; A A.Dr., Catal. North Amer. Dipt., 181. (1905).

leucocephala WiED., Zoolog. Magaz., I. 3., 55. 22. (1819) et Aussereurop. zweifl. Ins., II. 55. 2. (1830) ; ? MacQ., Dipt. exot., I. 1., 175. 1. (1838); WaLk., List Dipt. Brit. Mus., V. suppl. 1., 16. 2 et 303. (1854); Gerst., Linnaea Entomol., XI. 304. 20. (1857); HuxTER, Trans. Amer. Entom. Soc. Philad., XXVII. 129. (1901). albicaput WaLK., List Dipt. Brit. Mus., III. 521. [Clitellaria] (1849).

maculata FABr., Systema Antliat., 85. 30. [Stratiomys] (1805); Wied., Analecta Entomol., 13. (1824); Str.FARG. ET Serv., Encyclop. Méthod., X. 804. 1. (1825); WiEd., Aussereurop. zweifl. Ins., II. 57. 6. p. p. (1830); WaLk., List Dipt. Brit. Mus., V. suppl. 1., 17. 8. (1854); Gensт., Linnaea Entomol., XI. 308. 22. (1857) ; Huxter, Trans. Amer. Entom. Soc. Philad., XXVII. 129. (1901).

maculata Wied. (nec FABR.) p. p. = pulchella Gerst.

marginata Lw., Berlin. Entom. Zeitschr., IX. 148. 31. (1865); ALDr., Cuba.

Catal. North Amer. Dipt., 181. (1905).

ochracea Gigl.-Tos, Bollet. Mus. Zool. ed Anat. Comp. Torino, VI. Mexico. No. 102., 2. (1891) et Mem. R. Accad. Sci. Nat. Torino, sor. 2. XLIII. 106. 7. tab. I. fig. 4. (1893); ALDr., Catal. North Amer. Dipt., 181. (1905).

ornata WALK., Newman's Zool., Append., XCVI. (1850) et List Dipt. Brit. Mus., V. suppl. 1., 19. 14. (1854); Gerst., Limnaea Entomol., XI. 299. 17. (1857) ; Hunter, Trans. Amer. Entom. Soc. Philad., XXVII. 129. (1901).

picta Schis., Novara Reise, Dipt., 53. 8. (1868); Hunter, Trans. Americamer. Amer. Entom. Soc. Philad., XXVII. 129. (1901).

pilosissima Glerst., Linnaea Entomol., XI. 292. 11. (1857); Willist., Mexico. Biolog. Central. Amer., Dipt., I. 244. 5. (1900) ; Atdr., Catal. North Amer. Dipt., 181. (1905). 
pubiventris Rond., Archivio per la Zoolog., III. 86. [Ciphomia] Prom.bon.sp. (1863).

pulchella Gerst., Linnaea Entomol., XI. 309. 23. (1857).

Brasilia.

maculata WiED., Aussereurop. zweill. Ins., II. 57. 6. p. p. (1830).

rubra Lw., Berlin. Entom. Zeitschr., IX. 148. 30. (1865); AldDr., Cuba.

Catal. North Amer. Dipt., 181. (1905).

scalaris Bıt., Annal. Soc. Entom. France, sér. 5. IX. 487. 2. (1879); Ost.-SAck., Biolog. Central. Amer., Dipt., I. 35. (1886) ; Gigl.-Tos, Mem. R. Accad. Sci. Nat. Torino, ser. 2. XLIII. 105. 6. tab. I. fig. 3. (1893) ; Aldr., Catal. North Amer. Dipt., 181. (1905).

Schaefferi Coquili., Journ. New York. Entom. Soc., XII. 32. (1904). similis Bell., Saggio di Ditterol. Messic., T. 23 4. tab. I. fig. 7. (1859) ; Aldr., Catal. North Amer. Dipt., 181. (1905).

simplex WaLK., Trans. Entom. Soc. London, n. ser. V. 268. (1860)； Mexico. Aldr., Catal. North Amer. Dipt., 181. (1905).

tomentosa Gerst., Linnaea Entomol., XI. 294. 12. (1857); Belu., Mexico. Saggio di Ditterol. Messic., I. 22. 3. (1859) ; ALDr., Catal. North Amer. Dipt., 181. (1905).

varipes Gerst., Linnaea Entomol., XI. 283. 4. (1857) ; Scunn., Novara Reise, Dipt., 53. 7. (1868); Ost.-Sack., Biolog. Central. Amer., Dipt., I. 34. 1. (1886) ; Willist., Biolog. Central. Amer., Dipt., I. 243. (1900); Hunter, Trans. Amer. Entom. Soc. Philad., XXVII. 129. (1901) ; Alor., Catal. North Amer. Dipt., 181. (1905).

verticalis Genst., Linnaea Entomol., XI. 287. 6. (1857); Schin., Novara Reise, Dipt., 53. 6. (1868); Hunter, Trans. Amer. Entom. Soc. Philad., XXVII. 129. (1901).

cyanea Wied., Aussereurop. zweifl. Ins., II. 57. 7. excl. o7. (1830).

violacea Macq., Dipt. exot., suppl. 5., 40.5. (1855) ; Gerst., Linnaea Entomol., XI.' 290. 8. (1857) ; Hunter, Trans. Anier. Entom. Soc. Philad., XXVII. 129. (1901).

Wiedemanni Gerst., Linnaea Entomol., XI. 302. 19. (1857) ; Scuin., Novara Reise, Dipt., 53. 5. (1868); E. L. Arriß., Bolet. Acad. Nat. Cienc. Córdoba, IV. 129. 51. (1882); Huntrr, Trans. Amer. Entom. Soc. Philad., XXVII. 129. (1901).

albitarsis Wied. (nec FABR.), Analecta Entomol., 13. (1824); Str.FArg. et Serv., Encyclop. Méthod., X. 804. 2. (1825); WIED., Aussereurop. zweifl. Ins., II. 56. 5. tab. VIII. fig. 2. (1830) ; ? Macq., Dipt. exot., I. 1., 175. 2. (1838); WALK., List Dipt. Brit. Mus., V. suppl. 1., 16. 4 et 303. (1854). ?flavispinis MACQ., Dipt. exot., suppl. 5., 40. 6. (1855).

Anerica mer. et centr.

Anerica mer.

Brasilia.

Americamer. 


\section{Neorondania}

Ost.-SACK., Catal. Dipt. N. Amer., Ed. II. 50 et 225. 61. (1878).

Rondania Jaenn., Abhandl. Senckenb. Naturf. Ges., VI. 324. 14. (1867) (nec R.-D. 1850, nec Big. 1854).

Anchialus WALK. = chalybaea WiED.

chalybaea Wred., Analecta Entomol., 30. 36. [Clitellaria] (1824) et Antillae.

Aussereurop. zweifl. Ins., II. 49. 4. [Clitellaria] (1830); MacQ., Dipt. exot., I. 1., 191. [Ephippium] (1838); WaLk., List Dipt. Brit. Mus., V. suppl. 1., 60. 4. [Clitellaria] (1854); JAEnn., Abhandl. Senckenberg. Naturf. Ges., VI. 324. 14. tab. XLIII. fig. 4. [Rondania] (1867) ; Aldr., Catal. North Amer. Dipt., 182. (1905).

Anchialus WaLk., List Dipt. Brit. Mus., III. 522. [Clitellaria] (1849) et V. suppl. 1., 60. 3. [Clitellaria] (1854); As.dr., Catal. North Amer. Dipt., 189. [Clitellaria] (1905).

obscura Jaens., Abhandl. Senckenberg. Naturf. Ges., VI. 325. 15. Mexico. [Rondania] (1867); Aldr., Catal. North Amer. Dipt., 182. (1905).

\section{Alliocera}

SAUnd., Trans. Entomol. Soc. London, IV. 62. (1845).

clavicornis EGG. = graeca SAUND.

graeca SAUND., Trans. Entom. Soc. London, IV. 62. tab. IV. fig. 1, Europa mer. 1a-c. (1845); Walk., List Dipt. Brit. Mus., III. 526. (1849) et V. suppl. 1., 59. 1. (1854) ; Schin., Verh. zool.-bot. Ver. Wien, V. 619. 1. (1855) et VII. 7. (1857) et Fauna Austriaca, Dipt., I. 13. (1862); JAEnn., Berlin. Entom. Zeitschr., X. 228. 26b. (1866); KerT., Pótfüz. a Ṭermészettud. Közlönyhöz, XXXII. 126. (1900). clavicornis EGG., Verh. zool.-bot. Ver. Wien, IV. 3. tab. II. fig. 3-4. [Stratiomys] et Sitzgsber., 35. (1854).

\section{Hirtea}

Scop., Entomol. Carniol., 367. (1763).

Thyreodonta RonD., Archivio per la Zool. Modena, III. 77. [Thyreodontha].

Anubis WiEd., Aussereurop. zweifl. Ins., II. 60. 1. [Stratiomys] (1830); WalK., List Dipt. Brit. Mus., V. suppl. 1., 49. 85. [Stratiomys] (1854); Gerst., Linnaea Entomol., XI. 322. [Hoplomyia] (1857) ; Pleske, Sitzgsber. Naturf. Ges. Jurjeff, XII. 1900. 344, 349 et 363. 35. [Stratiomyia] (1901); Beck., Mitteil. Zoolog. Mus. Berlin, II. 10. 5. [Stratiomyia] (1902).

apicalis WALK., List Dipt. Brit. Mus., V. suppl. 1., 53. 105. [Stratio- China sejt. mys] (1854); v. D. Wulp, Catal. Dipt. South Asia, 51. [Stratiomyia]

Africa sept., Rossia mer., Persia. 
(1896) ; Pleske, Természetrajzi Füzetek, XXV. 414. 4. [Stratiomyia] (1902).

longicornis Scop., Entomol. Carniol., 367. 999. (1763) ; OuIv., Encyclop. Méthod., VII. 92. 1. (1792); WaLK., Insecta Britann., Dipt., I. 15. 1. [Stratiomys] (1851) et List Dipt. Brit. Mus., V. suppl. 1., 29. 8. [Stratiomys] (1854); ScHiv., Verh. zool.-bot. Ver. Wien, V. 622.8 et 667. [Stratiomys] (1855) et VI. 421. 999. [Stratiomys] (1856); Gerst., Linnaea Entomol., XI.-. tab. III. fig. 4. [Strationys] (1857); Schis., Fauna Austriaca, Dipt., I. 17. [Stratiomys] (1862); JAEnn., Berlin. Entom. Zeitschr., X. 229. 33. [Stratiomys] (1866); v. D. Wulp, Diptera Neerland., I. 452. 2. [Stratiomyia] (187i); ? Friedenfels, Verh. u. Mittheil. d. siebenbürg. Ver. f. Naturwiss. Hermannstadt, XXX. 164. [Stratiomys] (1879) ; Brau., Denkschr. Akad. Wien, XLVII.-. tab. II. fig. 23 b. [Stratiomys] (1883); Röp., Entomol. Nachricht., X. 253. [Stratiomyia] (1884); Neuhaus, Diptera Marchica, 81. 4. [Stratiomys] (1886); Girschn., Berlin. Entom. Zeitschr., XXXI.-. tab. III. fig. 6. [Stratiomyia] (1887); BRuNeTrI, The Entomologist, XXII. 86. 1. [Stratiomyia] (1889); Хсносн, Fauna Insect. Helvetiae, Dipt., 22. [Stratiomys] (1890); Hennegur et Binet, Annal. Soc. Entom. France, sér. 7. II. 309-316. tab. VI. fig. 1-6. [Stratiomys] (1892); É. \& L. Coucke, Annal. Soc. Entom. Belgique, XXXVII. 438. [Stratiomyia] (1893); StroвL, Mittheil. Naturwiss. Ver. Steiermark, XXIX. 1892. 10. [Stratiomyia, Thyreodonta] (1893) ; Pleske, Wien. Entom. Zeitg., XVIII. 262. 7. [Stratiomyia] (1899); Kert., Pótfüz. a Természettud. Közlönyhöz, XXII. 127. (1900) ; Pleske, Sitzgsber. Naturf. Ges. Jurjeff, XII. 1900. 344 et 348. [Stratiomyia] (1901).

nubeculosa MEIG., Klassif., I. 125. 3. [Stratiomys] (1804).

strigata FABR., Species Insect., II. 417.4.[Stratiomys] (1781) et

Mantissa Insect., II.330. 7. [Stratiomys] (1787); Rossi, Fauna Etrusca, II. 280. 1441. [Stratiomys] (1790); GueL., Systema Naturae, V. 2834. 153. [Musea] (1792) ; Panz., Fauna Germ., XII. tab. 20. [Stratiomys] (1793) ; FABr., Entomol. System., IV. 265. 10. [Stratiomys] (1794); Schrank, Fauna Boica, III. 94. 2377. [Stratiomys] (1803) ; Latr., Hist. Nat. d. Crust. et d. Ins., XIV. 339. 2. [Stratiomys] (1804); FABR., Systema Antliat., 80. 9. [Stratiomys] (1805); Rossi, Fauna Etrusca, Ed. II., 432. 1441. [Stratiomys] (1807) ; LAM., Hist. nat. anim. sans vert., III. 386. 1. [Stratiomys] (1816); MeIG., System. Beschreib., III. 139. 7. tab. XXVI. fig. 6-7. [Stratiomys] (1822) ; MAcQ., Recueil Soc. Sci. Agricult. Lille, 1826. 453. 4. [Stratiomys] (1826) et Suit. à Buffon, I. 242. 7. [Stratiomys] (1834) ; Duncan, Magaz. Zool. and Botany, I. 151. 5. [Stratiomys] (1837); Lw., Programm Posen, 1840. 25. 4. [Stratiomys] (1840) et Isis, 1840. VII. 556. 4. '[Stratiomys] (1840); Zетт., Dipt. Scand., I. 135. 3. [Stratiomys] (1842); Lw., Linnaea
Europa,

Asia min., Transcaucas. 
Entomol., I. 467. [Stratiomys] (1846); Giмм., Bull. Soc. Imp. Nat. Moscou, XX. 2., 169. 3. [Stratiomys] (1847); WALk., List Dipt. Brit. Mus., III. 529. [Stratiomys] (1849); Scholtz, Zeitschr. f. Entomol. Breslau, IV. 34. [Stratiomys] (1849); Zৈтт., Dipt. Scand., VIII. 2953. 3. [Stratiomys] (1849) et XI. 4260. 3. [Stratiomys] (1852) ; Rond., Archivio per la Zoolog., III. 77. [Thyreodontha] (1863) ; Meinert, Fluernes Munddeele, Trophi Dipt., 58. tab. IV. fig. 32-33. [Stratiomys] (1881). tenebricus Harris, Expos. Engl. Ins. 45. tab. XI. fig. 3. [Musca]

(1782).

thoracica FABr., Systema Antliat., 79. 7. [Stratiomys] (1805). villosa Meig., Klassif., I. 124. 1. [Stratiomys] (1804).

var. pallida Lw., Programm Posen, 1840. 25. 5. [Stratiomys] Europacentr. (1840) et Isis, 1840. VII. 557. 5. [Stratiomys] (1840).

nubeculosa Mrig. = longicornis Scop.

pallida $\mathrm{Lw} .=$ longicornis Scop. var.

strigata $\mathrm{FABR} .=$ longicornis ScOP.

tenebricus HARRIS = longicornis SCOP.

thoracica $\mathrm{FAPR}$. = longicornis ScOP.

villosa MEjG. = longicornis Scop.

\section{Stratiomyia}

GEofrr., Hist. Nat. d'Ins., II. 475. (1764) [Stratiomys]. Hoplomyia Zelu., Isis, 1842. XI. 882. [recte 828]. (1842).

Stratiomyia MacQ., Dipt. exot., I. 1., 179. 7. (1838).

Stratiotomyia E. L. Arriß., Bol. Acad. Nat. Cienc. Córdoba, IV. 128. 39. (1882).

Ahngeri Pleske, Sitzgsber. Naturf. Ges. Jurjeff, XII. 1900. 345 et Transcaspia. 364. 36. (1901).

Aleus WALK. = mutabilis FABR.

analis Fabr., Systema Antliat., 83. 21. [Stratiomys] (1805); Wied., Aussereurop. \%weifl. Ins., II. 76. 27. [Stratiomys] (1830); WALK., List Dipt. Brit. Mus., V. suppl. 1., 57. 122. [Stratiomys] (1854). angularis $\mathrm{LW}_{\mathrm{W}}=$ Meigenii WiED.

apicula Lw., Berlin. Entom. Zeitschr., IX. 136. 13. (1865) ; Jouns., Trans. Amer. Entom. Soc. Philad., XXII. 239. tab. III. fig. 23-24. (1895); Hart, Bull. Illinois State Labor. of Nat. Hist., IV. 249. (1895) ; Meland., Canad. Entomol., XXXVI. 21 et 22. 7. (1904) ; Aldr., Catal. North Amer. Dipt., 182. (1905).

armeniaca Bıg., Annal. Soc. Entom. France, sér. 5. IX. 213. 4. [Stratiomys] (1879) ; Pleske, Wien. Entom. Zeitg., XVIII. 243. 6. (1899), Sitzgsber. Naturf. Ges. Jurjeff, XII. 1900. 333. 8. (1901) et Természetrajzi Füzetek, XXV. 441. 1. (1902).

Badius WALK., List Dipt. Brit. Mus., III. 529. [Stratiomys] (1849) Americasept. et IV. 1157. [Stratiomys] (1849); Johns., Trans. Amer. Intom.

Transcaucas., Caucasus, Armenia. 
Soc. Philad., XXII. 243. tab. III. fig. 11-12. (1895); Meland., Canad. Entomol., XXXVI. 22. (1904) ; Audr., Catal. North Amer. Dipt., 182. (1905).

ischiaca WaLk., List Dipt. Brit. Mus., III. 529. [Stratiomys] (1849) et V. suppl. 1., 35. 35. [Stratiomys] (1854).

picipes Lw., Berlin. Entom. Zeitschr., X. 13. 21. (1866) ; v. D.

Wulp, Tijdschr. v. Entomol., XXIV. 154. 4. (1881).

barbata Lw., Berlin. Entom. Zeitschr., IX. 133. 9. (1865); Jonss.,

Trans. Amer. Entom. Soc. Philad., XXII. 231. tab. III. fig. 1-2. (1895) ; Meland., Canad. Entomol., XXXVI. 21. (1904); Aldr., Catal. North Amer. Dipt., 182. (1905).

calopus Bıg., Annal. Soe. Entom. France, sér. 6. VII. 23. 3. [Stratiomys] (1887).

dirulemata Brg., Annal. Soc. Entom. Franee, sér. 6. VII. 23. 2. [Stratiomys] (1887).

Barca Walk., List Dipt. Brit. Mus., III. 530. [Stratiomys] (1849) et

V. suppl. 1., 51. 94 et 312. [Stratiomys] (1854); v. D. Wulp, Catal. Dipt. South Asia, 51. (1896); Coquilu., Proc. Unit. Stat. Nat. Mus., XXI. 308. (1898).

Beckeri Pleske, Sitzgsber. Naturf. Ges. Jurjow, XII. 1900. 331. 7. Europacentr. (1901).

concinna Auct. Plur.; Jannn., Berlin. Entom. Zeitschr., X. 229. 31. [Stratiomys] (1866); BECK., Berlin. Entom. Zeitschr., XXXI. 10\% 14. [Stratiomys] (1887); Sспосн, Fauna Ins. Helvetiae, Dipt., 23. [Stratiomys] (1890); BEzzr, Wien. Entom. Zeitg., XV. 217. (1896); Röner, Wien. Entom. Zeitg., XV. 274. (1896); Pleske, Wien. Entom. Zeitg., XVIII. 243. 4. P. p. (1899).

Beresowskii PLiske, Wien. Entom. Zeitg., XVIII. 274. 28. (1899) et Sitzgsber. Naturf. Ges. Jurjew, XII. 1900. 351. (1901).

bimaculata Belu., Saggio di Ditterol. Messic., Append., 10. 4. tab. Mexico.

III. fig. 7. [Stratiomys] (1862); Jouns., Trans. Amer. Entom. Soc. Philad., XXII. 245. (1895); Wiluist., Biolog. Centr. Amer., Dipt., I. 248. 3. (1900); Meland., Canad. Entomol., XXXVT. 22. (1904) ; ALdr., Catal. North Amer. Dipt., 182. (1905).

bochariensis Pleske, Wien. Entom. Zeitg., XVIII. 278. 31. (1899) et Sitzgsber. Naturf. Ges. Jurjew, XII. 1900. 348, 352 et 356. 31. (1901).

brevicornis Pontscir., Horae Soc. Entom. Ross., XXI. 176. (1887); P'LESKE, Wien. Entom. Zeitg., XVIII. 267. 20. (1899) et Sitzgsber.

Naturf. Ges. Jurjew, XII. 1900. 343. notı 1 et 349. (1901).

Bruneri Jorrs., Trans. Amer. Entom. Soc. Philad., XXII. 233. tab. America sept.

III. fig. 7, 8. (1895); Meutnd., Canad. Entomol., XXXVI. 21.

(1904); Aldr., Catal. North Amer. Dipt., 182. (1905).

calopus $\mathrm{BiG}_{\mathrm{G}}=$ barbata $\mathrm{Lw}$.

caucasica PLeske = chamaeleon L. var. 
cenisia MeIG., System. Beschreib., III. 136. 2. [Strationys] (1822); Macq., Suit. à Buffon, I. 243. 2. [Stratiomys] (1834); Lw., Linnaea Entomol., I. 466. [Stratiomys] (1846); Macq. in Lucas: Explor. scient. de l'Algérie, Zool., III. 427. 37. tab. II. fig. 11. [Stratiomys] (1849); WALK., List Dipt. Brit. Mus., V. suppl. 1., 28. 4 [Stratiomys] (1854); Schin., Verh. zool.-bot. Ver. Wien, V. 620. 2 et 667. [Stratiomys] (1855) et Fauna Austriaca, Dipt., I. 16. 8. [Stratiomys] (1862); JAENN., Berlin. Entom. Zeitschr., X. 228. 28. [Stratiomys] (1866); Neuraus, Diptera Marclica, 81. 2. [Stratiomys] (1886); Sсносн, Fauna Ins. Helvetiae, Dipt., 23. [Slratiomys] (1890) ; E. \& L. Coucke, Annal. Soc. Entom. Belgique, XXXVII. 439. (1893); Pleske, Wien. Entom. Zeitg., XVIII. 266. 16. (1899); Kenт., Pótfüz. a Természettud. Közlönylü̈z, XXXII. 127. (1900); Pleske, Sitzgsber. Naturf. Ges. Jurjew, XII. 1900. 346 et 352 . (1901).

chamaeleon L., Systema Nat., Ed. X. 589. 3. [Musca] (1758), Fauna Suec., Edit. Alt., 440. 1780. [Musca] (1761); O. F. Múl., Fauna Ins. Fridrichsdal., 80. 706. [Musca] (1764); I., Systema Nat., Edit. XII., II. 979. 3. [Musca] (1767); FABR., Systema Entomol., 759. 1. [Stratiomys] (1775) ; Deg., Mém. pour serv. l'hist. d. Ins., VI. 151. 1. [Stratiomys] (1776) ; O. F. Mülu., Zoolog. Dan. Prodr., 177.2081. [Stratiomys] (1776) ; FA BR., Spec. Insect., II. 416. 1. [Stratiomys] (1781); Schrank, Enum. Ins. Austr., 436. 886. [Musca] (1781); Harris, Exop. Engl. Ins., 44. tab. XI. fig. 1. [Musca chamelion] (1782) ; FABR., Mantissa Insect., II. 330. 2. [Strutiomys] (1787) ; Rossi, Fauna Etrusca, II. 278. 1438. [Stratiomys] (1790) ; Gusc., Systema Naturae, V. 2833. 3. [Musca] (1792); PANz., Fauna Germ., VIII. tab. 24. [Stratiomys] (1793); FABr., Entomol. System., IV. 263. 3. [Stratiomys] (1794); Schrank, Fauna Boica, III. 93. 2376. [Stratiomys] (1803) ; MeIG., Klassif., I. 126. 4. p. p. tab. VII. fig. 19. ㅇ. [Stratiomys] (1804); Latr., Hist. Nat. d. Crust et Ins., XIV. 339. 1. tab. CXI. fig. 7. [Stratiomys] (1804) ; Fabr., Systema Antliat., 77. 1. [Stratiomys] (1805); Sparm., Kongl. Vet. Akad. Hanăl., XXVII. 239. [Stratiomys] (1806); G. Siraw, Gen. Zool., VI. 379. tab. CV. [Stratiomys] (1806); Rossi, Fauna Etrusca, Ed. II. 430. 1438. [Stratiomys] (1807); Isatr., Gen. Crust. et Ins., IV. 274. [Stratiomys] (1809) et Consid. génér., 442. [Stratiomys] (1810); ІАлм., Hist. nat. anim. sans vert., III. 386. 2. [Stratiomys] (1816); FaLL., Dipt. Suec., Stratiom., 7. 1. [Stratiomys] (1817); Cuv., Régne Animal., III. 623. [Stratiomys] (1817) ; MeIa., System. Beschreib., III. 134. 1. [Stratiomys] (1822); Dumér., Consid. gén. Ins., tab. XLVIII. fig. 6. [Stratiomys] (1823) ; MAcQ., Recueil Soc. Scient. Agricult. Lille, 4is1. 1. tab. III. fig. 2. [Stratiomys] (1826) ; Brulıé, Expéd. scient. do Morée, III. 1., 306. 662. [Stratiomys] (1832); Macq., Suit. à Buffon, I. 243. 1. [Stratiomys] (1834); Iuncan, Magaz. Zool. and Botany, I. 148. 1. [Stratiomys] (1837); BunNCH., Hist. d'Ins., III. 593. tab. II. fig'.
Europa centr. et mer.,

Africa sept., Asia min. et centr.

\section{Europa,}

Sibiria, Transcaspia. 
7. [Stratiomys] (1840); Westw., Introd., II. 532. [Stratiomys] (1840); Lw., Programm Posen, 1840. 25. 1. [Stratiomys] (1840) et Isis, 1840. VII. 556. 1. [Stratiomys] (1840) ; ZETT., Dipt. Scand., I. 134. 1. [Stratiomys] (1842); Lw., Linnaea Entomol., I. 467. [Stratiomys] (1846); WaLk., List Dipt. Brit. Mus., III. 528. [Stratiomys] (1849) ; ZETT., Dipt. Scand., VIII. 2951. 1. [Stratiomys] (1849) ; L. Duf., Mém. Acad. des Scienc., Matb. et Phys., XI. 171-360. tab. IV. fig. 44. [Stratiomys] (1850); Walk., Insecta Britann., Dipt., I. 15. 2. tab. I. fig. 3. [Stratiomys] (1851) ; EqGer, Verh. zool.-bot. Ver. Wien, IV. - - tab. II. fig. 5-6. [Stratiomys] (1854); Walk., List Dipt. Brit. Mus., V. suppl. 1., 28. 2. [Stratiomys] (1854); Schin., Verh. zool.-bot. Ver. Wion, V. 620. 1 et 667. [Stratiomys] (1855); Leydia, Archiv. f. Naturgeschr., XXVI. 1., 157. [Stratiomys] (1860) et Borlin. Entom. Zeitschr., V. pag. XXXIX. [Stratiomys] (1861); Scrin., Fauna Austriaca, Dipt., I. 16. 8. [Stratiomys] (1862); JAEnn., Berlin. Eintom. Zeitschr., X. 228. 27. [Stratiomys] (1866); v. D. Wulp, Diptera Neerland., I. 452. (1877); Meinert, Fluernes Munddeele, Trophi Dipt., 58. tab. IV. fig. 34-35. [Stratiomys] (1881); Becher, Denkschr. Akad. Wien, XLV. 140. tab. II. fig. 4. [Stratiomys] (1882); Brav., Denkschr. Akad. Wien, XLVII. - t tab. II. fig. 22. [Stratiomys] (1883); Neunuus, Diptera Marchica, 81. 1. [Stratiomys] (1886); Brunerti, The Entomologist, XXII. 86. 4. (1889); Schoci, Fauna Ins. Helvetiae, Dipt., 23. [Stratiomys] (1890); É.\& I. Coucke, Annal. Soc. Entom. Belgique, XXXVII. 438. (1893); SтroвL, Mittheil. Naturwiss. Ver. Steiermark, XXIX. 1892. 9. (1893); Mıк, Wien. Entom. Zeitg., XV. 11'). 52. (1896); P'leske, Wien. Entom. Zeitg., XVIII. 263. 10. (1899); Florentin, Annal. Scienc. Naturell., sér. 8. X. 274. [Stratiomys] (1899); Krnт., Pótfiiz. a Természettud. Közlönyhöz, XXXII. 127. (1900); PLeske, Sitzgsber.

Naturf. Ges. Jurjew, XII. 1900. 347 et 354. (1901). nigrodentata Meig., K’lassif., I. 127. 4. [Stratiomys] (1804). sellata Sulz., Gesch. d. Ins., 200. [Musca] (1776). ? spatula Scop., Entomol. Carniol., :41. 917. [Musca] (1763); Schin., Verh. zool.-bot. Ver. Wien, VI. 414. 917. [Stratiomys] (1856).

var. caucasica Pleske, Wien. Entom. Zeitg., XVIII. 264. 11. Transcaucas. (1899) et Sitzgsber. Naturf. Ges. Jurjew, XII. 1900. 353. (1901). var. rhaetica J AEnn., Berlin. Entom. Zeitschr., X. 228. 29. [Strutiomys] (1866); BEck., Berlin. Entom. Zeitschr., XXXI. 105. 16. [Stratiomys] (1887); Schocir, Fauna Ins. Helvetiae, Dipt., 23. [Stratiomys] (1890); PLeske, Wien. entom. Zeitg., XVIII. 264. 12. (1899) et Sitzgsber. Naturf. Ges. Jurjew, XII. 1900. 347 et 354. (1901).

?var. unguicornis Bнск., Berlin. Entom. Zeitschr., XXXI. 103. 15., fig. [Stratiomys] (1887); Scrocu, Fauna Ins. Helvetiae, Dipt., 23. [Stratiomys] (1890); PLeske, Wion. Ėntom. Zeitg.,

Europacentr.

Helvetia. 
XVIIJ. 165. 13. (1899) et Sitzgsber. Naturf. Ges. Jurjew, XII. 1900. 346. (1901).

chamacleon Meig. 1804. p. p. (nec L.) = Potamida Meta.

concinna Auct. Pluli. = Beckeri Pleske.

concinna MeIG., System. Beschreib., III. 137. 4. tab. XXVI. fig. 14. [Stratiomys] (1822) ; Mace., Suit. à Buffon, I. 242. 4. [Stratiomys] (1834) ; Gims., Bull. Soc. Imp. Nat. Moscou, XV. 667. [Stratiomys] (1842); Lw., Linnaea Entomol., I. 467. [Stratiomys] (1846); Walk., List Dipt. Brit. Mus., V. suppl. 1., 28. 5. [Stratiomys] (1854); Schin., Verh. zool.-bot. Ver. Wien, V. 623. 9 et 667. [Stratiomys] (1855) ; EgGer, Verh. zool.-bot. Ver. Wien, IX. 394. [Stratiomys] (1859); Scuns., Fauna Austriaca, Dipt., I. 15. 5. [Stratiomys] (1862); Pleske, Wien. Entom. Zeitg., XVIII. 243. 4. p. p. (1899); Kert., Pótfüz. a Természettud. Közlönyhöz, XXXII. 126. (1900) ; Pleske, Sitzgsber. Naturf. Ges. Jurjew, XII. 1900. 330. 6. (1901).

confertissima Walk., Proc. Linn. Soc. London, III. 79. 10. [Stratiomys] (1859); v. D. Wur.P, Catal. Dipt. South Asia, 51. (1896).

conica FABr., Systema Antliat., 81. 16. [Stratiomys] (1805); Wied., Aussereurop. zweifl. Ins., II. 67. 10. [Stratiomys] (1830); WALK., List Dipt. Brit. Mus., V. suppl. 1., 45. 68. [Stratiomys] (1854); Hunter, Trans. Amer. Entom. Soc. Philad., XXVII. 127. (1901). connexa HunTER = convexa v. D. WULP.

constans Lw., Berlin. Entom. Zoitschr., XVI. 55. 8. (1872) ; Johns.,

Trans. Amer. Entom. Soc. Philad., XXII. 244. tab. IV. fig. 1-2.

(1895) ; Meland., Canad. Entomol., XXXVI. 22. (1904); Aldr., Catal. North Amer: Dipt., 182. (1905).

constricta WALk., Trans. Entom. Soc. London, n. ser., V. 269. [Stratiomys] (1860); Johns., Trans. Amer. Entom. Soc. Philad., XXII. 245. (1895); Aldr., Catal. North Amer. Dipt., 182. (1905). convexa v. D. Wulp, Tijdschr. v. Entomol., XXIV. 154. 6. (1881).

comnexa Hunter, Trans. Amer. Entom. Soc. Pliilad., XXVII. 127. (1901) [lapsus].

cuprina Wied., Aussereurop. zweifl. Ins., II. 69. 14. [Stratiomys] (1830) ; Walk., List Dipt. Brit. Mus., V. suppl. 1., 48. 81. [Stratiomys] (1854) ; Hunter, Trans. Amer. Entom. Soc. Philad., XXVII. 127. (1901).

cypria Pleske, Természetrajzi Fiizetek, XXV. 413. (1902). dentata $\mathrm{BIG}_{\mathrm{G}}=$ maculosa $\mathrm{J} \mathrm{w}$. diademata $\mathrm{BIG} .=$ barbata Lw.

discalis Lw., Berlin. Entom. Zeitschr., IX. 136. 14. (1865) ; Jonns., Trans. Amer. Entom. Soc. Philad., XXII. 240. tab. III. fig. 27, 28. (1895) ; HAlт, Bull. Illinois Stato Laborat. of Nat. Hist., IV. 249. (1895); Mesand., Canad. Entomol., XXXVI. 21 et 22. 8. (1904) ; Aldor., Catal. Nortl Imer. Dipt., 182. (1905).

equestris MerG., System. Beschreib., VII. 106. 29. [Stratiomys] (1838); Lw., Linnaea Entomol., I. 462. 1. [Stratiomys] (1846); Gıмм.,

Europa centr. et mer.

Ins. Aru.

Americamer.

A merica sept.

Mexico,

Argentinia.

Brasilia.

Cyprus.

America sept.

Europa centr. et mer.,

Transbaikal. 
Bull. Soc. Imp. Nat. Moscou, XX. 2., 168. 1. [Stratiomys] (1847); WALK., List Dipt. Brit. Mus., V. suppl. 1., 29. 6 et 306. [Stratiomys] (185t); Scuns., Verh. zool.-bot. Ver. Wien, V. 623. 10 et 667. [Stratiomys] (1855) et Fauna Austriaca, Dipt., I. 15. 6. [Stratiomys] (1862); v. D. Wulp, Diptera Neerland., I. 453. (1877); Neunaus, Diptera Marelica, 81. 5. [Stratiomys] (1886); Schoch, Fauna Ins. Helvetiae, Dipt., 23. [Stratiomys] (1890); É. \& I. Coucke, Annal. Soc. Entom. Belgique, XXXVII. 438. (1893); Pleske, Wien. Entom. Zeitg., XVIII. 268. 23. p. p. (q) (1899); Kert., Pótfïiz. a Termószettud. Közlönyhöz, XXXII. 127. (1900); Pleske, Sitzgrsbor. Naturf. Ges. Jurjow, XII. 1900. 353. (1901) et Természetrajzi Füzetek, XXV. 411. 2. (1902).

hirtuosa MeIG., System. Beschreib., VI. 347. 26. [Stratiomys] (1830).

?tomenlosa Schrank, Fauna Boica, III. 94. 2378. [Stratiomys] (1803).

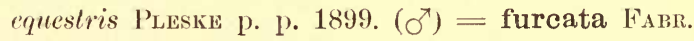

crythrocera EGa., Verh. zcol.-bot. Ges. Wien, IX. 393. [Stratiomys] (1859) ; Schin., Fauna Austriaca, Dipt., I. 14. 1. [Stratiomys] (1862); Bezzi, Wien. Entom. Zeitg., XV. 217. (1896); Rö̀., Wien. Entom. Zeitg., XV. 274. (1896); StrrobL, Glasnik Zem. Mus. Bosni i Hercegov., X. 390. (1898) ; PLeske, Wien. Entom. Zeitg., XVIII. 242. 3. p. p. (1899); Kert., Pótfüz. a Természettud. Közlönyhöz, XXXII. 126. (1900); Strobl, Wissenseh. Mittheil. aus Bosnien ù. Herzegov., VII. 554. (1900); Pleske, Sitzgsber. Naturf. Ges. Jurjew, XII. 1900. 329. 4. (1901).

erythrocera Pleske p. p. 1899. = pyrrhocera Lw.

fusciata FABR. 1787. = mutabilis FABR.

fenestrata GERst., I innaea Entomol., XI. 327. 4. [Stratiomys] (1857); Aıdr., Catal. North Amer. Dipt., 183. (1905).

festiva Walk., List Dipt. Brit. Mus., V. supp]. 1., 43. 63. [Stratiomys] (1854); Scuns., Novara Reise, Dipt., 61. 26. [Stratiomys] (1868); Hunter, Trans. Amor. Entom. Soe. Philad., XXVII. 127. (1901).

flavifrons MacQ., Dipt. exot., I. 1., 179. 1. (1838); WALK., List Dipt. Mesopotamia. Brit. Mus., V. suppl. 1., 5ย. 100. [Stratiomys] (1854); Pueske, Sitzgsber. Naturf. Ges. Jurjew, XII. 1900. 348 et 369. 39. (1901).

flaviventris Lw., Linnaea Entomol., I. 464. 2. [Stratiomys] (1846); WaLk., List Dipt. Brit. Mus., V. suppl. 1., 307. [Stratiomys] (1854) ; Pleske, Wien. Entom. Zeitg., XVIII. 266. 17. (1899) et Sitzgsber. Naturf. Ges. Jurjew, XII. 1900. 347, 352 et 355. 17. (1901).

flavolimbata A. Costa, Rendic. R. Accad. Napoli, ser. 2. VII. 101. Africa sept. [Strutiomys] (1893) et Atti R. Accad. Napoli, ser. 2. V. No. 14., 21. 2. tab. IV. fig. 6. [Stratiomys] (1893).

flavoscutellata v. D. Wulp, Notes Leyden Mus., VII. 60. 7. (1885) Java. et Catal. Dipt. South Asia, 51. (1896). 
furcata FABR., Entomol. System., IV. 264. 5. [Stratiomys] (1794) et Systema Antliat., 78. 3. [Stratiomys] (1805); OLiv., Encyclop. Méthod., VIII. 432. 1. [Odontomyia] (1811); Muig., System. Beschreib., III. . 138. 5. tab. XXVI. fig. 12-13. [Stratiomys] (1822); MacQ., Recueil Soc. Sci. Agricult. Lille, 1826. 452. 3. [Stratiomys] (1826) et Suit. à Buffon, I. 242. อ. [Stratiomys] (1834) ; Duncan, Magaz. Zoolog. and Botany, I. 150. 3. [Stratiomys] (1837); Lw., Programm Posen, 1840. 25. 2. [Stratiomys] (1840) et Isis, 1840. VII. 556. 2. [Stratiomys] (1840); ZETT., Dipt. Scand., I. 135. 2. [Stratiomys] (1842); Lw., Isinnaea Entomol., I. 467. [Stratiomys] (1846); Walk., List Dipt. Brit. Mus., III. 528. [Stratiomys] (1849); Zетт., Dipt. Scand., VIIJ. 2952. 2. [Stratiomys] (1849); Walk., Insecta Britann., Dipt., I. 16. 3. [Stratiomys] (1851) et List Dipt. Brit. Mus., V. suppl. 1., 30. 9. [Stratiomys] (1854); Schin., Verh. zool.-bot. Ver. Wien, V. 621. 6 et 667. [Stratiomys] (1855); Zетт., Dipt. Scand., XIII. 4952. 2. [Stratiomys] (1859); Bonsd., Finland tvaving. Ins., I. 116. 1. [Stratiomys] (1861); Scmin., Fauna Austriaca, Dipt., I. 16. [Stratiomys] (1862); Jaenn., Berlin. Entom. Zeitschr., X. 229. 32. [Stratiomys] (1866); v. D. Wulp, Diptera Neerland., I. 451. 1. tab. XIV. fig. 12-13. (1877) ; Big., Annal. Soc. Entom. France, sér. 5. IX. 186. [Thyreodontha] (1879) ; Brau., Denkschr. Akad: Wien, XLVII. tab. II. fig. 23a. [Stratiomys] (1883); Nevraus, Diptera Marchica, 81. 3. [Stratiomys] (1886); Ginschn., Berlin. Entom. Zeitschr., XXXI.tab.III.fig.7.(1887); BRunetTI, TLe Entomologist, XXII. 86. 3. (1889); Sсносн, Fauna Insect. Helvetiae, Dipt., 23. [Stratiomys] (1890) ; É. \& L. Coucke, Annal. Soc. Entom. Belgique, XXXVII. 438. (1893) ; Pleske, Wien. Entom. Zeitg., XVIII. 263. 8. (1899); Kert., Pótfüz. a Természettud. Közlönylüz, XXXII. 127. (1901) ; Pleske, Sitzgsber. Naturf. Ges. Jurjew, XII. 1900., 345 et 349. (1901); Meis., Zoolog. Jahrbüch., Anat. ete., XV. 676. tab. XXXIV. fig. 34. (1902); PLeske, Természetrajzi Füzetek, XXV. 412. (1902).

equestris Pleske, Wien. Entom. Zeitg., XVIII. 268. 23. p. p. [ơ] (1899) et Sitzgsber. Naturf. Ges. Jurjow, XII. 1900. 345. p. p. [ठ] (1901).

panthaleon FaLL., Dipt. Suec., Stratiom., 7. 2. [Stratiomys] (1817).

singularis Harris, Expos. Engl. Ins., 45. tab. XI. fig. 2. [Musca] (1782).

Gerstaeckeri Bell., Saggio di Ditterol. Messic., I. 31. 3. tab. I. fig. Mexico. 10. [Stratiomys] (1859); Johns., Trans. Amer. Entom. Soc. Philad., XXII. 245. (1895); Canad. Entomol., XXXVI. 22. (1904); Aldr., Catal. North Amer. Dipt., 183. (1905).

goniphora SAy, Journ. Acad. Nat. Sei. Philad., VI. 161. [Stratiomys] Mexico. (1829) et Compl. Writt., II. 356. [Stratiomys] (1859); WALK., List Dipt. Brit. Mus., V. suppl. 1., 114. [Stratiomys] (1854);

Europa, Sibiria. 
Jorns., Trans. Amer. Entom. Soe. Philad., XXII. 247. (1895);

ALDR., Catal. North Amer. Dipt., 183. (1905).

Herzi Pleske, Sitzogsber. Naturf. Ges. Jurjew, XII. 1900. 327. 2. Transcaucas. (1901).

hirtuosa MeIG. = equestris MeIG.

hispanica Pleske, Sitzgsber. Naturf. Ges. Jurjew, XII. 1900. 346, Hispania. 352 et 366. 38. (1901).

Hunteri Krng, Narrat. of Survey of Australasia, II, 467. 181. Australia. [Stratiomys] (1827); WIED., Aussereurop. zweill. Ins., II. 77. 28. [Stratiomys] (1830); Walk., List Dipt. Brit. Mus., V. suppl. 1., 55. 114. [Stratiomys] (1851).

inanimis WALk., Trans. Entom. Soc. London, n. ser. IV., 121. China. [Stratiomys] (1857); v. D. Wulp, Catal. Dipt. Sou h A sia, 51. (1896).

insignis $\mathrm{Lw} .=$ maculosa $\mathrm{Lw}$.

ischiaca WALK. $=$ Badius WALK.

japonica v. D. Wulp, Notes Leyden Mus., VII. 60. 6. (1885).

Koslowi Pleske, Sitzgsber. Natuif. Ges. Jurjew, XII. 1900. 344, 350 et 359. 33. (1901).

Kosnakowi Pleske, Sitzgsber. Naturf. Ges. Jurjew, XII. 1900. 346 et 365. 37. (1901).

lacerata $\mathrm{BIG}_{\mathrm{g}}=$ maculosa $\mathrm{Lw}$.

laevifrons Lw., Neue Beitr., II. 17. 34. [Hoplomyia] (1854) ; PLeske, Wien. Entom. Zeitg., XVIJI. 270. 25. (1899) et Sitzgsber. Naturf. Ges. Jurjew, XII. 1900. 347, 351 et 353. (1901).

lambessiana Big., Annal. Soc. Entom. France, sér. IX. 212. 3. Algeria. [Stratiomys] (1879).

laticeps Lw., Berlin. Entom. Zeitschr., X. 12. 20. (1866) ; Jonns., Trans. Amer. Entom. Soc. Philad., XXII. 234. tab. III. fig. 9. (1895) ; Meland., Canad. Entomol., XXXVI. 21. (1904) ; Aldr., Catal. North Amer. Dipt., 183. (1905).

? Nymphis Walk., List Dipt. Brit. Mus., III. 530. [Stratiomys] (1849) et V. suppl. 1., 36. 37. [Stratiomys] (1854).

lativentris Lw., Berlin. Entom. Zeitschr., IX. 132. 8. (1865) ; Johss., Trans. Amer. Entom. Soc. Philad., XXII. 223. tab. III. fig. 5-6. (1895) ; Merand., Catal. Entomol., XXXVI. 21 et 22. 2. (1904); Aldr., Catal. North Amer. Dipt., 183. (1905).

obesa Lw., Borlin. Entom. Zeitschr., IX. 134. 11. (1865) ; Har'T, Bull. Illinois State Labor. of Nat. Hist., IV. 249. (1895).

leucopsis WiED., Aussereurop. zweifl. Ins., II. 74. 22. [Stratiomys] (1830) ; Walk., Iist Dipt. Brit. Mus., V. suppl. 1., 48. 82. [Strcttiomys] (1854); Hunter, Trans. Amer. Entom. Soc. Philad., XXVII. 128. (1901).

lineata FABr., Systema Antliat., 83. 22. [Stratiomys] (1805); WIED., Aussereurop. zweifl. Ins., II. 70. 16. [Stratiomys] (1830); WALK., List Dipt. Brit. Mus., V. suppl. 1., 46. 75. [Stratiomys] (1854); Hunter, Trans. Amer. Entom. Soc. Philad., XXVII. 128. (1901).

Japonia.

China centr.

Mt. Altai.

Sibiria.

America sept.

America sept.

Brasilia.

America mer. 
lineolata MAcQ. = ? Meigenii Wied.

longifrons Rond. in Truqul: Studi Entomol., I. 101. 71. [Stratiomys] (1848) ; Walk., List Dipt. Brit. Mus., V. suppl. 1., 311. [Stratiomys] (1854) ; Hunter, Trans. Amer. Entom. Soc. Philad., XXVII. 128. (1901).

lugubris Lw., Beschreib. europ. Dipt., II. 36. 22. (1871); Pleske, Wien. Entom. Zeitg., XVIII. 268. 22. (1899) et Sitzgsber. Naturf. Ges. Jurjew, XII. 1900. 345 et 351. (1901).

maculosa Lw., Beılin. Entom. Zeitschr., X. 12. 19. (1866); Jonns., Trans. Amer. Entom. Soc. Philad., XXII. 241. tab. III. fig. 13-15. (1895); Meland., Canad. Entomol., XXXVI. 22. (1904); Aldr., Catal. North Amer. Dipt., 183. (1905).

dentata Big., Annal. Soc. Entom. France, sér. 5. IX. 210. 1. [Stratiomys] (1879).

insignis Lw., Berlin. Entom. Zeitschr., XVI. 54. 7. (1872); Ost.-Sack., Bullet. Unit. Stat. Geolog. Surv., III. 213. (1877). lacerata Big., Annal. Soc. Entom. France, sér. 5. IX. 211. 2. [Stratiomys] (1879).

marginalis Lw. = Meigenii WreD.

Meigenii Wied., Aussereurop. zweifl. Ins., II. 61. 2. tab. VIII. fig. 7. [Stratiomys] (1830); Walk., List Dipt. Brit. Mus., III. 532. [Stratiomys] (1849) et V. suppl. 1., 36. 39 et ? 310. [Stratiomys] (1854) ; Johss., Trans. Amer. Entom. Soc. Philad., XXII. 238. tab. III. fig. 21-22. (1895); MrLand., Canad. Entomol., XXXVJ. 21 et 22. 6. (1904); Aldr., Catal. North Amer. Dipt., 183. (1905). angularis Lw., Berlin. Entom. Zeitschr., IX. 138. 16. (1865). ? lineolata MAcq., Dipt. exot., suppl. 4., 48. 6. tab. III. fig. 5. (1849).

marginalis Lw., Berlin. Entom.' Zeitschr., IX. 138. 17. (1865); НАrт, Bull. Illinois State Laborat. of Nat. Hist., IV. 249. (1895).

melanopsis WIED., Aussereurop. zweifl. Ins., II. 70. 15. [Stratiomys] (1830) ; WALk., List Dipt. Brit. Mus., V. suppl. 1., 46. 73. [Stratiomys] (1854); Huntel, Trans. Amer. Entom. Soc. Philad., XXVII. 128. (1901).

melanostoma Lw., Berlin. Entom. Zeitschr., IX. 134. 10. [melastoma] (1865) ; Johns., Trans. Amer. Entom. Soc. Philad., XXII. 232. tab. III. fig. 3-4. [melastoma] (1895); Meland., Canad. Entomol., XXXVI. 21 et 22. 1. (1904); Aldr., Catal. North Amer. Dipt., 183. (1905).

melestoma $\mathrm{Lw}_{\mathrm{w}}=$ melanostoma $\mathrm{Lw}$.

mutabilis FAlR., Mantissa Insect., II. 331. 9. [Stratiomys] (1787), Entomol. System., IV. 266. 13. [Stratiomys] (1794) et Systema Antliat., 81. 14. [Stratiomys] (1805); Wied., Aussereurop. zweifl. Ins., II. 63. 4. tab. VIII. fig. $6 a-d$. [Stratiomys] (1830); Penty, Delect. animal. articul. Brasil., 184. tab. XXXVI. fig. 14. [Stratiomys] (1830-34); Wask., List Dipt. Brit. Mus., V. suppl.

America sept.

Brasilia.

America sept.

America. 
1., 42. 61 et 311. [Strationys] (1854); Belu., Saggio di Ditterol. Messic., I. 30. 1. [Strutiomys] (1859); Scuns., Novara Reise, Dipt., 61. 25. [Stratiomys] (1868); v. v. WuL1, Tijdsclır. v. Entomol., XXIV. 154. 3. (1881); E. L. Armis, Bolet. Acad. Nat. Cienc. Córdoba, IV. 128. 49. (1882); Ost.-SAck., Biolog. Central. Amer., Dipt., I. 37. 1. (1886); Willist., Trans. Amer. Entom. Soc. Philad., XV. 256. 30. p. p. ․ (1888); Johns., Trans. Amer. Entom. Soc. Philad., XXII. 243. tab. IV. fig. 3. (1895) ; Willıst., Biolog. Central. Amer., Dipt., I. 248. (1900); Hunter, Trans. Amer. Entom. Soc. Philad., XXVII. 128. (1901); Meland., Canad. Entomol., XXXVI. 22. (1904); Aldr., Catal. Nortl Amer. Dipt., 183. (1905).

Aleus Walk., List Dipt. Brit. Mus., III. 526. [Acrocheatu] (1849) et V. suppl. 1., 41. 58. [Stratiomys] (1854).

fasciata FAbr., Mantissa Insect., II. 331. 10. [Stratiomys] (1787); Gues., Systema Naturae, V. 2834. 156. [Musca] (1792); Fasr., Entomol. System., IV. 266. 14. [Stratiomys] (1794) et Systema Antliat., 81. 15. [Stratiomys] (1805).

sericeiventris Rond. in TruQu1: Studi Entomol., I. 102. 73. [Stratiomys] (1848); WaLK., List Dipt. Brit. Mus., V. supp]. 1., 311. [Stratiomys] (1854).

variabilis Gmel., Systema Naturae, V. 2834. 155. [Musca] (1792).

mutabilis WiLLIst. (nec. FABR.) p. p. $\sigma^{T}=$ subalba WALK.

Nevadae Bıg., Annal. Soc. Entom. France, sér. 6. VII. 24. 4. Americasept. [Stratiomys] (1887); Johss., Trans. Amer. Entom. Soc. Philad., XXII. 237. (1895) ; Meland., Canad. Entomol., XXXVI. 21. (1904); Aldr., Catal. North Amer. Dipt., 183. (1905).

nigrifrons WALK., List Dipt. Brit. Mus., III. 531. [Stratiomys] (1849) et V. suppl. 1., 36 36. [Stratiomys] (1854); Johns., Trans. Amer. Entom. Soc. Philad., XXII. 247. (1895); Aldr., Catal. North Amer. Dipt., 183. (1905).

nigriventris Lw., Berlin. Entom. Zeitschr., IX. 137. 15. (1865). nigrodentata MEIG. = chamaeleon $\mathrm{L}$.

nobilis Lw., Aeta Soc. Amic. Nat. Moscou, 1870. 54. 1. (1870) et Beschrejb. europ. Dipt., II. 38. 23. (1871); PLiske, Wien. Entom. Zeitg., XVIII. 266. 19. (1899) et Sitzgsber. Naturf. Ges. Jurjew, XII. 1900.345 et $350 .(1901)$.

norma Wied., Aussereurop. zweifl. Ins., II. 62. 3. [Strutiomys] (1830); Americasept.

WALK., List Dipt. Brit. Mus., III. 530. [Stratiomys] (1849) et V. suppl. 1., 37. 42. [Stratiomys] (1854); Jouns., Trans. Amer. Entom. Soe. Philad., XXII. 235. tab. III. fig. 17-18. (1895); НАгт, Bull. Illinois State Laborat. of Nat. Hist., IV. 249. tab. XIV. fig. 57 (1895) ; Meland., Canad. Entomol., XXXVI. 21 et 22. 4. (1904); Aldr., Catal. North Amer. Dipt., 183. (1905).

normula Lw., Berlin. Entom. Zeitschr., IX. 130. 5. (1865) ; JoHns., Ameriea sept. Trans. Amer. Entom. Soc. Philad., XXII. 235. tah. III. fig. 16. 
(1895) ; Meland., Canad. Entomol., XXXVI. 21 et 22. 3. (1904);

Aldr., Catal. North Amer. Dipt., 184. (1905).

var. notata Lw., Berlin. Entom. Zeitschr., IX. 139. 18. (1865).

var. quadrigemina Lw., Berlin. Entom. 'Zeitschr., IX. 129. 4.

(1865) ; v. D. Wulp, Tijdschr. v. Entomul., XXIV. 154. 5. (1881). nolata Lw. = normula Lw. var.

Nymphis WALK. = ? laticeps Lw

obcsa $\mathrm{Lw}$. = lativentris $\mathrm{Lw}$.

pallipes Fabr., Species Insect., II. 417. 5. [Stratiomys] (1781) et

Mantissa Insect., II. 331. 8. [Stratiomys] (1787); Guel., Systema

Naturae, V. 2834. 154. [Musca] (1792); FAbr., Entomol. System.,

IV. 265. 12. [Stratiomys] (1794) et Systema Antliat., 81. 13.

[Stratiomys] (1805); Wred., Aussereurop. zweifl. Ins., I. 76. 26.

[Stratiomys] (1830); WaLk., List Dipt. Bıit. Mus., III. 534. [Odon-

tomyia] (1849) et V. suppl. 1., 39. 50. [Stratiomys] (1854); Hunter,

Trans. Amer. Entom. Soc. Philad., XXVII. 128. (1901).

paludosa SiEbKE = validicornis $\mathrm{Lw}$.

panthalcon FALL. = furcata FABR.

parallela WaLK., Proc. Linn Soc. London, VIII. 107. 16. [Stratiomys]

(1865) ; v. D. Wulp, Catal. Dipt. South Asia, 51. (1896).

pellucida Rond. in Truqui : Studi Entomol., I. 102. 72. [Stratiomys]

(1848) ; Walk., List Dipt. Brit. Mus., V. suppl. 1., 311. [Stratio-

mys] (1854) ; Hunter, Trans. Amer. Entom. Soc. Philad., XXVII.

128. (1901).

picipes $\mathrm{Lw} .=$ Badius WALK.

pinguis WALK., Trans. Entom. Soc. London, n. ser. V. 270. [Strutio-

mys] (1860) ; Johns., Trans. Amer. Entom. Soc. Philad., XXII.

246. (1895); Aldr., Catal. North Amer Dipt., 184. (1905).

Pleskei WaGn., Sitzgsber. d. Naturf. Ges. Jurjew, XIII. 1901. 108. Turkestan. (1902).

Portschinskii Pleske, Sitzgsber. Naturf. Ges. Jurjew, XII. 19u0. Transcaucas. 328. 3. (1901).

pyrrhocera Pleske, Wien. Entom. Zeitg., XVIII. 242. 1. p. p. (1899).

Potamida Meig., System. Beschreib., III. 136. 3. [Stratiomys] (1822); Macq., Recueil Soc. Sci. Agricult. Lille, 1826. 442. 2. [Stratiomys] (1826) et Suit. à Buffon, I. 243. 3. tab. VI. fig. 4. [Stratiomys] (1834) ; Duncan, Magaz. Zoolog. and Botany, I. 149. 2. [Slratiomys] (1837) ; Lw., Linnaea Entomol., I. 467. [Straliomys] (1846); Walk., List Dipt. Brit. Mus., III. 528. [Stratiomys] (1849) ; ZeTT., Dipt. Seand., VIII. 2952. 1-2. [Straliomys] (1849); HaLı., Stettin. Entom. Zeitg., XI. 136. [Stratiomys] (1851); ZETT., Dipt. Scand., XI. 4260. 1-2. [Stratiomys] (1852); WaLK., List Dipt. Brit. Mus., V. suppl. 1., 28. 3. [Stratiomys] (1854); Schns., Verh. zool.-bot. Ver. Wion, V. 621. 5 et 667. [Stratiomys] (1855), VII. 7. [Straliomys] (1857) et Fauna Austriaca, Dipt., I. 15. 3. [Stratiomys] (1862); JAEnN., Berlin. Entom. Zeitschr., X. 229. 30.

Europa. 
[Stratiomys] (1866); Brunetti, The Entomologist, XXII. 86. 5. (1889); Schoci, Fauna Insect. Helvetiae, Dipt., 22. [Stratiomys] (1890) ; É. \& I. Coucke, Annal. Soc. Entom. Belgique, XXXVII. 438. (1893) ; Sтrobl, Mittheil. Naturwiss. Ver. Steiermark, XXIX. 1892. 10. (1893) ; Pleske, Wien. Entom. Zeitg., XVIII. 265. 15. (1899) et Sitzgsber. Naturf. Ges. Jurjew, XII. 1900. 345 et 351. (1901).

chamaeleon MeIG., Klassif., I. 126. 4. p. p. Ђ. tab. VII. fig. 18. [Stratiomys] (1804).

Potanini Pleske, Wien. Entom. Zeitg., XVIJI. 275. 29. (1899) et Sitzgsber. Naturf. Ges. Jurjew, XII. 1900. 353. (1901).

princeps Gerst., Linnaea Entomol., XI. 321. tab. III. fig. 1, 1^. [Stratiomys] (1857); Hunter, Trans. Amer. Entom. Soc. Philad., XXVII. 128. (1901).

Przewalskii Pleske, Wien. Entom. Zeitg., XVIII. 276. 30. (1899) et Sitzg'sber. Naturf. Ges. Jurjew, XII. 1900. 348 et 351. (1901). pyrrhocera Lw., Berlin. Entom. Zeitschr., XII. 370. 1. (1868); Bezzi, Wien. Entomol. Zeitg., XV. 217. (1896); Röder, Wien. Entom. Zeitg., XV. 274. (1896); Pleske, Wien. Entom. Zeitg., XVIII. 242. 1. p. p. (1899) et Sitzgsber. Naturf. Ges. Jurjew, XII. 1900. 326. 1. (1901).

erythrocera Pleske, Wion. Entom. Zeitg., XVIII. 242. 3. p. p. (1899).

pyrrhocera Pleske (nec Lw.) p. p. = Portschinskii Pleske.

quadrigemina Lw. = normula Lw. var.

quaternaria Lw., Berlin. Entom. Zeitschr., IX. 135. 12. (1865); Johns., Trans. Amer. Entom. Soc. Philad., XXII. 241. tab. III. fig. 25-26. (1895); HАRT, Bull. Illinois State Laborat. of Nat. Hist., IV. 249. (1895); Meland., Canad. Entomol., XXXVI. 21. (1904) ; Aldr., Catal. North Amer. Dipt., 184. (1905).

rhatica JAENN. = chamaeleon L. var.

riparia Meig., System. Beschreib., III. 138. 6. [Stratiomys] (1822); Macq., Suit. à Buffon, I. 242. 6. [Stratiomys] (1834); Duncan, Magaz. Zoolog. and Botany, I. 150. 4. [Stratiomys] (1837); Lw., Programm Posen, 1840. 25. 3. [Stratiomys] (1810) et Isis, 1840. VII. 556. 3. [Stratiomys] (1840); MACQ. in Lucas : Explor. scient. de l'Algérie, Zool., III. 428. 38. [Stratiomys] (1849); WaLk., Insecta Britaun., Dipt., I. 16. 4. [Stratiomys] (1851) et List Dipt. Brit. Mus., V. suppl. 1., 29. 7. [Stratiomys] (1854); Scunn., Verh. zool.-bot. Ver. Wien, V. 622. 7 et 667. [Strationys] (1855) et Fauna Austriaca, Dipt., I. 16. [Stratiomys] (1862); KawaLL, Stettin. Entom. Zeitg., XXVIII. 124. [Stratiomys] (1867); BIG., Annal. Soc. Entom. France, sér. 5. IX. 186. [Thyreodontha] (1879) ; Brunetti, The Entomologist, XXII. 86. 2. (1889) ; Schocil, Fauna Insect. Helvetiae, Dipt., 23. [Stratiomys] (1890); SтroвL, Mittheil. Naturwiss. Ver. Steiermark, XXIX. 1892. 10. (1893); Pleske, Wien. Entom. Zeitg., X VIII. 263. 9. (1899) ; Kert., Pót-

Europa centr. et mer., Africa sejt. 
fiiz. a Természettud. Közlönyhöz, XXXII. 127. (1900); Pleske, Sitzgsber. Naturf. Ges. Jurjew, XII. 1900. 345 et 349. (1901).

strigata MeIG., Klassif., I. 124. 2. [Stratiomys] (1804).

Roborowskii Pleske, Sitzgsber. Naturf. Ges. Jurjew, XII. 1900. China sept. 343,349 et 357.32 . (1901).

robusta WALK., List Dipt. Brit. Mus., V. suppl. 1., 37. 40. [Stratiomys] (1854); Johns., Trans. Amer. Entom. Soc. Philad., XXII. 247. (1895); Aldr., Catal. Nortl Amer. Dipt., 184. (1905).

rossica Grmm., Bullet. Soc. Imp. Nat. Moscou, XX. 2., 169. 4. Rossia, [Stratiomys russica] (1847); WaLk., List Dipt. Brit. Mus., V. suppl. 1., 31. 16. [Stratiomys] (1854); ScHin., Verh. zool.-bot. Ver. Wien, V. 632. 29. [Odontomyia] (1855); Pleske, Wien. Entom. Zeitg., XVIII. 265. 14. (1899) et Sitzgsber. Naturf. Ge s. Jurjew, XII. 1900. 355. (1901).

rubricornis BEzz1, Wien. Entom. Zeitg., XV. 215. (1896) et Bullet. Italia. Soc. Entom. Ital., XXX. 24. 10. (1898); Pleske, Wion. Entom. Zeitg., XVIII. 243. 5 (1899) et Sitzgsber. Naturf. Ges. Jurjew, XII. 1900. 333. 9. (1901).

ruficornis Macq., Dipt. exot., I. 1., 180.2. (1838); WalK., List Dipt. Mesopotamia. Brit. Mus., V. suppl. 1., 51. 99. [Stratiomys] (1854).

rufipennis MacQ., Dipt. exot., suppl. 5., 42. 7. (1855).

russice Grmn. = rossica Grm.

Sarudnyi Pleske, Sitzgsber. Naturf. Ges. Jurjew, XII. 1900. 350. Persia.

(1901).

Zarudnyi Pleske, Wien. Eutom. Zeito., XVIII. 267. 21. (1899). sellata SuLz = chamaeleon $\mathrm{L}$.

senaria Lw., Berlin. Entom. Zeitschr., JX. 132. 7. (1865) ; Jouns., Trans. Amer. Entom. Soc. Philad., XXII. 237. tab. III. fi:. 10. (1895); Meluand., Canad. Entomol., XXXVI. 21. (1904); Aldr., Catal. North Amer. Dipt., 184. (1905).

serica Pleske, Sitzgsber. Naturf. Ges. Jurjew, XII. 1900. 350 et 369. China.

40. (1901).

sericeiventris ROND. = mutabilis FABR.

simplex BıG., Annal. Soc. Entom. France, sér. 6. VII. 24. 5. [Stratiomys] (1887); Johns., Trans. Amer. Entom. Soc. Philad, XXII. 248. (1895); Aldn., Catal. North Amer. Dipt., 184. (1905).

sinensis Pleske, Sitzgsber. Naturf. Ges. Jurjew, XII. 1900. 344 et China sept. 35\%. 34. (1901).

singularis HARRIS = furcata FABR.

Sintenisi Pleske, Wien. Entom. Zeitg., XVIII. 273. 27. (1899), Rossia.

Sitzgsber. Naturf. Ges. Jurjew, XII. 1900. 344 et 352. (1901) et

Természetrajzi Fïzetek, XXV. 412. (1902).

spatula Scop. = chamaeleon L.

strigata MEIG. 1804. (nec FA BR.) = riparia MeIG.

subalba WALK., List Dipt. Brit. Mus., V. suppl. 1., 43. 62. [Stratiomys] (1854); Bers.., Saggio di Ditterol. Messic., I. 31. 2. var. [Stratiomys] (1859); Ost.-SAck., Biolog. Central, Amer., Dipt., I.

America mer. et centr. 
37. 2. (1886) ; Johns., Trans. Amer. Entom. Soc. Philad., XXII. 246. (1895) ; Wulısт., Biolog. Central. Amer., Dipt., I. 248. (1900);

Aldr., Catal. North Amer. Dipt., 184. (1905).

mutabilis Willist. (nee Farr.), Trans. Amer. Entom. Soc. Philad., XV. 256. 30. p. p. ơ. (1888).

sublunata Iw., Berlin. Entom. Zeitschr., XII. 370. Anmerk. 2. (1868); Bezzr, Wien. Entom. Zeitg., XV. 217. (1896); Pleske, Wien. Entom. Zeitg., XVIII. 242. 2. (1899); Kert., Pótfïz. a Természettud. Közlönyhöz, XXXII. 126. (1900) ; Pleske, Sitzgsber. Naturf. Ges. Jurjew, XII. 1900. 330. 5. (1901).

tomentosa Schrank = ? equestris Meig.

unguicornis BECK. = chamaeleon I. ?var.

unilimbata Lw., Berlin. Entom. Zeitschr., IX. 131. 6. (1865); Joнss., Trans. Amer. Entom. Soc. Philad., XXII. 236, tab. III. fig. 19-20. (1895) ; Meland., Canad. Entomol., XXXVI. 21 et 22. 5. (1904); Aldr., Catal. North Amer. Dipt., 184. (1905).

validicornis Lw., Neue Beitr., II. 17. 35. [Hoplomyia] (1854) ; Puesie, Wien. Entom. Zeitg., XVIII. 269. 24. (1899) et Sitzgsber. Naturf. Ges. Jurjew, XII. 1900. 347, 351 et 354. (1901).

paludosa Siebre, Nyt Magaz. f. Naturvidensk., XII. 149. [Stratiomys] (1864) et Catal. Dipt. Norvegiae, 6. 2. [Stratiomys] (1877).

variabilis GMeI. = mutabilis FABR.

velutina Big., Annal. Soc. Entom. France, sér. 5. IX. 213. 5. [Stratiomys] (1879); Hunter, Trans. Amer. Entom. Soc. Philad., XXVII. 128. (1901).

ventralis Lw., Stettin. Entomol. Zeitg., VIII. 369. 1. [Stratiomys] (1847); WaLK., List Dipt. Brit. Mus., V. suppl. 1., 51. 97. [Stratiomys] (1854); I.w., Nene Beitr., II. 17.33. [Odontomyia] (1854); Pleske, Wien. Entom. Zeitg., X VIII. 266. 18. (1899) et Sitzgsber.

Naturf. Ges. Jurjew, XII. 1900. 343 et 350. (1901).

virens Wied., Aussercurop. zweill. Ins., II. 73. 21. [Stratiomys] Brasilia.

(1830) ; Watk., List Dipt. Brit. Mus., V. 'suppl. 1., 47. 79 et 311.

[Stratiomys] (1854); Hunter, Trans. Amer. Entom. Soe. Philad., XXVII. 128. (1901).

Wagneri Pueske, Wien. Entom. Zeitg., XVIIT. 271. 26. (1899) et Mt. Altai.

Sitzgsber. Naturf. Ges. Jurjew, XII. 1900. :48 et 354. (1901). Zarudnyi PLeske $=$ Sarudnyi PJeske.

\section{Metabasis}

Watk., Insecta Saunders., Dipt., I. 81. (1851).

rostratus WAsk, Insecta Saunders., Dipt., I. 81. tab. III. fig. 4. Brasilia. (1851) et List Dipt. Brit. Mıs., V. suppl. 1., 84. 1. (1854).

Eiropa centr. et mer., Mt. Taurus.

America sept.

Europla sept., Sibiria, Mt. AItai.

Chile.

Sibiria. 


\section{Promeranisa}

Walk., List Dipt. Brit. Mus., V. suppl. 1., 305. (1854).

Promerisana Wilisist., Trans. Amer. Entom. Soc. Philad., XV. 253. (1888).

cylindricornis Willist., 'Trans. Amer. Entom. Soc. Philad., XV. Brasilia.

253. 24. [Promerisana] (1888); Hunter, Trans. Amer. Entom.

Soc. Philad., XXVII. 130. [Promerisana] (1901).

nasuta MAcQ., Dipt. exot., suppl. 4., 47. 5. tab. III. fig. 4. [Stratio-

myia] (1849); Willist., 'Trans. Amer. Entom. Soc. Philad., XV.

253. 23. [Promerisana] (1888); Hunter, Trans. Amer. Entom. Soc.

Philad., XXVII. 130. (1901).

vittata WALK., List Dipt. Brit. Mus., V. suppl. 1., 306. 1. (1854). Brasilia.

\section{Eulalia}

Meig., Nouvelle Classification, 21. 28. (1800).

Odontomyia Meig., Illiger's Magaz. f. Ins., II. 265. 29. (1803).

Opseogymnus A. Costa, Il Giamb. Vico, Napoli, II. 443. (1857).

Psellidotus Rond., Archivio per la Zool. Modena, III. 78. (1863).

adusta Lw., Öfvers. Kongl. Vet. Akad. Förhandl., XIII. 263. 7. Caffraria. [Odontomyia] (1856) et Dipt.-Fauna Südafrika's, I. 7. 1. [Odontomyia] (1860).

aequalis WALk., Proc. Linn. Soc. London, V. 271. 2. [Stratiomys] Batjan.

(1861) ; v. D. Wulp, Catal. Dipt. South Asia, 52. [Odontomyia] (1896).

affinis BeLt., Saggio di Ditterol. Messic., I. 35. 3. tal. I. fig. 12. Mexico.

[Odonthomyia] (1859); Jonns., Trans. Amer. Entom. Soc. Pliilad., XXII. 274. [Odontomyia] (1895) ; Áusn., Catal. Nort'L Amer. Dipt., 184. [Odontomyia] (1905).

albipennis Macq., Dipt. exot., I. 1., 184. 3. [Odontomyia] (1838); Senegal. Walk., List Dipt. Brit. Mus., V. suppl. 1., 50. 91. [Stratiomys] (1854).

albomaculata Macq., Dipt. exot., I. 1., 189. 12. [Odontomyia] (1838); Walk., List Dipt. Brit. Mus., V. suppl 1., 40. 55. [Odontomyia] (1854) ; Jouns., Trans. Amer. Eutom. Soc. Plilad., XXII. 276. [Odlontomyia] (1895); ALdr., Catal. North Amer. Dipt., 184. [Odontomyia] (1905).

Aldrichi Jomss., Trans. Amer. Entom. Soc. Philad., XXII. 262. tab. IV. fig. 17. [Odontomyia] (1895); Mel.and., Canad. Entomol., XXXVI. 24 et 53. 11. [Odontomyia] (1904); Aldr., Catal. North Amer. Dipt., 184. [Odontomyia] (1905).

alpina JAEN, = hydroleon L. var.

St. Domingo, Hayti.

America sept. 
altifrons v. D. WulP, Tijdschr. v. Entomol., XXXI. 363. 1. tab. IX. fig. Argentinia. 3-4. [Odontomyia] (1888); Hunter, Trans. Amer. Entom. Soc. Philad., XXVII. 126. [Odontomyia] (1901).

americana DAy, Proc. Acad. Nat. Scienc. Philad., 1882. 77. [Odontomyia] (1882) ; JoHns., Trans. Amer. Entom. Soc. Philad., XXII. 261. [Odontomyia] (1895); Meland., Canad. Entomol., XXXVI. 24. [Odontomyia] (1904); Aldr., Catal. North Amer. Dipt., 184. [Odontomyia] (1905).

Amyris Wark., List Dipt. Brit. Mus., III. 535. [Odontomyia] (1849) et V. suppl. 1., 54. 109. [Stratiomy/s] (1854); Hutron, Catal. New Zealand Dipt. etc., 39. [Odontomyia] (1881).

anchorata Bix., Annal. Soc. Entom. France. sér. 5. IX. 216. 4. Chile. [Odontomyia] (1879) ; Hunter, Trans. Aner. Entom. Soc. Ihilad., XXVII. 126. [Odontomyia] (1901).

- angulata Meig. 1804. (nee PANz.) = hydroleon $\mathrm{L}$.

angulata PAnz., Fauna Germ., LVIII. tab. 19. [Stratiomys] (1798);

Americasept.

Ouiv., Encyclop. Méthod., VIII. 435. 19. [Odontomyia] (1811); Lw., Linnaea Entomol., I. 483. 9. [Odontomyia] (1846); WALK., Iist Dipt. Brit. Mus., V. suppl. 1., 308. [Stratiomys] (1854); Scun., Verh. ziol.-bot. Ver. Wien, V. 629. 5 et 670. [Odontomyia] (1855) et VII. 8. [Odontomyia] (1857); Schis., Fauna Austr., Dipt., I. 19. 11. [Odontomyia] (1862); JaEnN., Berlin. Entom. Zeitschr., X. 231. 41. [Odontomyia] (1866); v. D. Wuli, Dipt. Neerland., I. 455. 5. [Otontomyia] (1877); JARoscheff., Trudy Kharkoff, XI. 340. 5. [Odontomyia] (1877); Neuinats, Diptera Marchiea, 82. 3. [Odontomyia] (1886) ; BRunetri, The Entomologist, XXII. 131. 5. [Odontomyia] (1889); ScHocir, Fauna Ins. Helvetiae, Dipt., 24. [Odontomyia] (1890); É. \& I. Covcke, Annal. Soc. Entom. Belgique, XXXVII. 441. [Odontomyia] (1893); Sтroњ Mittlieil. Naturwiss. Ver. Steiermark, XXIX. 1892. 10. [Odontomyia] (1893); Kкrт., Pótfïz. a Természettud. Közlönyhöz, XXXII. 128. [Odontomyia] (1900).

brevicornis Lw., Programm Posen, 25. 8. tab., fig. 37. [Stratiomys] (1840) et Isis, 1840. VII. 557. 8. tab., fig. 37. [Stratiomys] (1840).

hydropota MAcQ. (nec Meig.), Recueil. Soc. Seiene. Agricult. Lille, 447. 5. [Odontomyia] (1826) et Suit. à Buffon, I. 247. 8. [Odontomyia] (1834); Duncan, Magaz. Zoolog. and Botany, I. 153. 4. [Odontomyia] (1837); ? ZEтT., Dipt. Scand., I. 138. 8. [Stratiomys] (1842), VIII. 2954. 8. [Stratiomys] (1849) et XI. 4260. 8. [Stratiomys] (1852).

ruficornis Zетт., Dipt. Scand., I. 139. 9. [Stratiomys] (1842), VIII. 2954. 9. [Stratiomys] (1849) et XI. 4261. 9. [Strutiomys] (1852); Walk., List Dipt. Brit. Mus., V. suppl. 1., 34. 29. [Stratiomys] (1854); ZETT., Dipt. Scand., XII. 4554. 9. [Stratiomys] (1855) et XIII. 4953. 9. [Stratiomys] (1859).

angusta W WLK., List Dipt. Brit. Mus., V. suppI. 1., 57. 121. [Stratio-

N. Selandia. 
mys] (1854); Hutton, Catal. New Zealand Dipt. etc., 38. [Odontomyia] (1881) et Trans. New Zealand Instit., XXXIII. 9. [Odontomyia] (1901).

anilis SchrANK $=$ ? argentata FABR.

annulata Merg., System. Beschreib., III. 143. 11. [Stratiomys] (1822); MAcQ., Suit. à Buffon, I. 246. 3. [Odontomyia] (1834); Lw., Linnaea Entomol., I. 471. 3. [Odontomyia] (1846); WAsk., List Dipt. Brit. Mus., III. 529. [Stratiomys] (1849) et V. suppl. 1., 30.10 et 307. [Stratiomys] (1854); Schin., Verh. zool.-bot. Ver. Wien, V. 626. 7 et 668. [Odontomyia] (1855) et VII. 8. (1857) et Fauna Austr., Dipt., I. 18. [Odontomyia] (1862); JAENN., Berlin. Entom. Zeitschr., X. 230. 36. [Odontomyia] (1866); v. D. Wulp, Diptera Neerland., I. 456. [Odontomyia] (1877); É. \& L. Coucke, Annal. Soc. Entom. Belgique, XXXVII. 439. [Odontomyia] (1893) ; Kert., Pótfïz. a Természettud. Közlönyhöz, XXXII.128. [Odontomyia] (1900).

septemguttata Wien. apud Meia., System. Beschreib., III. 150. 20. [Stratiomys] (1822); WaLK., List Dipt. Brit. Mus., V. suppl. 1., 32. 19. [Stratiomys] (1854); Lw., Wien. Entom. Monatschr., VI. 163. 5. [Odontomyia] (1862).

annulipes M̄icq., Dipt. exot., suppl. 4., 52. 34. [Odontomyia] (1849). anodonta Mace., Dipt. exot., suppl. 1., 52. 18. [Odontomyia] (1846) ; Walk., List Dipt. Brit. Mus., V. suppl. 1., 49. 87. [Stratiomys] (1854).

araneifera Scinn., Novara Reise, Dipt., 58.20. [Odontomyia] (1868); Hunter, Trans. Amer. Entom. Soc. Philad., XXVII. 126. [Odontomyia] (1901).

arcuata Lw., Berlin. Entom. Zeitschr., XVI. 52. 4. [Odontomyia] (1872); Day, Proc. Acad. Nat. Scienc. Philad., 1882. 80. [Odontomyia] (1882) ; Jouss., Trans. Amer. Entom. Soc. Philad., XXII. 255. tab. III. fig. 35 et tab. IV. fig. 7, 8. [Odontomyia] (1895); Menand., Canad. Entomol., XXXVI. 23 et 53. 4. [Odontomyia] (1904); Aldr., Catal. North Amer. Dipt., 184. [Odontomyia] (1905).

argentata FAlR., Entomol. System., IV. 266. 15. [Stratiomys] (1794); Panz., Fauna Germ., LXXI. tab. 20. o'. [Stratiomys] (1799) et CVIII. tab. 10. ㅇ. [Odontomyia] (1809); Mera., Krlassif., I. 131. $a$. [Odontomyia] (1804); FAlr., Systema Antliat., 82. 17. [Stratiomys] (1805) ; Latr., Gen. Crust. et Ins., IV. 274. [Odontomyia] (1809); Ourv., Encyclop. Méthod., VIII. 434. 14. [Odontomyia] (1811); Fall., Dipt Suec., Stratiom., 9. 5. [Stratiomys] (1817); Mrig., System. Beschr., III. 141. 9. [Stratiomys] (1822); MAcQ., Recueil. Soc. Scienc. Agricult. Lille, 1826. 445. 2. tab. III. fig. 3. [Odontomyia] (1826) et Suit. à Buffon, I. 246. 4. tab. VI. fig. 5. [Odontomyia] (1834); Duncan, Magaz. Zool. and Botany, I. 152. 1. [Odontomyia] (1837); ZsLuen, Isis, 1842. XI. 287. [recte 827.] 10. [Stratiomys] (1842); ZETT., Dipt. Seand., I. 137. 6. [Stratiomys] (1842); Jacobsen, Naturhist. Tidsskr., ser. 2. I. 40. [Stratiomys] (1844); Lw., Linnaea Entomol., I.476.5. [Odontomyia] (1846);
Europacentr. et mer., Africa sept.

T'asmania.

Prom. bon.sp.

Chile.

America sept.

Europa,

Sibiria. 
WALk., List Dipt. Brit. Mus., III. 534. [Odontomyia] (1849) ; Zetr., Dipt. Scand., VIII. 2953. 6. [Stratiomys] (1849); Walk., Ins. Britannica, Dipt., I. 17. 6. [Stratiomys] (1851) ; Zetr., Dipt. Scand., XI. 4260. 6. [Stratiomys] (1852); WaLK., List Dipt. Brit. Mus., V. suppl. 1., 32. 18 et 307. [Stratiomys] (1854); Schis., Verh. zool.-bot. Ver. Wien, V. 627. 11 et 670. [Odontomyia] (185i) et Fauna Austr., Dipt., I. 20. [Odontomyia] (1862); JAENv., Berlin. Entom. Zeitschr., X. 232. 42. [Odontomyia] (1866); v. D. Wulp, Diptera Neerland., I. 454. 1. [Odontomyia] (1877); Big., Annal. Soc. Entom. France, sér. 5. IX. 186. [Oplodontha] (1879); Bruxetri, The Entomologist, XXII. 131. 2. [Odontomyia] (1889); SсHоch, Fau ıa Ins. Helvetiae, Dipt., 24. [Odontomyia] (1890); É. \& L. Coucke, Annal. Soc. Entom. Belgique, XXXYII. 439. [Odontomyia] (1893); Kent., Pótfüz. a Természettud. Közlönyliöz, XXXII. 128. [Odontomyia] (1900); Beck., Acta Soc. Scienc. Fenn., XXVI. No. 9., 5. 4. [Odontomyia] (1900).

? amilis Schravk, Fauna Boica, III. 97. 2388. [Stratiomys] (1803).

argentula Ginn., Bull. Soc. Imp. Nat. Moscou, XX. 2., 169. 2. [Stratiomys] (1847) lapsus.

paludosa Schumi., Übers. d. Arb. u. Veränd. d. schles. Ges. f. vaterl. Kultur, 1836. 85. 1. [Stratiomys] (1836).

argentula Giмn. = argentata FABR.

aterrima WALK., Insecta Saunders., Dipt., I. 78. [Odontomyia] (1851) et List Dipt. Brit. Mus., V. suppl. 1., 41. 59. [Stratiomys] (1854).

atraria WALk., Proc. Linn. Soc. London, VIII. 106. 15. [Stratiomys] NovaGuinea. (1865) ; v. D. Wulp, Catal. Dipt. South Asia, 52. [Odontomyia] (1896).

atrovirens Bic., Annal. Soc. Entom. France, sér. 5. IX. 214. 1. [Odontomyia] (1879); Hurros, Trans. New Zeeland Instit., XXXIII.

7. [Odontomyia] (1901).

australensis Schin., Novara Reiso Dipt., 59. 22. [Odontomyia] N. Selandia. (1868).

antstraliensis Hutron, Catal. New Zealand Dipt. etc., 38. [Odontomyia] (1881) et Trans. Now Zealand Instit., XXXIII. 9. [Odontomyia] (1901).

australiensis Hutтon = australensis Schin.

bieolor $\mathrm{I}_{\mathrm{AY}}=$ Truquii BeLL.

bifascia Walk., Proc. Linn. Soc. London, V. 232. 12. [Stratiomys] NovaGninca. (1861); v. D. Wulp, Catal. Dipt. South Asia, 52. [Odontomyia] (1896).

binotata Lw. = Truquii BELL.

bipunctata Bıg., Annal. Soc. Entom. France, sér. 3. VII. 130. Madagascar. [Odontomyia] (1859).

brevicomis L.: = aigulata PANz.

brevifacies MacQ., Dipt. exot., suppl.5., 43.38. [Odontomyia] (1855); Americamer.

Kertész: Catalogus Dipterorum. IIr. 
Hunter, Trans. Amer. Entom. Soc. Philad., XXVII. 126. [Odontomyia] (1901).

brevipennis OuIv. = obscura OuIv.

bucephala Gerst., Linnaea Entomol., XI. 326. 3. [Stratiomys] (1857);

E. L. Arrib., Bolet. Acad. Nat. Cienc. Córdoba, IV. 127. 45.

[Odontomyia] (1882) ; Hunter, Trans. Amer. Entom. Soc. Philad.,

XXVII. 126. [Odontomyia] (1901).

byzantina Strobl, Glasnik Zem. Mus. Bosni i Hercegov., XIV. 463. Europa mer.

[Odontomyia] (1902) et Wissensch. Mitteil. Bosnien u. Hercegow.,

JX. 521. [Odontomyia] (1904).

caloceps $\mathrm{BIG} .=$ ? nigrirostris Lw.

canadensis WALK., List Dipt. Brit. Mus., V. suppl. 1., 310. [Sratiomys] (1854); Day, Proc. Acad. Nat. Scienc. Plilad., 74. [Odontomyia] (1882) ; Jonss., 'Trans. Amer. Entom. Soc. Philad., XXII. 277. [Odontomyia] (1895); Aldr., Catal. North Amer. Dipt., 185. [Odontomyia] (1905).

carinata Mac'Q., Dipt. exot., suppl. 1., 5́2. 18. [Odontomyia] (1846); Walk., List Dipt. Brit. Mus., V. suppl. 1., 56. 115 et 312. [Stratiomys] (1854'.

carinifacies Macq., Di,t. exot., suppl. 4., 51. 32. [Odontomyia] (1849). carnifex Gerst., Linnaea Entomol., XI. 323. tab. 1II. fig $2 a, b$. Prom.bon.sp.

[Stratiomys] (1857).

cephalonica STRoвL = limbata Wied. var.

chathamensis Hutron, 'Trans. New Zealand Instit., XXXIII. 8. N. Sclandia.

[Odontomyia] (1901).

chloraspis WIED., Aussereurop. zweifl. Ins., II. 68. 12. [Stratiomys] (1830) ; WaLK., Iist Dipt. Brit. Mus., V. suppl. 1., 47. 78. [Stratiomys] (1854); Schin., Novara Reise, Dipt., 58. 19. [Odontomyia] (1868) ; Hunter, Trans. Amer. Entom. Soc. Philad., XXVII. 126. [Odontomyia] et 127. [Stratiomyia] (1901).

chloris Walk., List Dipt. Brit. Mus., V. suppl. 1., 57. 120 et 312. [Stratiomys] (1854); Hutron, Catal. New Zealand Dipt. etc., 37. [Odontomyia] (1881) et Trans. New Zealand Instit., XXXIII. 8. [Odontomyia] (1901).

hypochlora Now., Memorien d. krakauer k. k. Akad. d. Wiss., II. 15. [Odontomyia] (1875) et Beitr. z Kenntniss d. Dipterenfauna Neu-Seeland's, 15. [Odontomyia] (1875); Hutron, Catal. New Zealand Dipt. etc., 38. [Odontomyia] (1881).

cincta Ourv., Encyclop. Méthod., VIII. 432. 3. [Odontomyia] (1811) ; Macq., Dipt. exot., I.2., 189. [Odontomyia] (1839) ; WaLK., List Dipt. Brit. Mus., V. suppl. 1., 39. 48. [Stratiomys] (1854); Dax, Proc. Acad. Nat. Sc. Plilad., 1882. 87. [Odontomyia] (1882); Willis'r., Canad. Entomol., XVII. 128. [Odontomyia] (1885) ; JoHss., Trans. Amer. Entom. Soc. Philad., XXII. 253. [Odontomyia] (1895) ; Hart., Bull. Illinois State Labor. of Na!. Hist., IV. 255 et 256. tab. XIV. fig. 58-59. [Odontomyia] (1895); Meland., Canad. Entomol., XXXVI. 23 et 53. 2. [Odontomyia] (1904); ALDr.,

Catal. North Amer. Dipt., 185. [Odontomyia] (1905).

Canada.

Australia, Tasmania.

Tasmania.

Anerica mer.

N. Selandia.

America sept. 
extremis Day, Proc. Acad. Nat. Sc. Philad., 80. [Odontomyia] (1882).

cinctilinea Walk., Proc. Linn. Soc. London, VI. 4. 3. [Stratiomys] Gilolo. (1862); v. D. Wulp, Catal. Dipt. South Asia, 52. [Odontomyia] (1896).

claripennis Thомs., Eugenies Resa, Dipt., 456. 19. [Odontomyia] Manila. (1869); v. D. Wulp, Catal. Dipt. South Asia, 52. [Odontomyia] (1896).

clypeata Big., Annal. Soc. Entom. France, sér. 5. IX. 215. 3. [Odontomyia] (1879); Hunter, Trans. Amer. Entom. Soc. Philad., XXVII. 126. [Odontomyia] (1901).

? comzra Willist., Biolog. Centr. Amer., Dipt., I. 247. 9. [? Odontomyia] (1900); A Lyr., Catal. North Amer. Dipt., 185. [? Odontomyia] (1905).

concinnata Willist., Biolog. Central. Amer., Dipt., I. 247. 7. COdontomyia] (1900); Ardr., Catal. North Amer. Dipt., 185. [Odontomyia] (1905).

? confusa Rossr, Mantissa, II. 60. [Stratiomys] (1794).

connexa WALK., Insecta Britann., Dipt., I. 17. 7. [Stratiomys] et III. pag. XI. [Stratiomys] (1856) et List Dipt. Brit. Mus., V. suppl. 1., 33. 24. [Stratiomys] (1854); Schin., Verl. zool.-bot. Ver. Wien, V. 632. 28. [Odontomyia] (1855); Brunetti, The Entomologist, XXII. 131. [Odontomyia] (1889).

consobrina MACQ., Dipt. exot., suppl. 3., 16. 25. tab. I. fig. 8. [Odontomyia] (1818); WalK., List Dipt. Brit. Mus., V. suppl. 1., 52. 102. [Stratiomys]-(1854); v. D. Wulp, Sumatra Exped., Dipt., 14. 1. [Odontomyia] (1881) et Catal. Dipt. South Asia, 52. [Odontomyia] (1896).

coronata GUÉR = flavicornis OLIV.

cruciata MAcQ., Dipt. exot., I. 1., 188. 10. [Odontomyia] (1838); Blancir. in Gay: Hist. fis. y polit. de Chile, Zool., VII. 402. 2. [Odlontomyia] (1852); WALK., List Dipt. Brit. Mus., V. suppl.1., 44. 65. [Stratiomys] (1854); Rond., Archivio per la Zoolog., III. 78. [Psellidotus] (1863); PHIL., Verlı, zool.-bot. Ges. Wien, XV. 732. 2. [Odontomyia] (1865); Schin., .Novara Reise, Dipt., 58. 18. [Odontomyia] (1868) ; Hunter, Trans. Amer. Entom. Soc. Plilad., XXVII. 126. [Odontomyia] (1901).

decora WreD. = flavissima Rossi.

diffusa Walk., List Dipt. Brit. Mus., V. suppl. 1., 53. 106. [Stratiomys] (18j4); v. D. Wur., Sumatra Exped., Dipt., 14. 2. [Odontomyia] (1881) et Catal. Dipt. Soutl Asia, 52. [Odontomyia] (1896). discolor Lw., Linnaea Entomol., I. 473. 4. [Odontomyia] (1846); WaLK., List Dipt. B:it. Mus., V. suppl. 1., 307. [Stratiomys] (1854); Scrun., Verh. zool.-bot. Ver. Wien, V. 626. 8. [Odontomyia] (1855).

? limba!r MIscq., Explor. scient. de l'Algérie, Zool., III. 428. 40. tab. I. fig. 10. [Odontomyia] (1849).

Amazonia.

Mexico.

Mexico.

Italia.

Patriaignota.

Sumatra, Java.

Chile.

Java,

Sumatra.

Asia min., ?Africa sept. 
dissimilis Bell., Saggio di Ditterol. Messic., I. 35. 4. tab. I. fig. 13-14. [Odontomyia] (1859); Jomss., T'uans. Amer. Entom. Soc. Philad., XXII. 274. [Odontomyia] (1895); Aspr., Catal. North Amer. Dipt., 185. [Odontomyia] (1905).

dorsalis Fank., Systema Antliat., 82. 20. [Stratiomys] (1805); WiEn., Aussereurop. zweifl. Ins., II. 66. 9. [Stratiomys] (1830); WaLK., List Dipt. Brit. Mus., V. suppl. 1., 47. 80. [Stratiomys] (1854); Jonss., Trans. Amer. Entom. Soc. Philad., XXII. 254. tab. IV. fig. 9. [Odontomyia] (1895); Huntur, Thans. Aner. Entom. Soc. Philad., XXVII. 127. [Stratiomyia] (1901); Meraxd., Canad. Entomol., XXXVI. 23 et 533. 3. [Odontomyia] (1904); ALns., Catal. North Amer. Dipt., 185. [Odontomyia] (1905).

dorsalis Walk., List Dipt. Brit. Mus., III. 536. [Odontomyin] (1849) et V. stypl. 1., 56. 118. [Stratiomys] (1854); Butren, Zoolog. of the voyage of Erebus and Terror, Ins., 27. tab. VII. fig. 16. [Odontomyia] (1874); Now., Beitrag z. Kenntniss d. Dipterenfauna Neu Secland's, 17. Anmerk. [Stratiomyia] (1875): Hetros, Catal. New Zealand Dipt. ete., 37. [Odontomyia] (1881) et Trans. New Zealand Instit., XXXIII. 9. [Odontomyia] (1901).

elegans Macq., Dipt. exot., I. 1., 187. 9. [Odontomyia] (1838); Вгалсн. in GAY : Hist. fis. y polit. de Chile, Zool., VII. 401. 1. [Odontomyial (1852); Walk., List Dipt. Brit. Mus., V. suppl. 1., 45. 70. [Stratiomyls] (1854); Ronn., Archivio per la Zoolog., III. 78. [Psellidotus] (1863); Pннш., Verh. zool.-bot. Ges. Wien, XV. 732. 1. [Odontomyia] (1865); Huxter, Trans. Amer. Entom. Soc. Philad., XXVII. 126. [Odontomyia] (1901).

emarginata MAcQ., Dijt. exot., I. 1., 190. 14. [Odontomyia] (1838); Walk., List Dipt. Brit. Mus., V. suppl. 1., 40. 54. [Stratiomys] (1854); Jouns., Trans. Amer. Entom. Soc. Philad., XXII. 275. [Odontomyia] (1895); AlnR., Catal. North Amer. Dipt., 185. [Odontomyia] (1905).

euchlora Gerst. = Lefebvrei MACQ.

excocta WaLK., Insecta Saunders., Dipt., I. 78. (1851) et Iist Dipt. Brit. Mus., V. suppl. 1., 58. 124. [Stratiomys] (1854).

extremis DaY = cincta OLIV.

exul Walk., Inseeta Saunders., Dipt., I. 79. (1851) et List Dipt. Brit. Mus., V. suppl. 1., 58. 125. [Stratiomys] (1854).

fallax Johns., 'Trans. Amer. Entom. Soc. Philad., XXII. 257. [Odontomyia] (1895); Meland., Canad. Entomol., XXXVI. 23. [Odontomyia] (1904); Ardor., Catal. North Amer. Dipt., 185. [Odontomyia] (1905).

fasciata MAcq., Suit. à Buffon, I. 249. 16. [Odontomyia] (1834); Walk., List Dipt. Brit. Mus., V. suppl. 1., 44. 66. [Stratiomys] (1854).

fascifrons MACQ., Dipt. exot., suppl. 4., 50. 30. tab. III. fig. 6. [Odontomyia] (1849); Buanch. apud GaY : Hist. fis. y polit. de Chile, Zool., VII. 402. 3. [Odontomyia] (1852) ; PHir., Verh. zool.-
Mexico.

Ampricamer. SanDomingo, Hayti.

N. Selandia.

Chile.

Mexico.

Patria ignota.

Patria ignota. Americasept.

Americamer.

Chile. 
bot. Ges. Wien, XV.732. 3. [Odontomyia] (1865); Hunter, Trans. Amer. Entom. Soc. Philad., XXVII. 126. [Odontomyia] (1901).

felina Panz, Fauna Germ., LVIII. tab. 22. [Stratiomys] (1798); Europacentr.

Merg., Klassif., I. 132. f. [Odontomyia] (1804); OLIv., Encyclop. Métlıd., VIII. 435. 18. [Odontomyia] (1811); Muıg., System. Besclireib., III. 145. 14. [Stratiomys] (1822); Duxcan, Magaz. Zool. and Botany, 1. 153. 3. [Odontomyia] (1837); Grum., Bull. Soe. Imp. Nat. Moseou, XV. 667. [Stratiomys] (1842); Lw., Linnaea Entomol., I. 487. 11. [Odontomyia] (1846); WaLk., List Dipt. Brit. Mus., V. suppl. 1., 34. 27 et 309. [Stratiomys] (1854); Scrun., Verh. zool.-bot. Ver. Wien, V. 630. 22 et 669. [Odontomyia] (1855) et Fauna Austriaca, Dipt., I. 18. 8. [Odontomyia] (1862) ; JaENx., Berlin. Entom. Zeitsclir., X. 230. 37. [Odontomyia] (1866); v. D. Wulp, Diptera Neerland., I. 457. [Odontomyia] (1877); É. \& L. Covcke, Annal. Soc. Entom. Belgique, XXXViI. 439. [Odontomyia] (1893).

lydropote WALK. (nec MeIG.), Insecta Britann., Dipt., I. 18. 10. [Stratiomys] (1851) et List Dipt. Brit. Mus., V. suppl. 1., 34. 28. [Stratiomys] (1854).

marginata FABr., Systema Antliat., 84. 27. [Stratiomys] (1805); MeIG., System. Beschreib., VI. 348. 28. [Stratiomys] (1830). mycroleon Harris, Expos. Engl. Ins., 46. tab. XI. fig. 5. [M[uscu] (1782).

femorata BeLL., Saggio di Ditterol. Messic., I. 37. 7. [Odontomyia] (1859) ; Johns., Trans. Amer. Entom. Soc. Philad., XXII. 275. [Odontomyia] (1895); Aldr., Catal. North Amer. Dipt., 185. [Odontomyia] (1905).

fenestrata Thомs., Eugenies Resa, Dipt., 458. 22. [Odontomyia] (1869) ; E. L. Arris., Bolet. Acad. Nat. Ciene. Córdoba, IV. 127. 46. [Odontomyia] (1882); Hunter, Trans. Amer. Entom. Soc. Philad., XXVII. 126. [Odontomyia] (1901).

finalis WaLK., Proc. Linn. Soc. London, IV. 94. 15. [Stratiomys] (1860) et V. 258. 2. [Strationys] (1861); v. D. Wulp, Catal. I)ipt. South Asia, 52. [Odontomyia] (1896).

flava DAx, Proc. Acad. Nat. Scienc. Philad., 1882. 76. [Odontomyia] (1882); Willist., Canad. Entomol., XVII. 128. [Odontomyia] (1885); Johxs., Trans. Amer. Entom. Soc. Philad., XXII. 256. [Odontomyia] (1895); Meland., Canad. Entomol., XXXVI. 23. [Odontomyia] (1904); Al.pr., Catal. North Amer. Dipt., 185. [Odontomyia] (1905).

flaviceps MacQ. = flavicornis OLIV.

flavicornis OLiv., Encyclop. Méthod., VIII. 433. 9. [Odontomyia] (1811); Macq., Suit. à Buffon, I. 248. 14. [Odontomyia] (1834); WALK., List Dipt. Brit. Mus., V. suppl. 1., 38. 46. [Stratiomys] (1854); DAY, Proc. Acad. Nat. Sci. Philad., 1882. 87. LOdontomyia] (1882); Johns., Trans. Amer. Entom. Soc. Philad., XXII. 269. tab. IV. fig. 21-22. [Odontomyia] (1895); Meland., Canad.
Mexico.

Argentinia.

America sept.

America sept.
Celebes. 
Entomol., XXXVI. 24 et 53. 18. [Odontomyia] (1904); Aldr., Catal. North Amer. Dipt., 185. [Odontomyia] (1905).

coronata (ruĖr., Iconogr. cl. Régne Anim., VII. Ins., tab. XCVIII. fig. 6. [Stratiomys] (1835).

flaviceps MACQ., Suit. à Buffon, I. 245. 8. [Stratiomys] (1834); GuÉr., Iconogr. d. Régne Anim., VII., Ins., 544. tab. XCVIII. fig. 6. [Stratiomys] (1835); WaLk., List Dipt. Brit. Mus., V. suppl. 1., 37. 41. [Stratiomys] (1854) ; Genst., Linnaea Entomol., XI. 317. [Stratiomys] (1857).

lasiophthalma Lw., Berlin. Entom. Zeitschr., IX. 142. 23. [Odontomyia] (1865); Day, Proc. Acad. Nat. Sci. Philad., 1882. 81. [Odontomyia] (1882).

pulchella MavQ., Dipt. exot., I. 1., 180. 3. tab. XXII. fig. 2. [Stratiomyia] (1838); WALk., I.ist Dipt. Brit. Mus., V. supp]. 1., 38. 43. [Stratiomys] (1854); Gerst., Linnaea Entomol., XI. 318. [Stratiomys] (1857).

vicina MACQ., Dipt. exot., I. 1., 181. 4. [Stratiomyia] (1838);

WALK., List Dipt. Brit. Mus., V. suppl. 1., 38. 45. [Stratiomys] (1854).

flavifasciata Macq., Dipt. exot., suppl. 4., 53. 36. [Odontomyia] (1849) ; Johns., Trans. Amer. Entom. Soc. Philad., XXII. 275. [Odontomyia] (1895); Aldr., Catal. North Amer. Dipt., 185. [Odontomyia] (1905).

flavipalpis MACQ., Dipt. exot., suppl. 4, 49. 29. [Odlontomyia] (1849). flavissima Rossi, Fauna Etrusca, II. 280. 1444. tab. X. fig. 5. [Stratiomys] (1790); Fabr., Entom. System., IV. 265. 8. [Stratiomys] (1794) ; Panz., Fauna Germ., XXXV. tab. 24. [Stratiomys] (1796); Meig., Klassif., I. 131. b. [Odontomyia] (1804); Coqueb., Illustr. Icon. Insect., 101. tab. XXIII. fig. 5. [Stratiomys] (1804) ; FABR., Systema Antliat., 79. 6. [Stratiomys] (1805) ; Rossi, Fauna Etrusca, Ed. II., 434. 1444. [Stratiomys] (1807); Otiv., Encyclop. Méthod., VIII. 432. 4. [Odontomyia] (1811); MeIG., System. Beschreib., III. 153. 25. [Stratiomys] (1822) et VI. 347. [Stratiomys] (1830); Lw., Linnaea Entomol., I. 469. 2. [Odontomyia] (1846); WALK., List Dipt. Brit. Mus., V. suppl. 1., 33. 22 et 307. [Stratiomys] (1854); Schin., Verh. zool.-bot. Ver. Wien, V. 625 . 5 et 668. [Odontomyia] (1855), VII. 7. [Odontomyia] (1857) et Fauna Austriaca, Dipt., I. 17. 5. [Odontomyia] (1862); RöDER, Berlin. Entom. Zeitschr., XXXI. 73. [Odontomyia] (1887); Scноcr, Fauna Ins. Helvetiae, Dipt., 24. [Odontomyia] (1890); Bezzi, Bull. Soc. Entom. Ital, XXX. 24. 11. [Odontomyiä] (1898); Kert., Pótfüz. a Természettud. Közlönyhöz, XXXII. 128. [Odontomyia] (1900). decora Wied. apud Meig., System. Beschreib., III. 144. 12. [Stratiomys] (1822); MACQ., Suit. à Buffon, I. 245. 2. [Odontomyia] (1834); WaLk., List Dipt. Brit. Mus., V. suppl. 1., 32. 21. [Stratiomys] (1854).

infuscata Meig., System. Beschreib., VI. 347. 27. [Stratiomys]

Australia.

Europa mer., Asia min. 
(1830) ; Scrins., Verh. zool.-bot. Ver. Wien, V. 625. 6 et 668. [Odontomyia] (1855) et Fauna Austriaca, Dipt., I. 17. 5. [Odontomyia] (1862).

semiviolacea Brulcé, Expéd. scient. de Morée, III. 1., 307. 663. tab. XLVII. fig. 5. [Odontomyia] (1832); WaLK., List Dipt. Brit. Mus., V. suppl. 1., 309. [Stratiomys] (1854).

flavosignata A. Costa = limbata WiED.

foveifrons Thомs., Eugenies Resa, Dipt., 458. 23. [Odontomyia] (1869) ; Huntrer, Trans. Amer. Entom. Soc. Philad., XXVII. 126. [Odontomyia] (1901).

fratella Wilusst., Biolog. Central. Amer., Dipt., I. 246. 6. [Odontomyia] (1900) ; Aldr., Catal. North Amer. Dipt., 185. [Odontomyia] (1905).

frontalis MACQ., Dipt. exot., I. 1., 185. 4. [Odontomyia] (1838); WaLK., List Dipt. Brit. Mus., V. suppl. 1., 49. 86. [Stratiomys] (1854) ; Lw., Öfvers. Kongl. Vet. Akad. Förhandl., XIII. 263. 8. [Odontomyia] (1856) et Dipt.-Fauna Südafrika's, I. 8. 3. [Odontomyia] (1860).

fulviceps Walk., List Dipt. Brit. Mus., V. suppl. 1., 56. 119. [Stratiomys] (1854); Hurton, Catal. New Zealand Dipt. etc., 37. [Stratiomyia] (1881) et Trans. New Zealand Instit., XXXIII. 9. [Odontomyia] (1901).

furcata MeIG. = ornata MeIG.

fuscipennis MACQ., Dipt. exot., I. 1., 190. 15. [Odontomyia] (1838); WaLK., List Dipt. Brit. Mus., V. suppl. 1., 58. 126. [Stratiomys] (1854).

Garatas WaLK., List Dipt. Brit. Mus., III. 532. [Odontomyia] (1849) et V. suppl. 1., 51. 95. [Stratiomys] (1854); Big., Aunal. Soc. Entom. France, sér. 5. IX. 186. [Odontomyia] (1879); v. D. Wuur, Catal. Dipt. South Asia, 52. [Odontomyia] (1896).

Guerinii Macq., Dipt. exot., I. 1., 183. 1. [Odontomyra] (1838); WALK., List Dipt. Brit. Mus., V. suppl. 1., 50. 90. [Stratiomys] (1854).

halterata Schrank, Fauna Boica, III. 95. 2380. [Stratiomys] (1803); MeIG., System. Beschreib., III. 152. 23. [Stratiomys] (1822); Lw., Linnaea Entomol., I. 490. [Odontomyia] (1846); WaLk., List Dipt. Brit. Mus., V. suppl. 1., 31. 13. [Stratiomys] (1854) ; Schin., Verh. zool.-bot. Ver. Wien, V. 630. 20. [Odontomyia] (1855).

heteroneura M4CQ., Dipt. exot., I. 1. 186. 7. bis. tab. XXII. fig. 3. Brasilia. [Odontomyia] (1838); Walk., List Dipt. Brit. Mus., V. suppl. 1., 46. 74. [Stratiomys] (1854); Hunter, Trans. Amer. Entom. Soc. Philad., XXVII. 126. [Odontomyia] (1901).

Heydenii JAENv., Berlin. Entom. Zeitschr., X. 231. 40. [Odontomyia] (1866) ; Big., Annal. Soc. Entom. France, sér. 5. IX. 186. [Oplodontha] (1879); Beck., Berlin. Entom. Zeitschr., XXXI. 102. 13. [Odontomyia] (1887); Sсносн, Fauna Insect. Helvetiae, Dipt., 25. [Odontomyia] (1890).

hieroglyphica Ousv., Encyclop. Méthod., VIII. 434.11. [Odontomyia] Americasept. 
(1811); Day, Proc. Acad. Nat. Sci. Philad., 1882. 87. [Odontomyia] (1882) ; Johns., Trans. Amer. Entom. Soc. Philad., XXII. 267. tab. IV. fig. 4-5. [Odontomyia] (1895); HakT, Bull. Illinois State Laborat. of Nat. Hist., IV. 256 et 266. [Odontomyia] (1895); Melaxd., Canad. Entomol., XXXVI. 24 et 53. 17. [Odontomyia] (1904); Aldr., Catal. North Amer. Dipt., 185. [Odontomyia] (1905).

Snovi HarT, Bull. Illinois State Laborat. of Nat. Hist., IV. 256 et 266. [Odontomyia] (1895).

histrio WAIK., Insecta Saunders., I. 77. [Odontomyiu] (1851) et List Dipt. Brit. Mus., V. suppl. 1., 41. 60. [Stratiomys] (1854). holosericea Ourv., Encyclop. Méthod., VIII. 4:4. 15. [Odontomyia] (1811).

hoodiana Big., Annal. Soc. Entom. France, sér. 6. VII. 25. 2. [Odontomyia] (1887); Jouss., T'rans. Amer. Entom. Soc. Plilad., XXII. 266. [Odontomyia] (1895); Meland., Canad. Entomol., XXXVI. 24. [Odontomyia] (1904); Aldr., Catal. North Amer. Dipt., 186. [Odontomyia] (1905).

Hydrodromia MeIg., System. Beschreib., III. 146. 15. [Stratiomys] (1822) ; MACQ., Suit. à Buffon, I. 247. 7. [Odontomyira] (1834); Walk., List Dipt. Brit. Mus., IJI. 533. [Odontomyia] (1849), Insecta Britann., Dipt., I. 18. 11. [Stratiomys] (1851) et List Dipt. Dipt. Mus., V. suppl. 1., 31. 15. [Stratiomys] (1854).

hydroleon L., Systema Naturae, Ed. X. 589. 5. [Minsca] (1758) et Europa.

Fauna Suec., Ed. Alt., 44. 1782. [Musca] (1761); O. F. Múlu., Fauna Ins. Fridrichsdal., 80. 707. [Musca] (1764); L., Systema Naturae, Ed. XII. II., 980. 5. [Musca] (1767); Faвr., Systema Entomolog., 760. 5. [Stratiomys] (1775); O. F. Mélu., Zoolog. Dan. Prodr., 177. 2082. [Stratiomys] (1776); Deg., Mém. pour serv. l'hist. d. Ins., VI. 154. 3. tab. IX. fig. 4-5. [Stratiomys] (1776) ; Fabr., Species Insect., II. 417. 7. [Stratiomys] (1781); Schrank, Enum. Ins. Austr., 437. 888. [Musca] (1781); Fabr., Mantissa Insect., II. 331. 12. [Stratiomys] (1787); Rossi, Fauna Etrusca, II. 280. 1442. [Stratiomys] (1790) ; Giel., Systema Naturae, V. 2835. 5. [Musca] (1792); Panz., Fauna Germ., VII. tab. 21. [Stratiomys] (1793); FABr., Entomol. System., IV. 267. 17. [Stratiomys] (1794); Schraxk, Fauna Boica, III. 95. 2382. [Stratiomys] (1803) ; Schellexr., Gattungen d. Fliegen, 36 et 37. tab. XXIV. fig. 3. [Stratiomys] (1803) ; MeIG., Klassif., I. 131.c. [Odontomyia] (1804); Latr., Hist. Nat. d. Crust. et d. Ins., XIV. 340. [Odontomyia] (1804); FABr., Systema Antliat., 82. 19. [Stratiomys] (1805); Rossi, Fauna Etrusca, Ed. II. 433. 1442. [Stratiomys] (1807) ; Latr., Gen. Crust. et Ins., IV. 275. [Odontomyia] (1809); Ourv., Encyclop. Méthod., VIII. 433. 10. [Odontomyia] (1811); LAM., Hist. nat. anim. sans vert., III. 387. 5. [Stratiomys] (1816); Fall., Dipt. Suec., Stratiom., 8. 4. [Stratiomys] (1817); Meig., System. Beschreib., III. 148. 17. [Stratiomys] (1822); MacQ., Recueil Soc. Sci. Agricult. Lille, 1826. 448. 6. [Odontomyia] 
(1826) et Suit. à Buffon, J. 247.9. [Odontomyia] (1834); Duxcax, Naga\%. Zool, and Botany, I. 153. 5. [Odontomyia] (18:37); Lw., Programm Posen, 1840. 25. 7. [Odontomyia] (1840) et Isis, 1840. VII. 557. 7. [Odontomyia] (1840); ZeтT., Dipt. Seand., I. 140. 10. [Stratiomys] (1842); Lw., Linnaea Entomol., I. 481. 8. [Odonelomyia] (1846) ; Walk., List Dipt. Brit. Mus., III. 535. [Odontomyiu] (1849); Zetr., Dipt. Scand., VIII. 2954. 10. [Stratiomys] (1849); HaLid., Stettin. Lntom. Zeitg., XI. 136. [Odontomyia] (1851); WaLK., Insecta Britann., Dipt., I. 19. 12. [Stratiomys] (1851); Zeтт., Dipt. Scand., XI. 4261. 10. [Stratiomys] (1852); Wa1k., List Dipt. Brit. Mus., V. suppl. 1., 34. 60 it 308. [Straliomys:] (1854) ; Schns., Verh. zool.-bot. Ver. Wien, V. 628. 13 ct 670. [Odontomyia] (1855); ZeTT., Dipt. Scand., XII. 4555. 10. [Stratiomys] (1850); Lw., Zeitschr. f. d. ges. Naturwiss., X. 99. 7. [Odontomyia] (1857); Scmin., Verh. zool.-bot. Ver. Wien, VII. 8. [Odontomyia] (1857) ; ZeTT., Dipt. Seand., XIII. 4953. 10. [Str(ltiomys] (1859); Schin., Fauna Austriaca, Dipt., I. 19. 10. [Odontomyia] (1862); Jaknx., Perlin. Entom. Zeitschr., X. 230. 39. [Odontomyia] (1866); Sieskse, Catal. Dipt. Norveg., 6. 4. [Strutiomys] (1877); v. D. Wulp, Diptera Neerland., I. 456. 6. [(1)dontomyia] (18iT); Beck., Borlin. Entom. Zeitschr., XXXI. 102. 12. [Odontomyia] (1887); Scuocn, Falna Insect. Helvetiae, Dipt., 25. [Odontomyia] (1890); E. \& L. Coucke, Annal. Soc. Entom. Belgique, XXXVII. 440. [Odontomyia] (1893); Srrop., Mittheil. Naturwiss. Ver. Steiermark, XXIX. 1892. 10. [Psellidotus] (1893); Kerr., Pótfüz. a Természettud. Közlönylıöz, XXXII. 128. [Odontomyia] (1900).

angulate MeIG., Klassif., I. 133. h. [Odontomyia] (1804).

vulpina PaNz., Fauna Germ., LVIII. tab. 24. [Stratiomys]

(1798) ; Meig., Klassif., I. 132. d. [Odontomyia] (1804); Otr.,

Eincyclop. Méthod., VIII. 435. 20. [Odontomyia] (1811).

var. alpina J AEnN., Berlin. Entom. Zeitschr., X. 230. 39. [0,(ontomyia] (1866); Strobl, Mittheil. Naturwiss. Ver. Steiermark, XXIX. 1892. 10. [Psellidotus] (1893).

hydroleonoides Johns., Trans. Amer. Entom. Soc. Philad., XXII. 261. [Odontomyia hydrolenoides] tab. IV. fig. 10. (1895); Mlicand., Canad. Entomol., XXXVI. 24 et 53. 10. [Odontomyia] (1904); Aldr., Catal. North Amer. Dipt., 186. [Odontomyia] (1905).

hydrophila Lw., Linnaea Entomol., I. 486. 10. [Odontomyia] (1846); WaLK., List Dipt. Brit. Mus., V. suppl. 1., 308. [Stratiomys] (1854) ; Scmin., Verlı. zool.-bot. Ver. Wien, VII. 9. [Odontomyjia] (1857) ; Kent., Pótfüz. a Természettud. Közlönyhöz, XXXII. 128. [Odontomyia] (1900).

hydropota MACQ. (nee MEIG.) = angulata PANz.

Hydropota MeIG., System. Beschreib., III. 147. 16. [Stratiomys] (1822) ; ? Perris, Mém. Acad. d. Scienc. Lyon, II. 490. [Odontomyia] (1850) ; Schun., Fauna Austriaca, Dipt., I. 19. 11. [Odonto-

Europacentr.

Amerieasept.

Europa mer., Asia min.

Europacentr. 
myia] (1862) ; BruveTrI, The Entomologist, XXII. 131. 6. [Odontomyia] (1889); É. \& I. Cotcke, Amnal. Soc. Entom. Belgique, XXXVII. 440. [Odontomyia] (1893) ; Kert., Pótfüz. a Természettud. Közlöny höz, XXXII. 128. [Odontomyia] (1900).

hydropota Walk. (nec Meig.) = felina Panz.

hypochlora Now. = chloris Wark.

immiscens WALK., Proc. Limn. Soc. London, IV. 94. 14. [Stratiomys] (1860); Ost.-Sack., Annal. Mus. Civ. Genova, XVI. 411. [Odontomyia] (1E82); v. D. Wulp, Catal. Dipt. South Asia, 52. [Odontomyia] (1896).

inaequalis Lw., Berlin. Entom. Zeitschr., IX. 143. 24. [Odontomyia] (1865) ; DAY, Proc. Acad. Nat. Sci. Philad., 1882. 74. [Odontomyia] (1882) ; Johss., Trans. Amer. Eutom. Soc. Philad., XXII. 254. [Odontomyia] (1895); Meland., Canad. Entomol., XXXVI. 23. [Odontomyia] (1904); Aldr., Catal. North Amer. Dipt., 186. [Odontomyia] (190う).

inermis WIED, Ausscreurop. zweifl. Ins., II. 71. 18. [Stratiomys] (1830) ; Walk., List Dipt. Brit. Mus., V. suppl. 1., 48. 84. [Stratiomys] (1854); Gerst., Linnaea Entomol., XI. 330. 7. [Stratiomys] (1857); E. L. A riris., Bolet. Acıd. Nat. Cienc. Córdoba, IV. 127. 47. [Odontomyia] (1882); Hunter, Trans. Amer. Entom. Soc. Philad., XXVII. 126. [Odontomyia] (1901).

infuseata ME1G. = flavissima Rossi.

intermedia WıED. = interrupta OLIV.

interrupta Otiv., Encyclop. Méthod., VIII. 433. 8. [Odontomyia] (1811) ; D $\mathbf{y}$, Proc. Acad. Nat. Sei. Philad., 1882. 88. [Odontomyia] (1882); Johss., Trans. Amer. Entom. Soc. Plilad., XXII. 265. tab. IV. fig. 29-30. [Odontomyia] (1895) ; Meland., Canad. Entomol., XXXVI. 24 et 53. 16. [Odontomyia] (1904); Aldr., Catal. North Amer. Dipt., 186. [Odontomyia] (1905).

intermedia WiED., Aussereurop. zweifl. Ins., II. 64. 5. [S'tratiomys] (1830) ; WalK., List Dipt. Brit. Mus., III. 534. [Odontomyia] (1849) et V. suppl. 1., 38. 44. [Stratiomys] (1854); DAY, Proc. Acad. Nat. Sci. Philad., 1882. 85. [Odontomyia] (1882); Hart, Bull. Illinois State Laborat. of Nat. Hist., IV. 255 et 256. [Odontomyia] (1895).

Jalemus WaLK., List Dipt. B it. Mus., III. 533. [Odontomyia] (1849) et V. suppl. 1., 54. 110 et 312. [Stratiomys] (1854); Big., Annal. Soc. Entom. France, sér. 5. IX. 186. [Odontomyia] (1879).

Kirchneri JAenv., Abhandl. Senckenb. Naturf. Ges., VI. 323. 12. Australia. [Odontomyia] (1867).

lasiophthalma Lw. = flavicornis·Otıv.

lateremaculata MACQ., Dipt. exot., suppl. 4., 49. 27. [Odontomyia] Australia. (1849).

latifaciata MACQ. = latifasciata MACQ.

latifasciata Macq., Suit. à Buffon, I. 248. 11. [Odontomyia latifaciata] Europa osc. (1834); MeIg., System. Beschreib., VII.107.32. [Stratiomys] (1838); 
WaLk., List Dipt. Brit. Mus., V. suppl. 1., 33. 25. [S/ratiomys] (1854).

Lefeburei BELL. $=$ Lefebvrei MaCQ.

Lefebvrei MacQ., Dipt. exot., I. 1., 189. 13. [Odontomyia] (1838); WaLk., Iist Dipt. Brit. Mus., V. suppl. 1., 40.53 et 311. [Straliomys] (1854); Johvs., Trans. Amer. Entom. Soc. Philad., XXII. 272. [Odontomyia] (189i); Willist., Biolog. Central. Amer., Dipt., I. 245. 2. [Odontomyia] (1900); Aldr., Catal. North Amer. Dipt., 186. [Odontomyia] (1905).

euchlora GERst., Linnaea Entomol., XI. 328. 5. [Stratiomys] (1857).

Lefeburei Beul., Saggio di Ditterol. Messic., I. 33. 1. [Odontomyia] (1859).

prasina Jaknn., Abhandl. Senekenb. Naturf. Ges., VI. 324. 13. [Odontomyia] (1867); Johns., Trans. Amer. Entom. Soc. Philad., XII. 273. [Odontomyia] (1895).

Limae Guḱr., Voyagu de la Coquille, Zool., II. P. 2., 291. tab. XX. Pe 1. fig. 12. [Odontomyia] (1830); Walk., List Dipt. Brit. Mus., V. suppl. 1., 48. 83. [Stratiomys] (1854).

limbata MAcQ. (nee WIED.) = ? discolor Lw.

limbata Wied. apud MeIG., System. Beschreib., III. 151. 21. [Strcltiomys] (1822); Walk., List Dipt. Brit. Mus., V. suppl. 1., 33. 23. [Stratiomys] (18j4); Schın., Novara Reise, Dipt., 57. 16. [Odontomyia] (1868); Lw., Beschreib. europ. Dipt., III. 73. Anmerk. [Odontomyia] (1873); B1G., Anual. Soc. Entom. France, sér. 5. IX. 186. [Opseogymnus] (1879); StroßL, Wien. Entom. Zeitg., XVIII. 295. 2. [Odontsmyia] (1898); BEzzI, Bullet. Soe. Entom. Ital., XXXII. 77. 328. [Odontomyia] (1900).

flavosignata A. Costa, Il Giamb. Nico Napoli, II. 453. [Opseogymnus] (1857).

pacifica Wied. apud MEig., System. Beschreib., III. 121. ¿. [Clitellaria] (1822); WALK., List Dipt. Brit. Mus., V. suppl. 1., 65. 3. [Cyclogaster] (1854); Schin., Verh. zool.-bot. Ver. Wien, V. 639. 1. [Clitellaria] (1855); BIG., Annal. Soc. Entom. France, sér. 5. IX. 186. [Lasiopa] (1879).

var cephalonica StroвL, Wien. Entom. Zeitg., XVII. 297. [Odontomyia] (1898).

limbifacies Brg., Annal. Soc. Entom. France, sér. 3. VII. 129. tab. III. fig. 3. [Odontomyia] (1859).

limbipennis MACQ., Dipt. exot., suppl. 2., 30. 24. [Odontomyia] (1847) ; WaLK., List Dipt. Brit. Mus., V. suppl. 1., 39. 49. [Stratiomys] (1854) ; Ost.-Sack., Catal. Dipt. North America, Ed. II., 47 et 225. 57. [Odontomyia] (1878) ; DAy, Proc. Acad. Nat. Sci. Plilad., 1882. 74. [Odontomyia] (1882); Jorns., Trans. Amer. Entom. Soc. Philad., XXII. 276. [Odontomyia] (1895); Hunter, Trans. Amer. Entom. Soc. Philad., XXVII. 126. [Odontomyia] (1901); Aldr., Catal. North Amer. Dipt., 186. [Odontomyia] (1905). Europa mer., Africa sept.

Mexico.

Africa selt.

Graecia.

Madagascar.

?America. 
lunata OuIV., Encyclop. Méthod., VIII. 436. 21. [Odontomyia] (1811); MAce., Suit. à Buffon, I. 248. 12 [Odontomyia] (1834); MeIG., System. Besclireib., VII. 107. 31. [Stratiomys] (18:38); Lw., Linnaea Entomol., I. 490. [Odontomyia] (1846); WaLk., List Dipt. Brit. Mus., V. suppl. 1., 31. 17. [Stratiomys] (1854).

Inmlata Mace., Recueil Soc. Sci. Agricult. Lille, 1826. 450. 8. [Odontomyiu] (1826).

lumelate MAce. = lunata Ous.

Lutatius WALK., List Dipt. Brit. Mus., III. 532. [Odontomyia] (1849), V. suppl. 1., 58. 123. [Stratiomys] (1854) et Proc. Linu. Soc. London, I. 7. 10. [Stratiomys] (1856); ?? Big., Annal. Soc. Entom. France, sér. 5. IX. 186. [Odontomyia] (1879); v. D. Wulp, Catal. Dipt. South Asia, 52. [Odontomyia] (1896).

maculifrons Watk., List Dipt. Brit. Mus., III. 536. [Odontomyia] (1849) et V. supul. 1., 41. 57. [Stratiomys] (1854); Jurss., Trans. Amer. Entom. Soc. Philad., XXII. 277. [Odontomyia] (1895); Aldr., Catal. North Amer. Dipt., 186. [Odontomyia] (1905).

maculifrons v. D. W ULP, Tijdschr. v. Entomol., XXXI. 264. 2. tab.

IX. fig. 5. [Odontomyia] (1888); Huster, Trans. Amer. Entom.

Soc. Philad., XXVII. 126. [Odontomyia] (1901). marginate $\mathrm{F}_{\mathrm{ABR}}=$ felina PANz.

marginella MACQ., Dipt. exot., suppl. 4., 52. 33. /Odontomyia] (1849). megaceplace $\mathrm{L}_{\mathrm{w}} .=$ Truquii BeLL.

megacephala Osıv., Encyclop. Méthod., VIII. 432. 2. [Odontomyia] (1811); Lw., Dipt.-Fauna Siidafrika's, I. 7. (79). [Odontomyia] (1860). mexicana Jorns., Trans. Amer. Entom. Soc. Philad., XXII. 271.
[Odontomyia] (1895); Al.pr., Catal. North Amer. Dipt., 186. [Odontomyia] (1905).

microleon L., Systema Naturae, Ed. X. 589. 4. [Musca] (1758), Fauna Suec., Ed. Alt., 440. 1781. [Musca] (1761) et Ed. XII., II. 980. 4. [Musca] (1767); FABr., Systema Entomolog., 760. 3. [Stratiomys] (1775); Deg., Mèm. pour. serv. l'hist. d. Ins., VI. 152. 2. tab. IX. fig. 1-3. [Stratiomys] (1776); O. F. Moll., Zoolog. Dan. Prodr., 177. 2083. [Stratiomys] (1776) ; FABr., Species Insert., II. 417. 3. [Strutiomys] (1781); Schrank, Enum. Ins. Austr., 436. 887. [Muscu] (1781); IABr., Mantissa Insect., II. 330.6. [Strutiomys] (1787); Rossi, Fauna Etrusca, II. 279. 1440. [Stratiomys] (1790); Guer., Systema Naturae, V. 2834. 4. [Musca] (1792); Fabr., Entomol. System., IV. 265. 9. [Stratiomys] (1794); Latr., Hist. Nat. d. Crust et Ins., XIV. 342. 2. [Ephippium] (1804); Fabr., Systema Antliat., 80. 8. [Stratiomys] (1805); Rossi, Fauna Etrusea, Ed. II., 432. 1441. [Stratiomys] (1807); LAtr., Gen. Crust. et Ins., IV. 274. [Odontomyia] (1809); FALL., Dipt. Suec., Stratiom., 8. 3. [Stratiomys] (1817); Germ., Fauna Insect. Europ., VIII. 23. [Stratiomys] (1817); Wied. apud MeIG., System. Beschreib., III. 140. 8. [Stratiomys] (1822); Macq., Recueil Soc.
Europra oce.

Malacea,

?Senegambia.

Honduras.

Argentinia.

Tasmania.

Aegyptus.

Mexico.

Europa centr. et sept. 
Scient. Agricult. Lille, 1826. 444. 1. [Odontomyia] (1826); MeIG., System. Beschreib., VT. 346. [Stratiomys] (1830): Macq., Suit. à Buffon, I. 246.5. [Odontomyia] (1834) ; ZeTt., Dipt. Scand., I. 137. 5. [Stratiomys] (1842); O. G. Costa, Atti Accad. Sci. Napoli, V. 2., 105. 8. [Stratiomys] (1844); WALK., List Dipt. Brit. Mus., III. 532. [Odontomyia] (1849); ZетT., Dipt. Scand., VIII. 2953. 5. [Stratiomys] (1819); WaLk., Insecta Britamn., Dipt., I. 17. 5. [Stratiomys] (1851); ZETT., Dipt. Scand., IX. 4260. 5. [Stratiomys] (1852) ; Walk., List Dipt. Brit. Mus., V. suppl. 1., 32. 20. [Str(ttiomys] (1854); Scrrs., Verh. zool.-bot. Ver. Wien, V. 626. 9 et 670. [Odontomyia] (1855); Bossis., Finland traving. Ins., I. 116. 2. [Stratiomys] (1861); Schin., Fauna Austriaca, Dì)t., I. 20. [Odontomyia] (1862); v. D. Wulp, Diptera Neerland., J. 456. [Odontomyia] (187T); Muk, Wien. Entom. Zeitg., VI. 191. 33 et 238. 35. [Odontomyia] (1887); Bra., Annal. Soe. Entom. France, sér. 6. VII. Bullet., CCV. [Odontomyia] (1887); BrunetTi, The Entomologist, XXII. 130. 1. [Odontomyia] (1889); Scнocн, Fauna Ins. Helvetiae, Dipt., 24. [Odontomyia] (1890); É. \& L. Coucke, Anual. Soc. Entom. Belgique, XXXVII. 439. [Odontomyia] (189:3).

nigriceps Bıg., Annal. Soc. Entom. France, sér. 6. I. 363. [Odontomyia] (1881), sér. 6. VII. 22. 1. [Stratiomys] (1887) et Wien. Entom. Zeitg., VI. 215. [Stratiomys] (1887).

microstoma Lw., Berlin. Entom. Zeitschr., IX. 146. 28. [Odontomyia] (1865) ; DAY, Proc. Acad. Nat. Scienc. Philad., 1882. 77. [Odontomyia] (1882) ; Johss., Trans. Amer. Entom. Soc. Philad., XXII. 264. tab. IV. fig. 20. [Odontomyia] (1895); Melaxd., Canad. Entomol., XXXVI. 24 et 53. 14. [Odontomyia] (1904); ALpr., Catal. Nortl Amer. Dipt., 186. [Odontomyia] (1905).

minuta Fabr., Entom. System., IV. 268. 22. [Stratiomys] (1794) et Systema Antliat., 86. 32. [Stratiomys] (1805); Wren., Aussereurop. zweifl. Ins., II. 74. 23. [Stratiomys] (1830); WaLk., List Dipt. Brit. Mus., V. suppl. 1., 54. 107. [Stratiomys] (1854); v. D. Wulp, Catal. Dipt. South Asia, 51. [Odontomyia] (1896).

mutica v. D. Wur.P, Notes Leyden Mus., VII. 62. 9. [Odontomyia] (1885) et Catal. Dipt. South Asia, 59. [Odontomyia] (1896).

mycroleon HARRIS = felina PANz.

nidiceps Hunter $=$ nitidiceps v. D. WulP.

nigerrima Lw., Ber]in. Entom. Zeitschr., XVI. 53. 6. [Odontomyia]

Americasept.

(1872); Day, Proc. Acad. Nat. Sci. Philad., 1882. 82. [Odontomyia] (1882) ; Jorrs., Trans. Amer. Entom. Soc. Philad., XXII. 258. ? tab. IV. fig. 25. [Odontomyia] (1895) ; Meland., Canad. Entomol., XXXVI. 24 et 53. 6. [Odontomyia] (1904); Al.dr., Catal. North Amer. Dipt., 189. [Odontomyia] (1905). nigra $\mathrm{D}_{\mathrm{AY}}=$ Virgo $\mathrm{W}_{\mathrm{IED}}$. nigriceps $\mathrm{BlG} .=$ microleon $\mathrm{I}$.

nigripes MACQ., Dipt. exot., suppl. 2., 29. 22. [Odontomyia] (1847); Syria.

WaLk., List Dipt. Brit. Mus., V. suppl. 1., 51. 98. [Stratiomys] Ternate. Inclia orient.

America sept. (1854). 
nigrirostris Lw., Berlin. Entom. Zeitschr., IX. 140. 19. [Odontomyia] (1865); Day, Proc. Acad. Nat. Sci. Philad., 1882. 83. [Odontomyia] (1882) ; Johns., Trans. Amer. Entom. Soc. Philad., XXII. 257. tab. III. fig. 36-37. [Odontomyia] (1895) ; Meland., Canad. Entomol., XXXVI. 23 et 53. 5. [Odontomyia] (1904); Alsn., Calal. Nort'L Amer. Dipt., 186. [Odontomyia] (1905).

? caloceps Big., Annal. Soc. Entom. France. sér. 5. IX. 217. [Exochostoma] (1879); Willist., Entomol. Americ., I. 154. [? Odontomyia] (1885); Aldr., Catal. North Amer. Dipt., 185. [? Odontomyia] (1905).

nigrita $\mathrm{FALL}_{\mathrm{AL}}=$ tigrina $\mathrm{FABR}_{\mathrm{AB}}$

nitidiceps v. D. Wulp, Tijdschr. v. Entomol., XXXI. 365. 3. [Odontomyia] (1888).

nidiceps Hunter, Trans. Amer. Entom. Soc. Philad., XXVII. 126. [Odontomyia] (1901).

obscura Ouıv., Encyclop. Méthod., VIII. 433. 7. [Odontomyia] (1811); MACQ., Dipt. exot., I. 2., 189. [Odontomyia] (1839); ? WALK., List Dipt. Brit. Mus., V. suppl 1., 38. 47. [Stratiomys] (1854); DАY, Proc. Aead. Nat. Sci. Philad., 1882. 88. [Odontomyia] (1882); Johss., Trans. Amer. Entom. Soc. Philad., XXII. 270. [Odontomyia] (1895); Meland., Canad. Entumol., XXXVI. 24. [Odontomyia] (1904); Aldr., Catal. North Amer. Dipt., 186. [Odontomyia] (1905).

bievipennis Ourv., Encyclop. Méthod., VIII. 434. 13. [Odontomyia] (1811); Day, Proc. Acad. Nat. Sci. Philad., 1882. 86. [Odontomyia] (1882).

obscuripes Thous., Eugenies Resa, Dipt., 457. 20. [Odontomyia] (1869) ; Hunter, Trans. Amer. Entom. Soc. Philad., XXVII. 126. [Odontomyia] (1901).

occipitalis Johns., Trans. Amer. Entom. Soc. Philad., XXII. 268. tab. IV. fig. 23-24. [Odontomyia] (1895); Meland., Canad. Entomol., XXXVI. 24. [Odontomyia] (1904); ALDR., Catal. Nortl Amer. Dipt., 186. [Odontomyia] (1905).

ochropa Thous., Eugenies Resa, Dipt., 456. 18. [Odontomyia] (1869); v. D. Wurp, Catal. Dipt. South Asia, 52. [Odontomyia] (1896).

ornata MeIG., System. Besrhreib., IIJ. 144. 13. [Stratiomys] (1822); Macq., Recueil Soc. Sci. Agricilt. Lille, 1826. 446.3. [Odontomyia] (1826) ; Duncan, Magaz. Zool. and Botany, I. 152. 2. [Odontomyia] (1837) ; Lw., Programm Posen, 1840. 25. 6. [Straliomys] (1840) et Isis, 1810. VII. 557. 6. [Stratiomys] (1840); Zerr., Dipt. Scand., I. 136. 4. [Strutiomys] (1842); Lw., Linnaea Entomol., I. 476. 6. [Odontomyia] (1846); WAlk., List Dipt. Brit. Mus., III. 534. [Odontomyia] (1849); ZETr., Dipt. Scand., VIII. 2953. 4. [Stratiomys] (1849); Walk., Inser ta Britann., Dipt., I. 17. 8. [Stratiomys] (1851) et List Dipt. Brit. Mus., V. suppl. 1., 31. 14 et 308. [Stratiomys] (1854); Schin., Vell. zool.-bot. Ver. Wien, V. 624. 3 et 668. [Odontomyia] (1855) et Fauna Austriaca, Dipt., I. 18. [Odon-
America sept.

Argentinia.

America sept.

Puna.

Americasept

Manila.

Europa. 
tomyia] (1862); JAenn., Berlin. Entom. Zeitschr., X. 230. 35. [Odontomyia] (1866); v. D. Wulp, Dipt. Neerland., I. 455.3. [Odontomyia] (1877); Brau., Denkschr. Akad. Wien, XLVII. tab. II. fig. 23. c. [Odontomyia] (1883); Nevhaus, Diptera Marchica, 81. 2. [Odontomyia] (1886); Brune'Tu, The Entomologist, XXII. 131. 3. [Odontomyia] (1889); Schocr, Fauna Ins. Helvetiae, Dipt., 24. [Odontomyia] (1890) ; É.\& L. Coucke, Annal. Soc. Entom. Belgique, XXXVII. 439. [Odontomyia] (1893) ; Kert., Pótfüz. a Természettud. Közlönyhöz, XXXII. 128. [Odontomyia] (1900); MeIs., Zoolog. Jahrb., Abth. f. Anat., XV. 676. tab. XXXIV. fig. 33. [Odontomyia] (1902)

fireata Mera., Klassif., I. 129. 1. tab. VII. fig. 22. [Odontomyia] (1804); LAtr., Gen. Crust. et Ins., IV. 27j. [Odontomyia] (1809); LAM., Hist. Nat. anim. sans vertebr., III. 386. 3. [Stratiomys] (1816); MacQ., Suit. à Buffon, I. 245. 1. [Odontomyia] (1834).

pachycephala Schin., Novara Reise, Dipt., 6?. 24. [Odontomyia]

Columbia. (1868) ; Hunter, Trans. Amer. Entom. Soc. Philad., XXVII. 126. [Odontomyia] (1901).

pachyceps Bı́., Annal. Soc. Entom. France, sér. 5. IX. 215. 2. Brasilia.

[Odontomyia] (1879); Hunter, Trans. Amer. Entom. Soc. Pliilad., XXVII. 126. [Odontomyia] (1901).

pacifica Wied. = limbata Wied.

pallidiventris MaCQ., Dipt. exot., suppl. 1., 53. 20. [Odontomyia] (1846); Walk., List Dipt. Brit. Mus., V. suppl. 1., 44. 67. [Stratiomys] (1854); Hunter, Trans. Amer. Entom. Soc. Philad., XXVII. 126. [Odontomyia] (1901). paludosa Schumm. = argentata FABr. Paron WALK. = Virgo Wied.

pectoralis Thoms., Eugenies Resa, Dipt., 455. 17. [Odontomyia] (1869).

periscelis Lw., Berlin. Entom. Zeitschr., XVII. 36. 13. [Odontomyia] (1873) et Beschrcib. europ. Dipt., III. 98. 53. [Odontomyia] (1873) ; Kert., Pótfüz. a Természettud. Közlönyhöz, XXXII. 128. [Odontomyia] (1900).

personata Lw., Linnaca Entomol., I. 490. 12. [Odontomyia] (1846); Walk., List Dipt. Brit. Mus., V. suppl. 1., 309. [Stratiomys] (1854); Schin., Verh. ziol.-bot. Ver. Wien, V. 630. 21 et 669. [Odontomyia] (1855) et Fauna Austr., Dipt., I. 18. 8. [Odontomyia] (1862).

peruviana MAcQ., Dipt. exot., suppl. 5., 43. 37. [Odontomyia] (1855);

Hunter, Trans. Amer. Entom. Soc. Philad., XXVII. 126. [Odontomyia] (1901).

picea WALK., Insecta Saunders., Dipt., I. 78. (1851) et List Dipt. Tasmania.

Brit. Mus., V. suppl. 1., 55. 111. [Stratiomys] (1854).

pictifrons Lw., Neuo Beitr., II. 16. 32. [Odontomyia] (1854).

pilimana Lw., Berlin. Entom. Zeitschr., IX. 146. 27. [Odontomyia]

Sibiria.

America sept. 
(1865) ; Day, Proc. Acad. Nat. Sci. Philad., 1882. 83. [Odontomyia] (1882) ; Johss., Trans. Amer. Entom. Soc. Philad., XXII. 263. tab. IV. fig. 11-12. [Odontomyia] (1895); НАRT, Bull. Illinois State Labor. of Nat. Hist., IV. 255 et 256. [Odontomyia] (1895); Mriand., Canad. Entomol., XXXVI. 24 et 53. 13. [Odontomyia] (1904) ; Aldr., Catal. North Amer. Dipt., 186. [Odontomyia] (1905). pilosa DAy, Proc. Acad. Nat. Sci. Philad., 1882. 76. [Odontomyia] (1882); Johss., Trans. Amer. Entom. Soe. Philad., XXII. 258. tab. IV. fig. 13-14. [Odontomyia] (1895); Meland., Canad. Entomol., XXXVI. 24 et 53. 7. [Odontomyia] (1904); Atsr., Catal. North Amer. Dipt., 187. [Odontomyia] (1905).

pyrrhostoma Bia., Annal. Soc. Entom. France, sér. 6. VII. 25. 1. [Odontomyia] (1887).

plebeja L, = Virgo WiED.

prasina $\mathrm{J}_{\mathrm{AEN}}=$ Lefebvrei MacQ.

pubescens Day, Proc. Acad. Nat. Sci. Philad., 1882. 77. [Odontomyiu] (1882); Jouns., Trans. Amer. Entom. Soc. Philad., XXII. 264. tab. IV. fig. 15-16. [Odontomyia] (1895); HART, Bull. Illinois State Iaborat. of Nat. Hist., IV. 255 et 256. [Odontomyia] (1895) ; Mruand., Canad. Entomol., XXXVI. 24 et 53. 15. [Odontomyia] (1904); Aldr., Catal. North Amer. Dipt., 187. [Odontomyia] (1905).

pulchella MAcQ. = flavicornis Ouw.

pulchra Wied., Analecta Entomol., 28. 32. [Stratiomys] (1824) et Aussereurop. zweifl. Ins., II. 65. 6. táb. VIII. fig. 5. a-b. [Stratiomys] (1830) ; MacQ., Dipt. exot., I. 1., 190.7.[Odontomyia](1838) ; Wask., List Dipt. Brit. Mus., III. 532. [Odontomyia] (1849) et V. suppl. 1., 46. 76. [Stratiomys] (1854); Gers'., Linnaea Entomol., XI. 324. 1. [Stratiomys] (1857); Schin., Novara Reise, Dipt., 57. 17. [Odontomyia] (1868); E. L. Arrib., Bolet. Acad. Nat. Cienc. Córdoba, IV. 127. 48. [Odontomyia] (1882); Hunter, Trans. Amer. Entom. Soc. Philad., XXVII. 126 et 128. [Stratiomyia] (1901).

pulchriceps Lw., Öfvers. Kongl. Vet. Akad. Förhandl., XV. 335. 8 bis. [Odontomyia] (1858) et Dipt.-Faına Südafrika's, I. 8. 2. [Odontomyia] (1860).

punctifer Big., Annal. Soc. Entom. Francs, sér. 5. IX. 217. Ђ. Patriaignota. [Odontomyia] (1879).

pusilla FABr., Entomol. System., IV. 271. 5. [Nemotelus] (1794) et Tranquebaria Systerna Antliat., 89. 5. [Nemotelus] (1805); Or.rv., Encyclop. Méthod., VIII. 184. 6. [Nemotelus] (1811); Wird., Aussereurop. zweifl. Ins., II. 75. 24. [Stratiomys] (1830); WaIk., List Dipt. Brit. Mus., V. suppl. 1., 54. 108. [Stratiomys] (1854); v. D. Wulp, Catal Dipt. South Asia, 61. [Odontomyia] (1896).

pyrrhostoma BrG. = pilosa $\mathrm{DAY}_{\mathrm{X}}$.

quadrilineata Macq., Suit. à Buffon, I. 248. 13. [Odontomyia] (1834); Americamer. WALK., List Dipt. Brit. Mus., V. suppl. 1., 45. 71. [Stratiomys] 
(1854) ; Gerst., Linnaea Entomol., XI. 325. 2. [Stratiomys] (1857); Hunter, Trans. Amer. Entom. Soc. Philad., XXVII. 127. 「Stratiomyia] (1901).

quadrimaculata BeLI., Saggio di Ditterol. Messic., I. 37. 6. tab. I. fig. 15. [Odontomyia] (1859); Joнns., Trans. Amer. Entom. Soc. Philad., XXII. 274. [Odontomyia] (1895); Willist., Biolog. Central. Amer., Dipt., I. 246. 5. [Odontomyia] (1900); Aldr., Catal. North Amer. Dipt., 187. [Odontomyia] (1905).

quadrinotata Lw., Öfvers. Kongl. Vet. Akad. Förhandl., XIII. 263. 6. [Odontomyia] (1856) et Dipt.-Fauna Südafrika's, I. 9. 4. [Odontomyia] (1860).

rectifasciata MACQ., Dipt. exot., I. 1., 191. 16. [Odontomyia] (1838); WaLK., List Dipt. Brit. Mus., V. suppl. 1., 50. 92. [Stratiomys] (1854).

Regis Georgii Macq., Dipt. exot., suppl. 1., 186. 6. [Odontomyia] (1838) ; WalK., List Dipt. Brit. Mus., V. suppl. 1., 56. 116. [Stratiomys] (1854).

restricta WALK., Proc. Linn. Soc. London, VII. 203. 7. [Strutiomys] (1864); v. D. Wulp, Catal. Dipt. South Asia, 52. [Odontomyia] (1896).

rubicornis WILLIST, = rubricornis MACQ.

rubricornis MacQ., Dipt. exot., suppl. 1., 53. 21. [Odontomyia] (1846);

WaLK., List Dipt. Brit. Mus., V. suppl. 1., 45. 72. [Stratiomys] (1854); Johns., Trans. Amer. Entom. Soc. Philad., XXII. 276. [Orlontomyia] (1895); Aldr., Catal. Nortl Amer. Dipt., 187. [Odontomyia] (1905).

rubicornis Willist., Biolog. Central. Amer., Dipt., I. 247. 8. [Odontomyia] (1900).

rubrithorax MACQ., Dipt. exot., I. 1., 185. 5. [Odontomyia] (1838);

WaLK., List Dipt. Brit. Mus., V. suppl. 1., 52. 101. [Stratiomys] (1854) ; v. D. Wulp, Catal. Dipt. South Asia, 52. [Odontomyia] (1896).

ruficornis $\mathrm{MACQ}_{\mathrm{AC}}=$ viridana WIED.

ruficornis ZETT. = angulata PANZ.

ruffacies MACQ., Dipt. exot., suppl. 4., 51. 31. [Odontomyia] (1849). rufipes Lw., Berlin. Entom. Zeitschr., IX. 144. 25. [Odontomyia] (1865) ; Johss., Trans. Amer. Entom. Soc. Philad., XXII. 253. [Odontomyia] (1895); Meland., Canad. Entomol., XXXVI. 23. [Odontomyia] (1904); Aldr., Catal. North Amer. Dipt., 187. [Odontomyia] (1905).

scalairis Lw., Berlin. Entom. Zeitschr., IX. 145. 26. [Odontomyia] (1865).

scalaris $\mathrm{Lw},=$ rufipes $\mathrm{Lw}$.

scutellata MACQ., Dipt. exot., suppl. 1., 52. 19. tab. V. fig. 7. [Odontomyia] (1846) ; WALK., List Dipt. Brit. Mus., V. suppl. 1., 55. 112.

[Stratiomys] (18j4).

semiviolacea Brulté $=$ flavissima Rosst.

Mexico.

Mozambique.

Prom. bon.sp.

Australia.

I. Mysol.

Mexico.

Bengalia.

Tasmania. Cuba.

Tasmania. 6 
septemguttata WIED. = annulata MEIG.

siderogaster WrED., Analecta Entomol., 29. 33. [Stratiomys] (1824) Java.

et Aussereurop. zweifl. Ins., II. 65. 7. [Stratiomys] (1830); Walk., List Dipt. Brit. Mus., V. suppl. 1., 53. 104. [Stratiomys] (1854); v. D. Wulp, Catal. Dipt. South Asia, 52. [Odontomyia] (1896).

sidneyensis Schin., Novara Reise, Dipt., 60. 23. [Odontomyia] (1868). Australia. signuta JAENN. = tigrina FAlBR. var.

signaticornis Lw., Linnaea Entomol., I. 4i7. 7. [Odontomyia] (1846);

WAlK., List Dipt. Brit. Mus., V. suppl. 1., 108. [Stratiomys] (1854). similis Johns., Trans. Amer. Entom. Soc. Philad., XXII. 267. tab. IV. fig. 6. [Odontomyia] (1895); Meland., Canad. Entomol., XXXVI. 24. [Odontomyia] (1904); Aldr., Catal. Nortl Amer. Dipt., 187. [Odontomyia] (1905).

Snowi HaRT = hieroglyphica OLIv.

solennis WALK., Insecta Saunders., Dipt., I. 79. [Odontomyia] (1851) et List Dipt. Brit. Mus., V. suppl. 1., 51. 96. [Stratiomys] (1854); Big., Annal. Soc. Entom. France, sér. 5. IX. 186. [Odontomyia] (1879); v. D. Wulp, Catal. Dipt. South Asia, 52. [Odontomyia] (1896).

staurophora Schus., Novara Reise, Dipt., 59. 21. [Odontomyia] (1868) ; v. D. Wulp, Catal. Dipt. South Asia, 52. [Odontomyia] (1896); Coquill., Proc. Unit. Stat. Nat. Mus., XXI. 308. [Odontomyia] (1898).

stigmaticalis Thoмs., Eugenios Resa, Dipt., 457. 21. [Odontomyia] Puna. (1869) ; Hunter, Trans. Amer. Entom. Soc. Philad., XXVII. 127. [Odontomyia] (1901).

stricta Erichs., Archiv f. Naturgesch., VIII. 1., 272. 251. [Odontomyia] (1842); Walk., List Dipt. Brit. Mus., V. suppl. 1., 55. 113. [Stratiomys] (1854).

stylata MACQ., Dipt. exot., suppl. 2., 30. 23. [Odontomyia] (1847) et suppl. 4., 52. [Odontomyia] (1849) ; Walk., List Dipt. Brit. Mus., V. suppl. 1., 56. 117. [Stratiomys] (1854).

subcuprata WALK., List Dipt. Brit. Mus., V. suppl. 1., 47. 77. [Stratiomys] (1854).

subdentata MAcq., Dipt. exot., suppl. 4., 49. 28. [Odontomyia] (1849). texana ALDR. = texasiana JoHNs.

texasiana Jouss., Trans. Amer. Entom. Soc. Philad., XXII. 259. [Odontomyia] (1895); Meland., Canad. Entomol., XXXVI. 24. [Odontomyia] (1904).

texana ALdr., Catal. North Amer. Dipt., 187. [Odontomyia] (1905).

tigrina FABr., Systema Entomolog., 760. 4. [Stratiomys] (1775), Europa.

Species Insect., II. 417. 6. [Stratiomys] (1781) et Mantissa Insect., II. 331. 11. [Stratiomys] (1787); GmeL., Systema Naturae, V. 2835. 157. [Musca] (1792); FABR., Entomol. System., IV. 267. 16. [Stratiomys] (1794); PAnz., Fauna Germ., LVIII. tab. 20. [Stra-

Japonia, China.

Tasmania.

Australia, Tasmania.

Brasilia.

Australia.

America sept. 
tiomys] (1798) ; Schrank, Fauna Boica, III. 95. 2381. [Stratiomys] (1803) ; MeIG., Klassif., I. 130. 3. tab. VII. fig. 24-26. [Odontomyia] (1801) ; Farr., Systoma Antliat., 82. 18. [Stratiomys] (1805); LAtr., Gen. Crust. et Insect., IV. 275 [Odontomyia] (1809); OuIv., Encyclop. Méthod., VIII. 432. 6. [Odontomyia] (1811); MeIG., System. Beschreib., III. 152. 22. [Stratiomys] (1822); MıcQ., Recueil Soc. Sci. Agricult. Lille, 1826. 447. 4. [Odontomyia] (1826) et Suit. à Buffon, I. 246. 6. [Odontomyia] (1834); Duncan, Magaz. Zoolog. and Botany, I. 154. 7. [Odontomyia] (1837) ; Lw., Programm Posen, 1840. 26. 10. [Stratiomys] (1840), Isis, 1840. VII. 557. 10. [Stratiomys] (1840) et Linnaea Entomol., I. 468. 1. [Odontomyia] (1846); Walk., List Dipt. Brit. Mus., III. 534. [Odontomyia] (1849); L. Duf., Mém. Acad. d. Sci. Math. et Phys., XI. 171-360. tab. V. fig. 50. [Odontomyia] (1850) ; WaLK., Insecta Britann., Dipt., I. 18. 9. [Stratiomys] (1851) et List Dipt. Brit. Mus., V. suppl. 1., 30.12 et 307. [Stratiomys] (1854) ; Schin., Verh. zool.-bot. Ver. Wien, V. 623. 1 et 668. [Odontomyia] (1855) et Fauna Austriaca, Dipt., I. 17. 2. [Odontomyia] (1862); JAEnn., Berlin. Entom. Zeitschr., X. 230. 34. [Odontomyia] (1866); v. D. WulP, Diptera Neerland., I. 454. 2. [Odontomyia] (1877); JAROschefF., Trudy Kharkoff, XI. 340. 4. [Odontomyia] (1877); BIa., Annal. Soc. Entom. France, sér. 5. IX. 186. [Psellidotus] (1879); Neuhaus, Diptera Marchica, 81.1. [Odontomyia] (1886) ; Brunetri, The Entomologist, XXII. 131. 4. [Odontomyia] (1889); É. \& L. Coucke, Annal. Soc. Entom. Belgique, XXXVII. 439. [Odontomyia] (1893) ; Kert., Pótfüz. a Természettud. Közlönyhöz, XXXII 128. [Odontomyia] (1900).

nigrita Fall., Dipt. Suec., Stratiom., 9. 4. [Stratiomys] (1817);

Zetт., Dipt. Scand., I. 138. 7. [Stratiomys] (1842), VIII. 2953.

7. [Stratiomys] (1849) et XI. 4260. 7. [Stratiomys] (1852).

var. signata JAEnn., Berlin. Entom. Zeitschr., X. 230. 34. Europacentr. [Odontomyia] (1866).

tritaeniata BeLL. = trivittata $\mathrm{SAY}_{\mathrm{A}}$

trivittata SAY, Journ. Acad. Nat. Sci. Philad., VI. 160. [Strationys] (1829) et Compl. Writt., II. 356. [Stratiomys] (1859); WALK., List Dipt. Brit. Mus., V. suppl. 1., 114. [Stratiomys] (1854) ; Johns., Trans. Amer. Entom. Soc. Philad., XXII. 259. tab. IV. fig. 19. [Odontomyia] (1895); Willıs'., Biolog. Centr. Amor., Dipt., I. 245. 1. [Odontomyia] (1900); Meland., Canad. Entomol., XXXVI. 24 et 53. 8. [Odontomyia] (1904); Aldr., Catal. North Amer. Dipt., 184. [Stratiomyia] et 187. [Odontomyia] (1905).

tritaeniata BeLL., Saggio di Ditterol. Messic., I. 38. 9. tab. I. fig. 17. [Odontomyia] (1859); v. D. Wulp, Annal. Soc. Entom. Belgique, XXVIII. Compt. Rend., CCLXXXIX. 2. [Odontomyia] (1884); Ost.-Sack., Biolog. Central. Amer., Dipt., I. 36. 1. [Oduntomyia] (1886).

Truquii Brul., Saggio di Ditterol. Messic., I. 34. 2. tab. I. fig. 11. Americac.ets. 
[Odontomyia] (1859) ; Johns., Trans. Amer. Entom. Soc. Philad., XXII. 273. [Odontomyia] (1895) ; Wiluıst., Biolog. Central. Amer., Dipt., I. 246. 3. [Odontomyia] (1900) ; Alds., Catal. North Amer. Dipt., 187. [Odontomyia] (1905).

bicolor Day, Proc. Acad. Nat. Scienc. Plilad., 1882. 78. [Odontomyia] (1882).

binotata Lw., Berlin. Entom. Zeitschr., IX. 142. 22. [Odontomyia] (1865); Dax, Proc. Acad. Nat. Sci. Philad., 1882. 81. [Odontomyia] (1882); Johss., Trans. Amer. Entom. Soc. Philad., XXII. 251. tab. III. fig. 29-32. [Odontomyia] (1895); Hart, Bull. Illinois State Laborat. of Nat. Hist., IV. 255 et 262. [Odontomyia] (1895); Meland., Canad. Entomol., XXXVI. 23 et 53. 1. [Odontomyia] (1904).

megacephala Lw., Berlin. Entom. Zeitschr., IX. 140. 20. [Odontomyia] (1865) ; Day, Proc. Acad. Nat. Sci. Philad., 1882. 79. [Odontomyia] (1882); HaRT, Bull. Illinois State Laborat. of Nat. Hist., IV. 255 et 262. [Udontomyia] (1895).

varipes Lw., Berlin. Entom. Zeitschr., IX. 141. 21. [Odontomyia] (1865) ; Day, Proc. Acad. Nat. Sci. Philad., 1882. 84. [Odontomyia] (1882); Johns., Trans. Amer. Entom. Soc. Philad., XXII. 252. [Odontomyia] (1895); Meland., Canad. Entomol., XXXVI. 23. [Odontomyia] (1904); Aldr., Catal. North Amer. Dipt., 187. [Odontomyia] (1905).

vertebrata SAY, Long's Exped. to St. Peter's River, II. Append., 369. [Odontomyia] (1824) et Compl. Writt., I. 251. [Odontomyia] (1859) ; Wied., Aussereurop. zweifl. Ins., II. 73. 20. [Stratiomys] (1830) ; Walk., List Dipt. Brit. Mus., III. 532. [Odontomyia] (1849) et V. suppl. 1., 39. 51. [Stratiomys] (1854) ; BeLl., Saggio di Ditterol. Messic., I. 38. 8. [Odontomyia] (1859); Schin., Novara Reise, Dipt., 58. [Odontomyia] (1868); Dar, Proc. Acad. Nat. Sci. Philad., 1882. 85. [Odontomyia] (1882); JoHss., Trans. Amer. Entom. Soc. Philad., XXII. 260. tab. IV. fig. 26-28. [Oclontomyia] (1895) ; Hart, Bull. Illinois State Laborat. of Nat. Hist., IV. 255 et 262. tab. XIV. fig. 60. [Odontomyia] (1895) ; Meland., Canad. Entomol,, XXXVI. 24 et 53. 9. [Odontomyia] (1904); Aldr., Catal. North Amer. Dipt., 187. [O(lontomyia] (1905).

Willistoni Day, Proc. Acad. Nat. Sci. Philad., 1882. 78. [Odontomyia] (1882).

vicina MacQ., Dipt. exot., I. 1., 188. 11. [Odontomyia] (1838) ; WaLK., List Dipt. Brit. Mus., V. suppl. 1., 40. 56. [Stratiomys] (1854); Big. in SAgra : Hist. la Isla de Cuba, P. 2. VII. 335. [Odontomyia] (1856) ; Johns., Trans. Amer. Entom. Soc. Philad., XXII. 276. [Odontomyia] (1895); Aldr., Catal. North Amer. Dipt., 187. [Odontomyia] (1905).

vicina MAcQ. = flavicornis Orrv.

Virgo Wied., Aussereurop. zweifl. Ins., II. 69. 13. [Stratiomys] (1830); America sept WALK., List Dipt. Brit. Mus., V. suppl. 1., 39. 52. [Stratiomys]

Americasept. et centr.

Cuba. 
(1854) ; Day, Proc. Acad. Nat. Sci. Philad., 1882. 86. [Odontomyia] (1882) ; Johns., Trans. Amer. Entom. Soc. Philad., XXII. 262. tab. IV. fig. 31-35. [Odontomyia] (1895) ; Meland., Canad. Entomol., XXXVI. 24 ot 53. 12. [Odontomyia] (1904); ALDr., Catal. North Amer. Dipt., 187. [Odontomyia] (1905).

nigra DAY, Proc. Acad. Nat. Sci. Philad., 1882. 75. [Odontomyia] (1882).

Paron Walk., List Dipt. Brit. Mus., III. 536. (1819) et V. suppl. 1., 36. 38. [Stratiomys] (1854); BIG., Annal. Soc. Entom. France, sér. 5. IX. 186. [Odontomyia] (1879); DAY, Proc. Acad. Nat. Sci. Philad., 1882. 85. [Odontomyia] (1882).

plebeja Lw., Berlin. Entom. Zeitschr., XVI. 53.5. [Odontomyia] (1872) ; Day, Proc. Acad. Nat. Sci. Philad., 1882. 75. [Odontomyia] (1882); НАRT, Bull. Illinois State Laborat. of Nat. Hist., IV. 255 et 256. [Odontomyia] (1895).

viridana Wred., Analecta Entomol., 29. 34. [Stratiomys] (1824) et Ausereurop. zweifl. Ins., II. 66. 8. [Stratiomys] (1830); WALK., List Dipt. Brit. Mus., V. suppl. 1., 53. 103. [Strationys] (1854); v. D. Wulp, Notes Leyden Mus., VII. 61. 8. [Odontomyia] (1885) et Catal. Dipt. South Asia, 51. [Odontomyia] (1896).

ruficornis MacQ., Dipt. exot., suppl. 4., 48. 26. [Odontomyia] (1849).

viridis BeLl., Saggio di Ditterol. Messic., I. 36. 5. tab. I. fig.16.[Odontomyia] (1859) ; Johns., Trans. Amer. Entom. Soc. Philad., XXII. 270. tab. IV. fig. 18. [Odontomyia] (1895); Willist., Biolog. Centr. Amer., Dipt., I. 246. 4. [Odontomyia] (1900); Meland., Canad. Entomol., XXXVI. 23. [Odontomyia] (1904) ; Aldr., Catal. North Amer. Dipt., 188. [Odontomyia] (1905).

vittata MACQ., Dipt. exot., suppl. 4., 53. 35. [Odontomyia] (1849); Hunter, Trans. Amer. Entom. Soc. Philad., XXVII. 127. [Odontomyia] (1901).

vulpina $\mathrm{PANz}$. = hydroleon $\mathrm{L}$.

Willistoni $\mathrm{DAY}_{\mathrm{A}}=$ vertebrata $\mathrm{SAY}$.

Asia merid., Ternate.

America sept. et centr.

Brasilia.

\section{Cyrtopus}

Big., Annal. Soc. Entom. France, sér. 6. III. Bull., XCVI. (1883).

fastuosus Big., Annal. Soc. Entom. France, sér. 6. III., Bull. XCVI. Abyssinia. (1883).

\section{Euceromyia}

Big., Annal. Soc. Entom. France, sér. 5. VII. Bull., LXXIV. (1877). [Euceromys] v. D. WULP emend.

nexura Walk., Proc. Linn. Soc. London, III. 80. 11. [Stratiomys] I.Aru, Mysol. (1859); Big., Annal. Soc. Entom. France, sér. 5. VII. Bullet., LXXIV. [Euceromys] (1877) et sér. 5. IX. 188. [Euceromys] (1879); v. D. Wulp, Catal. Dipt. South Asia, 52. (1896). 


\section{Rhingiopsis}

Rö̀., Entomol. Nachricht., XII. 138. (1886).

rostrata WiED., Aussereurop. zweifl. Ins., II. 68. 11. [Stratiomys]

Brasilia. (1830) ; Macq., Suit. à Buffon, I. 249. 15. [Odontomyia] (1834) et Dipt. exot., I. 1., 186. 8. tab. XXII. fig. 4. [Odontomyia] (1838); Walk., List Dipt. Brit. Mus., V. suppl. 1., 45. 69. [Stratiomys] (1854) ; Gerst., Linnaea Entomol., XI. 329. 6. [Stratiomys] (1857); Willist., Trans. Amer. Entom. Soc. Philad., XV. 255. 29. (1888); ?, Kansas. Univ. Quart., I. - t tab. VII. fig. 6. (1892) ; Hunter, Trans. Amer. Entom. Soc. Philad., XXVII. 124. [Rhingiopsis] et 128. [Stratiomyia] (1901).

Tau Röder, Entomol. Nachricht., XII. 138 et 201. (1886) ; WiLlist., Brasilia. Trans. Amer. Entom. Soc. Philad., XV. 255. 28. (1888) ; Hunter, Trans. Amer. Entom. Soc. Philad., XXVII. 124. (1901).

\section{Hoplodonta}

Rond., Archivio per la Zool. Modena, IIT. 78. (1863) [Oploulontha].

Oplodonta Brav., Denkschr. Akad. Wien, XLIV. 69. (1882).

bimaculata MEIG. = viridula FABR.

canina PANZ. = viridula FABR.

dentata MEIG. = viridula FABR.

dispar MACQ., Dipt. exot., I. 1., 183. 2. [Odontomyia] (1838); WALK., Senegal.

List Dipt. Brit. Mus., V. suppl. 1., 49. 88. [Stratiomys] (1854);

Big., Annal. Soc. Entom. France, sér. 5. IX. 186. [Oplodontha]

(1879).

interrupta $\mathrm{Lw},=$ viridula $\mathrm{FABR}$.

jejuna $\mathrm{SchraNK}=$ viridula FABR.

subvittata MEIG. = viridula FABR.

viridula FABR., Systema Entomolog., 760. 6. [Stratiomys] (1775),

Species Insect., II. 418. 8. [Stratiomys] (1781) et Mantissa Insect.,

II. 331. 13. [Stratiomys] (1787); Rossi, Fauna Etrusea, II. 280. 1443. [Strationys] (1790); GmeL., Systema Naturae, V. 2835. 158. [Musca] (1792) ; Fabr., Entomol. System., IV. 267. 18. [Stratiomys] (1794); Panz., Fauna Germ., LVIII. tab. 18. [Stratiomys] (1798); Schrank, Fauna Boica, III. 96. 2383. [Stratiomys] (1803); Meig., Klassif., I. 133. g. [Odontomyia] (1804); FABr., Systema Antliat., 84. 25. [Stratiomys] (1805); Rossi, Fauna Etrusca, Ed. II., 433. 1443. [Stratiomys] (1807) ; Latr., Gen. Crust. et Ins., IV. 275. [Odontomyia] (1809) et Consid. génér., 442. [Odontomyia] (1810) ; Ourv., Encyclop. Méthod., VIII. 435. 16. [Odontomyia] (1811) ; Fall., Dipt. Suec., Stratiom., 10. 6. [Stratiomys] (1817); Meig., System. Beschreib., III. 149. 18. [Stratiomys] (1822); MACQ., Recueil Soc. Sci. Agricult. Lille, 1826. 449. 7. [Odontomyia] (1826); MeIG., System. Beschreib., VI. 347. [Stratiomys]

Europa, Africa sept., Asia min. 
(1830) ; Macq., Suit. à Buffon, I. 247. 10. [Odontomyia] (1834); Duncan, Magaz. Zoolog. and Botany, I. 154. 6. [Odontomyia] (1837); Lw., Programm Posen, 1840. 26. 9. [Stratiomys] (1840) et Isis, 1840. VII. 557. 9. [Stratiomys] (1840) ; Zerr., Dipt. Scand., I. 140. 11. [Stratiomys] (1842); Lw., Linnaea Entomol., I. 491. 13. [Odontomyia] (1846); Macq. in Lucas: Explor. Scient. de l'Algérie, Zool., III. 429. 41. [Odontomyia] (1849); WALK., List Dipt. Brit. Mus., III. 535. [Odontomyia] (1849) ; Scholtz, Zeitschr. f. Entomol. Breslau, IV. 34. [Odontomyia] (1849); Zetт., Dipt. Scand., VIII. 2954. 11. [Stratiomys] (1849); WALK., Insecta Britann., Dipt., I. 19. 13. [Stratiomys] (1851) et List Dipt. Brit. Mus., V. suppl. 1., 33. 26 et 309. [Stratiomys] (1854); Schin., Verh. zool.-bot. Ver. Wien, V. 630. 23 et 669. [Odontomyia] (1855); ZeтT., Dipt. Scand., XII. 4555. 11. [Stratiomys] (1855); EGgER, Verh. zool.-bot. Ver. Wien, VI. 392. [Odontomyia] (1856); Lw., Zeitschr. f. d. ges. Naturwiss., X. 99. 6. [Odontomyia] (1857); Schin., Verh. zool.-bot. Ver. Wien, VII. 11. [Odontomyia] (1857); BonsD., Finl. tvaving. Ins., I. 116. 3. [Stratiomys] (1861); Sch1N., Fauna Austriaca, Dipt., I. 19. [Odontomyia] (1862); Rond., Archivio per la Zoolog., III. 78. [Oplodontha] (1863); JAENN. ${ }^{7}$ Berlin. Entom. Zeitschr., X. 230. 38. [Odontomyia] (1866); v. D. WulP, Diptera Neerland., I. 455. 4. tab. XIV. fig. 14-15. [Odontomyia] (1877); Neuhaus, Diptera Marchica, 82. 4. [Odontomyia] (1886); Brunetti, The Entomologist, XXII. 131. 7. [Odontomyia] (1889); Schосн, Fauna Ins. Helvetiae, Dipt., 24. [Odontomyia] (1890); E. \& I. Coucke, Annal. Soc. Entom. Belgique, XXXVII. 440. [Odontomyia] (1893); StrnoвL, Mittheil. Naturwiss. Ver. Steiermark, XXIX. 1892. 10. [Odontomyia] (1893); Kert., Pótfüz. a Természettud. Közlönyhöz, XXXII. 128. [Odontomyia] (1900).

bimaculata MeIG., System. Beschreib., VII. 106. 30. [Stratiomys] (1838); WaLk., List Dipt. Brit. Mus., V. suppl. 1., 35. 33. [Stratiomys] (1854); Scmin., Verh. zool.-bot. Vor. Wien, V. 632: 27. [Odontomyia] (1855).

canina PANz., Fauna Germ., LVIII. tab. 23. [Stratiomys] (1798); MeIG., Klassif., I. 132. e. [Odontomyia] (1804); OuIv., Encyclop. Móthod., VIII. 435. 17. [Odontomyia] (1811).

dentata MeIr., Klassif., I. 130. 2. [Odontomyia] (180t); Ouiv., Encyclop. Méthod., VIII. 436. 22. [Odontomyia] (1811).

interrupta Lw., Linnaea Entomol., I. 493. 14. [Odontomyia] (1846); Walk., List Dipt. Brit. Mus., V. suppl. 1., 309. [Stratiomys] (1854); ScHin., Verh. zool.-bot. Ver. Wien, V. 632. 26 et 669. [Odontomyia] (1855) et VII. 10. [Odontomyia] (1857); Bıg., Annal. Soc. Entom.. France, sér. 5. IX. 186. [Oplodontha] (1879).

jejuna Schrank, Fauna Boica, III. 96. 2384. [Stratiomys] (1803) ;

MeIG., System. Beschreib., III. 153. 24. [Stratiomys] (1822);

WalK., List Dipt. Brit. Mus., V. suppl. 1., 35. 34. [Stratio- 
mys] (1854) ; Scmn., Verh. zool.-bot. Ver. Wien, V. 631. 25 et 668. [Odontomyia] (1855).

subvittata MeIG., System. Beschreib., III. 150. 19. tab. XXVI. fig. 15. [Stratiomys] (1822); Walk., Insecta Britann., Dipt., I. 19. 14. [Stratiomys] (1851) et List Dipt. Brit. Mus., V. suppl. 1., 35. 32. [Stratiomys] (1854); Scur., Verl. zool.-bot. Ver. Wien, V. 631. 24 et 669. [Odontomyia] (1855).

\section{Exochostoma}

Macq., Annal. Soc. Entom. France, XI. 42. (1842).

nitidum Macq., Annal. Soc. Entom. France, XI. 43. tab. 4. II. Europa occ. (1842); Walk., List Dipt. Brit. Mus., V. suppl. 1., 6. 1. (1854); Schin., Fauna Austriaca, Dipt., I. 20. (1862).

\section{Melanochroa}

RöD., Entomol. Nachricht., XII. 139. (1886).

dubia RöD., Entomol. Nachricht., XII. 140. (1886); Wiluıst., Trans. Brasilia. Amer. Entom. Soc. Philad., XV. 254. 25. (1888); Hunter, Trans. Amer. Entom. Soc. Pnilad., XXVII. 130. (1901).

\section{Myxosargus}

Brau., Denkschr. Akad. Wien, XLIV. 77. (1881).

Braueri Willist., Trans. Amer. Entom. Soc. Philad., XV. 254. 26. Brasilia. (1888) ; Hunțer, Trans. Amer. Entom. Soc. Philad., XXVII. 130. (1901).

fasciatus Brau., Denkschr. Akad. Wien, XLIV. 78. (1881); Röp., Americasept. Entomol. Nachricht., XII. 139. var. (1886); Gigl.-Tos, Mem. R. et centr. Accad. Scienc. Nat. Torino, ser. 2. XLIII. 109. 10. (1901); Willist., Biolog. Central. Amer., Dipt., I. 251. 1. (1901) ; Meland., Canad. Entomol., XXXVI. 20. (1904); Aldr., Catal. North Amer. Dipt., 188. (1905).

scutellatus Willist., Biolog. Central. Amer., Dipt., I. 251. 2. tab. Mexico. IV. fig. 18. (1901); Aldr., Catal. North Amer. Dipt., 188. (1905).

\section{Nothomyia}

Lw., Berlin. Entom. Zeitschr., XIII. 4. nota. (1869).

calopus Lw., Berlin. Entom. Zeitschr., XIII. 5. 5. (1869); ALDR., Cuba. Catal. North Amer. Dipt., 188. (1905).

scutellata Lw., Berlin. Entom. Zeitschr., XIII. 4. 4. (1869); Aldr., Cuba. Catal. North Amer. Dipt., 188. (1905). 


\section{Chrysochlorinae.}

\section{Cacosis}

WaLk., Ins. Saunders., Dipt., I. 83. (1851).

grandis Schin., Novara Reiso, Dipt., 67. 44. (1868); IIunter, Trans. Americamer. Amer. Entom. Soc. Philad., XXVII. 124. (1901).

niger W1ED., Zoolog. Magaz., I. 3., 52. 17. [Sargus]. (1819) et Brasilia.

Aussereurop. zweifl. Ins., 1I. 28. 2. [Sargus] (1830); Walk.,

Insecta Saunders., Dipt., I. 84. tab. III. fig. 1. (1851) et List

Dipt. Brit. Mus., V. suppl. 1., 86.1.(1854); Scms., Novara Rcise,

Dipt., 67. (1868) ; Willist., Trans. Amer. Entom. Soc. Philad.,

XV. 248. 9. (1888); Hunter, Trans. Amer. Entom. Soc. Philad.,

XXVII. 122. [Sargus] et 124. (1901).

\section{Chaetosargus}

Rö̀, Wien. Entom. Zeitg., XIII. 169. (1894).

hirticornis W1ed., Aussereurop. zweifl. Ins., II. 28. 3. [Sargus] Brasilia.

(1830) ; MACQ., Suit. à Buffon, I. 258. 3. [Chrysochlora] (183t);

GuEkr., Iconogr. d. Régne Anim., VII. Ins., 545. tab. XCVIII. fig. 10. [Chrysochlora] (1835); WALK., List Dipt. Brit. Mus., V. suppl. 1., 86. 1. [Chrysochlora] (1854); RöD., Wien. Entom. Zeitg., XIII. 169. (1894); IIUnter, Trans. Amer. Entom. Soc. Philad., XXVII. 122. [Sargus] (1901).

\section{Chrysochlora}

Latr., Régne Anim., V. 486. (189).

amethystina FABR., Systema Antliat., 258. 13. [Sargus] (1805);

I. Mauritius. Wied., Aussereurop. zweifl. Ins., II 27. 1. [Sargus] (1830); MACQ., Suit. à Buffon, I. 258. 1. (1834) et Dipt. exot., I. 1., 198. 1. (1838); Walk., List Dipt. Brit. Mus., III. 514. [Sargus] (1849) et V. suppl. 1., 86. 2. (1854) ; Lw., Dipt.-Fauna Siidafrika's, I. 5. (1860); Willist., Biolog. Centr. Amer., Dipt., I. 242. (1900).

annulipes WaLK., List Dipt. Brit. Mus., III. 515. [Sargus] (1849) et Patriaignota. V. suppl. 1., 86. 4. (1854).

baccoides Rond., Annal. Mus. Civico Genova, VII. 454. (1875); Bo:neo. v. D. Wulp, Catal. Dipt. South Asia, 48. (1896).

castanea MACQ., Dipt. exot., I. 1., 198. 1 bis, tab. XXV. fig. 1. Americamer. (1838) ; Rond. in Truqui: Studi Entomol., I. 103. 74. (1848); Walk., List Dipt. Brit. Mus., V. suppl. 1., 68. 8. [Acrochaeta] (1854) ; Hunter, Trans. Amer. Entom. Soc. Philad., XXVII. 124. (1901).

fasciata Thомs., Eugenies Resa, Dipt., 460. 25. (1869).

I. Galapagos. 
maculiventris Rond., Nuov. Aunal. Sei. Nat. Bologna, ser. 3. II. Venezuela. 190. 27. (1850); Hunter, Trans. Amer. Entom. Soc. Philad., XXVII. 124. (1901).

pluricolor Big., Annal. Soc. Entom. France, sér. 5. IX. 209. (1879); Brasilia.

Hunter, Trans. Amer. Entom. Soc. Philad., XXVII. 124. (1901).

pulchra Willist., Biolog. Central. Amer., Dipt., I. 243. 1. (1900); Mexico.

Aldr., Catal. North Amer. Dipt., 180. (1905).

purpurea WaLk., Trans. Entom. Soc. London, n. ser. V. 271. (1860); Mexico.

Aldr., Catal. North Amer. Dipt., 180. (1905).

quadrilineata Big., Annal. Soe. Entom. France, sér. 6. VII. 26. Cuba.

(1887); Aldr., Catal. North Amer. Dipt., 180. (1905).

similis MacQ., Dipt. exot., suppl. 5., 44. 41. (1855); IIunter, Trans. Americamer*

Amer. Entom. Soc. Philad., XXVII. 124. (1901).

vespertilio Fabr., Systema Antliat., 259. 14. [Sargus] (1805) ; Wied.,

Aussereurop. zweifl. Ins., II. 29. 4. [Sargus] (1830); MacQ.,

Suit. à Buffon, I. 258. 2. (1834) ; WALK., List Dipt. Brit. Mus.,

III. 515. [Sargus] (1849) et V. suppl. 1., 68. 6. [Acrochaela]

(1854) ; Jaens., Abhandl. Senckenb. Naturf. Ges., VI. 326. (1867) ;

Schiv., Novara Reise, Dipt., 67. 43. (1868); Willısт., Biolog.

Central. Amer., Dipt., I. 242. (1900); Hunter, Trans. Amer.

Entom. Soc. Philad., XXVII. 124. (1901).

vitripennis DoL., Natuurkund. Tijdschr. Nederl. Indie, n. ser. VII. Java.

(X). 408. 15. tab. XI. fig. 2. [Chrijsochloru] (1856); v. D. Wulp,

Catal. Dipt. South Asia, 48. (1896).

\section{Chromatopoda}

Brau., Denkschr. Akad. Wien, XLIV. 78. (1881).

bicolor MACQ., Dipt. cxot., suppl. 5., 44. 3. [Chrysochlora] (1855); Australia, Brav., Denkschr. Akad. Wien, XLIV. 78. (1881).

Ins. Tonga.

frontalis Thoms., Eugenies Resa, Dipt., 459. 24. [Chrysochlora] Taiti.

(1869) ; Brau., Donkschr. Akad. Wien, XliV. 78. (1881).

\section{Drasteria}

Brau., Denkschr. Akad. Wien, XLIV. 78. (1881) [praeocc. Héвs. Lep. 1816]. robusta BrAv., Denkschr."Akad. Wien, XLIV. 79. (1881).

Balia.

\section{Pelagomyia}

Willist., Manual of N. Amer. Dipt., Ed. II., 48. (1896).

albitalus WiLı.1sT., Trans. Entom. Soc. London, 1896. 299. 1. tab. I.St.Vincent, X. fig. 75. (1896) ; Hunter, Trans. Amer. Entom. Soc. Philad., I. Galapagos. XXVII. 124. (1901); Coquill., Proc. Washingt. Acad. Sci., III. 373. (1901) ; Aldr., Catal. North Amer. Dipt., 191. (1905). 


\section{Rhaphiocerinas.}

\section{Basentidema}

MacQ., Dipt. exot., I. 1., 197. 19. (1838).

syrphoides Macq., Dipt. exot., I. 1., 197. 1. tab. XXIV. fig. 3. Brasilia.

(1838) ; WaLK., List Dipt. Brit. Mus., V. suppl. 1., 85. 1. (185t);

Hunter, Trans. Amer. Entom. Soc. Philad., XXVII. 124. (1901).

\section{Hoplistes}

MacQ., Suit. à Buffon, I. 253. 9. (1834).

bispinosus WiEd, Aussereurop. zweifl. Ins., II. 32. 8. tab. VII. fig. 6. [Sargus] (1830); MACQ., Suit. à Buffon, I. 254. 1. (1834) et Dipt. exot., I. 1., 195. 1. tab. XXIII. fig. 2. (1838); WALK., List Dipt. Brit. Mus., V. suppl. 1., 76. 1. (1854); Hunter, Trans. Amer. Entom. Soc. Pliilad., XXVII. 122. [Sargus] et 123. (1901). hoplistes Wied., Aussereurop. zweifl. Ins., II. 30. 6. [Surgus] (1830). pomaceus Macq., Suit. à Buffon, I. 254. 3. (1834); Walk., List Dipt. Brit. Mus., V. suppl. 1., 76. 2. (1854).

hortulanus WIED., Aussereurop. zweifl. In-., II. 32. 9. [Sargus] (1830) ; MacQ., Suit. à Buffon, I. 254. 2. (1834); WALK., List Dipt. Brit. Mus., V. suppl. 1., 76. 3. (1854); Schin., Novara Reise, Dipt., 70. 47. (1868); Willist., Trans. Amer. Entom. Soc. Philad., XV. 251. 19. (1888); Hunter, Trans. Amer. Entom. Soc. Philad., XXVII. 122. [Sargus] et 123. (1901).

pomaceus MACQ. = hoplistes WIED.

\section{Rhaphiocera}

MacQ., Suit. à Buffon, I. 253. 8. (1834). [Raphiocera].

armata WiED., Aussereurop. zweifl. Ins., II. 29. 5. [Sargus] (1830); MACQ., Suit. à Buffon, I. 253. 1. (1834), Dipt. exot., I. 1., 196. 2. tab. XXIV. fig. 2. (1838) et suppl. 1., 54. (1846); Walk., List Dipt. Brit. Mus., V. suppl. 1., 78. 1. (1854); Schis., Novara Reise, Dipt., 70. 46.(1868); Willis'., Trans. Amer. Entom. Soc. Philad., XV. 251. 18. (1888) ; Hunter, Trans. Amer. Entom. Soc. Philad., XXVII. 122. [Sargus] et 123. (1901).

? Falléni Perty, Delect. animal. articul. Brasil., 184. tab. XXXVII. fig. 1. [Sargus] (1830-34); WaLK., List Dipt. Brit. Mus., V. suppl. 1., 78. 2. (1854); Hunter, Trans. Anier. Entom. Soc. Philad., XXVII. 122. [Sargus] (1901).

bipartita $\mathrm{W}_{\mathrm{ALK}}=$ mipartita $\mathrm{M}_{\mathrm{ACQ}}$.

? brevis Big., Annal. Soe. Entom. France, sér. 6. VII. 26. (1887). N. Caledonia. 
caloptera Ost.-Sack., Biolog. Centr. Amer., Dipt., I. 26. 2. (1886); Mexico. Aldr., Catal. North Amer. Dipt., 176. (1905).

Falléni Peuty = ? armata Wied.

mipartita Macq., Dipt. exot., suppl. 1., 54. 1. tab. VI. fig. 1. (1846); Columbia.

Hunter, Trans. Amer. Entom. Soc. Philad., XXVII. 123. (1901).

bipartita WaLK., List Dipt. Brit. Mus., V. suppl. 1., 79. 4. (1854).

ornata MACQ., Dipt. exot., suppl. 1., 55. 2. tab. VI. fig. 2. (1846);

WıLK., List Dipt. Brit. Mus., V. suppl. 1., 78. 3. (1854); Hunter,

Trans. Amer. Entom. Soc. Philad., XXVII. 123. (901).

pampina Ost.-SAck., Biolog. Central. Amer., Dipt., I. 25. 1. (1886). Panama. picta v. D. Wulp, Aninal. Soc. Entom. Belgique, XXII. Comp. Rend., Brasilia.

CLXI. [Rhapiocera] (1879).

\section{Dicranophora}

MacQ., Suit. à Buffon, I. 255. 10. (1834).

affinis Wıllıst., Trans. Amer. Entom. Soc. Philad., XV. 251. 16. Brasiiia.

(1888) ; Hunter, Trans. Amer. Entom. Soc. Philad., XXVII. 125.

(1901).

astuta Willist., Trans. Amer. Entom. Soc. Philad., XV. 250. 15. Brasilia.

(1888) ; Hunter, Trans. Amer. Entom. Soc. Philad., XXVII. 125. (1901).

furcifer WiEd., Analecta Entomol., 31. 40. tab., fig. 10. [Sargus] Brasilia. (1824) et Aussereurop. zweifl. Ins., II. 38. 20. tab. VII. fig. 5. [Sargus] (1830); MAcQ., Suit. à Buffon, I. 255. 1. (1834); Guḱr., Iconogr. d. Régne Anim., Ins., VII. 545. tab. XCVIII. fig. 12. (1835); MAcQ., Dipt. exot., I. 1., 195. 1. (1838) et suppl. 2., 31. (1847) ; WALK., List Dipt. Brit. Mus., V. suppl. 1, 77. 1 et 313. (1854); Hunter, Trans. Amer. Entom. Soc. Philad., XXVII. 125. (1901).

picta MAcQ., Suit. à Buffon, I. 255. 2. (1834) et Dipt. exot., I. 1., Brasilia. 196. 2. tab. XXIV. fig. 1. (1838) ; Walk., List Dipt. Brit. Mus., V. suppl. 1., 77. 2. (1854); Hunter, Trans. Amer. Entom. Soc. Philad., XXVII. 125. (1901).

\section{Histiodroma}

Scmun., Verh. zool.-bot. Ges. Wien, XVII. 308. (1867).

flaveola Big., Annal. Soc. Entom. France, sér. 5. IX. 205. (1879); Mexico. Aldr., Catal. North Amer. Dipt., 177. (1905).

inermis WiED., Aussereurop. zweifl. Ins., II. 31. 7. tab. VII. fig. 7. Brasilia. [Sargus] (1830); WaLk., List Dipt. Brit. Mus., V. suppl. 1., 79. 5. [Rhaphiocera] (185t); Lw., Verlı. zool.-bot. Ver. Wien, V. 141. [Raphiocera] (1855) ; Jaenn., Abhandl. Senckenb. Naturf. Ges., 
VI. 326. [Rhaphiocera] (1867); Schis., Novara Reise, Dipt., 70. 45. tab. II. fig. 8. (1868); Willist., Trans. Amer. Entom. Soc.' Philad., XV. 251. 17. (1888); Hunter, Trans. Amer. Entom. Soc. Philad., XXVII. 122. [Sargus] et 124. (1901).

\section{Sarginae.}

\section{Ptecticus}

Lw., Verh. zəol.-bot. Ver. Wien, V. 142. (1855).

Plecticus Lw., Verh. zool.-bot. Ver. Wien, V. 147. (1855).

affinis Schin., Novara Reise, Dipt., 65. 38. (1868); Willist., Trans. Americamer. Amer. Entom. Soc. Philad., XV. 248. 10. (1888); Hunter, Trans.

Amer. Entom. Soc. Philad., XXVII. 123. (1901).

apicalis Lw., Verh. zool.-bot. Ver. Wien, V. 142. tab., fig. 3-4.

(1855) ; v. D. Wucp, Catal. Dipt. Soutlı Asia, 49. (1896).

luridus Walk., Proc. Linn. Soc. Ivondon, I. 8. 19 et 110. 28. [Sargus] (1856) et Trans. Entom. Soc. London, n. ser. IV. 119. (1857).

apicalis v. D. WuLP, Notes Leyden Mus., VII. 62.10.(1885) et Catal. Dipt. South Asia, 50. (1896).

aurifer WaLk., List Dipt. Brit. Mus., V. suppl. 1., 96. 40. [Sargus] (1854) ; v. D. Wulp, Catal. Dipt. South A sia, 49. (1893) ; Coquill.; Proc. Unit. Stat. Nat. Mus., XXI. 308. [Sargus] (1898).

australis Schin., Novara Reise, Dipt., 65. 38. (1868); v. D. Wulp, Catal. Dipt. South Asia, 50. (1896).

brevipennis Rond., Annal. Mus. Civ. Genova, VII. 454. [? Sargus] (1875); v. D. Wulp, Notes Leyden Mus., VII. 63. 12. (1885) et Catal. Dipt. South Asia, 50. (1896).

cingulatus Lw., Verh. zool.-bot. Ver. Wien, V. 143. (1855); v. D. Pulo-Pinang. Wulp, Catal. Dipt. South Asia, 49. (1896).

complens Walk., Proc. Linn. Soc. London, III. 81. 15. [Sargus] (1859); Ins. Aru. v. D. Wulp, Catal. Dipt. South Asia, 49. (1896).

concinnus Willist., Biolog. Central. Amer., Dipt., I. 238. 7. (1900); Mexico. Aldr., Catal. Nortl Amer. Dipt., 177. (1905).

conopsoides Schin., Novara Reise, Dipt., 66. 42. (1868); Hunter, Columbia.

Trans. Amer. Entom. Soc. Philad., XXVII. 123. (1901).

cyanifrons Rond. in Truqui : Studi Entomol., I. 103. [Sargus] (1848); Gigl.-Tos, Mem. R. Accad. Sci. Nat. Torino, ser. 2. XLIII. 118. 23 (1893) ; Willist., Biolog. Central. Amer., Dipt., I. 237. 3. (1900); Aldr., Catal. North Amer. Dipt., 177. (1905).

testaceus MACQ. (nec FABR.), Dipt. exot., I. 1., 203. 5. [Sargiı] (1838) et suppl. 1., 57. [Sargus] (1846).

Doleschalii*) Brg., Annal. Soc. Entom. France, sér. 5. IX. 231. 2. Nova Guinea,

I. Mysol.

$\left.{ }^{*}\right)=$ ? rogans WALK.

Americamer.

et centr. 
[Plecticus] (1879) ; Ost.-Sack., Annal. Mus. Civ. Genova, XVI. 416. nota. (1882); v. D. Wulp, Catal. Dipt. South Asia, 50 (1896) et Természetrajzi Füzetek, XXI. 411. 7. (1898).

elongatus FAвr., Mantissa Insect., II. 348. 69. [Musca] (1787); Gмец., Systema Naturae, V. 2851. 220. [Musca] (1792); FABr., Entomol. System., IV. 338. 109. [Musca] (1794); Ourv., Encyclop. Méthod., VIII. 28. 92. [Musca] (1811); WALK., List Dipt. Brit. Mus., III. 517. [Sargus] (1849) et V. suppl. 1., 320. [Sargus] (1854); Lw., Verl.. zool.-bot. Ver. Wien, V. 142. (1855) et Öfver's. Kongl. Vet. Akad. Förhandl., XIII. 263. 1. (1856); Big. in Thomson: Arcluiv Entomol., II. 351. 658. [Sargus] (1858); Lw., Dipt.-Fauna Südafrika's, I. 6. 1. (1860).

ferrugineus Dol., Natuurkund. Tijdschr. Nederl. Indie, ser. 4. III. (XVII). 83. 13. [Sargus] (1858); v. D. Wulp, Catal. Dipt. South Asia, 48. [Sargus] (1896), Természetrajzi Füzetek, XXI. 410. 6. (1898) et Tijdschr. v. Entomul., XIII. 54. (1899).

figlinus Ost.-SAck., Biolog. Central. Amer., Dipt., I. 24. 1. (1886); Willıs'r., Biolog. Central. Amer., Dipt., I. 236. (1900) ; Aldr., Catal. North Amer. Dipt., 177. (1905).

flaviceps Bıg., Annal. Soc. Entom. France, sér. 5. IX. 230. 1. [Plecticus] (1879); Gigl.-Tos, Mem. R. Accad. Scienc. Nat. Torino, ser. 2. XIIII. 119. 24. (1893); Aldr., Catal. North Amer. Dipt., 177. (1905).

flavipennis MacQ., Dipt. exot., I. 1., 200. 1. [Sargus] (1838); WaLK., List Dipt. Brit. Mus., V. suppl. 1., 95. 35. [Sargus] (1854); BıG., Annal. Soc. Entom. Jirance, sér. 3. III. 131. [Sargus] (1859) et sér. 5. IX. 186. [Plecticus] (1879).

flavipes Lw., Dipt.-Fauna Siidafrika's, I. 5. 2. [Ptecticus] (1860). lapsus.

flavipes $\mathrm{Lw} .=$ flavipennis $\mathrm{MACQ}$.

illucens Schrn., Noval'a Reise, Dipt., 65. 40. (1868); v. D. Wulp, Notes Leyden Mus., VII. 63. 11. (1885) et Catal. Dipt. South Asia, 50. (1896).

illustris Schin., Novara Reise, Dipt., 64. 37. (1868); Hunter, Trans. Amer. Entom. Soc. Philad., XXVII. 123. (1901).

insignis MAcQ., Dipt. exot., suppl. 5., 46. 14. [Sargus] (1855) ; Big., Annal. Soc. Entom. France, sér. 5. IX. 186. [Plecticus] (1879).

latifascia WALk., Proc. Linı. Soc. London, I. 110. 28. [Sargıs] (1857); v. D. Wulp, Notes Leyden Mus., VII. 64. 13. (1885) et Catal. Dipt. South Asia, 49. (1896).

leoninus Rovd., Annal. Mus. Civ. Genova, VII. 454. [Sargus] (1875); Borneo. v. D. Wul. Catal. Dipt. South Asia, 50. (1896).

luridus $\mathrm{W}_{\mathrm{ALK}}$ = apicalis $\mathrm{Lw}$.

maculatus Willist., Biolog. Central. Amer., Dipt., I. 238. 6. (1900); Mexico. Aldr., Catal. North Amer. I)ipt., 177. (1905).

melanurus WALK., List Dipt. B.it. Mus., I. 78. [Clcnophora] (1848); Nepal, Ost.-SAck., Borliı. Entom. Zuitschr., XXX. 166. [Sargus] (1886); Bengalia. 
v. D. WulP, Tijdschr. v. Entomül, XXVIII. 81. (1884) et Catal. Dipt. South Asia, 38. nota. [Sargus] (1896); Mes., Bijdragen tot de Dierkunde, XVIII. 95. (1904).

nitidipennis Lw., Verh. z.ool.-bot. Ver. Wien, V. 143. (1855); WILList., Biolog. Central. Amer., Dipt., I. 237.5. (1900); Aldr., Catal. North Amer. Dipt., 177. (1905).

pallidus Macq., Dipt. exot., I. 1., 202. 3. [Sargus] (1838); WaI.K., List Dipt. Brit. Mus., V. suppl. 1., 95. 34. [Sargus] (1854); Lww., Dipt.-Fauna Südafrika's, I. 5. 3. (1860).

pomaceus Lw., Verh. zool.-bot. Ver. Wien, V. 144. (1855).

posticus Wied., Ausscreurop. zweifl. Ins., II. 34. 12. [Sargus] (1830) ; WaLk., List Dipt. Brit. Mus., V. suppl. 1., 94.31. [Sargus] (1854); Bıg., Annal. Soc. Entom. France, sér. 5. IX. 186. [Plectirus] (1879).

quadrifasciatus Walk., Proc. Iinn. Soc. London, V. 146. 8, 274. 15. [Sargus] (1861) et VII. 205. 14. [Sargus] (1864); v. D. Wurs, Sumatra Exped., Dipt., 15. 1. [Sargus] (1881); Osт.-SAck., Annal. Mus. Civ. Genova, XVI. 416. [Sargus] (1882); v. D. Wulp, Catal. Dipt. South Asia, 49. (1896).

remeans Walk., Proc. Linn. Soc. London, IV. 96. 20. [Sargus] (1860) ; Ost.-Sack., Annal. Mus. Civ. Genova, XVI. 416 et 417. [Sargus] (1882); v. D. Wulp, Catal. Dipt. South Asia, 49. (1896). repensans WaLk., Proc. Linn. Soc. London, IV. 96. 19. [Sargus] (1860) ; Ost.-Sack., Annal. Mus. Civ. Genova, XVI. 416. [Sargus] (1882); v. D. Wulp, Catal. Dipt. South Asia, 49. (1896).

rogans WALK., Proc. Linn. Soc. London, III. 81. 16. [Sargus] (1859); Ost.-Suck., Annal. Mus. Civ. Genova, XVI. 416. [Sargus] (1882); v. D. Wulp, Catal. Dipt. South Asia, 49. (1896).

rufus Dol., Natuurkund. Tijdschr. Nederl. Indie, ser. 4. III. (XVII). 83. 12. [Sargus] (1858); v. D. Wulp, Catal. Dipt. South Asia, 48. [Sargus] (1896).

rufescens v. D. Wulp, Tijdschr. v. Entomol., ser. 2. IJI. (XI). 104. 7. tab. III. fig. 7-9. [Sargus] (1868) et Catal. Dipt. South Asia, 50. (1896).

Sackenii Wıllist., Canad. Entomol., XVII. 124. (1885) et Biolog. Central. Amer., Dipt., I. 237. 4. (1900) ; Meland., Canad. Entomol., XXXVI. 19. (1904); Aldr., Catal. Nortl Amer. Dipt., 177. (1905).

testaceus Osт.-Sıck., Catal. Dipt. Nortlı Amer., 45. (18i8); E. L. Arrib., Bolet. Acad. Nat. Cienc. Córdoba, IV. 125. 42. (1882).

similis WILLIST. $=$ trivittatus SAY.

tarsalis WALK., Proc. Linn. Soc. London, V. 274. 16. [Sargus] (1861) et VI. 4. 5. [Sargus] (1862) ; v. D. Wulp, Catal. Dipt. South Asia, 50. (1896).

tenebrifer WALK., I.ist Dipt. Brit. Mus., III. 517. [Sargus] (1849) et V. suppl. 1., 95. 37 et 320. [Sargus] (1854); MacQ., Dipt. exot.,
Americacent.

Madagascar.

Chile.

Africa mer.

Asia mer., Arch.Indicus, Nova Guinea.

Celebes.

Celebes.

Nova Guinea, Ins. Aru.

Amboina.

I. Waigou.

America sept. et centr.

Arch.Indicus

Japonia,

China. 
suppl. 5., 45. 13. [Sargus] (1855); Big., Annal. Soc. Entom. France, sér. 5. IX. 187. [Macrosargus] (1879); v. D. Wulp, Notes Leyden Mus., VII. 63. (1885) et Catal. Dipt. South Asia, 49. (1896) ; Coquilı., Proc. Unit. Stat. Nat. Mus., XXI. 308. [Sargus] (1898).

tenuis Schin., Novara Reise, Dipt., 66. 41. (1868); Hunter, Trans. Columbia. Amer. Entom. Soc. Philad., XXVII. 123. (1901).

testaceus Fabr., Systema Antliat., 257. 6. [Sargus] (1805); Wied., Aussereurop. zweifl. Ins., II. 35. 15. [Sargus] (1830); Rovd. in Truqui : Studi Entomol., I. 103. 75. [Sargus] (1848); WaLK., List Dipt. Brit. Mus., III 517. [Sargus] (1849) et V. suppl. 1., 94. 29. [Sargus] (1854); Lw., Verh. zool.-bot. Ver. Wien, V. 142. (1855) ; Bell., Saggio di Ditterol. Messic., I. 45. 10. [Sargus] (1859) ; Lw., Dipt.-Fauna Südafrika's, I. 6. Anmerk. (1860) ; Scurv., Novara Reise, Dipt., 64. 36. (1868); Big., Annal. Soc. Entom. France, sér. 5. IX. 186. [Plecticus] (1879); v. D. WulP, Tijdsøhr. v. Entomol., XXIV. 156. 11. (1881); GigL.-Tos, Mem. R. Accad. Sci. Nat. Torino, ser. 2. XLIII. 118. 23. (1893); WILlisT., Biolog. Central. Amer., Dipt., I. 236. 2. (1900); Hunter, Trans. Amer. Entom. Soc. Philad., XXVII. 123. [Sargus ét Ptecticus] (1901); Aldr., Catal. North Amer. Dipt., 177. (1905).

testaceus MAcQ. (nec FABR.) = cyanifrons RoND. testaceus Ost.-SAck. (nec FABR.) = Sackenii Willist.

tricolor v. D. Wulp, (descriptio) apud Meis., Bijdrag tot de Dierkunde, Java.

XVIII. 95. tab. VIII. fig. 11. (1904).

trivittatus Gıal.'Tos, Bollet. Mus. Zool. ed Anat. comp. Torino, VI. Mexieo.

No. 102., 3. (1891) et Mem. R. Accad. Sci. Nat. Torino, ser. 2. XLIII. 120. 25. tab. I. fig. 8. (1893); Audr., Catal. North Amer. Dipt., 177. (1905).

trivittatus SAy, Journ. Acad. Nat. Sci. Philad., VI. 159. [Sargus] (1829) et Compl. Writt., II. 355. [Sargus] (1859); WaLk., List Dipt. Brit. Mus., V. suppl. 1., 90. 13. [? Sargus] (1854); Wilus'r., Entomol. Amer., I. 154. [? Ptecticus] (1885); Hine, Ohio Naturalist, I. 112. (1901); Meland., Canad. Entomol., XXXVI. 19. (1904); Aldr., Catal. North Amer. Dipt., 177. (1905).

similis Willist., Canad. Entomol., XVII. 124. (1885).

\section{Merosargus}

Lw., Verh. zool.-bot. Ver. Wien, V. 144. (1855).

antennatus Schin., Novara Reise, Dipt., 63. 33. (1868); Hunter, Trans. Amer. Entom. Soc. Philad., XXVII. 125 (1901).

bituberculatus Scuin., Novara Reise, Dipt., 64. 35.(1868); Hunter, Americamer. Trans. Amer. Entom. Soc. Philad., XXVII. 125. (1901).

bulbifrons Willist., Biolog. Centr. Amer., Dipt., I. 235. tab. IV. Mexico. fig. 8, 8a. (1900); Aldr., Catal. North Amer. Dipt., 177. (1905). 
calceolatus Big., Annal. Soc. Entom. France, sér. 5. IX. 229. 2. Mexico. (1879) ; Aldr., Catal. North Amer. Dipt., 177. (1905).

cingulatus Schis. $=$ stamineus FaBr.

concinnatus Wilurst., Biolog. Central. Amer., Dipt., I. 235. 4. tab. Mexico.

IV. fig. 9. (1900); Aldr., Catal. North Amer. Dipt., 178. (1905).

coriaceus Gigl.-Tos, Bollet. Mus. Zool. ed Anat. comp. Torino, Mexico.

VI. No. 102., 4. (1891) et Mem. R. Accad. Scienc. Nat. Torino, ser. 2. XLIII. 117. 22. (1893) ; AJ.Dr., Catal. North Amer. Dipt., 178. (1905).

dissimilis Gigl.-Tos, Bollet. Mus. Zool. ed Anat. comp. Torino, VI. Mexico.

No. 102., 3. (1891) et Mem. R. Accad. Scienc. Nat. Torino, ser.

2. XliII. 116. 20. tab. I. fig. 7. (1893); Aldr., Catal. North Amer., Dipt., 178. (1905).

fasciatus FA3r., Systema Antliat., 259. 15. [Sargus] (1805); WiED., Aussereurop. zweifl. Ins., II. 33. 10. [Sargus] (1830); WALK., List Dipt. Brit. Mus., V. suppl. 1., 93. 25. [Sargus] (1854) ; Lw., Verh. zool.-bot. Ver. Wien, V. 144. (1855); Hunter, Trans. Amer. Entom. Soc. Philad., XXVII. 122. [Surgus] (1901).

festivus Willist., Trans. Amer. Entom. Soc. Philad., XV. 250. 14. Brasilia. (1888); Hunter, Trans. Amer. Entom. Soc. Philad., XXVII. 125. (1901).

fraternus Big., Annal. Soc. Entom. France, sér. 5. IX. 228. 1. Mexico. (1879) ; Aldr., Catal. North Amer. Dipt., 178. (1905).

frontatus Schiv., Novara Reise, Dipt., 63. 34. (1868); Huvrer, Trans. Columbia. Amer. Entom. Soc. Philad,, XXVII. 12.5. (1901).

gracilis Willist., Trans. Amer. Entom. Soc. Philad., XV. 249. 13. Brasilia. (1888) ; Hunter, Trans. Amer. Entom. Soc. Philad., XXVII. 125. (1901).

hyalopterus Gigl.-Tos, Bullet. Mus. Zool. ed Anat. comp. Torino, VI. No. 102., 3. (1891) et Mem. R. Accad. Sci. Torino, ser. 2. XLIII. 115. 19. (1893); Audr., Catal. North Amer. Dipt., 178. (1905).

luridus Lw., Verh. zool.-bot. Ver. Wien, V. 145. (1855).

nigrifemoratus Macq., Dipt. exot., suppl. 2., 31. 10. [Sargus] (18t7);

Venezuela. WaLk., List Dipt. Brit. Mus., V. suppl. 1., 90. 12. [Sargus] (1854); Bell., Saggio di Ditterol. Messic., I. 42. 5. [Sargus] (1859); Big., Annal. Soc. Eitom. France, sér. 5. IX. 186. (1879); Meland., Canad. Entomol., XXXVI. 18. [Sargus] (1904).

obscurus Wred., Aussereurop. zweifl. Ins., II. 35. 14. [Sargus] (1830); Walk., List Dipt. Brit. Mus., V. suppl. 1., 94. 27. [Sargus] (1854); Lw., Verh. zool.-bot. Ver. Wien, V. 144. (1855); Hunter, Trans. Amer. Entom. Soc. Philad., XXVII. 122. [Sargus] (1901).

Orizabae Gigl.-Tos, Bollet. Mus. Zool. ed Anat. comp. Torino, VI. No. 102., 3. (1891) et Mem. R. Accad. Sci. Nat. Torino, ser. 2. XLIII. 116. 21. (1893); Aldn., Catal. North Amer. Dipt., 178. $(1905)$.

petiolatus Macq., Dipt. exot., I. 1., 202. 4. [Sargus] (1838); WalK., Brasilia. Kertész: Catalogus Dipterorum. III. 
List Dipt. Brit. Mus., V. suppl. 1., 94. 28. [Sargus] (1854) ; Schin., Novara Reise, Dipt., 64. (1868); Hunter, Trans. Amer. Entom. Soc. Philad., XXVII. 122. [Sargus] (1901).

spatulatus Willist., Biolog. Central. Amer., Dipt., I. 235. 2. (1900); Mexico.

Aldr., Catal. North Amer. Dipt., 178. (1905).

stamineus FABR., Systema Antliat., 253. 19. [Scacva] (1805); Wien., Analecta Entomol., 37. nota. [Sargus] (1824) et Aussereurop. zweifl. Ins., II. 39. 21. [Sargus] (1830); WaLK., List Dipt. Brit. Mus., III. 517. [Sargus] (1849) et V. suppl. 1., 92. 19 et 319. [Sargus] (1854); Ber.r., Saggio di Ditterol. Messic., I. 44. 8. [Sargus] (1859); Big., Annal. Soc. Entoin. France, sér. 5. IX. 187. [Macrosargus] (1879); Wilusst., Biolog. Central. Amer., Dipt., I. 234. 1. (1900); Huntel, Trans. Amer. Entom. Soc. Philad., XXVII. 122. [Sargus] (1901); Meland., Canad. Entomol., XXXVI. 18. [Sargus] (1904).

cingulatus Scurv., Novara Reise, Dipt., 62. 32. (1868); GigL.Tos, Mem. R. Accad. Sci. Nat. Torino, ser. 2. XIIII. 114. 17. (1893); Hunter, Trans. Amer. Entom. Soc. Philad., XXViI. 125. (1901); Aldr., Catal. North Aner. Dipt., 177. (1905).

subinterruptus Bes.., Saggio di Ditterol. Messic., I. 44. 9. tab. I. fig. 22. [Sargus] (1859); ? WaLk., Trans. Entom. Soc. London, n. ser. V. 271. [Sargus] (1860); Gigl.-Tos, Mem. R. Accad. Sci. Nat. Torino, ser 2. XLIII. 115. 18. (1893); Muland., Canad. Entomol., XXXVI. 16. nota. [Ptecticus] (1904); Aldr., Catal. North Amer. Dipt., 178. (1905).

tristis Lw., Verh. zool.-bot. Ver. Wien, V. 145. (1855).

America mor. et centr.

Mexico.

Venezuela.

\section{Compsosoma}

Brau., Denkschr. Akad. Wien, XLIV. 79. (1881) [pracocc. Serv., Col.].

chalconota Brau., Denkschr. Akad. Wien, XLIV. 79. (1881).

Venezuela.

picta Brau., Denkschr. Akad. Wien, XLIV. 80. (1881).

Venezuela.

\section{Chrysochroma}

WiLl., Manual of North Amer. Dipt., Ed. II., 47. (1896).

Chrysonotomyia Hunter, 'Trans. Amer. Entom. Soc. Philad., XXVII. 124. (1901).

Chrysonotus Lw., Verh. zool.-bot. Ver. Wien, V. 144. (1855)

[nec Swains, nec Amr.].

aeneiventre Grgl.-Tos, Mem. R. Accad. Sci. Nat. Torino, ser. 2. Mexico. XLIII. 121. 27. [Chrysonotus] (1893); Aldr., Catal. North Amer. Dipt., 178. (1905). 
albipes Adams, Kansas Univ. Sci. Bullet, II. 31. (1903); Aldr., Mexico.

Catal. North Amer. Dipt., 178. (1905).

anale Willist., Trans. Amer. Entom. Soc. Philad., XV. 251. 20. Americamer. [Chrysonotus] (1888); Gigl.-Tos, Mem. R. Accad. Sci. Nat. Torino, ser. 2. XLIII. 120. 26. [Chrysonotus] (1893); Hunter, Trans. Amer. Entom. Soc. Philad., XXVII. 124. [Chrysonotomyia] (1901); Aldr., Catal. North Amer. Dipt., 178. (1905).

bipunctatum O. G. CostA = bipunctatum Scop.

bipunctatum Scop., Entomol. Carniol., 341. 916. [Musca] (1763); Europacentr. WaLk., Ins. Britannica, Dipt., I. 32. 5. [Sargus] (1851) et List et mer. Dipt. Brit. Mus., V. suppl. 1., 89. 7. [Sargus] (1854); Lw., Verh. zool.-bot. Ver. Wien, V. 137. [Sargus] et 146. [Chrysonotus] (1855); Schis., Verh. zool.-bot. Ver. Wien, V. 645. 1 et 673. [Chrysonotus] (1855) et VI. 414. 916. [Sargus] (1856) et Fauna Austriaca, Dipt., I. 21. 1. [Sargus] (1862); JAENs., Berlin. Entom. Zeitschr., X. 232. 43. [Sargus] (1866); v. D. Wulp, Diptera Neerlandica, I. 461. 2. tab. XIV. fig. 20. [Sargus] (1877); Brunetri, The Entomologist, XXII. 132. 1. [Chrysonotus] (1889); ScHoch, Fauna Ins. Helvetiae, Dipt., 25. [Sargus] (1890); É. \& L. Coucke, Annal. Soc. Entom. Belgique, XXXVII. 441. [Sargus] (1893); Strobl, Mittheil. Naturwiss. Verein Steiermark, XXIX. 1902. 441. [Sargus] (1893) ; Ken'., Pótfüz. a Természettud. Közlönyhöz, XXXII. 129. [Chrysonotus] (1900).

bipunctatum O. G. Costa, Atti Accad. Sc. Napoli, V. 2., 88. 6. tab. II. fig. 4. [Sargus] (1844).

Réaumuri MeIG., Klassif., I. 142. 1. [Sargus] (1804); Faвr., Systema Antliat., 256. 2. [Sargus] (1805); Meig., System. Beschreib., III. 109. 6. [Sargus] (1822); MAcQ., Recueil Soc. Sc. Agricult. Lille, 429. 6. [Sargus] (1826); Curr., Brit.Entomol., VIII. 305. tab. [Sargus] (1830); MAcQ., Suit. à Buffon, I. 262. 6. tab.VI. fig. 12.[Sargus] (1834); GuĖr., Iconogr. d. Régne Anim., VII. Ins., 545. tab. XCVIII. fig. 9. [Sargus] (1835); Duncan, Magaz. Zool. and Botany, I. 163. 5. fig. 1-3. [Sargus]; WALK., List Dipt. Brit. Mus., III. 519. [Sargus] (1819).

calopus Big., Annal. Soc. Entom. France, sér. 5. IX. 227. 1. [Chrysonotus] (1879).

dichroum Schın., Novara Reise, Dipt., 62. 31. [Chrysonotus] (1868) ; Hunter, Trans. Amer. Entom. Soc. Philad., XXVII. 124. [Chrysonotomyia] (1901).

flavomarginatum Lw., Öfvers. Kongl. Vet. Akad. Förhandl., XIII. 263. 2. [Chrysonotus] (1856) et Dipt.-Fauna Südafrika`s, I. 6. 1. [Chrysonotus] (1860).

flavopilosum Big., Annal. Soc. Entom. France, sér. 5. IX. 227. 2. Mexico. [Chrysonotus] (1879); Willist., Biolog. Central. Amer., Dipt., I. 233. 2. (1900); Aldr., Catal. North Amer. Dipt., 178. (1905).

fulvithorax Bra., Annal. Soc. Entom. France, sér. 5. IX. 228. 3. Amazonia. [Chrysonotus] (1879); Hunter, Trans. Amer. Entom. Soc. Philad., XXVII. 124. [Chrysonotomyia] (1901). 
Hovas Bıg., Anual. Soc. Entom. France, sér. 3. VII. 133. [Sargus] Madagascar. (1859) et sér. 5. IX. 187. [Chrysonotus] (1879).

latifrons Willist., Biolog. Central. Amer., Dipt., I. 234. 3. (1900); Mexico. Aldr., Catal. North Amer. Dipt., 178. (1905).

nigricorne Lw., Berlin. Entom. Zeitschr., X. 9. 14. [Chrysonotus] (1866) ; Hine, Ohio Natural., I. 114. (1901); Aldp., Catal. North Aner. Dipt., 178. (1905).

pulchrum Willist., Biolog. Central. Amer., Dipt., I. 233. 1. (1900); Mexico. Aldr., Catal. North Amer. Dipt., 178. (1905).

Réaumuri MEig. = bipunctatum Scop.

ruficorne MAcQ., Dipt. exot., suppl. 1., 55. 8. [Sargus] (1846); WaLk., List Dipt. Brit. Mus., V. suppl. 1., 95. 32. [Sargus] (1854) ; Lw., Dipt., Fanna Südafrika's, I. 5. [Chrysonotus] (1860).

splendens Scus., Novara Reise, Dipt., 62. 30. [Chrysonotus] (1868); Hunter, Tıans. Amer. Entom. Soc. Philad., XXVII. 124. [Chrysonotomyia] (1901).

sulphureum Meig., System. Beschreib., III. 109. 7. [Sargus] (1822);

Walk., List Dipt. Brit. Mus., V. suppl. 1., 89. 8. [Sargus] (1854); Lw., Verl. zool.-bot. Ver. Wien, V. 137. [Sargus] (1855). viridiceps MAcQ., Dipt. exot., suppl. 5., 46. 16. [Sargus] (1855).

Caffraria.

Americamer.

Patria ignota.

China bor.

\section{Sargus}

FABR., Suppl. Entomol. Syst., 549. (1798).

affinis BIG., Thomson. Archiv. Entom., II. 351. 658. (1858).

albibarbus Lw., Verh. zool.-bot. Ver. Wien, V. 13ł. (1855) ; Schin., Verh. zool.-bot. Ver. Wien, V. 649. 14 et 673. (1855) et Fauna Austr., Dipt., I. 22. (1862).

analis MAcQ., Dipt. exot., I. 1., 204. 7. (1838); WALk., Iist Dipt. Brit. Patriaignota. Mus., V. suppl. 1., 97. 40. (1854).

angustifrons Isw., Verh. zool.-bot. Ver. Wien, V. 134. (185i); Schus., Verh. zool.-bot. Ver. Wien, V. 649. 13 et 673. (1855) et Fauna Austr., Dipt., I. 21. nota. (1862).

auratus Meig. 1804. (nec FABR.) =iridatus Scop.

australis Big., Annal. Soc. Entom. France, sér. 3. VII. 132. tab. Madagascar. IX. fig. 3. (1859).

Bagosas Walk., List Dipt. Brit. Mus., III. 518. (1849) et V. suppl. 1., 90. 15.(1854); Meland., Canad. Entomol., XXXVI. 17.(1904); Aldr., Catal. North Amer. Dipt., 178. (1905).

brasiliensis WieD., Aussereurop. zweifl. Ins., II. 37. 18. (1830); Walk., List Dipt. Brit. Mus., V. suppl. 1., 91. 17. (18i4); HuxTER, Trans. Amer. Entom. Soc. Philad., XXVII. 122. (1901).

ceriferus JaEns., Berlin. Entom. Zeitschr., X. 232. 47. (1866); Lw., Italia.

Verh. zool.-bot. Ver. Wien, V. 135. [speçies innominata] (1855). Chrysis Lw., Verh. zool.-bot. Vor. Wien, V. 136. (1855) et Dipt.- Nubia. Fauna Südafrika's, I. 5. (1860). 
claviventris Rond., Nuov. Annal. Scienc. Nat. Bologna, ser. 3. II. 190. 26. (1850); Hunter, Trans. Amer. Entom. Soc. Philad., XXVIY. 12:. (1901).

coarctatus MACQ., Dipt. exot., I. 1., 203. 6. tab. XXV. fig. 2. (1838); WaLK., Iist Dipt. Brit. Mus., V. suppl. 1., 92. 21. (1854); W1LList., Trans. Amer. Entom. Soc. Philad., XV. 249. 12. (1883); Gigl.-Tos, Mem. R. Accad. Scienc. Nat. Torino, ser. 2. XliII. 122. 28. (1893); Willist., Biolog. Centr. Amer., Dipt., I. 231. 3. (1900) ; Hunter, Trans. Amer. Entom. Soc. Philad., XXVII. 122. (1901); Meland., Canad. Entomol., XXXVI. 16. [Macrosargus] (1904) ; Aldpr., Catal. North Amer. Dipt., 179. (1905).

? notatus Wred., Aussereurop. zweifl. Ins., II. 34. 13. (1830); Walk., List Dipt. Brit. Mus., V. suppl. 1., 91. 18. (1854); Hunter, Trans. Amer. Entom. Soc. Philad., XXVII. 122. (1901).

cocrulcicollis MeIG. $=$ cuprarius $\mathrm{I}$.

coeruleifrons Johns., Entom. News Philad., XI. 325. (1900) ; Meland., Canad. Entomol., XXXVI. 17. (1904); Aldr., Catal. North Amer. Dipt., 178. (1905).

coevulescens VILLFRS = iridatus ScOP.

concinnus Ost.-Sack., Biolog. Central. Amer., Dipt., I. 23. 2. (1886); Willist., Trans. Amer. Entom. Soc. Philad., XV. 248. (1888); Meland., Canad. Entomol., XXXVI. 18. (1904); Alor., Catal. North Amer. Dipt., 179. (1905).

concisus WALK., Proc. Linn. Soc. London, V. 273. 18. (1861); v. D. Wulp, Catal. Dipt. South Asia, 49. (1896).

contractus WALK., List Dipt. Brit. Mus., V. suppl. 1., 319. (1854). cuprarius FALL. p p. (nec L.) = iridatus Scop.

cuprarius L., Systema Naturae, Ed. X., 598. 71. [Musca] (1758);

Pona, Ins. Mus. Graec., 115. 11. [Musca] (1761); L., Fauna Suec., Ed. Alt., 456. 1853. [Musca] (1761); O. F. Mút.., Fauna Ins. Fridrichsdal., 83. 740. [Musca] (1764); L., Systema Naturae, Ed. XII., II. 994. 92. [Musca] (1767); FABr., Systema Entomolog., 781. 39. [Musca] (1775); Deg., Mém. pour serv. l'hist. d. Ins., VI. 200. 16. tab. XII. fig. 4-5. [Nemotelus] (1776); O. F. Múll., Zoolog. Dan. Prodr., 172. 2023. [Musca] (1776); Fabr., Species Insect., II. 446. 52. [Musca] (1781); Schrank, Enum. Ins. Austr., 465. 944. [Musca] (1781); FABR., Mantissa Insect., II. 347. 62. [Musca] (1787); Rossi, Fauna Etrusca, II. 309. 1513. [Musca] (1790); GmeL., Systema Naturae, V. 2849. 92. [Musca] (1792) ; Farr., Entomol. System., IV. 335. 96. [Musca] (1794) et Supplem. Entom. System., 566. 1. (1798); Schraxk, Fauma Boica, III. 111. 2422. [Musca] (1803); Schellexb., Gattungen d. Fliegen, 64 et 65. tab. XV. fig. 1. (1803); Meig., Klassif., I. 143. 3. (1804); Latr., Hist. Nat. d. Crust. et d. Ins., XIV. 343. 3. (1804); Fabr., Systema Antliat., 256. 3. (1805); Rossi, Fauna Etrusea, Ed. II., 471. 1513. [Musca] (1807); Latr., Gen. Crust. ot Ins., IV. 278.
Venezuela.

America mer. et centr.

Americasept.

America mer. et centr.

Batjan.

Brasilia.

Europa, A merica sept. 
(1809) et Consid. génér., 442. (1810); Ouıv., Encyclop. Méthod., VIII. 27. 85. [Musca] (1811); LAM., Hist. nat. anim. sans vert., III. 388. 2. [Oxycera] (1816) ; Cuv., Règne Animal., III. 624. (1817); FAll., Dipt. Suec., Stratiom., 13. 2. ऊ. (1817); Mig., System. Beschreib., III. 106. 1. (1822); Dumér., Consid. génér., Ins., tab. L. fig. 8. (1823) ; Sт. FAng. Ет SErv., Encyclop. Méthod., X. 340. 2. (1825); MacQ., Recueil Soe. Sci. Agricult. Lille, 1826. 427. 1. tab. II. fig. 6. (1826); Cunt., Brit. Entomol., VIII. 305., 2. 1. (1830); Lyonn., Mém. posth., tab. XVII. fig. 21, 24-29. (1832) ; Boucué, Naturgesch. d. Ins., I. 48. 31. tab. IV. fig. 31--36. (1834) : MacQ., Suit. à Buffon, I. 260. 1. (1834); Grm., Bull. Soc. Imp. Nat. Moscou, VII. 109. tab. I. fig. 10-11. (1834); Duncan, Magaz. Zool. and Botany, I. 161. 1. (1837); ZeTT., Insecta Lappon., Dipt., 576. 1. (1838); Lw., Programm Posen, 1840. 23. 1. (1840) et Isis, 1840. VII. 553. 1. (1840); Westw., Introd., II. 533. (1840); Zetr., Dipt. Scand., I. 157. 1. (1842); L. Duf., Compt. Rent. Acad. Sci. Paris, XXII. 318. (1846); WAlK., List Dipt. Brit. Mus., III. 518. (1849); ZETT., Dipt. Scand., VIII. 2963. 1. (1849); I. Duv., Mém. Acad. des. Scienc., Math. et Phys., XI. 171-360. tab. IV. fig. 46. (1850); Halln., Stettin. Entom. Zeitg., XI. 136. (1851); WALK., Insecta Britann., Dipt., I. 30. 1. tab. I. fig. 9. (1851) et Iist Dipt. Brit. Mus., V. suppl. 1., 87. 1. (1854); Lw., Verh. zool.-bot. Ver. Wien, V. 131. (1855); Schrs., Verll. zool.-bot. Ver. Wien, V. 643. 3 et 673. (1855) et VI. 403. 11 et 414. 911. (1856); Iw., Zeitschr. f. d. ges. Naturwiss., X. 99. 5. (1857) ; BonsD., Finland traving. Ins., I. 119. 1. (1861); Schin., Fauna Austriaca, Dipt., I. 21.4. (1862); Jaenv., Perlin. Entom. Zeitschr., X. 232. 45. (1866); v. D. Wul.P, Diptera Neerland., I. 460. 1. tab. XIV. fig. 17-19. (1877); Beling, Archiv f. Naturgesch., XLVIII. 1., 187. 1. (1882); Becher, Denkschr. Akad. Wien, XLV. 140. tab. II. fig. 7. (1882); ? BraU., Denkschr. Akad. Wien, XLVII. - t tab. II. fig. 24. (1883); Neunaus, Diptera Marchica, 77. 1. (1886) ; Brunetri, The Entomologist, XXII. 132. 1. (1889); Sсносн, Fauna Insect. Helvetiae, Dipt., 25. (1890) ; É. \& L. Coucke, Annal. Soc. Entom. Belgique, XXXVII. 442. (1893); StroвL, Mittheil. Naturwiss. Ver. Steiermark, XXIX. 1892. 10. (1893); KerT., Pótfüz. a Természettud. Közlönyhöz, XXXII. 129. (1900); Meland., Canad. Entomol., XXXVI. 18 et 19. 2. (1904); Aldr., Catal. North Amer. Dipt., 179. (1905).

coeruleicollis MEIa., System. Beschreib., III. 107. 2. (1822);

MacQ., Recueil Soc. Sc. Agricult. Lille, 1826. 428. 3. (1826);

WalK., List. Dipt. Brit. Mus., V. suppl. 1., 88. 2. (1854); Lw., Verh. zool.-bot. Ver. Wien, V. 132. (1855); Scmin., Verh. zool.-bot. Ver. Wien, V. 647. 5 et 673. (1855), politus Schrank, Fauna Boica, III. 99. 2394. [Rhagio] (1803). violaceus Scop., Entomol. Carniol., 340. 915. [Musca] (1763); 
Schin., Verh. zool.-bot. Vor. Wien, VI. 414. 915. [Chrysomyia] (1856).

var. gracilis Lw., Programm Posen, 1810. 24. 3. (1840) et Europacentr. Isis, 1840. VII. 553. 3. (1840).

var. nubecolosus ZETT., Dipt. Scand., I. 157. 2. (1842) et VIII. Europacentr. 2963. 2. (1849); Walk., Insecta Britann., Dipt., I. 31. 2. (1851) et List Dipt. Brit. Mus., V. suppl. 1., 88. 4. (1854); Lw., Verh. zool.-bot. Ver. Wien, V. 131. (1855); Scuin., Verh. zool.-bot. Ver. Wien, V. 647. 4 et 673. (1855); Bonsd., Finl. tvaving. Ins., I. 120. 2. (1861); Scnis., Fauna Austriaca, Dipt., I. 21. 4. (1862); Jaens., Beilin. Entom. Zeitschr., X. 232. 46. (1866); Sсносн, Fauna Ins. Helvetiae, Dipt., 25. (1890) ; Strobl, Mittheil. Naturwiss. Ver. Steiermark, XXIX. 1892. 11. (1893); Johss., Entom. News Philad., VII. 9 Ł. (1896); KerT., Pótfüz. a Természettud. Közlönyhöz, XXXII. 129. (1900); ALDr., Catal. North Amer. Dipt., 179. (1905).

var. robustus Lw., Programm Posen, 1840. 23. 2. (1840) et Europacentr. Isis, 1840. VII. 553. 2. (1840).

debilis WaLk., Insecta Saund., Dipt., I. 83. (1851) et List Dipt. Brit. Mus., V. suppl. 1., 89. 9. (18.54); Meland., Canad. Entomol., XXXVI. 17.(1904); Aldr., Catal. North Amer. Dipt., 179. (1905). debilis WaLk., Proc. Linn. Soc. London, V. 274. 17. (1861); v. D. Wulp, Catal. Dipt. South Asia, 49. (1896).

decorus SAy, Long's Exped. to St. Peters River, II. Append., 376.

1. (1824) et Compl. Wr., I. 257. 1. (1859); WiEd., Aussereurop. zweifl. Ins., II. 38. 19. (1830) ; WALK., List Dipt. Brit. Mus., V. suppl.1., 89.10. (1854); v. c. WulP, Tijdschr. v. Entomol., XXIV. 156. 8. (1881); Willist., Canad. Entomol., XVII. 123. (1885) et Entomol. Amoric., I. 154. (1885); Meland., Canad. Entomol., XXXVI. 18 et 19. 3. (1904); Aldn., Catal. North Amor. Dipt., 179. (1905).

marginatus v. D. WuLP, Tijdschr. v. Entomol., ser. 2. II. (X). 1867: 134. 7. (1868); Lw., Zeitschr. f. d. ges. Naturwiss., N. F. II. (XXXVI). 119. (1870).

? xanthopus Wied., Aussereurop. zweifl. In's., II. 40. 23. (1830) ; WALK., List Dipt. Brit. Mus., V. suppl. 1., 99. 9. [Chrysomyia] (1854) ; Meland., Canad. Entomol., XXXVI. 18. (1904).

elegans Lw., Berlin. Entom. Zeitschr., X. 7. 10. (1866) ; Meland., Canad. Entomol., XXXVI. 17 et 19. 5. (1904); Aldr., Catal. North Amer. Dipt., 179. (1905).

festivus JaEnv., Abhaudl. Senckenb. Naturf. Ges., VI. 325. 16. (1867). flavipes Meıg., System. Beschreib., III. 108. 5. tab. XXV. fig. 14. (1822) ; Fall., Dipt. Suee, Supplem., 9. 2-3. (1826); MacQ., Recueil. Soc. Sc. Agricult. Lille, 1826. 429. 5. (1826); MEia., System. Beschreib., VI. 344. (1830); CurT., Brit. Entomol., VIII. 305., 2. 4. (1830); MACQ., Suit. à Buffon, I. 261. 4. (1834); DưCAn, Magaz. Zool. and Botany, I. 162. 4. (1837). Zetт., Insecta

et sept.,

America sept.

America sept.

Batjan.

America sept.

America sept.

Abyssinia.

Europa centr. et sept. 
Lappon., Dipt., 577. 3. (1838) et Dipt. Scand., I. 158. 4. (1842); WaLk., List Dipt. Brit. Mus., III. 518. (1849) ; Zetr., Dipt. Scand., VIII. 2963. 4. (1849); Walk., Insecta Britann., I)ipt., -I. 31. 4. (1851) et List Dipt. Brit. Mus., V. suppl. 1., 89. 6. (1854); Lw., Verh. zool.-bot. Ver. Wien, V. 134. (1855); Schis., Verh. zool.bot. Ver. Wien, V. 648. 11 et 673. (1855) ; ZETr., Dipt. Scand., XIII. 4957. 4. (1859); Schus., Fauna Austriaca, Dipt., I. 21. 5. (1862); v. D. Wulp, Diptera Neerland., I. 461. 3. (1877); BEck., Berlin. Entom. Zeitschr., XXXI. 97. 2. (1887); BrunetTr, The Entomologist, XXII. 132. 3. (1889) ; E. \& I. Coucke, Annal. Soc. Entom. Belgique, XXXVII. 441. (1893) ; Strobl, Mittheil. Naturwiss. Ver. Steiermark, XXIX. 1892. 11. (1893); Kert., Pótfüz. a Természettud. Közlönyhöz, XXXII. 129. (1900).

formicaeformis DoL. = metallinus FABr.

fortis Walk., Proc. Linn. Soc. London, VIII. 107. 17. (1865); v. D. NovaGuinea.

Wur.p, Catal. Dipt. South Asia, 49. (1896).

frontalis $\mathrm{Lw}$. = ? viridis $\mathrm{SAY}$.

gemmifer WaLk., List Dipt. Brit. Mus., III. 516. (1849) et suppl. 1., Silhet.

95. 36. (1854); v. D. Wulp, Catal. Dipt. South Asia, 48. (1896).

? glaucus Bir., Annal. Soc. Entom. France, sér. 3. VII. 134. [? Sar- Madagascar. gus] (1859).

gracilis $\mathrm{Lw}$. = cuprarius L. var.

inactus Walk., Proc. Linn. Soc. I London, IV. 97. 23. (1860); v. D. Celebes.

Wulp, Catal. Dipt. South Asia, 49. (1896).

indicus HarRIS = iridatus Scop.

inficitus Walk., Proc. Linn. Soc. London, V. 274. 18. (1861); v. D. Batjan.

Wulp, Catal. Dipt. South Asia, 49. (1896).

infuscatus MeIG. = iridatus Scop.

iridatus Scop., Entomol. Carniol., 340. 914. [Musca] (1763) ; Peragna,

Spec. Insect. ulta Calabr., 43. 229. [Musca] (1786); GMr.., Systema

Naturae, V. 2864. 312. [Musca] (1792) ; Otiv., Encỳclop. Méthod., VIII. 45. 214. [Musca] (1811); WALK., Insecta Britann., Dipt., I. 31. 3. (1851) et List Dipt. Brit. Mus., V. suppl. 1., 88. 3. (1854) ; Lw., Verl. zool.-bot. Ver. Wien, V. 133. (1855); Schin., Verh. zool.-bot. Ver. Wien, V. 647. 8 et 673. (1855) et VI. 414. 914. (1856) ; Lw., Zeitschr. f. d. ges. Naturwiss., X. 99. 4. (1857); Stroвl, Mittheil. Naturwiss. Ver. Steiermark, XXIX. 1892. 11. (1893).

auratus MeIg. (nec FABr.), Klassif., I. 143. 2. tab. VIII. fig. 10-15. (1804).

coerulescens Willers, Linn. Entom., III. 464. 141. tab. IX. fig. 26. [Musca] (1789).

cuprarius Fall. (nec L.), Dipt. Suec., Stratiom., 13. 2. ㅇ․ (1817).

indicus Harris, Expos. Engl. Ins., 47. tab. XI. fig. 7. 7. [Musca] (1782).

infuscatus Meia., System. Beschreib., III. 107. 3. (1822); ST. 
FARG. ET Serv., Encyclop. Méthod., X. 340. 1. (1825); Macq., Recueil Soc. Sci. Agricult. Lille, 1826. 428. 2. (1826); Herr.Schärf., Fauna Germ., 113. tab. 24. (1830); Curt., Brit. Entomol., VIII. 305., 2. 2. (1830); MAcQ., Suit. à Buffon, I. 261. 2. (1834); Duncan, Magaz. Zool. and Botany, I. 162. 2. (1837) ; Zетт., Insecta Lappon., Dipt., 577. 2. (1838) et Dipt. Scand., I. 158. 3. (1842) ; WALK., List Dipt. Brit. Mus., III. 518. (1849); ZетT., Dipt. Scand., VIII. 2963. 3. (1849) et XI. 4262. 3. (1852) ; Boxsd., Finl. tvaving. Ins., I. 120. 3. (1861); Schin., Fauna Austriaca, Dipt., I. 21. 3. (1862); JAEnn., Berlin. Entomol. Zeitschr., X. 232. 44. (1866); Siebre, Catal. Dipt. Norvegiae, 8. 3. (1877); v. D. Wulp, Diptera Neerland., I. 462. (1877) ; Neuhaus, Diptera Marchica, 78. 2. (1886) ; Beck., Berlin. Entom. Zeitschr., XXXI. 96. 1. (1887) ; Grischn., Berlin. Entom. Zeitschr., XXXI. tab. III. fig. 8. (1887); Brunerts, The Entomologist, XXII. 132. 2. (1889) ; Sсносн, Fauna Insect. Helvetiae, Dipt., 25. (1890); É. \& I. Coucke, Annal. Soc. Entom. Belgique, XXXVII. 442. (1893); Кект., Pótfüz. a Természettud. Közlöny̆höz, XXXII. 129. (1900).

jucundus WALK., Newman's Zool., Append., XCVI. (1850) et List Brasilia. Dipt. Brit. Mus., V. suppl. 1., 93. 24. (1854).

laetus v. D. Wulp, Notes Leyden Mus., VII. 66. 16. (1885) et Catal. Sumatra. Dipt. South Asia, 49. (1896).

lateritius Rond., Archivio per la Zoolog., III. 76. (1863); Conti, Annal. Mus. Civ. Genova, ser. 2. XV. (XXXV). 130. 1. (1895).

latus Bell., Saggio di Ditterol. Messic., I. 41. 3. tab. I. fig. 19. (1859); Melund., Canad. Eatomol., XXXVI. 18. (1904); Aldr., Catal. North Amer. Dipt., 179. (1905).

limbatus Macq., Dipt. exot., I. 1., 201. 2. (1838); Walk., List Dipt. Brit. Mus., V. suppl. 1., 95. 33. (1854) ; Lw., Dipt.-Fauna Südafrika's, I. 5. (1860).

Iongipennis WIED., Analecta Entomol., 31. 39. (182t) et Aussereurop. zweifl. Ins., II. 34. 11. (1830); Walk., List Dipt. Brit. Mus., V. suppl. 1., 96. 39. (1854) ; MacQ., Dipt. exot., suppl. 5., 47. 17. tab. 1. fig. 10-11. (1855); WALK., Proc. Linn. Soc. London, I. 8. 18. (1856); Rond., Amnal. Mus. Civ. Genova, VII. 454. (1875) ; Bıg., Annal. Soc. Entom. France, sér. 5. IX. 186. [Plecticus] (1879) ; v. D. Wulp, Catal. Dipt. South Asia, 48. (1896.)

longipes WALK., Proc. Linn. Soc. London, V. 232. 14. (1861); v. D. Wulp, Catal. Dipt. Soutl Asia, 49. (1896) et Természetrajzi Füzetek, XXI. 410. 5. (1898).

Macquartii Perty, Delect. animal. articul. Brasil., 185. tab. XXXVII. Brasilia. fig. 2. (1830-34); Hunter, Trans. Amer. Entom. Soc. Philad., XXVII. 122. (1901).

mactans Walk., Proc. Linn. Soc. London, IV. 97. 22. (1860) et V. 146. 9. (1861); Ost.-SAck., Annal. Mus. Civ. Genova, XVI. 417. (1882) ; v. D. Wulp, Notes Leyden Mus., VII. 65. 15. (1885), Catal.

Africa or., Madagascar. Mexico.

Madagascar.

Malacca, Java, Borneo.

Nova Guinea.

Asia mer., Arch.Indicus, Nova Guinea. 
Dipt. South Asia, 49. (1896) et Természetrajzi Fïzetek, XXI. 410. 4. (1898).

? magnificus Big., Annal. Soc. Entom. France, sér. 5. IX. 222. 3. Assam.

[? Sargus] (1879) ; v. D. Wulp, Catal. Dipt. South Asia, 49. (1896).

mandarinus Schin., Novara Reise, Dipt., 62. 29. (1868) : v. D. WulP, Hongkong.

Catal. Dipt. South Asia, 49. (1896).

marginatus v. D. WuLP $=$ decorus S.IY.

melleus Rond. in Truqui : Studi Entomol., I. 104. 76. (1848) ; Walk.,

List Dipt. Brit. Mus., V. suppl. 1., 319. (1854); Hunter, Trans.

Amer. Entom. Soc. Philad., XXVII. 122. (1901).

metallinus Fabr., Systema Antliat., 258. 11. (1805); Wied., Aussereurop. zweifl. Ins., II. 36. 16. (1830) ; WaLk., List Dipt. Brit. Mus., III. 517. (1849), V. suppl. 1., 96. 38 et 320. (1854); Proc. Linn. Soc. London, I. 110. 26. (1856) ct III. 80. 14. (1859); v. D Wulp, Notes Leyden Mus., VII. 65. 14. (1885) et Catal. Dipt. South Asia, 48. (1896).

formicaeformis Dou., Natuurkund. Tijdschr. Nederl. Indie, ser. 3. IV. (XIV). 403. tab. III. fig. 5. (1857).

minimus Zerr., Dipt. Scand., VIII. 2965. 6-7. (1849); WaLk., List Dipt. Brit. Mus., V. suppl. 1., 115. (1854); Lw., Verh. zool.-bot. Ver. Wien, V. 132. (1855) ; v. D. Wulp, Diptera Neerland., I. 462. (1877).

nitidus Zetт., Dipt. Scand., I. 159. 6. p. p. (1812).

nigribarbis $\mathrm{BIG} .=$ viridis $\mathrm{S}_{\mathrm{AY}}$.

nigripes Zetr., Dipt. Scand., I. 159. 5. (1842) et VIII. 2964. 5. Europa sept.

(1849) ; Walk., List Dipt. Brit. Mus., V. suppl. 1., 88. 5. (1854);

Lw., Verh. zool.-bot. Ver. Wien, V. 133. (1855); v. D. Wulp,

Diptera Neerland., I. 462. (1877).

niphonensis BIG., Annal. Soc. Entom. France, sér. 5. IX. 221. 1.

(1879) ; Coqurlu., Proc. Unit. Stat. Nat. Mus., XXI. 308. (1898).

nitidus MeIG., System. Beschreib., III. 108. 4. (1822); Macq., Recueil

Soc. Sci. Agricult. Lille, 1826. 428. 4. (1826) ; Curt., Brit. Entomol., VIII. 305., 2. 3. (1830); MACQ., Suit. à Buffon, I. 261. 3. (1834) ; Duncan, Magaz. Zoolog. and Botany, I. 162. 3. (1837) ; Lw., Programm Posen, 1840. 24. 4. (1840) et Isis, 1840. VII. 55̄3. 4. (1840) ; Zetr., Dipt. Scand., I. 159. 6. p. p. (1842) et VIII. 2964. 6. (1849); Lw., Verh. zool.-bot. Ver. Wien, V. 132. (1855); Schin., Verh. zool.-bot. Ver. Wien, V. 617.6. (1855) ; v. D. WulP, Diptera Neerland., I. 462. (1877); É. \& I/ Coucke, Annal. Soc.

Entom. Belgique, XXXVII. 442. (1893).

nitidus ZETT. p. p. = minimus ZETT.

notatus WIED. = ? coarctatus MACQ.

nubeculosus ZETT. = cuprarius L. var.

opulentus Walk., List Dipt. Brit. Mus., V. suppl. 1., 319. (1854). pallipes Big., Annal. Soc. Entom. France, sér. 5. IX. 222. 2. (1879);

v. D. Wutp, Catal. Dipt. South Asia, 49. (1896).

pallipes Bia., Annal. Soc. Entom. France, sér. 6. VII. 28. 3. (1887);

Brasilia.

Ceylon.

America sept. 
Mfeland., Canad. Entomol., XXXVI. 17. (1904); Aldr., Catal. North Amer. Dipt., 179. (1905).

? papuanus Big., Annal. Soc. Entom. France, sér. 5. IX. 223. 4. NovaGuinea. [? Sargus] (1879) ; v. D. Wulp, Catal. Dipt. South A sia, 49. (1896). picticornis Big., Annal. Soc. Entom. France, sér. 6. VII. 27. 2. Americasept. (1887) ; Meland., Canad. Entomol., XXXVI. 18. (1904); Aldr., Catal. North Amer. Dipt., 179. (1905).

pleuriticus Lw., Berlin. Entom. Zeitsehr., X. 8. 13. (1866); Merand., Cuba.

Canad. Entom., XXXVI. 17. (1904); Aldr., Catal. North A i er.

Dipt., 179. (1905).

politus Schrank = cuprarius I.

pubescens v. D. Wulp, Notes Leyden Mus., VII. 67. 17. (1885) et Gorontalo.

Catal. Dipt. South Asia, 49. (1896).

punctifer Big., Annal. Soc. Entom. France, sér. 6. VII. 27. 1. Americasept. [puntifer] (1887) ; Meland., Canad. Entomol., XXXVI. 17. (1904);

Aldr., Catal. North Amer. Dipt., 179. (1905).

puntifer $\mathrm{B} 1 \mathrm{G} .=$ punctifer $\mathrm{B} 1 \mathrm{G}$.

redhibens Walk., Proc. Linn. Soc. London, IV. 97. 21. (1860); Celebes.

v. D. Wulp, Catal. Dipt. South Asia, 48. (1896).

vobustus Lw. = cuprarius L. var.

rufibasis WaLk., Trans. Entom. Soc. London, n. ser. V. 271. (1860). Port Natal. rufipes WAHLBg., Öfvers. Kongl. Vet. Akad. Förhandl., XI. 213. 5. Europa sept. (1854) ; Zetr., Dipt. Scand., XII. 4558. 4-5. (1855).

Sallei Bell., Saggio di Ditterol. Messie., I. 43. 7. tab. I. fig. 21. Mexico.

(1859) ; Meland., Canad. Entomol., XXXVI. 18. (1904); Aisdr., Catal. North Amer. Dipt., 179. (1905).

sapphireus Big., Annal. Soc. Entom. France, sér. 6. VII. 28. 4. Cuba.

(1887) ; Meland., Canad. Entomol., XXXVI. 17. (1904) ; Aldr.,

Catal. North Amer. Dipt., 179. (1905).

speciosus MacQ., Dipt. exot., suppl. 1., 56. 9. (1846); WaLK., List

Dipt. Brit. Mus., V. suppl. 1., 92. 20. (1854); BeLL., Saggio di

Ditterol. Messic., I. 40. 2. (1859): Meland., Canad. Entomol.,

XXXVI. 18. (1904); Aldr., Catal. North Amer. Dipt., 179. (1905).

splendens Big., Annal. Soc. Entom. France, sér. 5. IX. 224. 5. Mexico.

(1879) ; Meland., Canad. Entomol., XXXVI. 17. (1904); Aldr.,

Catal. North Amer. Dipt., 179. (1905).

taeniatus W1ED., Aussereurop. zweifl. Ins., II. 37. 17. (1830); WALK.,

List Dipt. Brit. Mus., V. suppl. 1., 94. 30. (1854); Hunter, Trans.

Amer. Entom. Soc. Philad., XXVII. 123. (1901).

texanus Meland., Canad. Entomol., XXXVI. 17 et 19. (1904).

America sept.

thoracicus Macq., Suit. à Buffon, I. 261. 5 (1834); Walk., List Dipt. Americamer.

Brit. Mus., V. suppl. 1., 92. 22. (1854); Willist., Trans. Amer.

Entom. Soc. Philad., XV. 248. 11. (1888); Hunter, Trans. Amer.

Entom. Soe. Philad., XXVII. 123. (1901).

tibialis WaLk., Proc. Linn. Soc. London, V. 273. 14. (1861) et VI. Arch.Indieus.

4. 6. (1862); v. D. Wulp, Catal. Dipt. South Asia, 49. (1896). 
tricolor Lw., Berlin. Entom. Zeitschr., X. 8. 12. (1866) ; M LLAND., Canad. Entomol., XXXVI. 18. (1904); Aldr., Catal. North Amer. Dipt., 179. (1905).

tuberculatus Lw., Verh. zool.-bot. Ver. Wien, V. 135. (1855) et Nubia. Dipt.-Fauna Südafrika's, I. 5. (1860).

versicolor Bell., Saggio di Ditterol. Messic., Append., 13. 11. tab. Mexico.

III. fig. 8. (1862) ; Meland., Canad. Entomol., XXXVI. 17. (1904);

Aldr., Catal. North Amer. Dipt., 180. (1905).

violaceus Jaenn., Abhandl. Senckenb. Naturf. Ges., VI. 326. 17. Brasilia.

(1867) ; Hunter, Trans. Amer. Entom. Soc. Philad., XXVII. 123. (1901).

violaceus Scop. $=$ cuprarius $\mathrm{L}$.

viridis SAY, Journ. Acad. Nat. Sci. Philad., III. 87. (1823) et Compl. Writt., II. 77. (1859) ; Wied., Aussereurop. zweifl. Ins., II. 39. 22. (1830) ; WalK., List Dipt. Brit. Mus., III. 519. [Chrysomyia] (1849) et V. suppl. 1., 99. 8. [Chrysomyia] (1854); Ost.-SAck., Bullet. Unit. Stat. Geolog. Surv., III. 212. [Chloromyia] (1877); Big., Annal. Soc. Entom. France, sér. 7. IX. 186. [Microchryza] (1879) ; v. D. Wulp, Tijdschr. v. Entomol., XXIV. 156. 9. [Chloromifia] (1881); Wilusst., Canad. Entomol., XVII. 123. (1885) et Entomol. Americ., I. 153. (1885); Meland., Canad. Entomol., XXXVI. 18 et 19. 4. (1904); Aldr., Catal. North Amer. Dipt., 180. (1905).

? frontalis Lw., Verh. zool.-bot. Ver. Wien, VI. 133. (1855); Schin., Fauna Austriaca, Dipt., I. 22. (1862); v. D. Wulp, Diptera Neerland., I. 462. (1877).

nigribarbis Bıg., Annal. Soc. Entom. France, sér. 5. IX. 224. 6. (1879).

xanthopus WIED. $=$ ? decorus SAY.

\section{Chloromyia}

Duncan, Magaz. Zool. and Botany, I. 164. (1837).

Chrysomyia Macq., Suit. à Buffon, I. 262. 17. (1834).

Myiochrysa Verr. ap. Scudd. Nomencl., 204. (1882).

Myochrysa Rond., Dipterol. Ital. Prodr., IV. 11. (1861).

aurata $\mathrm{F}_{\mathrm{ABR}}$ = formosa Scop.

azurea $\mathrm{Lw} .=$ formosa Scop.

bella Lw., Öfvers. Kongl. Vet. Akad. Förhandl., XIII. 263. 3. [Chry-

Prom. bon.sp. somyia] (1856) et Dipt.-Fauna Südafrika's, I. 6. 1. [Chrysomyia] (1860).

Cicur HARR. = formosa Scop.

coerulea Bı̊a., Annal. Soc. Fintom. France, sér. 6. VII. 29. [Myochrisa] (1887) ; ALdr., Catal. North Amer. Dipt., 180. [Myiochrysa]

America sept. (1905). 
cupraria Scop. = formosa Scor.

flavogeniculata DEg. = formosa Scop.

formosa Scop., Entomol. Carniol., 933.910. [Musca] (1763); Schrank, Enum. Ins. Austr., 443. 899. [Musca] (1781); Petagna, Spec. Insect. ult. Calabr., 43. 228. [Musca] (1786) ; Rossi, Fama Estrusca, II. 309. 1514. [Musca] (1790); GMrL., Systema Naturae, V. 2836. 164. [Musca] (1792); Schrank, Fauna Boica, III. 99. 2395. [Rhagio] (1803); Rossi, Fauna Etrusca, Ed. II., 472. 1514. [Musca] (1807) ; Merg., System. Beschreib., III. 110. 8. [Sargus] (1822); S'r. Farg. ex Serv., Encyclop. Méthod., X. 340. 3. [Sargus] (1825); MacQ., Recueil Soc. Sci. Agricult. Iille, 1826. 4:0. 7. [Surgus] (1826) ; CurT., Brit. Entomol., VIII. 305., 2. 6. [Sargus] (1830); MAcq., Suit. à Buffon, I. 263. 1. [Chrysomyia] (1834); Duncan, Magaz. Zool. and Botany, I. 164. 1. fig. 1-3. (1837); Lw., Programm Posen, 1840. 24. 5. [Sargus] (1840) et Isis, 1840. VII. 553. 5. [Sargus] (1840); Zeller, Isis, 1842. XT. 826. [Sargus] (1842); MACQ. in Lucas : Explor. scient. de l'Algér., Zool., III. 430. 44. [Chrysomyia] (1849); Walk., List Dipt. Brit. Mus., III. 519. [Sargus, Chrysomyia] (1849); ZETT., Dipt. Scand., VIII. 2962. 1. [Chrysomyia] (1819); WALK., Ins. Britannica, Dipt., I. 28. 1. [Chrysomyia] (1851) et List Dipt. Brit. Mus., V. suppl. 1., 97. 1. [Chrysomyia] (1854); Lw., Verh. zjol.-bot. Ver. Wien, V. 138. [Chrysomyia] (1855); Scmin., Verh. zool.-bot. Ver. Wien, V. 649. 1 et 673. [Chrysomyia] (1855) et VI. 414. 910. [Chrysomyia] (1856) ; Lw., Zeitschr. f. d. ges. Naturwiss., X. 99. 2. [Chrysomyic] (1857) ; ZETT., Dipt. Scand., XIII. 4955. 1. [Chrysomyia] (1859); Connelius, Stettin. Entom. Zeitg., XXI. 202. tab. II. A. [Sargus] (1860) ; Schin., Fauna Austriaca, Dipt., I. 23. [Chrysomyia] (1862); JAENv., Berlin. Entom. Zeitschr., X. 233. 48. [Chrysomyia] (1866); v. D. Wulp, Diptera Neerland., I. 463. 1. tab. XIV. fig. 21. [Chrysomyia] (1877); Neuhaus, Diptera Marchica, 78. 3. [Chrysomyia] (1886) ; BrunetTi, The Entomologist, XXII. 133. 1. (1889) ; SсHоch, Fauna Ins. Helvetiae, Dipt., 26. [Chrysomyia] (1890); A. Costa, Atti R. Accad. Napoli, ser. 2. V. No. 14., 21. 3. [Chrysomyia] (1893) ; É. \& I. Coucke, Annal. Soc. Entom. Belgique, XXXVII. 442. [Chrysomyia] (1893); SRToвI, Mittheil. Naturwiss. Ver. Steiermark, XXIX. 1892.11. [Chrysomyia] (1893); Kert., Pótfüz. a Természettud. Közlönyhöz, XXXII. 130. [Chrysomyia] (1900); Wimmer, Rozpravy Ceske Akad., XIII. II. 1., 7. tab. II. fig. 4. [Chrysomyia] (1904).

aurata FABr., Mantissa Insect., II. 347. 63. [Musca] (1787); Gmel., Systema Naturae, V. 2850. 218. [Musca] (1792); Fabr., Entomol. System., IV. 335. 97. [Musca] (1794); Dovov., Brit. Ins., IV. 91. tab. CXIII. fig. 1. [Musca] (1795); FArR., Suppl. Entom. System., 566. 2. [Sargus] (1798); Schraxк, Fauna Boica, III. 111. 2421. [Muscu] (1803) ; FAdr., Systema Antliat., 257. 4. [Sargus] (1805); Otrv., Enyclop. Méthod., VIII. 27. 86. [Musca] (1811).

Europa,

Africa sept. 
azurea Lw., Programm Posen, 1810. 24. 7. [Sargus] (1840) et Isis, 1840. VII. 554. 7. [Sargus] (1840); Zeleer, Isis, 1842. XI. 826. [Sargus] (18.12); WaLk., List Dipt. Brit. Mus., V. suppl. 1., 98. 2. [Chrysomyia] (1854).

Cicur Harris, Expos. Engl. Ins., 47. tab. XI. fig. 8. [Musca] (1782).

cupraria Scop., Entomol. Carniol., 933. 911. [Musca] (1763). flavogeniculata Deg., Mém. pour serv. l'hist. d. Ins., VI. 201.

17. [Nemotelus] (1776).

xanthoptera MeIG., Klassif., I. 144. 5. tab. VIII. fig. 16-18. [Sargus] (1804); FABR., Systema Antliat., 255. 1. [Sargus] (1805) ; Latr., Gen. Crust. et Ins., IV. 278. [Sargus] (1809); Fald., Dipt. Suec., Stratiom., 14. 3. [Sargus] (181i).

melampogon Zelt., Isis, 1842. XI. 825. 8. [Sargus] (18t2); WALK., Europacentr.

List Dipt. Brit. Mus., V. suppl. 1., 98. 3. [Chrysomyia] (1854); et mer.

Lw., Verh. zool.-bot. Ver. Wien, V. 138. [Chrysomyia] (1855);

Scurv., Fauna Austr., Dipt., I. 22. 2. [Chrysomyia] (1862); v. D. Wulp, Diptera Neerland., I. 463. nota. [Chrysomyia]. (1877); Scноси, Fauna Ins. Helvetiae, Dipt., 26. [Chrysomyia] (1890); KerT., Pótfüz. a Természettud. Közlönyhöz, XXXII. 130. [Chrysomyia] (1900).

melanopogon FERM., Fauna Ins. Europ., XXIII. 21. [Sargus] (1842).

? speciosa MacQ., Suit. à Buffon, I. 263. 2. [Chrysomyia] (1834); Meig., System. Beschreib., VII. 104. 12. [Sargus] (1838); Lw., Programm Posen, 1840. 24. 6. [Sargus] (1810) et Isis, 1840. VII. 553. 6. [Sargus] (1840); Schin., Verh. zool.-bot. Ver. Wion, V. 650. 2 et 673. [Chrysomyia] (1855) et VI.

414. 915. [Chrysomyia] (1856).

melanopogon GERM. = melampogon ZELL.

sapphirina Walk., List Dipt. Brit. Mus., III. 519. [Sargus, Chrysomyia] (1849) et V. suppl. 1., 100.11. [Sargus, Chrysomyia] (1854);

v. D. Wutp, Catal. Dipt. South Asia, 50. (1896).

speciosa MACQ. = ? melampogon ZELL.

stigmatica v. D. Wurp, Természetrajzi Füzetek, XXI. 411. 8. (1898) NovaGuinea.

et Tijdschr. v. Entomol., XLII. 47. (1899).

xanthoptera MEIG. $=$ formosa Scop.

\section{Microchrysa}

Lww., Verh. zool.-bot. Ver. Wien, V. 146. (1855).

Chlorosoma Rosp., Diptorol. Ital. Prodr., I. 168. 3. (1856) [Clorisoma].

Chlorosia Rovd., Diptorol. Ital. Psodr., IV. 11. (1861).

affinis Wikd., Analecta Entomol., 31. 42. [Sargus] (1824) et Ausser- India orient. europ. zweifl. Ins., II. 41. 26. [Sargus] (1830); MAcQ., Dipt. exot., 
I. 1., 208. [Chrysomyia] (1838); WaLk., List Dipt. Brit. Mus.,

V. suppl. 1., 100. 13. [Chrysomyia] (1854); v. D. Wulp, Catal.

Dipt. South Asia, 50. (1896).

amulipes THомs. = flaviventris Wied.

anrata $\mathrm{DEG} .=$ polita $\mathrm{L}$.

bicolor Wied., Aussereurop. zweifl. Ins., II. 41. 28. [Sargus] (1830);

MacQ., Dipt. exot., I. 1., 205. [Chrysomyia] (1838); Walk., List

Dipt. Brit. Mus., V. suppl. 1., 93. 26. [Sargus] (1854); Schis.,

Novara Reise, Dipt., 61. 27. (1868); BIG., Annal. Soc. Entom.

France, sér. 5. IX. 186. (1879); Hunter, Trans. Amer. Entom.

Soc. Philad., XXVII. 127. (1901); Merand., Canad. Entomol.,

XXXVI. 17. [Sargus] (1904); Aldr., Catal North Amer. Dipt.,

178. [Sargus] (1905).

rufiventris MaCQ., Suit. à Buffon, I. 264. 5. [Chrysomyia] (18:34).

bipars Walk., Proc. Linn. Soc. London, V. 273. 12. [Chrysomyia] Batjan.

(1861) ; v. D. Wulp, Catal. Dipt. South Asia, 50. (1896).

circumscripta Lw., Öfvers. Kongl. Vet. Akad. Förhandl., XIII. 263. Caffraria.

4. (1856) et Dipt.-Fauna Südafrika's, I. 6. 1. (1860).

cyanea $\mathrm{FABr} .=$ polita $\mathrm{L}$.

cycucivcntris Zетт. = flavicornis MEIG.

dispar Scunv., Novara Reise, Dipt., 61. 28. (1868); Hunter, Trsns. Amer. Entom. Soc. Philad, XXVII. 127. (1901).

flavicornis Meig., System. Beschreib., III. 112. 10. [Sargus] (1822);

MacQ., Recueil Soc. Sci. Agricult. Lille, 1826. 431. 9. [Sargus]

(1826) ; Curt., Brit. Entomol., VIII. 305., 2. 8. [Sargus] (1830);

MaCQ., Suit à Buffon, I. 264. 4. [Chrysomyia] (1834); Duxcan,

Magaz. Zool. and Botany, I. 165. 3. [Chloromyia] (1837); WALk.,

List Dipt. Brit. Mus., III. 520. [Sargus, Chrysomyia] (1849),

Insecta Britann., Dipt., I. 29. 4. [Chrysomyia] (1851) et List

Dipt. Brit. Mus., V. suppl. 1., 99. 6. [Chrysomyia] (1854); Lw.,

Verh. zool.-bot. Ver. Wien, V. 139. [Chrysomyia] (1855); Schrn.,

Fauna Austriaca, Dipt., I. 23. [Chrysomyia] (1862); v. D. Wulp, Diptera Neerland., I. 464. 3. [Chrysomyia] (1877); Bıg., Annal. Soc. Entom. France, sér. 5. IX. 186. (1879) ; Brunetri, The Entomologist, XXII. 133. 2. (1889); Scnoch, Fauna Ins. Helvetiae, Dipt., 25. [Chrysomyia] (189门); E. \& L. Coucke, Annal. Soc. Entom. Belgique, XXXVII. 442. [Chrysomyia] (1893).

cyaneiventris Zetr., Dipt. Scand., I. 156. 4. [Chrysomyia] (1842) et VIII. 2962. 4. [Chrysomyia] (1849); DahlB., Kongl. Vet. Akad. Handlig., 1850. 159. [Chrysomyia] (1851); WALK., Ins. Britannica, Dipt., I. 29. 5. [Chrysomyia] (1851); ZетT., Dipt. Scand., XI. 4262. 4. [Chrysomyia] (1852); WALK., List Dipt. Brit. Mus., V. suppl. 1., 99. 7. [Chrysomyia] (1854); Lw., Verh. zool.-bot. Ver. Wien, V. 139. [Chrysomyia] (1855). pallipes Meia., System. Beschreib., VI. 344. 11. [Sargus] (1830) ; Zerr., Dipt. Scand., I. 155. 3. [Chrysomyia] (1842) et VIII. 2962. [Chrysomyia] (1849); WaL.K., Insecta Britann.,

Antillae, Brasilia, Guyana.

Americamer.

Europa centr. et sept. 
Dipt., I. 29. 3. [Chrysomyia] (1854); Lw., Verh. zool.-bot. Ver. Wien, V. 139. [Chrysomyia] (1855); Bonsd., Finl. tvaring. Ins., I. 119. 2. [Chrysomyia] (1861).

? parvulus Haris, Explor. Engl. Ins., 48. tab. XI. fig. 11. [Musca] (1782).

flaviventris WiED., Analecta Entomol., 31. 41. [Sargus] (1824) et Aussereurop. zweifl. Ins., II. 40. ¿5. [Sargus] (1830); MacQ., Dipt. exot., I. 1., 204. [Chrysomyia] (1838); WaLk., List Dipt. Brit. Mus., V. suppl. 1., 100. 12. [Chrysomyia] (1854); v. D. Wulp, Tijdschr. v. Entomol., XXIII. 16?. 11. [Chrysomyia] (1880); Ostr.-SAck., Annal. Mus. Civ. Genova, XVI. 417. (1882); v. ग. Wulp, Catal. Dipt. South Asia, 50. (1896), Természetrajzi Fiizetel, XXI. 411. 9. (1898) et Tijdschr. v. Entomol., XLII. 53. (1899) ; Meis., Bijdragen tot de Dierkunde, XVIII. 94. (1904).

anmulipes Thors., Eugenies Resa, Dipt., 461. 27. [Chrysomyia] (1869) ; Ost.-Sack., Annal. Mus. Civ. Genova, XVI. 417. (1882) ; v. D. Wulp, Catal. Dipt. Soutl Asia, 51. (1896).

gemma Big., Annal. Soc. Entom. France, sér. 5. IX. 231. [? Mirrochrysa] (1879) ; v. D. Wulp, Catal. Dipt. South Asia, 51. (1896). macula FABr., Systema Antliat., 258. 12. [Sargus] (1805); WiEd., Aussereurop. zweifl. Ins., II. 40. 24. [Sargus] (1830); WaLK., List Dipt. Brit. Mus., V. suppl. 1., 99. 10. [Chrysomyia] (1854); Schin., Novara Reise, Dipt., 62. (1868); Hunter, Trans. Amer. Entom. Soc. Philad, XXVII. 122. [Sargus] (1901).

nova Gigl.-Tos, Bollet. Mus. Zool. ed Anat. comp. Torino, VI. No. 102., 2. (1891) et Mem. R. Accad. Sci. Nat. Torino, ser. 2. XLIII. 112. 15. (1893) ; Aldr., Catal. North Amer. Dipt., 180. (1905). ? obscura Bıg., Annal. Soc. Entom. France, sér. 7. I. 370. 8. [Chrysomyia, ? Microchrysa] (1891).

pallipes MEIG. = flavicornis MEIG.

parvula HaRris = ? flavicornis MEIG.

polita I., Systema Naturae, Ed. X. 598. 72. [Musca] (1758), Fauna Suec., Ed. Alt., 457. 1854. [Musca] (1761) et Systema Naturae, Ed. XII., II. 994. 93. [Musca] (1767) ; FABR., Systema Entomolog., 781. 40. [Musca] (1775); O. F. Múlu., Zoolog. Dan. Prodr., 172. 2018 et 173. 3024. [Musca] (1776) ; FaBr., Species Insect., II. 446. 53. [Musca] (1781) et Mantissa Insect., II. 348. 64. [Musra] (1787) ; GMel., Systema Naturae, V. 2850. 93. [Musca] (1792); Fabr., Entomol. System., IV. 335. 99. [Musca] (1794) et Suppl. Entomol. System., 566. 4. [Sargus] (1798); Meig., Klassif., I. 145. a. [Sargus] (1804); Fabr., Systema Antliat., 257. 7. [Sargus] (1805); Ouıv., Eneyclop. Méthod., VIII. 27. 87. [Musca] (1811); Fali., Dipt. Suec., Stratiom., 14. 4. [Sargus] (1817); Mrig., System. Beschreib., III. 111. 9. [Sargus] (1822); Macq., Recueil Soc. Scient. Agricult. Lille, 1826. 430. 8. [Sargus] (1826); Herr.Schäff., Fauna Germ., CXII. tab. 24. [Sargus] (1829); Curt., Brit. Entomol.. VIII. 305., 2. 7. [Sargus] (1830); Bouche, Natur-

Asia mer., Manila, Arch.Indirus, Nova G sinea.

Ceylon.

America mer.

Mexico.

Assinia.

Europa, America sept. 
gesch. d. Ins., I. 49. 32. [Sargus] (1834); MAcQ., Suit. à Buffon, I. 263. 3. tab. VI. fig. 13. [Chrysomyia] (1834); Durcan, Magaz. Zoolog. and Botany, I. 165. 2. [Chloromyia] (1837); Zет'T., Insecta Lappon., Dipt., 577. 4. [Sargus] (1838); Lw., Programm Posen, 1840. 24. 8. [Sargus] (1840) et Isis, 1840. VII. 554. 8. [Sargus] (1840); ZetT., Dipt. Scand., I. 155. 2. [Chrysomyia] (1842) ; Scholtz, Zeitschr. f. Entomol. Breslau, III. 10. [Chrysomyia] (1848); Walk., List Dipt. Brit. Mus., III. 519. [Sargus, Chrysomyia] (1849) ; Zетт., Dipt. Seand., VIII. 2962. 2. [Chrysomyia] (18-19); WalK., Insecta Britann., Dipt., I. 28. 2. tab. I. fig. 8. [Chrysomyia] (1851); Halid., Stettin. En!om. Zeitg., XI. 136. [Chrysomyia] (1851); Zетт., Dipt. Scand., XI. 4262. 2. [Chrysomyia] (1852); Walk., List Dipt. Brit. Mus., V. suppl. 1., 98. 4. [Chrysomyia] (1854); Lw., Verh. zool.-bot. Ver. Wien, V. 139. [Chrysomyia] et 146. (1855); Scuin., Verh. zool.-bot. Ver. Wien, V. 650. 3 et 673. [Chysomyia] (1855) ; Lw., Zeitschr. f. d.ges. Naturwiss., X. 99. 3. (1857) ; Zетт., Dipt. Scand., XIII. 4956. 2. [Chrysomyia] (1859) ; BoxsD., Finland traving. Ins., I. 119. 1. [Chrysomyia] (1861); Schis., Fauna Austriaca, Dipt., I. 22. 1. [Chrysomyia] (1862) ; JAEnv., Berlin. Entom. Zeitschr., X. 233. 49. [Chrysomyia] (1866) ; v. D. Wulp, Diptera Neerland., I. 463. 2. [Chrysomyia] (1877) ; BIG., Annal. Soc. Entom. France, sér. 5. IX. 186. (1879); v. D. Wulp, Tijdsclir. v. Entomol., XXIV. 156. 10. (1881); Beling, Archiv f. Naturgesch., XLVIII. 1., 188. 2. [Chrysomyia] (1882); Neuraus, Diptera Marchica, 78. 4. [Chrysomyia] (1886); Beck., Berlin. Entom. Zeitschr., XXXI. 102. 11. (1887); Brunetri, The Entomologist, XXII. 133. 1. (1889); Sспосн, Fanna Ins. Helvetiae, Dipt., 25. [Chrysomyia] (1890); É. \& L. Coucke, Amal. Soc. Entom. Belgique, XXXVII. 442. [Chrysomyia] (1893); Sтrовг, Mittheil. Naturwiss. Ver. Steiermark, XXIX: 1892. 12. (1893); Verhoeff, Verh. Naturhist. Ver. d. preuss. Rheinl. und Westfal., III. 26-32. [Chrysomyia] (1895); Kent., Pótfüz. a Tormészettud. Közlönyhöz, XXXII. 130. (1900); Scraufuss, Insekten-Börse, XVIII. 100. (1901); AldDr., Catal. North Amer. Dipt., 180. (1905). aurata Jeg., Mém. pour serv. l'hist. d. Ins., VI. 202. 18. [Nemotelus] (1776).

cyanca Fabr., Systema Antliat., 258. 10. [Sargus] (1805). splendens Merg., Klassif., I. 144. 4. [Sargus] (1804).

vitrea Harris, Expos. Engl. Ins., 48. tab. XI. fig. 9-10. [Musca] (1782).

rufiventris MACQ. $=$ bicolor WIED.

scutellaris Lw., Öfvers. Kongl. Vet. Akad. Fïrlıandl., XIII. 263. 5. Caffraria. (1865) et Dipt.-Fauna Sïdafrika's, I. 7. 2. (1860).

splendens Mrig. = polita I.

vitrea HaRRIS = polita L. 


\section{Pedicella}

Big., Annal. Soc. Entom. France, sér. 3. IV. 63 et 83. (1856).

Macrosargus Big., Annal. Soc. Entom. France, sér. 5. IX. 187 et 225 . (1879).

Alchidas Walk., List Dipt. Brit. Mus., III. 517. [Sargus] (1849) et V. suppl. 1., 90. 14. [Sargus] (1854); Joinss., Proc. Acad. Nat. Scienc. Philad., 1894. 271. [Macrosargus] (1894); Msidand., Canad. Entomol., XXXVI. 17. [Macrosargus] (1904); Aldr., Catal. North Amer. Dipt., 180. [Macrosargus] (190氵).

aurea Bell., Saggio di Ditterol. Messic., I. 42. 6. tab. I. fig. 20. Mexico.

[Sargus] (1859); Big., Annal. Soc. Entom. France, sér. 5. IX. 187. [? Macrosargus] (1879) ; Meland., Canad. Entomol., XXXVI. 16. [Sargus, Macrosargus] (1904); A I.DR., Catal. North Amer. Dipt., 178. [Sargus] (1905).

caesia Bell., Saggio di Ditterol. Messic., I. 40. 1. tab. I. fig. 18. Mexico.

[Sargus] (18.)9) ; Willist., Biolog. Centr. Amer., Dipt., I. 232. 4. [Sargus] (1900) ; Meland., Canad. Entomol., XXXVI. 16. [Sargus] (1904); ALdr., Catal. Nortl Amer. Dipt., 178. (1905).

filiformis GigL.-Tos, Bollet. Mus. Zool. ed Anat. comp. Torino, VI. No. 102., 4. [Sargus, ? Macrosargus] (1891) et Mem. R. Accad. Scienc. Nat. Torino, ser. 2. XLIII. 124. 30. [Sargus] (1893).

clavata WALK., List Dipt. Brit. Mus., V. suppl. 1., 93. 23. [Sargus] (1854) ; Bell., Saggio di Ditterol. Messic., I. 41. 4. var. [Surgus] (1859) ; Hunter, Trans. Amer. Entom. Soc. Philad., XXVII. 122. [Sargus] (1901) ; Meland., Canad. Entomol., XXXVI. 16. [Sargus] (1904); Aldr., Catal. North Amer. Dipt., 179. (1905).

clavis Willist., Canad. Entomol., XVII. 123. [Macrosargus] (1885); Meland., Canad. Entomol., XXXVI. 16. [Macrosargus] (1904); Aldr., Catal. North Amer. Dipt., 180. [Macrosargus] (1905).

filiformis Gigl.-Tos = caesia BeLL.

lateralis Macq., Suit. à Buffon, I. 262. 7. [Sargus] (1834); WALK., List Dipt. Brit. Mus., V. suppl. 1., 90. 16. [Sargus] (1854); Big. in SAGRA: Hist. la Isla de Cuba, P. 2., VIII. 335. [Sargus] (1856); Meland., Canad. Entomol., XXXVI. 17. [Sargus, Macrosargus] (1904) ; Aldr., Catal. North Amer. Dipt., 179. [Sargus] (1905).

linearis WaLk., List Dipt. Brit. Mus., V. suppl. 1., 318. [Sargus] (1854) ; Meland., Canad. Entomol., XXXVI. 16. [Sargus, Macrosargu8] (1904); ALdr., Catal. North Amer. Dipt., 179. [Sargus] (1905).

lucens Lw., Berlin. Entom. Zeitschr., X. 7. 11. [Sargus] (1866); ? Gigl.-Tos, Mem. R. Accad. Sci. Nat. Torino, ser. 2. XLIII. 123. 29. [Sargus] (1893); Willist., Trans. Amor. Entom. Soc. London, 1896. 299. 1. [Sargus] (1896) et ? Biolog. Central. Amer., Dipt., I. 231. 1. [Sargus spec.] (1900); Hunter, Trans. Amer. Entom. Soc.

America mer. et centr.

America sopt.

Cuba.

Mexico.

America c., Antillae. 
Philad., XXVII. 122. [Sargus] (1901); Meland., Canad. Entomol., XXXVI. 17 et 18. 1. [Sargus, Macrosargus] (1904); Aldr., Catal. North Amer. Dipt., 179. [Sargus] (1905).

lumus Big., Annal. Soc. Entom. France, sér. 5. IX. 187. [? Macrosargus] (1879).

lunus Big. = lucens L $\mathrm{Lw}$.

natalensis MAcQ., Dipt. exot., suppl. 5., 45. 12. [Sargus] (1855); Port Natal.

Big., Annal. Soc. Entom. France, sér. 5. IX. 187. [Macrosargus] (1879).

rufibasis Big., Annal. Soc. Entom. France, sér. 5. IX. 225. 2. [Macrosargus] (1879); Hunter, Trans. Amer. Entom. Soc. Philad., XXVII. 123. [Macrosargus] (1901).

smaragdifera Big., Annal. Soc. Entom. France, sér. 5. IX. 226. 3. Mexico. [Macrosargus] (1879); Meland., Canad. Entomol., XXXVI. 16. [Sargus, Macrosargus] (1304); Aldr., Catal. North Amer. Dipt., 180. [Macrosargus] (1905).

tenuiventris Big., Annal. Soc. Entom. France, sér. 5. IX. 225. 1. Amazonia. [Macrosargus] (1879); Huvter, Trans. Amer. Entom. Soc. Philad., XXVII. 123. [Macrosargus] (1901).

\section{Hermetiinae.}

\section{Analcocerus}

Loew, Verh. zool.-bot. Ver. Wien, V. 160. (1855).

atriceps Lw., Verh. zool.-bot. Ver. Wien, V. 140. tab., fig. 1-2. Brasilia. (1855).

hortulanus Willist., Biolog. Centr. Amer., Dipt., I. 238. 1. tab. IV. Mexico. fig. 10. (1900); Aldr., Catal. North Amer. Dipt., 176. (1905).

\section{Acrochaeta}

Wied., Aussereurop. zweifl. Ins., II. 42. (1830).

breviseta WaLK., List Dipt. Brit. Mus., V. suppl. 1., 69. 10. (1854).

Echemon Walk., List Dipt. Brit. Mus., III. 515. [Sargus, Chrysochlora] (1849) et V. suppl. 1., 67. 1. (1854).

elegans Prerty, Delect. animal. articul. Brasil., 184. tab. XXXVI.fig. 15. Brasilia. (1830-34) ; WaLK., List Dipt. Brit. Mus., III. 526. (1819) et V. suppl. 1., 68. 5. (1854) ; Huntri, Trans. Amer. Entom. Soc. Philad., XXVII. 123. (1901).

fasciata Wied., Aussereurop. zweifl. Ins., II. 42. tab. IX. fig. 3. Americamer. (1830) ; MAcQ., Suit. à Buffon, I. 250. 1. tab. VI. fig. 11. (1834) et centr. et Dipt. exot., I. 1., 194. (1838); WaLK., List Dipt. Brit. Mus., V. suppl. 1., 68. 7. (1851); Gigl.-Tos, Mem. R. Accad. Scienc. Nat. Torino, ser. 2. XLIII. 112. 16. tab. I. fig. 6, 6a-b. (1893); WiL-

Brasilia.

Brasilia. 
urst., Biolog. Central. Âmer., Dipt., I. 232. 1. (1900) ; Hunter, Trans. Amer. Entom. Soc. Philad., XXVII. 123. (1901); Al.pr., Catal. North Amer. Dipt., 176. (1905).

Haterius WaLk., List Dipt. Brit. Mus., III. 516. [Sargus, Chrysc- Venezuela. chloraj (1849) et V. suppl. 1., 67. 2. (1854).

longiseta Walk., List Dipt. Brit. Mus., V. suppl. 1., 69. 9. (1854). Brasilia.

plana Wask., Insecta Saunders., Dipt., I. 82. [Chrysochlor ( ] (1851) Columbia.

et List Dipt. Brit. Mus., V. suppl. 1., 68. 4. (1854).

tincta Wлıк., Insecta Saunders., Dipt., I. 82. [? Chrysochlora] (18;1) Columbia.

et List Dipt. Brit. Mus.. V. suppl. 1., 67. 3. (1854).

\section{Eudmeta}

Wied., Aussereurop. zweifl. Ins., IJ. 43. (1830).

Toxocera NAcQ., Dipt. exot., suppl. 4., 44. (1849).

brunnea Meı., Bijdragen tot de Dierkunde, XVIII. 95. tab. VIII. Bengal.

fig. 10. (1904).

cingularis Gúk. = marginata FABR.

cingulatis GuÉr. = marginata FABR.

limbinervis OST.-SACK. = marginata FABR.

limbiventris $\mathrm{MACQ} .=$ marginata FABR.

marginata FABr., Systema Antliat., 63. 3. [Hermetia] (1805); WIED.,

Aussereurop. zweifl. Ins., II. 44. 1. tab. IX. fig. 4. (1830) ; MacQ., Asia mer., Arch.Indicus.

Suit. à Buffon, I. 259. 1. tab. VI. fig. 10. (1834), Dipt. exot., I. 1., 194. (1838) et suppl. 3., 16. tab. I. fig. 9. (1848); Walk., List Dipt. Brit. Mus., III. 514. (1849) et V. suppl. 1., 66. 1. (1854) et Proc. Linn. Soc. London, I. 8. 16. (1856); Dol., Natuurkund. Tijdschr. Nederl. Indie, ser. 4. III. (XVII). 82. 10. (1888); v. D. Wulp, Notes Leyden Mus., VII. 69. 20. (1885) et Catal. Dipt. South Asia, 47. (1896).

cingularis Gúk.r., Voyage de la Coquille, Zool., II. P. 2., 290. [Hermetia] (1830) et Iconogr. d. Régne Anini., VII., Ins., 543. [Hermetia] (1835).

limbinervis Ost.-SAck., Berlin. Entom. Zeitschr., XXVI. 367. (1882).

limbiventris Macq., Dipt. exot., suppl. 4., 45. 1. tab. V. fig. 3. [Toxocera] (1819).

singularis Guír., Iconogr. d. Régne Anim., Ins., tab. XCVIII.

fig. 1. [Hermetia] (1835).

singularis GuĖ. = marginata FABR.

\section{Campeprosopa}

Mıcq., Dipt. exot., suppl. 4., 46. (1849).

flavipes MacQ., Dipt. exot., suppl. 4., 46. 1. tab. V. fig. 4. (1849); Java. Ost.-SAck., Annal. Mus. Civ. Genova, XVI. 410. (1882); v. D. Wulp, Catal. Dipt. South Asia, 48. (1896). 
longicornis Wilusst., Biolog. Central. Amer., Dipt., I. 239. 1. tab. Mexico.

IV. fig. 11. (1900); ALDR., Catal. North Amer. Dipt., 175. (1905).

munda Ost.-SAck., Annal. Mus. Civ. Genova, XVI. 409.(1881); v. D. Sumatra.

Wulp, Catal. Dipt. South Asia, 48. (1896).

\section{Caenocephalus}

v. D. WuLp, T'ermészetrajzi Füzetek, XXI. 443. (1898).

melanarius Walk., Proc. Linn. Soc. London, V. 272. 7. [Salduba] (1861) ; v. D. Wulp, Catal. Dipt. South A sia, 51. [Saldubu] (1896), Természetrajzi Füzetek, XXI. 414. 14. tab. XX. fig. 4, $4 a-b$. (1898) et Tijdschr. v. Entomol., XLII. 54. (1899).

scapularis Walk., Proc. Linn. Soc. London, V. 272. 8. [Salduba] Batjan.

(1861); v. D. Wulp, Catal. Dipt. South Asia, 51. [Salduba] (1896)

et Tijdschr. v. Entomol., XLII. 54. (1899).

\section{Amphilecta}

Brav., Denkschr. Akad. Wien, XLIV. 80. (1881).

superba Brau., Denkschr. Akad. Wien, XLIV. 80. (1881); Karsch, Brasilia.

Berlin. Entom. Zeitschr., XXVIII. 171. I. (1884); Hunter, Trans.

Amer. Entom. Soc. Philad., XXVII. 121. (1901).

\section{Massicyta}

Walk., Proc. Linn. Soc. London, I. 8. (1856).

Lagenosoma Brav., Denkschr. Akad. Wien, XLIV. 81. (1881).

bicolor WALK., Proc. Linn. Soc. London, I. 8. 17. tah. I. fig. 1. Singapore.

(1856); Ost.-Sack., Annal. Mus. Civ. Genova, XVI. 411. (1882);

v. D. Wútp, Catal. Dipt. South Asia, 47. (1896).

dispar Brau., Denkschr. Akad. Wien, XLIV. 82. [Lagenosoma] (1881). Cap York. inflata WaLK., Proc. Linn. Soc. London, III. 78. 7. (1859); v. D. Ins. Aru.

Wulp, Catal. Dipt. South Asia, 47. (1896).

picta Brau., Denkschr. Akad. Wien, XLIV. 81. [Lagenosoma] (1881). Cap York. propinqua BraU., Denkschr. Akad. Wien, XLIV. 82. [Lagenosoma] Cap York. (1881).

\section{Hermetia}

Latr., Hist. Nat. d. Crust. et d. Ins., XIV. 338. CCCCXCIV. (1804).

Thorasena MacQ., Dipt. exot., I. 1., 177. 4. (1838).

aeneipennis GigL.-Tos, Mem. R. Accad. Sci. Nat. Torino, ser. 2. Mexico.

XLIII. 107. (1893); Wulist., Biolog. Central. Amer., Dipt., I.

241, 10. (1900); ALdr., Catal. North Amer. Dipt., 175. (1905). 
albitarsis FABr., Syst. Antl., 63. 4. (1805); Wred., Aussereurop. zweifl. Ins., II. 24. 4. tab. VII. fig. 4. (1830); MacQ., Dipt. exot., suppl. 4., 49. (1846); Rond. in Truqur : Studi Entomol., I. 100. 69. (1848) ; WaLK., List Dipt. Brit. Mus., V. suppl. 1., 21. 7. (1854); Schin., Novara Reise, Dipt., 71. 51. (1868) ; Wiluist., Trans. Amer. Entom. Soc. Philad., XV. 246. 5. (1888) et Biolog. Centr. Amer., Dipt., I. 240.9. (1900) ; Hunter, Trans. Amer. Entom. Soc. Philad., XXVII. 125. (1901) ; Aldpr., Catal. Nortl Amer. Dipt., 175. (1905). ? bimaculata MacQ., Suit. à Buffon, I. 228. 2. (1834); Walk., List Dipt. Brit. Mus., V. suppl. 1., 22. (1854).

planifroins MacQ., Dipt. exot., suppl. 1., 50. 3. (1846); Walk., List Dipt. Brit. Mus., V. suppl. 1., 22. 9. (1854).

? sexmaculata MACQ., Suit. à Buffon, I. 229. 4. (1834); WaLK., List Dipt. Brit. Mus., V. suppl. 1., 22. (1854).

albitarsis v. D. WulP (ncc FABr.), Természetrajzi Füzetek, XXI. 409. 3. (1898) et Tijdschr. v. Entomol., XLII. 47. (1899).

Andreas WALK. = flavipes WIED.

apicalis Wied., Aussereurop. zweifl. Ins., II. 25. 5. (1830); Walk., List Dipt. Brit. Mus., V. suppl. 1., 21. 6 et 304. (1854); Scmn., Novara Reise, Dipt., 70. 50. (1868); Willist., Trans. Amer. Entom. Soc. Philad., XV. 246. 6. (1888); Hunter, Trans. Amer. Entom. Soc. Philad., XXVII. 125. (1901).

armata v. D. Wulp, Notes Leyden Mus., VII. 68. 19. (1885) et Catal. Dipt. South Asia, 47. (1896).

aurata Bell., Saggio di Ditterol. Messic., I. 27. 4. tab. I. fig. 8. (1859) ; Osт.-SAck., Berlin. Entom. Zeitschr., XXVII. 297. (1883); Willist., Canad. Entomol., XVII. 125. (1885); Ost.-SAck., Biolog. Centr. Amer., Dipt., I. 28. 1. (1886) ; Towns., Proc. Calif. Acad. Scienc., ser. 2. IV. 594. 4. (1895); Willist., Biolog. Centr. Amer., Dipt., I. 240. (1900) ; Meland., Canad. Entomol., XXXVT. 20. 2. (1904); Aldr., Catal. North Amer. Dipt., 175. (1905).

batjanensis v. D. WULP = cerioides WALK.

bimaculata MACQ. = ? albitarsis FABR.

ceria Willist., Biolog. Central. Amer., Dipt., I. 242. 13. tab. IV. fig. 13. (1900); Aldr., Catal. North Amer. Dipt., 175. (1905).

ceriogaster Willist., Trans. Amer. Entom. Soc. Philad., XV. 246. 7. (1888); Hunter, Trans. Amer. Entom. Soc. Philad., XXVII. 125. (1901).

cerioides WALk., Proc. Linn. Soc. London, III. 78. 8. [Massicyta] (1859), V. 271. 4. [Massicyta] (1861) et VI. 5. 7. [Massicyta] (1862) ; Ost.-Sack., Annal. Mus. Civ. Genova, XVI. 411. (1882); v. D. Wulp, Catal. Dipt. South Asia, 47. (1896) et Természetrajzi Füzetek, XXI. 409. 2. (1898).

batjanensis v. D. Wulr, Tijdschr. v. Entomol., XXIII. 161. 8. (1880) et Notes Leyden Mus., VII. 67. 18. (1885).

melanaesiae Bıg., Annal. Soc. Entom. France, sér. 5. IX. 202. 4. (1879).
Americamer. et centr., Antillae.

NovaGuinea.

America mer.

I. Morotai.

Americasept. et centr.

\section{Mexico.}

Brasilia.

Arch.Indicus, Nova Guinea. 
chrysopila Lw., Berlin. Entom. Zeitschr., XVI. 56. 11.(1872); ALdr., Catal. North Amer. Dipt., 175. (1905).

coarctata Mucq., Dipt. exot., suppl. 1., 50. 2. tab. V. fig. 4. (1846); Walk., List Dipt. Brit. Mus., V. suppl. 1., 20. 1. (1854); BeLl., Saggio di Ditterol. Messic., I. 24. 1. (1859); Schin., Novara Reise, Dipt., 70. 49. (1868); Ost.-SAck., Biolog. Centr. Amer., Dipt., I. 32. 5. (1886) ; Hunter, Trans. Amer. Entom. Soc. Philad., XXVII. 125. (1901); Aldr., Catal. North Amer. Dipt., 175. (1905).

Comstocki Winlist., Canad. Entomol., XVII. 125. (1885̃) ; Ost.-SAck., Biolog. Centr. Amer., Dipt., I. 28. 2. tab. I. fig. 3. (1886) ; Aldr., Catal. North Amer. Dipt., 175. (1905).

concinna Willıst., Biolog. Central. Amer., Dipt., I. 241. 12. tab. IV. fig. 12. (1900); Alnr., Catal. North Amer. Dipt., 175. (1905). lativentris Willist. (nec Beis.), Canad. Entomol., XVII. 125. (1885).

crabro Ost.-SAck., Biolog. Centr. Amer., Dipt., I. 29. 3. tab. I. fig. 4. (1886) ; Aldr., Catal. North Amer. Dipt., 175. (1905).

Eiseni Towns., Proc. Calif. Acad. Scienc., ser. 2. IV. 594. 5. (1895) ; Aldr., Catal. North Amer. Dipt., 175. (1905).

fenestrata MeiJ., Bijdragen tot de Dierkunde, XVIII. 93. tab. VIII. fig. 9. (1904).

flavipes Wred., Aussereurop. zweifl. Ins., II. 26. 6. (1830); WALK., List Dipt. Brit. Mus., V. suppl. 1., 21.5 et 304. (1854); GigL.Tos, Mem. R. Acead. Sc. Nat. Torino, ser. 2. XIIII. 107. 8. (1893); Hunter, Trans. Amer. Entom. Soc. Philad., XXVII. 125. (1901); Aldr., Catal. North Amer. Dipt., 175. (1905).

Andreas WALK., List Dipt. Brit. Mus., III. 515. [Sargus, Chrysochlora] (1849) et V. supp]. 1., 86. 3. [Chrysochlora] (1854).

flavoscutata Big., Annal. Soc. Entom. France, sér. 5. IX. 201. 2. (1879) ; Aldr., Catal. Nortl Amer. Dipt., 175. (1905).

formica Os'r.-SAck., Biolog. Central. Amer., Dipt., I. 32. 7. (1886) ; Aldr., Catal. North Amer. Dipt., 175. (1905).

fulva WALk., List Dipt. Brit. Mus., V. suppl. 1., 22. 10. (1854).

geniculata MACQ., Dipt. exot., suppl. 5., 41. 5. tab. I. fig. 9. (1855) ; Hunter, Trans. Amer. Entom. Soc. Philad., XXVII. 125. (1901).

illucens L., Systema Naturae, Ed. X. 589. 2. [Musca] (1758) et Ed. XII., II. 979. 2. [Musca] (1767); DeG., Mém. pour serv. l'hist. d. Ins., VI. 205. 3. tab. XXIX. fig. 8-10. [Nemotelus] (1776); FABr., Species Insect., II. 412. 2. (recte 1). [Bibio] (1781) et Mantissa Insect., II. 328. 2. [Bibio] (1787); GreL., Systema Naturae, V. 2830. 2. [Musca] (1792); FABr., Entomol. System., IV. 253. 2. [Mydas] (1794); Latr., Hist. Nat. d. Crust. et d. Ins., XIV. 338. (1804) ; Fabr., Systema Antliat., 62. 1. (1805); LaM., Hist. nat. anim. sans vertebr., III. 385. 2. [Xylophagus] (1816); Sт. FARG. ET Serv., Encyclop. Méthod., X 802. 1. (1825); MACq., Suit. à Buffon, I. 228, 1. (1834) et Dipt, exot., I. 1., 177.1, tab. XXI. fig.
America sept.

America mer. et centr.

America sept. et centr.

America sept.

Guatemala.

America sept.

Palembang.

Brasilia.

Mexico.

Panama.

Brasilia.

America mer.

America,

Antillae. 
2. (1838) ; WaLk., List Jipt. Brit. Mus., HII. 514. (1849) et V. suppl. 1., 20. 2 et 304. (1854); BIG. in SAGliA: Hist. la Isla de Cuba, P. 2., VII. 335. (1856); BELl., Saggio di Ditterol. Messic., I. 25. 2. (1859) ; Scuin., Novara Reise, Dipt., 70. 48. (1868); E. L. Arrib., Bolet. Acad. Nat. Cienc. Córdoba, IV. 126. 43. (1882); Rö̀., Stettin. Entom. Zeitg., XLVI. 339. (1885); Ost.-SAck., Biolog. Central. Amer., Dipt., I. 32. 6. (1886); Whulist., Trans. Amer. Eutom. Soc. Philad., XV. 245. 4. (1888); v. D. Wulp, Tijdschr. v. Entomol., XXXI. 360. tab. IX. fig. 1-2. (1888) ; Jonss., Proc. Acad. Nat. Sci. Philad., 1894. 271. (1894) ; Wills's., 'Trans. Entom. Soc. London, 1896. 299. 1. (1896) et Biolog. Centr. Amer., Dipt., I. 240. (1900) ; Hunter, Trans. Amer. Entom. Soc. Plilad., XXVII. 125. (1901); Meland., Canad. Entomol., XXXVI. 20. 1. (1904); Aldr., Catal. North Amer. Dipt., 175. (1905).

lencopa L., Systema Naturae, Ed. XII., II. 983. 23. [Musca] (1767); Fabr., Species Insect., II. 428. 35. [Syrphus] (1781) et Mantissa Insect., H. 338. 41. [Syrphus] (1787); GMei., Systema Naturae, V. 2837. 23. [Musca leucopus] (1792). mucens Riley \& Howard, Insect Life, İ. 353. (1889).

nigrifacies Bra., Annal. Soc. Entom. France, sér. 5. IX. 200. 1. (1879).

pellucens MacQ., Suit. à Buffon, Expl. des Pl., 4. tab. V. fig. 11-12. (1834).

vufiventris Fabr., Systema Antliat., 63. 2. (1805); Wred., Aussereurop. zweifl. Ins., II. 23. 2. (1830) ; PEnTy, Delect. animal. articul. Brasil., 183. tab. XXXVI. fig. 12. (1830-34) ; Rond. in Truqui: Studi Entomol., I. 101. 70. (1848); Walk., List Dipt. Brit. Mus., V. suppl. 1., 21. 4. (1854); v. D. WulP, Tijdschr. v. Entomol., XXIV. 154. 2. (1881); E. L. ArriB., Bolet. Acad. Nat. Cienc. Córdoba, IV. 126. 44. (188ะ); HunTER, Trans. Amer. Entom. Soc. Philad., XXVII. 126. (1901). laeta Meis., Bijdragen tot de Dierkunde, XVIII. 93. tab. VIII. fig. Bengal. 8. (1904).

Laglaizei Bıg., Annal. Soc. Entom. France, sér. 6. VII. 21. (1887); v. D. Wulp, Catal. Dipt. South Asia, 47. (1896).

lativentris BeLL., Saggio di Ditterol. Messic., I. 27. 3. tab. I. fig. 9. Mexico. (1859) et Append., 8. 3. var. (1862); Willist., Biolog. Central. Amer., Dipt., I. 241.11. (1900) ; ALDr., Catal. North Amer., Dipt., 176. (1905).

lativentris Willist. 1885. (nec BrLl.) = concinna Willist.

leucopa L. = illucens L. melanaesiae $\mathrm{B}_{1 \mathrm{G}}=$ =erioides WALK. mucens RILEY \& Howard = illucens L. nigrifacies $\mathrm{BIa} .=$ illucens $\mathrm{L}$.

pectoralis WJED., Analecta Entomol., 19. 2. (1824); S'T. FARG. ET Guinea. Serv., Encyclop. Méthod., X. 802.5.(1825); Wred., Aussereurop. zweifl. Ins., II. 26. 7. (1830); MAcQ., Suit. à Buffon, I. 229. 3. 
(1834) et Dipt. exot., I. 1., 178. 1. tab. XXI. fig. 3. [Thorasena] (1838); WaLk., List Dipt. Blit. Mus., V. suppl. 1., 23. 1. [Thoresena] (1854); Osт.-Sack., Berlin. Entom. Zeitschr., XXVI. i71. [Thorasena] (1882).

pellucens $\mathrm{MACQ}_{\mathrm{A}}=$ illucens $\mathrm{L}$.

planifrons MACQ. $=$ albitarsis FARR.

pterocausta Ost.-Sack., Biolog. Central. Amer., Dipt., I. 33. 8. tah. Panama.

I. fig. 5. (1886); Aldr., Catal. North Amer. Dipt., 176. (1905)).

pulchra Wied., Aussereurop. zweifl. Ins., II. 24. 3. (1830); Walk., Brasilia, List Dipt. Brit. Mus., III. 514. (1849) et V. suppl 1., 22.8. (1854);

Hunter, Trans. Amer. Entom. Soc. Philad., XXVII. 126. (1901). relicta Ost.-SAck., Biolog. Central. Amer., Dipt., I. 30. 4. (1886); Panama. Aldr., Catal. North Amer. Dipt., 176. (1905).

remittens Walk., Proc. Linn. Soc. London, IV. 94. 13. (1860); v. D. Celebes. Wulp, Catal. Dipt. South Asia, 47. (1896).

rufitarsis Macq., Dipt. exot., suppl. 1., 49. 1. (1846); WaLk., List Columbia. Dipt. Brit. Mus., V. suppl.1., 21.3. (1854) ; IIunter, Trans. Amer.

Entom. Soc. Philad., XXVII. 126. (1901). rufiventris $\mathrm{FABR} .=$ illucens $\mathrm{L}$.

rufiventris WALK., Proc. Linn. Soc. London, V. 145. 7. (1861); v. 1. Amboina. Wulp, Catal. Dipt. South Asia, 47. (1896).

scutellata Mavq., Dipt. exot., suppl. 5., 41. 41. (1855); Hunter, Amazonia.

Trans. Amer. Entom. Soc. Pliilad., XXVII. 126. (1901).

sexmaculata MACQ. = ? albitarsis FABR.

variipennis Bıg., Annal. Soc. Entom. France, sés. 5. IX. 202. 3. Brasilia.

(1879); Hunter, Trans. Amer. Entom. Soe. Philad., XXVII. 126.

(1901).

\section{Tracana}

Walk., Proc. Linn. Soc. London, IV. 99. (1860).

iterabilis WALk., Proc. Linn. Soc. London, IV. 99. 27. (1860); v. D. Celebes. Wulp, Catal. Dipt. South Asia, 48. (1896).

\section{Antissinae.}

\section{Cyanauges}

Phil., Verh. zool.-bot. Ges. Wien, XV. 732. (1865).

ruficornis Schin., Novara Reise, Dipt., 54. 10. (1868); Ost.-Stck., Chile. Berlin. Entom. Zeitschr., XXVI. 374. (1882); Braver, Offenes Schreiben etc., 7. 21. (1883); Hunter, Trans. Amer. Entom. Soc. Philad., XXVII. 127. (1901). valdivianus PHIL. $=$ ? valdivianus RonD. 
valdivianus Rond., Arehivio per la Zoolog., III. 88. [Oplacantha] Chile.

(1863) ; Ost.-SAck., Berlin. Entom. Zeitschr., XXVI. 374. (1882);

Hunter, Trans. Amer. Entom. Soc. Plilad., XXVII. 122. [Beris] et 127. (1901).

? valdivianus Pril., Verh. zool.-bot. Ges. Wien, XV. 733. tab. XXVI. fig. 34. (1865).

\section{Antissa}

WALK., List Dipt. Brit. Mus., V. suppl. 1., 63. (1854). cuprea Walk., List Dipt. Brit. Mus., III. 524. [Clitellaria, ? Cyclogaster] (1849) et V. suppl. 1., 63. 15. (1854); Ost.-Sack., Borlin. Entom. Zeitschr., XXVI. 374. (1882); Brau., Offenes Schreiben etc., 7. 21. (1883).

\section{Anacanthella}

MAcQ., Dipt. exot., suppl. 5., 33. (1855).

splendens Macq., Dipt. exot., suppl. 5., 39. tab. I. fig. 8. (1855)

Australia.

\section{Beridiinae.}

\section{Beris}

Latr., Hist. Nat. Crust. et Ins., III. 447. (1802).

Hexacantha Lioy, Atti Instit. Veneto, ser. 3. IX. 586. 6. (1864). Hoplacantha Rond., Archivio per la Zool., Modena, III. 87. (1863). [Oplachantha].

Octacantha Lıox, Atti Instit. Veneto, ser. 3. IX. 586. 7. (1864). albitarsis MacQ., Dipt. exot., suppl. 1., 47. 3. (1846); WALK., List Dipt. Brit. Mus., V. suppl. 1., 11. 14. (1854); Hunter, Trans. Amer. Entom. Soe. Philad., XXVII. 121. (1901).

annulifera Bıg., Annal. Soc. Entom. France, sér. 6. VII. 21. [Oplacantha] (1887); Coquilı., Proc. Waslingt. Acad. Scienc., II. 406. (1900) et Harriman's Alaska Exped., IX. Pt. II. 20. (1904); Alpr., Catal. North Amer. Dipt., 173. (1905).

atra MEig. = chalybeata Forst.

bellula WiLL. = mexicana BrLL.

brevicornis HEYD. = fuscipes MEIG.

chalybeata Forst., Nov. Spec. Ins., Cent. I. 95. 95. [Musca] (1771); Europacentr. GMel., Systema Naturae, V. 2837. 165. [Musca] (1792); Meig., Americamer.

America sept.

System. Beschreib., II. 4. 4. (1820); St. FARG. et SERv., Encyclop. et sept. 
Méthod., X. 803. 1. (1825); Macq., Recueil Soc. Scienc. Agricult. Lille, 458. 3. (1826); CurT., Brit. Entomol., VIII. 337. (1830) ; Macq., Suit. à Buffon, I. 232. 5. (1834); WALK., Insecta Britann., Dipt., I. 11. 3. tab. I. fig. 1. (1851) et List Dipt. Brit. Mus., V. sul11. 1., 9. 3. (1854) ; Schis., Verh. zool.-bot. Ver. Wien, V. 65). "̈, 674 et 675. (1855) et Fauna Austriaca, Dipt., I. 24. (1862); J Aenv., Berlin. Entom. Zeitschr., X. 234. 52. (1866); v. D. Wur.r, Dipt. Neerlandica, I. 442. 1. (1877); Neuilaus, Diptera Maruhica, 40. 3. (1886); Beck., Berlin. Entom. Zeitsehr., XXXI. 99. 8. (1887) ; Brunetri, The Entomologist, XXII. 134. 1). (1889): Schoch, Fauna Ins. Helvetiae, Dipt., 26. (1890); É. \& L. Cotcke, Annal. Soc. Entom. Belgique, XXXVII. 435. (1893); Strobi, Mittheil. Naturwiss. Ver. Steiermark, XXIX. 1892. 12. (1893); Kert., Pótfüz. a Természettud. Közlönyhöz, XXXII. 131. (1900). atra Meig., Klassif., I. 118. 3. [Actina] (1804).

favipes Macq., Recueil Soc. Scient. Agricult. Lille, 460. 8. (1826); S't. Farg. ex Serv., Encyclop. Méthod., X. 803. 3. (? 1825) ; MACQ., Suit. à Buffon, I. 233. 9. (1834); Meig., System. Beschreib., VII. 56. 14. (1838).

obscura MeIG., System. Beschreib., II. 4. 3. (1820); ST. Farc. ET Serv., Encyclop. Méthod., X. 803. 3. (1825); MAcq., Suit. à Buffon, I. 232. 4. (1834); Zетт., Dipt. Scand., I. 133. 5. (1842) et VIII. 2950. 5. (1849).

sexdentata Fabr., Species Insect., II. 418. 12. [Stratiomys] (1781) et Mantissa Insect., II. 332. 18. [Stratiomys] (1787); Guel., Systema Naturae, V. 2836. 162. [Musca] (1792); Fab1., Entomol. System., IV. 269. 25. [Stratiomys] (1794); MeiG., Klassif., I. 118 a. [Actina] (1804); Latr., Dict. Hist. Nat., XXIV. 192. 552. (1804); FABr., Systema Antliat., 87. 36. [Stratiomys] (1805); MeIG., System. Beschreib., II. 8. Anmerk. (1820); St. Farg. ext Serv., Encyclop. Méthod., X. 803. 1. (1825) ; Meig., System. Beschreib., VI. 318. 12. (1830) ; Zетт., Insecta Lappon., Dipt., 812. 2. (1838) et Dipt. Scand., I. 132. 2. (1842) ; Lw., Stettin. Entom. Zeitg., VII. 261. 3. (1846); Walk., List Dipt. Brit. Mus., I. 125. (1848); Zetr, Dipt. Scand., VIII. 2948. 2. (1849) et XII. 4551. 2. (1855); BonsD., Finland. tvaving. Ins., I. 300. 1. (1861).

clavipes MeIG. 1804. (nee L.) = vallata Forst.

clavipes L., Systema Naturae, Ed. XII., II. 981. 12. [Musca] (1767); Farr., Systema Entomol., 761. 9. [Stratiomys] (1775); Schrank, Enum. Ins. Austr., 440. 894. [Musca] (1781); FABr., Spec. Insect., II. 418. 11. [Stratiomys] (1781) et Mantissa Insect., II. 332. 17. [Stratiomys] (1787); GMel., Systema Naturae, V. 2836. 12. [Musca] (1792); Panz., Fauna Germ., IX. tab. 19. [Stratiomys] (1793); FABr., Entomol. Syst., IV. 268. 24. [Stratiomys] (1794) et Systema Antliat., 86. 35. [Stratiomys] (1805) ; Latr., Consid. génér., 442. (1810) ; Fall., Dipt. Suec., Stratiom., 12. 10. [Stratiomys] (1817);

Europa centr. et sept. 
Cur., Régne Animal., III. 622. (1817) ; Meia., Sษstem. Beschreib., II. 5. 5. (1820) ; St. FArg. et Serv., Encyclop. Méthod., X. 803. 2. (1825) ; Macq., Recueil Soc. Sei. Agricult. Lille, 458. 4. tab. III. fig. 4. (1826) ; CunT., Brit. Entomol., VIII. 337. (1830); Macq., Suit. à Buffon, I. 233. 6. (1834) ; Guḱr., Iconogr. d. Régne Anim., VII. Ins., 544. tab. XCVIII. fig. 4. (1835); ZETT., Dipt. Scand., I. 132. 3. (1842); Lw., Stettin. Entom. Zeitg., VII. 259. 2. (1846); Grmm., Bull. Soc. Imp. Nat. Moscou, XX. 1., 176. 2. (1847); WaLk., List Dipt. Brit. Mus., I. 124. (1848) ; ZeTT., Dipt. Scand., VIII. 2950. 3. (1849); WaLK., Insecta Britann., Dipt., I. 11. 1. (1851) ; Halm., Stettin. Entom. Zeitg., XV. 1:8. (1851); ZerT., Dipt. Scand., XI. 4260. 3. (1852) ; WaLk., List Dipt. Brit. Mus., V. suppl. 1., 8. 1. (1854); Schin., Verh. zool.-bot. Ver. Wien, V. 653. 1 et 674. (1855); ZeTr., Dipt. Scand., XIII. 4950. 3. (1859) ; Schin., Fauna Austriaca, Dipt., I. 24. 2. (1862); J Aesv., Berlin. Entom. Zeitschr., X. 233. 50. (1866) ; v. D. Wulp, Diptera Neerland., I. 433. 2. (1877) ; JaroschefF, 'Trudy Kharkoff, XI. 341. 6. (1877); Neunaus, Dipt. Marchica, 39. 2. (1886); Brunettr, The Entomologist, XXII. 133. 1. (1889); Scroch, Fauna Ins. Helvetiae, Dipt., 26. (1890); E. \& L. Coucke, Annal. Soc. Entom. Belgique, XXXVII. 435. (1893); Strobl, Mittheil. Naturwiss. Ver. Steiermark, XXIX. 1892. 12. (1893); Kent., Pótfüz. a 'Természettud. Közlönylı̈̈z, XXXII. 180. (1900).

nigroptera Fourcr., Entomol. Paris., 469. 8. [Stratiomys] (1785). coerulescens Schin., Novara Reise, Dipt., 71. 53. (1868); Hunter, Trans. Amer. Entom. Soc. Philad., XXVII. 121. (1901). cuprea Hutron, Trans. New Zealand Instit., XXXIII. 6. (1901). filipalpis MACQ., Dipt. exot., suppl. 4., 41. 7. tab. III. fig. 2. (1849). flavipes MACQ. = chalybeata Forst.

flavispinosa MacQ., Dipt. exot., suppl. 5., 38. 6. (1855); Huster, Trans. Amer. Entom. Soc. Philarl., XXVII. 121. (1901).

fuscipes Meig., System. Beschreib., II. 8. 11. (1820); St. FARG. ET SErv., Encyclop. Méthod., X. 803. 2. (1825); Macq., Recueil Soc. Sci. Agricult. Lille, 1826. 460. 7. (1826) ; Curt., Brit. Entomol., VIII. 337. (1830) ; MAcQ., Suit. à Buffon, I. 233. 8. (1834); Lw., Stettin. Entom. Zeitg., VII. 282. 4. (1846) ; WaLK., List Dipt. Brit. Mus., I. 125. (1848) ; ZeT'., Dipt. Seand., VIII. 2949. 2-3. (1849) ; Walk., Insecta Britann., Dipt., I. 12. 4. (1851) et List Dipt. Brit. Mus., V. suppl. 1., 9. 4. (1854); Scmin., Verh. zool.-bot. Ver. Wien, V. 455. 4, 674 et 675. (1855) et Fauna Austriaea, Dipt., I. 24. (1862); BEck., Berlin. Entom. Zeitschr., XXXI. 100. 9. (1887) ; Brunetti, The Entomologist, XXII. 134. 4. (1889); ScHoch, Fauna Insect. Helvetiae, Dipt., 26. (1890); Sтrobr, Mittheil. Naturwiss. Ver. Steiermark, XXIX. 1892. 12. (1893); Kert., Pótfüz. a Természettud. Közlönyhöz, XXXII. 131. (1900); Beck., Acta Societ. Sci. Fenn., XXVI. No. 9., 5. 3. (1900); Verrall, Entom. Monthly Mag., ser. 2. XV. (XL). 139. (1904).

Columbia.

N. Selandia.

Tasmania.

America mer.

Europa centr. et sept., Sibiria. 
brevicornis HeYD, in litt. apud Lw.

nigra Meig., System. Beschreib., II. 7. 8. (1820); St. FARG.. ET Serv., Encyclop. Mrilliorl., X. 803. 5. (1825); Macq., Reeueil Soc. Sci. Agricult. Lille, 1826. 459. 6. (1826); Curt., Brit. Entomol., VIII. 337. (1830).

fusciventris Macq., Dipt. exot., suppl. 4., 42. 9. (1849).

geniculata CurT., Brit. Entomol., VIII. 337. fig. (1830); Walk., Insceta Britann., Dipt., I. 12. 6. (1851); WahLBg., Öfvers. Kongl. Vet. Akad. Förhandl., XI. 212. 2. (1854); WALK., List Dipt. Brit. Mus., V. suppl. 1., 10. 7. (1854); Schin., Verh. zool.-bot. Ver. Wien, V. 656. 6 et 674. (1855) ; Zeтr., Dipt. Scand., XII. 4552. 2-3. (1855); Verr., Entom. Monthly Mag., ser. 2. XV. (XL). 139. (1904).

Guerinii Macq., Dipt. exot., suppl. 1., 48. 4. tab. V. fig. 3. (1846); Walk., List Dipt. Brit. Mus., V. suppl. 1., 11. 15. (1854); Hunter, Trans. Amer. Entom. Soc. Philad., XXVII. 121. (1901). incisuralis MACQ., Dipt. exot., suppl. 2., 28. 5. (1847) et suppl. 4., 42. (1849); Walk., List Dipt. Brit. Mus., V. suppl. 1., 12. 19. (1854).

javana MAcq., Dipt. exot., I. 2., 188. (1838); WaLk., List Dipt. Brit. Mus., V. suppl. 1., 11. 16. (1854) ; Rond., Archivio per la Zoolog., III. 87. [Chorisops] (1863) ; v. D. WulP, Tijschr. v. Entomol., XXIII. 163. 9. (1880), Sumatra Exped., 1)ipt., 13. 1. (1881) et Catal. Dipt. South Asia, 58. (1896).

limbata Brg., Annal. Soc. Entom. France, sér. 5. IX. 196. [Oplacantha] (1879); Gigl.-Tos, Mem. R. Accad. Sc. Nat. Torino, ser. 2. Xlili. 102. 2. tab. I. fig. 1. (1893); Aldr., Catal. North Amor. Dipt., 173. (1905).

longicornis Prir., Verh. zool.-bot. Ges. Wien, XV. 731. 6. (1865); IIUnter, Trans. Amer. Entom. Soc. Philad., XXVII. 121. (1901).

luctifera P'Hı., Verh. zool.-but. Ges. Wien, XV. 729. 2. (1865); Hunter, Trans. Amer. Entom. Soc. Philad., XXVII. 121. (1901).

luteiventris Pril., Vesh. zool.-bot. Ges. Wien, XV. 731. 7. (1865) ; Hunter, Trans. Amer. Entom. Soc. Philad., XXVII. 121. (1901).

maculipennis BLANCH. in GaY: Hist. fis. y polit. de Chile, Zool., VII. 400. 1. (1852); Rond., Archivio per la Zool., III. 87. [Oplachantha] (1863); PHil., Verh. zool.-bot. Ges. Wien, XV. 729. 1. (1865) ; Hunter, Trans. Amer. Entom. Soe. Philad., XXVII. 121. (1901).

mexicana Bell., Saggio di Ditterolog. Messic., I. 20. 1. tab. I. fig. 6. (1859) ; Roxd., Archivio per la Zoolog., III. 87. [Oplachantha] (1863) ; ? Willist., Canad. Entomol., XVII. 123. (1885); Gigl.Tos, Bollet. Mus. Zool. ed Anat. comp. Torino, VI. No. 108., 2. (1891) et Mem. R. Accad. Sci. Nat. Torino, ser. 2. XI.IIl. 101. 1. (1893) ; Hunter, Trans. Amer. Entom. Soc. Philad., XXVII. 121. (190I); Meland., Canad. Entomol., XXXVI. 15. (1904); Aldor., Catal. North Amer. Dipt., 173. (1905).

Tasmania.

Europa centr. et sept.

America mer.

Australia,

Tasmania.

Arch.Indicus.

Mexico.

Chile.

Chile.

Chile.

Chile.

America. 
bellula Willist., Trans. Amer. Entom. Soc. Philad., XV. 245. 2. (1888).

micans Hutron, Trans. New Zealand. Instit., XXXIII. 6. (1901) et XXXIV. 169. (1901).

modesta PhiL., Verh. zool.-bot. Ges. Wien, XV. 730. 4. (1865); Hunter, Trans. Amer. Entom. Soc. Plilad., XXVII. 122. (1901).

Morrisii DALE, Entomologist, 1842. 175. 75. (1842); WALK., Insecta Britann., Dipt., I. 12. 5. (1851); WahlbG., Öfvers. Kongl. Vet. Akad. Förhandl., XI. 211. 1. (1854); WALK., List Dipt. Brit. Mus., V. suppl. 1., 9. 5. (1854); Schin., Verh. zool.-bot. Ver. Wien, V. 656. 5 et 674. (1855) ; ZeTT., Dipt. Scand., XII. 4552. 2-3. (1855) ; Lw., Zeitschr. f. d. Ges. Naturwiss., X. 99. 1. (1857) ; Schin., Fauna Austriaca, Dipt., I. 24. (1862) ; JAENN., Berlin. Entom. Zeitschr., X. 234. 53. (1866); v. D. Wulp, Tijdschr. v. Entomol., XXIV. 153. 1. (1881); BuUnerTi, The Entomologist, XXII. 134. 5. (1889); Schocr, Fauna Insect. Helvetiae, Dipt., 26. (1890); É. \& L. Coucke, Annal. Soc. Entom. Belgique, XXXVII. 435. (1893); SтroвL, Mittheil. Naturwiss. Ver. Steiermark, XXIX. 1892. 12. (1893) et Glasnik Zem. Mus. Boni i Herzegov., X. 391. (1898) ; KEnT., Pótfüz. a Természettud. Közlönyhöz, XXXII. 131. (1900) ; StroвL, Wissensch. Mittheil. aus Bosnien u. Herzegov., VII. 555. (1900); Aldr., Catal. North Amer. Dipt., 173. (1905).

pallipes Lw., Stettin. Entom. Zeitg., VII. 284. 5. (1846); Schоцтz, Zeitschr. f. Entom. Breslau, IV. No. 16., 35. 2. (1850).

nigra MEIG. = fuscipes MeIG.

nigritarsis LATR. = vallata FoRsT.

nigroptera Fourcr. = clavipes L.

nitidithorax MACQ., Dipt. exot., suppl. 4., 41. 8. tab. III. fig. 3. (18ł9). Tasmania. obscura NEIG. = chalybeata ForST.

pallipes $\mathrm{Lw} .=$ Morrisii DALE.

parvidentata Macq., Dipt. exot., suppl. 4., 40. 6. tab. III. fig. 1. Tasmania. (1819).

pulchella Wilisist., Trans. Amer. Entom. Soc. Philad., XV. 245. 3. Brasilia. (1888) ; Hunter, Trans. Amer. Entom. Soc. Philad., XXVII. 122. (1901).

quadridentata WaLk., List Dipt. Brit. Mus., I. 127. (1848) et V. suppl. 1., 10. 8. (1854).

quinquecella Macq., Dipt. exot., suppl. 1., 47. 2. tab. V. fig. 2. Tasmania.

(1846) ; WaLk., List Dipt. Brit. Mus., V. suppl. 1., 12. 17. (1854): rex PODA $=$ ? vallata Fonst.

sexdentata FABr. = chalybeata Forst.

substituta Walk., List Dipt. Brit. Mus., V. suppl. 1., 12. 18. (1854); Hutron, Catal. New Zealand Dipt. etc., 34. [Actina] (1881) et Trans. New Zealand Instit., XXXIII. 6. (1901).

thoracica Puil., Verh. zool.-bot. Ges. Wien, XV. 730. 5. (1865) ; Hunter, Trans. Amer. Entom. Soc. Philad, XXVII. 122. (1901).

N. Selandia.

Chile. 
trichonota PHrL, Verb. zool.-bot. Ges. Wien, XV. 730. 3. (1865) ; Hunter, Trans. Amer. Entom. Soc. Philad., XXVII. 122. (1901).

tricolor Wied., Aussereurop. zweifl. Ins., I. 831 et 575. 5. (1828); Lw., Stettin. Entom. Zeitg., VII. 305. 9. (1846); WaLK., List Dipt. Brit. Mus., V. suppl. 1., 11. 13. (1854); Hunter, Trans. Amer. Entom. Soc. Philad., XXVII. 122. (1901).

vallata Forst., Nov. Spec. Insect., Cent. I. 96. 96. [Musca] (1771) ; Gmel., Systema Naturae, V. 2837. 166. [Musca] (1792); Meig., Klassif., I. 119. b. [Actina] (180t) et System. Beschreib., II. 5. 6. (1820); St. Farg. et Sirv., Encyclop. Méthod., X. 803. 3. (1825) ; MacQ., Recueil Soc. Sci. Agricult. Lille, 1826. 459. 5. (1826) ; Curr., Brit. Entomol., VIII. 337. (1830) ; Macq., Suit. à Buffon, I. 233. 7. (1834); Zetr., Dipt. Scand., I. 133. 4. (1842); Lw., Stettin. Entom. Zeitg., VII. 222. 1. (1846); Gimm., Bull. Soc. Imp. Nat. Moscou, XX. 1., 175. 1. (1847); WalK., List Dipt. Brit. Mus., I. 124. (1848) ; Zетт., Dipt. Scand., VIII. 2950. 4. (1849); L. Duf., Mém. Acad. des Sci., Math. et Phys., XI. 171-360. tab. V. fig. 47-49, 51. (1850); Schoutz, Zeitschr. f. Entomol. Breslau, IV. No. 16., 36. (1850); Walk., Insecta Britann., Dipt., I. 11. 2. (1851) et List Dipt. Brit. Mus., V. suppl. 1., 8. 2. (1854) ; Schin., Verlı. zool.-bot. Ver. Wien, V. 654. 2 et 674. (1855) ; Buanch., Hist. d'Ins., III. 592. (1860); Schin., Fauna Austriaca, Dipt., I. 24. 2. (1862); Jaknv., Berliu. Entom. Zeitschr., X. 234. 51. (1866) ; v. D. Wulp, Diptera Neerland., I. 443. 3. tab. XIV. fig. 1-2. (1877); JaroschefF., Trudy Kharkoff, XI. 341. 7. (1877); Neuhaus, Diptera Marchica, 39. 1. (1886) ; Brunetir, The Entomologist, XXII.133.2. (1889); Schoch, Fauna Insect. Helvetiae, Dipt., 26. (1890); Grard, Compt. Rend. Soc. Biolog. Paris, n. sér. IV. 43. (1892) ; É. \& L. Covcke, Aunal. Soc. Entom. Belgique, XXXVII. 435. (1893).

clavipes MeIG., Klassif., I. 117. 2. [Actina] (1804).

nigritarsis Latr., Hist. Nat. d. Crust. et d. Ins., XIV. 340. 1. (1804) ; Lam., Hist. Nat. anim. sans vertébr., III. 385. 3. [Xylophagus] (1816).

? rex Poda, Ins. Mus. Graec., 114. 2. [Musea] (1761); Schin., Verh. zool.-bot. Ver. Wien, VI. 402. (1856).

violacea Hutron, Trans New Zealand Instit., XXXIII. 6. (1901). viridiventris PhIL., Verh. zool.-bot. Ges. Wien, XV. 731. 8. (1865) ; Hunrer, 'Trans. Amer. Entom. Soc. Philad., XXVII. 122. (1901).

Chile.

Brasilia.

Europa.

N. Selandia. Chile.

\section{Hadrestia}

Thoms., Eugenies Resa, Dipt., 453. (1869).

aenea Thoмs., Eugenies Resa, Dipt., 454. 15. tab. IX. fig. 3. (1869); Patagonia.

E. L. Arrin., Bolet. Acad. Nat. Cienc. Córdoba, IV. 125. 41. (1882) ; IIUnter, Trans. Amer. Enton. Soc. Philad., XXVII. 122. (1901). 


\section{Berismyia}

Gigi.,'Tos, Bollet. Mus. Zool. ed Anat. comp. Torino, VI. No. 108., 2. fig. (1891). fusca Gigl.-Tos, Mem. R. Accad. Sci. Nat. Torino, ser. 2. XIIII. 103. Mexico.

3. tab. I. fig. 2. (1893); Aldr., Catal. North Amer. Dipt., 174. (1905).

nigrofemorata Willis', Biolog. Central. Amer., Dipt., I. 230. 1. tab.

IV. fig. 7. (1900); A A.dr., Catal. North Amer. Dipt., 174. (1905).

\section{Heteracanthia}

Macq., Dipt. exot., suppl. 4., 43. (1849).

mexicana Gigl.-Tos, Bullet. Mus. Zool. ed Anat. comp. Torino, VI. Mexico.

No. 102., 1. (189l) et Mem. R. Accad. Sci. Nat. Torino, ser. 2.

XLIII. 104. 4. (1893) ; Aldr., Catal. North Amer. Dipt., 174. (1905).

ruficornis MACQ., Dipt. exut., suppl. 4., 44. tab. V. fig. 2. (1849); Columbia.

Schin., Novara Reise, Dipt., 71.52. (1868) ; Hunter, Trans. Amer.

Entum. Soc. Philad., XXVII. 121. (1901).

\section{Allognosta}

Ost.-SACK., Borlin. Entom. Zeitschr., XXVII. 297. (1883).

Metoponia Lw.(nee MAcq.), Dipt.-Fauna Südafrika's, I.1.(1860).

brevis WaLK, = fuscitarsis SAY.

dorsalis $\mathrm{SAY}=$ fuscitarsis $\mathrm{SAY}$.

fuscitarsis SAY, Journ. Acad. Nat. Sc. Philad., III. 29. [Beris] (1823) America sept.

et VI. 155. [Beris] (1829) et Compl. Writt., II. 52 et 353. [Beris]

(1859) ; Meland., Canad. Entomol., XXXVI. 15. (1904); Aldr.,

Catal. North Amer. Dipt., 174. (1905).

brevis Walk., List Dipt. Brit. Mus., I. 127. [Beris] (1818) et V. suppl. 1., 10. 10. [Beris] (1854).

dorsalis SAY, Long's Exped. to St. Peter's River, II. Append., 377. 2. [Sargus] (1824) et Compl. Writt., I. 257. 2. [Sargus] (1859) ; Wied., Aussereurop. zweill. Ins., I. 540. 3. [Beris] (1828); Lw., Stettin. Entom. Zeitg., VII. 306. 11. [Beris] (1846); Walk., List Dipt. Brit. Mus., V. suppl. 1., 10. 12. [Beris] (1854).

lata Walk., List Dipt. Brit. Mus., I. 127. [Beris] (1848) et V. suppl. 1., 10. 9. [Beris] (1854); v. D. WuLP, Tijdschr. v. Entomol., ser. 2. II. (X). 1867. 12. [Beris] (1868).

pallipes W1ED., Aussereurop. zweifl. Ins., II. 41. 27. [Sargus] (1830); Walk., List Dipt. Brit. Mus., V. suppl. 1., 90. 11. [Sargus] (1854). 
obscuriventris Lw., Berlin. Entom. Zeitschr., VII. 299. 45. [Metoponia] (1863); Meland., Canad. Entomol., XXXVI. 15. (1904); Aldr., Catal. North Amer. Dipt., 174. (1905).

pallipes WIED. = fuscitarsis SAY.

similis Lw., Berlin. Entom. Zeitschr., VII. 299. 44. [Metoponia] (1863) ; Meland., Canad. Entomol., XXXVI. 15. (1904); Aldr., Catal. North Amer. Dipt., 174. (1905).

vagans Lw., Beschreib. europ. Dipt., III. 71. 35. [Metoponia] (1873). Europacentr. et sept.

\section{Hexodonta}

Rond., Dipterol. Ital. Prodr., I. 169. 1. (185̌6). [Exodontha]. Acanthomyia Schis., Wien. Entom. Monatschr., IV. 49. (1860). Exodonta Verr. ap. Scudd., Nomencl., 124. (1882).

Hexodonta Mı, Wien. Entom. Zeitg., XVI. 40. (1897).

coerulans WiED. = dubia ZETT.

dubia Zeтr., Insecta Lappon., Dipt., 512. 1. [Beris] (1838) et Dipt. Europa. Scand., I. 131. 1. [Beris] (1842); Lw., Stettin. Entom. Zeitg., VII. 286. 6. [Beris] (1846); Zetr., Dipt. Scand., VIII. 2948. 1. [Beris] (1849); Scholtz, Zeitschr. f. Entomol. Breslau, IV. No. 16., 1. [Beris] (18j0) ; Walk., List Dipt. Brit. Mus., V. suppl. 1., 9. 6. [Beris] (1854); Scurn., Verh. zool.-bot. Ver. Wien, V. 656. 7 et 674. [Beris] (1855), Wien. Entom. Monatschr., IV. 49. [Acanthomyia] (1860) et Fauna Austriaca, Dipt., I. 23. [Acanthomyia] (1862) ; Slebкe, Nyt Magaz. f. Naturvid., XIX. 76. [Acanthomyia] (1872) ; v. D. Wulp, Diptera Neerland., I. 444. [Acanthomyia] (1877); Beck., Berlin. Entom. Zeitschr., XXXI. 105. 17. [Acanthomyia] (1887) ; Poк., Verh. zool.-bot. Ges. Wien, XXXIX. 547. [Acanthomyia] (1889); Sсносн, Fauna Ins. Helvetiae, Dipt., 26. [Acanthomyia] (1890); Griff., Bollet. Mus. Zool. ed Anat. comp. Torino, XI. No. 245., 3. [Exodontha] (1896); Mıк, Wien. Entom. Zeitg., XVI. 40. [Acanthomyia] (1897); Kert., Pótfüz. a Természettud.

Közlönyhöz, XXXII. 130. [Acanthomyia] (1900).

coerulans WiED, in collect.

pedemontana BELL. apud Rond.

pedemontana BELL. = dubia ZE'TT.

\section{Actina}

MeIG., Klassif., I. 116. XXVI. (1804).

chalybea MEIG. = nitens LATr.

elegans Schis., Nuvara Reise, Dipt., 71. 54.(1868); Hunter, Trans. Chile.

Amer. Entom. Soc. Philad., XXVII. 121. (1901).

femoralis MEIG. = nitens LATR.

flavofemorata MFIG. = nitens LATR.

Kertész: Catalogus Dipterorum. III. 
hirsuta MacQ. $=$ nitens LATR.

nigripes MEIG. = nitens LATR.

nitens Latr., Hist. Nat. d. Crust. et d. Ins., XIV. 341. 2. [Beris] Europa. (1804) ; MeIg., System. Beschreib., II. 2. 1. [Beris] (1820); Sr. Farg. ex Serv., Encyclop. Méthod., X. 802. 1. [Beris] (1825); Macq., Recueil Soc. Sci. Agricult. Lille, 1826. 457. 1. [Beris] (1826) et Suit. à Buffon, I. 231. 1. [Beris] (1834); Lw., Stettin. Entom. Zeitg., VII. 287 et 301. 7. [Beris] (1846); WALK., List Dipt. Brit. Mus., I. 126. [Beris] (1848) ; Zetr., Dipt. Scand., VIII. 2950. Obs. 2. [Beris] (1849) ; Schin., Verh. zool.-bot. Ver. Wien, V. 657. 8 et 675. [Beris] (1855) et Fauna Austriaca, Dipt., I. 25. (1862) ; Jaenn., Berlin. Entom. Zeitschr., X. 235. 54. (1866); v. D. Wulp, Diptera Neerland., 444. nota. (1877); Becher, Denkschr. Akad. Wien, XLV. 140. tab. II. fig. 10. (1882); Scноch, Fauna Insect. Helvetiae, Dipt., 26. (1890); É \& L. Coucke, Annal. S̄oc. Entom. Belgique, XXXVII. 436. (1893); Kent., Pótfüz. a Természettud. Közlönyhöz, XXXII. 131. (1900).

chalybea Meig., Klassif., I. 117. 1. (1804).

femoralis Meig., System. Beschreib., II. 6. 7. [Beris] (1820);

St. Farg. et Serv., Encyclop. Méthod., X. 803. 4. [Beris] (1825) ; Curt., Brit. Entomol., VIII. 337. [Beris] (1830).

flavofemoratr MeIG., System. Beschreib., II. 8. 10. tab. XII. fig. 6. [Beris] (1820) ; St. Farg. Ет Serv., Encyclop. Méthod., X. 803. 1. [Beris] (1825).

hirsuta MacQ, Suit. à Buffon, I. 232. 3. [Beris] (1834) ; Meig., System. Bescinreib., VII. 56. 13. [Beris] (1838).

nigripes Meig., System. Beschreib., II. 7. 9. [Beris] (1820); S't. Farg. et Serv., Encyclop. Méthod., X. 803. 6. [Beris] (1825).

octodentata MEg. in litt. apud MeIG.

scutellata Meig., Klassif., I. 119. c. (1804).

? similis Fonst., Nov. Spec. Ins., Cent., I. 97. 97. [Musca]

(1771) ; Grel., Systema Naturae, V. 2837. 167. [Musca] (1792);

Curt., Brit. Entomol., VIII. 337. [Beris] (1830); WaLk., List

Dipt. Brit. Mus., V. suppl. 1., 14. 3. (1854).

octodentata MEG. = nitens LATr.

scutellata MEIG. = nitens LATR.

similis FoRst. = ? nitens LATR.

viridis SAY, Long's Exped to St. Peter's River, II. Append., 368.

America sept.

[Beris] (1824) et Compl. Writt., I. 251. [Beris] (1859); Wied., Aussereurop. zweifl. Ins., I. 83. 2. [Beris] (1828); Lw., Stettin. Entom. Zeitg., VII. 305. 10. [Beris] (1846); WaLK., List Dipt. Brit. Mus., V. suppl. 1., 10. 11. [Beris] (1854); Meland., Canad. Entomol., XXXVI. 15. [Beris] (1904); Aldr., Catal. North Amer. Dipt., 174. (1905). 


\section{Scoliopelta}

Willis's., Entomol. Amer., I. 152 et 154. (1885).

luteipes Wils.1st., Entomol. Amer., I. 154. (1885); Aldr., Catal. Americasept. North Amer. Dipt., 175. (1905).

\section{Chorisops}

Rond., Archiv. per la Zool. Modena, III. 87. (1863).

Chlorisops Brau., Denkschr. Akad. Wien, XLIV. 72. (1882).

hyaliniventris A. CostA = tibialis MeIG.

Philippii Rond., Archivio per la Zoolog., III. 88. (1863); Hunter, Chile.

Trans. Amer. Entom. Soc. Philad., XXVII. 121. (1901).

tibialis MeIG., System. Beschreib., II. 3. 2. tab. XII. fig. 8. [Beris] Europacentr. (1820); St. Farg. et Serv., Encyclop. Méthod., X. 802. 2. [Beris] et mer. (1825) ; Macq., Recueil Soc. Sci. Agricult. Lille, 1826. 457. 2. tab. III. fig. 5. (1826) ; CurT., Brit. Entomol., VIII. 337. [Xylophagus] (1830) ; Macq., Suit. à Buffon, I. 232. 2. tab. V. fig. 15. [Beris] (1834) ; Lw., Stettin. Entom. Zeitg., VII. 304. 8. [Beris] (1846); Walk., List Dipt. Brit. Mus., I. 126. [Beris] (1848), Insecta Britann., Dipt., 'I. 13. 1. tab. I. fig. 2. [Actina] (1851) et List Dipt. Brit. Mus., V. suppl. 1., 13. 1. [Actina] (1854); Schin., Verh. zool.-bot. Ver. Wien, V. 658. 9 et 675. [Beris, Actina] (1855) et Fauna Austriaca, Dipt., I. 25. [Actina] (1862); JAENi., Berlin. Enton. Zéitschr., X. 235. 55. [Actina] (1866) ; v. D. WulP, Diptera Neerland., I. 444. tab. XIV. fig. 3. [Actina] (1877); A. Handu., Verh. zool.-bot. Ges. Wien, XXXIII. 243. fig., 1-3. [Chlorisops] (1883) ; BRunettr, The Entomologist, XXII. 134. 1. [Actina] (1889) ; É. \& L. Coucke, Annal. Soc. Entom. Belgique, XXXVII. 436. [Actina] (1893); Kert., Pótfüz. a Természettud. Közlönyhöz, XXXII. 77. (1900).

hyaliventris A. Costa, Il Giamb. Vico Napoli, II, 455. [Actina] (1857).

\section{Neoexaereta}

Ost.-SAck., Catal. Dipt. N. Amer. Ed. II. 44. (1878). [Neoexaireta].

Diphysa Macq., Dipt. exot., I. 1., 172. 3. (1838), praeoce.

Exaereta Schiv., Verh. zool.-bot. Ges. Wien, XVII. 309. (1867)

[Exaireta], praeocc.

albimacula $\mathrm{W}_{\mathrm{ALK}}=$ spiniger $\mathrm{W}_{\mathrm{IED}}$.

alpina Hutros, Trans. New Zealand Instit., XXXIII. 5. '[Exaireta] N. Selandia. (1901).

analis Nov. = apicalis WALK.

apicalis BUTLER = apicalis WALK. 
apicalis WaLK., List Dipt. Brit. Mus., IV. 1151. [Diphysa] (1849) et V. suppl. 1., 7. 2. [Diphysa] (1854); Hutron, Catal. New Zealand Dipt. etc. 34. [Diphysa] (1881) et Trans. New Zealand Instit., XXXIII. 4. [Exaireta] (1901).

analis Now., Memoiren der Krakauer k. k. Akad. d. Wiss., II. $p$ ? [Exaireta] (1875) et Beitr. z. Kenntn. d. Dipterenfauna Neu-Seelands, 11. [Exaireta] (1875); Hutron, Catal. New Zealand Dipt. etc., 36. [Exaireta] (1881); Mıк, Wien. Entom. Zeitg., IX. 290. [Exaereta] (1890).

apicalis ButLer, Zool. of the Voyage of Errebus \& Terror, Ins., 27. [Diphysa], tab. VII. fig. 17. [Beris] (1874).

eupodata Bı́., Annal. Soc. Entom. France, sér. 5. IX. 198. 1. Chile. [Exaireta] (1879) ; Hunter, Trans. Amer. Entom. Soc. Philad., XXVII. 122. [Exaireta] (1901).

hyacinthina Big., Annal. Soc. Entom. France, sér. 5. IX. 199. 2. Chile. [Exaireta] (1879) ; Hunter, Trans. Amer. Entom. Soc. Philad., XXVII. 122. [Exaireta] (1901).

longicornis Schin., Novara Reise, Dipt., 73. 56. [Exaireta] (1868); IIunten, Trans. Amer. Entom. Soc. Philad., XX VII. 122. [Exaireta] (1901).

? maculiventris*) Macq., Dipt. exot., suppl. 4., 43. 1. tab. V. fig. Natal. 5. [Diphysa] (1849).

opposita WALK., List Dipt. Brit. Mus., V. supnl. 1., 13. 2. [Actina] (1854) ; Hutron, Catal. New Zealand Dipt. etc., 35. [Actina] (1881) et Trans. New Zealand Instit., XXXIII. 5. [Exaireta] (1901).

Philippii Brg., Annal. Soc. Entom. France, sér. 5. IX. 199. 3 [Exaireta] (1879); Hunter, Trans. Amer. Entom. Soc. Philad., XXVII. 122. [Exaireta] (1901).

rufipalpis Wied., Aussereurop. zweifl. Ins., II. 619. 10. [Xylophagus] (1830) ; Macq., Dipt. exot., I. 1., 172. [Diphysa] (1838); Lw., Stettin. Entom. Zeitg., VII. 306. 13. [Beris] (1846); WALK., List Dipt. Brit. Mus., V. suppl. 1., 7. 3. [? Diphysa] (1854); Scmun., Novara Reise, Dipt., 72. [Exaireta] (1868); ALDR., Catal. North Amer. Dipt., 174. (1905).

Servillei MACQ. = spiniger WIED.

? sexspinosa**) MACQ., Dipt. exot., suppl. 1., 46.1. tab. V. fig.1. [Diphysa] (1846); WaLk., List Dipt. Brit. Mus., V. suppl. 1., 7. 4. [? Diphysa] (1854); Lw., Dipt.-Fauna Südafrika's, I. 2. [??Diphysa] (1860) ; Hunter, Trans. Amer. Entom. Soc. Philad., XXVII. 122. [Beris] (1901).

spiniger WiED., Aussereurop. zweifl. Ins., II. 618. 2. [Xylophagnes] (1830) ; Macq., Dipt. exot., J. 1., 172. [Diphysa] (1838); Lw.,

*) Species verosimiliter ad genus novum subfam. Pachygastrinarum pertinet.

**) Species verosimiliter ad genus novum subfam. Pachygastrinarum pertinet.
N. Selandia.

Chile.

N. Selandia.

Chile.

Mexico.

Brasilia.

Australia,

N. Selandia,

I. Hawaii. 
Stettin. Entom. Zeitg., VII. 306. 12. [Beris] (1816); WALK., List Dipt. Brit. Mus., IV. 1152. [Diphysa] (1849) et V. suppl. 1., 7. 1. [Diphysa] (1854) ; Lw., Dipt.-Fauna Südafrika's, I. 2. [Diphysa] (1860); Schiv., Novara Reise, Dipt., 73. [Exaireta] (1868); Hutton, Catal. New Zealand Dipt. etc., 35. [Exaireta] (1881); Kirby, Annal. Mag. Nat. Hist., ser. 5. XIII. 457. 1. [Sargus] (1884); Hudson, Manual of New Zealand Entom., 56. tab. VI. fig. 5. [Exaireta] (1892); Hutton, Trans. New Zealand Instit., XXXIII. 4. [Exaireta] (1901); Grimshaw, Fauna Hawaiiensis, III. 79. 1. (1902). albimacula WaLk., List Dipt. Brit. Mus., I. 126. [Beris] (1848). Servillei Macq., Dipt. exot., I. 1., 172. 1. tab. XXI. fig. 1.

[Beris] (1838) ot suppì. 1., 47. 1. [Beris] (1846); Rond., Archivio per la Zoolog., III. 87. [Chorisops] (1863); J AENN., Abhandl. Senckenb. Naturf. Ges, VI. 326. [? Actina] (1867).

Straznitzkii Now., Memoiren d. Krakauer Akad. d. Wiss., II. (1875) et Beitr. z. Kenntniss d. Dipterenfauna Neu-Seelands, 14. [Exaireta] (1875); Hutron, Catal. New Zealand Dipt. etc., 36. [Exaireta] (1881); Mik, Wien. Entom. Zeitg., IX. 290. (1890); Hutron, Trans. New Zealand Instit., XXXIII. 4. [Exaireta] (1901).

\section{ERINNIDAE.}

\section{Rhachicerus}

Walk., List Dipt. Brit. Mus., V. suppl. 1., 103. 1. [Rachicerus] (1854).

Antidoxion Sn. v. Vollenh., Versl. en Meded. d. Kongl. Akad. van Wetensch., Afd. Natuurk., XV. 1. (1863).

? Rhyphomorpha WALk., Proc. Linn. Soc. London, V. 275. (1861).

bellus Ost.-SAck., Biolog. Central. Amer., I. 62. 1. (1886); Aldr., America centr. Catal. North Amer. Dipt., 211. (1905).

bilinea Walk., Proc. Linn. Soc. London, V. 275. 19. [Rhyphomorpha] Batjan. (1861) ; v. D. Wulp, Catal. Dipt. South Asia, 46. [Rhyphomorpha] (1896).

fulvicollis WaLk., List Dipt. Brit. Mus., V. suppl. 1., 104. 1. (1854); America sept. Aldr., Catal. North Amer. Dipt., 212. (1905).

fulvicornis Snell. v. Vollenh., Versl. en Meded. d. Kongl. Akad. Java, van Wetensch., Afd. Natuurk., X.V. 6. tab., fig. 1-3. [Antidoxion] (1863) ; Ost.-SAck., Annal. Mus. Civ. Genova, XVI. 409. nota. (1882) ; v. D. Wulp, Catal. Dipt. South Asia, 46. (1896).

honestus Osт.-SAck., Bullet. Unit. Stat. Geolog. Surv., III. 211. Americasept. (1877) ; Aldr., Catal. North Amer. Dipt., 212. (1905).

nigrinus WANdolL., Entomol. Nachricht, XXIII. 290. (1897). Sumatra.

nigripalpus Lw.; Berlin. Entom. Zeitschr., XVIII. 378. 1. (1874); Mexico. Aldr., Catal. North Amer, Dipt., 212. (1905). 
nitidus Johns., Entomol. News Philad., XIV. 22. (1903); ALdr., America sept. Catal. North Amer. Dipt., 212. (1905).

obscuripennis Lw., Berlin. Entom. Zeitschr., VII. 4. 6. (1863) et XVIII. 378. Anmerk. (1874); Aldr., Catal. North Amer. Dipt., 212. (1905).

tristis Lw., Beschreib. europ. Dipt., I. 24. 23. (1869).

Hispania.

varipes Lw., Berlin. Entom. Zeitschr., VII. 5. 7. (1863) ; Aldr., Catal. North Amer. Dipt., 212. (1905).

zonatus Ost.-Sack., Annal. Mus. Civ. Genova, XVI. 408. (1881); Sumatra. v. D. Wulp, Catal. Dipt. South Asia, 46. (1896).

\section{Erinna}

Meig., Nouvelle Classification, 21. 26. (1800).

Pachystomus Latr., Gen. Crust. et Ins., IV. 286. DCLXVIII. (1809).

Xylophagus Me1G., Illig. Magaz. f. Ins., II. 266. 37. (1803).

abdominalis Lw., Berlin. Entom. Zeitschr., XIII. 163. 64. [Xylophagus] (1869) ; Johnson, Entomol. News Philad., XIV. 23. fig. 1-2. [Xylophagus] (1903); Adams, Kansas Univ. Sci. Bullet., II. 435. [Xylophagus] (1904); Aldr., Catal. North Amer. Dipt., 212. [Xylophagus] (1905).

atra FALL. p. p. = cincta DEG.

atra MeIG., Klassif., I. 154. 1. tab. IX. fig. 1-2. [Xylophagus] (1804); FABr., Systema Antliat., 64. 1. [Xylophagus] (1805); Fall., Dipt. Suec. Xylophagei, 13. 1. p. p. [Xylophagus] (1817); Cuv., Règno Animal., III. 622. [Xylophagus] (1817) ; Meig., System. Beschreib., II. 11. 1. tab. XII. fig. 14. [Xylophagus] (1820) ; St. FArG. ET SERv., Encyclop. Méthod., X. 800.1. [Xylophagus] (1825); MacQ., Recueil Soc. Sci. Agricult. Lille, 1826. 462.1. [Xylophagus] (1826); MeIG., System. Beschreib., VI. 318 et 319. [Xylophagus].(1830) ; Ruthe, Isis, 1831. XI. 1213. [Xylophagus] (1831); MAcQ., Suit. à Buffon, I. 229. 1. tab. V. fig. 13. [Xylophagus] (1834); ZETT., Insecta Lappon., Dipt., 511.1. [Xylophagus] (1838) ; BLanch., Hist. d'Ins., III. 592. [Xylophagus] (1840); ZETT., Dipt. Scand., I. 128. 1. [Xylophagus] (1842); Drewsen, Naturhist. Tidsskr., IV. 103. [Xylophagus] (1842) ; Gimm., Bullet. Soc. Imp. Nat. Moscou, XX. 1., 176. 1. [Xylophagus] (1847); Lw., Stettin. Entom. Zeitg., VIII. 70. 9. [Xylophagus] (1847); WAlk., Annal. Mag. Nat. Hist., ser. 2. II. 75. [Xylophaga] (1848) et List Dipt. Brit. Mus., I. 128. [Xylophagus] (1848); ZETT., Dipt. Scand., VIII. 2947. 1. [Xylophagus] (1849); Scholtz, Zeitschr. f. Entomol. Breslau, IV. No. 16., 36. [Xylophagus] (1850); WaLk., Insecta Britann., Dipt., I. 33. 1. tab. I. fig. 10. [Xylophagus] (1851) et List Dipt. Brit. Mus., V. suppl. 1., 105. 1. [Xylophagus] (1854) ; Schin., Verh. zool.-bot. Ges. Wien, V. 659. 1 et 675. [Xylophagus] (1855); Kaltenb.,

America sept.

Europa. 
Verhandl. naturhist. Ver. preuss. Rheinl. u. Westfal., XVI. 241. 6. [Xylophagus] (1859); ZEтT., Dipt. Scand., XIIJ. 4949. 1. [Xylophagus] (1859) ; Bossd., Finland. tvaving. Ins., I. 114. 1. [Xylophagus] (1861) ; Schin., Fauna Austriaca, Dipt., I. 27. [Xylophagus] (1862); JAEnv., Berlin. Entom. Zeitschr., X. 235. 59. [Xylophagus] (1866); Damianitsch, Verh. zool.-bot. Ges. Wien, XVIII. 117. fig. [Xylophagus] (1868); Frauenteld, Verh. zool.-bot. Ges. Wien, XVIII. 166. [Xylophagus] (1868); BeLivg, Archiv f. Naturgesch., XLI. 1., 31. 1. [Xylophagus] (1875); White, Entom. Monthly Mag., XIII. 162. [Xylophagus] (1876); Becher, Denkschr. Alsad. Wien, XI.V. 141. tab. II. fig. 11. [Xylophagus] (1882); Schocr, Fauna Ins. Helvetiae, Dipt., 27. [Xylophagus] (1890); L. Covcke, Annal. Soc. Entom. Belgique, XXXVII. 133. [Xylophagus] (1893); Kerт., Pótfüz. a Természettud. Közlönyhöz, XXXII. 132. [Xylophagus] (1900); Leonard, Insetti Nocivi, III. 367. fig. 199. [Xylophagus] (1900).

compedita Wred. ap. Meig., System. Beschreib., II. 13. 3. [Xylophagus] (1820); S'r. FarG. et Serv., Encyclop. Méthod., X. 800. 3. [Xylophayus] (1825); Ruthe, Isis, 1831. XI. 1214. [Xylophagus] (1831); WaLK., List Dipt. Brit. Mus., V. suppl. 1., 105. 3. [Xylophagus] (1854).

basipunctata Watk., Trans, Entom. Soc. London, n. ser. IV. 121. Australia. [Xylophagus] (1857).

carbonaria Phis., Verh. zool.-bot. Ges. Wien, XV. 727. 1. [Xylophagus] (1865); Hunter, Trans. Amer. Entom. Soc. Philad., XXVII. 132. [Xylophagus] (1901).

cincta Deg., Mém. pour serv. l'hist. d. Ins., VI. 183. 6. tab. IX. fig. 19-21. [Nemotelus] (1776); Meig., Klassif., I. 155. a. [Xylophagus] (1804); FABr., Systema Antliat., 65. 2. [Xylophagus] (1805); LAtr., Gen. Crust. et Ins., IV. 272. [Xylophagus] (1809); Fal.., Dipt. Sueciae, Xylophagei, 14. [Xylophagus] (1817); Merg., System. Beschreib., IJ. 12. 2. [Xylophagus] (1820); ST. FARG. ET SERv.; Encyclop. Méthod., X. 800. 2. [Xylophagus] (1825); MacQ., Recueil Soc. Sci. Agricult. Lille, 1826. 462. 2. [Xylophagus] (1826); MeIG., System. Beschroib., VI. 318. [Xylophagus] (1830) ; Ruv11e, Isis, 1831. XI. 1213. [Xylophagus] (1831); Macq., Suit. à Buffon, I. 230. 2. [Xylophäarus] (1834); Zetт., Insecta Lappon., Dipt., 511. 2. [Xylophagus] (1838) et Dipt. Scand., I. 128. 2. [Xylophagus] (1842); Grмм., Bullet. Soc. Imp. Nat. Moscou, XX. 1., 177. 2. [Xylophagus] (1847); Lw., Stettin. Entom. Zeitg., VIII. 70. 9. [Xylophagus] (1847); ZETT., Dipt. Scand., VIII. 2947. 2. [Xylophagus] (1849); Scноцтz, Zeitschr. f. Entomol. Breslau, IV. No. 16., 36. [Xylophagus] (1850); WaLK., List Dipt. Brit. Mus., V. suppl. 1., 105. 2. [Xylophagus] (1854); Schis., Verh. zool.-bot. Ver. Wien, V. 629. 2 et 675. [Xylophagus] (1855); Bonsd., Finl. tvaving. Ins., I. 114. 2. [Xylophagus] (1861); Schin., Fauna Austriaca, Dipt., I. 27. [Xylophagus] (1862); KALTENB., Verh. d. naturhist. Ver. d. preuss. Rheinl. u. Westphal., 
XXI. 289. 131 et 336. 155. [Xylophagus] (1864); JAEnN., Berlin. Entom. Zeitschr., X. 235. 60. [Xylophagus] (1866); Perris, Annal. Sos. Entom. France, sér. 4. X. 202. 13. tab. III. fig. 70-79. [Xylophagus] (1870); Beling, Archiv f. Naturgesch., XLI. 1., 34. 2. [Xylophagus] (1875); White, Entom. Monthly Mag., XIII. 160. [Xylophagus] (1876) ; BraU., Denkschr. Akad. Wien, XLVII. tab. IV. fig. 80-83. [Xylophagus] (1883); Nevhaus, Diptera Marchica, 40. 1. [Xylophagus] (1886); L. Coucke, Annal. Soc. Entom. Belgique, XXXVII. 134. [Xylophagus] (1893) ; Kent., Pótfüz. a Természettud. Közlönyhöz, XXXII. 132. [Xylophagus] (1900).

atra FALl., Dipt. Sueciae, Xylophagei, 13. 1. var. 3. [Xylophagus] (1817).

subulata Panz., Fauna Germ., LIV. 23. [Empis] (1798); Meig., Klassif., I. 235. Anmerk. [? Rhagio] (1804); I.atr., Encyelop. Méthod., VIII. 623. 2. [Pachystomus] (1811).

syrphoides Panz., Fauna Germ., LXXVII. 19. [Rhagio] (1801); LAtr., Gen. Crust. et Ins., IV. 287. [Pachystomus] (1809) et Encyclop. Méthod., VIII. 623 1. [Pachystomus] (1811) ; LaM., Hist. nat. anim. sans vert., III. 422. 1. [Pachystoma] (1816); Cuv., Règne Animal., III. 616. [Pachystomus] (1817); Meig., System. Beschreib., II. 12. [Xylophagus] (1820) et VI. 319. [Xylophagus] (1830); Macq., Suit. à Buffon, I. 226. 1. [Pachystomus] (1834); MeIG., System. Beschreib., VII. 57. 1. [Pachystomus] (1838); Walk., List Dipt. Brit. Mus., V. suppl. 1., 114. 1. [Pachystomus] (1854); Schin., Verh. zool.-bot. Ver. Wien; V. 660. 1. [Pachystomus] (1855) et Fauna Austriaca, Dipt., I. 27. [Pachystomus] (1862) ; Kaltenb., Ver. d. naturhist. Ver. d. preuss. Rheinl. u. Westphal., XXI. 289. 132. [Pachystomus] (1864).

compedita WIED. = atra MEIG.

decora Wustist., Canad. Entomol., XVII. 121. [Xylophagus] (1885) ; Adams, Kansas Univ. Sci. Bullet., II. 435. [Xylophagus] (1904); Aldr., Catal. North Amer. Dipt., 212. [Xylophagus] (1905).

fasciata WAlk., List Dipt. Brit. Mus., I. 128. [Xylophagus] (1848) et V. suppl. 1., 110. 4. [Subula] (1854); Adams, Kansas Univ. Sci. Bullet., II. 435. [Xylophagus] (1901); Aldr., Catal. North Amer. Dipt., 212. [Xylophagus] (1905).

gracilis WrLLIST., Canad. Entomol., XVII. 121. [Xylophagus] (1885) ; Adams, Kansas Univ. Sci. Bullet., II. 435. [Xylophagus] (1904); Aldr., Catal. North Amer. Dipt., 212. [Xylophagus] (1905).

longicornis Lw., Berlin. Entom. Zeitschr., XIII. 163. 62. [Xylophagus] (1869) ; Adams, Kansas Univ. Sci. Bullet., II. 435. [Xylophagus] (1904); Aldr., Catal. North Amer. Dipt., 212. [Xylophagus] (1905).

lugens Lw., Berlin. Entom. Zeitschr., VII. 6. 8. [Xylophagus] (1863); Adams, Kansas Univ. Sci. Bullet., II. 435. [Xylophagus] (1904); Aldr., Catal. North Amer. Dipt., 212. [Xylophagus] (1905).

nitida ADAMs, Kansas Univ. Sci. Bullet., II. 435. [Xylophagus] (1904). persequa WALK., Insecta Saunders., Dipt., I. 1. [Xylophagus] (1850)

America sept.

America sept.

America sept

America sept.

America sept.

America sept. America sept. 
et List Dipt. Brit. Mus., V. suppl. 1., 106. 6. [Xylophagus] (1854); Adsмs, Kansas Univ. Sci. Bullet., II. 435. [Xylophagus] (1904); Aldr,, Catal. North Amer. Dipt., 212. [Xylophagus] (1905).

reflectens WaLk., List Dipt. Brit. Mus., I. 129. [Xylophagus] (1848) et V. suppl. 1., 110. 5. [Subula] (1854); Adams, Kansas Univ. Sci. Bullet., II. 435. [Xylophagus] (1904); Aldr., Catal. North Amer. Dipt., 212. [Xylophagus] (1905).

rufipes Lw., Berlin. Entom. Zeitschr., XIII 163. 63. [Xylophagus] (1869) ; Adavs, Kansas Univ. Sci. Bullet., II. 435. [Xylophagus] (1904); Aldr., Catal. North Amer. Dipt., 212. [Xylophagus] (1905). subulata PANz. = cincta DEG.

syrphoides PANz. = cincta DEG.

triangularis SAY, Journ. Acad. Nat. Sci. Philad., III. 30. [Xylophagus] (1823) et Compl. Wr., II. 52. [Xylophagus] (1859); Wied., Aussereurop. zweifl. Ins., I. 85. 2. [Xylophagus] (1828); WaLk., List Dipt. Brit. Mus., J. 128. [Xylophagus] (1848) et V. suppl.1., 106. 5. [Xylophagus] (1854); Apass, Kansas Univ. Sci. Bullet., II. 435. [Xylophagus] (1904); Aldr., Catal. North Amer. Dipt., 212 [Xylophagus] (1905).

? vetusta W ALK., List Dipt. Brit. Mus., V. suppl. 1., 106. 7. [Xylophagus] (1854).

\section{Solva}

WALk., Proc. Linn. Soc. London, IV. 98. (1860).

Macroceromys Big., Annal. Soc. Entom. France, sér. 5. VII. Bull., I,XXIII. (1877).

Subula Meg. ap. MeIG., System. Beschreib., II. 15. Anmerk. (1820) nес Sсhum., Moll., 1817.

Subula Omyia Willist., Manual of N. Amer. Dipt., 43 et IV. (1896).

Xylomyia Rond., Dipterol. Ital. Prodr., IV. 11. [Xylomya] (1861).

americana Wied., Dipt. exot., 51. [Xylophagus] (1821); ST. FARG. ET Serv., Encyclop. Méthod., X. 800. [Xylophagus] (1825); Wied., Aussereurop. zweifl. Ins., I. 84. 1. [Xylophagus] (1828); MacQ., Dipt. exot., I. 1., 171. [Subula] (1838); WALK., List Dipt. Brit. Mus., I. 128. [Xylophagus] (1843) et V. suppl. 1., 106. 4. [Xylophagus] (1854); Lw., Zeitschr. f. d. ges. Naturwiss., N. F. II. (XXXVI). 115. [Xylophagus] (1870); Jounson, Entomol. Nows Philad., XIV. 24. [Xylomyia] (1903); ALdr., Catal. North Amer. Dipt., 212. [Xylomyia] (1905).

aterrima Johns., Entomol. News Philad., XIV. 25. [Xylomyia] (1903) ; Aldr., Catal. North Amer. Dipt., 212. [Xylomyia] (1905).

atra LATr. = varia MEIG.

caffra Bia., Annal. Soc. Entom. France, sér. 5. IX. 195. 2. [Subula] (1879).
America sept.

America sept.

America sept.

Brasilia.

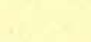


calopodata Big., Annal. Soc. Entom. France, sér. 5. IX. 195. 3. [Subula] (1879) ; v. D. Wurp, Catal. Dipt. South Asia, 46. [Subula] (1896). citripes $\mathrm{I}$. Duf. = varia MeIG.

elongata Ost.-SAck., Biolog. Central. Amer., Dipt., T. 22. 1. [Subula] (1886) ; Ar.Dr., Catal. North Amer. Dipt., 212. [Aylomyia] (1905).

fasciata SAY, Journ. Acad. Nat. Sci. Philad., VI. 155. [Xylophagus] (1829) et Compl. Wr., II. 353. [Xylophagus] (1859); Aldr., Catal. North Amer. Dipt., 212. [Xylomyia] (1905)

flavipes Dol., Natuurkund. Tijdschr. Nederl.Indie, ser. 4. III. (XVII). 85. 18. [Subula] (1858); Ost.-SAck., Annal. Mus. Civ. Genova, XVI. 408. [Subula] (1882); v. D. Wulp, Notes Leyden Mus., VII. 70. [Subula] (1885), Catal. Dipt. Soutl A sia, 46. [Subula] (1893) et Természetrajzi Füzetek, XXI. 409. 1. [Subula] (1898).

fulviventris Big., Annal. Soc. Entom. France, sér. 5. IX. 187. Mexico. [Macroceromys] (1879); Aldn., Catal. North Amer. Dipt., 213. [Xylomyia] (1905).

gracilicomis MuG. = maculata MeIG.

hybotoides Walk., Proc. Linn. Soc. London, VI. 5. 8. (1862) ; v. D. Wulp, Catal. Dipt. South Asia, 46. [Subula] (1896).

inamoena WALK., Proc. Iinn. Soc. London, IV. 98. 25. (1860); Ost.-Sack., Annal. Mus. Civ. Genova, XVI. 407. [Subula] (1882); v. D. Wulp, Notes Leyden Mus., VII. 70. 21. [Subula] (1885) et Catal. Dipt. South Asia, 46. [Subula] (1896).

maculata LATR. $=$ varia MEIG.

maculata Meig., Klassif., I. 154. 2. [Xylophagus] (1804); FABr., Systema Antliat., 65. 3. [Xylophagus] (1805); LAм., Hist. nat. anim. sans vert., III. 385. 1. [Xylophagus] (1816); FALL., Dipt. Sueciae, Xyloplıagei, 14. 2. [Xylophagus] (1817); Meig., System. Beschreib., II. 13. 4. tab. XII. fig. 15. [Xylophagus] (1820); Sт. FArg. ET Serv., Encyclop. Méthod., X. 800. 1. [Xylophagus] (1825); Macq., Recueil Soc. Sci. Agricult. Lillo, 1826. 463. 3. [Xylophagus] (1826) ot Suit. à Buffon, I. 230. 1. tab. V. fig. 14. [Subula] (1834) ; Wesmael, Annal. Soc. Entom. France, VI. Bullet. IXXXIX. [Xylophagus] (1837); Sahlbera, Acta Soc. Scien. Fenn., fase. 1. [Xylophagus] (1841); Zeтt., Dipt. Scand., I. 129. 3. [Xylophagus] (1842) et VIII. 2947. 3. [Xylophagus] (1849); Walk., Insecta Britann., Dipt., I. 34. 1. tab. I. fig. 11. [Subula] (1851) et List Dipt. Brit. Mus., V. suppl. 1., 109. 1. [Subulu] (1854); Schin., Verh. zool.-bot. Ver. Wien, V. 661. 2 et 675. [Subula] (1855) ; Bonsd., Finl. tvaving. Ins., I. 115. 3. [Xylophagus] (1861); Sculv., Fauna Austriaca, Dipt., I. 26. 1. [Subula] (1862) ; JAEnN., Berlin. Entom. Zeitschr., X. 235. 56. [Subula] (1866) ; Mernert, Fluernes Munddeele, Trophi Dipt., 51. tab. IV. fig. 1-5. [Subula] (1881); É. \& I. Coucke, Annal. Soc. Entom. Belgique, XXXVII. 435. [Subula] (1893); Austen, Annal. Mag. Nat. Hist., ser. 7. III. 181. [Xylomyia] (1899); Gorhan, Entom. Monthly Mag., XXXV. 71. [Xylomyia] (1899); Kert., Pótfüz. a Természettud. Közlönyhöz, XXXII. 132. [Subula] (1900).
Ternate.

Guatemala.

America sept.

Amboina,

Nova Guinea.

Gilolo.

Celebes, Java.

Europacentr. et sept. 
gracilicornis Meg. in litt. apud MeIa.

scutellata StEPH. in coll.

marginata MeIG., System. Beschreib., II. 15. 6. [Xylophagus] (1820);

St. FARG. ET SERv., Encyclop. Méthod., X. 800. 3. [Xylophagus]. (1825) ; Ruthe, Isis, 1831. XI. 1214. [Xylophagus] (1831); MacQ., Suit. à Buffon, I. 231. 3. [Subula] (1834); Wesu., Bullet. Acad. Sci. Bruxelles, IV. [Xylophagus] (1837); Duf., Annal. d. Scienc. Naturell., sér. 3. VI. tab. XVII. fig. 19, 21 et VII. 12. [Subula] (1847); Scholtz, Zeitschr. f. Entomol. Breslau, IV. No. 16., 36. [Xylophagus] (1850); Walk., List Dipt. Brit. Mus., V. suppl. 1., 110. 3. [Subula] (1854); Schin., Verh. zool.-bot. Ver. Wien, V. 661. 3 et 675. [Subula] (1855) et Fauna Austriaca, Dipt., I. 26. 2. [Subula] (1862); Kaltв., Verh. d. naturhist. Ver. d. preuss. Rheinl. u. Westphal., XXI. 336. 156. [Xylophagus] (1864); JAEnv., Berlin. Entom. Zeitschr., X. 235. 57. [Subula] (1866); Goureau, Annal. Soc. Entom. France, sér. 4. VII. Bull. LXXXVII. [Subula] (1867); Becher, Denkschr. Akad. Wien, XLV. 141. tab. II. fig. 12. [Subula] (1882); Neuhaus, Diptera Marchica, 40. 2. [Xylophagus] (1886); É. \& L. Coucke, Annal. Soc. Entom. Belgique, XXXVII. 435. [Subula] (1893) ; Austen, Annal. Mag. Nat. Hist., ser. 7. III. 183. nota. [Xylomyia] (1899); Kent., Pótfüz. a Természettud. Közlönyhöz, XXXII. 132. [Subula] (1900).

varia MacQ. (nec Meig.), Recueil Soc. Sci. Agricult. Lille, 1826. 463. 4. [Xylophagus] (1826).

nigritibialis Macq. in WeBB et Berth. : Hist. Natur. d'iles Canar., Entom., Dipt., 102. 12. [Subula] (1838) et Dipt. exot., I. 1., 171. 1. [Subula] (1838); WaLK., List Dipt. Brit. Mus., V. suppl. 1., 111. 7. [Subula] (1854).

pallipes Lw., Berlin. Entom. Zeitschr., VII. 6. 9. [Subula] (1863); Willist., Canad. Entomol., XVII. 122. [Subula] (1885); Towss., Entomol. News Philad., IV. 163. [Subula] (1893); BAKER, Entomol. News Philad., VI. 173. [Subula] (1895); Willist., Biolog. Central. Amer., Dipt., I. 230. 2. [Xylomyia] (1900); Aldr., Catal. North Amer. Dipt., 213. [Xylomyia] (1905).

parens Wrilist., Canad. Entomol., XVII. 122. [Subula] (1885); Hine, Canad. Entomol., XXXVI. 86. [Xylomyia] (1904); Alpr., Catal. North Amer. Dipt., 213. [Xylomyia] (1905).

rufiventris Big., Annal. Soc. Entom. France, sér. 5. IX. 194. 1. Natal. [Subula] (1879).

scutellata STEPH. = maculata MEIG.

tenthredinoides v. D. WuLP, Tijdschr. v. Entomol., ser. 2. II. (X). 1867. 132. 5. tab. III. fig. 5-7. [Subula] (1868); JoHsson, Entomol. News Philad., XIV. 24. [Xylomyia] (1903); Aldr., Catal. North Amer. Dipt., 213. [Xylomyia] (1905).

trinotata Big., Annal. Soc. Entom. France, sér. 5. X. 148. [Subula] (1880).

I. Canariae.

America sept. et centr.

Americasept.

America sept.

? Persia, ? Calleasus. varia MACQ. (nec MeIG.) = marginata MeIG. 
varia Meig., System. Beschreib., II. 14. 5. [Xylophagus] (1820); St. Europacentr. FArG. ET Serv., Encyclop. Méthod., X. 800. 2. [Xylophagus] et mer. (1825) ; v. Roser, Tübing. naturhist. Zeitschr., II. [Xylophagus] (1828); Feruss., Bullet. Sci. Natur., p.? [Xylophagus] (1829); MeIG., System. Beschreib., VI. 319. [Xylophagus] (1830) ; MacQ., Suit. à Buffon, I. 230. 2. [Subula] (1834); Walk., Insecta Britann., Dipt., I. 34. 2. tab. I. fig. 12. [Subula] (1851) et List Dipt. Brit. Mus., V. suppl. 1., 109. 2. [Subula] (1854); Scurv., Verh. zool.-bot. Ver. Wien, V. 660. 1 et 675. [Subula] (1855); HeEg., Sitzgsber. Akad. Wien, XXXI. 307. tab. III. fig. 1-9. [Xylophagus] (1858); Scuin., Fauna Austriaca, Dipt., I. 26. 2. [Subula] (1862); Jaenn., Berlin. Entom. Zeitschr., X. 235. 58. [Subula] (1866) ; LAвoulb., Annal. Soc. Entom. France, sér. 6. II. 313. [Subula] (1882); A. Handu., Verh. zool.-bot. Ges. Wien, XXXIII. 244. fig. 4. [Subula] (1883) ; É. \& L. Coucke, Annal. Soc. Entom. Belgique, XXXVII. 435. [Subula] (1893); Austen, Annal. Mag. Nat. Hist., ser. 7. III. 183. nota. [Xylomyia] (1899); Kert., Pótfüz. a Természettud. Közlönyhöz, XXXII. 132. [Subula] (1900); GiaRd, Bull. Soc. Entom. France, 1904. 88. [Subula] (1904).

atra Latr., Gen. Crust. et Ins., IV. 272. tab. XVI. fig. 9-10. [Xylophagus] (1809).

citripes L. Duf., Annal. d. Scienc. Natur., sér. 3. VI. tab. XVII. fig. 12-18 et VII. 5-14. [Subula] (1847); Walk., List Dipt. Brit. Mus., V. suppl. 1., 110. [Subula] (1854); Schrs., Verh. zool.-bot. Ver. Wien, V. 662. 4. [Subula] (1855); Laboulb., Annal. Soc. Entom. France, sér. 6. II. Bullet., XCVI. [Subula] (1882).

maculata Litr., Gen. Crust. et Ins., IV. 272. [Xylophagus] (1809).

varicolor BIG., Bullet. Soc. Zool. France, XVI. 276. [Subula] (1891). I. Canariae. vittata Dol., Natuurkund. Tijdschr. Nederl. Indie, ser. 4. III. (XVII). Amboina.

86. 19. [Subula] (1858); v. D. Wulp, Catal. Dipt. South Asia, 46. [Subula] (1896).

vittata WALK., Trans. Linn. Soc. London, XVII. 338. 20. [Xylo- Brasilia. phagus] (1837) et List Dipt. Brit. Mus., V. suppl. 1., 110. 6. [Subula] (1854).

\section{Bolbomyia}

Lw., Programm d. k. Realschule Meseritz, 1850, 39. (1850).

nana Lw., Berlin. Entom. Zeitschr., VI. 188. 5. (1862); Aldr., Catal. America sept, North Amer. Dipt., 213. (1905). 


\section{COENOMYIIDAE.}

\section{Coenomyiinae.}

\section{Coenomyia}

Latr., Précis d. caract. génér. d. Ins., 159. XV. (1796).

Caenomyia LATr. 1804. et auct. plur.

aurea MeIG. = ferruginea Scop.

australis Gnes. = ? ferruginea Scop.

bicolor $\mathrm{FABR}_{\mathrm{AB}}=$ ferruginea SCOP.

bidentata $\mathrm{FABR}_{\mathrm{AB}}=$ ferruginea Scop.

bispinosa $\mathrm{FABR}_{\mathrm{ABR}}=$ ferruginea ScoP.

cinereibarbis $\mathrm{BIG} .=$ ferruginea $\mathrm{Scop}$.

Crucis $\mathrm{FABR}_{\mathrm{AB}}=$ ferruginea SCOP.

errans FABR. = ferruginea Scop.

ferruginea Scop., Entomol. Carniol., 340. 913. [Musca] (1763) ; FABr.,

Supplem. Entomol. Syst., 555. 2. [Sicus] (1798); Schellenb.,

Europa,

Gattungen d. Fliegen, 32 et 33. tab. XXVI. fig. 1. [Sicus] (1803);

America

Meig., Klassif., I. 121. 1. tab. VII. fig. 11. [Sicus] (1804); Latr., Hist. Nat. d. Crust. et Ins., XIV. 326. (1804); FABr., Systema Antliat., 75. 2. [Sicus] (1805); LAM., Hist. nat. anim. sans vert., III. 417. 1. (1816); FALL., Dipt. Suec., Xylophagei, 12. 1. [Sicus] (1817) ; Cuv., Règno Animal., III. 616. [Sicus] (1817); MeIG., System. Beschreib., II. 18. 1. tab. XII. fig. 16-25. (1820) ; Dumér., Consid. Génér. Ins., tab. XLVIII. fig. 3. [Sicus] (1823); MAcQ., Recueil Soc. Sci. Agricult. Lille, 1826. 466. (1826) et Suit. à Buffon, I. 225. 1. tab. V. fig. 10. (1834); Blanch., Hist. d'Ins., III. 591. tab. II. fig. 5. (1840); ZETr., Dipt. Scand., I. 130. 1. [Sicus] (1842); Grmm., Bull. Soc. Imp. Nat. Moscou, XX. 1., 178. 1. (1847); WaLk., List Dipt. Brit. Mus., V. suppl. 1., 130. (1848) ; Zetr.; Dipt. Scand., VIII. 2948. 1. [Sicus] (1849); Scholtz, Zeitschr. f. Entomol. Breslau, IV. No. 16., 36. (1850); WaLK., List Dipt. Brit. Mus., V. suppl. 1., 113. 1. (1854); Schıs., Verh. zool.-bot. Ver. Wien, V. 662. 1. (1855) et VI. 414. 913. (1856) et Fauna Austriaca, Dipt., I. 28. (1862); Lw., Silliman's Amer. Journ. of Sci. and Arts, XXXVII. 317. (1864); JAENN., Berlin. Entom. Zeitschr., X. 236. 61. (1866) ; v. D. Wulp, Diptera Neerlandica, I. 465. tab. XIV. fig. 22-23. (1877); Beling, Verh. zool.-bot. Ges. Wien, XXX. 343., fig. (1880); Becher, Denkschr. Akad. Wien, XIV. 141. tab. II. fig. 13. (1882); Neurinus, Diptera Marchica, 41. 1. (1886); Mıк, Wien. Entom. Zeitg., V. 278. (1886); Rö̀., Wien. Entom. Zeitg., VIII. 10. (1889); Schосı, Fauna Insect. Helvetiae, Dipt., 27. (1890); L. Coucke, Annal. Soc. Entom. Belgique, XXXVII. 134. (1893); StronL, Mittheil. Naturwiss. Ver. Steiermark, XXIX. 1892. 12. (1893); Kent., Pótfüz. a Természettud. Közlönyhöz, XXXII. 131. (1900); LeonardI, Insetti 
Nocivi, III. 367. fig. 200. (1900) ; Aldr., Catal. North Amer. Dipt., 211. (1905).

aurea MeIG., Klassif., I. 122. b. [Sicus] (1804).

? australis Gmel., Systema Naturae, V. 2833. 149. [Musca] (1792).

bicolor FABR., Supplem. Entomol. System., 555. 3. [Sicus]

(1798) ; Meig., Klassif., I. 122. a. [Sicus](1804); Fabr., Systema

Antliat., 76. 3. [Sicus] (1805); LAM., Hist. nat. anim. sans vert., III. 418. 2. (1816).

bidentata FABR., Entomol. System., IV. 372. 40. [Tabanus] (1794).

bispinosa FABr., Entomol. System., IV. 372. 41. [Tabanus] (1794); Grel., Systema Naturae, V. 2885. 34. [Tabanus] (1792).

cinereibarbis Bıg., Annal. Soc. Entom. France, sér. 5. IX. 194. (1879) ; Wili.ist., Canad. Entomol., XVII. 122. (1885) ; RöD., Wien. Entom. Zeitg., VIII. 10. (1889); ALDr., Catal. North Amer. Dipt., 211. (1905).

Crucis FaBr., Mantissa Insect., II. 330. 5. [Stratiomys] (1787); Guel., Systema Naturae, V. 2834. 152. [Musca] (1792); FABR., Entomol. System., IV. 264. 7. [Stratiomys] (1794), Supplem. Entomol. System., 555. 5. [Sicus] (1798) et Systema Antliat., 76. 5. [Sicus] (1805); WiED., Aussereurop. zweifl. Ins., I. 86. Anmerk. (1828).

errans FABR., Entomol. System., IV. 263. 2. [Stratiomys] (1794), Supplem. Entomol. System., 555. 4. [Sicus] (1798); Panz., Fauna Germ., LVIII. 17. [Stratiomys] (1798); FABR., Systema Antliat., 76. 4. [Sicus] (1805).

grandis Schrank, Fauna Boica, III. 92. '2373. [Stratiomys] (1803).

macroleon Panz., Fauna Germ., IX. 20. [Stratiomys] (1793). major Schrank, Fauna Boica, III. 93. 2374. [Stratiomys] (1803). olens Herbst, Gemeinnütz. Naturgesch., VIII. 108. tab. 340. fig. 6. [Musca] (1787).

palatina Schrank, Fauna Boica, III. 93. 2375. [Stratiomys] (1803).

pallida SAY, Long's Exped. to St. Peter's River, II. Append., 369. (1824), Amer. Entomol., 42. tab. XX. (1825) et Compl. Wr., II. 42. tab. XX. (1859); WIED., Aussereurop. zweifl. Ins., I. 86. 1. (1828); Walk., List Dipt. Brit. Mus., I. 131. (1848) et V. suppl. 1., 113. 2. (1854) ; MAcQ., Dipt. exot., suppl. 5., 38. 1. (1855) ; Ost.-SAck., Catal. Dipt. North Amer., Ed. II., 43. (1878); Rßv., Wien. Entom. Zeitg., VIII. 10. (1889) ; Hart, Bull. Illinois State Laborat. of Nat. Hist., IV. 267. (1895).

? testacea FABR., Mantissa Insect., II. 330. 1. [Stratiomys] (1787), Entomol. System., IV. 262. 1. [Stratiomys] (1794), Supplem. 
Entomol. System., 554. 1. [Sicus] (1798) et Systema Antliat.,

75. 1. [Sicus] (1805).

unguiculata Paxz., Fauna Germ., XII. 22. [Stratiomys] (1793).

unicolor MeIG., Klassif., I. 122. 2. [Sicus] (1804).

grandis Schrank = ferruginea Scop.

macroleon PANz. = ferruginea Scop.

major Schrank = ferruginea Scop.

olens Herbst = ferruginea Scop.

palatina SchraNk = ferruginea Scop.

pallida $\mathrm{SAY}=$ ferruginea $\mathrm{Scop}$.

testacea $\mathrm{FABr} .=$ ? ferruginea Scop.

unguiculata PANz. = ferruginea Scop.

unicolor MeIG. = ferruginea Scop.

\section{Heterostomus}

Big., Annal. Soc. Entom. France, sér. 3. V. 283. (1857), nec Duv. Col., 1858.

Heterostomyia Bıg., Annal. Soc. Entom. France, sér. 5. IX.

186. (1879). cl ciso\%

Heterostomatomyia Scudd., Nomend. Zool., II. Univ. Ind., 148. (1884).

curvipalpis Bıg., Annal. Soc. Entom. France, sér. 3. V. 285. tab. Chile.

VI. fig. 5. (1857); Phil., Verh. zool.-bot. Ges. Wien, XV. 727.

1. (1865) ; Schin., Novara Reise, Dipt., 74. (1868); Brau., Sitzgsber. Akad. Wien, XCI. 149. tab., fig. 6, $a-c$. (1885); Ми, Wien. Entom. Zeitg., IX. 291. (1890); Hunter, Trans. Amer. Entom. Soc. Philad., XXII. 132. (1901).

flexipalpis Big., Annal. Soc. Entom. France, sér. 5. IX. 186. [Heterostomyia] (1879).

inflexipalpis Big., Annal. Soc. Entom. France, sér. 5. IX. 186. [Heterostomyia] (1879).

flexipalpis $\mathrm{BIG} .=$ curvipalpis $\mathrm{BIG}$.

inflexipalpis $\mathrm{BIG} .=$ curvipalpis $\mathrm{BIG}$.

\section{Arthropeas}

Lw., Stettin. Entomol. Zeitg., XI. 304. (1850).

Arthropaeas Marsch., Nomencl. Zool., 322. (1873).

americana Lw., Berlin. Entomol. Zeitschr., V. 316. 16. (1861); America sept.

Röper, Wien. Entom. Zeitg., VIII. 10. (1889); ALDR., Catal. North Amer. Dipt., 211. (1905).

leptis Ost.-SAck., Catal. Dipt. North Amer., Ed. II., 43 et 223. 48. Americasept.

(1878) et Berlin. Entom. Zeitschr., XXVI. 365. (1882); Rö.,

Wien. Entom. Zeitg., VIII. 10. (1889); Aldr., Catal. North Amer.

Dipt., 211. (1905). 
sibirica Lw., Stettin. Entom. Zeitg., XI. 305. tab. I. fig. 43-46. Sibiria. (1850) ; WalK., List Dipt. Brit. Mus., V. suppl. 1., 322. (1854); Röd., Wien. Entom. Zeitg., VIII. 10. (1889); BEck., Entomol. Nachricht., XXVI. 115. (1900).

tessella Morsch., Bullet. Soc. Imp. Nat. Moscou,- XXXII. 2., 505. [Leptis] (1859).

tessella MотsсH. = sibirica Lw.

\section{Anacanthaspis}

Rő̀., Wien. Entomol. Zeitg., VIII. 8. (1889).

bifasciata Rö̀., Wien. Entomol. Zeitg., VIII. 8. (1889).

Sibiria.

\section{Chiromyzinae.}

\section{Chiromyza}

Wied., Nova Dipt. Gen., 19. (1820).

? Inopus Walk., Ins. Saund., Dipt., I. 2. (1850).

? Nonacris WalK., Ins. Saund., Dipt., I. 7. (1850).

Xenomorpha Macq., Dipt. exot., I. 1., 193. 12 et I. 2., 190. (1838).

despecta WALK. = ? fuscana WIED.

dispecta WALK. = ? fuscana WIED.

fuscana Wied., Dipt. exot., 115. 2. (1821); St. Farg. ET Serv., Encyclop. Méthod., X. 431. (1825); WiEd., Aussereuıop. zweifl. Ins., I. 238. 2. tab. I. fig. 5. (1828) ; Schin., Novara Reise, Dipt., 76. 2. (1868); v. D. Wulp, Tijdsehr. v. Entomol., XXIV. 156. (1881) ; Ost.-Sack., Biolog. Central. Americ., Dipt., I. 60.1. (1886); Hunter, Trans. Amer. Entom. Soc. Plilad., XXVII. 132. (1901); Aldr., Catal. North Amer. Dipt., 211. (1905).

? despecta WALK., Insecta Saundeıs., Dipt., I. 2. tab. I. fig. T. [Inopus] (1850).

? dispecta Walk., List Dipt. Brit. Mus., V. suppl. 1., 112. 1. [Inopus] (1854).

leptiformis MACQ. = vittata WIED.

ochracea Wied., Nova Dipt. Gen., 20. (1820) et Dipt. exot., 115. 3

Americamer.

(1821); St. Farg. ex Serv., Encyclop. Méthod., X. 431. (1825);

Wied,, Aussereurop. zweifl. Ins., I. 238. 3. (1828); Schin., Novara Reise, Dipt., 76. 3. (1868); Hunter, Trans. Amer. Entom. Soc. Philad., XXVII. 132. (1901).

Americamer. et centr. 
vittata Wied., Nova Dipt. Gen., 20. fig. VIII. (1820) et Dipt. exot., Americamer. 114. 1. (1821); St. Farg. et Serv., Encyclop. Méthod., X. 431. (1825) ; Wred., Aussereurop. zweifl. Ins., I. 237. 1. (1828); MacQ., Suit. à Buffon, I. 422. 1. tab. X. fig. 10. (1834); Gúér, Iconogr. d. Régne Anim., VII. Ins., 543. tab. XCVII. fig. 7. (1835); WALK., Trans. Linn. Soc. London, XVII. 347. 43. (1837); MAcQ., Dipt. exot., II. 1. tab. V. fig. 6. (1840) ; WaLK, List Dipt. Brit. Mus., I. 227. (1848) ; Lw., Dipt.-Fauna Sïdafrika's, I. (1860); Schin., Novara Reise, Dipt., 76. 1. (1868); E. L. Arris., Bolet. Acad. Nat. Cienç. Córdoba, IV. 124. 40. (1882); Hunter, Trans. Amer. Entom. Soc. Philad., XXVII. 132. (1901).

leptiformis MACQ., Dipt. exet., J. 1., 193. 1. tab. XXIIJ. fig. 1. [Xenomorpha] (1838) et I. 2., 190. [Xenmorpha] (1838); Walk., List Dipt. Brit. Mus., V. suppl. 1., 66. 1. [Xenomorpha] (1854).

\section{Metoponia}

MACQ., Dipt. exot., suppl. 2., 28. (1847).

australis MAcQ., Dipt. exot., suppl. 4., 54. 2. tab. III. fig. 7. [Xenomorpha] (1849); Willis'., Trans. Amer. Entom. Soc. Philad., XV. 244. (1888).

fulvicaput WALK. = rubriceps MACQ.

rubriceps MACQ., Dipt. exot., suppl. 2., 28. 1. tab. I. fig. 4. (1847) et suppl. 3., 15. 1. (1848); W ALK., List Dipt. Brit. Mus., V. suppl. 1., 113. 1. (1854); Ost.-SAck., Berlin. Entom. Zeitschr., XXVII. 297. (1883).

fulvicaput Walk., Insecta Saunders., Dipt., I. 163. (1852).

vicina Bıg., Annal. Soc. Entom. France, sér. 5. IX. 200. [Chiromyza] (1879); Willist., Trans. Amer. Entom. Soc. Philad., XV. 244. (1888).

Australia.

Australia,

Tasmania.

\section{Lagarus Tana Reed}

Phil., Verh. zool.-bot. Ges. Wien, XV. 728. (1865) praeocc. Chaud., Col. 1838.

Paulseni Phıl., Verh. zool.-bot. Ges. Wien, XV. 729. (1865); Hunter, Chile.

Trans. Amer. Entom. Soc. Philad., XXVII. 132. (1901).

\section{Hylorus}

PurL., Verh. zool.-bot. Ges. Wien, XV. 728. (1865).

Krausei PhiL., Verh. zool.-bot. Ges. Wien, XV. 728. tab. XXVI. fig. Chile. 33. (1865); Ost.-SACK., Berlin. Entom. Zeitschr., XXVI. 368. (1882); Hunter, Trans. Amer. Entom. Soc. Philad., XXVII. 133. (1901). 


\section{Tanystoma.}

\section{TABANIDAE.}

\section{Pangoniinae.}

\section{Dicranomyia}

Hunter, Trans. Amer. Entom. Soc. Philad., XXVII. 135. (1901).

Dicrania Macq., Suit. à Buffon, I. 195. (1834) [nec Lesp. Serv., Col., 1825].

Cervus Wied., Aussereurop. zweifl. Ins., I. 94. 15. [Pangonia] Brasilia.

(1828) ; Macq., Suit. à Buffon, I. 196. 1. [Dicrania] (1834), Annal.

Soc. Entom. France, V. 438. tab. XV. [Dicrania] (1837) et Dipt. exot., I. 1., 110. 1. tab. XV. fig. 3. [Dicrania] (1838); WaLK., List Dipt. Brit. Mus., V. suppl. 1., 131. 66. var. [Pangonia] (1854) ; Gigr.-Tos, Bollet. Mus. Zool. ed Anat. comp. Torino, XII. No. 276. [Dicrania]. (1897); Rrcardo, Annal. Mag. Nat. Hist., sor. 7. V. 99 et 100. [Dicrania] (1900); Hunrer, Trans. Amer. Entom. Soc. Philad., XXVII. 135. (1901).

comprehensa WaLK., Insecta Saunders., Dipt., I. 11. tab. I. fig.

2. [Pangonia] (1850).

comprehensa WALK. = Cervus WIED.

furcata Wred., Aussereurop. zweifl. Ins., I. 99. 23. [Pangonia] (1828);

Brasilia.

MAcq., Suit. à Buffon, I. 196. 2. [Dicrania] (1834) et Annal. Soc.

Entom. France, VI. 438. tab. XV. fig. 18. [Dicrania] (1837);

Walk., List Dipt. Brit. Mus., V. suppl. 1., 132. 67. [Pangonia]

(1854); Ricırdo, Annal. Mag. Nat. Hist., ser. 7. V. 100. [Dicronia]

(1900) ; Hunter, Trans. Amer. Entom. Soc. Philad., XXVII. 134.

[Pangonia] (1901).

\section{Apocampta}

Schin., Verh. zool.-bot. Ges. Wien, XVII. 310. (1867).

melas $\mathrm{BIG}_{\mathrm{IG}}=$ subcana WALK.

nigra ScHin. = subcana WALK.

subcana WALK., List Dipt. Brit. Mus., I. 20t. [Chrysops] (1848); Australia.

Ricando, Anual. Mag. Nat. Hist., ser. 7. VIII 287. (1901) et ser.

7. XIV. 357. (1904).

melas Big., Mém. Soc. Zool. France, V. 625. [Diachlorus] (1892).

nigra Schis., Novara Reise, Dipt., 96. 40. (1868); Ricardo, Annal. Mag. Nat. Hist., ser. 7. V. 99. (1900). 


\section{Pityocera}

Gigl.-Tos, Bollet. Mus. Zool. ed Anat. comp. Torino, XI. No. 224., 3. (1896).

Festae Gigr.-Tos, Bollet. Mus. Zool. ed Anat. comp. Torino, XI. No. Panama.

- 224., 3. fig. (1896) ct XII. No. 276. (1897); Ricardo, Annal. Mag.

Nat. Hist., ser. 7. V. 99. (1900); Hunter, Trans. Amer. Entom.

Soc. Philad., XXVII. 135. (1901) ; Aldor., Catal North Amer. Dipt., 200. (1905).

\section{Goniops}

Aldr., Psyche, VI. No. 191., 236. (1892).

c’arysocoma Ost.-Sack., M $\rightarrow$ n. Boston Soc. Nat. Hist., II. 368. [Pangonia] (1876); Willist., Trans. Kansas Acad. Sci., X. 130. [Pangonia] (1887); Ricardo, Annal. Mag. Nat. Hist., ser. 7. V. 170. [Pangonia] (1900); Hine, Entom. News Philad., XI. 392. (1900) ; Al.dr., Entom. News Philad., XI. 531. (1900); Hine, Ohio Naturalist, II. 169. (1902) et Ohio State Acad. Sci., Spec. Pap., No. 5., 46. (1903) ; Aldr., Catal. North Amer. Dipt., 194. (1905).

hippoboscoides Aldr., Psyche, VI. No. 191., 237. (1892); Ricardo, Annal. Mag. Nat. Hist., ser. 7. V. 99. (1900).

hippoboscoides ALDR. = chrysocoma Ost.-SACK.

\section{Cadicera}

MACQ., Dipt. exot., suppl. 5., 22. (1855).

chrysostigma Wied., Aussereurop. zweifl. Ins., I. 100. 24. [Pangonia] (1828) ; Walk., List Dipt. Biit. Mus., V. suppl. 1., 136. 88. [Pangonia] (1854); Ricarco, Annal. Mag. Nat. Hist., ser. T. V. 101. (1900).

crassipalpis MacQ., Annal. Soc. Entom. France, VI. 429. tab. XV. Prom.bon.sp. fig. 12. [Pangonia] (1837) et Dipt. exot., I. 1., 98. 3. tab. XIV. fig. 1. [Pangonia] (1838); WalK., List Dipt. Brit. Mus., I. 137. [Pangonia] (1848) et V. suppl. 1., 137. 9. [Pangonia] (1854); Ricardo, Annal. Mag. Nat. Hist., ser. 7. V. 101. (1900).

melanopyga Wied., Zoolog. Magaz., I. 3., 31. 46. [Pangonia] (1819), Dipt. exot., I. 56. 8. [Pangonia] (1821) et Aussereurop. zweifl. Ins., I. 98. 20. [Pangonia] (1828); Macq., Annal. Soc. Entom. France, VI. 429. tab. XV. fig. 13. [Pangonia] (1837) et Dipt. exot., I. 1., 97. 2. [Pangonia] (1838); WaLk., List Dipt. Brit. Mus., I. 136. [Panjonia] (1849) et V. suppl. 1., 138. 97. [Pangonia] (1854) ; Lw., Dipt.-Fauna Suidafrika's, I. 19. 6. [Pangonia] (1860); Griff., Bollet. Mus. Zool. ed Anat. comp. Torino, X. No. 218., 6. [Pangonia] (1895); Ricardo, Annal. Mag. Nat. Hist., ser. 7. V. 101. (19j0). 
nigrescens Ricardo, Annal. Mag. Nat. Hist., ser. 7. VI. 161. (1900). rubramarginata MACQ., Dipt. exot., suppl. 5., 23. tah. I. fig. 7.

(1855); Ricardo, Annal. Mag. Nat. Hist., sel. 7. V. 100. (1900).

\section{Pelecorrhynchus}

MAcQ., Dipt. exot., suppl. 4., 28. (1850).

Caenopmyga Thoms., Eugenies Resa, Dipt., 449. (1868).

aurantiacus Ricardo, Annal. Mag. Nat. Hist., ser. 7. V. 10?. tah. Chile.

I. fig. 2. (1900).

Darwini Ricardo, Ammal. Mag. Nat. Hist., ser. 7. V. 102. tab. I. fig. 1. (1900).

eristaloides WalK., List Dipt. Brit. Mus., I. 193. [? Silvins] (1848) et V. suppl. 1., 267. 4. [Dasybusis] (1854); Ricırdo, Annal, Mag. Nat. Hist., ser. 7. V. 102. (1900).

fusconiger Wark., List Dipt. Brit. Mus., I. 192. [? Silvius] (1848) et V. suppl. 1., 267. 2. [T)asybasis] (1854); Ricnnno, Annal. Mag.

Nat. Hist., ser. 7. V. 102. (1900).

maculipennis Macq., Dipt. (xot., suppl. 4., 28. 1. tab. II. fig. 6. (1850); Iicardo, Annal. Mag. Nat. Hist., ser. 7. V. 102. (1900). maculipennis 'Trous., Eugenies Resa, Dipt., 450. 11. tal. IX.

fig. 2. [Caenopnyga] (1868).

maculipennis 'Тном. = maculipennis MACQ.

ornatus Schis. $=$ personatus WALK.

personatus WALK., List Dipt. Brit. Mus., I. 192. [? Silvius] (1818) et V. suppl. 1., 267. 3. [Dasybasis] (1854); Ricardo, Annal. Mag. Nat. Hist., ser. 7. V. 102. (1900).

ormatus Schis., Novara Reise, Dipt., 98, 44. (1868); Hutton, Catal. New Zealand Dipt. ete,, 23. (1881); WILLIsT., Kansas Univ. Quart., III. 192. (1895).

\section{Coenura}

Big., Annal. Soc. Entom. France, sér. 3. Y. 286. (1857).

albopunctata Schis.; Novara Reise, Dipt., 77.4. (1868); Rö̀., Wirn.

Entom. Zeitg., VIII. 10. (1889); Hunter, Trans. Amer. Entom.

Soc. Philad., XXVII. 133. (1901).

biguttata PIIIL., Verh, zool.-bot. Ges. Wien, XV. 726. 3. (1865); Chile.

Röp., Wien. Entom. Zeitg., VIII. 10. (1889); Huvtel, Trans.

Amer. Entom. Soc. Philad., XXVII. 133. (1901).

elegans PhIL., Verh. zool.-bot. Gies. Wien, XV. 727. 4. (186i); Rö̀., Chile.

Wien. Entom. Zeitg., VIJI. 10. (1889); Huxter, Trans. Amer.

Entom. Soc. Philad., XXVII. 133. (1901).

longicauda Bı́., Annal. Soc. Entom. France, sér. 3. V. 287. tah. Chile.

VI. fig. 2. (1857); PHI., Verh. zool.-bot. Ges. Wien, XV. 726.

Chile.
Africa centr.

Oceania,

Africa mer.

Americamer.

Australia,

Tasmania.

Australia.

Australia,

Tasmania.

Australia, N. Selandia. 
1. (1865) ; RöD., Wien. Entom. Zeitg., VIII. 10. (1889) : Hunter,

Trans. Amer. Entom. Soc. Philad., XXVII. 133. (1901).

xanthopleura Pun., Verh. zool.-bot. Ges. Wien, XV.726. 2. (1865);

Röd., Wien. Entom. Zeitg., VIII. 10. (1889); Hunter, 'Trans. Amer.

Entom. Soc. Philad., XXVII. 133. (1901).

\section{Apatolestes}

Willist., Entomol. Americ., I. 12. (1885).

comastes Willist., Entomol. Americ., I. 12. (1885); 'Towns., Proc. Calif. Acad. Sci., ser. 2. IV. 596. 8. (1895); Ricardo, Annal. Mag. Nat. Hist., ser. 7. V. 99. (1900); Hrne, Ohio Naturalist, V. 230. (1904); Aldr., Catal. North Amer. Dipt., 194. (1905).

Eiseni Towss., Proc. Calif. Acad. Sci., ser. 2. IV. 596. (1895); Ricardo, Annal. Mag. Nat. Hist., ser. 7. V. 99. (1900); Aldr., Catal. North Amer. Dipt., 194. (1905).

Eiseni Towss. = comastes WILLIST.

lutulentus Hutron, Trans. New. Zealand Instit., XXXIII. 18. (1901).

Chile.

America sept.

\section{Snowiellus}

Hine; Olnio Naturalist, V. 230. (1904).

atratus HıNe, Ohio Naturalist, V. 230. (1904).

America sept.

\section{Scione}

WaLk., Insecta Saunders., Dij)t., I. 10. (1850).

Diclisa Scmix., Verh. zool.-bot. Ges. Wien, XVII. 311. (1867). albifasciata MAcQ., 1)ipt. exot., suppl. 1., 28. 36. tab. III. fig. 8. [Pangonia] (1845); WaLK., List Dipt. Brit. Mus., V. suppl. 1., 126. 43. [Pangonia] (1854); Rıcando, Annal. Mag. Nat. Hist., ser. 7. V. 104. (1900); Hunter, Trans. Amer. Entom. Soc. Philad., XXVII. 133. [Pangonia] (1901); Ricardo, Annal. Mag. Nat. Hist., ser. 7. IX. 435. (1902).

Alluaudi Gıgl.-Tos, Annal. Soc. Entom. France, LXIV. 357. (1895); Ricardo, Annal. Mag. Nat. Hist., ser. 7. V. 104. (1900) et ser. 7. IX. 435. (1902).

claripennis Ricardo, Annal. Mag. Nat. Hist., ser. 7. VI. 292. (1900) et ser. 7. IX. 435. (1902).

distincta Schun., Novara Reise, Dipt., 102. 58. [Diclisa] (1868);

I. Seychelles.

Ricardo, Aunal. Mag. Nat. Hist., ser. 7. V. 104. (1900); Huxter, Trans. Amer. Entom. Soc. Philad., XXVII. 135. [Diclisa] (1901); Ricardo, Annal. Mag. Nat. Hist., ser. 7. IX. 435. (1902).

flavohirta Ricardo, Annal. Mag. Nat. Hist., ser. 7. IX. 437. (1902). Bolivia. 
fulva Ricardo, Annal. Mag. Nat. Hist., ser. 7. IX. 436. (1902).

Bolivia.

fusca Ricarno, Annal. Mag. Nat. Hist., ser. 7. VI. 291. (1900) et ser. Bolivia. 7. I.X. 434. (1902).

incompleta MACQ., Dipt. exot., suppl. 1., 27. 35. tab. III. fig. 7. Columbia.

[Pangonia] (1845); WALK., Insecta Saunders., Dipt., I. 20. [Pangonia] (1850); Macq., Dipt. exot., suppl. 4., 25. [Pangonia] (1850); Walk., List Dipt. Brit. Mus., V. suppl. 1., 124. 33. [Pangonia] (1854) ; Schrn., Novara Reise, Dipt., 101. 59. [Diclisa] (1868); Gigl.-Tos, Annal. Soc. Entom. France, LXIV. 357. [Diclisa] (1895) ; Ricardo, Annal. Mag. Nat. Hist., ser. 7. V. 104. (1900); Hunter, Trans. Amer. Entom. Soc. Philad., XXVII. 134. [Pan. gonia] et 135. [Diclisa] (1901); Ricardo, Annal. Mag. Nat. Ilist., ser. 7. IX. 435. (1902).

maculipennis Scurn., Novara Reise, Dipt., 102. 57. tab. II. fig. 7. [Diclisa] (1868) ; RöD., Stettin. Entom. Zeitg., XLVII. 262. [Diclisa] (1886); Ost.-SAck., Biolog. Central. Americ., Dipt., I. 47.1.[Diclisa] (1886) ; Rö̀., Dipt. gesammelt in Süd-Amerika von A. Stübel, 8. [Diclisı] (1892); Ricardo, Annal. Mag. Nat. Hist., ser. 7. V. 104. (1900) ; Hunter, Trans. Amer. Entom. Soc. Philad., XXVII. 135. [Diclisa] (1901); Ricardo, Annal. Mag. Nat. Hist., ser. 7. IX. 435. (1902); Aldr., Catal. North Amer. Dipt., 192. (1905).

misera Ost.-SAck., Biolog. Contral. Americ., Dipt., I. 47. 2. [Diclisa] (1386) ; Ricardo, Annal. Mag. Nat. Hist., ser. 7. V. 104. (1900) et ser. 7. IX. 435. (1902); Aldpr., Catal. North Amor. Dipt., 192. [Diclisa] (1905).

singularis MACQ., Dipt. exot., suppl. 1., 23. 27. tab. III. fig. 2. Australia. [P(angonia] (1845); Walk., List Dipt. Brit. Mus., V. supp]. 1., 124. 30. [Pangonia] (1854); Ricardo, Annal. Mag. Nat. Hist., ser. 7. V. 104. (1900) et ser. 7. IX. 435. (1902).

\section{Pangonia}

Latr., Hist. Nat. Crust. et Ins., III. 437. (1802) [Pangonius].

Clanis Walk, Insecta Saunders., Dipt., I. 9. (1850).

Erephopsis Rond., Archivio per la Zool. Modena, III. 85. (1863).

Erephrasis Ricardo, Annal. Mag. Nat. Hist., ser. 7. V. 114. (19̀00).

Fidena WaLk., Insecta Saunders., Dipt., I. 8. (1850).

Lilaea WALK., Insecta Saunders., Dipt., I. 11. (1850).

Melpia WaLk., Insecta Saunders., Dipt., I. 8. (1850).

Nuceria WalK., Insecta Saunders., Dipt., I. 7. (1850).

Osca Walk., Insecta Saunders., Dipt., I. 10. (1850).

Phara Walk., Insecta Saunders.; Dipt., I. 9. (18j0).

Philoliche Wied, Aussereurop. zweifl. Ins., I. 95. B. (1828).

Americamer. et centr.

Guatemala. 
Plinthina WaLk., Insecta Saunders., Dipt., I. 10. (1850).

Sackenimyia Bıg., Annal. Soc. Entom. France, sér. 5. IX. Bullet., L. (1879).

Scaptia Walk., Insecta Saunders., Dipt., I. 8. (1850).

Searphia WaLK., Insecta Saunders., Dipt., I. 10. (1850).

Tacina Walk., Insecta Saunders., Dipt., I. 9. (1850).

Tanyglossa Meig., Klassif., I. 174. XLII. (1804).

\section{Pangonias. str.}

adjuncta Walk., List Dipt. Brit. Mus., I. 135. (1848); Ricardo, Africa mer. Annal. Mag. Nat. Hist., ser. 7. V. 106 et 108. (1900). aethiopica Tuuns $_{4}=$ rostrata $\mathrm{L}$.

affinis Lw., Neue Peiträge, VI. 29. 8. (1859); Ricardo, Annal. Mag. Hispania. Nat. Hist., ser. 7. V. 180. (1900).

alboatra WALK., Insecta Saunders., Dipt., I. 13. tab. I. fig. 3. (1850); Ricardo, Annal. Mag. Nat. Hist., ser. 7. V. 106 et 107. (1900). amboinensis FABr., Systema Antliat., 91. 7. (1805); L.xTr., Lineyclop. Méthod., VIII. 704. 10. (1811); WiEd., Dipt. exot., I. 53. 4. (1821) et Aussereurop. zweifl. Ins., I. 92. 10. (1828); WaLK., List Dipt. Brit. Mus., I. 131. (1848) et V. suppl. 1., 139. 102; 324. (1854); v. D. Wulp, Catal. Dipt. South Asia, 64. (1896); Ricardo, Annal. Mag. Nat. Hist., ser. 7. V. 167. (1900).

analis Fabr., Systema Antliat., 91. 6. (1805); Latr., Encyclop. Méthod., VIII. 703. 4. (1811); Wied., Dipt. exot., I. 57. 11. (1821) et Aussereurop. zweifl. Ins., I 93. 12. (1828); WALK., List Dipt. Brit. Mus., V. suppl. 1., 126. 40. (1854); Ricando, Amnal. Mag. Nat. Hist., ser. 7. V. 173. (1900); Hunter, Trans. Amer. Entom. Soc. Philad., XXVII. 133. (1901).

angulata FABR., Systema Antliat., 91. 5. (1805); LATr., Encyclop. Méthod., VIII. 704. 9. (1811); WIED., Dipt. exot., I. 55. 7. (1821) et Aussereurop. zweifl. Ins., I. 97. 19. (1828); MacQ., Annal. Soc. Entom. France, VI. 429. tab. XV. fig. 4, 24. (1837); Lw., Dipt.-Fauna Südafrika's, I. 20. 9. (1860); Schis., Novara Reise, Dipt., 99. 49. (1868) ; Ricardo, Annal. Mag. Nat. Hist., ser. 7. V. 105 et 107 . (1900) et ser. 7. VI. 162. (1900).

obesa WaLk., Insecta Saunders., Dipt., I. 14. (1850); Johss., Proc. Acad. Nat. Sci. Philad., 1898. 157. (1898).

appendiculata MACQ., Dipt. exot., I. 1., 97. 1. tab. XIII. fig. 2. (1838) ; WaLk., List Dipt. Brit. Mus., I. 134. (1848) et V. suppl. 1., 137. 94. (1854); Johss., Proc. Acad. Nat. Sci. Philad., 1898. 157. (1898); Ricardo, Annal. Mag. Nat. Hist., ser. 7. V. 107. (1900).

arcuata Willist., Kansas Univ. Quart., III. 190. (1895); Ricıreo, Annal. Mag. Nat. Hist., ser. 7. V. 171 et 174. (1900); Huster,

Trans. Amer. Entom. Soe. Philad., XXVII. 133. (1901).

Africa mer.

India or., Amboina.

America mer.

Africa mer.

Brasilia. 
aterrima L. Dur., Anual. Soc. Entom. France, sér. 3. I. 388. 23. (1853). atra Phir., Verl.. zool.-bot. Ges. Wien, XV. 709. 13. (1865) ; Rıcardo, Annal. Mag. Nat. Hist., ser. 7. V. 173. (1900); Hunten, Trans. Amer. Entom. Soc. Philad., XXVII. 133. (1901).

atricornis WiEd., Dipt. exot., I. 58. 13. (1821) et Aussereurop. zweifl. Ins., I. 103. 28. (1828); I w., Dipt.-Fauna Südafrika's, I. 19. 7. (1860) ; Ricalddo, Annal. Mag. Nat. Hist., ser. 7. V. 105. (1900).

cingulata WiEn., Aussereurop. zweifl. Ins., I. 103. 29. (1828). atrifera WALK., Trans. Entom. Soc. London, n. ser. V. 272. (1860); Ricardo, Annal. Mag. Nat. UIist., ser. 7. V. 169 et 170. (1900); Aldr., Catal. North Amer. Dipt., 193. (1905).

aurulans WIED., Aussereurop. zweifl. Ins., II. 620. 12. (1830) ; WA ג., List Dipt. Brit. Mus., V. suppl. 1., 120. 12. (1854); Ricardo, Annal. Mag. Nat. Hist., ser. 7. V. 170. (1900); Aldr., Catal. Nortl Amer. Dipt., 193. (1905).

australis Pmu., Verh. \%ool.-bot. Ges. Wien, XV. 710. 16. (1865); E. I. Arrib., Bol. Acad. Nat. Ciene. Córdoba, IV. 130. 53. (1882); Ricardo, Annal. Mag. Nat. Hist., ser. 7. V. 173. (1900); Hunter, Trans. Amer. Entom. Soc. Philad., XXVII. 133. (1901).

basalis MACQ., Dipt. exot., suppl. 2., 10. 38. (1847); WALK., List Dipt. Brit. Mus., V. suppl. 1., 138. 98. (nec pag. 322); Ricardo, Annal. Mag. Nat. Hist., ser. 7. V. 105. (1900).

basilaris WiED., Aussereurop. zweifl. Ins., J. 554. 25. (1828) ; Röper, Stettin. Entom. Zeitg., XLVII. 261. (1886) et Dipteren gesammelt in Süd-Amerika von A. Stuibel, 7. (1892); Ricardo, Annal. Mag. Nat. Hist., ser. 7. V. 173. (1900); Hunter, Trans. Amer. Entom. Soc. Philad., XXVII. 133. (1901).

basilaris W1ED. 1830. = Wiedemanni BELL.

Beckeri Bezzi, Bull. Soc. Entom. Ital., XXXIII. 10. 3. (1901).

tricolor Austen, Proc. Zool. Soc. London, 1900. 7. tab. I. fìg. 8. (1900).

biclausa Isw., Öfvers. Kongl. Vet. Akad. Förhandl., XIV. 1857. 337. 3. (1858) et Dipt.-Fauna Südafrika's, I. 19. 5. (1860); RicArdo, Annal. Mag. Nat. Hist., ser. 7. V. 106. (1900) et ser. 7. VI. 162. (1900).

bicolor MAcQ. = semiflava W1ED.

bifasciata WIED., Dipt. exot., I. 58. 12. (1821) et Aussereurop. zweifl. Ins., I. 102. 27. (1828) ; WALk., List Dipt. Brit. Mus., I. 137. (1848) ; Ricardo, Annal. Mag. Nat. Hist., ser. 7. V. 105 et 108. (1900).

binotata Latr., Encyclop. Méthod., VIII. 703. 7. (1811).

brevis Lw., Wien. Entom. Monatschr., VII. 9. 2. (1863); Ricakdo, Annal. Mag. Nat. Hist., ser. 7. V. 106 et 109. (1900).

Bricchettii BezzI, Annal. Mus. Civ. Genova, ser. 2. XII. (XXXII). Somali. 181. 1. (1892); Ricardo, Annal. Mag. Nat. Hist., ser. 7. V. 106. (1900).
Algeria.

Chile.

Prom. bon.sp.

Mexico.

Mexico.

Chile.

Africa sept.

Brasilia, Ecuador.

Africa or.

Africa mer.

Prom.bon.sp.

Australia.

Africa mer. 
bullata WıLıIst., Kansas Univ. Quart., III. 191. (1895); Ricaluo, Africa mer. Annal. Mag. Nat. Hist., ser. 7. V. 106. (1900).

brunnea Thusв., Nova Acta Upsal., IX. 69. [Tanyglossa] (1820).

caffra Mace., Dipt. exot., suppl. 2., 11. 39. (1847); Lw., Dipt.-Fauna Südafrika's, I. 20. 10. (1860) ; Ricurdo, Annal. Mag. Nat. Hist., ser. 7. V. 106. (1900).

castanea Perty, Delect. Anim. Artic. Brasil., 182. tab. XXXVI. fig. 8. (1833); WaLk., List Dipt. Brit. Mus., V. suppl. 1., I29. 56. (1854); Hunter, Trans. Amer. Entom. Soc. Philad., XXVII. 1:33. (1901). caustica Ost.-SAck., Biolog. Central. Amer., Dipt., I. 44. 2. (1886); Rıcardo, Annal. Mag. Nat. Hist., ser. 7. V. 169. (1900); Al,sR., Catal. North Amer. Diplt., 193. (190i).

cellulata $\mathrm{BRLL} \mathrm{E}=$ marginata $\mathrm{F}_{\mathrm{ABR}}$.

chlorogastra Punt., Verh. zool.-bot. Ges. Wien, XV. 708. 11. (186i); Ricardo, Annal. Mag. Nat. Hist., ser. 7. V. 173. (1900); IIuxter, Trans. Amer. Entom. Soc. Philad, XXVII. 133. (1901).

chrysopila Macq., Suit. à Buffon, I. 194. 9. (1834); WALK., List Dipt. Brit. Mus., V. suppl. 1., 136. 89. (185ł); Ricardo, Annal. Mag. Nat. Hist., ser. 7. V. 107. (1900) et ser. 7. VI. 162. (1900). cingulata Thừe., Nova Acta Upsal., IX. 70. tab. I. fig. 8. [Tamyglossa] (1827).

cingulata $\mathrm{W}_{\mathrm{IED}}$ = atricornis W1WD.

collaris PhIL., Verh. zool.-bot. Ges. Wien, XV. 709. 14. (1865); Ricardo, Annal. Mag. Nat. Hist., ser. 7. V. 173. (1900); Hunter, Trans. Amer. Entom. Soc. Philad., XXVII. 133. (1901).

conjuncta WALK., List Dipt. Brit. Mus., I. 135. (1848); Ricardo, Annal. Mag. Nat. Hist., ser. 7. V. 106 et 107. (1900).

conjungens WAlK., List Dipt. Brit. Mus., I. 140. (1848); Ruckno, Annal. Mag. Nat. Hist., ser. 7. V. 114 et 121. (1900).

cornuta WALK., Trans. Linn. Soc. London, XVII. 337. 16. (1837).

diaphana Schin., Novara Reise, Dipt., 99. 51. (1868); WiLlist., Kansas Univ. Quart., II. 190. (1895); Ricardo, Annal. Mag. Nat. Hist., ser. 7. V. 173. (1900); Hun'en, Trans. Amer. Entom. Soc. Plilad., XXVII. 133. (1901).

dimidiata Lw., Neue Beitr., VI. 50. (1859); Rıcardo, Annal. Mag. Hispania. Nat. Hist., ser. 7. V. 180. (1900).

dorsalis Latr., Encyclop. Méthod., VIII. 704. 11. (1811).

dorsalis Mavq., Dipt. exot., I. 1., 100. 6. (1838); Walk., List Dipt. Brit. Mus., V. suppl. 1., 144. 131. (1854) ; Rıcardo, Annal. Mage. Nat. Hist., ser. 7. V. 114. (1900).

fasciata Latr., Encyclop. Méthod., VIII. 706. 16. (1811).

Chile.

Patiaignota. Prom. bon.sp.

Brasilia.

Mexico.

Africa mer.

Prom.bon.sp.

Chile.

Africa mer.

A ustralia oec.

Brasilia.

America mer.

He de Fiance. Australia.

Aegyilus.

fasciata MAcQ., Suit. à Buffon, I. 194. 8. (1834); WALK., List Dipłt. Patria ignota. Brit. Mus., V. suppl. 1., 116. 142. (1854).

fasciata Macq., Dipt. exot., I.1., 103. 14. (1838); WaLk., List Dipt. Brasilia. Brit. Mus., V. suppl. 1., 127. 46. (1854); Ricardo, Annal. Mag. Nat. Hist., ser. 7. V. 173. (1900) ; Huserer, Trans. Amer. Entom. Soc. Philad., XXVII. 133. (1901). 
fascipennis MaCQ., Dipt. exot., I. 1., 106. 20. (1838); BLANCI. in Chile. GaY : Hist. fis. y polit. de Chile, Zool. VII. 387. 2. tab. I. fig. 9. (1854) ; WalK., List Dipt. Brit. Mus., V. suppl. 1., 131. 65. (1854) ; Phil., Verh. zool.-bot. Ges. Wien, XV. 707. 2. (1865); Schin., Novara Reise, Dipt., 98. 45. (1868); Ricardo, Annal. Mag. Nat. Hist., ser. 7. V. 173. (1900) ; Hunter, Trans. Amer. Entom. Soc. Philad., XXVII. 133. (1901).

ferruginea MACQ., Dipt. exot., I. 2., 179. (1839); WaLK., List Dipt. Brit. Mus., V. suppl. 1., 129. 54. (1851); Ricardo, Annal. Mag. Nat. Hist., ser. 7. V. 171. (1900); Hunter, Trans. Amer. Entom. Soc. Philad., XXVII. 134. (1901).

ferruginea Meis., Klassif., I. 175. 1. tab. X. fig. 2. [Tanyglossa] (1801) ; Latr., Gen. Crust. et Insect., IV. 282. (1809) et Encyclop. Méthod., VIII. 706. 17. (1811); Meig., System. Beschreib., II. 24. 3. (1820) ; Walk., List Dipt. Bıit. Mus., V. suppl. 1., 118. 2. (1854); Lw., Neue Beitr., VI. 28. 7. (1859); Schis., Novara Reise, Dipt., 99. 48. (1868); Ricando, Annal. Mag. Nat. Hist., ser. 7. V. 180 et 181. (1900); Villen., Annal. Soc. Entom. France, LXXIV. 309. 3. (1905).

filipalpis Willıst., Kansas Univ. Quart., III. 190. (1895); RICARdo, Annal. Mag. Nat. Hist., ser. 7. V. 171 et 174. (1900); Hunter, Trans. Amer. Entom. Soc. Philad., XXVII. 134. (1901).

flava Meig., System. Beschreib., II. 22. 1. (1820); MacQ., Suit. à Buffon, I. 193. 4. (1834); WaLk., List Dipt. Brit. Mus., V. supll. 1., 118. 3. (1854); Gobert, Mém. Soc. Linn. Nord France, 1881. 44. (1881); Pand., Revue d'Entom., II. 227. (1883); Ricardo, Annal. Mag. Nat. Hist., ser. 7. V. 180. (1900) ; Vilı.ex., Annal. Soc. Entom. France, IXXIV. 309. 2. (1905).

flavescens Ricardo, Annal. Mag. Nat. Hist., ser. 7. V. 174. tab. I. Brasilia. fig. 6, 6 a. (1900).

flavipes MacQ., Dipt. exot., I. 1., 98. 4. (1838); WaLk., List Dipt. Brit. Mus., I. 137. (1848) ; Ricardo, Annal. Mag. Nat. Hist., ser. 7. V. 107. (1900).

flavohirta BeLl., Saggio di Ditterol. Messic., I. 49. 5. (1859); Ricardo, Annal. Mag. Nat. Hist., ser. 7. V. 169. (1900); Willist., Biolog. Central. Americ., Dipt., I. 254. 6. (1901); Aldr., Catal. North Amer. Dipt., 193. (1905).

fuliginosa WALK. = lasiophthalma BorsD.

fulvipes Lw., Neue Beitr., VI. 26. 3. (1859) et Berlin. Entom. Zeitschr., XII. 371. 2. (1868) ; Ricardo, Annal. Mag. Nat. Hist., ser. 7. V. 167. (1900).

fulviventris MacQ., Nipt. exot., I. 1., 109. 25. (1838); WaLK., List Dipt. Brit. Mus., V. suppl. 1., 144. 132. (1854); Ricardo, Annal. Mag. Nat. Hist., ser. 7. V. 112. (1900).

fumida Lw., Neue Beitr., VI. 28. 6. (1859); Rrcardo, Annal. Mag. Hispania. Nat. Hist., ser. 7. V. 180. (1900).

funebris Macq., Dipt. exot., suppl. 1., 23. 26. tab. III. fig. 1. (1845) Algeria. 
et in Lucas: Explor. scient. de l'Algérie, Zool., III. 421. 18. tab. I. fig. 5. (1849); Walk., List Dipt. Brit. Mus., V. suppl. 1., 137. 91. (1854); Rrcardo, Annal. Mag. Nat. Hist., ser. 7. V. 107. (1900). fuscanipennis MACQ., Dipt. exot., suppl. 5., 18. 58. tab. I. fig. 5. (1855) ; Ricardo, Annal. Mag. Nat. Hist., ser. 7. V. 112. (190J). fuscipennis.Wred., Aussereurop. zweifl. Ins., I. 95. 16, (182S); MacQ., Suit. à Buffon, I. 194. 10. (1834) et Dipt. exot., I. 1., 102. 12. (1838); Walk., Iist Dipt. Brit. Mus., V. suppl. 1., 128. 23. (1854); Ricardo, Annal. Mag. Nat. Hist., ser. 7. V. 171 et 174. (1900) ; Hunter, Trans. Amer. Entom. Soc. Philad., XXVII. 134. (1901).

fusiformis WALK., Insecta Saunders., Dipt., I. 19. (1850) et List Dipt. Brit. Mus., V. suppl. 1., 119. 10. (1854); Ricardo, Annal. Mag. Nat. Hist., ser. 7. V. 169 et 170. (1900) ; Aldr., Catal. North Amer. Dipt., 193. (1905).

griseipennis Lw., Neue Beitr., VI. 31. 11. (1859); Ricardo, Annal. Hispania.

Mag. Nat. Hist., sor. 7. V. 180. (1900).

gulosa Wied., Aussereurop. zweifl. Ins., I. 99. 22. (1828); Ricardo, Prom.bon.sp.

Annal. Mag. Nat. Ilist., ser. 7. V. 105 et 108. (19j0).

haustellata $\mathrm{F}_{\mathrm{ABR}}=$ marginata $\mathrm{F}_{\mathrm{ABR}}$.

haristellata Oı.1v. = micans Meig.

hebes Walk., List Dipt. Brit. Mus., I. 137. (1848); Ricardo, Annal. Mag. Nat. Hist., ser. 7. V. 182. (1900).

hera Ost.-SAck., Bullet. Unit. Stat. Geolog. Surv., III. 214. (1877); Ricardo, Annal. Mag. Nat. Hist., ser. 7. V. 170. (1900); Hine, Ohio Naturalist, V. 227. (1904); BeutenmëLl., Bullet. Amer. Mus. Nat. Hist., XX. 87. (1904); Aldr., Catal. North Amer. Dipt., 193. (1905).

? hirsuta Thuns., Nova Acta Lpsal., IX. 67. [Tanyglossa] (1827). illota Willistr., Biolog. Central. Americ., Dipt., I. 254. 7. (1901); Aldr., Catal. North Amer. Dipt., 193. (1905).

incerta BELL. = nigronotata MACQ.

incisa W1ED, = incisuralis SAY.

incisuralis MACQ., Dipt. exot., suppl. 2., 12. 41. (1847); WALK., List Dipt. Brit. Mus., V. suppl. 1., 127. 45. (1854); Ricardo, Annal. Mag. Nat. Hist., ser. 7. V. 171. (1900); Hunter, Trans. Amer. Entom. Soc. Philad., XXVII. 134. (1901).

incisuralis SAY, Journ. Acad. Nat. Sci. Philad., III. 31. (1823), Americ. Entom., 75. tab. XXXIII. (1825) et Compl. Writt., I. 75. tab. XXXIII. (1859); Wrilist., Trans. Kansas Acad. Sci., X. 130. (1887) ; Aldr., Catal. North Amer. Dipt., 193. (1905).

incisa Wied., Aussereurop. zweifl. Ins., I. 90. 6. (1828); Waıк., List Dipt. Brit. Mus., V. suppl. 1., 120. 11. (1854); Ost.SAck., Bull. Unit. Stat. Geolog. Surv., III. 214. (1877); Ricardo, Annal. Mag. Nat. Hist., ser. 7. V. 169. (1900); Hine, Ohio Naturalist, V. 228. (1904).

inconspicua WALK., List Dipt. Brit. Mus., I. 136. (1848) et V. suppl. Patria ignota.
Brasilia.

Honduras.

? Brasilia.

America sept. 
1., 141. 116. (1854) ; Ricanuo, Annal. Mag. Nat. Hist., ser. ז. V. 182. (1900).

isabellina Wrid., Aussereurop. zweill. Ins., I. 112. 3. [Silvius] (1828);

Walk., List Dipt. Brit. Mus., I. 192. (1848) et V. suppl. 1., 274.

2. [Silvius] (1854); Os'r.-SAck., Catal. Dipt. North America, Ed.

II., 52 et 225. 65. (1878); Ricardo, Annal. Mag. Nat. Hist., ser.

7. V. 169. (1900); Aldr., Catal. North Amer. Dipt., 193. (1905).

lasiophthalma Borsn., Voyage de l'Astrolabe, Zool., II. 666. tab. XII.

fig. 13. (1832); MACQ., Suit. à Buffon, I. 193. 7. (1834); WALK.,

List Dipt. Brit. Mus., V. suppl. 1., 139. 103. (1854). v. I. Wulp,

Catal. Dipt. South Asia, 64. (1896); Ricsndo, Ammal. Mag. Nat. Hist., ser. 7. V. 154. (1900).

fuliginosø Walk., Iist Dipt. Brit. Mus., V. suppl. 1., 140. 108. (1854).

leucomelas WiED., Aussereurop. zweill. Ins., I. 50. 7. (1828); WaLk.,

List Dipt. Brit. Mus., I. 136. (1848) et V. suppl. 1., 138. 96. (1854);

Ricando, Anual. Mag. Nat. Hist., ser. 7. V. 107. (1900).

ligens HurTer $=$ lingens WiED.

lineala $\mathrm{F}_{\mathrm{ABR}}$. = rostrata $\mathrm{L}$.

lingens Wied., Aussereurop. zweifl. Ins., I. 87. 2. (1828); MAcQ.,

Dipt. exot., I. 1., 102. 11. (1838) ; BLANCH. in GaY: Hist. fis. y polit. de Chile, Zool. VII. 387. 1. tab. I. fig. 10. (1854); WA1.K., List Dipt. Brit. Mus., V. suppl. 1., 129. 58. (1854); P'Hu., Verh. zool.-bot. Ges. Wien, XV. 707. 1. (1865); Ricardo, Annal. Mag. Nat. Hist., ser. 7. V. 173. (1900).

ligens Hunter, Trans. Amer. Entom. Soc. Philad., XXII. 134. (1901) [lapsus].

lugubris MacQ., Dipt. exot., I. 1., 104. 15. tab. XIV. fig. 2. (1838); WALk., Iist Dipt. Brit. Mus., V. suppl. 1., 123. 28. (1854); Ricardo, Ammal. Mag. Nat. Hist., ser. 7. V. 173. (1900) ; Hunter, 'Trans. Amer. Entom. Soc. Pliilad., XXVII. 134. (1901).

? lurida*) WalK., List Dipt. Brit. Mus., I. 140. (1848); Ricardo, Annal. Mag. Nat. Hist., ser. 7. V. 121. [? gen.] (1900).

Macquarti Guér., Voyage de la Coquille, Zool. II. 2., 288. (1830); Ricsndo, Annal. Mag. Nat. Hist., s(r. 7. V. 173. (1900).

macroglossa Westw., London and Ediuburgh Philos. Maga\%. aud Journ. of Sei., VI. 449. (1835); Ost.-Sick., Mem. Boston Soc. Nat. Hist., II. 368. (1876) ; Ricardo, Annal. Mag. Nat. Hist., ser. 7. V. 169. (1900); Aldr., Catal. North Amer. Dipt., 193. (1905). maculata FABr., Systema Antliat., 90. 3. (1805); LAtr., Gen. Crust. et Ins., IV. 282. tab. XV. fig. 4. (1809), Consid. génér. 443. (1809) et Encyclop. Méthod., VIII. 706. 15. (1811); LAм., Hist. nat. anim. sans vertebr., III. 419. 1. (1816); Meig., System. Beschreib., II. 22. 2. (1820) ; Macq., Suit à Buffon, I. 192. 1. tab. V. fig.

Africa mes.

Americamer.

Brasilia.

Australia.

Chile.

America sept.

\#) Secundum G. RIcsindo l. e. ad subfamiliam Tabaninarum referenda.

Europa mer., Africa sept. 
1-2. (1834) et Annal. Soc. Entom. France, VI. 429. tab. XV. fig. 26. (1837); Blanch., Hist. Nat. Ins., III. 589. 1. (1840); WaLK., List Dipt. Brit. Mus., I. 134. (1848); Macq. in Lucas: Explor. scient. de l'Algérie, Zool. III. 480. 16. (1849); WaLk., List Dipt. Brit. Mus., V. sujpl. 1., 118. 1. (1854); I,., Neue Beitr., VI. 24. 1. (1859); Scunv., Fauna Austriaca, I. 44. (1862); Pand., Revue d'Entomol., II. 226. 1. (1883) ; Griff., Bollet. Mus. Zool. ed Anat. comp. Torino, X. No. 218., 6. (1895); Ricardo, Annal. Mag. Nat. Hist., ser, 7. V. 180 et 181. (1900).

proboscidea Fabr., Entomol. System., IV. 363. 3. [Tabaums] (1794); Meig., Klassif., I. 175. b. [Truyglossa] (1804); Cogüв., Illustr. Iconogr. Ins., 111. tab. XXV. fig. 1. [Tabamus] (1804). tabaniformis Latr., Hist. Nat. Crust. et Ins., XIV. 318. (1805), Gen. Crust. et Ins., I. tab. XV. fig. 4. (1809) et Encyclop. Méthod., VIII. 705. 13. (1811); Lıм., Hist. nat. anim. sans vertebr., III. 419. 2. (1816).

varipennis Latr., Eneyclop. Méthod., VIII. 705. 14. (1811). maculata RossI = ? variegata FABR.

maculiventris Westw., London and Edinburgh Philos. Magaz. and Journ. of Sci., VI. 449. (1835) et Isis, 1838. II. 87. (1838); Ricalido, Annal. Mag. Nat. Hist., ser. 7. V. 114. (1900).

Magrettii Bezz, Bullet. Soc. Ent. Ital., XXXIII. 7. 2. (1901). marginalis Wied., Aussereurop. zweifl. Ins., II. 620, 11. (1830); WaLK., List Dipt Brit. Mus., V. suppl. 1., 128. 50. (1854); Ricardo, Annal. Mag. Nat. Hist., ser. 7. V. 173. (1900); Hunter, Trans. Amer. Entom. Soc. Philad., XXII. 134. (1901).

marginata Farr., Systema Antliat., 90. 2. (1805); Latr., Gen. Crust. et Ins., IV. 282. (1809) et Encyclop. Méthod., ViII. 705. 12. (1811) ; Meig., System. Beschreib., II. 24. 4. (1820); MaCq., Suit. à Buffon, I. 192. 2. (1231); Buavch., Hist. Nat. Ins., III. 589. 2. (1840); Gıмм., Bull. Soc. Imp. Nat. Moscou, XX. 2., 154. 1. (1847) ; Walk., List Dipt. Brit. Mus., I. 133. (1848); MAcQ. in Lucss: Explor. Seient. de l'Algérie, Zool. III. 421. 17. (1849); L. Dư., Mém. Acad. d. Sci., Math. et Phys., XI. 173-360. tab. IV. fig. 40. (1850) ; Walk., List Dipt. Brit. Mus., V. suppl. 1., 118. 4. (1854); Lw., Neue Beitı’, VI. 30. 9. (1859); Goвert, Mém. Soe. Linn. Nord France, 1881. 43. (1881); Pand., Revue d'Entomol., II. 227. 2. (1888) ; GrifF., Boll. Mus. Zool. ed Anat. comp. Torino, X. No. 218., 6. (1895) : Ricarno, Annal. Mag. Nat. Hist., ser. T. V. 179 et 181 . (1900).

cellulata Brutsié, Exped. scient. d. Morée, III. 303. 657. tab. XLVII. fig. 1. (1832); WAlk., List Dipt. Brit. Mus., V. suppl. 1., 118. 5. (1854).

haustellata FABr., Spec. Insect., II. 455. 2. [Tabamus] (1781); Vilt., Entomol. Linn., III. 558. 18. [Tabanus] (1789); FABr., Fntonol. System., IV. 362. 2. [Tabanus] (1791); CoQuen., Illustr. Icon. Ins., III. 120. tab. XXVII. fig. 4. [Tabanus] (1804) ; Meit., Klassif., I. 175. a. [Tt(myglossa] (1804).

Europa mer., Africa sept., Asia min.

Erythraca.

Brasilia. 
? mauritana L., Systema Naturae, Ed. XII., II. 999. 3. [Tabanus]

(1767) ; Ousv., Encyclop. Méthod., IV. 330. 26. [Bombylius] (1789) ; GMel., Systema Naturae, V. 2881. 3. [Tabanus] (1792). mauritanica Meig., Klassif., I. 176. c. [T(ınyglossa] (1804); Latr., Encyclop. Méthod., VIII. 703. 3. (1811).

mauritana $\mathrm{L}$. $=$ ? marginata $\mathrm{F}_{\mathrm{ABR}}$. mauritanica MEIG. = marginata FABR.

micans Meig., System. Beschreib., II. 25. 5. (1820); Macq., Suit. à Europa mer. Buffon, I. 192. 3. (1834) ; Walk., List Dipt. Brit. Mus., I. 133. (1848) et V. suppl. 1., 119. 7. (1854); Lw., Neue Beitr., VI. 31. 10. (1859) ; Schin., Novara Reise, Dipt., 99. 47. (1868); Gobert, Mém. Soc. Linn. Nord France, 1881. 43. (1881); Ricardo, Annal. Matg. Nat. Hist., ser. 7. V. 180 et 181. (1900).

lıaustellata Ouıv., Encyclop. Méthod., IV. 329. 24. [Bombylius] (1789).

ornata Meig., System. Beschreib., IJ. 26. 6. tab. XIII. fig. 7. (182(1) ; MACQ., Suit. à Buffon, I. 193. 5. (1834); WALk., List Dipt. Brit. Mus., V. suppl. 1., 119. 8. (1854) ; Villen., Annal. Soc. Entom. France, LXXIV. 309. 1. (1905).

molesta Wied., Aussereurop. zweifl. Ins., I. 91. 9. (1828); WALK., List Dipt. Brit. Mus., V. suppl. 1., 127. 48. (1854); Ricardo, Annal. Mag. Nat. Hist., ser. 7. V. 173. (1900); Hunter, Trans. Amer. Eutom. Soc. Philad, XXVIJ. 134. (1901).

multifaria WalK., List Dipt. Brit. Mus., I. 134. (1848); Ricardo, Annal. Mag. Nat. Hist., ser. 7. V. 106 et 108. (1900).

nigripennis Guér., Voyage de la Coquille, Zool. II. 2., 288. (1832)

et Iconogr. d. Régne Anim., VII. Ins., 542. tab. XCVII. fig. 2.

(1835); Ricardo, Annal. Mag. Nat. Hist., ser. 7. V. 173. (1900). nigronotata MACQ., Dipt. exot., suppl. 4., 27. 56. tab. II. fig. 5. (1850) ; Belu., Saggio di Ditterol. Messic., I. 51. 8. (1859); Ricardo, Annal. Mag. Nat. Hist., ser. 7. V. 169. (1900); Willist., Biolog. Central. Americ., Dipt., I. 254. 9. (1901); Aldr., Catal. Nortlı Amer. Dipt., 193. (1905).

incerta BeLL., Saggio di Ditterol. Messic., I. 52. 9. (1859);

Ricardo, Annal. Mag. Nat. Hist., ser. 7. V. 169. (1900);

Aldr., Catal. North Amer. Dipt., 193. (1905).

nobilis Wied., Aussereurop. zweifl. Ins., II. 622. 15. (1830) ; WALK.,

List Dipt. Brit. Mus., V. suppl. 1., 138. 99. (1854); Ricardo,

Annal. Mag. Nat. Hist., ser. 7. V. 107. (1900).

notabilis Walk., Insecta Saunders., Dipt., 18. (1850); Ricardo, Americamer. Annal. Mag. Nat. Hist., ser. 7. V. 171 et 174. (1900). o'jesc WALK. = angulata $\mathrm{F}_{\mathrm{ABR}}$.

obscura Tiunb., Nova Acta Upsal., IX. 73. tab. I. fig. 10. [Tany- Patriaignota. glossa] (1827).

obscurata Lw., Ne:se Beitr., VI. 27.5. (1859); Ricardo, Annal. Mag. Asia min., Nat. Hist., ser. 7. V. 167. (1900).

I. Rhodus. obscuripennis Phil., Verh. zool.-bot. Ges. Wien, XV.710. 17. (1865); Chile. 
Ricardo, Annal. Mag. Nat. Hist., ser. 7. V. 173. (1900); Hunter,

Trans. Amer. Entom. Soc. Philad., XV. 134. (1901).

? ocellus*) Walk., List Dipł. Brit. Mus., I. 143. (1848); Ricardo, Ecuador.

Annal. Mag. Nat. Hist., ser. 7. V. 179. [? gen.]

ornata MEIG. = micans MEIG.

pavida Wilusts., Biolog. Central. Americ., Dipt., I. 253. 3. (1901); Mexico.

Aldr., Catal. North Amer. Dipt., 193. (1905).

picta Macq., Suit. à Buffon, I. 195. 12. (1834); Meig., System. Europa mer.

Beschreib., VII. 58. 8. (1838); WaLK., List Dipt. Brit. Mus., V.

suppl. 1., 119. 9. (1854); Lw., Neue Beitr., VI. 32. 14. (1859);

Goв., Mém. Soc. Linn. Nord France, 1881. 45. (1881); Pand.,

Revue d'Entomol., II. 227. 4. (1883); Ricardo, Annal. Mag. Nat.

Hist., ser. 7. V. 180. (1900).

pigra Ost.-SAck., Mem. Boston Soc. Nat. Hist., II. 367. (1876) ; Ricardo, Annal. Mag. Nat. Hist., ser. 7. V. 170. (1900) ; Aldpr., Catal. Nortlı Amer. Dipt., 193. (1905).

planiventris MaCQ., Dipt. exot., suppl. 4., 23. 55. (1850); Ricardo, Annal. Mag. Nat. Hist., ser. 7. V. 169. (1900); Aldr., Catal. North Amer. Dipt., 193. (1905).

prasiniventris MacQ., Dipt. exot., suppl. 1., 29. 37. tab. III. fig. 9. (1845) ; Wal., List Dipt. Brit. Mus., V. suppl. 1., 130. 62. (1854); Schin., Novara Reise, Dipt., 100. 53. (1868); Ost.-Sack., Biolog. Central. Amer., Dipt., I. 45. 3. (1886); RiCakdo, Annal. Mag. Nat. Hist., ser. 7. V. 171 et 173. (1900) ; Alnr., Catal. North Amer. Dipt., 193. (1905).

prasiventris Hunter, Trans. Amer. Entom. Soc. Philad., XXVII.

134. (1901) lapsus.

prasiventris HunTER = prasiniventris MACQ.

proboscidca $\mathrm{F}_{\mathrm{ABR}}=$ maculata $\mathrm{F}_{\mathrm{ABR}}$.

pyritosa Lw., Neue Beitr., VI. 27. 4. (1859) et Wien. Entom.

Monatschr., VI. 163. 7. (1862); JARowsch., Trudy Kharkoff, XVII. 299. 1. (1883); Ricardo, Annal. Mag. Nat. Hist., ser. 7. V. 180. (1900).

ramulifera Lw., Wien. Entom. Monatschr., VII. 9. 3. (1863); RiCArdo, Annal. Mag. Nat. Hist., ser. 7. V. 107. (1900).

rasa Lw., Berlin. Entom. Zeitschr., XIII. 5. 7. (1869); Ost.-SAcK., Mem. Boston Soc. Nat. Hist., II. 366. (1876); Willist., Trans. Kansas Acad. Sci., X. 130. (1887); Ricardo, Annal. Mag. Nat. Hist., ser. 7. V. 170. (1900) ; Hine, Ohio Naturalist, II. 169. (1902) et Ohio State Acad. Sci., Spec. Pap. No. 5., 45. (1903); Aldr., Catal. North Amer. Dipt., 193. (1905).

Roei King, Narr. Surv. Coasts of Austral., II. 467. 185. (1827); Australia. WiED., Aussereurop. zweill. Ins., II. 647. 72. (1830); WALK.,

America mer. et centr.

Europa centr. et mer., Asia min.

Africa mer.

America sept.

*) Secundum G. Ricardo 1. c. ad subfamiliam Tabaninarum referenda. 
List Dipt. Brit. Mus., I. 145. (1848) et V. suppl. 1., 145. 136. (1854) ; Ricardo, Annal. Mag. Nat. Hist., ser. 7. V. 114. (1900).

Rondanii Bertol., Mem. Aecad. Sci. Istit. Bologna, XII. 18. 20. Mozambique. tab. I. fig. 8. [Róndoni] (1862).

rostrata L., Mus. Lud. Ulr. Reg., 421. 1. [Tabanus] (1764) et Sy- Prom.bon.sp. stema Naturae, Ed. XII., II. 999. 1. [Tabanus] (1767); FABr., Species Insect., II. 455. 1. [Tabanus] (1781); Ouxv, Eneyclop. Méthod., IV. 330. 25. [Bombylius] (1789); Farr., Entomol. System., IV. 362. 1. [Tabamus] (1794); W1ED., Dipt. exot., I. 54. 6. (1821) et Aussereurop. zweifl. Ins., I. 96. 17. tab. I. fig. 2. (1828); Mace., Annal. Soc. Entom. France, VI. 429. tab. XV. fig. 11. (1837) ; WalK., List Dipt. Brit. Mus., I. 131. (1848) ; Lw., Dipt.Fauna Siidafrika's, I. 20. 8. (1860); Rrcanpo, Annal. Mag. Nat. Hist., ser. 7. V. 105 et 103. (1905).

aethiopica Thexp., Nova Acta Upsal., IX. 67. tab. I. fig. 6. [Tanyglossa] (1827).

lincata Fabr., Systema Antliat., 89. 1. (1805); Latrr., Eneyclop.

Méthod., VIII. 703. 1. (1811).

tabaniformis grisea Dea., Mém. pour serv. à l'hist. d. Ins.,

VI. 270. 1. tab. XXX. fig. 9--10. [Bombylius] (1776).

rostrata Thusr., Nova Acta Upsal., IX. 75. tab. I. fig. 13. [Tanyglossa] (1827).

rufa Macq., Dipt. exot., suppl. 4., 18. 41. (1850); v. D. Wulp, Catal. Dipt. South Asia, 64. (1896); Ricurdo, Annal. Mag. Nat. Hist., ser. T. V. 167. (1900).

Rüppellii JaEnn., Abhandl. Senckenb. Naturforsch. Ges., VI. 329. 21. (1867) ; Ricardo, Annal. Mag. Nat. Hist., ser. 7. V. 106. (1900).

Sallei Bes..., Saggio di Ditterol. Messic., I. 50. 6. (1859); Ricardo, Annal. Mag. Nat. Hist., ser. 7. V. 169. (1900); Aldr., Catal. North Amer. Dipt., 193. (1905).

Saussurei Beli., Saggio di Ditterol. Messic., I. 49. 4. tab. II. fig. 4. (1859) ; Ricardo, Annal. Mag. Nat. Hist., ser. 7. V. 169. (1900); Willist., Biolog. Central. Amer., Dipt., I. 254. 8. (1901); Hine, Ohio Naturalist, V. 228. (1904); ALDR., Catal. North Amer. Dipt., 193. (1905).

semiflava WiEd., Ausscreurop. zweifl. Ins., II. 622. 16. (1830); Wark., List Dipt. Brit. Mus., V. suppl. 1., 120. 14. (1854); Beld., Saggio di Ditterol. Messic., I. 51. 7. tab. II. fig. 2. (1859); Ricurdo, Annal. Mag. Nat. Hist., ser. 7. V. 169. (1900); Willist., Biolog. Central. Amer., Dipt., I. 253. 4. (1901); Ator., Catal. North Amer. Dipt., 193. (1905).

bicolor MAcQ., Dipt. exot., suppl. 4., 27. 57. (1850).

semilivida Bı̈., Annal. Soc. Entom. France, sér. 7. I. 366. 3. Assinia. [Sackenimyia] (1891); Rrcurdo, Annal. Mag. Nat. Hist., ser. 7. V. 107. (1900).

seminuda Coquil.., Journ. New York Entom. Soc., X. 137. (1902); Mexico. Aı.Dr., Catal. North Amer. Dipt., 194. (1905). 
semiviridis RICARDo, Annal. Mag. Nat. Hist., ser. 7. V. 181. tab. Hispania. I. fig. 7. (1900).

senegalensis Macq., Suit. à Buffon, I. 193. 6. (1834); Walk., List Senegal. Dipt. Brit. Mus., V. suppl. 1., 137. 92. (1854); Ricardo, Annal. Mag. Nat. Hist., ser. 7. V. 107. (1900).

sexfasciata WaLK., List Dipt. Brit. Mus., I. 136. (1848); Ricardo, Annal. Mag. Nat. Hist., ser. 7. V. 106 et 108. (1900).

spiloptera WiEd., Dipt. exot., I. 57. 10. (1821) et Aussereurop. zweifl. Ins., I. 102. 26. (1828); WaLK., List Dipt. Brit. Mus., I. 136. (1848) et V. suppl. 1., 138. 100. (1854); Ricardo, Annal. Mag. Nat. Hist., ser. 7. V. 107. (1900).

subandina PHm., Verh. zool.-bot. Ges. Wien, XV. 710. 15. (1865) ; Ricardo, Annal. Mag. Nat. Hist., ser. 7. V. 173. (1900); Hunter, Trans. Amer. Entom. Soc. Philar., XXVII. 134. (1901).

subfasciata WALK., The Entomologist, V. 257. 7. (1871); RiCARDO, Annal. Mag. Nat. Hist., ser. 7. V. 167. (1900).

subvaria Walk., List Dipt. Brit. Mus., I. 150. [Tabanus] (1848); Ricardo, Annal. Mag. Nat. Hist., ser. 7. V. 171 et 175. (1900).

suturalis Rond. in Truqui : Studi Entomol., I. 107. 83. (1848); Ricardo, Annal. Mag. Nat. Hist., ser. 7. V. 173. (1900); Hunter, Trans. Amer. Entom. Soc. Philad., XXVII. 134. (1901).

tabaniformis LATR. = maculata FABR.

tabaniformis grisea $\mathrm{DEG} .=$ rostrata L.

tenuirostris WALK., Trans. Entom. Soc. London, n. ser. V. 272. (1860); Ricardo, Annal. Mag. Nat. Hist., ser. 7. V. 169 et 170. (1900) ; Aldr., Catal. Nortl Amer. Dipt., 194. (1905).

testaceiventris MAcq., Dipt. exot., suppl. 3., 9. 39. (1847); WaLK., List Dipt. Brit. Mus., V. suppl. 1., 125. 37. (1854); Schin., Novara Reise, Dipt., 99. 50. (1868); Ric^RDo, Annal. Mag. Nat. Hist., ser. 7. V. 171. (1900); Hunter, Trans. Amer. Entom. Soc. Philad., XXVII. 134. (1901).

thoracica GuÉr., Voyage de la Coquille, Zool. II. 2., 288. (1832) ; Rucardo, Annal. Mag. Nat. Hist., ser. 7. V. 173. (1900).

thoracica Thusb., Nova Acta Upsal., IX. 71. [Tanyglossa] (1827). thoracica WiED., Aussereurop. zweifl. Ins., I. 89. 5. (1828); WALK., List Dipt. Brit. Mus., V. suppl. 1., 138. 95. (1854); Ricardo, Annal. Mag. Nat. Hist., ser. 7. V. 107. (1900).

tranquilla Ost.-SAck., Mem. Boston Soc. Nat. Hist., II. 367. (1874); Willist., Trans. Kansas Acad. Sci., X. 130. (1887); Ricardo, Annal. Mag. Nat. Hist., ser. 7. V. 170. (1900); Aldr., Catal. North Amer. Dipt., 194. (1905).

trauslucens MacQ., Dipt. exot., suppl. 1., 26. 33. tab. III. fig. 5. Brasilia. (1845); Walk., List Dipt. Brit. Mus., V. suppl. 1., 130. 61. (1854) ; Ricardo, Annal. Mag. Nat. Hist., ser. 7. V. 171. (1900); Hunter, Trans. Amer. Entom. Soc. Philad., XXVJr. 134. (1901). tricolor Austen $=$ Beckeri BezzI. 
umbra Walk., Insecta Saunders., Dipt., I. 19. (1850); Ricardo, Annal. Mag. Nat. Hist., ser. 7. V. 171 et 175. (1900).

variegata FABR., Systema Antliat., 92. 8. (1805); LATr., Encyclop. Méthod., VIII. 703. 2. (1811); Walk., List Dipt. Brit. Mus., V. suppl. 1., 119. 6. (1854); Lw., Neue Beitr., VI. 25. 2. (1859).

? maculata Rossi, Mantissa, 75. [Tabanus] (1792); Meig., System. Besclireib., IJ. 22. 2. p. p. (1820); Goв., Mém. Sос. Linn. Nord France, 1881. 42. (1881).

variegata MAcq., Suit. à Buffon, I. 195. 11. (1834); Mrig., System. Beschreib., VII. 57. 7. (1838); Lw., Neue Beitr., VI. 32. 13. (1859) ; Gos., Mém Soc. Linn. Nord France, 1881. 44. (1881); Pand., Revue d'Entomol., II. 227. 3. (1883); Ricardo, Annal. Mag. Nat. Hist., ser. 7. V. 180. (1900).

varipennis $\mathrm{LATR} .=$ maculata $\mathrm{FABR}$.

venosa Wied., Dipt. exot., I. 52. 1. (1821) et Aussereurop. zweifl.

Ins., I. 87. 1. (1828) ; Walk., List Dipt. Brit. Mus., V. 126. 42. (1854) ; WillisT., Kansas Univ. Quart., III. 189. (1895); Ricnirdo, Annal. Mag. Nat. Hist., ser. 7. V. 173. (1900); Huneren, Trans. Amer. Entom. Soc. Philad., XXVII. 135. (1901).

vittata PHIL., Verh. zool.-bot. Ges. Wien, XV. 711. 18. (1865);

Ricardo, Annal. Mag. Nat. Hist., ser. 7. V. 173. (1900); Hunter, Trans. Amer. Entom. Soc. Philad., XXVII. 135. (1901).

Walkeri Newm., Trans. Entom. Soc. London, 11. ser. IV. 56. 1. (1857). Wiedemanni Bell., Saggio di Ditterol. Messic., I. 48. 3. tab. II. fig.

3. (1859) ; Ricardo, Annal. Mag. Nat. Hist., ser. 7. V. 169. (1900);

Willist., Biolog. Central. Amer., Dipt., I. 254. 5. (1901) ; Aldr., Catal. North Amer. Dipt., 194. (1905).

basilaris WIED., Aussereurop. zweifl. Ins., II. 621. 13. $0^{7}$. (1828) ; Walk., List Dipt. Brit. Mus., V. 120. 13. (1854).

zonata Walk., The Entomologist, V. 256. (1870); Ricardo, Annal. Arabia.

Mag. Nat. Hist., ser. 7. V. 167. (1900).

\section{Erephopsis}

Adrel Walk., List Dipt. Brit. Mus., I. 141. (sine descr.) [Pangonia] (1848) et Insecta Saunders., Dipt., I. 16.[Pangonia] (1850) ; Butler, Cistula Entom., I. 356. [Pangonia] (1876); Hutron, Catal. New. Zealand Dipt. etc., 22. [Pangonia] (1881) ; Ricardo, Annal. Mag. Nat. Hist., ser. 7. V. 112 et 115. [Erephrosis] (1900); Hutton, Trans. New Zealand Instit., XXXIII. 15. [Pangonia] (1901).

albifrons MacQ., Dipt. exot., I. 1., 108. 24. [Pangonia] (1838); Blanch. in Gay : Hist. fis. y polit. de Chile, Zool. VII. 389. tab. I. fig. 11. [Pangonia] (1854); Walk., List Dipt. Brit. Mus., V. suppl. 1., 125. 34. [Pangonia] (1854) ; Pril., Verh. zool.-bot. Ges. Wien, XV. 708. 7. [Pangonia] (1865); Ricardo, Annal. Mag. Nat. Hist., ser. 7. V. 171. [Erephrosis] (1900) ; Huncer, Trans. Amer. Entom. Soc. Pliilad., XXVII. 133. [Pangonia] (1901).
Cliile.

Europa mer.

Europa mer.

Brasilia.

Chile.

Australia .

Mexico.

N. Selandia.

Chile. 
albonotata MacQ., Dipt. exot., suppl. 5., 20. 61. [Pangonia] (1855); Ricardo, Annal. Mag. Nat. Hist., ser. 7. V. 112. [Erephrosis] (1900).

ardens MACQ., Dipt. exot., I. 1., 103. 13. [Pangonia] (1838) ; WALK., List Dipt. Brit. Mus., V. 130. 59. [Pangonia] (1854); Ricardo, Annal. Mag. Nat. Hist., ser. 7. V. 171. [Erephrosis] (1900); Hunter, Trans. Amer. Entom. Soc. Philad., XXVII. 133. [Pangonia] (1901).

atripes RöD., Stettin. Entom. Zeitg., XLVII.261.[Pangonia] et Dipteren gesammelt in Südamerika von A. Stübel, 7. [Pangonia] (1892); Ricardo, Annal. Mag. Nat. Hist., ser. 7. V. 172. [Erephrosis] (1900) ; Hunter, Trans. Amer. Entom. Soc. Philad., XXVII. 133. [Pangonia] (1901).

aureohirta Ricardo, Annal. Mag. Niit. Hist., ser. 7. V. 116. fab. I. Australia. fig. 10. [Erephrosis] (1900).

aurimaculata MAcq., Dipt. exot., I. 1., 105. 17. [Pangonia] (1838) ; WaLk., List Dipt. Brit.' Mus., V. 125. 38. [Pangonia] (1854); Ricardo, Annal. Mag. Nat. Hist., ser. 7. V. 171. [Erephrosis] (1900) ; Hunter, Trans. Amer. Entom. Soc. Philad., XXVII. 133. [Pangonia] (1901).

auripes Ricardo, Annal. Mag. Nat. Hist., ser. 7. V. 176. tab. I. fig. 12. [Erephrosis] (1900).

badia Walk., List Dipt. Brit. Mus., I. 132. [Pangonia] (1848) et V. suppl. 1., 120. 15. [Pangonia] (1854) ; RiCARdo, Annal. Mag. Nat. Hist., ser. 7. V. 172 et 176. [Erephrosis] (1900).

bahiana Bı́., Mém. Soc. Zool. France, V. 612. [Pangonia] (1892); Ricardo, Annal. Mag. Nat. Hist., ser. 7. V. 172. [Erephrosis] (1900).

basalis WALK., List Dipt. Brit. Mus., I 133. [Pangonia] (1848) et V. suppl. 1., 322. var. [Pangonia] (1854); Ricardo, Annal. Mag. Nat. Hist., ser. 7. V. 172 et 178. [Erephrosis] (1900).

Besckii Wied., Aussereurop. zweifl. Ins., I. 97. 18. [Pangonia] (1828) ; WalK., List Dipt. Brit. Mus., V. suppl. 1., 124. 29. [Pangonia] (1854); Ricardo, Annal. Mag. Nat. Hist., ser. 7. V. 171 et 176. [Erephrosis] (1900); Hunter, Trans. Amer. Entom. Soc. Philad., XXVII. 133. [Pangonia] (1901).

bicolor MACQ., Dipt. exot., suppl. 1., 24. 29. [Pangonia] (1845) ; WALK., List Dipt. Brit. Mus., V. suppl. 1., 143. 127. [Pangonia] (1854); Ricardo, Annal. Mag. Nat. Hist., ser. 7. V. 112. [Erephrosis] (1900).

contigua Walk., List Dipt. Brit. Mus., I. 138. [Pangonia] (1848); Ricardo, Annal. Mag. Nat. Hist., ser. 7. V. 112 et 116. [Evephrosis] (1900).

crocata JAENN. = lata GUÉR.

depressa MACQ. = lata Gún.

dilatata Jaenn., Abhandl. Senckenberg. Naturfor'sch. Ges., VI. 328. Australia. 20. [Pangonia] (1867); Ricardo, Amnal. Mag. Nat. Hist., ser. 7. V. 112. [Erephrosis] (1900). 
divisa WaLk., Insecta Saunders., Dipt., I. 17. [Pangonia] (1850); Ricardo, Amnal. Mag. Nat. Hist., ser. 7. V. 112 et 117 . [Erephrosis] (1900).

eriomera Macq., Dipt. exot., I. 1., 105. 18. tab. XIV. fig. 3. [Pangonia] (1838) ; Blanch. in GaY : Hist fis. y polit. de Chile, Zool. VII. 389. 5. 1ab. I. fig. 12. [Pangonia] (1854); Walk., List Dipt. Brit. Mus., V. suppl. 1., 124. 32. [Pangonia] (1854); Phis., Verh. zool.-bot. Ges. Wien, XV. 708. 5. [Pangonia] (1865); Ricardo, Annal. Mag. Nat. Hist., ser. 7. V. 171. [Erephrosis] (1900); IIunter, Trans. Amer. Entom. Soc. Philad., XXVII. 133. [Pangonia] (1901).

fenestrata MacQ., Dipt. exot., suppl. 1., 26. 32. [Pangonia] (1845); Walk., List Dipt. Brit. Mus., V. suppl. 1., 125. 36. [Pangonia] (1854) ; Ricardo, Annal. Mag. Nat. Hist., ser. 7. V. 172. [Erephrosis] (1900) ; Hunter, Trans. Amer. Entom. Soc. Philad., XXVII. 134. [Pangonia] (1901).

fulvithorax WiEd., Dipt. exot., I. 52. 2. [Pangonia] (1821) et Aussereurop. zweifl. Ins., I. 89. 4. [Pangonia] (1828); MACQ., Annal. Soc. Entom. France, VI. 429. tab. XV. fig. 21. [Pangonia] (1837); Walk., List Dipt. Brit. Mus., I. 132. [Pangonia] (1848) et V. suppl. 1., 126. 41. [Pangonia] (1854); Brg. in SAGRA: Hist. la Isla de Cuba, P. 2. VII. 334. [Pangonia] (1856); Willist., Kansas Univ. Quart., III. 189. [Pangonia] (1895); Ricardo, Annal. Mag. Nat. Hist., ser. 7. V. 171 et 176. [Erephrosis] (1900); Hunter, Trans. Amer. Entom. Soc. Philad., XXVII. 134. [Pangonia] (1901) ; Aldr., Catal. North Amer. Dipt., 193. [Pangonia] (1905).

fulvitibialis RicsRdo, Annal. Mag. Nat. Hist., ser. 7. V. 177. tab. I. fig. 11. [Erephrosis] (1900).

fumifera Walk., list Dipt. Brit. Mus., V. 323. [Pangonia] (1854); Ricardo, Annal. Mag. Nat. Hist., ser. 7. V. 172 et 178. [Erephrosis] (1900).

fusca Ricurdo, Annal. Mag. Nat. Hist., ser. 7. IX. 431. [Erephrosis] (1902).

gemina Walk., List Dipt. Brit. Mus., I. 138. [Pangonia] (1848); Ricardo, Annal. Mag. Nat. Hist., ser. 7. V. 112 et 117. [Erephrosis] (1900).

gibbula WALK., List Dipt. Brit. Mus., I. 140. [Pangonia] (1848); Ricardo, Annal. Mag. Nat. Hist., ser. 7. V. 112 et 117. [Erephrosis] (1900).

guttata Donov., Gener. illustr. of Entom., I. Tab. Hymen. et Dipt. fig. ? [Tabanus] (1805) ; LAtr., Encyclop. Méthod., VIII. 703. 5. [Pangonia] (1811); WIED., Aussereurop. zweifl. Ins., I. 194. 124. ['Tabanus] (1828); Gú̉r., Voyage de la Coquille, Zool. II. 2., 289. tab. XX. fig. 10. [Tabamus] (1830); Walk., List Dipt. Brit. Mus., I. 141. [Pangonia] (1848) et V. suppl. 1., 142. 121. [Pangonicl (18j4); Ricardo, Annal. Mag. Nat. Hist., ser. 7. V. 112 et 114. [Erephrosis] (1900).
Australia occ.

Brasilia, Chile.

Brasilia.

Brasilia, Cuba.

Brasilia.

Brasilia.

Brasilia.

A ustralia occ.

A ustralia occ.

Asia mer., Australia. 
margaritifera WiEn., Aussereurop. zweifl. Ins., I. 88. 3. [Pangonia] (1828); MaCQ., Anral. Soc. Entom. France, VI. 429. tab. XV. fig. 8. [Pangonia] (1837) et Dipt. exot., suppl. 3., 8. [Pangonia] (1847); Willist., Kansas Univ. Quart., III. 191. [Pangonia] (1895).

Jacksonii MACQ., Dipt. exot., I. 1., 102. 10. [Pangonia] (1838); Walk., List Dipt. Brit. Mus., I. 140. [Pangonia] (1848), Insecta Saunders., Dipt., I. 14. [Pangonia] (1850) et List Dipt. Brit. Mus., V. suppl. 1., 143. 126. [Pangonia] (1854); Ricardo, Annal. Mag. Nat. Hist., ser. 7. V. 112 et 117. [Erephrosis] (1900).

lata Guḱr., Voyage de la Coquille, Zool. II. 2., 289. [Tabanus] (1832) et Iconogr. Régn. Anim., VII. Ins., 542. tab. XCVII. fig. 1. [Tabanus] (1835); WaLK., Trans. Linn. Soc. London, XVII. 336. 15. [Tabanus] (1837); RöDer, Stettin. Entom. Zeitg., XIIII. 510. [Pangonia] (1882); Hunter, Trans. Amer. Entom. Soc. Philad., XXVII. 134. [Pangonia] (1901).

crocata Jaenn., Abhandl. Senckenberg. Naturf. Ges., VI. 330. 22. [Pangoniu](1867).

depressa Macq., Annal. Soc. Entom. France, VI. 429. tab. XV. fig. 22. [Pangonia] (1837) et Dipt. exot., I. 1., 107. 21. [Pangonia] (1838) ; WaLK., List Dipt. Brit. Mus., I. 143. [Pangonia] (1848) ; Macq., Dipt. exot., suppl. 4., 25. [Pangonia] (1850) ; Walk., List Dipt. Brit. Mus., V. suppl. 1., 126. 39. [Pangonia] (1854); Blanch. in GaY: Hist. fis. y polit. de Chile, Zool. VII. 388. 4. tab. I. fig. 8. [Pangonia] (1854); PHIL., Verh. zool.-bot. Ges. Wien, XV. 707. 4. [Pangonia] (1865); Scuin., Novara Reise, Dipt., 100. 54. [Pangonia] (1868); v. D. Wulp, Tijdschr. v. Entomol., XXIV. 157. 2. [Pangonia] (1881) ; Hansen, Fabrica oris Dipt., tab. II. fig. 14. [Pangonia] (1883) ; Rrcardo, Annal. Mag. Nat. Hist., ser. 7. V. 171 et 177. [Erephrosis] (1900).

laterina Rond., Nuov. Annal. Sci. Nat. Bologna, ser. 3. II. 370. 27. [Pangonia] (1850); Ricardo, Annal. Mag. Nat. Hist., ser. 7. V. 172. [Erephrosis] (1900); Hunter, Trans. Amer. Entom. Soc. Pliilad., XXVII. 134. [Pangonia] (1901).

leucopogon Wied., Aussereurop. zweifl. Ins., I. 92. 11. [Pangonia] (1828); Macq., Annal. Soc. Entom. France, VI. 429. tab. XV. fig. 3, 10. [Pangonia] (1837); Watk., List Dipt. Brit. Mus., I. 133. [Pangonia] (1848) et V. suppl. 1., 128. 49. [Pangonia] (1854); Ricardo, Annal. Mag. Nat. Hist., ser. 7. V. 171 et 178. [Erephrosis] (1900); Hunter, Trans. Amer. Entom. Soc. Philad., XXVII. 134. [Pangonia] (1901).

limbinervis MACQ., Dipt. exot., suppl. 5., 21. 62. [P(nngonia] (1855); Ricardo, Annal. Mag. Nat. Hist., ser. 7. V. 112. [Erephrosis] (1900).

longirostris MACQ., Dipt. exot., suppl. 2., 12. 42. [Pangonia] (1847); WALK., List Dipt. Brit. Mus., V. suppl. 1., 128. 52. [Pangonia]

Australia.

Chile.

America aeq.

Brasilia.

Australia.

? Brasilia. 
(1854); Ricardo, Annal Mag. Nat. Hist., ser. 7. V. 172. [Erephrosis] (1900); Hunten, Trans. Amer. Entom. Soc. Philad., XXVII. 134. (1901).

nigripes RöD., Dipteren gesamm. in Siid-Amerika von A. Stübel, 8. [Pangonia] (1892).

macroporum MacQ., Dipt. exot., I. 1., 101. 8. tab. XV. fig. 2. [Pangonia] (1838); WaLK., List Dipt. Brit. Mus., V. suppl. 1., 143. 129. [Pangonia] (1854); Rrcardo, Annal. Mag. Nat. Hist., ser. 7. V. 112 et 117. [Erephrosis] (1900).

maculipennis MacQ., Dipt. exot., suppl. 4., 20. 45. [Pangonia] (1850); Schin., Novara Reise, Dipt., 99. 46. [Pangonia] (1868); Rrcardo, Annal. Mag. Nat. Hist., ser. 7. V. 106. [Erephrosis] (1900). margaritifera WIED. = guttata Dosov.

media WalK., List Dipt. Brit. Mus., I. 42. [Pangonia] (1818); Ricardo, Annal. Mag. Nat. Hist., ser. 7. V. 112 et 114. [Erephrosis] (1900). minor Macq., Dipt. exot., suppl. 2., 13. 43. [Pangonia] (1847); Walk., List Dipt. Brit. Mus., V. suppl. 1., 124. 31. [Pangonia] (1854); Ricardo, Amnal. Mag. Nat. Hist., ser. 7. V. 172. [Erephrosis] (1900) ; Hunter, Trans. Amer. Entom. Soc. Philad., XXVII. 134. [Pangonia] (1901).

nana Walk., Insecta Saunders., Dipt., I. 11. [Pangonia] (1850); Ricardo, Annal. Mag. Nat. Hist., ser. 7. V. 172 et 178. [Erephrosis] (1900).

nigra RiCARdo, Annal. Mag. Nat. Hist., ser. 7. VI. 292. [Erephrosis] (1900).

nigripes RöD. = longirostris MACQ.

nigrivittata Marq., Dipt. exot., suppl. 4., 23. 51. [Pangonia] (1850);

Ricando, Annal. Mag. Nat. Hist., ser. 7. V: 172. [Erephrosis] (1900) ; Hunter, Trans, Amer. Entom. Soc. Philad., XXVII. 134. (1901).

nigrohirta WaLk., List Dipt. Brit. Mus., I. 132. [Pangonia] (1848); Ricardo, Annal. Mag. Nat. Hist., ser. 7. V. 172 et 176. [Erephrosis] (1900).

? picea Thuns., Nova Acta Upsal., IX. 66. [Tanyglossa] (1827).

piceohirta WALK., List Dipt. Brit. Mus., I. 132. [Pangonia] (1848); Ricardo, Annal. Mag. Nat. Hist., ser. 7. V. 172 et 175. [Erephrosis] (1900).

? pulchra Thuns., Nova Acta Upsal., IX. 72. t. I. f. 9. [Tanyglossa] (1827).

quadrimacula WALK., List Dipt. Brit. Mus., I. 138. [Pangonia] (1848); Ricardo, Annal. Mag. Nat. Hist., ser. 7./ V. 112 et 116. [Erephrosis] (1900).

rostrifera BeLl., Saggio di Ditterol. Messic., I. 47. 2. [Pangonia] (1859); Ricardo, Annal. Mag. Nat. Hist., ser. 7. V. 169. [Erephrosis] (1900); Willist., Biolog. Central. Americ., Dipt., 1. 253. 2. [Pangonia] (1901); Aldr., Catal. North Amer. Dipt., 193. [Pangonia] (1905).

I. Kanguroos.

Australia.

Australia.

?America

Brasilia.

Surinam.

Brasilia.

Brasilia.

Brasilia. Brasilia.

Brasilia.

Australia.

Mexico. 
rufescens Rrcarno, Annal. Mag. Nat. Hist., ser. 7. VI. 294. [Ere- Peru. phrosis] (1900).

rufoaurea PhiL., Verh. zool.-bot. Ges. Wien, XV. 709. 12. [Pangonia] (1865); Richrdo, Annal. Mag. Nat. Hist., ser. 7. V. 172 et 177. [Erephrosis] (1900); Hunter, Trans. Amer. Entom. Soc. Philad., XXVII. 134. [Pangonia] (1901).

rufohirta Walk., List Dipt. Brit. Mus., I. 131. [P(mgonia] (1848); Ricardo, Annal. Mag. Nat. Hist., ser. 7. V. 172 et 175. [Erephrosis] (1900).

rufopilosa Ricardo, Annal. Mag. Nat. Hist., ser. 7. VI. 293. [Erephrosis rufopilosis] (1900).

sorbens WIED., Aussereurop. zweifl. Ins., I. 93. 13. [Pangonia] (1828); WaLk., List Dipt. Brit. Mus., I. 133. [Pangonia] (1848) et V. suppl. 1., 128. 51, 323. [Pangonia] (1854); E. L. Arrib., Bolet. Acad. Nat. Cienc. Córdoba, IV. 130. 54. [Pangonia] (1882); Becher, Denkschr. Akad. Wien, XLV. 142. tab. II. fig. 19. [Pangonia] (1882); Hansex, Fabrica oris Dipt., tab. II. fig. 12-13, 15-17. [Pangonia] (188j); Rrcardo, Annal. Mag. Nat. Hist., ser. 7. V. 171 et 177. [Erephrosis] (1900); IIunter, Trans. Amer. Entom. Soc. Philad., XXVII. 134. [Pangonia] (1901).

submacula WALk., List Dipt. Brit. Mus., I. 142. [Pangonia] (1848) et Insecta Saunders., Dipt., I. 13. [Pangonia] (1850); Ricardo, Annal. Mag. Nat. Hist., ser. 7. V. 112 et 115. [Erephrosis] (1900). tenuistria WALK., List Dipt. Brit. Mus., I. 143. [Pangonia] (1848); Ricardo, Annal. Mag. Nat. Hist., ser. 7. V. 172 et 178. [Erephrosis] (1900).

tricolor WaLk., List Dipt. Brit. Mus., I. 139. [Pangonia] (1848); Ricardo, Annal. Mag. Nat. Hist., ser. 7. V. 112 et 117. [Erephrosis] (1900).

vertebrata Bı́., Mém. Soc. Zool. France, V. 618. [Erephosis] (1892); Ricardo, Annal. Mag. Nat. Hist., ser. 7. V. 112. [Erephrosis] (1900).

Winthemi Wied., Zoolog. Magaz., I. 3., 44. 5. [Pangonia] (1819), Dipt. exot., I. 53. 3. [Pangonia] (1821) et Aussereurop. zweifl. Ins., I. 91. 8. [Pangonia] (1828) ; WaLk., List Dipt. Brit. Mus., V. suppl. 1., 127. 47. [Pangonia] (1854); Ricardo, Annal. Mag. Nat. Hist., ser. 7. V. 171 et 178. [Erephrosis] (1900); Hunter, Trans. Amer. Entom. Soc. Philad., XXVII. 135. [Pangonia] (1901).

xanthopogon MAcQ., Dipt. exot., I. 2., 179. [P(angonia] (1839); Walk., List Dipt. Brit. Mus., V. suppl. 1., 129. 55. [Pangoniu] (1854); Ricardo, Annal. Mag. Nat. Hist., ser. 7. V. 172. [Erephrosis] (1900); Hunter, Trans. Amer. Entom. Soc. Philad., XXVII. 135. [Pangonia] (1901).

Australia oce.

Brasilia.

Australia.

Australia.

Brasilia.

Brasilia. 


\section{Diatomineura}

Rond., Archivio per la Zool. Modena, III. 84. (1863).

Corizoneura Rond., Archivio per la Zool. Modena, III. 85. (1863).

\section{Diatomineura s. str.}

albicostata MAcq., Dipt. exot., supp]. 1., 24. 28. [Pangonia] (1845); Walk., List Dipt. Brit. Mus., V. suppl. 1., 144. 130. [Pangonia] (1854); Ricando, Annal. Mag. Nat. Hist., ser. 7. V. 113. (1900). albithorax Macq., Dipt. exot., 1. 1., 107. 22. [Pangonia] (1838) ; Walk., List Dipt. Brit. Mus., I. 143. [Pangonia] (1848); Branch. in GAY : Hist. fis. y polit. do Chile, Zool., VII. 388. 3. tab. I. fig. 7. [Pangonia] (1854); Walk., List Dipt. Brit. Mus., V. suppl.1., 125. 35. [Pangonia] (1854); P1II., Verb. zool.-bot. Ges. Wien, XV. 707. 3. [Pangonia] (1865); Schrn., Novara Reise, Dipt., 101. 55. [Pangonia] (1868); Ricardo, Annal. Mag. Nat. Hist., ser. 7. V.172. (1900); Hunter, Trans. Amer. Entom. Soc. Philad., XXVII. 133. [Pangonia] (1901).

amaena BiG. = latipalpis MacQ.

aperta Lw., Noue Beitr., VI. 32. 12. [Pangonia] (1859); Ricardo, Lusitania.

Annal. Mag. Nat. Hist., ser. 7. V. 180. (1900).

aurata MACQ., Dipt. exot., I. 1., 100. 7. tab. XV. fig. 1. [Pangonia] (1838) et suppl. 2., 12. [Pangonia] (1847) et Annal. Soc. Entom. France, VI. 429. tab. XV. fig. 7. [Pangonia] (1837); WaLk., List Dipt. Brit. Mus., I. 141. [Pangonia] (1848) et V. suppl. 1., 142. 123. [Pangonia] (1854); Ricardo, Annal. Mag. Nat. Hist., ser. 7. V. 113. (1900).

auriflua Doxov., Gen. Illustr. of Entom., I. Hymen. et Dipt., fig. [Tabanus] (1805); Wied., Aussereurop. zweifl. Ins., I. 194. 125. [Tabanus] (1828); WatK., List Dipt. Brit. Mus., V. suppl. 1., 142. 122. [Pángonia] (1854); Ricardo, Annal. Mag. Nat. Hist., ser. T. V. 112 et 119 . (1900).

solida WalK., List Dipt. Brit. Mus., I. 141. [Pangonia] (1848).

barbata L., Mus. Lud. Ulr. Reg., 422. 2. [Tabanus] (1764) et Systema Naturae, Ed. XII., II. 999. 2. [Tabanus] (1767); OLIV., Encyclop. Méthod., IV. 330. 27. [Bombylius] (1789); Latr., Encyclop. Méthod., VIII. 703. 6. [Pangonia] (1811); Wikd., Zool. Magaz., I. 3., 7. 10. [Pangonia] (1819) et Aussereurop. zweifl. Ins., I. 94. 14. [Pangonia] (1828); MacQ., Annal. Soc. Entom. France, VI. 429. tab. XV. fig. 17. [Pangonia] (1837); Walk., List Dipt. Brit. Mus., I. 135. [Pangonia] (1848); Ricardo, Annal. Mag. Nat. Hist., ser. 7. V. 106 et 109. (1900).

bicolor Hudson, Manual of New Zealand Entom., 54. tab. VI. fig. N. Selandia. 2. [Comptosia] (1892).

Ricarloi Hutron, Trans. New Zealand Instit., XXXIII. 16. [Pangonia] (1901).

Australia, Tasmania.

Australia.

Prom.bon.sp.

Australia.
Australia

Chile. 
brevirostris MAcQ., Dipt. exot., suppl. 4., 22. 48. [Pangonia] (1850); Australia. Ricardo, Annal. Mag. Nat. Hist., ser. 7. V. 113. (1900).

californica BIG. = dives WiLlis's.

caliginosa Walk., Proe. Linn. Soc. London, VIII. 108. 19. [Pangonia] (1865) ; v. D. Wulp, Catal. Dipt. South Asia, 64. [Pangonia] (1896); Ricardo, Annal. Mag. Nat. Hist., ser. 7. V. 113 et 118. (1900). carnea Big., Mém. Soc. Zool. France, V. 619. (1892); Ricardo, Annal. Mag. Nat. Hist., ser. 7. V. 113. (1900).

clavata MACQ., Dipt. exot., I. 1., 101. 9. [Pangonia] (1838); Walk., List Dipt. Brit. Mus., V. suppl. 1., 143. 125. [Pangonia] (1854); Ricardo, Annal. Mag. Nat. Hist., ser. 7. V. 113. (1900).

concolor WALK., Insecta Saunders., Dipt., I. 14. [P'ıngonia] (1850); Ricardo, Annal. Mag. Nat. Hist., ser. 7. 'V. 113 et 118. (1900). constans WALK., Insecta Saunders., Dipt., I. 15. [Pangonia] (1850); Ricardo, Annal. Mag. Nat. Hist., ser. 7. V. 113 et 118. (1900). crassa WaLk., List Dipt. Brit. Mus., I. 14t. [Pangonic!] 1848); Ricardo, Annal. Mag. Nat. Hist., ser. 7. V. 113 et 120. (1900). dives Willist., Trans. Kansas Acad. Sci., X. 130. [Pangoniı] (1887); Ricardo, Annal. Mag. Nat. Hist., ser. 7. V. 169. (1900); Hine, Ohio Naturalist, V. 227. [Pangonia] (1904); ALDr., Catal. North Amer. Dipt., 193. [Pangonia] (1905).

californica Bı̈., Mén. Soc. Zool. France, V. 618. (1892);

Ricardo, Annal. Mag. Nat. Hist., ser. 7. V. 169. (1900); Aldor, Catal. Nortl 1 Amer. Dipt., 193. [P(ungonia] (1905).

dorsoguttata Macq., Dipt. exot., suppl. 4., 24. 53. [Pangonia] (1850); Blaxch. in Gay : Hist. fis. y polit. de Chile, Zool. VII. 390. 9. [Pangonia] (1854); Pinl., Verh. zool.-bot. Ges. Wien, XV. 708. 9. [Pangonia] (1865); Ricardo, Amal. Mag. Nat. Hist., ser. 7. V. 172. (1900); Hunter, Trans. Amer. Entom. Soe. Philad., XXVII. 133. [Pangonia] (1901).

dorsomaculata MACQ., Dipt. exot., suppl. 4., 21. 47. [Pangonia] (1850); Ricardo, Annal. Mag. Nat. Hist., ser. 7. V. 113. (1900). exeuns WaLk., Insecta Saunders., Dipt., I. 12. [P(1ngonia] (1850); Ricardo, Annal. Mag. Nat. Hist., ser. 7. V. 172 et 179. (1900). fulvifascia WALK., List Dipt. Brit. Mus., I. 137. [Pangonia] (1848); Ricardo, Annal. Mag. Nat. Hist., ser. 7. V. 106 et 109. (1900). ? gagatina BiG., Mém. Soc. Zool. France, V. 620. (1892); Rrcardo, Annal. Mag. Nat. Hist., ser. 7. V. 113. (1900).

gemella WaLK., List Dipt. Brit. Mus., I. 139. [Pangonia] (1848); Ricardo, Annal. Mag. Nat. Hist., ser. 7. V. 113 et 118. (1900). grisea JAEnn., Abhandl. Senckenberg. Naturforsch. Ges., VI. 331. 23. [P(ıngonia] (1867); Ricardo, Annal. Mag. Nat. Hist., ser. 7. V.172. (1900); Hunter, Trans. Amer. Entom. Soc. Plilad., XXVII. 134. [Pangonia] (1901).

hirticeps Now., Memoiren d. Krakauer Akad., II. 17. [Pangonia] N. Selandia. (1875); Hutron, Catal. New Zealand Dipt. ete, 22. [Pangonia] (1901) et Trans. New Zealand Instit., XXXIII. 16. (1901). 
hirtipalpis Bıg., Mém. Soc. Zool. France, V. 619. (1892); Rıcardo, Chile. Annal. Mag. Nat. Hist., ser. 7. V. 172. (1900).

jaksoniensis Guér., Voyage de la Coquille, Zool., II. 2., 288. tab. Australia.

XX. fig. 3. [Pangonia] (1832); Ricardo, Annal. Mag. Nat. Hist., ser. 7. V. 113 et 118 . (1900).

jucunda Jaknn., Abhandl. Senckenberg. Naturf. Ges., VI. 327. 18. [Pangonia] (1867); Ricardo, Aunal. Mag. Nat. Hist., ser. 7. V. 172. (1900) ; Hunter, Trans. Amer. Entom. Soc. Philad., XXVII. 134. [Pangonia] (1901).

Landbecki PHIL. = latipalpis MACQ.

lasiophthalma v. D. Wulp, Tijdschr. v. Entonı., XXXI. 365. 4. tab. IX. fig. 6. [Pangonia] (1888); Rıcardo, Annal. Mag. Nat. Hist., ser. 7. V. 172. (1900); Hunter, Trans. Amer. Eutom. Soc. Philad., XXVII. 134: [Pangonia] (1901).

latipalpis Macq., Dipt. exot., suppl. 4., 25. 54. tab. II. fig. 4. [Pangonia] (1850); Blanch. in GaY : Hist. fis. y polit. de Chile, Zool. VII. 390. [Pungonia] (1854); PhiL., Vorlı. zool.-bot. Ges. Wien, XV. 708. 8. [Pangonia](1865); Röner, Stettin. Entom.Zeitg., XLIII. 511. [Pangonia] (1882); Ricardo, Annal. Mag. Nat. Hist., ser. 7. V. 172. (1900); Hunter, 'Trans. Amer. Entom. Soc. Philad., XXVII. 134. [Pangonia] (1901).

amaena Brg., Annal. Soc. Entom. France, sér. 6. I. 20. [Trichophthalma] (1881).

Landbecki PHIL., Verh. zool.-bot. Ges. Wien, XV. 658. 11. [Hermoneura] (1865).

Lerda WaLK., List Dipt. Brit. Mus., I. 141. [Pangonia] (1848) [sinc descr.] et Insecta Saunders., Dipt., I. 16. [Pangonia] (1850); Buțler, Cistula Entomol., I. 355. [Pangonica] (1876); Hutton, Catal. New Zealand Dipt. etc., 21. [Pangonia] (1881); Rıcardo, Annal. Mag. Nat. Hist., ser. 7. V. 113 et 118. (1900); Hutron, Trans. New Zealand Instit., XXXIII. 16. [Pangonia] (1901).

leucothorax Ricardo, Annal. Mag. Nat. Hist., ser. 7. V. 179. tab. I. fig. 8. (1900).

limbithorax MACQ., Dipt. exot., suppl. 5., 22. 63. [Pangonia] (1855) ; Ricardo, Annal. Mag. Nat. Hist., ser. 7. V. 182. (1900).

longipennis Ricardo, Annal. Mag. Nat. Hist., ser. 7. IX. 433. (1902). minima Ricardo, Annal. Mag. Nat. Hist., ser. 7. V. 119. tab. I. fig. $4-5$. (1900).

morio v. D. Wulp, Tijdschr. v. Entomol., XXIV. 156. 1. [Pangonia] (1881) ; Ricardo, Annal. Mag. Nat. Hist., ser. 7. V. 172. (1900); IIunter, Trans. Amer. Entom. Soc. Philad., XXVII. 134. [Pangonia] (1901).

patula WaLK., List Dipt. Brit. Mus., I. 144. [Pangonia] (1848); RICARDo, Annal. Mag. Nat. Hist., ser. 7. V. 113 et 120. (1900).

plana WaLk., List Dipt. Brit. Mus., I. 144. [Pangonia] (1848); Ricardo, Annal. Mag. Nat. Hist., ser. 7. V. 113 et 118. (1900).

pyrausta Ost.-SAck., Biolog. Central. Americ., Dipt., I. 43. 1. [Pan-Americacentr. 
gonia] (1886) ; Willist., Kansas Univ. Quart., III. 189. [Pangonia] (1895) ; Ricardo, Annal. Mag. Nat. Hist., ser. 7. V. 172. (1900); Willist., Biolog. Central. Americ., Dipt., I. 253. 1. [Pangonia] (1901) ; Aldr., Catal. North Amer. Dipt., 193. [Pangonia] (1905). rhinophora Bell., Saggio di Ditterol. Messic., I. 46. 1. tab. II. fig.

1. [Pangonia] (1859); Ost.-SAck., Biolog. Central. Americ., Dipt.,

I. 44. [Pangonia] (1886); Ricardo, Annal. Mag. Nat. Hist., ser.

7. V. 169. (1900); Aldr., Catal. North Amer. Dipt., 193. [Pangonia] (1905).

Ricardo $i$ HutTon $==$ bicolor Hudson.

rufa Macq., Dipt. exot., I. 1., 106. 19. [Pangonia] (1838); WaLk., List Dipt. Brit. Mus., V. suppl. 1., 130. 60. [Pangonia] (1854); Ricardo, Annal. Mag. Nat. Hist., ser. 7. V. 172. (1900); Hunter, Trans. Amer. Entom. Soc. Philad., XXVII. 134. [Pangonia] (1901).

ruficornis MacQ., Dipt. exot., suppl. 1., 25. 31. tab. III. fig. 3. [Pangonia] (1845); Walk., List Dipt. Brit. Mus., V. suppl. 1., 143. 128. [Pangonia] (1854); Ricardo, Annal. Mag. Nat. Hist., ser. 7. V. 113. (1900).

seminigra Ricardo, Annal. Mag. Nat. Hist., ser. 7. IX. 432. (1902). Brasilia. solida WALK. = auriflua Donov.

subappendiculata Macq., Dipt. exot., suppl. 4., 19. 44. tab. II. fig. Tasmania.

2. [Pangonia] (1850); Ricardo, Annal. Mag. Nat. Hist., ser. 7. V. 113. (1900).

tabanipennis Macq., Dipt. exot., I. 1., 104. 16. [Pungonia] (1838); WaLk., List Dipt. Brit. Mus., V. suppl. 1., 131. 64. [Pangonia] (1854); Ricardo, Annal. Mag. Nat. Hist., ser. 7. V. 172. (1900); Hunter, Trans. Amer. Entom. Soc. Plilad., XXVII. 134. [Pangonia] (1901).

testacea Macq., Dipt. exot., I. 1., 99. 5. [Pangonia] (1838); WALK., List Dipt. Brit. Mus., V. suppl. 1., 145. 135. [Pangonia] (1854); Ricardo, Annal. Mag. Nat. Hist., ser. 7. V. 113. (1900); Hunter, Trans. Amer. Entom. Soc. Pliilad., XXVII. 134. [Pangonia] (1901).

testaceomaculata Macq., Dipt. exot., suppl. 4., 21. 46. [Pangonia] (1850); Ricardo, Annal. Mag. Nat. Hist., ser. 7. V. 113. (1900). unicolor Macq., Dipt. exot., suppl. 1., 27. 34. tab. III. fig. 6. [Pangonia] (1845); WALK., List Dipt. Brit. Mus., V. suppl. 1., 129. 57. [Pangonia] (1854); Willist., Kansas Univ. Quart., IJI. 189. [Pangonia] (1895); Ricardo, Annal. Mag. Nat. Hist., ser. 7. V. 172. (1900); Hunter, Trans. Amer. Entom. Soc. Pliilad., XXVII. 135. [Pangonia] (1901).

varia WALK., List Dipt. Brit. Mus., I. 209. [Diabasis] (1848) et V. Chile. suppl. 1., 269. 1. [Diabasis] (1854); Ricardo, Annal. Mag. Nat. Hist., ser. 7. XIV. 357. (1904).

violacea MAcQ., Dipt. exot., suppl. 4., 22. 49. tab. II. fig. 3. [PanTasmania. gonia] (1850); Ricardo, Annal. Mag. Nat. Hist., ser. 7. V. 113. (1900). 
viridiventris MAcQ., Dipt. exot., I. 1., 108. 23. [Pangonia] (1838); Walk., List Dipt. Brit. Mus., I. 137. [Pangonia] (1848) et V. suppl. 1., 130. 63. [P'angonia] (1854); Blanch. in Gay : Hist. fis. y polit. de Chile, Zool. VII. 389. 6. [Pangonia] (1854); PHiL., Verh. zool.-bot. Ges. Wien, XV. 708. 6. [Pangonia] (1865); Scuin., Novara Reise, Dipt., 100. 52. [Pangonia] (1868); Ricardo, Annal. Mag. Nat. Hist., ser. 7. V. 172 et 179. (1900); Hunter, Trans. Amer. Entom. Soc. Philad., XXVII. 135. [Pangonia] (1901). vulpes MACQ., Dipt. exot., suppl. 4., 23. 52. [Pangonia] (1850); Blanch. in GaY : Hist. fis. y polit. de Chile, Zool. VII. 391. 10. [Pangonia] (1854); PHIL., Verl. zool.-bot. Ges. Wien, XV. 708. 10. [Pangonia] (1865); Rrcardo, Annal. Mag. Nat. Hist., ser. 7. V. 173. (1900) ; Huntrk, Trans. Amer. Entom. Soc. Philad., XXVII. 135. [Pangonia] (1901).

\section{Corizoneura}

albifrons BıG., Mém. Soc. Zool. France, V. 613. (1892); Rıcardo, Annal. Mag. Nat. Hist., ser. 7. V. 106. (1900).

alternans MACQ., Dipt. exot., suppl. 5., 19. 59. [Pangonia] (1855); Ricardo, Ammal. Mag. Nat. Hist., ser. 7. V. 113. (1900).

angusta Big., Mém. Soc. Zool. France, V. 617. [? Corizoneura] (1892); Ricardo, Annal. Mag. Nat. Hist., ser. 7. V. 114. (1900).

angusta MAcQ., Dipt. exot., suppl. 2., 11. 40. [Pangonia] (1847); Walk., List Dipt. Brit. Mus., V. suppl. 1., 144. 133. [Pangonia] (1854) ; Ricardo, Aunal. Mag. Nat. Hist., ser. 7. V. 113. (1900). annulata Big., Mém. Soc. Zool. France, V. 612. (1892); Ricardo, Annal. Mag. Nat. Hist., ser. 7. V. 180. (1900).

anthracina MACQ., Dipt. exot., suppl. 4., 23. 50. tal. II. fig. 3. [Pangonia] (1850); Ricardo, Annal. Mag. Nat. Hist., ser. 7. V. 113. (1900).

aurofasciuta $\mathrm{J}_{\mathrm{AENN}}=$ chrysophila WALK. $_{\text {. }}$

brachyrrhyncha Big., Mém. Soc. Zool. France; V. 614. (1892); Ricardo, Annal. Mag. Nat. Hist., ser. 7. V. 106. (1900).

brevipalpis MacQ., Dipt. exot., suppl. 3., 8. 38. [Pangonia] (1847); Walk., List Dipt. Brit. Mus., V. suppl. 1., 144. 134. [Pangonia] (4854); Ricardo, Annal. Mag. Nat. Hist., ser. 7. V. 113. (1900). brunnipennis Lw., Öfvers. Kongl. Vet. Akad. Förhandl., XIV. 1857. 337. 2. [Pangonia] (1858) et Dipt.-Fauna Südafrika's, I. 18. 4. [Pangonia] (1860) ; Ricardo, Annal. Mag. Nat. Hist., ser. 7. V. 106 et $110 .(1900)$.

chrysophila WaLk., List Dipt. Brit. Mus., I. 155. [Tabanus] (1848); Ricardo, Annal. Mag. Nat. Hist., ser. 7. V. 113 et 120. (1900). aurofasciata JaEnv., Abhandl. Sonckenberg. Naturf. Ges., VI. 327. 19. tab. XLIII. fig. 5. [Pangonia] (1867).

directa WALK., Insecta Saunders., Dipt., I. 20. [Pangonia] (1850); Ricardo, Annal. Mag. Nat. Hist., ser. 7. V. 106 et 111. (1900).
Venezuela, Chile.

Chile.

Prum. bon.sp.

Oceania.

Australia.

Australia.

Europa mer.

Tasmania.

Prom. bon.sp.

Australia.

Africa mer.

Australia.

Prom.bon.sp. 
dives Macq., Dipt. exot., suppl. 1., 25. 30. tab. III. fig. 3. [Pangonia] (1845) ; Walk., List Dipt. Brit. Mus., V. suppl. 1., 142. 124. [P(ungonia] (1854); Ricardo, Ammal. Mag. Nat. Hist., ser. 7. V.113.(1900).

erratica Walk., List Dipt. Brit. Mus., I. 189. [T'abanus] (1848); Ricardo, Annal. Mag. Nat. Hist., ser. 7. V. 113 et 121. (1900).

fera Wili.jst., Trans. Kansas. Acad. Sci., X. 130. [Pangonia] (1887); Ricaldo, Annal. Mag. Nat. Hist., ser. 7. Y. 169. (1900); Hine, Ohio Naturalist, V. 227. [Pangonia] (1904); AldR., Catal. North Amer. Dipt., 193. (1905).

fulva Macq., Dipt. exot., suppl. 4., 19. 43. [Pangonia] (1850); Ricardo, Annal. Mag. Nat. Hist, ser. 7. V. 113 et 120. (1900).

lateralis Fabr., Systema Antliat., 91. 4. [Pangonia] (1805); LATr., Encyclop. Méthod., VIII. 704. 8. [Pangonia] (1811); Wied., Dipt. exot., I. 56. 9. [Pangonia] (1821) et Aussereurop. zweifl. Ins., I. 101. 25. [Pangonia] (1828); WaLK., List Dipt. Brit. Mus., I. 134. [Pangonia] (1848); Lw., Dipt.-Fauna Südafrika's, I. 17. 2. [Pangonia] (1860); RICARJo, Annal. Mag. Nat. Hist., ser. 7. V. 106 et 109. (1900) et ser. 7. VI. 163. (1900).

tabaniformis rufa Deg., Mém. pour. serv. à l'hist. d. Ins., VI. 272. 2. tab. XXX. fig. 11. [Bombylius] (1776).

leucopicta Big., Mém. Soc. Zool. France, V. 616. (1892); Ricurdo, Annal. Mag. Nat. Hist., ser. 7. V. 114. (1900).

longipalpis MAcQ., Dipt. exot., suppl. 3., 9. 40. tab. I. fig. 3. [Pangonia] (1847); WALk., List Dipt. Brit. Mus., V. 123. 17. [Pangonia] (1854); Ricardo, Annal. Mag. Nat. Hist., ser. 7. V. 173. (1900) ; Hunter, 'Trans. Amer. Entom. Soc. Plillad., XXVII. 134. [Pangonia] (1901).

longirostris HaRDw., Trans. Linn. Soc. London, XIV. 135. tab. VI. fig. 5, 6. [Pangonia] (1823); Wied., Ausserenrop. zweill. Ins., II. 621. 14. [Panyonia] (1830); Gay in GrifF.: Cuvier Anim. Kingd., XV. tab. CXIV. fig. 3. [Pangonia] (1832); MacQ., Annal. Soc. Entom. France, VI. 429. tab. XV. fig. 20, 23. [P'angonia] (1837) ; Walk., List Dipt. Brit. Mus., I. 131. [Pangonia] (1848) et V. suppl. 1., 139. 101. [Pangonia] (1854); Röder, S̈tettin. Entom. Zeitg., XLII. 384. [Pangonia] (1886); v. D. Wulp, Catal. Dipt. South Asia, 60 [Tabanus] et 64. [Pangonia] (1896); Ricardo, Annal. Mag. Nat. Hist., ser. 7. V. 167 et 168. (1900).

montana Hutton, Trans. New Zealand Instit., XXXIII. 17. [Pangonia] (1901).

neocaledonica Big., Annal. Soc. Entom. France, sér. 5. VIII. Bull., CXI.V. [P(angonia] (1878), sér. 5. IX. Bullet., IX. [Pangonia] •(1879) et sér. 6. III. Bull., CXXXVIII. [Philoliche] (1884); Ricando, Annal. Mag. Nat. Hist., ser. 7. V. 113. (1900).

nigricornis Big., Mém. Soc. Zool. France, V. 614. (1892) ; Ricardo, Annal. Mag. Nat. Hist., ser. 7. V. 114. (1900).

nigrosignata Troms., Eugenies Resa, Dipt., 451. 12. [P(angonia] (1868) ; Ricardo, Aunal. Mag. Nat. Hist., ser. 7. V. 11\%. (1900).
Tasmania.

Australia.

America sept.

Australia.

Africa mer.

N. Caledonia. Brasilia.

India or.

N. Selandia.

N. Caledonia.

Australia.

Australia. 
pallidipennis Ricardo, Annal. Mag. Nat. Hist., ser. 7. V. 110. tab. Africa occ. I. fig. 3. (1900).

parva Walk., List Dipt. Brit. Mus., I. 145. [Pangonia] (1848); Ricardo, Africa mer. Annal. Mag. Nat. Hist., ser. 7. V. 106 et 111. (1900).

? rubiginosa Bıg., Mém. Soc. Zool. France, V. 617. (1892); Rrcardo, Australia.

Annal. Mag. Nat. Hist., ser. 7. V. 114. (1900).

ruficornis Bıg., Mém. Soc. Zool. France, V. 615. (1892); Ricardo, Annal. Mag. Nat. Hist., ser. 7. V. 169. (1900); Hins, Ohio Naturalist, V. 228. [Pangonia] (1904); Aldr., Catal. North Amer. Dipt., 193. [Pangonia] (1905).

rufovittata Macq., Dipt. exot., suppl. 4., 19. 42. tab. II. fig. 1. [Pangonia] (1850); Ricardo, Aunal. Mag. Nat. Hist., ser. 7. V. 113. (1900).

suavis Lw., Öfvers. Kongl. Vet. Akad. Förhadl., XIV. 1857. 337. 1. [Pangonia] (1858) et Dipt.-Fauna Süiafrika's, I. 17. 3. [Pangonia] (1860) ; Ricardo, Anmal. Mag. Nat. Hist., ser. 7. .V. 106 et 110. (1900) et ser. 7. VI. 163. (1900).

subfascia WALK., List Dipt. Brit. Mus., V. suppl. 1., 136. 87. [Pangonia] (1854); Ricardo, Annal. Mag. Nat. Hist., ser. 7. V. 106 et 110 . (1900).

sulcifrons MacQ., Dipt. exot., suppl. 5., 19. 60. [Pangonia] (1855); Ricardo, Annal. Mag. Nat. Hist., ser. 7. V. 113. (1900).

tabaniformis rufa DEG. = lateralis FABR.

Taprobanes Walk., List Dipt. Brit. Mus., V. suppl. 1., 324. [Pangonia] (1854) ; v. D. Wulp, Catal. Dipt. South Asia, 64. [Pangonia] (1896) ; Ricardo, Annal. Mag. Nat. Hist., ser. 7. V. 167 et 168. (1900).

tigris Big., Annal. Soc. Entom. France, sér. 5. X. 143. [Pangonia] (1880); Ricardo, Annal. Mag. Nat. Hist., ser. 7. V. 167. (1900).

Americasept.

Tasmania.

Africa mer.

Port Natal.

Oceania.

Ceylon.

? Persia sept., ?Caucasus. trichocera Big., Mém. Soc. Zool. France, V. 616. (1892); Ricardo, Australia. Annal. Mag. Nat. Hist., ser. 7. V. 114. (1900).

umbratipennis Rucardo, Annal. Mag. Nat. Hist., ser. 7. V. 111. tab. I. fig. 9. (1900).

varicolor WiED., Aussereurop. zweifl. Ins., I. 98. 21. [Pangonia] (1828) ; WALK., List Dipt. Brit. Mus., I. 134. [Pangonia] (1848) et V. suppl. 1., 137. 93. [Pangonia] (1854); Lw., Dipt.-Fauna Südafrika's, I. 17. 1. [Pangonia] (1860); Ricardo, Annal. Mag. Nat. Hist., ser. 7. V. 106 et 110. (1900) et ser. 7. VI. 162. (1900). velutina Bıg., Mém. Soc. Zool. France, V. 615. (1892); Ricardo, Annal. Mag. Nat. Hist., ser. 7. V. 169. (1900); Hine, Ohio Naturalist, V. 228. [Pangonia] (1904); ALdr., Catal. North Amer. Dipt., 194. [Pangonia] (1905).

zigzag Macq., Dipt. exot., suppl. 5., 20. 61. tab. I. fig. 6. [Pangonia] (1855) ; Karscr, Berlin. Entom. Zeitschr., XXVIII. 171. [P(angonia] (1884); Rrcsldoo, Annal. Mag. Nat. Hist., ser. 7. V. 106. (1900).

? Oceania, Madagascar. 


\section{Mycteromyia}

Phil., Verh. zool.-bot. Ges. Wien, XV. 712. (1865).

albipectus BiG., Mém. Soc. Zool. France, V. 611. (1892); Ricardo, Brasilia. Annal. Mag. Nat. Hist., ser. 7. V. 99. (1900).

brevirostris Phil., Verh. zool.-bot. Ges. Wien, XV. 713. 3. (1865); Chile.

Ricardo, Annal. Mag. Nat. Hist., ser. 7. V. 99. (1900); Hunter,

Trans. Amer. Entom. Soc. Philad., XXVII. 138. (1901).

cinerascens Big., Mém. Soc. Zool. France, V. 610. (1892); Ricardo, Chile.

Annal. Mag. Nat. Hist., ser. 7. V. 99. (1900).

conica Bis., Annal. Soc. Entom. France, sér. 3. V. 278. tab. VI. fig. Chile.

4. [Pangonia] (18ī7); PhiL., Verh. zool.-bot. Ges. Wien, XV. 712.

1. (1865) ; Ricardo, Annal. Mag. Nat. Hist., ser. 7. V. 99. (1900);

Hunter, Trans. Amer. Entom. Soc. Philad., XXVII. 133. [Pangonia] et 138. (1901).

elegans Big., Mém. Soc. Zool. France, V. 608. (1892); Ricurdo, Prom.bon.sp. Annal. Mag. Nat. Hist., ser. 7. V. 99. (1900).

ensata Big., Mém. Soc. Zool. France, V.608. (1892); Ricardo, Annal. Prom.bon.sp. Mag. Nat. Hist., ser. 7. V. 99. (1900).

erythronotata Bı́., Mém. Soc. Zool. France, V.612. (1892); RıCARdo, Brasilia.

Annal. Mag. Nat. Hist., ser. 7. V. 99. (1900).

fusca Phil., Verh. zool.-bot. Ges. Wien, XV.712. 2. (1865); Ricardo, Chile.

Annal. Mag. Nat. Hist., ser. 7. V. 99. (19j0); Hunter, Trans.

Amer. Entom. Soc. Philad., XXVII. 138. (1901).

murina Phil., Verh. zool.-bot. Ges. Wien, XV. 713. 4. (1865);

Ricırdo, Annal. Mag. Nat. Hist., ser. 7. V. 99. (1900); Hunter,

Trans. Amer. Entom. Soc. Philad., XXVII. 138. (1901).

nigrifacies Bı̈., Mém. Soc. Zool. France, V.607. (1892); v. D. Wulp,

Catal. Dipt. South Asia, 64. (1896); Ricardo, Annal. Mag. Nat.

Hist., ser. 7. V. 99. (1900).

nitens Big., Mém. Soc. Zool. France, V. 609.(1892); Rrcardo, Annal. Brasilia. Mag. Nat. Hist., ser. 7. V. 93. (1900).

penicillata Bıg., Mém. Soc. Zool. France, V. 610. (1892); Rıcurdo, Brasilia.

Annal. Mag. Nat. Hist., ser. 7. V. 99. (1900).

\section{Scepsis}

Walk., Insecta Saunders., Dipt., I. 71. (1850).

nivalis WaLk., Insecta Saunders., Dipt., I. 71. tab. II. fig. 7. (1850); America mer.

Lw., Dipt.-Fauna Südafrika's, I. 15. (1860); E. L. Arrib., Bol. Acad. Nat. Cienc. Córdoba, IV. 131. 57. (1882) ; Ricardo, Annal. Mag. Nat. Hist., ser. 7. VIII. 286. (1901); Hunter, Trans. Amer. Entom. Soc. Pliilad., XXVII. 136. (1901). 


\section{Pronopes}

Lw., Öfvers. Kongl. Vet. Akad. Förhandl., XIV. 1857. 339. 4. (1858).

nigricans Lw., Öfrers. Kongl. Vet. Akad. Förhandl., XIV. 1857. Prom.bon.sp. 339. 13. (1858) et Dipt.-Fauua Suidafrika's, I. 27. 1. tab. I. fig. 12-16. $(1860)$.

\section{Hinea}

Adans, Kansas Unir. Sci. Bullet., III. 150. (1905).

flavipes Apans, Kansas Univ. Sei. Bullet., III. 150. [Hincia] (1905). Rhodesia.

\section{Rhinomyza}

W'ied., Nova Dipt. Gell., 8. (1820).

Erodiorhynchus Macq., Dipt. exot., I. 1., 110. (1838).

Tabanocella Bı̈., Annal. Soc. Entom. France, sér. 3. IV.76.(1856).

binolata MACQ. = denticornis WIED.

costata Iw., Dipt.-Fauna Siidafrika's, I. 26. 1. (1860); Ricardo, Prom.bon.sp. Annal. Mag. Nat. Hist., ser. 7. VIII. 290. (1901).

denticornis WruD., Aussereurop. zweifl. Ins., I. 111. 2. [Silvius] Africa mes.

(1828); Walk., Iist Dipt. Brit. Mus., I. 192. [Silvius] (1848) et

V. suppl. 1., 275. 6. [Silvius] (1854); Bıg., Annal. Soc. Entom. France, sér. 3. IV. 62. [Tabanocella] (1856); Lw., Öfvers. Kongl. Vet. Akad. Förhandl., XIV. 1857. 337. 4. [Silvius] (1858) et Dipt.Fauma Südafrika's, I. 21. 1. [Silvius] (1860); Ricardo, Annal. Mag. Nat. Hist., ser. 7. VI. 163. (1900) et ser. 7. VIII. 290. (1901). binotata MAcQ., Dipt. exot., I. 1., 113. 1. [Dichelacera] (1838); Walk., List Dipt. Brit. Mus., V. suppl. 1., 154. 29. [Dichelacera] (1854).

edentula W1ED., Aussereurop. zweifl. Ins., I. 105. 2. (1828); Walk.,

List Dipt. Brit. Mus., V. suppl. 1., 146. 2. (1854) ; Lw., Dipt.Fanna Suidafrika's, I. 15. 26. (1860) ; Scmin., Novara Reise, Dipt., 97. 43. [Erodiorhynchus] (1868); Rıcardo, Annal. Mag. Nat. Hist., ser. 7. VIII. 289. (1901).

eristaloides Macq., Dipt. exot., I. 1., 111. 1. tab. VI. fig. 1. [Erodiorhynchus] (1838); WALK., List Dipt. Bıit. Mus., I. 145. [Erodiorhynchus] (1848) et V. suppl. 1., 147. 1. [Erodiorhynchus] (1854).

eristaloides MACQ. = edentula Wred.

fusca WreD., Nova Dipt. Gen., 8. (1820) et Dipt. exot., I. 59. 1. (1821); Java.

S'tr. Farg. et Serv., Encyclop. Méthod., X. 544. (1825); Wied., Aussereurop. zwoifl. Ins., I. 104. 1. tab. I. fig. 3. (1828) ; Jwssen, Bullet. Soc. Imp. Nat. Moscou, IV. 323. (1832); MACQ., Suit. à 
Buffon, I. 196. 1. (1834) ; WALK., List Dipt. Brit. Mus., V. suppl. 1., 146. 1. (1854); v. D. Wulp, Catal. Dipt. South Asia, 64. (1896); Ricardo, Annal. Mag. Nat. Hist., ser. 7. VIII. 289. (1901). pusilla Schin., Novara Reise, Dipt., 97. 42. [Erodiorlynnchus] (1868); Prom.bon.sp.

Ricardo, Annal. Mag. Nat. Hist., ser. 7. VIII. 290. (1901).

\section{Silvius}

Meig., System. Beschreib., II. 27. (1820).

Ectenopsis Macq., Dipt. exot., I. 1., 111. (1838).

Esenbeckia Rovd., Archivio per la Zool. Modena, III. 83. (1863).

Mesomyia Macq., Dipt. exot., suppl. 4., 38. (1850).

Veprius Rosd., Archivio per la Zool. Modena, III. 83. (1863).

\section{Silvius s. str.}

algerus $\mathrm{MACQ}_{\mathrm{AC}}=$ appendiculatus MACQ.

algirus MEIG. = ?appendiculatus MacQ.

alpinus Drap., Annal. Génér. d. scienc. phys., 1819. 136. tab. VII. Europacentr.

fig. 7. [Tabanus] (1819); Bergroth, Entomol. Nachricht., XIII.

150. (1887); Ricardo, Annal. Mag. Nat. Hist., ser. 7. VIII. 291. (1901).

hirtus Lw., Wien. Entom. Monatschr., II. 350. (1858); Schin., Fauna Austriaca, Dipt., I. 43. (1862) ; PAND., Revue d'Entom., II. 221. (1883).

alpinus ScOP. = ?vituli FABR.

appendiculatus MACQ., Dipt. exot., suppl. 1., 45. 2. tab. IV. fig. 10.

(1845) et in Lucas: Explor. Scient. de l'Algér., Zool. III. 426. 34. tab. I. fig. 8. (1849) ; WaLk., List Dipt. Brit. Mus., V. suppl. 1., 275. 8. (1854); Schis., Novara Reise, Dipt., 97. 41. (1868); Ricardo, Annal. Mag. Nat. Hist., ser. 7. VIII. 291. (1901).

algerus Macq., Suit. à Buffon, I. 214. 2. (1834) et in Lucas:

Explor. Scient. de l'Algér., Zool., III. 427. 35. (1849).

? algirus Meig., System. Beschreib., VI. 319. 2. (1830); WaLk., List Dipt. Brit. Mus., V. suppl. 1., 275. 7. (1854); Lw., Wien. Entom. Monatschr., II. 350. (1858); Ricardo, Annal. Mag. Nat. Hist., ser. T. VIII. 291. (1901).

? italicus FABr., Spec. Insect., II. 457. 15. [Tabanus] (1781) et Entomol. Systemat., IV. 367. 21. [Tabanus] (1794); Соqueb., Illustr. Iconogr. Insect., III. 112. tab. XXV. fig. 4. [Tabanus] (1804); Meig., Klassif., I. 172. c. [Tabanus] (1804); FABR., Systema Antliat., 98. 24. [Tabanus] (1805); MeIG., System. Beschreib., II. 28. [Tabanus] (1820).

barbatus Bıł., Mém. Soc. Zool. France, V. 622. [Diuchlorus] (1892); Bє«z, Katal. đ. paläarkt. Dipt., II. 54. notı. [Gen. ?] (1903); RICARdo, Annal. Mag. Nat. Hist., sex. 7. XIV. 357. (1904).

Kertész: Catalogus Dipterorum. III.

Europa mer., Africa sept.

Gallia. 
bicolor Biq., Mém. Soc. Zool. France, V. 625. (1892); Ricardo, Annal. Marocco.

Mag. Nat. Hist., ser 7. VIII. 291. (1901).

confluens Lw., Öfvers. Kongl. Vet. Akad. Förhandl., XIV. 1857. Caffraria.

338. 8. (1858) et Dipt.-Fauna Südafrika's, I. 24. 5. tab. I. fig. 11.

(1860) ; Ricardo, Annal. Mag. Nat. Hist., ser. 7. VIII. 294. (1901).

cuneatus Lw., Öfvers. Kongl. Vet. Akad. Förhandl., XIV. 1857. 338.

7. (1858) et Dipt.-Fauna Südafrika's, I. 23. 4. (1860) ; Ricardo,

Annal. Mag. Nat. Hist., ser. 7. VIII. 294. (1901).

decipiens Lw., Öfvers. Kongl. Vet. Akad. Förhandl., XIV. 1857.

338. 9. (1858) et Dipt.-Fauna Südafrika's, I. 25. 6. (1860); RICARdo,

Annal. Mag. Nat. Hist., ser. 7. VIII. 294. (1901).

? decorus MacQ., Dipt. exot., suppl. 4., 38. 1. tab. II. fig. 10.

[Mesomyia] (1850); Ricardo, Annal. Mag. Nat. Hist., ser. 7.

VIII. 294. (1901).

decisus WAIK. = vituli FABR.

decorus MACQ. = ? decipiens Lw.

dimidiatus v. D. Wulp, Tijdschr. v. Entomol., XI. 102. 5. tab. III. fig. 3-5. (1868) ; Ost.-Sack., Annal. Mus. Civ. Genova, XVI. 418. [? Silvius] (1880) ; v. D. Wuı., Catal. Dipt. South Asia, 65. (1896) ; Ricardo, Annal. Mag. Nat. Hist., ser. 7. VIII. 295. (1901). dorsalis Coquill., Proc. Unit. Stat. Nat. Mus., XXI. 309. (1898); Ricardo, Annal. Mag. Nat. Hist., ser. 7. VIII. 291. (1901).

gigantulus Lw., Berlin. Entom. Zeitschr., XVI. 57. 12. [Chrysops] (1861) ; Ost.-SAcK., Bullet. Unit. Stat. Geolog. Surv., III. 215. (1877) et Catal. Dipt. North Amer., Ed. II., 58 et 226. 71. (1878); Willist., Trans. Kansas Acad. Sci., X. 131. (1887); Towns., Proc. Calif. Acad. Sci., ser. 2. IV. 595. 7. (1895); Ricardo, Annal. Mag. Nat. Hist., ser. 7. VIII. 293. (1901); Hine, Ohio Naturalist, V. 229. (1904) ; Aldr., Catal. Norh Amer. Dipt., 194. (1905).

trifolium Ost.-Sack., Mem. Boston Soc. Nat. Hist., II. 395. (1876).

glandicolor Lw., Öfvers. Kongl. Vet. Akad. Förhandl., XIV. 1857. 338. 6. (1858) et Dipt.-Fauna Südafrika's, I. 23. 3. tab. I. fig. 8-10. (1860); Ricardo, Annal. Mag. Nat. Hist., ser. 7. VIII. 294 et 295. (1901).

hirtus Lw. = alpinus Drap.

innotatus Karsch, Berlin. Entom. Zeitschr., XXXI. 372. 11. tab. IV. fig. 6. (1887); Ricardo, Annal. Mag. Nat. Hist., ser. 7. VIII. 294. (1901).

irritans Ricardo, Annal. Mag. Nat. Hist., ser. 7. VIII. 292. (1901). italicus FABR. = ?appendiculatus MACQ.

longirostris Big., Annal. Soc. Entom. France, sér. 3. VII. 128. [Dichelacera] (1859) ; Ricardo, Annal. Mag. Nat. Hist., ser. 7. XIV. 368. (1904).

marginatus MACQ. = Sylveirii MACQ.

marginatus WaLk., List Dipt. Brit. Mus., I. 189. [Tabanus] (1848); Australia.

Ricardo, Annal. Mag. Nat. Hist., ser. 7. VIII. 296. (1901).

Caffraria.

Africa mer.

I. Salavatti.

Japonia.

America sept.

Africa mer.

Africa or.

Afganistan.

Madagascar. 
maroccanus Bıg., Mém. Soc. Zool. France, V. 623. [Diachlorus] (1892); Ricardo, Annal. Mag. Nat. Hist., ser 7. XIV. 357. (1904).

? nitescens Walk., Trans. Entom. Soc. London, n. ser. IV. 124. (1857) ; Ricardo, Annal. Mag. Nat. Hist., ser. 7. VIII. 296 ot 297. (1901).

notatus $\mathrm{BrG} .=$ quadrivittatus SAY.

nubipennis Rond., Nuov. Annal. Sci. Nat. Bologna, ser. 3. II. 371. 30. (1850) ; Ricardo, Annal. Mag. Nat. Hist., ser. 7. VIII. 293. (1901) ; Hunter, Trans. Amer. Entom. Soc. Philad., XXVII. 136. (1901).

ochraceus Lw., Wien. Entom. Monatschr., II. 351. (1858); Ricardo, Annal. Mag. Nat. Hist., ser. 7. VIII. 291. (1901).

oestroïdes Karsch, Berlin. Entom. Zeitschr., XXXI. 371. 10. tab. IV. fig. 1. (1887) ; Ricardo, Annal. Mag. Nat. Hist., ser. 7. VIII. 294. (1901).

pertusus Lw., Öfvers. Kongl. Vet. Akad. Förhandl., XIV. 1857. 337. 5. (1858) et Dipt.-Fauna Südafrika's, I. 22. 2. (1860); Ricardo, Annal. Mag. Nat. Hist., ser. 7. VIII. 294. (1901); Marshal., Trans. Entom. Soc. London, 1902. tab. XXII. fig. 18. (1902).

pollinosus Willist., Trans. Connect. Acad., IV. 244. (1880) et Trans. Kansas Acad. Sci., X. 131. (1887); Hine, Ohio Naturalist, V. 229. [pollinosis] (1904); Aldr., Catal. North Amer. Dipt., 194. (1905).

presbiter Rond., Archivio per la Zoolog. Modena, III. 84. [Veprius] (1863); Ricardo, Annal. Mag. Nat. Hist., ser. 7. VIII. 293. (1901); Hunter, Trans. Amer. Entom. Soc. Philad., XXVII. 136. [Veprius] (1901).

quadrivittatus SAY, Journ. Acad. Nat. Sci. Philad., III. 33. 1. [Chrysops] (1823) et Americ. Entomol., Ed. 1859., II. 54. [Chrysops] (1859); WiEd., Aussereurop. zweifl. Ins., I. 200. 9. [Chrysops] (1828); Walk., List Dipt. Brit. Mus., V. suppl. 1., 283. 30. [Chrysops] (1854); Ost.-Sack., Mem. Boston Soc. Nat. Hist., II. 395. 3. [Chrysops] (1876) et Catal. Dipt. North America, Ed. II., 54 et 226. [Chrysops] (1878); Willist., Trans. Kansas Acad. Sci., X. 131. (1887); Towns., Trans. Amer. Entom. Soc. Philad, XXII. 57. 7. (1895); Ricardo, Annal. Mag. Nat. Hist., ser. 7. XIV. 357. (1904); Hine, Ohio Naturalist, V. 229. (1904); ALdr., Catal. North Amer. Dipt., 194. (1905).

notatus Bı́., Mém. Soc. Zool. France, V. 623. [Diachlorus] (1892); IIne, Ohio Naturalist, V. 245. [Diachlorus] (1904). rufipes MACQ., Dipt. exot., suppl. 4., 37. 1. tab. IX. fig. 19. (1850); E. L. Arrib., Bolet. Acad. Nat. Cienc. Córdoba, IV. 131. 56. (1882) ; Ricardo, Annal. Mag. Nat. Hist., ser. 7. VIII. 293. (1901); Hunter, Trans. Amer. Entom. Soc. Philad., XXVII. 136. (1901). rufopilosus Bıঞ., Mém. Soc. Zool. France, V. 620. [Veprius] (1892); Ricardo, Annal. Mag. Nat. Hist., ser. 7. VIII. 293 et 294. (1901). silvester Bergr., Stettin. Entom. Zeitg., LV. 71. (1894).
Marocco.

Australia.

America aeq.

Asia min.

Africa or.

Caffraria.

America sept.

Chile.

America sept.

Brasilia.

Chile.

Australia. 
Sylveirii MacQ., Dipt. exot., I. 1., 155. 1. (1838); WaLK., List Dipt. Brit. Mus., V. suppl. 1., 275. 5. (1854); Ricardo, A mnal. Mag. Nat. Hist., ser. 7. VIII. 293. (1901); Huxter, Trans. Amer. Entom. Soc. Philad., XXVII. 136. [silverii] (1901).

marginatus MacQ., Dipt. exot., I. 1., tab. XIX. fig. 1. (1838). trifolium OsT.-SACK. = gigantulus $\mathrm{Lw}$.

vituli Fabr., Systema Antliat., 97. 19. [Tabanus] (1805); MeIG., System. Beschreib., II. 27. 1. tab. XIII. fig. 13. (1820) et VI. 319. (1830); Macq., Suit. à Buffon, I. 213. 1. tab. V. fig. 7. (1834); Schumm., Übers. d. Arb. u. Veränd. d. schles. Ges. f. Vaterländ. Kultur, 1837. 107. (1837); Blaxch., Hist. Nat. d'Ins., III. 590. (1840) ; Grmm., Bull. Soc. Imp. Nat. Moscou, XX. 1., 179. (1847); WaLk., List Dipt. Brit. Mus., I. 192. (1848) ; Schol.rz, Zeitschr. f. Entomol. Breslau, IV. No. 16., 36. (1850); WaLk., List Dipt. Brit. Mus., V. suppl. 1., 274. 1. (1854); Lw., Wien. Entom. Monatschr., II. 350. (1858); Schix., Fauna Austriaca, Dipt., I. 43. (1862); Gов., Mém. Soe. I.inn. Nord France, 1881. 45. (1881); Paxd., Revue d'Entomol., II. 221. (1883) ; S'trobs, Mittheil. Naturwiss. Ver. Steiermark, XXIX. 1892. 19. (1893); Grifr., Bollet. Mus. Zool. ed Anat. comp. Torino, X. No.218., 6. (1895) ; Ricardo, Annal. Mag. Nat. Hist., ser. 7. VIII. 291. (1901); ViLI.en., Annal. Soe. Entom. France, LXXIV. 309. 1. (1905).

?alpinus Scop., Entomol. Carniol., 372. 1010. [T'abanus] (1763);

Vilt., Entom. Linn., III. 556. 12. [Tabanus] (1789); Scurank, Fauna Boica, III. 154. 2534. [Tabanus] (1803); Schin., Verh. zool.-bot. Ges. Wien, VI. 422. 1010. (1856).

decisus Walk., List Dipt Brit. Mus., I. 171. [Tabanus] (1848) et V. suppl. 1., 27t. [T'abanus] (1854).

Vulpecula Wien., Aussereurop. zweifl. Ins., I. 195. 1. [Chrysops] (1828); MAcQ., Dipt. exot., I. 1., 112. 1. [Ectenopsis] (1838); Walk., List Dipt. Brit. Mus., I. 205. [Ectenopsis] (1848) et V. suppl. 1., 147. 1. [Eetenopsis] (18.34); Isw., Dipt.-Fauna Südafrika's, I. 15. (1860) ; Ricardo, Annal. Mag. Nat. Hist., ser. 7. VIII. 297. (1901).

\section{Esenbeckia}

Esenbeckii WIED., Aussereurop. zweifl. Ins., II. 623. 19. [Silvius] (1830); Walk., List Dipt. Brit. Mus., V. suppl. 1., 274.4. [Silvius] (1854).

pangonia Hunter, Trans. Amer. Entom. Soc. Philad., XXVII. 136. (1901) [lapsus].

pangonina Rovi., Archiv. per la Zool. Modena, III. 83. (1863);

E. L. Arrib., Bolet. Aead. Nat. Cienc. Córdoba, IV. 131. 55.

(1882); Ricardo, Annal. Mag. Nat. Hist., ser. 7. VIII. 297. (1901).

pangonic HuNTER = Esenbeckii W IED. $_{\text {. }}$

pangonina RoND. = Esenbeckii WrED.
Brasilia.

Europacentr. et mer.

Patriaignota.

Brasilia. 
Vulpes WiED., Aussereurop. zweifl. Ins., I. 111. 1. [Silvius] (1828); Walk., List Dipt. Brit. Mus., V. suppl. 1., 274. 3. [Silvius] (1854); Rond., Archivio per la Zool. Modena, III. 89. (1863); Rıcando, Annal. Mag. Nat. Hist., ser. 7. VIII. 297. (1901); Hunrer, Trans. Amer. Entom. Soc. Philad., XXVII. 136. [Silvius] (1901).

\section{Gastroxides}

SAUnd., Trans. Entom. Soc. London, III. 59. (1841).

Ditylornyia BıG., Rev. et Magaz. de Zool., sér. 2. XI., 305. (1859).

ater SAUNd., Trans. Entom. Soe. London, III. 59. tab. V. fig. 4 . $q$ (1841) et IV. 233. tab. XIV. fig. 3. $\sigma^{\top}$. (1847); WaLK., List Dipt. Brit. Mus., I. 209. (1848) et V. suppl. 1., 293. 1. (1854); Lw., Dipt.-Fauna Südafrika's, I. 15. (1860); v. D. Wulp, Catal. Dipt. South Asia, 64. (1896) ; Ricardo, Annal. Mag. Nat. Hist., ser. 7. VIII. 298. (1901).

ornatus BıG., Revue et Magaz. de Zool., sér. 2. XI. 306. [Ditylomyia] (1859); v. D. Wulp, Catal. Dipt. South Asia, 63. [Ditylomyia] (1896); Ricardo, Annal. Mag. Nat. Hist., ser. 7. XIV. 372. (1904).

\section{Chrysops}

Meıg., Nouvelle Classification, 23. 33. (1800).

aestuans v. D. WULP $=$ moerens WALK.

affinis Belu., Saggio di Ditterol. Messic., I. 70. 2. tab. II. fig. 14. Mexico. (1859); Aldr., Catal. North Amer. Dipt., 195. (1905).

afflictus Wisd., Aussereurop. zweifl. Ins., I. 204. 15. (1828); Walk., List Dipt. Brit. Mus., V. suppl. 1., 288. 50. (1854); Hunter, Trans. Amer. Entom. Soc. Philad., XXVII. 135. (1901); Ricardo, Annal. Mag. Nat. Hist., ser. 7. VIII. 310. (1901).

albicinctus v. D. WuLP, Tijdschr. v. Entom., XI. 103. 6. tab. III. fig. 6. (1868) ; Ostr-SAck., Annal. Mus. Civ. Genova, XVI.418. (1882); v. D. Wulp, Catal. Dipt. South Asia, 66. (1896); Ricardo, Annal. Mag. Nat. Hist., ser. 7. IX. 371. (1902).

alter Rond., Annal. Mus. Civ. Genova, VII. 460. (1875); v. D. Wulp, Catal. Dipt. South Asia, 66. (1896); Ricardo, Annal. Mag. Nat. Hist., ser. 7. IX. 373. (1902).

altivagus Os'r.-SACK., Biolog. Centr. Amer., Dipt., I. 45. 1. tab. I. fig. 6, 7. (1886); Ricardo, Annal. Mag. Nat. Hist., ser. 7. VIII. 300. (1901); AldDr., Catal. North Amer. Dipt., 195. (1905).

amazon DAEcke, Entom. News Philad., XVF. 250. fig. (1905). amazonius RoND. = costatus FABR.

apicalis BeLL., Saggio di Ditterol. Messic., I. 73. 6. (1859); Aldr., Mexico. Catal. North Amer. Dipt., 195. (1905).
Brasilia.

India or.

Ceylon.

Brasilia.

Nova Guinea,

I. Salavatti, Hattam.

Borneo.

Mexico.

America sept. 
areolatus WALK. $^{=}$vittatus WIED.

ater $\mathrm{MACQ}$. $=$ ? carbonarius WALK. aterrimus $\mathrm{KIRBY}=$ japonicus WIED. atricornis $\mathrm{BIG} .=$ proclivis OsT.-SACK. atropos Ost.-SACK. $=$ divisus $\mathrm{W}_{\text {ALK. }}$

aurantiacus JAENN., Berlin. Entom. Zeitschr., X. 88. 36. (1866); Hispania. Ricardo, Annal. Mag. Nat. Hist., ser. 7. IX. 427. (1902).

bifasciatus MACQ., Dipt. exot., I. 1., 157. 3.(1838); v. D. Wulp, Catal. Bengal. Dipt. South Asia, 65. (1896); Ricardo, Annal. Mag. Nat. Hist., ser. 7. IX. 373. (1902).

bimaculatus WIED., Aussereurop. zweifl. Ins., I. 201. 10. (1828); WaLk., List Dipt. Brit. Mus., V. suppl. 1., 287. 47. (1854); Ricardo, Annal. Mag. Nat. Hist., ser. 7. VIII. 310. (1901); HuvTER, Trans. Amer. Entom. Soc. Philad., XXVII. 135. (1901). bipunctatus МотsсH. $=$ parallelogrammus ZeLL.

bistellatus DaECKe, Entom. News Philad., XVI. 249. fig. (1905). brasiliensis Ricardo, Annal. Mag. Nat. Hist., ser. 7. VIII. 314. (1901). Brimleyi Hine, Canad. Entomol., XXXVI. 55. (1904).

brunneus Hise, Ohio Stat. Acad. Sci., Spec. Pap. No. 5., 34. (1903); Aldr., Catal. North Amer. Dipt., 195. (1905).

? caeculus O.F.MüLL., Zoolog. Dan. Prodr., 180. 2119.[Tabanus](1776). caecutiens L., Fauna Sues., 464. 1888. [Tabanus] (1761); PodA, Insect. Mus. Graec., 117. 2. [Tabanus] (1761); Scop., Entomol. Carniol., 373. 1014. [Tabanus] (1763); O. F. Múlu., Fauna Ins. Fridrichsdal., 86. 770. [Tabanus] (1764); L., Systema Naturae, Ed. XII., II. 1001. 17. [Tabanus] (1767); Deg., Mém. pour serv. à l'hist. d. Ins., VI. 224. 6. tab. XIII. fig. 3-5. [Tabanus] (1776); O. F. MüLL., Zoolog. Dan. Prodr., 180. 2117. [1abanus] (1776); FABR., Spec. Insect., II. 459. 27. [Tabamus] (1781); Vul., Entomol. Linn., III. 555. 8. [Tabamus] (1789) ; GueL., Systema Naturae, V. 2885. 17. [Tabanus] (1792); FABR., Entomol. System., IV. 372. 42. [Tabanus] (1794); Donov., Brit. Ins., IV. 61. tab. CXXXI. [Tabanus] (1795) ; ScheLLend., Gattung. d. Fliegen, 72. tab. XXVII. fig. 2. [Tabanus] (1803) ; Meıa., Klassif., I. 159. 4. (1804) ; FABR., Systema Antliat., 110. 1. (1805); LATr., Gen. Crust. et Ins., IV. 285. (1809); FALL., Kongl. Vet. Akad. Handl., XXX. 247. 1. (1809) ; Latr., Consid. génér., 443. (1810); Lam., Anim. sans vertébr., III. 421. 4. [Tabanus] (1816); Cuv., Règne Animal., III. 615. (1817); MeIG., System. Beschreib., II. 67. 2. (1820); Dum., Consid. gén. d. Ins., tab. XLVI. fig. 6. (1823); MacQ., Recueil Soc. Sci. Agricult. Iille, 1826. 485. 1. tab. III. fig. 6. (1826) et Suit. à Buffon, I. 214. 1. tab. V. fig. 5. (1834); DAнLв., Ins. Scand. och nytta, 307. 26. (1837); Duncax, Magaz. Zoolog. and Botan., I. 454. fig. 2. (1837) ; ZETT., Insecta Lappon., Dipt., 518. 1. (1838) ; Blanch., Hist. d. Ins., III. 591. tab. II. fig. 3. (1840); Lw., Progr. Posen, 1840. 5. 1. (1840) et Isis, 1840. VII-VIII. 518. 1. (1840); ZeLL., Isis, 1842. 823. tab. I. fig. 33-34. (1842);

America sept.

Brasilia.

America sept.

America sept.

Europa sept.

Europa,

Sibiria. 
Zetr., Dipt. Scand., I. 123. 1. (1842); Gimm., Bull. Soc. Imp. Nat. Moscou, XX. 1., 188. 1. (1847); Walk., List Dipt. Brit. Mus., I. 193. (1848); ZeTT., Dipt. Scand., VIII. 2942. 1. (1849); WАLK., Insecta Britann., Dipt., I. 43. 1. tab. I. fig. 15. (1851) et List Dipt. Brit. Mus., V. suppl. 1., 276 et 277. 1. (1854); Zетт., Dipt. Scand., XII. 4550. 1. (1855); Lw., Verh. zool.-bot. Ges. Wien, VIII. 628. 16. (1858); Assuuss, Wien. Entom. Monatschr., II. 181. 33. (1858) ; BonsD., Finl. tvaving. Ins., I. 112. 1. (1861); Schin., Fauna Austriaca, Dipt., I. 42. (1862); JAENv., Berlin. Entom. Zeitschr., X. 90.40. (1866) ; Goв., Mém. Soc. Linn. Nord France, 1881. 50. (1881); Becher, Denkschr. Akad. Wien, XLV. 142. tab. II. fig. 16. (1882); PAND., Revue d'Entom., II. 224. 2. (1883) ; Neuhaus, Diptera Marchica, 44. 1. (1886) ; Girschn., Berlin. Entom. Zeitschr., XXXI. - . tab. III. fig. 5. (1887) ; BeLing, Verh. zool.-bot. Ges. Wien, XXXVIII. 1. 1. (1888); E. \& L. Covcke, Annal. Soc. Entom. Belgique, XXXVI. 6. (1892); STrobl, Mittheil. Naturwiss. Ver. Steiermark, XXIX. 1892. 19. (1893); Griff., Bollet. Mus. Zool. ed. Anat. comp. Torino, X. No. 218., 6. (1895) ; Austen, Entom. Monthly Mag., ser.2. VIII. 227. (1897); Leonardi, Insetti Nocivi, III. 377. fig. 207, 208. (1900); Ricardo, Annal. Mag. Nat. Hist., ser. 7. IX. 425 et 428. (1902) ; Villen., Annal. Soc. Entom. France, LXXIV. 309. 1. (1905).

lugubris L., Fauna Suec., 464. 1889. [Tabanus] (1761); FABR., Spec. Insect., II. 460. 28. [Tabanus] (1781) ; Villers, Entomol. Linn., IIT. 556. 9. tab. X. fig. 12. [Tabanus] (1789); FABR., Entomol. System., IV. 373. 46. [Tabanus] (1794); Schellenb., Gattung. d. Fliegen, 72 et 73. tab. XXVIII. fig. 1 A, $a-c$. (1803); MeIG., Klassif., I. 160. 5. (1804); FABr., Systema Antliat., 113. 9. (1805); Fall., Kongl. Vet. Akad. Handl., XXX. 249. 4. (1809); Villen., Annal. Soc. Entom. France, LXXIV. 310. 4. (1905).

maritimus Scop., Entomol. Carniol., 374. 1016. [Tabanus] (1763) ; Vill., Entom. Linn., III. 557. 15. [Tabanus] (1787).

viduatus FABR., Entomol. System., IV. 374. 47. [Tabanus] (1794) et Systema Antliat., 113. 10. (1805); FALL., Kongl. Vet. Akad. Handl., XXX. 248. 2. (1809).

caecutiens PANz. = relictus MeIG.

calidus Walk., List Dipt. Brit. Mus., I. 205. (1848); Ricardo, Annal. Mag. Nat. Hist., sor. 7. IX. 367 et 368. (1902).

callidus Ost.-SAck., Mem. Boston Soc. Nat. Hist., II. 379. (1876); Towns., Trans. Amer. Entom. Soc. Philad., XXII. 56. (1905); Hart, Bullet. Illin. State Labor. of Nat. Hist., IV. 227. (1895); Hine, Ohio State Acad. Sci., Spec. Pap. No. 5., 35. (1903) ; Aldr., Catal. North Amer. Dipt., 195. (1905).

calogaster Schin., Novara Reise, Dipt., 103. 61. (1868); Ricardo, Americamer. Annal. Mag. Nat. Hist., ser. 7. VIII. 310. (1901); Hunter, Trans. Amer. Entom. Soc. Philad., XXVII. 135. (1901). 
calopterus Hine, Ohio Natural., VI. 392. (1905).

Guatemala. canifrons WALK. = flavidus WIED.

carbonarius WaLk., List Dipt. Brit. Mus., I. 203 et 204. var. $\gamma$. Amoricasept.

(1848) ; Hine, Ohio Naturalist, V. 220. (1904).

? ater MACQ., Dipt. exot., suppl. 4., 40. 18. (1850).

fugax Ost.-Sack., Mem. Boston Soc. Nat. Hist., II. 375. (1876) ;

Willist., Trans. Kansas Acad. Sci., X. 132. (1887); Aldr.,

Catal. North Amer. Dipt., 196. (1905).

niger Walk. (nec Macq.), List Dipt. Brit. Mus., I. 202. (18t8). provocans WaLK., Insecta Saunders., Dipt., I. 73. (1850).

carbonarius WALK. p. p. = niger MACQ.

celer*) Ost.-Sack., Mem. Boston Soc. Nat. Hist., II. 376. 5. (1876);

Ricardo, Annal. Mag. Nat. Hist., ser. 7. VIII. 305. (1901); Hive,

Ohio State Acad. Sci., Spec. Pap. No. 5., 36. (1903); Aldr.,

Catal. North Amer. Dipt., 195. (1905).

ceras Towns., Psyche, VIII. No. 251., 38. (1897); Ricardo, Annal.

Mag. Nat. Hist., ser. 7. VIlI. 300. (1901) ; Hine, Ohio Naturalist,

V. 220. (1904); Aldr., Catal. North Amer. Dipt., 195. (1905).

ciliaris Lw., Öfvers. Kongl. Vet. Akad. Förhandl., XIV. 1857. 338.

10. (1857) et Dipt.-Fauna Siidafrika's, I. 28. 2. tab. I. fig. 14

(nec 17) (1860) ; Ricsndo, Annal. Mag. Nat. Hist., ser. 7. IX. 367. (1902).

cincticornis Walk., List Dipt. Brit. Mus., I. 201. (1848); Ricardo, Annal. Mag. Nat. Hist., ser. 7. VIII. 302. (1901).

cinctus Bı̈., Mém. Soc. Zool. France, V. 602. (1892); v. D. Wulp, Catal. Dipt. South Asia, 66. (1896); Ricardo, Amnal. Mag. Nat. Hist., ser. 7. IX. 378. (1902).

? cluvicrus Thомs., Eugenies Resa, Dipt., 452. 14. (1868); v. D.

Wulp, Catal. Dipt. South Asia, 66. (1896); Ricardo, Annal.

Mag. Nat. Hist., ser. 7. IX. 373. (1902).

clavicrus 'Тномs. $=$ ? cinctus BIG.

coloradensis Bı́., Mém. Soc. Zool. France, V. 605. p. p. (1892); Ricardo, Annal. Mag. Nat. Hist., ser. 7. VIII. 307. (1901) ; Hine, Ohio Naturalist, V. 220. (1904); Aldr., Catal. Nurth Amer. Dipt., 195. (1905).

coloradensis BIG. p. p. = fulvaster OsT.-SAck.

concavus Lw., Yerh. zool.-bot. Ges. Wien, VIII. 622. 8. (1858); Rossia.

Ricardo, Annal. Mag. Nat. Hist., ser. 7. IX. 427. (1902).

confluens Lw., Öfvers. Kongl. Vet. Akad. Förhandl., XIV. 1857. Africa mer. 338. 12. (1858) et Dipt.-Fauna Südafrika's, I. 30. 4. tab. I. fig. 19. (1860); Ricardo, Annal. Mag. Nat. Hist., ser. 7. IX. 367 et 368. (1902).

connexus Lw., Verh. zool.-bot. Ges. Wien, VIII. 629. 18. (1858); Gallia.

Ricardo, Annal. Mag. Nat. Hist., ser. 7. IX. 427. (1902).

Coquillettii Hine, Ohio Naturalist, V. 220. (1904).

California.

$\left.{ }^{*}\right)=$ ? cincticornis WALK. 
costatus FARr., Entomol. System., IV. 373. 45. [Tabanus] (1794) et Systema Antliat., 112. 8. (1805); PaL. Beauv., Ins. recueill. en Afrique et en Amérique, 223. tab. III. fig. 7. (1805-1821); WiED., Dipt. exot., 101. 4. (1821) et Aussereurop. zweifl. Ins., I. 198. 6. (1828) ; MacQ., Dipt. exot., I. 1., 160. 8. (1838) ; Big. in SAGRA: Hist. la Isla de Cuba, P. 2. VII. 334. (1856); Ost.-Sack., Berlin. Entom. Zeitschr., XXVII. 297. (1883); Röd., Stettin. Entom. Zeitg., XLVI. 339. (1885) ; Ost.-Sack., Biolog. Central. Americ., Dipt., I. 46. 2. (1886); Willist., Kansas Acad. Sci., X. 134. (1887) ; Towns., Trans. Amer. Entom. Soc. Philad., XXII. 56. 3. (1895) et Annal. Mag. Nat. Hist., ser. 6. X1.. 18. 3. (1897); Ricardo, Annal. Mag. Nat. Hist., ser. 7. VIII. 309 et 313. (1901); IIunter, Trans. Amer. Entom. Soc. Philad., XXVII. 135. (1901); Willist., Biolog. Central. Amer., Dipt., I. 255. (1901); Aldk., Catal. North Amer. Dipt., 195. (1905). amazonius Rond., Archivio per la Zool. Modena, III. 81. (1863). molestus Guèr. (nec. Wred.), Jeonogr. d. Régne Anim., VII. Ins., 542. tab. XCVII. fig. 3. (1835).

?varicgatus Deg., Mém. pour serv. à l'hist. d. Ins., VI. 230.

7. tab. XXX. fig. 7. [Tabanus] (1776).

vulneratus Rond. in Truqui : Studi Entomol., I. 104. 77. (1848);

Ost.-SAck., Berlin. Entom. Zeitschr., XXVII. 292. (1883).

crassicornis v. D. WULP. = virgulatus BELL.

crucians Wied., Aussereurop. zweifl. Ins., I. 211. 24. (1828); WALK., List Dipt. Brit. Mus., V. suppl. 1., 287. 48. (1854); JAENn., Abhandl. Senckenberg. Naturf. Ges., VI. 312. (1867); Rıcardo, Annal. Mag. Nat. Hist., ser. 7. VIII. 310. (1901); Hunter, Trans. Amer. Entom. Soc. Philad., XXVII. 135. (1901); Aldr., Catal. Nortlı Amer. Dipt., 196. (1905).

crudelis Wied., Aussereurop. zweifl. Ius., I. 195. 2. (1828); WaLK., List Dipt. Brit. Mus., V. suppl. 1., 293. 72. (1854); Hunrer, Trans. Amer. Entom. Soc. Philad., XXVII. 135. (1901); Ricardo, Annal. Mag. Nat. Hist., ser. 7. IX. 431. (1902).

euchix RICARDO = cuclux WhITNEY.

cuclux Whitney, Canad. Entomol., XI. 35. (1879) ; Willist., Trans. Kansas Acad. Sci., X. 131, 134. (1887); Aldr., Catal. North Amer. Dipt., 196. (1905).。

euchix Ricardo, Annal. Mag. Nat. Hist., ser. 7. VIII. 300. (1901) [lapsus].

cursim WHITNEY = pudicus OST.-SACK.

delicatulus Ost.-SAck., Mem. Boston Soc. Nat. Hist., II. 380. (1876) ; Aldr., Catal. North Amer. Dipt., 196. (1905).

dimidiatus v. D. Wulp, Notes Leyden Mus., VII. 80. 34. (1885); Ricardo, Annal. Mag. Nat. Hist., ser. 7. IX. 367. (1902).

Dimmocki Hine, Ohio Naturalist, VI. 393. (1905).

discalis Willist., Trans. Connecticut Acad., IV. 245. (1880) et Trans. Kansas Acad. Sci., X. 132. 134. (1887); Ricardo, Aunal. Mag.
A merica mer. et centr., India occ.

Brasilia,

Cuba.

Patriaiguota.

Americasept.

America sept.

Africam. oce. Americasept. A merica sept. 
Nat. Hist., ser. 7. VIII. 300. (1901) ; Hine, Ohio Naturalist, V. 221. (190t) ; ArdDr., Catal. North Amer. Dipt., 196. (1905).

dispar FABR., Entomol. System. Supplem., 567. [Tabanus] (1798) et Systema Antliat., 112. 5. (1805); Wied., Dipt. exot., 102. 1. (1821) et Aussereurop. zweifl. Ins., I. 196. 3. (1828) ; MacQ., Dipt. exot., I. 1., 159. 5. (1838) ; WALk., List Dipt. Brit. Mus., I. 195. (1848) ; MACQ., Dipt. exot., suppl. 3., 14. (1848); WALK., List Dipt. Brit. Mus., V. suppl. 1., 229. 67. (1854) ; v. D. Wul.P, Sumatra Exped., Dipt., 19. 1. (1881); Ost.-SAck., Annal. Mus. Civ. Genova, XVI. 418. (1882) et Berlin. Entom Zeitschr., XXVI. 97. (1882); Röder, Entomol. Nachricht., XIX. 234. 3. (1893); v. D. Wulp, Catal. Dipt. South Asia, 65. (1896); Ricardo, Annal. Mag. Nat. Hist., ser. 7. IX. 374. (1902).

impar Rond., Annal. Mus. Civ. Genova, VII. 460. (187a).

ligatus WaLK., List Dipt. Brit. Mus., I. 195. (1848); v. D. WuL1, Catal. Dipt. South Asia, 65. (1896).

lunatus Gray in Grifriti : Anim. Kingdom., XV. 696. tab. CXIV. fig. 4. [Hacmatopota] (1832).

terminalis WalK., List Dipt. Brit. Mus., I. 195. (1848); v. D.

Wulp, Catal. Dipt. South Asia, 65. (1896).

dissectus Lw., Verh. zool.-bot. Ges. Wien, VIII. 618. 4. (1858); Ricardo, Annal. Mag. Nat. Hist., ser. 7. IX. 427. (1902).

divaricatus Lw., Verh. zool.-bot. Ges. Wien, VIII. 624. 12. (1858); Ricardo, Annal. Mag. Nat. Hist., ser. 7. IX. 427. (1902).

divisus Walk., List Dipt. Brit. Mus., I. 204. (1848); Ricardo, Annal. Americasept. Mag. Nat. Hist., ser. 7. VIII. 303. (1901).

atropos Ost.-SAck., Mem. Boston Soc. Nat. Hist., II. 372. (1876);

Alur., Catal. North Amer. Dipt., 195. (1905).

excitans WaLk., Insecta-Saunders., Dipt., I. 72. (1850) et List Dipt. Brit. Mus., V. suppl. 1., 281. (1854); Osr.-SAck., Mem. Boston Soc. Nat. Hist., II. 373. (1876) ; WiLLIst., Trans. Kansas Acad. Sci., X. 132. (1887) ; Ricardo, Annal. Mag. Nat. Hist., ser. 7. VIII. 304. (1901); Hine, Ohio Naturalist, V. 222. (1904); AlDr., Catal. Norh Amer. Dipt., 196. (1905).

facialis Towns., Psyche, VIII. No. 251. 39. (1897); Hine, Ohio Naturalist, V. 222. (1904); Aldr., Catal. North Amer. Dipt., 196. (1905).

fascialis Ricardo, Annal. Mag. Nat. Hist., ser. 7. VIII. 300. (1901) [lapsus].

fallax Ost.-SAck., Mem. Boston Soc. Nat. Hist., II. 392. (1876); America sept. Hine, Ohio State Acad. Sci., Spec. Pap. No. 5., 36. (1903); Aldr., Catal. North Amer. Dipt., 196. (1905).

fascialis RICARDO = facialis Towns.

fasciatus WiED., Dipt. exot., 103. 3. (1821) et Aussereurop. zweifl. Ins., I. 198. 5. (1828); WaLk., List Dipt. Brit. Mus., I. 195. (1848) Asia mer., et V. suppl. 1., 291. 66. (1854); Dol., Natuurkund. Tijdschr. Arch.Indirus. Nederl. Ind., XVII. 84. (1858); Walk., Proc. Linn. Soc. London,

Asia mer.,

I.Philippinae,

Honglkong, Arch.Indicus.

Sibiria.

Sibiria.

America sept.

Americasept. 
IV. 104.. 38. (1860) ; v. D. Wulp, Catal. Dipt. South A sia, 65. (1896) ; Ricardo, Annal. Mag. Nat. Hist., ser. 7. IX. 375. (1902). fascipennis MacQ. = ? univittatus MACQ.

fenestratus FABR., Entomol. System., IV. 373. 44. [Tabanus] (1794); Meig., Klassif., I. 160. a. (1804); FABr., Systema Autliat., 111. 4. (1805) ; MeIG., System. Beschreib., II. 71. 7. (1820); MAcQ. in Lucas: Explor. Scient. de l'Algérie, Zool. III. 427. 36. (1849); WaLK., List Dipt. Brit. Mıs., V. suppl. 1., 277 et 279. 8. (1854); Goв., Mém. Soc. Linn. Nord France, 1881. 48. (1881); Pand., Revue d'Entom., II. 226. (1883); Ricardo, Annal. Mag. Nat. Hist., ser. 7. IX. 425. (1902).

fenestratus LATR. = marmoratus Rossi.

fixissimus Walk., Proc. Linn. Soc. London, I. 112. 37. (1856); v. D. Wulp, Catal. Dipt. South Asia, 65. (1896); RieArdo, Annal. Mag. Nat. Hist., ser. 7. IX. 376. (1902).

unizonatus Rond., Annal. Mus. Civ. Genova, VII. 459. (1873); Ost.-SACK., Berlin. Entom. Zeitschr., XXVI. 97. (1882).

flavidus Wued., Dipt. exot., 105. 5. (1821) et Aussereurop. zweifl. Ins., I. 199. 7. (1828); WaLK., List Dipt. Brit. Mus., V. suppl. 1., 283. 32. (1854) ; Ost.-SAck., Mem. Boston Soc. Nat. Hist., II. 385. (1876); Ricardo, Annal. Mag. Nat. Hist., ser. 7. VIII. 300. (1901); Hine, Ohio State Acad. Sci., Spec. Pap. No. 5., 37. (1904); Aldr., Catal. North Amer. Dipt., 196. (1905).

canifrons WaLk., List Dipt. Brit. Mus., I. 197._(1848) et V. suppl. 1., 283. (1854).

pallidus BkLl., Saggio di Ditterol. Messic., I. 73. 7. tab. II. fig. 16. (1859).

flavipes Meig., Klass., I. 159. 3. tab. IX. fig. 13. (1804); Villen., Annal. Soc. Entom. France, LXXIV. 310. 6. (1905).

perspicillaris Lw., Neue Beitr., IV. 25. 5. (1856) et Verh. zool.-bot. Ges. Wein, VIII. 633. 20. (1858); Schrs., Fauna Austriaca, Dipt., I. 41. (1862) ; Goв., Mém. Soc. Linn. Nord France, 1881. 48. (1881); Pand., Revue d'Entonı., II. 226. (1883) ; Griff., Boll. Mus. Zool. ed Anat. comp. 'Torino, X. No. 218., 6. (1895) ; Ricardo, Annal. Mag. Nat. Hist., ser. 7. IX. 426 et 430 . (1902).

flaviventris MACQ., Dipt. exot., suppl. 1., 44. 13. (1845); WALK., List Dipt. Brit. Mus., V. suppl. 1., 292. 68. (1854); Ricardo, Annal. Mag. Nat. Hist., ser. 7. IX. 373. (1902).

flavocinctus Ricardo, Annal. Mag. Nat. Hist., ser. 7. IX. 380. (1902).

Frazari Willist., Trans. Kansas Acad. Sci., X. 133. (1887); Ricardo, Annal. Mag. Nat. Hist., ser. 7. VIII. 311. (1901); Aldr., Catal. North Amer. Dipt., 196. (1905).

frigidus Ostr.-SAck., Mem. Boston Soc. Nat. Hist., II. 384. 14 et 474. (1876) ; Ricardo, Annal. Mag. Nat. Hist., ser. 7. VIII. 305. (1901); Hrne, Ohio State Acad. Sci., Spec. Pap. No. 5., 37. (1903) et Ohio Naturalist, V. 223. (1904); ALdr., Catal. North Amer. Dipt., 196. (1905).
Europa mer., Africa sept.

Asia mer.,

Arch.Indicus.

America sept. et centr.

Europa centr. et mer., Africa sept., Asia min.

India or.

Assam, Born. S. Domingo.

America sept. 
frontalis MACQ., Dipt. exot., I. 1., 160. 7. (1838); WALK., List Dipt. Brit. Mus., V. suppl. 1., 284. 34. (1854); Ricardo, Ammal. Mag. Nat. Hist., ser. 7. VIII. 310. (1901); Aldr., Catal. Nortl Amer. Dipt., 196, (1905).

fugax: Ost.-SAck. = carbonarius WALK.

fuliginosus WIED., Dipt. exot., 109. 11. (1821) et Aussereurop. zweifl. Ins., I. 210. 23. (1828); Watk., List Dipt. Brit. Mus., I. 204. (1848) et V. suppl. 1., 282. 25. (1854).

plangens WiED., Aussereurop. zweill. Ins., I. 210. 22. (1828);

Walk., List Dipt. Brit. Mus., I. 204. (1848) et V. suppl. 1., 282. 24. (1854); Ost.-SAck., Mem. Boston Soc. Nat. Hist., II. 393. (1876) et Catal. Dipt. North Amer., Ed. II., 53 et 225. 67. (1878); Willıst., Trans. Kansas Acad. Sci., X. 134. (1887); Ricardo, Annal. Mag. Nat. Hist., ser. 7. VIII. 301. (1901); Aldr., Catal. North Amer. Dipt., 197. (1905).

fulvaster Osт.-S৯ск., Bullet. Unit. Stat. Geolog. Surv., III. 221. 2. (1877); Wrllist., Trans. Kansas Acad. Sci.,. X. 134. (1887); Ricardo, Annal. Mag. Nat. Hist., ser. 7. VIII. 306. (1901); Hine, Ohio Naturalist, V. 223. (1904); Alnr., Catal. North Amer. Dipt., 196. (1905).

coloradensis Bı́., Mém. Soc. Zool. France, V. 605. p. p. (1892).

fulviceps WaLk., List Dipt. Brit. Mus., V. suppl. 1., 285. 40. (1854). fulvistigma Hine, Canad. Entomol., XXXVI. 55. (1904).

furcatus WaLk., List Dipt. Brit. Mus., I. 199. (1848); Ost.-SACK., Mem. Boston Soc. Nat. Hist., II. 391. (1876); Rrcardo, Annal. Mag. Nit. Hist., ser. 7. VIII. 302. (1901); Hıxe, Ohio Naturalist, V. 223. (1904).

fuscipennis Richreo, Annal. Mag. Nat. Hist., ser. 7. IX. 370. (1902). fuscuis Ricardo, Annal. Mag. Nat. Hist., ser. 7. IX. 368. (1902). geminatus MAcQ. (nee W1ED, $=$ virgulatus BELL.

geminatus Wied., Aussereurop. zweifl. Ins., I 205. 16. (1828); Walk., List Dipt. Brit. Mus., I. 200. (1848) et V. suppl. 1., 293. 73. (1854); Rıcardo, Annal. Mag. Nat. Hist., ser. 7. VII. 299. (1901); Aldr., Catal. North Amer. Dipt., 196. [excl. sym. Macq.] (1905).

guttula Wied., Aussereurop. zweill. Ins., I. 203. 13. (1828) ; WALK., List Dipt. Brit. Mus., V. suppl. 1., 286. 42. (1854); Ricardo, Annal. Mag. Nat. Hist., scr. 7. VHII. 310. (1901); Hunter, Trans. Amer. Entom. Soc. Philad., XXVII. 135. (1901).

hamatus Lw., Verh. zool.-bot Ges. Wien, VIII. 617. 3. (1858); Asia min. Ricardo, Annal. Mag. Nat. Hist., ser. 7. IX. 427. (1902).

hilaris Ost.-SAck., Mem. Boston Soc. Nat. Hist., II. 391. (1876); America sept. Aldr., Catal. North Amor. Dipt., 196. (1905).

immaculatus WinD., Ausscreurop. zweill. Ins., I. 102. 11. (1828); ?Brasilia. Ricardo, Annal. Mag. Nat. Hist., ser. 7. VIII. 310. (1901). impar ROND. $=$ dispar FABR.

incisus MAcq., Dipt. exot., suppl. 1., 44. 15. tab. IV. fig. 12. (1845); N. Granada.
India occid., Brasilia.

America sept.

America sept.

Brasilia.

America sept.

America sept.

Maslionaland.

Africa.

Patria ignota.

Brasilia.

$$
\text { Prasilia. }
$$


Walk., List Dipt. Brit. Mus., V. suppl. 1., 285. 39. (1854); Schus., Novara Reise, Dipt., 104. 62. (1868); Ricardo, Anual. Mag. Nat. Hist., ser. 7. VIII. 310. (1901); Hunter, Trans. Amer. Entom. Soe. Philad., XXVII. 135. (1901).

indianus Rrcardo, Annal. Mag. Nat. Hist., ser. 7. IX. 379. (1902).

indus Osт.-SAck., Mem. Boston Suc. Nat. Hist., JI. 383. 13. (1876);

Hine, Ohio Naturalist, II. 168. (1902) et Obio State Acad. Sci., Spec. Pap., No. 5., 38. (1903) ; Aldr., Catal. North Amer. Dipt., 196. (1905).

intrudens WiLIIst., Kansas Univ. Quart., III. 191. (1895) ; Ricardo,

Annal. Mag. Nat. Hist., ser. T. VIII. 311.(1901); Hunter, Trans.

Amer. Eutom. Soc. Philad., XXVII. 136. (1901).

iranensis BıG. = Mlokosiewiczi Bı.

italicus Lw. = marmoratus Rossi.

italicus Meig., Klassif., I. 158. 1. (1804) et System. Beschreib., II. 67. 1. tab. XIV. fig. 7. (1820); MACQ., Suit. à Buffon, I. 216. 6. (1834); Walk., List Dipt. Brit. Mus., I. 194. (1848) et V. suppl. 1., 277 et 2ع0. 11. (1854); Gos., Mém. Soe. Linn. Nord France, 1881. 49. (1881) ; Pand., Revue d'Entom., II. 225. (1883); Ricardo, Annal. Mag. Nat. Hist., ser. 7. IX. 425. excl. syn. (1902); Villen., Annal. Soc. Entom. France, LXXIY. 310. 3. (1905).

japonicus WIED., Aussersurup. zweifl. Ins., I. 203. 14. (1828); WALK., List Dipt. Brit. Mus., V. suppl. 1., 291. 63. (1854); Lw, Verlı. zool.-bot. Ges. Wien, VIII. 621. Anmerk. (1858); Ost.-Sack., IVien. Entom. Zeitg., III. 316. (1884) ; Ricardo, Annal. Mag. Nat. IIist., ser. 7. IX. 428 et 430. (1902).

aterrimus limby, Annal. Mag. Nat. Hist., ser. 5. XIII. 457. 3. (1884).

laetus FABr., Systema Antliat., 112. 7. (1805); WiEd., Dipt. exo ., 108. 9. (1821) et Aussereurop. zweifl. Ins., I. 207. 19. (1828); Walk., List Dipt. B: it. Mus., V. suppl. 1., 286. 44. (1854); Schin., Novara Reise, Dipt,, 103. 60. (1868); Ricalido, Anmal. Mag. Nat. Hist., ser. 7. VIII. 310 et 313. (1901); Hunter, Trans. Amer. Entom. Soc. Philad., XXVII. 136. (1901).

laniger Lw., Dipt.-Fauna Südafrika's, I. 28. 1. (1860); Ricardo, Prom.bon.sp. Annal. Mag. Nat. Hist., ser. 7. IX. 367. (1902).

lapponicus Lw., Verh. zool.-bot. Ges. Wien, VIII. 624. 11. (1858); Europa sept. Ricardo, Annal. Mag. Nat. Hist., ser. 7. IX. 427. (1902).

lateralis WIED. = ? scalaratus BELL.

latifasciatus Betr., Saggio di Ditterol. Messic., I. 71. 4. tab. II. fig. Americacentr. 15. (1859) ; Ost.-SAck., Biolog. Central. Amer., Dipt., I. 46. 3. (1886); Ricardo, Annal. Mag. Nat. Hist., ser. 7. Vill. 310. (1901); Willist., Biolog. Central. Amer., Dipt., I. 256. (1901); Aldr., Catal. Nortl Ame:. Dipt., 197. (1905).

leucospilus Wied., Aussereurop. zweifl. Ins., I. 202. 12. (1828); Brasilia,

WAr.k., List Dipt. Brit. Mus., V. suppl. 1., 286. 41. (1854); Schin., Columbia. Novara Reise, Dipt., 104. 63. (1868); Ricardo, Aumal. Mag. Nat.
America sept.

Japonia,

Amur.

Brasilia.
India or. 
Hist., ser. 7. VIII. 310. (1901); Hunter, Trans. Amer. Entom.

Soc. Philad., XXVII. 136. (1901).

ligatus WALK. $=$ dispar $\mathrm{F}_{\mathrm{ABR}}$.

lineatus $\mathrm{J}_{\mathrm{AENN}}=$ vittatus WIED.

longicornis MACQ., Dipt. exot., I. 1., 156. 1. tab. XIX. fig. 2. (1838);

Walk., List Dipt. Brit. Mus., V. suppl. 1., 290. 58. (1854) ; Gerst.,

Archiv f. Naturgesch., XXXVII. 1., 362. 1. (1871) et Decken's

Reise in Ost-Afrika, III. 2., 384. 1. (1873); Ricardo, Annal. Mag.

Nat. Hist., ser. 7. IX. 366 et 368. (1902).

tarsalis WaLK., List Dipt. Brit. Mus., I. 200. (1848); Ricardo,

Annal. Mag. Nat. Hist., ser. 7. IX. 367 et 368. (1902).

trimaculatus Big., Mém. Soc. Zool. France, V. 607. (1892);

Ricardo, Annal. Mag. Nat. Hist., ser. 7. IX. 367 et 371. (1902).

ludens Lw., Verh. zool.-bot. Ges. Wien, VIII. 628. 17. (1858);

Ricardo, Annal. Mag. Nat. Hist. ser., 7. IX. 427. (1902).

lugens Wied., Dipt. exot., 109. 12. (1821) et Aussereurop. zweifl.

Ins., I. 212. 26. (1828) ; Walk., List Dipt. Brit. Mus., V. suppl.

1., 282. 27. (1854); Hine, Ohio Stat. Acad. Sci., Spec. Pap. No.5.,

39. (1903) ; Aldr., Catal. North Amer. Dipt., 197. (1905).

morosus Ost.-SAck., Mem. Boston Soc. Nat. Hist., II. 389. 18

et 474. (1876) ; Ricardo, Annal. Mag. Nat.Hist., ser. 7. VIII.

305. (1901); Alpr., Catal. North Amer. Dipt., 197. (1905).

? trinotatus MACQ., Dipt. exot., I. 1., 161. 9. (1838); WALK., List

Dipt. Brit. Mus., V. suppl. 1., 282. 26. (1854).

lugubris $\mathrm{L}$. = caecutiens $\mathrm{L}$.

lugubris MacQ., nipt. exot., suppl. 1., 44. 14. tab. IV. fig. 11. (1846);

Walk., List Dipt. Brit. Mus., V. suppl. 1., 285. 38. (1854) ; Ricardo,

Annal. Mag. Nat. Hist., ser. 7. VIII. 310. (1901); Hunter, Trans.

Amer. Entom. Soc. Philad., XXVII. 136. (1901).

lunctus GRAY $=$ dispar FABR.

lupus Whitney, Canad. Entomol., XXXVI. 205. (1904); Hine, Ohio

Naturalist, V. 224. (1904).

madagascarensis Ricardo, Annal. Mag. Nat. Hist., ser. 7. IX 369. (1902).

manilensis Scrin., Novara Reise, Dipt., 104. 65. (1868); v. D. Wur.P, Catal. Dipt. South Asia, 66. (1896); Ricardo, Annal. Mag. Nat.

Hist., ser. 7. IX. 373. (1902).

maritimus ScOP. $=$ caecutiens $\mathrm{L}$.

marmoratus Rossi, Frauna Etrusca, II. 322. 1552. [Tabanus] (1790);

Paxz., Fauna Germ., CX. tab. 20. (? 1813); Mrig., System.

Beschreib., II. 73. 8. (1820); MacQ., Recueil Soc. Sci. Agricult.

I ille, 1826. 488. 6. (1826) et Suit. à Buffon, I. 215. 5. (1834) ;

Schumm., Übers. d. Arb. u. Veränd. d. schles. Ges. f. vaterl.

Kultur, 1837. 107. 2. (1837); Gimm., Bull. Soc. Imp. Nat. Moscou,

XX. 1., 191. 6. (1847); Schor,z, Zeitschr. f. Entomol. Breslau, JV. No. 16., 38. (1850); Walk., List Dipt. Brit. Mus., V. suppl. 1., 277 et 279. 10. (1854); Scrin., Verl., zool.-bot. Ges. Wien,

Africa or. et occid.

Asia min.

America sept.

Brasilia.

America sept.

Madagasear.

Manila.

Europacentr. et mer., Asia min. 
VII. 19. 4. (1857) et Fauna Austriaca, Dipt., I. 40. (1862); Rond., Archiv. per la Zool. Modena, III. 82. (1863); Goв., Mém. Soc. Linn. Nord France, 1881. 47. (1881); PAND., Revue d'Entomol., II. 225. 3. (1883); Ricardo, Annal. Mag. Nat. Hist., ser. 7. IX. 426. (1902).

fenestratus Latr., Gen. Crust. et Ins., IV. 285. (1809).

italicus Lw., Verh. zool.-bot. Ges. Wien, VIII. 630. 19. (1858);

Ricardo, Annal. Mag. Nat. Hist., ser. 7. IX. 425 et 428. (1902).

nigriventris Lw., Neue Beitr., IV. 26. 6. (1854).

? salinarius Scop., Entomol. Carniol., 373. 1015. [Tabanus](1763);

Vill., Entom. Linn., III. 557. 14. [Tabanus] (1789).

mauritanicus A. Costa, Rendic. Accad. Napoli, ser. 2. VII. 101. (1893) et Atti Accad. Napoli, ser. 2. V. No. 22., 14. tab. IV. fig. 7-8. (1893); Ricardo, Annal. Mag. Nat. Hist., ser. 7. IX. 427 et 431. (1902).

maurus Siebke, Nyt Magaz. f. Naturvid., XII. 108. (1863) ; RiCARdo, Annal. Mag. Nat. Hist., ser. 7. IX. 427. (1902).

megaceras Bell., Saggio di Ditterol. Mossic., I. 74. 9. tab. II. fig. Mexico. 18. (1859); Aldr., Catal. North Amer. Dipt., 197. (1905).

melanopleurus WahlbG., Öfvers. Kongl. Vet. Akad. Förhandl., V. Europa sept. No. 9., 200. (1848) ; Zетт., Dipt. Scand., VIII. 2943. 2-3. (1849); Lw., Verh. zool.-bot. Ges. Wien, VIII. 627. Anmerk. (1858); ZEтT., Dipt. Scand., XIII. 4948. 2-3. (1859); Bonsd., Finl. tvaving. Ins., I. -113. 3. (1861); Ricardo, Annal. Mag. Nat. Hist., ser. 7. IX. 426. (1902).

morio Zfтт., Dipt. Scand., VIII. 2944. Obs. (1849).

melanopterus Hine, Ohio Naturalist, VI. 391. (1905).

Melicharii Mıк, Wien. Entom. Zeitg., XVII. 158. 9. (1898); Ricardo, Annal. Mag. Nat. Hist., ser. 7. IX. 427. (1902).

merula PhIL., Verl. zool.-bot. Ges. Wien, XV. 724. 2. (1865); Ricardo, Annal. Mag. Nat. Hist., ser. 7. VIII. 310. (1901); Hunter, Trans. Amer. Entom Suc. Philad., XXVII. 136. (1901). mitis Ost.-SAck., Mem. Boston Soc. Nat. Hist., II. 374. 3. (1876); Willist., 'Trans. Kansas Acad. Sci., X. 131, 134. (1887); HaRT, Bull. Illin. Stat. Labor. of Nat. Hist., IV. 226. (1895) ; RicArdo, Annal. Mag. Nat. Hist., ser. 7. VIII. 305. (1901); Hıse, Ohio Naturalist, V. 224. (1904); Aldr., Catal. North Amer. Dipt., 197. (1905).

Mlokosiewiczi Bıt., Annal. Soc. Entom. France, sér, 5. X. 146. (1880) ; Ricardo, Annal. Mag. Nat. Hist., ser. 7. IX. 378. (1902). iranensis Bıg., Mém. Soc. Zool. France, V. 602. (1891).

moechus Ost.-SAck., Mem. Boston Soc. Nat. Hist., II. 387. (1876) ; Hine, Ohio Naturalist, II. 168. (1901) et Ohio State Acad. Sci., Spec. Pap. No. 5., 39. (1903) ; ALDr., Catal. North Amer. Dipt., 197. (1905).

moerens Walk., List Dipt. Brit. Mus., I. 201. (1848); Ricardo, America sept. 
Annal. Mag. Nat. Hist., ser. 7. VIII. 302. (1901); Hıne, Ohio St.

Acad. Sci., Spec. Pap. No, 5., 40. (1903).

aestuans v. D. WulP, Tijdschr. v. Entom., X. 135. 8. tab. III. fig. 8-9. (1867); Ost.-SAck., Mem. Boston Soc. Nat. Hist., II. 378. 8. (1876); Willist., Trans. Kansas Acad. Sci., X. 132. (1887); Hart, Bull. Illin. Stat. Laborat. of Nat. Hist., IV. 227. tab. X. fig. 42. (1895); Lugger, 2nd Report Entom. Minnesota, 196. (1897) ; Lécalllon, Annal. Soc. Entom. France, LXXIV. 21. (1905); Aldr., Catal. North Amer. Dipt., 195. (1905).

molestus GukR. (nec WIED.) = costatus FABR.

molestus Wied., Aussereurop. zweifl. Ins., I. 205. 17.(1828) ; WALk.,

Jist Dipt. Brit. Mus., V: suppl. 1., 288. 51. (1854); Ricaldo, Annal. Mag. Nat. Hist., ser. 7. VIII. 310. (1901); Hunter, Trans. Amer. Entom. Soc. Philad., XXVII. 136. (1901).

montanus Osт.-Sack., Mem. Boston Soc. Nat. Hist., II. 382. (1876) ;

Towns., Trans. Amer. Entom. Soc. Philad., XXII. 57. (1895);

Hine, Ohio State Acad. Sei., Spec. Pap. No.5., 40. (1903); Aldr.,

Catal. North Amer. Dipt., 197. (1905).

morio ZETT. = melanopleurus WAHLig.

morosus Ost.--SAck. = lugens WiFD.

natalis MAcQ., Dipt. exot., suppl. 1., 43. 12. tab. IV. fig. 13. (1845);

Port Natal.

Walk., List Dipt. Brit. Mus., V. suppl. 1., 290. 57. (185t);

Ricardo, Annal. Mag. Nat. Hist., ser. 7. IX. 367. (1902).

neglectus Willis'r., Biolog. Central. Amer., Dipt., I. 256. 5. tab. IV.

fig. 20. (1901) ; Aldr., Catal. North Amer. Dipt., 197. (1905).

niger Macq., Dipt. exot., I. 1., 161. 10. (1838); WALK., List Dipt. Americasept.

Brit. Mus., I. 202. (1848) et V. suppl. 1., 282. 28. (1854) ; Ost.-

Sack., Mem. Boston Soc. Nat. Hist., II. 377. 7.(1876); v. D. Wulp, 'Tijdsclır. v. Entomol., XXIV. 161. 15. (1881); Towns., 'Trans. Amer. Entom. Soc. Philad., XXII. 57. 5. (1895); Hart, Bullet. Illin. State Laborat. of Nat. Hist., IV. 227. (1895); RicArdo, Annal. Mag. Nat. Ifist., ser. 7. VIII. 301. (1901); HıNe, Ohio State Acad. Sci., Spec. Pap. No. 5., 41. (1903); Aldr., Catal. North Amer. Dipt., 197. (1905).

carbonarius WaLk., List Dipt. Brit. Mus., I. 203. var. $\beta$. $q$. (1848).

niger WALK. (nec MACQ.) = carbonarius WALK.

nigribimbo ALDR. = nigrilimbo WhitNeY.

nigrilimbo Whitney, Canad. Entomol., XI. 36. (1879); Willist.,

Trans. Kansas Acad. Sci., X. 131. (1887); Ricardo, Annal. Mag.

Nat. Hist., ser. 7. VIII. 300. (1901).

nigribimbo Aldr., Catal. North Amer. Dipt., 197. (1905).

nigripes ZETT., Insecta Lappon., Dipt., 519. 3. (1838) et Dipt. Scand.,

I. 125. 3. (1842) , t VIII. 294t. 3. (1849); WaLK., List Dipt. Brit. Mus., V. suppl. 1., 277 et 278. 4. (1854); Lw., Verh. zool.-bot. Europa bor., Alaska, Sitka.

Ges. Wien, VIII. 623. 10. (1858) ; BonsD., Finland tvaving. Ins., 
I. 113. 4. (1861); Ost.-Sack., Mem. Boston Soc. Nat. Hist., II. 394. 2. (1876) ; Coquill., Proc. Washington Acad. Sci., II. 406. (1900) ; Ricardo, Annal. Mag. Nat. Hist., ser. 7. IX. 426. (1902); Hrne, Ohio Naturalist, V. 224. (1904); CoquitL., Harriman Alaska Exped., IX. Pt. II. 20. (1904) ; Aldor., Catal. North Amer. Dipt., 197. (1905).

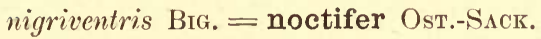

nigriventris Lw. = marmoratus RossI.

noctifer Ost.-SAck., Bull. Unit. Stat. Geolog. Survey, III. 220. 1. (1877); Hine, Ohio Naturalist, V.224. (1904); Aldr., Catal. North Amer. Dipt., 197. (1905).

nigriventris Bı̈., Mém. Soc. Zool. France, V. 604. (1892); ALDr., Catal. North Amer. Dipt., 197. (1905).

pertinax Willist., Trans. Kansas Acad. Sci., X. 132́.(188i);

Ricardo, Annal. Mag. Nat. Hist., ser. 7. VIII. 307. (1901);

Aldr., Catal. North Amer. Dipt., 197. (1905).

novus Scmn., Novara Reise, Dipt., 103. 59. (1868); Ricnrdo, Annal. Hispania.

Mag. Nat. Hist., ser. 7. IX. 427. (1902).

mubilosus HaRris $=$ relictus MEIG.

obliquefasciatus MACQ., Dipt. exot., I. 1., 157. 2. (1838); WALK., List Dipt. Brit. Mus., I. 195. (1848) et V. suppl. 1., 289. 56. (1854); Iw., Dipt.-Fauna Siidafrika's, I. 30. (1860); Ricardo, Annal. Mag. Nat. Hist., ser. T. IX. 367. (1902).

obsoletus WiEd., Dipt. exot., 108. 10. (1821) et Aussereurop. zweifl. Ins., I. 211. 25. (1828); WaLk., List Dipt. Brit. Mus., I. 200. (1848) et V. suppl. 1., 283. 31. (1854); Ost.-SAcK., Mem. Boston Soc. Nat. Hist., II. 393. (1876) et Catal. Dipt. North Amer., Ed. II., 53 et 226. 68.(1878); Hine, Ohio State Acad. Sci., Spec. Pap., No. 5., 42. (1903) ; Aldr., Catal. North Amer. Dipt., 197. (1905).

oculatus Big., Mém. Soc. Zool. France, V. 606. (1892); Ricardo, Annal. Mag. Nat. Hist., ser. 7. VIII. 311 et 313. (1901).

pachycera Willist., Trans. Kansas Acad. Sci., X. 134. p. p. [ㅇ]. (1887); Towns., Proc. Calif. Acad. Sci., ser. 2. IV. 596. 10. (1895);

Ricardo, Annal. Mag. Nat. Hist., ser. 7. VIII. 300. (1901) ; Hine, Ohio Naturalist, V. 224. (1904); AdAms, Kansas Univ. Sci. Bullet., IJ. 442. (1904) ; Aldor., Catal. North Amer. Dipt., 197. (1905). pachycera Willist. p. p. $\left[\sigma^{\top}\right]=$ proclivis Ost.-SAcK.

pachyenemius Hine, Ohio Naturalist, VI. 391. (1905). pallidus BeLL. = flavidus Wied.

pallidus MACQ., Dipt. exot., I. 1., 162. 11. (1838); WALK., List Dipt. Brit. Mus., V. suppl. 1., 280. 12. (1854); Ricardo, Annal. Mag. Nat. Hist., ser. 7. IX. 426. (1902).

parallelogrammus Zet., Isis, 1842. XI. 823. tab. I. fig. 31. (1842); Zетт., Dipt. Scand., VIII. 2943. 2. (1849); WALK., List Dipt. Brit. Mus., V. suppl. 1., 277 et 278. 3. (1854); Lw., Verh. zool.-bot. Ges. Wien, VIII. 621. 7. (1858) ; Scurs., Fauna Austriaca, Dipt.,

Africa mer.

America sept.

Brasilia.

America sept.

Guatemala.

? Gallia.

Europa centr. et sept., Sibiria. 
I. 42. (1862) ; Brav., Horae Soc. Entom. Ross., -XXIII. 111. (1882) ; Pand., Revue d'Entomol., II. 225. (1883); Ricardo, Annal. Mag. Nat. Hist., ser. 7. IX. 426. (1902).

bipunctatus Мотsch., Bull. Soc. Imp. Nat. Moscou, XXXII: 2., 505. (1859).

? parallelus WALK., Proc. Linn. Soc. London, V. 276. 23. (1861); v. D. Wulp, Catal. Dipt. South Asia, 66. (1896); Ricardo, Annal. Mag. Nat. Hist., ser. 7. IX. 372. [? Diachlorus] (1902) et ser. 7. XIV. 356. [? Udenocera] (1904).

pellucidus Fabr., Systema Antliat., 113. 11. (1805); Wied., Dipt. India or. exot., 107. 8. (1821) et Aussereurop. zweifl. Ins., I. 206. 18. (1828) ; Walk., List Dipt. Brit. Mus., I. 200. (1848) ; Ricardo, Annal. Mag. Nat. Hist., ser. 7. IX. 373. (1902).

perspicillaris $\mathrm{Lw}_{\mathrm{w}}=$ flavipes MeIG.

pertinax WILLIST. $=$ noctifer OsT.-SACK.

pictus MeIG., System. Beschreib., II. 70. 4. (1820); Macq., Recueil Soc. Sci. Agricult. Lille, 1821. 487. 3. (1826) et Suit. à Buffon, I. 215. 3. (1834) ; Duncan, Magaz. Zool. and Botan., I. 456. (1837); Lw., Programm Posen, 1840. 6. 4. (1840) et Isis, 1840. VII-VIII. 519. 4. (1840) ; Zelu., Isis, 1842. XI. 823. tab. I. fig. 28-29. (1842) ; Givm., Bull. Soc. Imp. Nat. Moscou, XX. 1., 189. 3. (1847); WAlK., List Dipt. Brit. Mus., I. 194. (1848) ; ZeTt., Dipt. Scand., VIII. 2945. 4. (1849); WALK., List Dipt. Brit. Mus., V. suppl. 1., 276 et 278. 6. (1854); Bonsd., Finl. tvaving. Ins., I. 113. 5. (1861); Neunaus, Diptera Marchica, 45. 3. (1886); Griff., Boll. Mus. Zool. ed Anat. comp. Torino, X. No. 218., 7. (1895); Villen., Feuille jeun. Natural., XXXIV. 227. 3. (1904) et Annal. Soc. Entom. France, LXXIV. 310. 7. (1905).

quadratus Meig., System. Beschreib., II, 70. 5. (1820); MacQ., Recueil Soc. Sci. Agricult. Lille, 1826. 488.4. (1826) et Suit. à Buffon, I. 215. 4. (1834); Zexт., Insecta Lappon., Dipt., 519. 4. (1838); Lw., Programm Posen, 1840. 6. 3. (1840) et Isis, 1840. VII-VIII. 519. 3. (1840); Grmy., Bull. Soc. Imp. Nat. Moscou, XV. 664. (1842); Zeтr., Dipt. Scand., I. 126. 5. (1842); Grmu., Bull. Soc. Imp. Nat. Moscou, XX. 1., 189. 4. (1847); Walk., List Dipt. Brit. Mus., I. 194. (1848) ; ZetT., Dipt. Scand., VIII. 2945. 5. (1849); WalK., List Dipt. Brit. Mus., V. suppl. 1., 277 et 278. 5. (1854); Lw., Verh. zool.bot. Ges. Wien, VIII. 626. 14. (1858) ; Schin., Fauna Austriaca, Dipt., I. 41. (1862) ; Gов., Mém. Soc. Linn. Nord France, 1881. 50. (1881); Hansen, Fabrica oris Dipt., tab. II. fig. 10-11. (1883) ; Pand., Revue d'Entomol., II. 224. (1883); É. \& L. Coucke, Annal. Soc. Entom. Belgique, XXXVI. 6. (1892); Austen, Entom. Monthly Mag., ser. 2. VIII. 227. (1897) ; Ricardo, Annal. Mag. Nat. Hist., ser. 7. IX. 425 et 429. (1902); Villen., Annal. Soc. Entom. France, LXXIV. 310. 5. (1905). 
viduatus MeIG., Klassif., I. 158. 6. var. (1804).

Pikei Whitney, Canad. Entomol., XXXVI. 205. (1904); Hine, Ohio America sept. Naturalist, V. 224. (1904).

plangens WIED. = fuliginosus WiED.

proclivis Ost.-SAck., Bullet. Unit. Stat. Geolog. Surv., III. 222. 3. America sept.

(1877); Willist., Trans. Kansas Acad. Sci., X. 132, 134. (1887);

Ricardo, Annal. Mag. Nat. Hist., ser. 7. VIII. 306. (1901) ; Hine, Canad. Entomol., XXXV. 244. (1903) et Ohio Naturalist, V. 225. (1904) ; Adams, Kansas Univ. Sci. Bullet., II. 442. (1904) ; Beutexmưlu., Bullet. Amer. Mus. Nat. Hist., XX. 88. (1904); Aldr., Catal. North Amer. Dipt., 198. (1905).

atricornis Bı́., Mém. Soc. Zool. France, V. 603. (1892).

pachycera Willist., Trans. Kansas Acad. Sci., X. 134. p. p.

[O] $]$ (1887).

provocans $\mathrm{WALK}_{\text {ALK }}=$ carbonarius WALK.

pudicus Ost.-Sack., Mem. Boston. Soc. Nat. Hist., II. 381 et 474 . America sept.

(1876) ; Alpr., Catal. North Amer. Dipt., 198. (1905).

cursim Whitney, Canad. Entomol., XI. 36. (1879); Willist.,

Trans. Kansas Acad. Sci., X. 134. (1887); Rrcurdo, Annal.

Mag. Nat. Hist., ser. 7. VIII. 300. (1901).

punctifer Lw., Neue Beitr., IV. 24. 4. (1856) et Verh. zool.-bot. Ges. Wien, VIII. 633. 21. (1858); Ricardo, Annal. Mag. Nat. Hist., ser. 7. IX. 426. (1902).

quadratus MeIG. = pictus MeIG.

relictus Meig., System. Beschreib., II. 69. 3. (1820) ; Macq., Recueil Soc. Sci. Agricult. Lille, 1826. 487. 2.(1826) et Suit. à Buffon, I. 215. 2.(1834); Duncan, Magaz. Zoolog. and Botan., I. 455. (1837); Zетт., Insecta Lappon., Dipt., 518. 2. (1838) ; Lw., Programm Posen, 1840. 6. 2. (1840) et Isis, 1840. VII-VIII. 518. 2. (1840); ZеTт., Dipt. Scand., I. 124. 2. (1842) ; ZeLL., Isis, 1842. XI. 825. tab. I. fig. 32. (1842); Grмm., Bull. Soc. Imp. Nat. Moscou, XX. 1., 188. 2. (1847); Walk., List Dipt. Brit Mus., I. 194. (1848); Zett., Dipt. Scand., VIII. 2943. 2. (1849); Walk., Insecta Britann., Dipt., I. 44. 2. (1851) et List Dipt. Brit. Mus., V. suppl. 1., 276 et 277. 2. (1854) ; Lw., Verh. zool.-bot. Ges. Wien, VIII. 627. 15. (1858); ZerT., Dipt. Scand., XIII. 4948. 2. (1859); Bonsd., Finland tvaving. Ins., I. 112. 2. (1861); Schin., Fauna Austriaca, Dipt., I. 42. (1862) ; Goв., Mém. Soc. Linn. Nord France, 1881. 51. (1881); Beling, Archiv f. Naturgesch., XLVIII. 1., 189. 3. (1882) ; PAnd., Revue d'Entomol., II. 225. (1883); Neuhaus, Diptera Marchica, 44. 2. (1886); É. \& L. Coucke, Annal. Soc. Entom. Belgique, XXXVI. 6. (1892); Strobl, Mittheil. Naturwiss. Ver. Steiermark, XXIX. 1892. 19. (1893); Griff., Bollet. Mus. Zool. ed Anat. comp. Torino, X. No. 218., 7. (1895); Austen, Entom. Monthly Mag., ser. 2. VIII. 227. (1897); Ricardo, Annal. Mag. Nat. Hist., ser. 7. IX. 425 et 429. (1902); Villen., Annal. Soc. Entom. France, LXXIV. 309. 2. (1905). 
caecutiens Panz., Fauna Germ., XIII. tab. 24. [Tabanus] (1794). mubilosus Harris, Expos. Engl. Ins., 28. tab. VII. fig. 5. [Tabanus] (1782).

viduatus Meig., Klass., I. 158. 6. tab. IX. fig. 12. (1804); FALL., Dipt. Suec., Taban., 10. 2. (1817).

rufipes Meig., System. Beschreib., II. 71. 6. (1820); MACQ., Recueil Soc. Sci. Agricult. Lille, 1826. 488. 5. (1826); Sснимм., Übers. d. Arb. u. Veränd. d. schles. Ges. f. vaterl. Kultur, 1837. 107. 1. (1837); Lw., Programm Posen, 1840. 6. 5. (1840) et Isis, 1840. VII-VIII. 519. 5. (1840) ; Zetт., Dipt. Scand., I. 126. 6. (1842); Zell., Isis, 1842. XI. 823. tab. I. fig. 30. (1842); Zetт., Dipt. Scand., VIII. 2945. 6. (1849) et XI. 4260. 6. (1852); WALK., List Dipt. Brit. Mus., V. suppl. 1., 277 et 279. 7. (1854); Lw., Verh. zool.-bot. Ges. Wien, VIII. 625. 13. (1858) ; Schin., Fauna Austriaca, Dipt., I. 41. (1862); Goв., Mém. Soc. Linn. Nord France, '1881. 49. (1881) ; Pand., Revue d'Entomol., II. 224. 1. (1883) ; Neuhaus, Diptera Marchica, 45. 4. (1886); Griff., Bollet. Mus. Zool. ed Anat. comp. Torino, X. No. 218., 7. (1895) ; É. \& L. Coucke, Annal. Soc. Entom. Belgique, XL. 229. (1896); Ricardo, Annal. Mag. Nat. Hist., ser. 7. IX. 426. (1902); Vilulen., Annal. Soc. Entom. France, LXXIV. 310. 9. (1905).

sepulchralis Meig., System. Beschreib., II. 74. 20. p. p. (1820). rufitarsis MACQ., Dipt. exot., suppl. 3., 14. 16. (1847); WALK., List Dipt. Brit. Mus., V. suppl. 1., 292. 69. (1854); v. D. Wulp, Catal. Dipt. South Asia, 65. (1896); Ricardo, Annal. Mag. Nat. Hist., ser. 7. IX. 373. (1902).

Sackeni Hine, Ohio State Acad. Sci., Spec. Pap. No. 5., 42. (1903); Aldr., Catal. North Amer. Dipt., 198. (1905).

salinarius Scop. = ? marmoratus Ross.

scalaratus BeLr., Saggio di Ditterol. Messic., I. 72. 5. tab. II. fig. 19. (1859); Rrcardo, Annal. Mag. Nat. Hist., ser. 7. VIII. 304. (1901) ; Aldr., Catal. North Amer. Dipt., 198. (1905).

? lateralis Wied., Aussereurop. zweifl. Ins., I. 209. 21. (1828); Walk., List Dipt. Brit. Mus., I. 200. (1848) et V. suppl. 1., 286. 43. (1854); Ricardo, Annal Mag. Nat. Hist., ser. 7. VIII. 310. (1901) ; Hunter, Trans. Amer. Entom. Soc. Philad., XXVII. 136. (1901) ; Aldr., Catal. North Amer. Dipt., 196. (1905).

semicirculus Walk., List Dipt. Brit. Mus., I. 196. (1848) ; v. D. WulP, India or. Catal. Dipt. South Asia, 65. (1896); Ricardo, Annal. Mag. Nat. Hist., ser. 7. IX. 375. (1902)

sepulchralis FABR., Entomol. System., IV. 374. 48. [Tabanus] (1794); Meig., Klassif., I. 161. b. (1804); FABR., Systema Antliat., 113. 12. (1805) ; FAlL., Kongl. Vet. Akad. Handl., XXX. 248. 3. (1817); MeIG., System. Beschreib., II. 74. 20. p. p. (1820) ; Kırby, Fauna Boreal. Americ., IV. 314. 1. (1837); Zетт., Insecta Lappon., Dipt., 519. 5. (1838) ; Iw., Programm Posen, 1840. 6. 6. (1840) et Isis, 1840. VII-VIII. 520. 6. (1840) ; Zетт., Dipt. Scand., I.

Europa.

Java.

America sept.

Americamer. et centr.

Europa centr. et sept., America sept. 
126. 7. (1842); Grum., Bull. Soc. Imp. Nat. Moseou, XX. 1., 190. 5. (1847) ; Walk., List Dipt. Brit. Mus., I. 202. (1848); Zetт., Dipt. Scand., VIII. 2945. 7. (1849); Scholtz, Zeitschr. f. Entomol. Breslau, IV. No. 16., 38. (1850); WaLk., List 'Dipt. Brit. Mus., V. suppl. 1., 277 et 279. 9. (1854); ZETT., Dipt. Scand., XII. 4550. 7. (1855); Lw., Verh. zool.-bot. Ges. Wien, VIII. 622. 9. (1858) ; Bonsd., Finland tvaving. Ins., I. 113. 6. (1861); Schis., Fauna Austriaca, Dipt., I. 41. (1862); Ost.-SAck., Mem. Boston Soc. Nat. Hist., II. 395. 4. (1875); Betuune, Canad. Entomol., XIII. 167. 442. (1881) ; Pand., Revue d'Entomol., II. 225. (1883); Neuraus, Diptera Marchica, 45. 5. (1886); Austen, Entom. Monthly Mag., ser. 2. VIII. 227. (1897) ; Ricardo, Amnal. Mag. Nat. Hist., ser. 7. VIII. 306. (1901) et ser. 7. IX. 425 et 429. (1902); Villen., Annal. Soc. Entom. France, LXXIV. 310. 8. (1905); Aldr., Catal. North Amer. Dipt., 198. (1905).

sepulchralis MeIG. p. p. = rufipes Meig.

sequax Willist., Trans. Kansas Acad. Sci., X. 133. (1887); Ricardo, Annal. Mag. Nat." Hist., ser. 7. VIII. 306. (1901); Hine, Ohio Naturalist, V. 225. (1904); Aldr., Catal. North Amer. Dipt., 198. (1905).

signifer Walk., Proc. Linn. Soc. London, V. 276. 22. (1861); Ost.SACK., Berlin. Entom. Zeitschr., XXVI. 97. (1882); v. D. Wulp, Catal. Dipt. South Asia, 65. (1892); Ricardo, Annal. Mag. Nat. Hist., ser. 7. IX. 376. (1902).

sinensis WALK., Insecta Saunders., Dipt., I. 453. (1856) ; v. D. WulP, Catal. Dipt. South Asia, 65. (1896); Ricardo, Annal. Mag. Nat. Hist., ser. 7. IX. 377. (1902).

sordidus Ost.-Sack., Mem. Boston Soc. Nat. Hist., II. 376. 6. (1876); Willist., Trans. Kansas Acad. Sci., X. 134. (1887); Ricardo, Annal. Mag. Nat. Hist., ser. 7. VIII. 305. (1901); ALDr., Catal. North Amer. Dipt., 198. (1905).

stigmaticalis Lw., Öfvers. Kongl. Vet. Akad. Förhandl., XIV. 1857. 338. 11. (1858) et Dipt.-Fauna Südafrika's, I. 29. 3. fab. I. fig. 18. (1860) ; Johss., Proc. Acad. Nat. Sci. Philad., 1898. 157. (1898) ; Ricardo, Annal. Mag. Nat. Hist., ser. 7. VI. 163. (1900) et ser. 7. IX. 367 et 368. (1902).

stimulans WALK., Insecta Saunders,, Dipt., I. 73. (1850) et List Dipt. Brit. Mus., V. suppl. 1., 265. (1854); v. D. Wulp, Catal. Dipt. South Asia, 65. (1896); Ricardo, Annal. Mag. Nat. Hist., ser. 7. IX. 376. (1902).

striatus Ost.-SAck., Mem. Boston Soc. Nat. Hist., II. 391. 20. (1876); Ricardo, Annal. Mag. Nat. Hist., ser. 7. VIII. 299. (1901); Hine, Ohio State Acad. Sci., Spec. Pap. No. 5., 43. (1903); Aldr., Catal. North Amer. Dipt., 198. (1905).

vittatus Bell. (nec Wied.), Saggio di Ditterol. Messic., I. 74. 8. (1859).

striatus v. D. Wulp, Notes Leyden Mus., VII. 79. 33. (1885) et Arch.Indicus، 
Catal. Dipt. South Asia, 66. (1896); Ricardo, Annal. Mag. Nat. Hist., ser. 7. IX. 379. (1902).

suavis Lw., Wien. Entom. Monatschr., II. 103. 4. (1858) et Verh. Sibiria, zool.-bot. Ges. Wien, VIII. 620. 6. (1858); Ricardo, Annal. Mag. Japonia. Nat. Hist., ser. 7. IX. 427 et 430 . (1902).

subcaecutiens Bell., Saggio di Ditterol. Messic., I. 69. 1. tab. II. Mexico. fig. 13. (1859) ; Aldr., Catal. North Amer. Dipt., 198. (1905).

subfascipennis MacQ., Dipt. exot., suppl. 5., 35. 19. (1855); Ricardo, Annal. Mag. Nat. Hist., ser. 7. VIII. 310. (1901); Hunter, Trans. Amer. Entom. Soc. Philad., XXVII. 136. (1901).

surdus Ost.-ŠAck., Bull. Unit. Stat. Geolog. Surv., III. 223. 4. (1876); Willist., Trans. Kansas Acad. Sci., X. 134. (1887); Hine, Ohio Naturalist, V. 226. (1904); Aldr., Catal. North Amer. Dipt., 198. (1905).

tanycerus Ost.-SAck., Biolog. Central. Amer., Dipt., I. 46. 4. (1886); Ricardo, Annal. Mag. Nat. Hist., ser. 7. VIII. 310. (1901); Aldr., Catal. North Amer. Dipt., 198. (1905).

tardus Wied., Aussereurop. zweifl. Ins., I. 577. 8. (1828) ;"WALK., List Dipt. Brit. Mus., V. suppl. 1., 285. 37. (1854); Ricardo, Annal. Mag. Nat. Hist., ser. 7. VIII. 310. (1901); Hunter, Trans. Amer. Entom. Soc. Philad., XXVII. 136. (1901).

tarsalis WALK. = longicornis MACQ.

terminalis MAcq., Dipt. exot., suppl. 5., 36. 20. (1855); Ricardo, Annal. Mag. Nat. Hist., ser. 7. VIII. 310. (1901); Hunter, Trans. Amer. Entom. Soc. Philad., XXVII. 136. (1901).

terminalis WALK. = dispar FABR.

testaceus MAcQ., Dipt. exot., suppl. 4., 38. 17. (1850); Rrcardo, Tasmania.

Annal. Mag. Nat. Hist., ser. 7. IX. 371. (1902).

translucens MacQ., Dipt. exot., I. 1., 158. 4. (1838); WaLK., List Dipt. Brit. Mus., V. suppl. 1., 291. 65. (1854) ; v. D. Wulp, Catal. Dipt. South Asia, 65. (1896); Ricardo, Annal. Mag. Nat. Hist., ser. 7. IX. 375. (1902).

trifarius Macq., Dipt. exot., I. 1., 159. 6. (1838); Blanch. in GaY : Hist. fis. y polit. de Chile, Zool. VII. 399. 1. (1854); WaLK., List Dipt. Brit. Mus., V. suppl. 1., 287. 45. (1854); Pril., Verh. zool.bot. Ges. Wien, XV. 724. 1. (1865); Ricardo, Annal. Mag. Nat. Hist., ser. 7. VIII. 310. [trifariam] (1901); Hunter, Trans. Amer. Entom. Soc. Philad., XXVII. 136. (1901).

trimaculatus $\mathrm{BIG}$. = longjeornis MAcQ.

trinotatus MACQ. = ? lugens WIED.

tristis F^BR., Entomol. System. Supplem., 567. 44. [Tabanus] (1798) Cayenne. et Systema Antliat., 112. 6. (1805); WiED., Dipt. exot., I. 103. 2. (1821) et Aussereurop. zweifl. Ins., I. 197. 4. (1828); WALK., List Dipt. Brit. Mus., V. suppl. 1., 288. 49. (1854); Ricardo, Annal. Mag. Nat. Hist., ser. 7. VIII. 310. (1901); Hunter, Trans. Amer. Entom. Soc. Philad., XXVII. 136. (1901).

univittatus MacQ., Dipt. exot., suppl. 5., 36. 21. (1855); Ost,-SAck., Americasept. 
Mem. Boston Soc. Nat. Hist., II. 387. (1876); H^kт, Bull. Illinois State Lahorat. of Nat. Hist., JV. 228. (1895); Hine, Ohio State Acad. Sci., Spec. Pap. No. 5., 44. (1903); Atdr., Catal. North Amer. Dipt., 198. (1905).

? fascipennis MACQ., Suit. à Buffon, I. 216. 7. (1834); WALK., List Dipt. Brit. Mus., I. 197. var? (1848) et V. suppl. 1., 283. 29. (1854).

unizonatus RoND. = fixissimus WALK.

validus Lw., Verh. zool.-bot. Ges. Wien, VIII. 619. 5. (1858); RICARLo, Annal. Mag. Nat. Hist., ser. 7. IX. 427. (1902).

varians Wied., Aussereurop. zweifl. Ins., I. 208. 20. (1828) ; WaLK.,

List Dipt. Brit. Mus., V. suppl. 1., 287.46. (1854); Schin., Novara Reise, Dipt., 104. 64. (1868); Willıst., Kansas Univ. Quart., III. 192. (1895); Ricardo, Annal. Mag. Nat. Hist., ser. 7. VIII. 310. (1901); Hunter, Trans. Amer. Entom. Soc. Philad., XXVII. 136. (1901).

variegatus DEG. = ? costatus FABR.

viduatus $\mathrm{F}_{\mathrm{ABR}}$ = caecutiens $\mathrm{I}$.

viduatus MEIG. = relictus MEIG.

viduatus MeIg. var. = pictus MeIG.

virgulatus BeLt., Saggio di Ditterol. Messic., I. 71. 3. tab. II. fig. Mexico.

17. (1859); Willist., Biolog. Central. Americ., Dipt., I. 255. 1.

(A) (1901); Hine, Ohio Naturalist, V. 226. (1904); Aldr., Catal.

North Amer. Dipt., 193. (1905).

crassicornis v. D. Wulp, Wien. Entom. Zeitg., III. 141. (1884)

et Annal. Soc. Entom. Belgique, XXVIII. Compt. Rend. pag.

CCLXXXIX. 5. (1884); Ricardo, Annal. Mag. Nat. Hist., ser. 7. VIII. 300. (1901); Hunter, Trans. Amer. Entom. Soc. Philad., XXVII. 136. nota. (1901); ALDR., Catal. North Amer. Dipt., 196. (1905).

geminatus MACQ., Dipt. exot., suppl. 4., 39. excl. syn. Wied. (1850).

vittatus BELL. (nec WIED.) = striatus Ost.-SAcK.

viltatus WIED., Dipt. exot., 106. 7. (1821) et Aussereurop. zweifl.

Ins., I. 200. 8. (1828); Harris, Ins. of New England, 406. (1841);

Walk., List Dipt. Brit. Mus., I. 196. (1848) et V. suppl. 1., 284.

33. (1854); Macq., Dipt. exot., suppl. 5., 37. 22. (1855); Osт.-

SAcK., Mem. Boston Soc. Nat. Hist., II. 309. (1876) ; Towns., Trans. Amer. Entom. Soc. Philad., XXII. 57. 6. (1895); Hart, Bullet. Illin. State Labor. of Nat. Hist., IV. 228. tab. X. fig. 41. (1895) ; Ricardo, Annal. Mag. Nat. Hist., ser. 7. VIII. 300. (1901); Hrne, Olico State Acad. Sci., Spec. Pap., No. 5., 44. (1903);

Aldr., Catal. North Amer. Dipt., 198. (1905).

areolatus WALK., List Dipt. Brit. Mus., I. 197. (1848).

lineatus JaEnn., Abhandl. Senckenberg. Naturf. Ges., VI. 334. 26. (1867).

vulneratus ROND. = costatus FABR. 


\section{Nemorius}

Roxd., Dipterol. Ital. Prodr., I. 171. 4. (1856).

Haematophila Verr., Scudder Nomencl., 152. (1882).

Haemophila Kriechs., Verh. zool.-bot. Ges. Wien, XXIII. 70. (1873).

Fallottii Kriecrib. = vitripennis MeIG.

Ranzonii SchIs. = vitripennis MeIG.

singularis MeIG., System. Beschreib., VII. 60. 12. [Chrysops] (1838);

WALK., List Dipt. Brit. Mus., V. suppl. 1., 277 et 280. 13. [Chrys-

ops] (1854) ; RoNd., Dipterol. Ital. Prodr., I. 171. (1856); Lw.,

Verh. zool.-bot. Ges. Wien, VIII. 617. 2. [Chrysops] (1858);

Pand., Revue d'Entomol., II. 222. 2. (1883); Ricardo, Annal.

Mag. Nat. Hist., ser. 7. IX. 426. [Chrysops] (1902); Villen.,

Annal. Soc. Entom. France, LXXIV. 310. 11. (1905).

vitripennis MeIG., System. Beschreib., II. 74. 11. [Chrysops] (1820);

MACQ., Suit. à Buffon, I. 216. 8. [Chrysops] (1834); WALK., List

Dipt. Brit. Mus., Y. suppl. 1., 277 et 280. 14. [Chrysops] (1854);

Schix., Verl. zool.-bot. Ges. Wien, VIII. 34. [Chrysops] (1858); Lw., Verh. zool.-bot. Ges. Wien, VIII. 616. 1. [Chrysops] (1858) ; Schis., Fauna Austriaca, Dipt., I. 39. (1862); Paxd., Revue d'Entomol., II. 222. 1. (1883); Stroвl, Mittheil. Naturwiss. Ver. Steiermark, XXIX. 1892. 19. (1893); Griff., Bollet. Mus. Zool. ed Anat. comp. Torino, X. No. 218., 7. (1895); Ricardo, Annal. Mag. Nat. Hist., ser. 7. IX. 426. [Chrysops] (1902); Villex., Bullet. Soc. Entom. France, 1904. 235. III. (1904) et Annal. Soc. Entom. France, LXXIV. 310. 10. (1905).

Fallottii Kriechr., Verh. zool.-bot. Ges. Wien, XXIII. 70. [Haemophila] (1873).

Ranzonii Schin., Verh. zool.-bot. Ges. Wien, VIII. 34. [Chrysops] (1858).

\section{Tabaninae.}

\section{Heptatoma}

MEIG., Illiger's Magaz., II. 266. 38. (1803).

Hexatoma Meig., System. Beschreib., II. 83. (1820) [nec Latr., Dipt. 1809].

albipes Schrank = pellucens FABR.

bimaculata MEIG. = pellucens FABR.

pellucens FABr., Mantissa Insect., II. 355. 12. [Tabamus] (1776) et Spec. Insect., II. 457. 2. [Tabamus] (1781) ; VıLL., Entomol. Linn.,

Europa centr. et sept.

III. 559. 19. [Tabanus] (1789); GMel., Systema Naturae, V. 2883. 23. [Tabanus] (1792); FABR., Entomol. System., IV. 365. 15.

Europacentr. et mer. 
[Tabanus] (1794) ; Schellwns., Gattung. d. Fliegen, tab. XXVIII. fig. 3. [Tabanus] (1803); -MeIG., Klassif., I. 172. e. [Tabanus] (1804) et System. Beschreib., II. 83. 1. tab. XIV. fig. 17-24. [Hexatoma] (1820); Schrn., Fauna Austriaca, Dipt., I. 37. [Hexcttoma] (1862) ; Marno, Verh. zool.-bot. Ges. Wien, XVIII. Sitzgsber., 74. [Hexatoma] (1868); Gов., Mém. Soc. Linn. Nord France, 1881. 40. [Hexatoma] (1881); Becher, Denkschr. Akad. Wien, XLV. 141. tab. II. fig. 18. [Hexatoma] (1882); PANd., Revue d'Entomol., II. 193. [Hexatoma] (1883); Brauer, Denkschr. Alsad. Wien, XLVII. 42. tab. II. fig. $35-37$, tab. III. fig. 38-40. [Hexatoma] (1883) ; Neunaus;'Diptera Marchiea, 45. 1. [Hexatoma] (1886) ; Portsch., Horae Soc. Entom. Ross., XXI. 182. [Hexatoma] (1887) ; Strobl, Mittheil. Naturwiss. Ver. Steiermark, XXIX. 1892. 18. [Hexatoma] (1893); Ricardo, Annal. Mag. Nat. Hist., ser. 7. XIV. 350. [Hexatoma] (1904).

albipes Schrank, Enum. Insect. Austr., 480. 977. [Tabamus] (1781) ; Vir.L., Entomol. Linn., III. 561. 26. [Tabanus] (1789); Schrank, Fauna Boica, III. 154. 2531. [Tabanus] (1803).

bimaculata MeIG., Klass., I. 156. 1. tab. IX. fig. 5-10. (1804); Fabr., Systema Antliat., 105. 1. (1805); Latr., Gen. Crust. et Ins., IV. 284. (1809) et Consid. génér., 443. (1810) ; Panz., Fauna Germ., CX. tab. 19. (1813); MacQ., Recneil Soc. Sci. Agricult. Lille, 1826. 493. tab. III. fig. 7. [Hexatoma] (1826) et Suit. à Buffon, I. 213. 1. tab. V. fig. 6. [Hexatoma] (1834); Blanch., Hist. Nat. d. Ins., III. 590. [Hexatoma] (1840); Grмn., Bull. Soc. Imp. Nat. Moscou, XX. 1., 192. 1. [Hexatoma] (1847); WAHLBG., Öfvers. Kong. Vet. Akad. Förhandl., V. No. 9., 198. [Hexatoma] (1848); Walk., List Dipt. Brit. Mus., I. 205. [Hexatoma] (1848); Zетт., Dipt. Scand., VIII. 2946. 1. [Hexatoma] (1849); WALK., List Dipt. Brit. Mus., V. suppl. 1., 299. 1. [Hexatoma] (1854); Zerr., Dipt. Scand., XII. 4551. 1. [Hexatoma] (1855); Bonsd., Finland tvaving. Ins., I. 114. [Hexatoma] (1861); Villen., Annal. Soc. Entom. France, LXXIV. 310. [Hexatoma] (1905).

\section{Chrysozona}

Meig., Nouvelle Classification, 23. 34. (1800).

Haematopota Meig., Illiger's Magaz., II. 267. 40. (1803).

albihirta KARsCH, Berlin. Entom. Zeitschr, XXXI. 371. 8. tab. IV. fig. 4. [Haematopota] (1887) ; Johns., Proc. Acad. Nat. Sci. PhiAfrica or., Somali. lad., 1898. 158. [Haematopota] (1898).

americana Ost.-SAck., Mem. Boston Soc. Nat. Hist., II. 395. [HaeAmerica sept. matopota] (1876); Willist., Trans. Kansas Acad. Sci., X. 135. [Haematopota] (1887); BIG., Bullet. Soc. Zool. France, XVI. 76. [Haematopota] (1891); Hine, Ohio Naturalist, V. 231. [Haemato- 
pota] (1904); Alor., Catal. North Amer. Dipt., 199. [Haematopota] (1905).

? arctica O.F. Múlu., Fauna Insect. Fridrichsdal., 86. 771. [Tabanus]

Europa sept. (1764) et Zoolog. Dan. Prodr., 180. 2118. [Tabanus] (1776) ; ViLL., Entomol. Linn., III. 561. 27. [Tabanus] (1789).

usiatica RoND. = javana WIED.

atomaria WALk., Proc. Iinn. Soc. London, I. 112. 39. [Hacmatopota] (1856) ; Bra., Bullet. Soc. Zool. France, XVI. 79. [Haematopota] (1891) ; v. D. Wulp, Catal. Dipt. South Asia, 66. [Haematopota] (1896).

Bigoti Gов. = pluvialis L. var.

bistrigata Lw., Öfvers. Kongl. Vet. Akad. Förhandl., XIV. 1857. 341. 32. [Haematopota] (1868) et Dipt.-Fauna Südafrika's, I. 51. 6. tab. I. fig. 27. [Haematopota] (1860); Schin., Novara Reise, Dipt., 81. 2. [Haematopota] (1868) ; Big., Bull. Soc. Zool. France, XVI. 78. [Hacmatopota] (1891).

borneana Rovd., Annal. Mus. Civ. Genova, VII. 461. [Haematopota] (1875) ; BIG., Bull. Soc. Zool. France, XVI. 78. [Haematopota] (1891) ; v. D. Wulp, Catal. Dipt. South Asia, 66. [Haematopota] (1896).

cana Walk., List Dipt. Brit. Mus., I. 207. [Haematopota] (1848); Big., Bullet. Soc. Zool. France, XVI. 79. [Haematopota] (1891); v. D. Wulp, Catal. Dipt. South Asia, 66. [Haematopota] (1896).

cilipes Bıg., Nouv. Archiv Mus. d'Hist. Nat. Paris, sér. 3. II. 205. Ins. Laos.

[Haematopota] (1890) et Bullet. Soc. Zool. France, XVI. 77.

[Hacmatopota] (1891); v. D. Wulp, Catal. Dipt. South Asia, 66. [Haematopota] (1896).

cingulata WiED., Aussereurop. zweifl. Ins., I. 216. 5. [Haematopota] (1828) ; Walk., List Dipt. Brit. Mus., V. suppl. 1., 297. 11. [Haematopota] (1854) ; v. D. WULP, Tijdschr. v. Entomol., XXXIV. 197. [Haematopota] (1891); BIG., Bullet. Soc. Zool. France, XVI. 79. [Haematopota] (1891); v. D. WulP, Catal. Dipt. South Asia, 66. [Hacmatopota] (1896).

circumscripta Lw., Öfvers. Kongl. Vet. Akad. Förhandl., XIV. 1857. 342. 33. [Hacmatopota] (1858) cet Dipt.-Fauna Südafrika's, I. 51. 7. tab. I. fig. 31. [Haematopota] (1860); Big., Bullet. Soc. Zoolog. France, XVI. 76. [Haematopota] (1891).

concentralis WaLK., List Dipt. Brit. Mus., I. 207. [Haematopota] Patriaignota. (1848) ; Big., Bullet. Soc."Zool. France, XVI. 78. [Haematopota] (1891).

cortigera $\mathrm{BIG} .=$ guineensis $\mathrm{BIG}$.

cordigera Big., Bull. Soc. Zool. France, XVI. 76. [Haematopota] (1891) et Mém. Soc. Zool. France, V. 626. [Hacmatopota] (1892); v. D. Wurs, Catal. Dipt. South A sia, 67. [Haematopota] (1896).

crassicornis WAHLBG., Öfvers. Kong. Vet. Akad. Förhandl., V. No. 9., 200. [Haematopota] (1848); ZeTr., Dipt. Scand., VIH. 2941. 3. [Haematopota] (1849) et XI. 4259. 3. [Haematopota] (1852) ; BonsD.,

Bengal, Hindostan.

Europacentr. et sept. 
Finl. tvaving. Ins., I. 112. 3. [Haematopota] (1861); Lw., Jahrb. Krakau. Gel. Ges., XLI. 12. 7. [Haematopota] (1870); Verr., Entom. Monthly Mag., XIX. 224. 1. [Haematopota] (1883); Kow., Wien. Entom. Zeitg., II. 110. nota. [Haematopota] (1883); Strobl, Mittheil. Naturwiss. Ver. Steiermark, XXIX. 1892. 18. nota. [Haematopota] (1893).

decora Walk., Insecta Saunders., Dipt., I. 454. [Haematopota] (1856); BıG., Bull. Soc. Zool. France, XVI. 79. [Haematopota] (1891). dorsalis Lw., Öfvers. Kongl. Vet. Akad. Förhandl., XIV. 1857. 342. 34. [Haematopota] (1857) et Dipt.-Fauna Südafrika's, I. 52. 8. tab. I. fig. 32-34. [Haematopota] (1860); Brg., Bullet. Šc. Zool. France, XVI. 79. [Huematopota] (1891). dorsalis Lw. = decora WALK.

duplicata Lw., Öfvers. Kongl. Vet. Akad. Förhandl., XIV. 1857. 341. 28. [Haematopota] (1858) et Dipt.-Fauna Südafrika's, I. 47.

1. tab. I. fig. 24. [Haematopota] (1860); Big., Bullet. Soe. Zool.

France, XVI. 77. [Haematopota] (1891). elongata St. FARG. ET SERv. = italica Meig. equorum $\mathrm{F}_{\mathrm{ABR}}$ = pluvialis L.

globulifera Schumm., Übers. d. Arb. u. Veränd. d. schles. Ges. f. Europacentr.

vaterl. Kult., 108. [Haematopota] (1837).

grandis MACQ. = italica MEIG.

guineensis BiG., Bull. Soc. Zool. France, XYI. 76. [Haematopota] (1891).

cordigera Big., Annal. Soc. Entom. France, sér. 7. I. 369. 7. [Haematopota] (1891).

gymnonota BRuLlé = italica MetG.

hieroglyphica Gerst., Archiv f. Naturgesch., XXXVII. 1., 362. 2.

[Haematopota] (1871) et Decken's Reisen in Ost-Afrika, III. 2. 385. 2. [Haematopota] (1873); Big., Bullet. Soc. Zool. France, XVI. 77. [Haematopota] (1891).

hyentomantis SuHIN. = pluvialis $\mathrm{L}$.

hyetomantis Schrank = pluvialis $\mathrm{L}$.

imbrium Wien., Aussereurop. zweifl, Ins., I. 215. 4. [Haematopota] (1828) ; MacQ., Dipt. exot., suppl. 1., 46. [Haematopota] (1845); WALK., List Dipt. Brit. Mus., I. 208. [Haematopota] (1848) et V. suppl. 1., 295. 5. [Hacmatopota] (1854).

inappendiculata Bı̈. in Tномs.: Archiv Entomol., II. 350. 657. [Haematopota] (1858) et Bullet. Soc. Zool. France, XVI. 78. [Haematopota] (1891).

indiana BIG., Bullet. Soc. Zool. France, XVI. 78. [Haemátopota] (1891) et Mém. Soc. Zool. France, V. 626. [Haematopota] (1892); v. D. Wulp, Catal. Dipt. South Asia, 67. [Hrematopota] (1896). irrorata MACQ., Dipt. exot., I. 1., 163. 1. tab. XIX. fig. 3. [Haematopota] (1838); Walk., List Dipt. Brit. Mus., V. suppl. 1., 298. 15. [Hacmatopota] (1854); v. D. Wulp, Sumatra Exped., Dipt., 19. 2. tab. I. fig. 13. [Haematopota] (1881); Ost.-SACK., Annal.

Port Natal, Caffraria.

Prom.bon.sp.

Assinia.

Zanzibar.

Africa mer.

Gabon.

Bengal.

Sumatra, Java. 
Mus. Civ. Genova, XVI. 419. [Haematopota] (1882); Big., Bullet. Soc. Zool. France, XVI. 75. [Haematopota] (1891); v. D. WutP, Catal. Dipt. South Asia, 66. [Haematopota] (1896).

? pungens DoL., Natuurkund. Tijdschr. Nederl. Indie, X. 407.

12. tab. IV. fig. 3. [Haematopota] (1856)

italica Meig., Klassif., I. 163. 2. tab. IX. fig. 17. [Haematopota] Europa. (1804) ; Curt., Brit. Entomol., VIII. 525. fig. [H(uematopota] (1834) ; Schin., Fauna Austriaca, Dipt., I. 38. [Haematopota] (1862); Goв., Mém. Soc. I.inn. Nord France, 1881. 39. 4. [Haematopota] (1881) ; Kow., Wien, Entom. Zeitg., V. 110. nota. [Haematopota] (1883) ; Pand., Revue d'Entomol., II. 195. 1. [Haematopota] (1883) ; Ginschn., Berlin. Entom. Zeitschr., XXXI. -. tab. III. fig. 4. [Haematopota] (1887); É. \& L. Coucke, Annal. Soc. Entom. Belgique, XXXVI. 5. [Haematopota] (1892); StroвL, Mittheil. Naturwiss. Ver. Steiermack, XXIX. 1892. 18. [Haematopota] (1893); Villen., Feuille jeun. Natural., XXXIV. 228. 2. var. [Haematopota] (1904) et Annal. Soc. Entom. France, LXXIV. 310. 2. [Haematopota] (1905).

elongata St. Farg. ex Serv., Encyclop. Méthod., X. 543. 1. [Haematopota] (1825) ; CurT., Brit. Entomol., VIII. 525. [Haematopota] (1834) ; Micq., Suit. à Buffon, I. 211. 5. [Haematopota] (1834).

grandis MACQ., Suit. à Buffon, I. 211. 4. [Haematopota] (1834); VIllex., Annal. Soc. Entom. France, LXXIV. 310. 1. [Haematopota] (1905).

gymnonota Brullé, Exped. scient. de Morée, III. 306. 661. tab. XLVII. fig. 4. [Haematopota] (1832).

longicornis MACQ., Suit. à Buffon, I. 211. 3. [Haematopota] (1834) ; Grmy., Bull. Soc. Imp. Nat. Moscou, XX. 3., 192. [Haematopota] (1847); ZETT., Dipt. Scand., VIII. 2941. 2. [Haematopota] (1849) et XI. 4259. 2. [Haematopota] (1853). nigricornis Gов., Mém. Soc. Linn. Nord France, 1881. 38. 2. [Hacmatopotı] (1881); Bug., Bullet. Soc. Zool. France, XVI. 76. [Haematopota] (1891); Villen., Feuille jeun. Natural., XXXIV. 225. 2. [Hacmatopota] (1904) et XXXV. 59. 2. [Haematopota] (1905).

tenuicornis MACQ., Suit. à Buffon, I. 210. 2. [Haematopota] (1834) ; Grmm., Bull. Soc. Imp. Nat. Moscou, XX. 3., 192. [H(cematopota] (1847).

javana Wied., Dipt. exot., 100. 5. [Haematopota] (1821) et Ausser- Archip.Indic., europ. zweifl. Ins., I. 218. 7. [Haematopota] (1828) ; WALK., List Dipt. Brit. Mus., I. 208. [Haematopota] (1848) et V. suppl. 1., 297. 13. [Haematopota] (1854); v. D. Wulp, Sumatra Exped., Dipt., 19. 1. tab. I. fig. 12. [Haematopota] (1881); Big., Bullet. Soc. Zool. France, XVI. 77. [Haematopota] (1891); v. D. Wulp, Catal. Dipt. South Asia, 66. [Haematopota] (1896).

asiatica Rovd., Annal. Mus. Civ. Genova, VII. 461. [Haematopota] (1875). 
nigra WiED., Dipt. exot., 101. 7. [Haematopota] (1821).

limbata Brg., Bull. Soc. Zool. France, XVI. 78. [Haematopota] (1891)

et Mém. Soc. Zool. France, V. 626. [Haematopota] (1892);

Bengal.

v. D. Wulp, Catal. Dipt. South Asia, 66. [Haematopota] (1896). longicornis MACQ. = italica MEIG.

Junulata MACQ., Dipt. exot., suppl. 3., 15. 4. tab. I. fig. 6. [Haematopota] (1847); Walk., List Dipt. Brit. Mus., V. suppl. 1., 297. 14. [Haematopota] (1854); v. D. Wulp, Sumatra Exped., Dipt., 20. 3. tab. I. fig. 14. [Haematopota] (1881); Ost.-SAck., Berlin. Entom. Zeitschr., XXVI. 97. [Haematopota] (1882); v. D. WulP, Catal. Dipt. South Asia, 66. [Haematopota] (1896).

lusitanica Gúkr., Iconogr. d. Régne Anim., VII. Ins., 542. tab. Lusitania. XCVIII. fig. 4. [Haematopota] (1835).

maculiplena Karsch, Berlin. Entom. Zeitschr., XXXI: 371. 9. tab.

IV. fig. 5. [Haematopota] (1887); Johsson, Proc. Acad. Nat. Sci.

Pliilad., 1898. 158. [Haematopota] (1898).

marginula MEG. = pluvialis $\mathrm{L}$.

meteorica Corti, Annal. Mus. Civ. Genova, ser. 2. XV. (XXXV). Africa or.

131. 2. [Haematopota] (1895).

nigra WIED. = javana WIED.

nigricornis Gor. = italica MeIG.

obscura Big. (nee Lw.) = obscurata Big.

obscura Lw., Öfvers. Kongl. Vet. Akad. Förhandl., XIV. 1857. 341.

29. [Haematopota] (1858) et Dipt.-Fauna Südafrika's, I. 48. 2.

tab. I. fig. 25. [Haematopota] (1860); Big., Bull. Soc. Zool. France,

XVI. 77. [Haematopota] (1891).

obscurata BIG., Bull. Soc. Zool. France, XVI. 77. [Haematopota] (1891).

obscura Big., Annal. Soc. Entom. France, sér. 5. X. 147. [Haematopota] (1880).

ocellata MEG. $=$ pluvialis $\mathrm{I}$.

ocellata Wied., Zoolog. Maga\%., I. 3., 38. 56. [Haematopota] (1819),

Prom.bon.sp.

Dipt. exot., 100. 6. [Haematopota] (1821) et Aussereurop. zweifl. Ins., I. 217. 6. [Haematopota] (1828); WALk., List Dipt. Brit. Mus., V. suppl. 1., 296. 6. [Haematopota] (1854); Schin., Novara Reise, Dipt., 81. 1. [Haematopota] (1868); Big., Bull. Soc. Zool. France, XVI. 76. [Haematopota] (1891).

pachycera Bra., Nuov. Archiv Mus. d'Hist. Nat. Paris, sér. 3. II. Ins. Laos. 206. [Haematopota] (1890) et Bull. Soc. Zool. France, XVI. 76. [Haematopota] (1891); v. D. Wulp, Catal. Dipt. South Asia, 66. [Hacmatopota] (1896).

pallens Lw., Schrift. Ges. Freund. d. Nat. Moskau, 1870. 54. 3. Turkestan. [Haenatopota] (1870) et Beschreib. europ. Dipt., II. 61. 36. [Haematopota] (1871).

pluvialis L., Fauna Suec., 463. 1887. [Tabanus] (1761); PoDA, Insect. Mus. Graec., 117. 3. [Tabamus] (1761); Geofrr., Hist. d'Ins., II. 461. 5. [Tabanus] (1762); Scop., Entomol. Carniol., 372. 1012.

Europa,

Africa sept., Japonia. 
[Tabanus] (1763); O. F. Mưller, Fauna Ins. Fridrichsdal., 86. 769. [Tabanus] (1764); L., Systema Naturae, Ed. XII., II. 1001. 16. [Tabanus] (1767) ; O. F. Múller, Zoolog. Dan. Prodr., 180. 2116. [Tabanus] (1776) ; Deg., Mém. pour serv. à l'hist. d. Ins., VI. 223. 5. tab. XIII. fig. 1-2. [Tabanus] (1776); FABR., Species Insect., II. 459. 23. [Tabanus] (1781); Vilı., Entomol. Linn., III. 554. 7. [Tabanus] (1789); GNeL., Systema Naturae, V. 2885. 16. [Tabanus] (1792); PAnz., Fauna Germ., XIII. 23. [Tabanus] (1794) ; FABr., Entomol. System., IV. 369. 32. [Tabanus] (1794); Donov., Brit. Ins., V. 21. tab. CLI. fig. 3. [Tabanus] (1796) ; Schrank, Fauna Boica, III. 155. 2535. [Tabanus] (1803) ; Meig., Klassif., I. 162. 1. tab. IX. fig. 20-21. [Haematopota] (1804); FıBR., Systema Antliat., 106. 1. [Haematopota] (1805); LAtr., Gen. Crust. et Ins., IV. 284. [Haematopota] (1809) ; FALL., Kongl. Vet. Akad. Handl., XXX. 245. 1. [Haematopota] (1809); LAtr., Consid. génér., 443. [Haematopota] (1810) ; LAM., Hist. nat. anim. sans vertébr., III. 421. 5. [Tabanus] (1816); FALL., Dipt. Suec., Taban., 9. 1. [Haematopota] (1817); MeIg., System. Beschreib., II. 78. 1. tab. XIV. fig. 16. [Haematopota] (1820); MAcQ., Recueil. Soc. Sci. Agricult. Lille, 1826. 490. 1. tab. III. fig. 8. [Haematopota] (1826) ; Curt., Brit. Entomol., VIII. 525. [Haematopota] (1834) ; MACQ., Suit. à Buffon, I. 210. 1. tab. V. fig. 4. [Haematopota] (1834); DAнцв., Ins. Skand. skäd. och nytta, 307. 205. [Haematopota] (1837) ; Duncan, Magaz. Zool. and Botan., I. 457. fig. 3. [Haematopota] (1837) ; Zeтt., Insecta Lappon., Dipt., 517. 1. [Haematopota] (1838); Blanch., Hist. Nat. d. Ins., IJI. 590. tab. II. fig. 2. [Haematopota] (1840); Lw., Programm Posen, 1840. 6. 1. [Hacmatopota] (1840) et Isis, 1840. VII-VIII. 520. 1. [Haematopota] (1842); Grmm., Bull. Soc. Imp. Nat. Moscou, XX. 1., 191. 1. [Haematopota] (1847) ; Walk., List Dipt. Brit. Mus., I. 206. [Haematopota] (1848); MacQ. in Lucas: Explor. scient. de l'Algérie, Zool. III. 425. 33. [Hacmatopota] (1849) ; Zetr., Dipt. Scand., VIII. 2940. 1. [Haematopota] (1849); Scholtz, Zeitschr. f. Entomol. Breslau, IV. No. 16., 38. [Haematopota] (1850); WALK., Insecta Britann., Dipt., I 42. 1. tab. I. fig. 14. [Haematopota] (1851) et List Dipt. Brit. Mus., V. suppl. 1., 294. 1. [Haematopota] (1854); Zetr., Dipt. Scand., XII. 4549. 1. [Haematopota] (1855); Bonsd., Finland. tvaving. Ins., I. 111. 1. [Haematopota] (1861); Scuns., Fauna Austriaca, Dipt., I. 39. [Haematopota] (1862); Fedtsch., Schrift. Ges. Freund. d. Nat. Moskau, VI. 61. 1. [Haematopota] (1868); Brav., Verh. zool.-bot. Ges. Wien, XIX. 921. tab. XIII. fig. 7-12. [Haematopota] (1869); Perris, Annal. Soc. Entom. France, sér. 4. X. 196. 12. tab. II. fig. 62-69. [Huematopota] (1870); Lw., Rocznik towarz. nauk. Krakow, P. 3. XIX. (XLII). 173. 7. [Haematopota] (1871); Pontsch., Horae Soc. Entom. Ross., VIII. Bullet., XII. [Haematopota] (1872); Belixg, Archiv f. Naturgesch., XLI. 1., 37. 4. [Hacmatopota] 
(1875) ; Goв., Mém. Soc. Linn. Nord France, 1881. 33 et 39. 5. [Haematopota] (1881); Becher, Denkschr. Akad. Wien, XLV. 141. tab. II. fig. 17. [Haematopota] (1882) ; Pand., Revue d'Entomol., II. 195. 2. [Haematopota] (1883); Kow., Wien. Entom. Zeitg., II. 110. nota. [Haematopota] (1883); Mik in Becker: Fauna von Hernstein, II. 2., 53. [Haematopota] (1885) ; Neuraus, Diptera Marchica, 45. 1. [Haematopota] (1886); Big., Bull. Soc. Zool. France, XVI. 76. [Haematopota] (1891); É. \& L. Coucke, Annal. Soc. Entom. Belgique, XXXVI. 5. [Haematopota] (1892); Strobl, Mittheil. Naturwiss. Ver. Steiermark, XXIX. 1892. 18. [Haematopota] (1893); Leonard, Insetti nocivi, III. 375. fig. 201. A. B., 205, 206. [Haematopota] (1900); Wesché, Trans. Linn. Soc. London, Zool., ser. 2. IX. pt. 4. 219-230. tab. VIII. fig. 1. [Haematopota] (1904).

equorum FABR., Entomol. System., IV. 370. 35. [Tabanus] (1794); Meig., Klassif., I. 163. a. [Haematopota] (1804); FABR., Systema Antliat., 109. 8. [Haematopota] (1805); MeIG., System. Beschreib., II. 80. 2. [Haematopota] (1820); Big., Bull. Soc. Zool. France, XVI. 78. [Haematopota] (1891).

hyentomantis ScHin., Fauna Austriaca, Dipt., I. 39. [Haematopota] (1862).

hyetomantis Schrank, Fauna Boica, IJI. 155. 2536. [Tabanus] (1803).

marginula MEg. in litt. ap. MeIG.

ocellata Meg. in litt. ap. Meig.

tristis Bia., Bull. Soc. Zool. France, XVI. 77. [Haematopota] (1891) et Mém. Soc. Zool. France, V. 628. [Haematopota] (1892).

var. Bigoti Goв., Mém. Soc. Linn. Nord France, 1881. 38. 3. [Haematopota] (1881) ; PAND., Revue d'Entomol., II. 196. [Haematopota] (1883); Villen., Feuille joun. Natural., XXXV. 59. 1. [Hacmatopota] (1905).

var. subcylindrica PAND., Revue d'Entomol., II. 196. [Haematopota] (1883).

punctifera Big., Bull. Soc. Zool. France, XVI. 79. [Haematopota] (1891) et Mém. Soc. Zool. France, V. 629. [Haematopota] (1892); v. D. Wulp, Catal. Dipt. South Asia, 67. [Haematopota] (1896).

punctulata MACQ., Dipt. exot., I. 1., 163. 2. [Hacmatopota] (1838); Walk., List Dipt. Brit. Mus., V. suppl. 1., 295. 2. [Hacmatopota] (1854); Bit., Bull. Soc. Zool. France, XVI. 77. [Haematopota] (1891); Aldr., Catal. North Amer. Dipt., 199. [Haematopota] (1905).

pungens DoL. $=$ ? irrorata MACQ.

recurrens Lw., Öfvers. Kongl. Vet. Acad. Handl., XIV. 1857. 341. Port Natal. 31. [Haematopota] (1858) et Dipt.-Fauna Südafrika's, I. 50. 4. tab. I. fig. 26. [Haematopota] (1860); Bia., Bullet. Soc. Zool. France, XVI. 76. [Hacmatopota] (1891). 
roralis FABr., Systema Antliat., 107. 2. [Haematopota] (1805); WiEd., Dipt. exot., 97. 1. [Haematopota] (1821) et Ausserenrop. zweifl. Ins., I. 215. 3. [Haematopota] (1828) ; WALK., List Dipt. Brit. Mus., I. 208. [Haematopota] (1848) et V. suppl. 1., 297. 12. [Haematopota] (1854) ; Big., Bullet. Soc. Zool. France, XVI. 78. [Hacmatopota] (1891) ; v. D. Wulp, Catal. Dipt. South Asia, 66. [Hacmatopota] (1896).

ruficornis MAcQ., Dipt. exot., suppl. 1., 45. 3. [Haematopota] (1845); Walk., List Dipt. Brit. Mus., V. suppl. 1., 296. 7. [Haematopota] (1854) ; Bıg., Bull. Soc. Zool. France, XVI. 75. [Haematopota] (1891). ruficornis WALK., Inseeta Saunders., Dipt., I. 453. [Haematopota] (1856).

rufipennis Big., Bullet. Soc. Zool. France, XVI. 77. [Hacmatopota] (1891) et Mém. Soc. Zool. France, V. 628. [Hacmatopota] (1892). scutellaris Lw., Öfvers. Kongl. Vet. Akad. Förhandl., XIV. 1857. 341. 30. [Haematopota] (1858) et Dipt.-Fauna Südafrika's, I. 49. 3. [Haematopota] (1860); BiG., Bullet. Soc. Zool. France, XVI. 77. [Hacmatopota] (1891).

serpentina Wied., Aussereurop. zweifl. Ins., I. 219. 8. [Haematopota] (1828); Walk., List Dipt. Brit. Mus., V. suppl. 1., 298. 17. [Haematopota] (1854); Big., Bullet. Soc. Zool. France, XVI. 79. [Haematopota] (1891).

strigipennis Karsch, Entomol. Nachricht., XV. 240. [Haematopota] (1889) ; Bıg., Bullet. Soc. Zool. France, XVI. 79. [Hacmatopota] (1891).

subcylindrica PAND. = pluvialis L. var.

tenuicornis MACQ. = italica MeIG.

tristis $\mathrm{BIG} .=$ pluvialis $\mathrm{L}$.

variegata FABr., Systema Antliat., 109. 6. [Haematopota] (1805); MeIG., System. Beschreib., II. 81. 3. [Haematopota] (1820); MAcq., Suit. à Buffon, I. 211. 6. [Haematopota] (1834); WALk., List Dipt. Brit. Mus., I. 206. [Haematopota] (1848) et V. suppl. 1., 295 et 296. 8. [Haematopota] (1854); Schin., Fauna Austriaca, Dipt., I. 38. [Haematopota] (1862); Gox., Mém. Soc. Linn. Nord France, 1881. 36. 1. [Haematopota] (1881); Pand., Revue d'Entomol., II. 195. 1. [Haematopota] (1883) ; Big., Bull. Soc. Zool. France, XVI. 79. [Haematopota] (1891).

vittata Lw., Öfvers. Kongl. Vet. Akad. Förhandl., XV. 336. 32. bis. [Haematopota] (1858) et Dipt.-Fauna Südafrika's, I. 50. 5. tab. I. fig. 28-30. [Haematopota] (1860); Big., Bullet. Soc. Zool. France, XVI. 76. [Haematopota] (1891).
Tranquebar, Borneo, Java.

A frica.

Port Natal.

Japonia.

Caffraria.

Patriaignota.

Gabon.

Europa mer., Africa sept.

Africa mer. 


\section{Dasybasis}

MacQ., Lipt. exot., suppl. 2., 25. (1846).

appendiculata MACQ., Dipt. exot., suppl. 2., 25. 1. tab. I. fig. 1. Australia. (1846) ; Walk., List Dipt. Brit. Mus., V. suppl. 1., 266. 1. (1854);

Ricardo, Annal. Mag. Nat. Hist., ser. 7. XIV. 350. (1904).

tristis Big., Mém. Soc. Zool. France, V. 621. (1892); Ricardo, Annal. Chile.

Mag. Nat. Hist., ser. 7. XIV. 350. (1904).

\section{Bolbodimyia}

Big., Wien. Entom. Zeitg., XI. 62. (1892).

bicolor BıG., Wien. Entom. Zeitg., XI. 62. (1892); Röp., Wien. Venezuela.

Entom. Zeitg., XI. 237. (1892); Ricardo, Annal. Mag. Nat. Hist., ser. 7. XIV. 351. (1904).

\section{Lepidoselaga}

MacQ., Dipt. exot., I. 1., 153. (1838) [Lepiselaga].

Hadrus Perty, Delect. Anim. Articul. Brasil., 183. (1833) [nec Des., Col. 1833].

albitarsis MACQ., Dipt. exot., suppl. 4., 36. 1. [Lepiselaga] (1850); v. D. Wul.P, Tijdschr. v. Entom., XXIV. 161. 14. (1881); E. L. Arris., Bol. Acad. Nat. Cienc. Córdoba, IV. 131. 58. [Hadrus] (1882); Hunter, Trans. Amer. Entom. Soc. Philad., XXVII. 136. [Hadrus] (1901) ; Willist., Biolog. Central. Americ., Dipt., I. 262. (1901) ; Ricardo, Annal. Mag. Nat. Hist., ser. 7. XIV. 352. (1904). parva Willist., Kansas Univ. Quart., III. 192. [Hadrus] (1895);

Hunter, Trans. Amer. Entom. Soc. Philad., XXVII. 137.

[Hadrus] (1901); Willist., Biolog. Central. Americ., Dipt., I. 262. [Hadrus] (1901).

crassipes Fabr., Systema Antliat., 108. 4. [Haematopota] (1805); Wied., Dipt. exot., 97. 2. [Haematopota] (1821) et Aussereurop.

- zweifl. Ins., I. 220. 10. [Haematopota] (1828); WaLK., List Dipt. Brit. Mus., V. suppl. 1., 270. 8. [? Diabasis] (1854) ; E. L. Arrib., Bol. Acad. Nat. Cienc. Córdoba, IV. 131. 59. [Hadrus] (1882); Huntrer, Trans. Amer. Entom. Soc. Philad., XXVII. 137. [Hadrus] (1901).

lepidota Wied., Aussereurop. zweifl. Ins., I. 193. 123. [Tabanus] (1828) ; Perty, Delect. Anim. Artic. Brasil., 183. tab. XXXVI. fig. 9. [Hadrus] (1833); MACQ., Dipt. exot., I. 1., 154. 1. tab. XVIII. fig. 3. (1838); WaLK., List Dipt. Brit. Mus., I. 209. [Hadrus] (1848) et V. suppl. 1., 272. 1. [Hadrus] (1854); Ber.t., Saggio di Ditterol. Messic., I. 75. 1. [Hadrus] (1859); Schin., Novara Reise, Dipt., 96. 39. (1868); Lw., Berlin. En-

Argentinia, Paraguay.

Americamer. ot centr., Antillae. 
tom. Zeitschr., XIII. 6. nota. (1869); Ost.-SAck., Mem. Boston Soc. Nat. Hist., II. 475. (1876); Wrlusst., Kansas Univ. Quart., III. 192. [Hadrus] (1895); Towss., Annal. Mag. Nat. Hist., ser. 6. XIX. 19. 4. [Hadrus] (1897); Willist., Biolog. Central. Americ., Dipt., I. 262. 1. (1901); Ricardo, Annal. Mag. Nat. Hist., ser. 7. XIV. 331 et 352. (1904); Aldr., Catal. North Amer. Dipt., 199. (1905).

recta Lw., Berlin. Entom. Zeitschr., XIII. 6. (1869); Ost.-SAck.,

Biolog. Central. Americ., Dipt., I. 57. 1. (1886); Hunter, Trans. Amer. Entom. Soc. Philad., XXVII. 137. [Hadrus] (1901); Ricardo, Annal. Mag. Nat. Hist., ser. 7. XIV. 352 et 353. (1904).

lepidota WiED. = crassipes FA

parva WILLIST. = albitarsis M MCQ.

rect $\iota \mathrm{LW} .=$ crassipes $\mathrm{FABR}$.

\section{Selasoma}

Macq., Dipt. exot., I. 2., 187. (1839).

chalybeum PERTY = tibiale FАBR.

cyancum WALK. = tibiale FABR.

cyaneum Wied., Aussereurop. zwoifl. Ins., I. 152. 65. [Tabanus] (1828) ; MAcQ., Dipt. exot., I. 2., 188. (1838); WАLK., List Dipt. Brit. Mus., V. suppl. 1., 273. 2. (1854); Ricardo, Annal. Mag. Nat. Hist., ser. 7. XIV. 354. (1904).

tibiale FABr., Systema Antliat., 102. 39. [Tabamus] (1805); WIED., Dipt. exot., I. 89. 47. [Tabanus] (1821) et Aussereurop. zweifl. Ins., I. 164. 82. [Tabanus] (1828); Macq., Dipt. exot., I. 2., 188. (1839) ; WaLk., List Dipt. Brit. Mus., V. suppl. 1., 273. 1. (1854); Röper, Wien. Entom. Zeitg., XI. 237. (1892); Willist., Kansas Univ. Quart., IIJ. 192. (1895); Hunter, Trans. Amer. Entom. Soc. Philad., XXVII. 139. (1901); Ricardo, Annal. Mag. Nat. Hist., ser. 7. XIV. 354. (1904).

chalybeum Perty, Delect. Animal. Articul. Brasil., 183. tab. XXXVI. fig. 10. [Hadrus] (1.830).

cyaneum WaLK., List Dipt. Brit. Mus., I. 208. [Haclrus] (1848).

\section{Udenocera}

Ricardo, Annal. Mag. Nat. Hist., ser. 7. XIV. 354. (1904).

brunnea Ricardo, Annal. Mag. Nat. Hist., ser. 7. XIV. 355. (1901). Ceylon. 


\section{Diachlorus}

Ost.-SACK., Mom. Boston Soc. Nat. Hist., II. 475. (1876).

Diabasis MacQ., Suit. à Buffon, I. 207. (1834) [nec HoFrm. Col., 1819).

americanus PAL. BeAUV. = ferrugatus FABR.

approximans WALK. = ferrugatus FABR.

ataenia $\mathrm{MACQ} .=$ ferrugatus $\mathrm{F}_{\mathrm{ABR}}$.

bicinctus Fabr., Systema Antliat., 102. 42. [Tabanus] (1805); W'ied.,

Brasilia,

Dipt. exot., 105. 6. [Chrysops] (1821) et Aussoreurop. zweifl.

Ins., I. 191. 120. [Tabanus] (1828); MAcQ., Suit. à Buffon, I. 207.

1. [Diabasis] (1834); Walk., List Dipt. Brit. Mus., V. suppl. 1., 270. 4. [Diabasis] (1854); IIunter, Trans. Amer. Entom. Soc. Philad., XXVII. 139. [Tabamus] (1901); Ricardo, Annal. Mag.

Nat. Hist., ser. 7. XIV. 358. (1904).

bivittatus W1ED., Aussereurop. zweifl. Ins., I. 193. 122. [Tabanus] (1828); Macq., Suit. à Buffon, I. 212. 8. [Hacmatopota] (1834); WaLK., List Dipt. Brit. Mus., V. suppl. 1., 270. 5. [Diabasis] (1854); Ricardo, Annal. Mag. Nat. Hist., ser. 7. XIV. 358 et 360. (1904).

inornatus WALK., List Dipt. Brit. Mus., I. 199. [Chrysops] (1848) ; Ricardo, Annal. Mag. Nat. Hist., ser. 7. VIII. 309. [Chrysops] (1901); A ldr., Catal. North Amer. Dipt., 196. [Chrysops] (1905). convergens WALK. = ferrugatus FABR.

curvipes Fabr., Systema Antliat., 107. 3. [Hacmatopota] (1805); Wied., Dipt. exot., 90. 49. [Tabanus] (1821) et Aussoreurop. zweifl. Ins., I. 176. 98. [Tabanus] (1828) ; Macq., Suit. à Buffon, I. 208. 3. [Diabasis] (1834); Walk., List Dipt. Brit. Mus., V. suppl. 1., 271. 12. [Diabasis] (1854); Ẉ̣LL1st., Kansas Univ. Quart., III. 193. (1895); Hunter, Trans. Amer. Entom. Soc. Philad., XXVII. 138. (1901); Ricardo, Annal. Mag. Nat. Hist., ser. 7. XIV. 358 et 359 . (1904).

varipes WaLk., List Dipt. Brit. Mus., V. suppl. 1., 289. 53.

[Chrysops] (1854); Ricardo, Annal. Mag. Nat. Hist., ser. 7.

VIII. 309. [Chrysops] (1901).

diversipes MACq., Dipt. exot., supp'. 3., 13. 2. tab. I. fig. 5. [Diabasis] (1847) ; WALK., List Dipt. Brit. Mus., V. suppl. 1., 270. 7. [Diabasis] (1854); v. D. Wulp, Notes Leyden Mus., VII. 81. 35. (1885) ; Hunter, Trans. Amer. Entom. Soc. Philad., XXVII. 138. (1901) ; Ricardo, Annal. Mag. Nat. Hist., ser. 7. XIV. 358. (1904).

ferrugatus FABR., Systema Antliat., 111.2. [Chrysops] (1805); WiED., America centr.

Dipt. exot., 94. 56. [Tabanus] (1821) et Aussereurop. zweifl. Ins., I. 186. 113. [Tabanus] (1828) ; WaLK., List Dipt. Brit. Mus., et sept., India oce.

I. 191. [Dichelacera] (1848) et V. suppl. 1., 148. 1: [Dichelacera] (185!) ; Ost.-S.Ack., Mem. Boston Soc. Nat. Hist., II. 396 et 475. [Diabasis] (1876) et Biolog. Central. Americ., Dipt., I. 57.1. (1886); 
Hunter, Trans. Amer. Entom. Soc. Philad., XXVII. 138. (1901); Willist., Biolog. Central. Americ., Dipt., I. 263. (1901) ; Ricardo, Annal. Mag. Nat. Hist., ser. 7. XIV. 358 et 359. (1904); Banks, Entomol. News Philad., XV. 290. (1904); Aldr., Catal. North Amer. Dipt., 199. (1905).

americanus PaL.Beauv., Insect. recueill. en Afrique et en Amérique, 223. tab. III. fig. 6. [Tabanus] (1805-21).

approximans WaLK., List Dipt. Brit. Mus., I. 198. [Chrysops] (1848).

ataenia MACQ., Dipt. exot., I. 1., 152. 3. [Diabasis] (1838); Walk., List Dipt. Brit. Mus., V. suppl. 1., 271. 11. [Diabasis] (1854).

convergens WaLk., List Dipt. Brit. Mus., I. 198. [Chrysops] (1848).

Rondanii Bell., Saggio di Ditterol. Messic., I. 68. 22. tab. II. fig. 11. [Tabanus] (1859).

flavipennis MACQ., Dipt. exot., I. suppl. 4., 35. 8. [Diabasis] (1850); v. D. Wulp, Catal. Dipt. South Asia, 64. (1896); Ricardo, Annal. Mag. Nat. Hist., ser. 7. XIV. 359. (1904).

fuscipennis MacQ., Dipt. exot., I. 1., 152. 2. [Diabasis] (1838); WALK., List Dipt. Brit. Mus., V. suppl. 1., 271. 13. [Diabasis] (1854); Hunter, Trans. Amer. Entom. Soc. Philad., XXVII. 138. (1901); Ricando, Annal. Mag. Nat. Hist., ser. 7. XIV. 358. (1904).

glaber Wied., Aussereurop. zweifl. Ins., I. 192. 121. [Tabanus] (1828); Macq., Suit. à Buffon, I. 208. 2. [Diabasis] (1834); WALk., List Dipt. Brit. Mus., V. suppl. 1., 270. 6. [Diabasis] (1854) ; Hunter, Trans. Amer. Entom. Soc. Philad., XXVII. 141. [Tabanus] (1901); Ricardo, Annal. Mag. Nat. Hist., ser. 7. XIV. 358. (1904).

globicornis Wied., Dipt. exot., 96. 59. [Tabanus] (1821) et Aussereurop. zweifl. Ins., I. 190. 118. [Tabanus] (1828); MAcQ., Suit. à Buffon, I. 208. 4. [Diabasis] (1834) ; WaLK., List Dipt. Brit. Mus., V. suppl. 1., 271. 9. [Diabasis] (1854); Hunter, Trans. Amer. Entom. Soc. Philad., XXVII. 141. [Tabanus] (1901); Ricardo, Annal. Mag. Nat. Hist., ser. 7. XIV. 658. (1904).

inornatus WALK. = bivittatus WIED.

interruptus MACQ., Dipt. exot., I. 1., 152.4. [Diabasis] (1838); WALK., List Dipt. Brit. Mus., V. suppl. 1., 271. 10. [Diabasis] (1854); Hunter, Trans. Amer. Entom. Soc. Philad., XXVII. 138. (1901); Ricardo, Annal. Mag. Nat. Hist., ser. 7. XIV. 358. (1904).

ochraceus MACQ., Dipt. exot., suppl. 4., 36. 4. [Diabasis] (1850); E. L. Arrib., Bolet. Acad. Nat. Cienc. Córdoba, IV. 133. 64. (1882); Hunter, Trans. Amer. Entom. Soc. Philad., XXVII. 138. (1901) ; Ricardo, Annal. Mag. Nat. Hist., ser. 7. XIV. 359. (1904). podagricus FABr., Systema Antliat., 108. 5. [Hacmatopota] (1805); Wied., Dipt. exot., 98. 3. [Haematopota] (1821) et Aussereurop. zweifl. Ins., I. 219. 9. [Hacmatopota] (1828); MAcQ., Suit. à Buffon, I. 212. 9. [Haematopotı] (1834); WaIK., List Dipt. Brit. Mus.,

I.Philippinae.

Brasilia.

Brasilia.

Brasilia.

Brasilia.

Brasilia.

Brasilia. 
V. suppl. 1., 269. 3. [? Diabasis] (1854); Huxter, Trans. Amer. Entom. Soc. Philad., XXVII. 137. [Hacmatopota] (1901); Ricardo, Annal. Mag. Nat. Hist., ser. 7. XIV. 358. (1904).

Rondanii BELL. = ferrugatus FABR.

scutellatus MAcq., Dipt. exot., I. 1., 151. 1. tab. XVIII. fig. 2. [Dicbasis] (1838); WaLk., List Dipt. Brit. Mus., V. suppl. 1., 269. 2. [Diabasis] (1854); Hunter, Trans. Amer. Entom. Soc.'Philad., XXVII. 138. (1901); Ricardo, Annal. Mag. Nat. Hist., ser. 7. XIV. 358 et 360 . (1904).

varipes Rond. in Truqur: Studi Entomol., I. 105. 78. [Diabasis] (1848) ; Huntel, Trans. Amer. Entom. Soc. Philad., XXVII. 138. (1901); Ricardo, Annal. Mag. Nat. Hist., ser. 7. XIV. 359. (1904). varipes WALK. $_{\text {ALvipes }} \mathrm{FABR}_{\mathrm{A}}$

\section{Stibasoma}

Schis., Verh. zool.-bot. Ges. Wien, XVII. 310. (1867).

bicolor Bı̈., Mém. Soc. Zool. France, V. 636. (1892); Rıcardo, Annal. Brasilia.

Mag. Nat. Hist., sor. 7. XIV. 361 et 362. (1904).

compactum WALK. = fulvohirtum WiED.

dives Walk., List Dipt. Brit. Mus., I. 166. [Tabanus] (1848); Ricardo, Annal. Mag. Nat. Hist., ser. 7. XIV. 361. (1904).

fulvohirtum Wied., Aussereurop. zweifl. Ins., I. 155. 70. [Tabanus] (1828); Walk., List Dipt. Brit. Mus., V. suppl. 1., 150. 13. [Tabanus] (1854) ; Schın., Novara Reise, Dipt., 94. 35. (1868); Ost.SAck., Biolog. Central. Americ., Dipt., 1. 57. 1. (1886); Hunter, Trans. Amer. Entom. Soc. Philad., XXVII. 137. (1901); Ricardo, Annal. Mag. Nat. Hist., ser. 7. XIV. 360 et 361. (1901); Aldr., Catal. North Amer. Dipt., 199. (1905).

compactum Walk., List Dipt. Brit. Mus., V. suppl. 1., 222. 293. [Tabanus] (1854).

pachycephalum Big., Mém. Soc. Zool. France, V. 636. (1892); Ricardo, Annal. Mag. Nat. Hist., ser. 7. XIV. 361. (1904); Aldr., Catal. North Amer. Dipt., 199. (1905).

theotaenia Wied., Aussereurop. zweifl. Ins., I. 136. 38. [Tabanus] (1828); Walk., List Dipt. Brit. Mus., V. suppl. 1., 200. 194. [Tabanus] (1854); Scins., Novara Reise, Dipt., 94. 34. tab. II. fig. 6. (1868) ; E. L. Arrib., Bolet. Acad. Nat. Cienc. Córdoba, IV. 133. 65. (1882); WirList., Kansas Univ. Quart., III. 194. (1895); Hunter, Trans. Amer. Entom. Soc. Philad., XXVII. 137. (1901); Ricardo, Annal. Mag. Nat. Hist., ser. 7. XIV. 360 et 361. (1904).

triste Wied., Aussereurop. zweifl. Ins., I. 164. 81. [Tabanus] (1828); Walk., List Dipt. Brit. Mus., I. 200. 195. [Tabanus] (1854) ; Scmrx., Novara Reise, Dipt., 94. (1868); Hunter, Trans. Amer. Entom. Soc. Philad., XXVII. 144. [Tabanus] (1901); RICARdo, Annal, Mag. Nat. Hist., ser. 7. XIV. 360. (1904).

Brit. Guiana, Cayenne.

Brasilia.

Amazon.

America mer. et centr.

Mexico.

Brasilia.

Brasilia. 


\section{Acanthocera}

MAce., Suit. à Buffon, I. 209. (1834).

coarctata WIED., Aussereurop. zweifl. Ins., I. 578. 9. [Hacmatopota] (1828) ; WaLK., List Dipt. Brit. Mus., V. suppl. 1., 295. 3. [Hacmatopota] (1854); Big., Bullet. Soc. Zool. France, XVI. 74. [Hacmatopota] (1891); Hunter, Trans. Amer. Entom. Soc. Philad., XXVII. 137. [Haematopota] (1901); Ricardo, Annal. Mag. Nat. Hist., ser. 7. XIV. 363. (1904).

exstincta Wies., Aussereurop. zwoifl. Ins., I. 214. 2. [Hacmatopota] (1828) ; WaLK., List Dipt. Brit. Mus., I. 295. 4. (1854); Lw., Dipt.-Fauna Südafrika's, I. 46. (1860) ; E. I. ArriB., Bolet. Acad. Nat. Cienc. Córdoba, IV. 132. 60. (1882) ; Hunter, Trans. Amer. Entom. Soc. Philad., XXVII. 138. (1901); Ricardo, Annal. Mag. Nat. Hist., ser. 7. XIV. 363. (1904).

longicornis Fabr., Entomol. System., IV. 371. 38: [Tabanus] (1794) et Systema Antliat., 103. 45. [Tabanus] (1805) ; Wied., Dipt. exot., 98. 4. [Haematopota] (1821) et Aussereurop. zweifl. Ins., 213. 1. [Hacmatopota] (1828); MACQ., Suit. à Buffon, I. 209. 1. (1834) et Dipt. exot., I. 1., 114. 2. tab. XVI. fig. 2. [Dichelacera], 158. [Acanthocera] (1838); Walk., List Dipt. Brit. Mus., V. suppl. 1., 150. 12. [Dichelacera] et 267. 1. (1854); Hunter, Trans. Amer. Entom. Soc. Philad., XXVII. 137. [Dichelacera] et 138. (1901); Ricardo, Annal. Mag. Nat. Hist., ser. 7. XIV. 363 et 364. (1904).

triangularis WIED., Zoolog. Magaz., I. 3., 44. 6. [Hacmatopota] (1849).

marginalis WaLk., List Dipt. Brit. Mus., V. suppl. 1., 268. 2. (1854);

Brasilia.

Ricardo, Annal. Mag. Nat. Hist., ser. 7. XIV. 363. (1904).

triangularis WIED. = longicornis FABR.

trigonifera Schis., Novara Reise, Dipt., 95. 38. (1868); Hunter, Trans. Amer. Entom. Soc. Philad., XXVII. 138. (1901); Ricardo, Annal. Mag. Nat. Hist., ser. 7. XIV. 363 et 364. (1904).

\section{Dichelacera}

MACQ., Dipt. exot., I. 1., 112. (1838).

alcicornis WIED., Aussereurop. zweifl. Ins., I. 158. 74. [Tabanus] Brasilia. (1828) ; Walk., List Dipt. Brit. Mus., I. 190. (1848) et V. suppl. 1., 154. 27. (1854); Hunter, Trans. Amer. Entom. Soc. Philad., XXVII. 139. [Tabanus] (1901); Ricardo, Annal. Mag. Nat. Hist., ser. 7. XIV. 366. (1904).

?tetrapuncta Thunb., Nova Acta Upsal., IX. 57. tab. I. fig. 2. (1827).

bifacies WaLK., List Dipt. Brit. Mus., I. 191. (1848); Ricardo, Annal. Brasilia. Mag. Nat. Hist., ser. 7. XIV. 367 et 370, (1904).

brasiliensis RoND. = ? rufa MACQ. 
Capreolus Wied., Aussereurop. zweifl. Ins., I. 162. 78. [Tabanus] (1828); Walk., List Dipt. Brit. Mus., V. suppl. 1., 152. 18. (1854); Hunter, Trans. Amer. Entom. Soc. Philad., XXVII. 140. [Tabanus] (1901) ; Ricardo, Annal. Mag. Nat. Hist., ser. 7. XIV. 367. (1904). cervicornis FABr., Systema Antliat., 100. 35. [Tabanus] (1805); WiED., Dipt. exot., 79. 32. [Tabanus] (1821) et Aussereurop. zweifl. Ins., I. 157. 73. [Tabanus] (1828); Macq., Suit. à Buffon, I. 197. 1. [Tabanus] (1834); WaLK., List Dipt. Brit. Mus., V. suppl. 1., 153. 24. (1854); Schin., Novara Reise, Dipt., 94. 36. (1858) ; Ost.-SAck., Biolog. Central. Americ., Dipt., I. 58. 1. (1886); Hunter, Trans. Amer. Entom. Soc. Philad., XXVII. 137. (1901); Willist., Biolog. Central. Americ., Dipt., I. 263. (1901); Ricardo, Amnal. Mag. Nat. Hist., ser. 7. XIV. 366 et 369. (1904) ; ALdr., Catal. North Amer. Dipt., 199. (1905).

fasciata WaLk., Insecta Saunders., Dipt., I. 68. (1850) ; Ost.SAck., Catal. Dipt. North America, Ed. II., 55. observ. (1878); Aldr., Catal. North Amer. Dipt., 200. (1905).

multifascia WaLk., Insecta Saunders., Dipt., I. 68. (1850).

multifasciata Ricardo, Annal. Mag. Nat. Hist., ser. 7. XIV. 366. (1904) [lapsus].

damicornis FABR., Systema Antliat., 101. 36. [Tabamus] (1805); Wred., Dipt. exot., 87. 45. [Tabanus] (1821) et Aussereurop. zweifl. Ins., I. 159. 75. [Tabanus] (1828); MAcQ., Suit. à Buffon, I. 197. 2. [Tabanus] (1834) et Dipt. exot., I. 1., 112. (1838); Walk., List Dipt. Brit. Mus., V. suppl. 1., 151. 15. (1854); HuNTer, Trans. Amer. Entom. Soc. Philad., XXVII. 140. [Tabanus] (1901); Ricardo, Annal. Mag. Nat. Hist., ser. 7. XIV. 366 et 369. (1904).

fusciata $\mathrm{W}_{\Lambda \mathrm{LK}}$ = = ervicornis FABR.

flava Wied., Aussereurop. zweifl. Ins., I. 163. 80. [Tabanus] (1828); WALK., List Dipt. Brit. Mus., V. suppl. 1., 153. 25. (1854); E. L. A rris., Bolet. Acad. Nat. Cienc. Córdoba, IV. 132. 61. (1882); Hunter, Trans. Amer. Entom. Soc. Philad., XXVII. 137. (1901); Ricardo, Annal. Mag. Nat. Hist., ser. 7. XIV. 367. [?Dichelacera] (1904).

fuscipennis MacQ., Dipt. exot., suppl. 2., 14. 7. (1847); WALK., List Dipt. Brit. Mus., V. suppl. 1., 151. 14. (1854); Hunter, Trans. Amer. Entom. Soc. Philad., XXVII. 137. (1901); Ricardo, Annal. Mag. Nat. Hist., ser. 7. XIV. 367. [? Dichelacera] (1904).

grandis Ricardo, Annal. Mag. Nat. Hist., ser. 7. XIV. 369 et 371 . Honduras. (1904).

himulus WALK. = marginata MACQ.

immaculata MACQ., Dipt. exot., I. 1., 115. 4. (1838); WALK., List Brasilia. Dipt Brit. Mus., V. suppl. 1., 152. 17. (1854); Huster, Trans. Amer. Entom. Soc. Philad., XXVII. 137. (1901): RicARdo, Annal Mag. Nat. Hist., ser. 7. XIV. 367. (1904).

Januarii Wied., Zoolog. Magaz., I, 3., 43. 4. [Tabanus] (1819), Dipt. Brasilia.
Americamer. et centr.

America mer,

Urug'nay.

Brasilia. 
exot., 94. 55. [Tubanus] (1821) et Aussereurop. zweifl. Ins., I. 162. 79. [Tubanus] (1828); MACQ., Dipt. exot., suppl. 3., 10. (1847) ; Walk., List Dipt. Brit. Mus., V. suppl. 1., 153. 23. (1854); Scunx, Novara Reise, Dipł., 95. 37. (1868); Hunter, Trans. Amer. Entom. Soc. Philad., XXVII. 137. (1901) ; Ricardo, Annal. Mag. Nat. Hist., ser. 7. XIV. 366. (1904).

marginata MACQ., Dipt. exot., suppl. 2., 14. 8. (1847); WALK., List Dipt. Brit. Mus., V. suppl. 1., 152. 20. (1854); Hunter, Trans. Amer. Entom. Soc. Philad., XXVII. 137. (1901); Ricardo, Annal. Mag. Nat. Hist., ser. 7. XIV. 367 et 370. (1904).

hinnulus Walk., Newman Zoologist, VIII. Append., CXXII. (1850) et List Dipt. Brit. Mus., V. suppl. 1., 153. 22. (1854). .multifuscia WALK. = cervicornis FABR. multifasciata RICARDo = cervicornis FABR.

nubipennis Rond., Annuar. Soc. Nat. Modena, III. 39. 37. (1868);

E. L. Arrib., Bolet. Acad. Nat. Cienc. Córdoba, IV. 133. 62. (1882) ; Hunter, Trans. Amer. Entom. Soc. Philad., XXVII. 137. (1901) ; Ricardo, Annal. Mag. Nat. Hist., ser. 7. XIV. 367. [? Dichelacera] (1904).

pulchra Willist., Biolog. Central. Amer., Dipt., I. 263. 2. tab. IV. fig. 22, 22a. (1901); Ricardo, Annal. Mag. Nat. Hist., ser. 7. XIV. 367. (1904) ; Aldr., Catal North Amer. Dipt., 200. (1905).

rufa MACQ., Dipt. exot., suppl. 2., 13. 6. (1847); WALK., List Dipt. Brit. Mus., V, suppl. 1., 152. 21. (1854); Hunter, Trans. Amer. Entom. Soc. Philad., XXVII. 137. (1901).

? brasiliensis Rond., Nuov. Annal. Sci. Nat. Bologna, ser. 3. II. 192. 29. [Tabanus] (1850).

scapularis MacQ., Dipt. exot., suppl. 2., 15. 9. (1847); WALK., List Dipt. Brit. Mus., V. suppl. 1., 148. 3. (1854); Bell., Saggio di Ditterol. Messic., I. 53. 1. tab. II. fig. 12. (1859); Ost.-SAck., Biolog. Central. Americ., Dipt., I. 59. (1886); Ricardo, Annal. Mag. Nat. Hist., ser. 7. XIV. 367. (1904); Aldr., Catal. North Amer. Dipt., 200. (1905).

? scutellata Willist., Kansas Univ. Quart., III. 193. [? Diachlorus] Brasilia.

(1895) ; Hunter, Trans. Amer. Entom. Soc. Philad., XXVII. 138. (1901); Ricardo, Annal. Mag. Nat. Hist., ser. 7. XIV. 357 et 367. [? Dichelacera] (1904).

? testacea MACQ., Dipt. exot., suppl. 1., 29. 5. tab. III. fig. 10. America mer. et centr.

Argentinia.

Mexico.

Brasilia.

Mexico. (1845) ; Walk., List Dipt. Brit. Mus., V. suppl. 1., 153. 26. (1854); Hunter, Trans. Amer. Entom. Soc. Philad., XXVII. 138. (1901); Ric^rdo, Annal. Mag. Nat. Hist., ser. 7. XIV. 367. [?Dichelacera] (1904).

tetrapuncta THUNB. = alcicornis Wien.

T nigrum FABR., Systema Antliat., 101. 38. [Tabanus] (1805); W'IED., Brasilia.

Aussereurop. zweifl. Ins., I. 160. 76. [Tabanus] (1828); MACQ., Dipt. exot., I. 1., 112. (1838) ; Walk., List Dipt. Brit. Mus., V. suppl. 1., 152. 19 et 325 var? (1854); Willist., Kansas Univ. 
Quart., III. 195. [Tubumus] (1895); Huster, Trans. Amer. Entom.

Soe. Philad., XXVII. 142. [T'tubnus nigmem] et 14t. [Tubroms] (1901) ; Ricando, Amnal. Mag. Nat. Hist., ser. T. XIV. i66 et 370. (1904).

unifasciata MAcQ., Dipt. exot., I. 1., 115. 3. (1838) ; WalK., List

Brasilia.

Dipt. Brit. Mus., V. suppl. 1., 154. 28. (1854) ; E. L. Arum.,

Bolet. Acad. Nat. Cienc. Córdoba, IV. 133. 63. (1882); Huxtur,

Trans. Amer. Entom. Soc. Philad., XXVII. 138. (1901); Ricarno,

Annal. Mag. Nat. Hist., ser. 7. XIV. 367. (1904).

\section{Tabanus}

L., Fauna Suec., 462. (1761).

Agelanius Rond., Archivio per la Zool. Modena, III. 79 (1863).

Atylotus Ost.-Sack., Mem. Boston Soc. Nat. Hist., II. 426. (1876).

Bellarlia Rond., Archivio per la Zool. Modena, III. 81. (1863).

Brachytomus A. Costa, Il Giamb. Vico Napoli, II. 445. (1857).

Therioplectes ZeLL., Isis, 1842. XI. 819. (1842).

abazus Biø., Annal. Soc. Entom. France, sér. 5. X. 146. (1880). ?Persia,? Caucas. abbreviatus Bıg., Mém. Soc. Zool. France, V. 670. (1892); v. D. Wul.r, Jara.

Catal. Dipt. South Asia, 63. (1896).

abdominalis Fabr., Systema Antliat, 96. 15. (1805); ? PAL. BEAUv., Americasept.

Ins. recueil. en Afrique et en Amérique, 101. tab. II. fig. 4.

-(1805-1821); WaLK., List Dipt. Brit. Mus., I. 146. (1848) et V. suppl. 1., 186. 129. (1854); Ost.-Sack., Mem. Boston Soc. Nat. Hist., II. 434. 6 et 557. (1876) et Catal. Dipt. North America, Ed. II., 57 et 227. (1878); HART, Illinois State Laborat. of Nat. Hist., IV. 233. (1895) ; ALDR., Catal. North Amer. Dipt., 200. (1905). abdominalis WIED. (nec FABR.) = exul Ost.-SACK.

abiens WaLK., List Dipt. Brit. Mus., I. 191. [Dichelacera] (1848);

India occid.

Ost.-SAck., Catal. Dipt. North Amer., Ed. II., 55. [Dichelacera] (1878) ; Rrcardo, Annal. Mag. Nat. Hist., ser. 7. XIV. 367. (1904); Aldr., Catal. North Amer. Dipt., 199. [Dichelacera] (1905).

abscondens WALK., Trans. Entom. Soc. London, n. ser. V. 275. Birmania. (1860) ; v. D. Wutp, Catal. Dipt. South Asia, 270. (1896).

abstersus WALK., Insecta Saunders., Dipt., I. 58. (1850); Schin., Novara Reise, Dipt., 85. 14. (1868).

Actaeon Ost.-SAck., Mem. Boston Soc. Nat. Hist., II. 443. 15. (1876); ALDr., Catal. North Amer. Dipt., 200. (1905).

acuminatus Lw., Verh. zool.-bot. Ges. Wien, VIII. 590. 16. (1858); Schin., Fauna Austriaca, Dipt., I. 30. (1862); Braver, Denkschr. Akad. Wien, XLII. 157. 19. (1880); Pand., Revue d'Entom., II. 211. 30. (1883).

Australia.

America sept.

Europa centr. et mer., Sibiria. 
acupunctutus ROND. = bonariensis MACQ.

acutidens Pıil., Verl. zool.-bot. Ges. Wien, XV. 715. 13. (1865);

Hunter, Trans. Amor. Entom. Soc. Philad., XXVII. 139. (1901).

acutipalpis MacQ., I)ipt. exot., I. 1., 131. 20. (1838); WaLk., List Oceania.

Dipt. Brit. Mus., V. suppl. 1., 251. 433. (1854).

acutus Bıs., Mém. Soc. Zool. France, V. 660. [Atylotus] (1892); Aldr., America sept.

Catal. North Amer. Dipt., 200. (1905).

administrans Schin., Novara Reise, Dipt., 83. 9. (1868); v. D. Wulp, Hongkong.

Catal. Dipt. South Asia, 61. (1896).

adustus WALK., Insecta Saunders., Dipt., I. 34. (1850).

advena WALK., Newman Zoologist., VIII. Append., LX1X. (1850) Patriaignota.

et List Dipt. Brit. Mus., V. suppl. 1., 263. 493. (1854).

aegrotus Ost.-SAck., Bullet. Unit. Stat. Geolog. Surv., III. 219. 6.

(1878); Willist., Trans. Kansas Acad. Sci., X. 139. (1887);

Towrs., Proc. Calif. Acad. Sci., ser. 2. IV. 597. 13. (1895); Hrse,

Ohio Naturalist, V. 234. (1904); Beutenm., Bull. Amer. Mus. Nat.

Hist., XX. 88. (1904); Aldr., Catal. North Amer. Dipt., 200. (1905).

aequetinctus Beck., Acta Soc. Sci. Fenn., XXVI. No. 9., 8. 8. Sibiria.

[Therioplectes] (1900).

aethereus BıG., Mém. Soc. Zool. France, V. 637. [Therioplectes] (1892). ?Europa.

acthiops LuUngu. = aterrimus MeIG.

affinis Kirby, Fauna Boreal. Americ., Ins. IV. 313. 1. (1837); WALK.,

List Dipt. Brit. Mus., I. 156. (1848); Ost.-SAck., Mem. Boston

Soc. Nat. Hist., II. 466. 48. (1876) ; Bethune, Canad. Entomol., XIII. 166. 440. (1881); WrLlist., Trans. Kansas Acad. Sci., X. 137. (1887); Hine, Ohio Naturalist, V. 234. (1904); Aldr., Catal. North Amer. Dipt., 200. (1905).

triligatus WALK., List Dipt. Brit. Mus., V. suppl. 1., 183. 121.

(1854) ; Ost.-SAck., Mem. Boston Soc. Nat. Hist., II. 474. (1876).

africanus GraY = latipes MACQ.

agrestis WleD., Aussereurop. zweifl. Ins., I. 557. 32. (1828); WALK.,

List Dipt. Brit. Mus., V. suppl. 1., 232. 338. (1854).

agricola Wied., Aussereurop. zweifl. Ins., I. 556. 29. (1828); WALK.,

List Dipt. Brit. Mus., V. suppl. 1., 234. 346. (1854); Scmin., Novara Reise, Dipt., 82. 6. (1868); v. D. Wulp, Catal. Dipt. South Asia, 59. (1896).

alazinus Bí., Mém. Soc. Zool. France, V. 647. [Atylotus] (1892).

albibarbis Wied., Analecta Entomol., 21. 6. (1824) et Aussereurop.

America sept.

zweifl. Ins., I. 125. 22. (1828); WaLא., List Dipt. Brit. Mus., V. suppl. 1., 216. 262. (1854); Hunter, Trans. Amer. Entom. Soc. Philad., XXVII. 139. (1901).

albicans MAcQ., Suit. à Buffon, I. 204. 24. (1834); WALK., List Dipt. Senegal.

Brit. Mus., V. suppl. 1., 235. 351. (1854).

albicans MACQ., Dipt. exot., I. 2., 183. (1839); WALK., List Dipt. Arabia.

Brit. Mus., V. suppl. 1., 234. 347. (1854). 
albicans M.ce., Dipt, exot., suppl. 1., 37. 77. (1845); WALK, List Columbia.

Dipt. Brit. Mus., V. suppl. 1., 215. 260. (1854); Hunter, Trans.

Amer. Entom. Soc. Philad., XXVII. 139. (1901).

albicollis Hunter = albidicollis MACQ.

albidicollis MAcQ., Dipt: exot., suppl. 4., 32. 103. (1850).

Guyana.

albicollis Hunter, Trans. Amer. Entom. Soc. Philad., XXVII. 139. (1901).

albidocinctus Big., Mém. Soc. Zool. France, V. 686. (1892).

Brasilia.

albifacies Lw., Neue Beitr., IV. 27. 8. (1856) ; Ascherson, Sitzgosber. d. Ges. naturf. Freunde Berlin, 1887. No.8., 181. (1887) et 1891. No.69. (1891) ; Ricdrdo, Annal. Mag. Nat. Hist., ser. 7. XVI. 200. (1905). albilateralis MACQ., Dipt. exot., I. 1., 129. 17. (1838); WALk., List Dipt. Brit. Mus., V. suppl. 1., 242. 388. (1854); v. D. Wulp, Catal. Dipt. South Asia, 59. (1896).

albilinea WaLK., List Dipt. Brit. Mus., I. 176. (1848).

Aegyptus.

albimedius WALK., Insecta Saunders., Dipt., 48. (1850); v. D. WulP, Catal. Dipt. South Asia, 60. (1896).

albipalpis Rond., Annuar. Soc. Nat. Modena, III. 36. 33. tab. IV. fig. 12. [Agelanius] (1868); E. L. Arrir., Bol. Acad. Nat. Ciene. Córdoba, IV. 133. 66. (1882); Hunter, Trans. Amer. Entom. Sor. Philad., XXVII. 139. (1901).

albipalpus WALK., Insecta Saunders., Dipt., I. 44. (1850).

Gambia.

albipectus Big., Annal. Soc. Entom. France, sér. 3. VII. 125. tab. Madagascar. IX. fig. 2. (1859).

albipes $\mathrm{F}_{\mathrm{ABR}}=$ = gigas HerBst.

albiscutellatus MACQ., Dipt. exot., suppl. 4., 34. 107. tab. II. fig. 9. Mexico. (1850) ; Aldr., Catal. North Amer. Dipt., 200. (1905).

albitibialis MacQ., Dipt. exot., I. 1., 125. 8. (1838); Walk., List Dipt. Madagascar. Brit. Mus., V. suppl. 1., 236. 354. (1854); Big., Annal. Soc. Entom. France, sér. 3. VII. 125. (1859).

albivittatus $\mathrm{MACQ}_{\mathrm{A}}=$ ? spectabilis $\mathrm{Lw}$.

alboater WaLk. in Newman: Zoologist, VIII. Append. LXVI. (1850) et List Dipt. Brit. Mus., V. suppl. 1., 198. 186. (1854).

albocostatus Big., Mém. Soc. Zool. France, V. 649. [Atylotus] India. (1892); v. D. Wulp, Catal. Dipt. South Asia, 63. (1896).

albohirtus WALK., Trans. Linn. Soc. London, XVII. 338. 18. (1837); E. L. Arris., Bolet. Acad. Nat. Cienc. Córdoba, IV. 133. 67. (1882) ; Honter, Trans. Amer. Entom. Soc. Philad., XXVII. 139. [anbohirtus] (1901).

albomaculatus WALK., List Dipt. Brit. Mus., V. suppl. 1., 207. 224. Brasilia. (1854).

albomaculatus ZETT. = lapponicus WAHLBG. var.

albonotatus BeLL., Saggio di Ditterol. Messic., I. 56. 3. tab. II. fig. 5. (1859); Osт.-Sack., Biolog. Centr. Americ., Dipt., I. 55. 11. Amorica mer. (1885) ; Hunter, Trans. Amer. Entom. Soc. Philad., XXVII. 139. et centr. (1901); Willist., Biolog. Centr. Americ., Dipt., I. 260. 11. (1901); Aldr., Catal. North Amer. Dipt., 200. (1905).

bipartitus Walk., List Dipt. Brit. Mus., I. 158. (1848). 
oculus Watk., List Dipt. Brit. Mus., I. 157. (1848). albonotatus Bıg., Mém. Soc. Zool. France, V. 670. [Atylotus] (1892). N. Caledonia. albopictus*) Big., Móm. Soc. Zool. France, V. 633. [Dichelacera] (1892); Rrcardo, Annal. Mag. Nat. Hist., ser. 7. XIV. 368. (1904).

alboscutatus Rond., Annal. Mus. Civ. Genova, VII. 456. (1875); Borneo.

v. D. Wulp, Catal. Dipt. South Asia, 62. (1896).

albovarius WALk., List Dipt. Brit. Mus:, V. suppl. 1., 206. 219. Amazon. (1854).

albulus WALK, Insecta Saunder's., Dipt., I. 46. (1850); v. D. WuLr', India or. Catal. Dipt. South Asia, 60. (1896).

alcis Willist., Trans. Entom. Soc. London, 1896. 302. 1. tab. X. fig. 77. (1896) ; Hunter, Trans. Amer. Entom. Soc. Philad., XXVII. 139. (1901); Aldr., Catal. North Amer. Dipt., 201. (1905).

alene Towss., Trans. Amer. Entom. Soc. Plilad., XXII. 59. 12. (1895); Aldr., Catal. North Amer. Dipt., 201. (1905).

alexandrinus WiLD., Aussereurop. zweifl. Ins., II. 624. 23. (1830); Walk., List Dipt. Brit. Mus., V. suppl. 1., 225 et 231. 330. (1854); Jaenn., Berlin. Entom. Zeitschr., X.91.(1866); Brauer, Denkschr. Akad. Wien, XLII. 161. 23. tab. II. fig. 23. (1880); Paxd., Revue d'Entom., II. 217. 41. (1883); Ricardo, Annal. Mag. Nat. Hist., ser. 7. XIV. 197. [Atylotus] (1905).

carbonatus Macq., Suit. à Buffon, I. 199. 8. (1834); Meig., System. Besclır., VII. 58. 44. (1838); Wa Mus., V. suppl. 1., 156 et 162. 24. (1854); A. Costa, Atti R. Accad. Napoli, ser. 2. V. 101. 4. (1893).

alfourensis Big., Mém. Soc. Zool. France, V. 672. [Atylotus] (1892); v. D. Wulp, Catal. Dipt. South Asia, 63. (1896).

algivicus Trune, = lunatus FABR.

algirus MACQ. = ? ater Rossi.

Allynii Martex, Canad. Entomol., XV. 110. (1883); Willist., Trans.

I. St. Vincent.

Brasilia.

Jamaica.

Europa,

Africa sept., Asia min.

Nova Guinea.

America sejt.

Kansas Acad. Sci., X. 136. (1887); ALdr., Catal. North Amer.

Dipt., 201. (1905).

alpinus Paxz. = fulvus Meig.

alpinus ZะTT. = nigricornis ZETT.

alteripennis WALK., Trans. Entom. Soc. London, n. ser. V. 274. Mexico.

(1860) ; Aldr., Catal. North Amer. Dipt., 201. (1905).

amabilis WaLK., List Dipt. Brit. Mus., I. 154. (1848).

Patria ignota.

amaenus Walk., List Dipt. Brit. Mus., I. 163. (1848); v. D. WulP, Hongkong.

Catal. Dipt. South Asia, 60. (1896).

cmericanus DRURY = atratus FABR.

americanus Forster, Nov. Spec. Ins., Cent. I. 100. 100. (1771); Americasept. Ost.-SAck., Mem. Boston Soc. Nat. Hist., II. 457. 36. (1876); Aldr., Catal. Nortlı Amer. Dipt., 201. (1905).

limbatus Pal. de Beauv., Ins. recueill. on Afrique et en Amérique, 54. tab. I. fig. 2. (1805-1821).

*) cf. marmoratus BIG. 
plumbeus Drury, Illustr. of Nat. Hist. Exot. Ins., I. 103. II. tab. XLIV. fig. 2. (1770); Panzer, Drury's Abbild. u. Beschr. exot. Ins., 170. tab. XIIT. fig. 2. (1785-88).

ruficomis FABR., Systema Entomol., 789. 8. (1775), Entomol. System., IV. 365. 14. (1794) et Systema Antliat., 96. 14. (1805); Wied., Dipt. exot., I. 62. 1. (1821) et Aussereurop. zweifl. Ins., I. 112. 1. (1828); WalK., List Dipt. Brit. Mus., I. 146. (1848) et V. suppl. 1., 181. 110. (1854).

anachoreta PHiL., Verh. zool.-bot. Ges. Wien, XV. 717. 20. (1865); Schrn., Novara Reise, Dipt., 84. 11. (1868); Hunter, Trans. Amer. Entom. Soc. Philad., XXVII. 139. (1901).

andamanicus Big., Mém. Soc. Zool. France, V. 668. [Atylotus] (1892) ; v. D. Wulp, Catal. Dipt. South Asia, 63. (1896).

andicola Phul., Verh. zool.-bot. Ges. Wien, XV. 716. 18. (1865); Hunter, Trans. Amer. Entom. Soc. Philad., XXVII. 139. (1901). angusticornis Lw., Wien. Entom. Monatschr., II. 104. 6. (1858). angustifrons MaCQ., Dipt. exot., suppl. 3., 12. 93. (1847); WALK., List Dipt. Brit. Mus., V. suppl. 1., 209. 231. (1854); Hunter, Trans. Amer. Entom. Soc. Philad., XXVII. 139. (1901).

angustifrons Towss., Trans. Amer. Entom. Soc. Philad., XXII. 59. 13. (1895) ; Aldr., Catal. North Amer. Dipt., 201. (1905).

angustus Big., Mém. Soc. Zool. France, V. 652. [Atylotus] (1892); v. D. Wulp, Catal. Dipt. South Asia, 63. (1896).

angustus Macq., Dipt. exot., I. 1., 136. 29. tab. XVII. fig.1. (1838); Walk., List Dipt. Brit. Mus., V. suppl. 1., 208. 226. (1854); E. L. Arrib., Bolet. Acad. Nat. Cienc. Córdoba, IV. 134. 68. (1882); Hunter, Trans. Amer. Entom. Soc. Philad., XXVII. 139. (1901). annamitus Bıg., Mém. Soc. Zool. France, V. 630. [Bellardia] (1892); v. D. Wulp, Catal. Dipt. South Asia, 62. (1896).

annulatus SAY, Journ. Acad. Nat. Sci. Philad., III. 32. 2. (1823) et Compl. Writt., II. 53. (1859) ; WiED., Aussereurop. zweifl. Ins., I. 185. 111. (1828); Walk., List Dipt. Brit. Mus., V. suppl. 1., 181. 109. (1854); Ost.-Sack., Mem. Boston Soc. Nat. Hist., II. 473 et 555. (1876); Willist., Trans. Kansas Acad. Sci., X. 142. (1887) ; Hine, Ohio Naturalist, V. 234. (1904); Aldr., Catal. North Amer. Dipt., 201. (1905).

annulicornis Phu., Verh. zool.-bot. Ges. Wien, XV. 721. 30. fig. (1865) ; Hunter, Trans. Amer. Entom. Soc. Philad., XXVII. 139. (1901).

antarcticus L., Systema Naturae, Ed. XII., II. 1001. 15. (1767); FABr., Entomol. System., IV. 369. 31.(1794); Wred., Aussereurop. zweifl. Ins., I, 577. 7. (1828); WalK., List Dipt. Brit. Mus., V. suppl. 1., 223. 294. (1854); Hunter, Trans. Amer. Entom. Soc. Philad., XXVII. 139. (1901).

antecedens WALk., List Dipt. Brit. Mus., I. 178. (1848).

Chile.

I. Andaman.

Chile.

Japonia.

Cayenne.

Jamaica.

India or.

Uruguay.

Cochinchina.

America sept.

Chile.

Americamer.

Australia.

antecedens WaLk., List Dipt. Brit. Mus., V. suppl. 1., 253. 440. (1854). Tasmania. anthophilus $\mathrm{I}_{4} \mathrm{w}$. = lunatus $\mathrm{F}_{\mathrm{ABR}}$. 
anthracinus Meig., System. Beschreib., II. 36. 7. (1820) ; ? WaLK., Insecta Britannica, Dipt., I. 37. 3. (1851) et List Dipt. Brit. Mus., V. suppl. 1., 157 et 171. 61. (1854); Brauer, Denksclir. Akad. Wien, XLIJ. 160. 22. tab. II. fig. 22. (1880); Pand., Revue d'Entomol., II. 216. 39. (1883).

atropos JAExw., Berlin. Entom. Zeitschr., X. 87. 33. (1866).

corsicamus Macq. ap. Pand., Revue d'Entomol., II. 216. (1883). obscurus Lw., Verh. zool.-bot. Ges. Wien, VIII. 612. 43. (1858). aphanopterus Wied., Aussereurop. zweifl. Ins., I. 148. 59. (1828) ; WALK., List Dipt. Brit. Mus., V. suppl. 1., 204. 213. (1854); HuNTER, Trans. Amer. Entom. Soc. Philad., XXVII. 139. (1901). apiarius $\mathrm{J}_{\mathrm{AENN}}=$ tomentosus $\mathrm{M} \Lambda \mathrm{CQ}$. apicalis MACQ. $=$ Bigoti BeLL.

apicalis Rosd., Annal. Mus. Civ. Genova, VII. 459. (1875); v. D. Wulp, Catal. Dipt. South Asia, 62. (1896).

apicalis WALK. = finalis WALK.

apicalis Wied., Aussereurop. zweifl. Ins., I. 142. 49. (1828); WALK., Jist Dipt. Brit. Mus., V. suppl. 1., 221. 286. (1854); Hunter, Trans. Amer. Entom. Soc. Philad., XXVII. 139. (1901).

apoecilus Scinn., Novara Reise, Dipt., 85. 13. (1868); Hunter, Trans. Amer. Entom. Soc. Philad., XXVII. 139. (1901).

apricus Meig., System. Beschreib., II. 37. 9. (1820); WaLK., List Dipt. Brit. Mus., V. suppl. 1., 156 et 169. 52. (1854); Brauer, Denkschr. Akad. Wien, XLII. 176. 38. tab. III. fig. 38. (1880); Becier, Denkschr. Akad. Wien, XLV. 141. tab. II. fig. 14-15. (1882) ; Beck., Berlin. Entom. Zeitschr., XXXI. 106. 27. (1887); Stroß, Mittheil. Naturwiss. Ver. Steiermark, XXIX. 1892. 15. (1893); Villen., Feuille jeun. Natural., XXXIV. 226. 5. (1904) et XXXV. 59. 5. (1905) et Annal. Soc. Entom. France, LXXIV. 306. 2 et 308. 14. (1905).

infuscutus Lw., Verh. zool.-bot. Ges. Wien, VIII. 608. 39. (1858) ; Schis., Fauna Austriaca, Dipt., I. 34. (1862); Gов., Mém. Soc. Linn. N. France, 1881. 22. 8. (1881); Mrk in BEck. : Fauna von Hernstein, II. 2., 54. (1885).

tropicus Meig., System. Beschreib., II. 57. 54. p. p. ㅇ․ (1820). arabicus Mavq., Dipt. exot., I. 2., 182. (1839); Wilk., List Dipt. Arabia.

Brit. Mus., V. suppl. 1., 238. 358. (1854).

ardens Wied., Dipt. exot., 78. 31. (1821) et Aussereurop. zweifl.

Ins., I. 154. 68. (1828); Mucq., Dipt. exot., I. 1., 129. 16. (1838);

Walk., List Dipt. Brit. Mus., V. suppl. 1., 245. 401. (1854); v. D.

Wulp, Catal. Dipt. South Asia, 59. (1896).

argentifrons Walk., List Dipt. Brit. Mus., I. 186. (1848).

argyrophorus Schin., Novara Reise, Dipt., 90. 25. (1868); Hunter,

Trans. Amer. Entom. Soc. Philad., XXVII. 139. (1901).

assamensis BıG., Mén. Soc. Zool. France, V. 653. [Atylotus] (1892); Assam.

v. D. Wulp, Catal. Dipt. South Asia, 63. (1896).

aspahanicus RoND. = ispahanicus RoND.
Europa mer.

Brasilia.

Borneo.

Brasilia.

Chile.

Europa centr. et mer.

Java.

Patriaignota. America mer. 
Astur Erichs., Middendorff's Reise in Sibirien, Zool., 1. II. 66. 143. Sibiria.

(1851); Brauer, Denkschr. Akad. Wien, XLII. 140. 4. tab. I. fig.

4. (1880).

spilopterus Lw., Verh. zool.-bot. Ges. Wien, VIII. 581. 6. (1858).

astutus Ost.-SACK., Mem. Boston Soc. Nat. Hist., II. 471. 53. (1876);

Towns., Trans. Amer. Entom. Soc. Philad., XXII. 57. 8. [The-

rioplectes] (1895); Aldr., Catal. North Amer. Dipt., 201. (1905).

aler PAL. BEAUV. = lugubris MACQ.

ater Rossi, Fauna Etrusca, II. 320. 1547. tab. I. fig. 11. (1790); MfIG., System. Beschreib., II. 32. 1. tab. XIII. fig. 24. (1820); Macq., Recueil Soc. Sci. Agricult. Lille, 473. 1. (1826); L. Duf., Mém. Acad. d. Scienc., Math. et Phys., XI. 171-360. tab. IV. fig. 41. (1850); Lw., Verh. zool.-bot. Ges. Wien, VIII. 610. 42. (1858); Scmun., Fauna Austriaca, Dipt., I. 33. (1862) ; Brav., Denkschr. Acad. Wien, XLII. 159. 21. tab. 2. fig. 21. (1880); Goв., Mém. Soc. Linn. Nord France, 1881. 18. 1. (1881); Pand., Revue d'Entonı., II. 216. 40. (1883) ; Villes., Annal. Soc. Entom. France, LXXIV. 309. 27. (1905).

? algirus MACQ., Dipt. exot., I. 2., 180. (1839) et in Lucis: Explor scient. d. l'Algérie, Zool., III. 422. 22. (1849); WaLK., List Dipt. Brit. Mus., V. 225 et 231. 331. (1854); Schin., Novara Reise, Dipt., 87. 20. (1868).

fuscatus Macq., Recueil Soc. Sci. Agricult. Lille, 1826. 473. 2. (1826) ; MelG., System. Beschreib., VII. 59. 45. (1838); Walk., List Dipt. Brit. Mus., V. suppl. 1., 157 et 171. 60. (1854). morio ? L., Systema Naturae, Ed. XII., II. 1001. 19. (1767); ? VilL., Entomol. Linn., III. 556. 10. (1789); Fabr., Entom. System., IV. 363. 6. (1794); MeIG., Klassif., I. 167. 4. (1804); Fabr., Systema Antliat., 94. 4. (1805); IAatr., Gen. Crust. et Insect., IV. 283. (1809); LAM., Hist. nat. anim. sans vertebr., III. 420. 2. (1816); Dumér., Consid. génér. d'Ins., tab. XL. fig. 9. (1823); MACQ., Suit. à Buffon, I. 198. 5. (1834) et in Lucas: Explor. Scient. de l'Algérie, Zool., III. 422. 19. (1819); WaLK., List Dipt. Brit. Mus., V. suppl. 1., 157 et 170. 58. (1854); Leonardi, Insetti Nocivi, III. 374. [Atylotus] (1900).

nigrita MeIG. (nec FABR.), Klassif., I. 172. d. (1804).

? transiens WALK., List Dipt. Brit. Mus., I. 174. (1848); RicaRdo, Annal. Mag. Nat. Hist., ser. 7. XVI. 200. (1905).

ater Wred. p. p. = Wiedemanni Ost.-SAcK.

aterrimus Meig., System. Beschreib., II. 33. 3. (1820); WALK., List Dipt. Brit. Mus., V. suppl. 1., 156 et 169. 51. (1854); Lw., Verh. zool.-bot. Ges. Wien, VIII. 580. 4. (1858); Braver, Denkschr. Akad. Wien, XIII. 142. 6. tab. I. fig. 6. (1880); Pand, Revuo d'Entom., II. 215. 37. (1883); Mik in BEck.: Fauna von Hernstein, II. 2., 53. [Therioplectes] (1885); ВEck., Berlin. Entom. Zeitschr., XXXI. 105. 18. [Therioplectes] (1887); StronL, Mittheil.

Europacentr. et mer., Africa sept.

America sept.

$$
\text { A }
$$


Naturwiss. Ver. Steiermark, XXIX. 1892. 13. (1893); SPEISER, Zeitschr. f. wissensch. Insectenbiol., I. 407. 153. [Therioplectes] (1904) ; Villen., Annal. Soc. Entom. France, LXXIV. 308. 17. (1905) ; Speiser, Schrift. d. Phys.-ökon. Ges. Königsberg, XLVI. 163. [Therioplectes] (1905).

acthiops Luuvgh., Vetensk. Akad. Nya Handl., 1823. 265. tab.

III. fig. 3. (1823); ZETT., Dipt. Scand., I. 110. nota. (1842). austriacus Wied. ap. Meig., System. Beschreib., II. 35. 5. p. p. (1820).

Heydenianus Jaknv., Berlin. Entom. Zeitschr., X. 68. 6. (1866). nigerrimus Zetт., Dipt. Scand., I. 115. 15. (1842) et VIII. 2937.

15. (1849); Scholtz, Zeitschr. f. Entomol. Breslau, IV. No. 16., 37. (1850); WaLK., List Dipt. Brit. Mus., V. suppl. 1., 156 et 162 . 27. (1854).

signatus Wied. ap. Meig., System. Beschreib., II. 34. 5. (1820); Ginn., Bull. Soc. Imp. Nat. Moseou, XX. 1., 180. 2. (1847); Walk., I ist Dipt. Brit. Mus., I. 170. (1848), Insecta Britannica, Dipt., I. 39. 10. (1851) et List Dipt. Brit. Mus., V. suppl.

1., 155 et 161. 20. (1854).

var. auripilus Meig., System. Beschreib., II. 41. 15. (1820); MAcQ., Recueil Soc. Sci. Agricult. Lille, 1826. 475. 5. (1826); Zетт., Insecta Lappon., Dipt., 514. 3. (1838); ZeLL., Isis, 1842. XI. 821. 3. (1842); ZETT., Dipt. Scand., I. 109. 8. (1842) et VIII. 2937. 8. (1849); Scholtz, Zeitschr. f. Entomol. Breslau, IV. No. 16., 37. (1850) ; Walk., List Dipt. Brit. Mus., V. suppl. 1., 156 et 167. 41. (1854); Lw., Verh. zool.-bot. Ges. Wien, VIII. 579. 2. (1858) ; Bonsd., Finland tvaving. Ins., I. 108. 5. (1861); Schr., Fauna Austriaca, Dipt., I. 29. (1862); Goв., Mém. Soc. Linn. Nord France, 1881. 12. 2. [Therioplectes] (1881); Beck., Berlin. Entom. Zeitschr., XXXI. 105. 19. [Therioplectes] (1887); StroвL, Mittheil. Naturwiss. Ver. Steiermark, XXIX. 1892. 13. [Therioplectes] (1893); Villew., Annal. Soc. Entom. France, LXXIV. 308. 16. (1905) ; Speıser, Schrift. d. Physik.-ökon. Ges. Königsberg, XLVI. 163. (1905).

var. lugubris Zетт., Insecta Lappon., Dipt., 515. 6. (1838), Europacentr. Dipt. Seand., I. 114. 14, (1842) et VIII. 2937. 14. (1849); Lw., et sept. Verh. zool.-bot. Ges. Wien, VIII. 580. 3. (1858); BonsD., Finl. tvaving. Ins., I. 109. 9. (1861); StroвL, Mittheil. Naturwiss. Ver. Steiermark, XXIX. 1892: 13. [Therioplectes] (1893).

atratus FABr., Systema Entomol., 709. 9. (1775), Entom. System., IV. 366. 16. (1794) et Systema Antliat., 96. 16. (1805); WiED., Dipt. exot., 63. 2. (1821) et Aussereurop. zweifl. Ins., I. 114. 3. (1828) ; Macq., Dipt. exot., I. 1., 142. 41. (1838); WALK., List Dipt. Brit. Mus., I. 145. (1848) et V. suppl. 1., 177. 89. (1854); Besc., Saggio di Ditterol. Messic., I. 58. 5. (1859); Harris, Ins. of new Engl., E.. III., 602. (1862); Walsi, Proc. Boston Soc. Nat. Hist., IX. 304. (186t); РАСкаRD, Injurious Ins., 24. (1871);

America sept. et centr. 
RILEy, Second Ann. Rep. Ins. Minn., 128. (1871); Ost.-SAck., Mem. Boston Soc. Nat. Hist., II. 454. 31. (1876); Sмптн, Trans. Amer. Entom. Soc. Philad., XVII. 327. fig. 6. (1890) ; Towss., Trans. Amer. Entom. Soc. Philad., XXII. 60. 14. (1892) ; HaRT, Bull. Illinois State Laborat. of Nat. Hist., IV. 242. tab. XI. fig. 50. tab. XII. fig. 51-54, tab. XIII. fig. 55, 56. (1895); Towns., Journ. New York Entom. Soc., VI. 50. (1898); WiLLIst., Biolog. Central. Amer., Dipt., I. 259. 6. (A). (1901); Hine, Ohio State Acad. Sci., Spec. Pap. No. 5., 48. (1903), Ohio Naturalist, V. 234. (1904) et Bullet. Unit. Stat. Deptm. of Agrie., Div. of Entom., No. 44., 57. (1904); Lécarllon, Annal. Soc. Entom. France,LXXIV. 21. fig. (1905) ; Aldr., Catal. North Amer. Dipt., 201. (1905).

americanus Druny, Illustrat. of Nat. Hist. Exot. Ins., I. 104.

III. tab. XLIV. fig. 3. (1770) ; Panzer, Drury's Abbild. und Beschr. exot. Ins., 170. tab. XLIV. fig. 3. (1785-88).

niger PaL. Beauv., Ins. recueill. en Afrique et en Amérique, 54. tab. I. fig. 1. (1805-21).

validus WiED., Aussereurop. zweifl. Ins., I. 113. 2. (1828);

WALK., List Dipt. Brit. Mus., I. 145. (1848).

atricornis Big., Mém. Soc. Zool. France, V. 683. (1892).

America mer.

atricornis MeIG. p. p. = bromius L.

atricornis MeIG. p. p. = cordiger MeIG.

atricornis ${ }^{-M e I G}$. p. p. = nemoralis MeIG.

atrimanus Lw., Öfvers. Kongl. Vet. Akad. Förhadl., XIV. 340. 21.

(1857) et Dipt.-Fauna Südafrika's, I. 40. 11. tab. I. fig. 22. (1860). atripes v. D. Wulp, Notes Leyden Mus., VII. 75. 29. tab. V. fig. 4. (1885).

atropos $\mathrm{J}_{\mathrm{AENN}}$ = anthracinus Mkig.

attenuatus WALK., List Dipt. Brit. Mus., I. 159. (1848).

anctumnalis ZELL. = autumnalis $\mathrm{L}$.

aurantiacus Bell., Saggio di Ditterol. Messic., I. 67. 20. tab. II. fig.

9. (1859); Willist., Biolog. Central. Amer., Dipt., I. 259. 1. (A). (1901) ; Aldr., Catal. North Amer. Dipt., 201. (1905).

auribarbis MacQ., Dipt. exot., suppl. 3., 12. 94. (1847); WalK., List Dipt. Brit. Mus., V. suppl. 1., 198. 187. (1854); Röd., Stettin. Entom. Zeitg., XLVII. 263. (1886) et Dipteren gesammelt in Süd-Amerika von A. Stübel, 8. (1892); Hunwer, Trans. Amer. Entom. Soc. Philad., XXVII. 139. (1901).

auricinctus MAcQ., Dipt. exot., I. 1., 130.18. tab. XVII. fig. 3. (1838) ; WALK., List Dipt. Brit. Mus., V. suppl. 1., 242. 386. (1854); v. D. Wulp, Catal. Dipt. South Asia, 59. (1896).

auriflamma WALK., List Dipt. Brit. Mus., I. 155.(1848); v. D. WulP, India or.

Catal. Dipt. South Asia, 59. (1896).

auripilus Meig. = aterrimus MelG. var.

auripunctatus MACQ., Dipt. exot., I. 2., 180. (1839) et in LuCas: Algeria.

Expor. Seient. de l'Algérie, Zool., III. 422. 21. (1849); Walk.,

List Dipt. Brit. Mus., V. suppl. 1., 230. 327. (18г̃).

Kertész: Catalogus Dipterorum. III. 
aurisquammatus Bı̈., Mém. Soc. Zool. France, V. 665. [Atylotus] Brasilia. (1892).

aurora MAcq., Dipt. exot., I. 1., 138. 32. (1838) ; WALK., List Dipt. Brit. Mus., V. suppl. 1., 214. 253. (1854); Huv'er, Trans. Amer. Entom. Soc. Philad., XXVII. 139. (1901).

aurotestaceus WALK., List Dipt. Brit. Mus.,' V. suppl. 1., 247. 408. (1854) ; v. D. Wulp, Catal. Dipt. South Asia, 60. (1896).

austriacus $\mathrm{F}_{\mathrm{ABR}}$. = micans MeIG.

anstriacus WIED. = aterrimus MEIG.

autumnalis L., Fauna Sues., 462. 1883. (1761); Georrr., Hist. d'Ins., II. 460. 2. tab. XVII. fig. 2. (1762); Scop., Entomol. Carniol., 370. 1007. (1763) ; L., Systema Naturae, Ed. XII., II. 1000. 5. (1767) ; Fabr., Spec. Insect., II. 456. 5. (1781); GmeL., Systema Naturae, V. 2882. 5. (1788); Vill., Entomol. Linn., III. 552. 2. (1789) ; Fabr., Entomol. System., IV. 364. 7. (1794); Schellexvi., Gattung. d. Fliegen, 72. tab. XXVII. fig. 1. (1803) ; Meig., Klassif., I. 167. 6. tab. IX. fig. 27. (1804); FABR., Systema Antliat., 94. 5. (1805) ; Fall., Kongl. Vet. Akad. Handl., XXX.243.5.(1809); Panz., Fauna Germ., CX.21. (1809); LaM., Hist. nat. anim. sans vertébr., III. 420. 3. (1816) ; FatL., Dipt. Suec., Taban., 6. 6. (1817); MacQ., Recueil Soc. Sci. Agricult. Iille, 1826. 474. 4. (1826) et Suit. à Buffon, I. 204. 25. (1834); Duxcan, Magaz. Zoolog. and Botan., I. 363. (1837) ; Zетт., Dipt. Scand., I. 106. 2. (1842); Guмm., Bull. Soc. Imp. Nat. Moscou, XX. 1., 181.3. (1847) ; ZeTr., Dipt. Scand., VIII. 2936. 2. (1849); MacQ. in Lucas : Explor. Scient. de l'Algérie, Zool. III., 424. 27. (1849); Sсноцтz, Zeitsclır. f. Entom. Breslau, IV. No. 16., 36. (1850) ; WaLk., Insecta Britanu., Dipt., I. 37. 2. (1851) et List Dipt. Brit. Mus., V. suppl. 1., 156 et 167. 42. (1854); Kollar, Sitzgsber. Akad. Wien, XIII. 533. (1854); Zетт., Dipt. Scand., XII. 4547. 2. (1855); Lw., Verl., zool.-bot. Ges. Wien, VIII. 605. 33. (1858) ; Schin., Fauna Austriaca, Dipt., I. 35. (1862) ; JaENx., Berlin. Entom. Zeitschr., X. 84. 27. (1866); Friedenfels, Verh. und Mittheil. d. siebenbürg. Ver. f. Naturwiss. Hermannstadt, XXX. 168. (1879) ; Kraus, Zoolog. Anzeig., II. 229. (1879) ; Graber, Archiv f. mikroskop. Anat., XX. 506. (1880) ; Brauer, Denkschr. Akad. Wien, XLII. 192. 49. tab. VI. fig. 49. (1880); Gов., Mém. Soc. Linn. Nord France, 1881. 24. 11. (1881) ; Pand., Revue d'Entom., II. 205. 11. (1883) ; Neviiaus, Diptera Marchica, 42. 3. (1886); L. Coucke, Annal. Soc. Entom. Belgique, XXXVI. 136. (1892); Sтrовц, Mittleil. Naturwiss. Ver. Steiermark, XXIX. 1892. 16. (1893); LEoNARDI, Insetti Nocivi, III. 373. fig. 204. (1900) ; Ricardo, Annal. Mag. Nat. Hist., ser. 7. XIV. 199. (1905); Villen., Annal. Soc. Entom. France, LXXIV. 308. 13. (1905).

auctumnalis ZeLL., Isis, 1842. XI. 816. 3. tab. I. fig. 9. (1812)

[lapsus].

autumnalis Schrank, Fauna Boica, III. 153. 2530. (1803). 
bovinus Harris (nec L.), Expos. Engl. Ins., 27. tab. VII. fig. 1. (1782).

autumnalis Schrank = autumnalis $\mathrm{L}$.

avidus Big., Mém. Soc. Zool. France, V. 673. [Atylotus] (1892).

baal Towns., Trans. Amer. Entom. Soc. Philad., XXII. 58. 11. [Aty-

Australia.

lotus] (1895) ; Aldr., Catal. North Amer. Dipt., 201. (1905).

baltimorensis MAcQ. = costalis WiED.

barbarus CoqueB., Illustr. Iconogr. Insect., 111. tab. XXV. fig. 2. (1804); Brauer, Denkschr. Akad. Wien, XLII. 166. 29. tab. II. fig. 29. (1880) ; Pand., Revue d'Entom., II. 201. 1. (1883).

maroccanus F $\Lambda$ Br., Systema Antliat., 93. 2. (1805); Cuv., Régrie Anim., III. 614. (1817); Walk., List Dipt. Brit. Mus., V. suppl. 1., 155 et 161. 19. (1854).

tuurinus Meig., Klassif., I. 165. 1. (1804) et System. Beschreib., II. 42. 17. tab. XIII. fig. 23. (1820); Lw., Verh. zool.-bot. Ges. WVien, VIII. 596. 25. (1858).

barbarus Thưв. = nemoralis MeIG.

basalis Macq., Dipt. exot., I. 1., 126. 10. (1838); WalK., List Dipt. Brit. Mus., V. suppl. 1., 243. 391.(1848) ; v. D. Wulp, Notes Leyden Mus., VII. 72. 26. (1885) et Catal. Dipt. South Asia, 59. (1896).

basalis WaLK., List Dipt. Brit. Mus., I. 182. (1848).

basirufus WaLK., Insecta Saunders., Dipt., I. 32. (1850).

basivitta WALK., Newman Zoologist, VIII. Append., LXVIII. (1850) et List Dipt. Brit. Mus., V. suppl. 1., 206. 220. (1854).

batnensis Bı́., Mém. Soc. Zool. France, V. 639. [Therioplectes] (1892).

benedictus Whitney, Canad. Entomol., XXXVI. 206. (1904); Hine, Ohio Naturalist, V. 235. (1904).

bicolor MAcQ. = giganteus DEG.

bicolor Thunb., Nova Acta Upsal., IX. 55. (1827).

bicolor Wied., Dipt. exot., 96. 58. (1821) et Aussereurop. zweifl. Ins., I. 188. 115. (1828) ; WaLK., List Dipt. Brit. Mus., I. 163. (1848) et V. suppl. 1., 184. 122. (1854) ; Ost.-SAck., Mem. Boston Soc. Nat. Hist., II. 460. 39. (1876) et Catal. Dipt. N. America, Ed. II., 62 et 229. (1878) ; v. D. WulP, Tijdschr. v. Entom., XXIV. 158. 6. (1881); Hine, Ohio State Acad. Sci., Spec. Pap. No. 5., 48. (1903); Aldr., Catal. North Amer. Dipt., 201. (1905).

fulvescens Walk., List Dipt. Brit. Mus., I. 171. (1848); Ost.-

SAck., Mem. Boston Soc. Nat. Hist.; II. 460. 40. (1876).

ruficeps MacQ., Dipt. exot., suppl. 5., 35. 130. (1855).

bifarius I,w., Verh. zool.-bot. Ges. Wien, VIII. 595. 21. (1858); Scuin., Fauna Austriaca, Dipt., I. 36. (1862) ; Brauer, Denkschr. Akad. Wien, XLII. 172. 35. tab. III. fig. 35. (1880) ; Pand., Revie ¿'E:ntom., II. 210. 25. (1883).

bifasciatus*) Fourcr., Entom. Paris., II. 458. 10. (1785); Villers, Europa. Entom. Linn., III. 557. 16. (1789).

India or., Sumatra.

Australia.

Columbia.

Brasilia.

Algeria.

America sept.

Patria ignota.

America sept.

Europa centr. et mer., Asia min.

*) ? Chrysops. 
bifasciatus MacQ., Suit. à Buffon, I. 201. 17. (1834); WALK., List Australia. Dipt. Brit. Mus., V. suppl. 1., 254. 447. (1854).

bifenastratus Ost.-SАск., Biolog. Central. Amer., Dipt., I. 52. 7. tab. Mexico.

I. fig. 9. (1886) ; Astdr., Catal. North Amer. Dipt., 201. (1905).

Bigoti Bell., Saggio di Ditterol. Messic., I. 58. 6. (1859) ; Ost.-SAck.,

Biolog. Centr. Amer., Dipt., I. 48. 2. (1886); Hunter, Trans.

Amer. Entom. Soc. Philad., XXVII. 139. (1901); Alvr., Catal.

North Amer. Dipt., 201. (1905).

apicalis Macq., Dipt. exot., II. 1., 20. 102. (1841) ; WaLK., List

Dipt. Brit. Mus., V. suppl. 1., 188. 141. (1854).

Macquarti Schin., Novara Reise, Dipt., 89. 24. (1868).

biguttatus WIED., Aussereurop. zweifl. Ins., II. 623. 20. (1830) ;

WaLk., List Dipt. Brit. Mus., V. suppl. 1., 231. 332. (1854); Lw.,

Öfv. Kongl. Vet. Akad. Förhandl., XIV. 1857. 339. 16. (1858) et

Dipt.-Fauna Südafrika's, I. 37. 6. (1860) ; Karsch, Berlin. Entom.

Zeitsclır., XXXI. 370. 5. (1887); Johrs., Proc. Acad. Nat. Sci.

Philad., 1898. 158. (1898) ; Ricardo, Annal. Mag. Nat. Hist., ser.

7. VI. 163. (1900).

Cerberus Walk., List Dipt. Brit. Mus., I. 149. (1848).

cilipes MACQ., Dipt. exot., I. 1., 120. 2. (1838); WALk., List

Dipt. Brit. Mus., V. suppl. 1., 236. 352. (1854).

noctis WaLK., Insecta Saunders., Dipt., I. 42. (1850).

tripunctifer Walk., Newman Zoologist, VIII. Append., XCV.

(1850) et List Dipt. Brit. Mus., V. suppl. 1., 227. 313. (1854).

bimaculatus MacQ. = ? tropicus Panz.

bipartitus WALK. = albonotatus BELL.

bipartitus WaLk., Insecta Saunders., Dipt., I. 451. (1856).

Natal.

bipunctatus v. D. WulP, Notes Leyden Mus., VII. 75. 30. tab. V. Africa or.

fig. 5. (1885).

birmanicus Bıg., Mém. Soc. Zool. France, V. 653. [Atylotus] (1892);

v. D. Wulp, Catal. Dipt. South Asia, 63. (1896).

bisignatus JAENN., Berlin. Entom. Zeitschr., X. 74. 14. (1866) ; VILIEN., Feuille jeun. Natural., XXXV. 59. 6. /Therioplectes] (1905).

bitinctus WALK., Trans. Entom. Soc. London, n. ser. IV. 123. (1857).

bituberculatus Big., Mém. Soc. Zool. France, V. 659. [Atylotus] (1892); v. D. Wulp, Catal. Dipt. South Asia, 63. (1896).

bivittatus MaCQ., Dipt. exot., suppl. 1., 35. 72. (1845); Walk., List

Dipt. Brit. Mus., V. suppl. 1., 209. 229. (1854); Hunter, Trans.

Amer. Entom. Soc. Plilad., XXVII. 139. (1901).

bonariensis MACQ., Dipt. exot., I. 1., 138. 33. (1838); WALK., List Dipt. Argentinia.

Brit. Mus., V. suppl. 1., 209. 230. (1854); E. L. Arrib., Bol. Acad.

Nat. Cienc. Córdoba, IV. 134. 69. (1882); Hunter, Trans. Amer.

Entom. Soc. Philad., XXVII. 139. (1901).

acupunctatus Rosn., Annuar. Soc. Nat. Modena, III. 38. 36. [Agelanius] (1868).

borealis FaBr.=? lapponicus WaHLi,

borealis $\mathrm{J}_{\mathrm{AENN}}$ = = tropicus PANz. 
borealis MEIG., System. Beschreib., II. 37. 10. p. p. (1820); Zетт., Insecta Lappon., Dipt., 515. 5. (1838) et Dipt. Scand., I. 113. 13. (1842); Zelu., Isis, 1842. XI., 821. 4. (1842); Gimм., Bull. Soc. Imp. Nat. Moscou, XX. 1., 183. 11. (1847); WALK., List Dipt. Brit. Mus., V. suppl. 1., 156 et 165. 38. (1854); Lw., Verlı. zool.-bot. Ges. Wien, VIII. 586. 13. (1858); Bonsd., Finl. tvaving. Ins., I. 109. 8. (1861); Schis., Fauna Austriaca, Dipt., I. 30. (1862) ; Brauer, Denkschr. Akad. Wien, XLII. 143. 7. tab. I. fig. 7. (1880) ; Pand., Rovue d'Entomol., II. 213. 34. (1883); STrolsi, Mittheil. Naturwiss. Ver. Steiermark, XXIX. 1892. 14. (1893); Beck., Acta Soc. Sci. Fenn., XXVI. No. 9., 6. 5. (1900) ; Villex., Annal. Soc. Entom. France, LXXIV. 307. 6. (1905).

borealis MEIG. p. p. = maculicornis ZETT.

borealis ZETT. p. p. = luridus FALL.

bovinus HARRIS $=$ autumnalis $\mathrm{L}$.

bovinus L. Ет AUCт. PLUR. = bovinus Lw., spodopterus MeiG. et sudeticus ZeLL.

bovinus Lw., Verh. zool.-bot. Ges. Wion, VJII. 606. 36. (1858), Öfv. Kongl. Vet. Akad. Förhandl., XIV. 1857. 340. 18. (1858) et Dipt.Fauna Südafrika's, I. 38. 8. (1860); Brauer, Denkschr. Akad. Wien, XLII. 185. 44. tab. III. fig. 44. (1880); Goв., Mém. Soc. Linn. Nord France, 1881. 20. 5. (1881); PaND., Revue d'Entom., II. 203. 7. (1883); Hansen, Fabricia oris Dipt., tab. II. fig. 5-6. (1883); L. Coucke, Annal. Soc. Entom. Belgique, XXXVI.137 (1892); Strobl, Mittheil. Naturwiss. Ver. Steiormark, XXIX. 1892. 16. (1893); Leonard, Insetti Nocivi, III. 372. fig. 201. C., 202. 203. (1900); Villex., Annal. Soc. Entom. France, LXXIV. 309. 24. (1905).

p. p. bovinus L., Fauna Suec., 462. 1882. (1761); Poda, Ins. Mus. Graec., 117. 1. (1761); Scop., Entom. Carniol., 370. 1006. (1763); O. F. Mutl., Fauna Ins. Fridrichsdal., 86. 767. (1764); L., Systema Naturae, Ed. XII., II. 1000.4. (1767); FABr., Spec. Insect., II. 455. 3. (1781); Schrank, Enum. Insect. Austr., 478. 974. (1781); Deg., Mém. pour serv. à l'hist. d. Ins., VI. 219. 1. tab. XII. fig. 10-11. (1776); Herbst, Gemeinnütz. Naturgesch., VIII. tab. CCCXIII. fig. 1. (1787); Gred., Systema Naturae, V. 2882. 4. (1788); Vill., Entom. Linn., III. 551. 1. (1789) ; Panz., Fauna Germ., II. 20..(1793) ; FABR., Entom. System., IV. 363. 4. (1794); Schrank, Fauna Boica, III. 153. 2529. (1803); MeIg., Klassif., I. 165. ․ (1804) ; FABr., Systema Antliat., 92. 1. (1805); LAtr., Gen. Crust. et Ins., IV. 283. (1809) ; Fall., Kongl. Vet. Akad. Handl., XXX. 239.1.(1809) ; LAM., Hist. nat. anim. sans vertébr., III. 420. 1. (1816) ; Fall., Dipt. Suec., Taban., 4. 1. (1817) ; Cuv., Régne Anim., III. 614. (1817); MeIG., System. Beschreib., II. 43. 18. (1820); Macq., Recueil Soc. Sci. Agricult. Lille, 1826. 476. 2. tab. III. fig. 7. (1826) et Suit. à Buffon, I. 199. 9. (1834); GuÉr., Iconogr. d. Régne Anim., VII. Ins., 542. tab. XCVII. fige,
Europacentr. et sept., Sibiria.

Europa, Africa. 
1, a-d. (1835) ; Daнlв., Ins. Scand. skäd. och nytta, 306. 203. (1837) ; Duncan, Magaz. Zoolog. and Botan., I. 362. (1837); ZeтT., Insecta Lappon., Dipt., 513. 1. (1838) ; Westw., Introd., II. 541. fig. 128., 9. 10. (1840); Blancir., Hist. Nat. d'Ins., III. 589. tab. I. fig. 9. (1840); ZeTr., Dipt. Scand., I. 105. 1. (1842); ZELL., Isis, 1842. XI., 814. 1. tab. I. fig. 2-4. (1842); G1мм., Bull. Soc. Imp. Nat. Moscou, XX. 1., 179. 1. (1847); MACQ. in IuCAs : Explor. Scient. de l'Algérie, Zool., III. 424. 28. (1849) ; ZETT., Dipt. Scand., VIII. 2936. 1. (1849); L. Duf., Mém. Acad. des Sci. Math. et Phys., XI. $171-360$. tab. I. fig. $3-5$, tab. II. fig. 15-16, tab. IV. fig. 38-39, 42. (1850); WALK., Ins. Britann., Dipt., I. 37. 1. tab. I. fig. 13. (1851) et List Dipt. Brit. Mus., V. suppl. 1., 156 ot 166. 40. (1854) ; Zетт., Dipt. Scand., XII. 4547. 1. (1855); Bonsd., Finl. tvaving. Ins., I. 107. 1. (1861); Scurn., Fauna Austriaca, Dipt., I. 34. (1862); JAExn., Berlin. Entom. Zeitschr., X. 80. (1866); Nevhuus, Diptera Marchica, 42. 2. (1886).

Bratnankii Now., Memoiren d. Krakauer Akad., II. 19. (1875) et Beitr. z. Kenntn. d. Dipt.-Fauna Neu-Seeland's, 19. (1875); Hutron, Catal. New Zealand Dipt. etc., 20. (1881) et Trans. New Zealand Instit., XXXIII. 12. [bratranchii] (1901).

Braneri JAENN. = cordiger MEIG.

brevidentatus MacQ., Dipt. exot., suppl. 5., 28. 118. (1855).

brevior WaLk., List Dipt. Brit. Mus., I. 188. (1848).

brevis Lw., Verh. zool.-bot. Ges. Wien, VIJI. 584. 7. (1858); Braver, Denkschr. Akad. Wien, XLII. 145. 8. a. (1880).

breviusculus Walk., Proc. Linn. Soc. London, VIII. 109. 22. (1865). brevivitta Walk., List Dipt. Brit. Mus., I. 157. (1848).

brevivitta WALK., Newman Zoologist, VIII. Append., XCVI. (1850) et List Dipt. Brit. Mus., V. suppl. 1., 265. 509. (1854).

bromius L., Fauna Suec., 463. 1885. (1761) ; Scop., Entomol. Carniol., 372. 1011. (1763); L., Systema Naturae, Ed. XII., II. 1001. 12. (1767) ; O. F. Múlleer, Zoolog. Dan. Prodr., 180. 2114. (1776); Fabr., Spec. Insect., II. 457. 14. (1781); Herest, Gemeinnütz. Naturgesch., VIII. 112. tab. LXVII. fig. 3. (1787) ; VILL., Entomol. Linn., III. 553. 5. (1789); GMel., Systema Naturae, V. 2884. 12. (1792) ; Fabr., Entomol. System., IV. 366. 17. (1794); Coqueb., Illustr. Iconogr., Dec. III. 119. tab. XXVII. fig. 3. а. (1799); Meig., Klassif., I. 169. 9. (1804); Fabr., Systema Antliat., 97. .18. (1805) ; Fall., Kongl. Vet. Akad. Handl., XXX. 243. 6. (1809) et Dipt. Suec., Taban., 7. 7.(1817); Meig., System. Beschreib., II. 52. 29. (1820); Macq., Recueil Soc. Sci. Agricult. Lille, 1826. 479. 11. (1826) et Suit. à Buffon, I. 20う̌. 28. (1834); Duncan, Magaz. Zoolog. and Botan., I. 365. (1837); ZETT., Insecta Lappon., Dipt., 516. 8. (1838) ; Zelu., Isis, 1842. XI., 817. 5. tab. I. fig. 14-19. (1842); Zетт., Dipt. Scand., I. 107. 5. (1842); WaLK., List Dipt. Brit. Mus., I. 169. (1848) ; ZeTT., Dipt. Scand., VIII. 2936. 5. (1849); Macq. in Lucas: Explor. Scient. de

Australia.

Australia. Sibiria.

Nova Guinea. Australia. Patriaignota.

Europa. 
l'Algérie, Zool. III., 424. 29. (1849); WaLk., Insecta Britann., Dipt., I. 38. 5. (1851) ot List Dipt. Brit. Mus., V. suppl. 1., 156 et 164. 35. (1854); ZETT., Dipt. Scand., XII. 4547. 5. (1855) ; Assuuss, Wien. Entom. Monatschr., II. 181. 34. (1858); Zetr., Dipt. Scand., XIII. 4944. 5. (1859); BonsD., Finl, tvaving. Ins., I. 107. 3. (1861); Schin., Fauna Austriaca, Dipt., I. 36. (1862); Beling, Archiv f. Naturgesch., XLI. 1., 35. 3. (1875); Brauer, Denkschr. Akad. Wien, XLII. 187. 45. tab. VI. fig. 45. (1880); Gов., Mém. Soc. Linn. Nord France, 1881. 25. 13. (1881); Pand., Revue d'Entom., II. 207. 16. (1883) ; Hansex, Fabrica oris Dipt., tab. I. fig. $8,9,12,14$; tab. II. fig. 2, 7, 8. (1883); Nevinaus, Diptera Marchica, 42. 8. (1886) ; Beck., Berlin. Entom. Zeitschr., XXXI. 106. 26. (1887); L. Coucke, Annal. Soc. Entom. Belgique, XXXVI. 137. (1892); StrobL, Mittheil. Naturwiss. Ver. Steiermark, XXIX. 1892. 16. (1893); Yerbury, Proc. Entom. Soc. London, 1900. pag. XXII. (1900); LeONaRd, Insecti Nocivi, III. 373. (1900); Villen., Feuille jeun. Natural., XXXV. 60. 2. var. (1905) et Annal. Soc. Entom. France, LXXIV. 308. 12 et 309. 21. (1905).

atricornis MeIG., System. Beschreib., VII. 59. 47. p. p. (1838). bronicus Grmm., Bull. Soc. Imp. Nat. Moscou, XX. 1., 182. 6. (1847).

connexus WaIK., Insecta Saunders., Dipt., I. 62. (1850).

connexans Ricardo, Annal. Mag. Nat. Hist., ser. 7. XVI. 200. (1905).

glaucus MeiG., System. Beschreib., II. 51. 28. p. p. (1820); Grms., Bull. Soc. Imp. Nat. Moscou, XX. 1., 187. 20. (1847); WaLk., Ins. Britann., Dipt., I. 38. 7. (1851) et List Dipt. Brit. Mus., V. suppl. 1., 156 et 164. 32. (1854); Neunaus, Diptera Marchica, 42. 7. (1886); SтroвL, Mittheil. Naturwiss. Ver. Steiermark, XXIX. 1892. 16. (1893); Viulen., Annal. Soc.

Entom. France, LXXIV. 309. 23. (1905).

maculatus Deg., Mém. pour serv. à l'hist. d. Ins., VI. 221. 3. (1776).

nemoralis MeIG., System. Beschreib., II. 50. 26. p. p. (1820). scalaris Meig., System. Beschreib., II. 38. 11. (1820); WaLK.,

List Dipt. Brit. Mus., V. suppl. 1., 155 et 159. 12. (1854).

var. glaucescens Schis., Fauna Austriaca, Dipt., I. 36. p. p. (1862).

bromius Meig. p. p.= lunatus Fabr.

bronicus Ginм. = bromius L.

brunneus MAcQ., Suit. à Buffon, I. 203. 22..(1834) et Dipt. exot., I. India or.,

1., 129. 15. (1838); WaLk., List Dipt. Brit. Mus., V. suppl. 1., Java.

243. 392. (1854); v. D. Wulp, Catal. Dipt. South Asia, 59. (1896).

brunneus Thuns., Nova Acta Upsal., IX. 56. (1827).

Prom. boll.sp.

bubali DoL., Natuurkund. Tijdschr. Neder]. Indie, X. 407. 11. tab. Java.

III. fig. 3. (1856); v. D, Wưp, Catal. Dipt. South Asia, 60. (1896), 
bucolicus Scmn., Novara Reise, Dipt., 81. 4. (1868); v. D. Wulp, Hongkong. Catal. Dipt. South Asia, 61. (1896).

Buddha Portscir., Horae Soc. Entom. Ross., XXI. 181. [Budda] (1887) et XXVI. 202. (1891).

Mongolia chinensis.

caesiofasciatus MaCQ. = giganteus DEG.

caesius WaLK., List Dipt. Brit. Mus., I. 180. (1848).

caiennensis Fabr., Entomol. System., IV. 366. 19. (1794) et Systema Antliat., 98. 22. (1805); Wred., Dipt. exot., 91. 52. (1821) et Aussereurop. zweifl. Ins., I. 178. 101. (1828); WАI.K., List Dipt. Brit. Mus., V. suppl. 1., 200. 193. (1854) ; Hunter, Trans. Amer. Entom. Soc. Philad., XXVII. 140. (1901); Thenese Prixz. v. BAYERn, Berlin. Entom. Zeitschr., XLVII. 244. 4. [Therioplectes] (1902).

calens L., Systema Naturae, Ed. XII., II. 1000. 6. (1767); FABr., Entomol. System., IV. 364. 9. (1794) et Systema Antliat., 94. 7. (1805) ; Wred., Dipt. exot., 72. 19. (1821) et Aussereurop. zweifl. Ins., I. 134. 36. (1828); WALK., List Dipt. Brit. Mus., V. suppl. 1., 181 112. (1854); Ost.-Sack., Mem. Boston Soc. Nat. Hist., II. 472. (1876); Aldr., Catal. North Amer. Dipt., 202. (1905).

calidus WALK., Insecta Saunders., Dipt., I. 57. (1850); v. D. Wul., ? Asia.

Catal. Dipt. South Asia, 60. (1896).

californicus MARTEN = epistates Ost.-SAck.

caliginosus BeLr., Saggio di Ditterol. Messic., I. 68. 21. tab. II. fig. Americacentr.

10. (1859); Willist., Biolog. Central. Amer., Dipt., I. 259. 4.

(1901) ; Aldr., Catal. North Amer. Dipt., 202. (1905).

? cbrius Ost.-Sack., Biolog. Central. Amer., I. 49. 4. (1886);

Aldr., Catal. North Amer. Dipt., 203. (1905).

callicera Big., Mém. Soc. Zool. France, V. 686. (1892).

callosus MacQ., Nipt. exot., suppl. 3., 11. 91. (1847); WaLk., List

Brasilia.

Dipt. Brit. Mus., V. suppl. 1., 200. 196. (1854); Hunter, Trans.

Amer. Entom. Soc. Philad., XXVII. 140. (1901).

calopsis Big., Mém. Soc. Zool. France, V. 639. [Therioplectes] (1892). calopterus Schin., Novara Reise, Dipt., 91. 27. (1868); Hunter, Trans. Amer. Entom. Soc Philad., XXVII. 140. (1901).

camaronensis Big., Mém. Soc. Zool. France, V. 644. [Atylotus] Madeira. (1892).

campechianus Towns., Canad. Entomol., XXIX. 197. 1. (1897); Mexico.

ALdr., Catal. North Amer. Dipt., 202. (1905).

canipalpis Bı̈., Mém. Soc. Zool. France, V. 649. [Atylotus] (1892). Persia.

canus Karsch, Zeitsclir. f. d. ges. Naturwiss., 3. F. IV. (III). 377. Africa occid. 1. tab. IV. fig. 1. (1879).

capensis WIED., Dipt. exot., 91. 51. (1821) et Aussereurop. zweifl. Prom.bon.sp. Ins., I. 177. 100. (1828); Walk., List. Dipt. Brit. Mus., V. suppl. 1., 228. 318. (1854).

captonis Martex, Canad. Entomol., XIV. 211. [Therioplectes] (1882); Americasept. Hine, Ohio Naturalist, V. 235. (1904).

comastes Wiluıst., Trans. Kansas Acad. Sci., X. 137. (1887); 
Towns., Trans. Amer. Entom. Soc. Philad, XXII. 58. 9. [Therioplectes] (1895) et Proc. Calif. Acad. Sci., ser. 2. IV. 597. 11. [Therioplectes] (1895); Aldr., Catal. North Amer. Dipt., 202. (1905).

carabaghensis Pon'tscir., Trudy Russk. Entom., X. 154. tab. 111. fig. 2. [Atylotus] (1877) et Horae Soc. Eutom. Ross., XVIII. 127. [Atylotus] (1883) ; Osт.-SAcK., Wien. Entom. Zeitg., III. 255. [Atylotus] (188t).

carbo Macq., Dipt. exot., suppl. 4., 33. 104. (1850); BLANCr. in GAY: Hist. fis. y polit. de Chile, Zool. VII. 397. 11. (1854); PHiL., Verh. zool.-bot. Ges. Wien, XV. 714. 11. (1865) ; Scmus, Novara Reise, Dipt., 92. 29. (1868); Hunter, Trans. Amer. Entom. Soc. Philad., XXVII. 140. (1901).

carbonaritus Merg. = nigrita FABr.

carbonatus $\mathrm{MACQ}_{\mathrm{C}}=$ alexandrinus $\mathrm{W}_{\mathrm{IED}}$.

carneus Ber.., Saggio di Ditterol. Messic., I. 62. 12. (1859); AlnR., Catal. North Amer. Dipt., 202. (1905).

carolinensis MAcq., Dipt. exot., I. 1., 145. 47. (1838); WaLK., List Dipt. Brit. Mus., V. suppl. 1., 179. 99. (1854); Ost.-SAck., Mem. Boston Soc. Nat. Hist., II. 473. (1876) et Catal. Dipt. North America, Ed. II., 56 et 226. (1878) ; Hrne, Ohio State Acad. Sci., Spec. Pap. No. 5., 49. (1903); Aldr., Catal. North Amer. Dipt., 202. (1905).

castaneus Bı́., Mém. Soc. Zool. France, V. 633. [Dichelaccra] (1892);

Ricardo, Annal. Mag. Nat. Hist., ser. 7. XIV. 368. (1904).

castaneoventris MACQ., Dipt. exot., I. 1., 148. 55. (1838); WA1K., Patriaignota. List Dipt. Brit. Mus., V. suppl. 1., 264. 506. (1854).

castaneus MacQ., Suit. à Buffon, I. 198.4. (1834); Walk., List Dipt. Cayenne.

Brit. Mus., V. suppl. 1., 209. 228. (1854).

catenatus OST.-SACK. $=$ recedens $\mathrm{W}_{A \mathrm{LK}}$.

catenatus WALK. p. p. = giganteus DEG.

catenatus WALK. p. p. = turbidus WIED.

centron Marten, Canad. Entomol., XIV. 211. (1882); Hine, Oliio Americasept.

Naturalist, V. 235. (1904)

cerastes Ost.-Sack. = hirtioculatus Maci.

Cerberus WALK. = biguttatus WiED.

cereolus Big., Mém. Soc. Zool. France, V. 644. [Atylotus] (1892). Africa or. ceylonicus Schin., Novara Reise, Dipt., 93. 33. (1868); v. D. Wulp, Ceylon.

Catal. Dipt. South Asia, 61. (1896).

cheliopterus Rond. = ? fronto Ost.-SAck.

chilensis Macq., Dipt. exot., I. 1., 141. 38. (1838); Brancir. in GaY: Chile.

Hist. fis. $y$ polit. de Chile, Zool., VII. 395. 7. tab. II. fig. 4.

(1854); Walk., List Dipt. Brit. Mus., V. suppl. 1., 198. 184.

(1854); PhIL., Verh. zool.-bot. Ges. Wien, XV. 714. 7. (1865);

Hunter, Trans. Amer. Entom. Soc. Philad., XXVII. 140. (1901).

chinensis Thunb., Nova Acta Upsal., IX. 61. (1827). China, Pr.b.sp. chionostigma Ost.-SAck., Biolog. Central. Amer., Dipt., I. 54. 9. Americacentr. 
tab. I. fig. 11. (1886); Wil.ıst., Biolog. Central. Amer., Dipt., I.

259. 9. (1901); Aldr., Catal. Nortlı Amer. Dipt., 202. (1905).

chlorophthalmus Meig. = glaucopis MeIG.

chrysoleucus WaLk., List Dipt. Brit. Mus., V. suppl. 1., 327. (1854). chrysurus Lw., Wien. Entom. Monatschr., II. 103. 5. (1858); Poktsci.,

Horae Soc. Entom. Ross., XXVI. 202. (1891); Coquil., Proc.

Unit. Stat. Nat. Mus., XXI. 309. (1898).

cilipes $\mathrm{M}_{\Lambda \mathrm{CQ}}$. = biguttatus $\mathrm{W}_{\mathrm{IED}}$.

cinctus Fari., Entom. System., IV. 366. 18. (1794) et Systema

Antliat., 97. 20. (1805); Meig., System. Beschreib., II. 42. 16. (1820); Wied., Dipt. exot., 67. 10. (1821) et Aussereurop. zweifl. Ins., I. 119. 12. (1828); WaLK., List Dipt. Brit. Mus., I. 153. (1848) et V. suppl. 1., 179. 102. (1854); Harris, Ins. of New England., Ed. III., 602. fig. 261. (1862); Ost.-SAck., Mem. Boston Soc. Nat. Hist., II. 464. 45. (1876); Willist., Trans. Kansas Acad. Sci., X. 138. (1887); Aldr., Catal. North Amer. Dipt., 202. (1905).

cinerarius WiEd., Aussereurop. zweifl. Ins., I. 121. 15. (1828); WaLK., List Dipt. Brit. Mus., V. suppl. 1., 212. 242. (1854) ; Hunter, Trans. Aner. Entom. Soc. Philad., XXVII. 140. (1901).

glaueus Wied., Zoolog. Magaz., I. 3., 42. 3. (1819) et Dipt. exot., 69. 13. (1821).

cinerascens Bıg., Mém. Soc. Zool. France, V. 669. [Atylotus] (1892); v. D. Wulp, Catal. Dipt. South Asia, 63. (1896).

cinerascens King, Nar. Survey, Coats of Austral., II. 467. 181. (1827) ; Wied., Aussereurop. zweifl. Ins., II. 647. 71. (1830); Walk., List Dipt. Brit. Mus., I. 150. (1848) et V. suppl. 1., 253. 443. (18כ̃4).

cinereus WalK., List Dipt. Brit. Mus., I 167. (1848).

cinereus WiED., Dipt. exot., 89. 48. (1821) et Aussereurop. zweifl. Ins., I. 167. 85. (1828); WaLk., List Dipt. Brit. Mus., V. suppl. 1., 199. 189. (1854) ; Hunter, Trans. Amer. Entom. Soc. Philad., XXVII. 140. (1901).

cingulatus MACQ., Dipt. exot., I. 1., 144. 46. (1838); WaLk., List Dipt. Brit. Mus., V. suppl. 1., 183. 118. (1854); Ost.-SAck., Mem. Boston Soc. Nat. Hist., II. 473. (1876); Aldor., Catal. North Amer. Dipt., 202. (1905).

cingulatus Thunb., Nova Acta Upsal., IX. 58. tab. I. fig. 3. (1827). cingulifer Walk, Trans. Entom. Soc. London, n. ser. IV. 123. (1857). cinnamomeus Schin., Novara Reise, Dipt., 90. 26. (1868); Huxter, Trans. Amer. Entom. Soc. Philad., XXVII. 140. (1901).

cinnamoneus Dol., Natuurkund. Tijdschr. Nederl. Indie, XVII. 84. Amboina. 16. (1858); v. D. Wulp, Catal. Dipt. South Asia, 61. (1896). circumdatus WaLk., List Dipt. Brit. Mus., I. 185. (1848).

circumfusus Wied., Aussereurop. zweifl. Ins., II. 624. 21. (1830) ; Walk., List Dipt. Brit. Mus., V. suppl. 1., 188. 140. (1854); Aldr., Catal, North Amer. Dipt., 202. (1905),

Brasilia. Japonia.

America sept. et centr.

Brasilia.

Java.

Australia.

Australia oce.

America mer.

America sept.

Patria ignota.

Reg.Amazon.

America mer.

Patriaignota, Mexico. 
claripennis Bıt., Mém. Soc. Zool. France, V. 675. [Alylolus] (1892). clarus Scuiv., Novara Reise, Dipt., 88. 21. (1868); Hunter, 'Trans. Amer. Entom. Soc. Philad., XXVII. 140. (1901).

clausacella MAcq., Dipt. exot., suppl. 5., 25. 113. (1855); v. D. Wui.P, China. Catal. Dipt. South Asia, 60. (1896).

clausus Macq., Dipt. exot., suppl. 2., 17. 95. (1847); WaLK., List Dipt. Brit. Mus., V. suppl. 1., 201. 199. (1854); Hunter, 'Trans. Amer. Entom. Soc. Philad., XXVII. 140. (1901).

cochaerens Walk., Proc. Linn. Soc. London, VIII. 108. 20. (1865); v. D. Wulp, Catal. Dipt. South Asia, 61. (1896).

coerulescens Macq., Dipt. exot., I. 1., 128. 14. (1838); WalK., List Dipt. Brit. Mus., V. suppl. 1., 243. 390. (1854) ; v. D. Wulp, Catal. Dipt. South Asia, 59. (1896).

coffeatus MAcQ., Dipt. exot., suppl. 2., 23. 109. (1847); WALK., List Dipt. Brit. Mus., V. suppl. 1., 178. 94. (1854); Ost.-SAck., Mem. Boston Soc. Nat. Hist., II. 441. 13. (1876) ; Hrne, Ohio Naturalist, V. 235. (1904) ; ALDR., Catal. North Amer. Dipt., 202. (1905).

? nigripes Wred., Dipt. exot., 75. 24. (1821) et Aussereurop. zweifl. Ins., I. 142. 50. (1828); WaLk., List Dipt. Brit. Mus., V. suppl. 1., 177. 91. (1854); Ostr.-SAck., Mem. Boston Soc. Nat. Hist., II. 473. (1876).

cognatus Lw. = glaucopis MeIG. var.

colombensis Mace., Dipt. exot., suppl. 1.,. 37. 76. tab. IV. fig. 2. (1845); Walk., List Dipt. Brit. Mus., V. suppl. 1., 203. 208. (1854) ; Huster, Trans. Amer. Entom. Soc. Philad., XXVII. 140. (1901).

colon Thuns., Nova Acta Upsal., IX. 54. (1827).

comastes WiLJist. = captonis Marten.

combustus Big.; Annal. Soc. Entom. France, sér. 7. I. 368. 5. (1891). comes Walk., List Dipt. Brit. Mus., IV. 1152. (1849); Ost.-SAck., Mem. Boston Soc. Nat. Hist., II. 473. (1876); ALdr., Catal. Nortlı Amer. Dipt., 202. (1905).

inscitus Walk., List Dipt. Brit. Mus., I. 172. (1848).

- comitans Wied., Aussereurop. zweifl. Ins., I. 175. 96. (1828); WaLK.,

List Dipt. Brit. Mus., V. suppl. 1., 197. 180. (1854); Hunter,

Trans. Amer. Entom. Soc. Philad., XXVII. 140. (1901).

commixtus WALK. = lineola FABR.

compactus ALDR. = lineola FABr.

completus*) WaLk., List Dipt. Brit. Mus., I. 185. (1848); Ost.-SAck.,

Biolog. Central. Amer., Dipt., I. 53. (1886); Aldr., Catal. North Amer. Dipt., 202. (1905).

concolor WaLK., List Dipt. Brit. Mus., I. 179. (1848).

concurrens WaLk., List Dipt. Brit. Mus., V. suppl. 1., 216. 265. (1854).

confinis Walk., List Dipt. Brit. Mus., I. 160. (1848).
Australia.

America mer.

Brasilia.

Nova Guinea.

Java.

America sept.

Columbia.

Brasilia.

Assinia.

America sept.

Brasilia.

I. St. Thomas.

Australia.

Ameriea oce.

America mer.

*) $=$ ?stigma FABR. 
conflnis Zkтт., Insecta Lappon., Dipt., 516. 10. (1838) et Dipt. Scand., I. 118. 19. (1842); Walk., List Dipt. Brit. Mus., V. suppl. 1., 155 et 159. 13. (1854) ; Beck., Acta Soc. Sei. Fenn., XXVI. No. 9., 7. 7. [Therioplectes] (1900).

confligens WALK., List Dipt. Brit. Mus., V. suppl. 1., 326. (1854). tenens WaLk., Newman Zoologist, VIII. Append, LXV. (1850) et List Dipt. Brit. Mus., V. suppl. 1., 213. 247. (1854).

conformis WaLK., List Dipt. Brit. Mus., I. 150. (1848).

Confucius MACQ., Dipt. exot., suppl. 5., 26. 114. (1855).

confusus WaLk., List Dipt. Brit. Mus., I. 147. (1848); Osт.-SAck., Mem. Boston Soc. Nat. Hist., II. 473. (1876); ALdr., Catal. Nortl Amẹr. Dipt., 202. (1905).

conicus Big., Mém. Soc. Zool. France, V. 650. [Atylotus] (1892); v. D. Wurp, Catal. Dipt. South Asia, 63. (1896).

comnexans RICARDO $=$ bromius L.

comexus WALK. $=$ bromius $\mathrm{L}$.

consanguineus MacQ., Dipt. exot., I. 1., 127. 11. (1838); WaLk., List Dipt. Brit. Mus., V. suppl. 1., 244. 393. (1854); v. D. Wulp, Catal. Dipt. South Asia, 59. (1896).

consequa WaLk., Newman Zoologist, VIII. Append., CXXI. (1850) et List Dipt. Brit. Mus., V. suppl. 1., 213. 248. (1854).

consocius W WLK., Insecta Saunders., Dipt., I. 56. (1850); v. D. WULP, Catal. Dipt. South Asia, 60. (1896).

constans Wilk., Jist Dipt. Brit. Mus., I. 186. (1848).

contactus Walk., Insecta Saunders., Dipt., I. 63. (1850).

conterminus WaLK., Insecta Saunders., Dipt., I. 24. (1850) ; Ost.SAck., Mem. Boston Soc. Nat. Hist., II. 473. (1876) ; Aldr., Catal. North Amer. Dipt., 202. (1905).

coracinus Phil., Verh. zool.-bot. Ges. Wien, XV. 718. 23. (1865); Hunter, Trans. Amer. Entom. Soe. Philad., XXVII. 140. (1901). corax Lw., Wien. Entom. Monatschr., VII. 10. 5. (1863).

cordiger MeIG., System. Besehreib., II. 47. 23. (1820); MACQ., Recueil Soc. Sci. Agricult. Lille, 1826. 477. 8. (1826); ZeLL., Isis, 1842. XI., 817. 4. tab. I. fig. 10-13. (18t2); G1мм., Bull. Soc. Imp. Nat. Moscou, XX. 1., 181. 5. (1847); ? Schourz, Zeitschr. f. Entom. Breslau, IV. No. 16., 37. (1850); WALK., Insecta Britann., Dipt., I. 40. 12. (1851) et List Dipt. Brit. Mus., V. suppl. 1., 156 et 163. 28. (1854); Lw., Verh. zool.-bot. Ges. Wien, VIII. 599. 28. (1858); Schis., Fauna Austriaca, Dipt., I. 35. (1862); Brauer, Denkschr. Akad. Wien, XIII. 201. 55. tab. IV. fig. 55. (1880); Gob., Mém. Soc. Linn. Nord France, 1881. 23. 10. (1881); Pand., Revue d'Entom., II. 205. 13. (1883); Brauer, Denkschr. Akad. Wien, XLVII.-. tab. II. fig. 32-34. (1883); Nevhaus, Diptera Marehica, 42. 5. (1886) ; Stropl, Verh. Naturwiss. Ver. Steiermark, XXIX. 1892. 17. (1893) ; Ricardo, Annal. Mag. Nat. Hist., ser. 7. XVI. 199. (1905); Villew., Annal. Soc. Entom, France, LXXIV, 309. 20. (1905).
Eurepa bor., Sibiria.

Brasilia.

Congo.

China bor.

America sept.

India or.

India or.

Brasilia.

India or.

Australia.

Patria ignota.

America sept.

Chile.

Africa mer.

Europa centr.

et mer.,

Africa sept., Asia min. 
atricornis Meıa., System. Beschreib., VII. 59. 47. p. p. (1838);

Zетт., Dipt. Scand., I. 107. 4. (1842) et VIII. 2936. 4. (1849);

WaLK., Insecta Britannica, Dipt., I. 38. ค. (1851); ZeTr., Dipt.

Scand., XI. 4259. 4. (1852) ; WaLk., List Dipt. Brit. Mus., V. suppl. 1., 156 et 162. 25. (1854); Villex., Annal. Soc.

Entom. France, LXXIV. 308. 19. (1905).

Braneri JaEns., Berlin. Entom. Zeitschr., X. 82. 25. a. (1866). latifrons ZETT., Dipt. 'Scand., I. 106. 3. (1812) et VIII. 2936. 3. (1849) ; WALK., List Dipt. Brit. Mus., V. suppl. 1., 156 et 163. 31. (1854); Bovsn., Finl. tvaving. Ins., I. 107. 2: (1861). megacephalus JAenn., Berlin. Entom. Zeitschr., X. 82. 25. (1866). vicinus Egg. (nec MeIg.), Verl. zool. bot. Ges. Wien, IX. 391.

(1859); Schin., Fauna Austriaca, Dipt., I. 35. (1862).

corone Ost.-SAck., Biolog. Central. Amer., Dipt., I. 51. 6. (1886) ; Aldr., Catal. Nortl Amer. Dipt., 202. (1905).

corsicanus MACQ. apud PAND. = anthraeinus MeIg.

costalis Wied., Aussereurop. zweifl. Ins., I. 173. 94. (1828) ; MacQ., Dipt. exot., I. 1., 146. 50. (1838); WaLK., List Dipt. Brit. Mus., V. suppl. 1., 185. 126. (1854); ? BeLL., Saggio di Ditterol. Messic., I. 63. 14. (1859); Ostr-SAck., Mem. Boston Soc. Nat. Hist., JI. 450. 25. (1876); НАRт, Bullet. Illinois State Laborat. of Nat. Hist., IV. 236. (1895); Hine, Ohio State Acad. Sci., Spec. Pap. No. 5., 50. (1903) et Bullet. Unit. Stat. Deptm. of Agricult., Divis. of Entomol., No.44., 58. (1904); Aldr., Catal. Norh Amer. Dipt., 202. (1905).

baltimorensis MacQ., Dipt. exot., suppl. 5., 34. 129. (1855);

Ost.-SAck., Mem. Boston Soc. Nat. Hist., II. 473. (1876).

vicarius WaLK., List Dipt. Brit. Mus., I. 187. (1848).

crassicornis WiED., Dipt. exot., 71. 18. (1821) et Aussereurop. zweifl. Ins., I. 130. 29. (1828); Macq., Suit. à Buff., I. 197. 3. (1834); Walk., List Dipt. Brit. Mus., V. suppl. 1., 181. 111. (1854).

crassus WALK., Insecta Saunders., Dipt., I. 50. (1850); v. D. WulP, India or. Catal. Dipt. South Asia, 60. (1896).

Craverii Bell., Saggio di Ditterolog. Messic., I. 60. 9. (1859) ; Ost.SAck., Catal. Dipt. North Amer., Ed. II., 62 et 229. [?Atylotus] (1878) ; Aldr., Catal. North Amer. Dipt., 202. (1905).

cribellum Osт.-Sack., Biolog. Central. Amer., Dipt., I. 52. 8. tab. I. fig. 10. (1886); Hine, Ohio Naturalist, V. 236. (1901); Alnr., Catal. North Amer. Dipt., 202. (1905).

guttatulus Towns., Trans. Kansas Acad. Sci., XIII. 134. [Dicchlorus] (1892) et Psyche, VIII. No. 260., 147. 1. (1897);

Ricardo, Annal. Mag. Nat. Hist., sor. 7. XIV. 358. (1904); Aldr., Catal. North Amer. Dipt., 204. (1905).

cyaneoviridis Macq., Dipt. exot., suppl. 4., 31. 101. (1850).

Tasmania.

cyaneus MAcQ., Dipt. exot., suppl. 4., 30. 99. tab. II. fig. 7. (1850). Tasmania. cyanops Brauer, Denkschr. Akad. Wien, XLII. 153. 15. 1ab. V. fig. Syria. 15. $(1880)$. 
cymatophorus Ost.-Sack., Mem. Boston Soc. Nat. Hist., II. 444. 16. America sept. (1876) ; Aldr., Catal. North Amer. Dipt., 202. (1905).

decorus Lw., Verh. zool.-bot. Ges. Wien, VIII. 588. 15.(1858); Bravir, Asia min.,

Denkschr. Akad. Wien, XLII. 152. 14. tab. II. fig. 14. (1880); Creta.

Röder, Berlin. Entom. Zeitschr., XXXI. 73. (1887).

De-Filippii Belr., Saggio di Ditterol. Messic., I. 57. 4. (1859); Mexico.

Aldr., Catal. North Amer. Dipt., 202. (1905).

depressus WALK. = luridus FALL.

derivatus WALK., List Dipt. Brit. Mus., I. 151. (18t8) ; Ost.-SACK.,

Mem. Roston Soc. Nat. Hist., II. 473. (1876); Aldr., Catal. North

Amer. Dipt., 202. (1905).

desertus WALK., Newman Zoologist, VIII. Append., LXIX. (1850) Brasilia.

et List Dipt. Brit. Mus., V. suppl. 1., 206. 221. (1854).

detersus Walk., Insecta Saunders., Dipt., I. 31. (1850).

Deyrollei Big. in Thomson: Archiv Entomol., II. 349. 656. (1868). difficilis Wred., Aussereurop. zweifl. Ins., I. 165. 83. (1828) ; Walk.,

List Dipt. Brit. Mus., V. suppl. 1., 262. 492. (1854).

dimidiatus Merg., System. Beschreib., VI. 320. 43. (1830); WALK., List Dipt. Brit. Mus., V. suppl. 1., 156 et 165. 37. (1854).

diminutus WALK., List Dipt. Brit. Mus., I. 183. (1848).

discifer Big., Mém. Soc. Zool. France, V. 684. (1892).

discifer WALK., Insecta Saunders., Dipt., I. 35. tab. II. fig. 2. (1850).

Discus Wred., Aussereurop. zwoifl. Ins., I. 123. 18. (1828); WaLK.,

List Dipt. Brit. Mus., V. suppl. 1., 212. 245. (1854) ; Hunter, Trans.

Amer. Entom. Soc. Philad., XXVII. 140. (1901).

ditaenia Wied., Aussereurop. zweifl. Ins., I. 182. 107.(1828); WALK.,

List Dipt. Brit. Mus., V. suppl. 1., 262. 493. (1854).

ditaeniatus MACQ., Dipt. exot., I. 1., 126. 9. (1838); WALK., List

Dipt. Brit. Mus., V. suppl. 1., 236. 355. (1854); Bezzr, Annal.

Mus. Civ. Genova, ser. 2. XII. (XXXII). 184. 3. (1892).

diurnus WaLK., Insecta Saunders., Dipt., I. 43. (1850).

diversipennis WaLK., List Dipt. Brit. Mus., I. 165. (1848).

dives Rond., Annal. Mus. Civ. Genova, VII. 457. (1875) ; v. D. Wulp, Catal. Dipt. South Asia, 62. (1896).

Dodgei Whitney, Canad. Entomol., XI. 37. (1879); WiLlist., Trans. Kansas Acad. Sci., X. 135. (1887); Hine, Ohio Naturalist, V. 236. (1904) ; Alpr., Catal. North Amer. Dipt., 202. (1905).

doreicus Walk, Proc. Linn. Soc. London, V. 233. 19. (1861); v. D. Wulp, Catal. Dipt. South Asia, 61. (1896).

dorsifer Walk., Trans. Entom. Soc. London, n. ser. V. 273. (1860); Mexico.

Aldr., Catal. North Amer. Dipt., 202. (1905).

dorsiger WiLD., Dipt. exot., 83. 38. (1821) et Aussereurop. zweifl. Ins., J. 172. 92. (1828); WaLk., List Dipt. Brit. Mus., I. 180. (1848) et V. suppl. 1., 202. 202. (1854) ; E. L. Arrir., Bolet. Acad.

Columbia.

Gabon.

Patria ignota.

Patriaignota.

Australia.

Amazonia.

Brasilia.

Brasilia.

Patriaignota.

Ile de France,

Somali.

Prum.hon.sp.

Chile.

Borneo.

America sept.

Nat. Cienc. Córdoba, IV. 13 t. 70. (1882); Hunter, Trans. Amer.

Entom. Soc. Philad., XXVII. 140. (1901).

dorsilinea $\mathrm{W}_{\mathrm{IED}}=$ striatus FABR.

Brasilia, Uruguay. 
dorsivitta WALK., Insecta Saunders., Dipt., I. 39. (1850).

?America mer.

dorsivitta Walk., List Dipt. Brit. Mus., V. suppl. 1., 231. 334.

Africa occid.

(1854) ; Fowler, Proc. Zoolog. Soc. London, 1900. 971. (1900);

?Austen in Johnston: Uganda Protectorate, I. 465. (1902).

dorsobimaculatus MaCQ., Di ${ }_{\mathrm{i}}$ t. exot., suppl. 4., 29. 97. (1850).

dorsomaculatus MacQ., Dipt. exot., suppl. 2., 16. 91. (1847); WaLk., List Dipt. Brit. Mus., V. suppl. 1., 226 et 231. 333. (1854).

dorsonotatus MACQ., Dipt. exot., suppl. 2., 22. 106. (1847); WaLk., List Dipt. Brit. Mus., V. suppl. 1., 178. 98. (1854); Ost.-SAck., Mem. Boston Soc. Nat. Hi st., II. 473. (1876); Aldr., Catal. North Amer. Dipt., 203. (1905).

dorsovittatus MAcQ., Dipt. exot., suppl. 5., 30. 123. (1855); Hunter, Trans. Amer. Entom. Soc. Philad., XXVII. 140. (1901).

dubius MacQ., Dipt. exot., I. 1., 150. 58. (1838); Walk., List Dipt. Brit. Mus., V. suppl. 1., 261. 489. (1854).

duplex Wark., List Dipt. Brit. Mus., V. suppl. 1., 173. 74. (1854); Ost.-SAck., Mem. Boston Soc. Nat. Hist., II. 474. (1876); Aldr., Catal. North Amer. Dipt., 203. (1905). imitans Watk., List Dipt. Brit. Mus., I. 173. (1848).

duplovittatus Rond., Annuar. Sor. Nat. Modena, III. 37. 34. [Agelanius] (1868); E. L. Arris., Bolet. Acad. Nat. Cienc. Córdoba, IV. 134. 71. (1882); Hunter, Trans. Amer. Entom. Soc. Philad., XXVII. 140. (1901).

Eatoni Ricardo, Annal. Mag. Nat. Hist., ser. 7. XVI. 198. [Atylotus] (1905).

ebrius Ost.-SAcK. = ? caliginosus BELL.

edentulus MACQ., Dipt. exot., suppl. 1., 34. 68. tab. III. fig. 13. (1845); Walk., List Dipt. Brit. Mus., V. suppl: 1., 251. 432. (1854). Eggeri Schin., Novara Reise, Dipt., 81. (1868).

gallorum Schrs. in litt.

intermedius EGG., Verh. zool.-bot. Ges. Wien, IX. 389. (1859);

Braver, Denkschr. Akad. Wien, XLII. 182. 42. tab. III et VI. fig. 42. (1880); Goв., Mém. Soc. Linn. Nord France, 1881. 21. 6. (1881); Pand., Revue d'Entomol., II. 202. 4. (1883). elegans Thuns. = quadripunctatus FABR.

elongatus MACQ., Dipt. exot., suppl. 1., 38. 79. tab. IV. fig. 4. (1845); Walk., List Dipt. Brit. Mus., V. suppl. 1., 210. 234. (1854); Hunter, Trans. Amer. Entom. Soc. Philad., XXVII. 140. (1901). elongatus WIED., Aussereurop. zweifl. Ins., I. 124. 19. (1828); WaLK., List Dipt. Brit. Mus., V. suppl. 1., 266. 515. (1854).

Endymion Ost.-Sack., Mem. Boston Soc. Nat. Hist., II. 556. (1876); Aldr., Catal. North Amer. Dipt., 203. (1905).

cngarinensis J JENN. = nigricornis ZETT.

epistates Ost.-SAck., Mem. Boston Soc. Nat. Hist., II. 555. (1876) ;

Hine, Ohio State Acad. Sci., Spec. Pap. No. 5., 50. (1903) et Ohio Naturalist, V. 236. (1901); Aldpr., Catal. North Amer. Dipt., 203. (1905).

Australia.

Algeria.

America sept.

America mer.

Patriaignota.

America sept.

Argentinia.

Algeria.

Tasmania.

Europa mer., Aegyptus, Asia min.

N. Grenada.

Patria ignota.

America sept.

America sept.t. 
californicus Marten, Canad. Entomol., XIV.210. [Therioplectes] (1882).

socius Ost.-SAck., Mem. Boston Soc. Nat. Hist., II. 467. 49. (1876). equestris v. D. Wulp, Notes Leyden Mus., VII. 77. 31. tab. V. fig. Sumatra,Java,

6. (1885) et Catal. Dipt. South Asia, 62. (1896).

Borneo.

Erberi Braver, Denkschr. Akad. Wien, XLII. 151. 13. tab. II. fig. Europa mer.

13. (1880) ; Pand., Revue d'Entomol., II. 212. 31. (1883); Villen.,

Feuille jeun. Natural., XXXV. 60. 1. var. (1905).

erebús Ost.-SAck., Biolog. Central. Amer., Dipt., I. 50. 5. (1886); Americacentr.

Aldr., Catal. Nortl Amer. Dipt., 203. (1905).

erraticus Bı̈., Mém. Soc. Zool. France, V. 676. [Atylotus] (1892). Patriaignota. erythraeus Big., Mém. Soc. Zool. France, V. 661. [Atylotus] (1892); Mexico.

Aldr., Catal. North Amer. Dipt., 203. (1905).

erythraeus Big., Mém. Soc. Zool. France, V. 687. (1892).

erythrocephalus Bıg., Mém. Soc. Zool. France, V. 668. [Atylotus]

Argentinia.

(1892) ; Aldr., Catal. Nortl Amer. Dipt., 203. (1905).

erythrocephalus v. D. WuLp, Tijdschr. v. Entom., XI. 99. 2. (1868) I.Halmalieira.

et Catal. Dipt. South Asia, 61. (1896).

erythroletus WALK. = Reinwardtii WIED.

eutaeniatus Bı́., Mém. Soc. Zool. France, V. 664. [Atylotus] (1892). Brasilia. cxaestuans $\mathrm{L} .=$ ? melanocerus WIED.

exagens WalK., Proc. Linn. Soc. London, VII. 205. 15. (1864); I. Mysol.

v. D. Wulp, Catal. Dipt. South Asia, 61. (1896).

exclamationis GiARD, Journ. Sc. Acad. Lisboa, VIII. 230. (1900); Angola.

Capello et Ivens: De Benguella às Terras de Jácca, II. 367. (1900).

exclusus Pand., Revue d'Entom., II. 208. 19. (1883).

explicatus WaLk., List Dipt. Biit. Mus., V. suppl. 1., 328. (1854); India or.

v. D. Wulp, Catal. Dipt. South Asia, 60. (1896).

expollicatus PAND., Revue d'Entom., II. 218. 45. (1883).

expulsus WalK., List Dipt. Brit. Mus., V. suppl. 1., 329. (1854).

extricans WALK., Proc. Linn. Soc. London, V. 275. 20. (1861); v. D.

Wulp, Catal. Dipt. South Asia, 61. (1896).

exul Ost.-SAck., Mem. Boston Soc. Nat. Hist., II. 557. (1876) ;

Willist., Trans. Kansas Acad. Sci., X. 138. (1887); Towss.,

Trans. Amer. Entom. Soe. Philad., XXII. 60. 15; var? 16. (1895);

HArT, Bullet. Illinois State Eaborat. of Nat. Hist., IV. 233. (1895);

Jouxs., Entom. News Philad., IX. 126. (1898); Aldr., Catal. North

Amer. Dipt., 203. (1905).

abdominalis Wied. (nec FABr.), Dipt. exot., 65. 6. (1821) et.

Aussereurop. zweifl. Ins., I. 116. 7. (1828).

exulans ERscus., Archiv f. Naturgesch., VIII. 1., 270. 247. (1842); Tasmania.

Walk., List Dipt. Brit. Mus., V. suppl. 1., 252. 435. (1854).

facilis Walk., Proc. Linn. Soc. London, VII. 206. 16. (1864); v. D. I. Mysol.

Wulp, Catal. Dipt. South Asia, 61. (1896).

factiosus Wruk., Proc. Linn. Sor. London, IV. 102. 33. (1860); v. D. Celebes.

Wul1, Catal. Dipt. South Asia, 61. (1896). 
fallax MACQ., Dipt. exot., suppl. 1., 32. 63. (1845); WALK., Lișt Dipt. Caffraria. Brit. Mus., V. suppl. 1., 227. 312. (1854).

fallax MACQ., Dipt. exot., suppl. 2., 20. 101. (1847); WALK., List Cayenne. Dipt. Brit. Mus., V. suppl. 1., 212. 244. (1854); Hunter, Trans. Amer. Entom. Soc. Philad., XXVII. 140. (1901).

? fasciatus Deg., Mém. pour serv. à l'hist. d. Ins., VI. 222. 4. (1776); Europa. Vill., Entomol. Linn., III. 560. 25. (1789).

fasciatus FABr., Systema Entomol., 788. 3. (1775), Entomol. System., IV. 364. 8. (1794) et Systema Antliat.," 94. 6. (1805); Wied., Dipt. exot., 73. 21. (1821) et Aussereurop. zweifl. Ins., I. 133. 35. (1828); Walk., List Dipt. Brit. Mus., I. 166. (1848) et V. suppl. 1., 226. 311. (1854).

fascipennis MacQ., Dipt. exot., suppl. 1., 35. 71. tab. IV. fig. 1. (1845) ; Walk., List Dipt. Brit. Mus., V. suppl. 1., 221. 285. (1854); Schin., Novara Reise, Dipt., 93. 32. (1868); Hunter, Trans. Amer. Entom. Soc. Philad., XXVII. 140. (1901).

Felderi v. D. Wulp, Notes Leyden Mus., VII. 78. 32. tab. -V. fig. 7. (1885) et Catal. Dipt. South Asia, 62. (1896).

fenestra Wiluıst., Trans. Kansas Acad. Sci., X. 141. (1887); Aldr., Catal. North Amer. Dipt., 203. (1905).

fenestratus MAcQ., Dipt. exot., I. 1., 135. 28. tab. XVI. fig. 3. (1838); Walk., List Dipt. Brit. Mus., V. suppl. 1., 219. 280. (1854); Hunter, Trans. Amer. Entom. Soc. Philad., XXVII. 140. (1901). fenestratus WALK, = latipes $\mathrm{MACQ}$.

ferreus Walk., List Dipt. Brit. Mus., I. 151. (1848).

ferrifer Walk., Insecta Saunders., Dipt., I. 30.(1850); Aldr., Catal. North Amer. Dipt., 203. (1905).

ferrugineus MEIG. 1804. = glaucopis MeIG.

ferrugineus Meig. 1820. = graecus FABR.

ferrugineus Pal. Beauv., Insect. recueill. en Afrique et en Amérique, 221. tab. III. fig. 2. (1805-1821); Ost.-SAck., Mem. Boston Soc. Nat. Hist., II. 472. (1876); Aldr., Catal. North Amer. Dipt., 203. (1905).

ferrugineus THuns. $=$ ? unicolor WIED.

ferruginosus Walk., Insecta Saunders., Dipt., I. 40. (1850). ferus ScOP. $=$ ? fulvus MEIG.

fervens L., Systema Naturae, Ed. XII., II. 1000. 9. (1767) ; DEg., Mém. pour serv. à l'hist. d. Ins., VI. 228. 4. tab. XXX. fig. 4. (1776) ; FABr., Entomol. System., IV. 365. 12. (1794) et Systema Antliat., 95. 11. (1805); Wied., Aussereurop. zweifl. Ins., I. $18 \pi$. 112. (1828) ; WaLK., List Dipt. Brit. Mus., V. suppl. 1., 209. 227. (1854) ; Hunter, Trans. Amer. Entom. Soc. Philad., XXVII. 140. (1901).

fervidus Walk., Insecta Saunders., Dipt., I. 55. (1850); v. D. Wulr, Asia. Catal. Dipt. South Asia, 60. (1896).

festivus Wied., Aussereurop. zweifl. Ins., I. 135. 37. (1828); WaLk., Brasilia. List Dipt. Brit. Mus., V. suppl. 1., 222. 292. (1854) ; Wilist., Kertész: Catalogus Dipterorum. III. 
Kansas Univ. Quart., III. 195. (1895); Hunter, Trans. Amer.

Entom. Soc. Philad., XXVII. 140. (1901).

fezianus Big., Mém: Soc. Zool. France, V. 638. [Therioplectes] (1892). Marocco.

filiolus Willist., Biolog. Central. Americ., Dipt., I. 261. 19. (1901) ;

Aldr., Catal. North Amer. Dipt., 203. (1905).

? rufiventris Macq., Dipt. exot., I. 1., 141. 39. (1838); WaLk., List Dipt. Brit. Mus., I. 180. (1848) et V. suppl. 1., 189. 150. (1854); Big. in Sagra : Hist. la Isla de Cuba, P. 2. VII. 334. (1856).

finalis Walk., List Dipt. Brit. Mus., V. suppl. 1., 258. 469. (1854). Patriaignota. apicalis Walk., List Dipt. Brit. Mus., I. 176. (1848).

flammans WaLK., List Dipt. Brit. Mus., I. 153. (1818).

flavescens 'Thuns., Nova Acta Upsal., IX. 57. (1827).

flavibarbis MAcQ., Dipt. exot., suppl. 1., 41. 85. (1845); WALK., List

Dipt. Brit. Mus., V. suppl. 1., 204. 211. (1854); Hunter, Trans.

Amer. Entom. Soc. Philad., XXVII. 140. (1901).

flavicans ZELL. = glaucopis MEIG.

flaviceps $\mathrm{ZETT}$. = ? rusticus $\mathrm{I}$.

flavidus Hine, Ohio Naturalist, V. 236. (1904).

Americac.ets.

flavifacies MacQ., Dipt. exot., suppl. 1., 36. 73. (1845); WaLk., List

Dipt. Brit. Mus., V. suppl. 1., 197. 182. (1854); Hunter, Trans.

Amer. Entom. Soc. Philad., XXVIJ. 140. (1901).

flavipes Wied., Aussereurop. zweifl. Ins., I. 137. 41. (1828); WALK., Americasept.,

List Dipt. Brit. Mus., I. 156. (1848) et V. suppl. 1., 177. 93. Sibiria or.

(1854) ; Ost.-Sack., Mem. Boston Soc. Nat. Hist., II. 462. 43.

(1876) ; Bruuer, Denkschr. Akad. Wien, XIII. 141. 5. tab. I. fig.

5. (1880); Aldr., Catal. North Amer. Dipt., 203. (1905).

flaviventris Bıg., Mém. Soc. Zool. France, V. 657. [Atylotus] (1892); India or.

v. D. Wulp, Catal. Dipt. South Asia, 63. (1896).

flaviventris Macq., Dipt. exot., suppl. 3., 11.90. tab. I. fig. 4. (1847); Brasilia.

WALK., List Dipt. Brit. Mus., V. suppl. 1., 211. 238. (1854);

Hunter, Trans. Amer. Entom. Soc. Philad., XXVII. 140. (1901).

flavocinctus BELL. = zonalis KIRBY.

flavus $\mathrm{MACQ} .=$ mexicanus $\mathrm{L}$.

flexilis WaLk., Proc. Linn. Soe. London, IV. 104. 37. (1860) et V.

259. 4. (1861); v. D. Wulp, Catal. Dipt. South Asia, 61. (1896).

formosus WALK., List Dipt. Brit. Mus., I. 148. (1848).

fratellus Willist., Trans. Kansas Akad. Sci., X. 140. (1887); Ricırdo,

Annal. Mag. Nat. Hist., ser. 7. XIV. 357. (1904); Hive, Ohio Naturalist, V. 237. (1904); Aldr., Catal. North Am.r. Dipt., 203. (1905).

hacmatopotides Bia., Mém. Soc. Zool. France, V. 624. [? Dicchlorus] (1892).

fraterculus MacQ., Dipt. exot., suppl. 4., 30. 98. (1850).

Tasmania.

fraterculus WIED, = Gerkei BRAU.

fraternus MAcQ., Dipt. exot., suppl. 1., 31. 61. (1845); INALK., List Caffraria.

Dipt. Brit. Mus., V. suppl. 1., 232. 235. (1854). 
Frenchi Marten, Canad. Entomol., XV. 111. [Therioplectes] (1883); Willist., Trans. Kansas Acad. Sci., X. 136. (1887); Hine, Ohio Naturalist, V. 237. (1904); AtdDR., Catal. North Amer. Dipt., 203. (1905).

frontalis WALK., List Dipt. Brit. Mus., I. 172. (1848); Ost.-SACk., Mem. Boston Soc. Nat. Hist., II. 474. (1876); Aldr., Catal. North Amer. Dipt., 203. (1905).

fronto Ost.-SAck., Mem. Boston Soc. Nat. Hist., II. 431.2 et 473. (1876); Willist., Trans. Kansas Acad. Sci., X. 138. (1887); Alpr, Catal. North Amer. Dipt., 203. (1905).

? cheliopterus Ronc., Nuovi Annal. Sci. Nat. Bologna, ser. 3. II. 192. 30. (1850); Ost.-SAck., Mem. Boston Soc. Nat. Hist., II. $473 .(1876)$.

fullo WaLk., Newman Zoologist, VIII. Append., LXVII. (1850) et Patriaignota. List Dipt. Brit. Mus., V. suppl. 1., 265. 512. (1854).

fulvescens $\mathrm{W}_{\mathrm{ALK}}=$ bicolor $\mathrm{W}_{1 \mathrm{ED}}$.

fulvianus Lw., Öfvers. Kongl. Vet. Akad. Förhandl., XIV. 1857. Caffraria. 339. 15. (1858) et Dipt.-Fauna Südafrika's, I. 35. 3. (1860).

fulvicornis MEIG. = montanus Meig. var.

fulvifasciatus Suit. à Buffon, I. 206. 33. (1834); WaLk., List Dij)t. Patriaignota. Brit. Mus., V. suppl. 1., 262. 494. (1854).

fulviger WALk., Insecta Saunders., Dipt., I. 65. (1850).

fulvilateralis MAcQ., Dipt. exot., I. 1., 133. 24. (1838); WALK., List Dipt. Brit. Mus., V. suppl. 1., 216. 261. (1854); Hunter, Trans. Amer. Entom. Soc. Philad., XXVII. 140. (1901).

fulvimedius WALK., List Dipt. Brit. Mus., I. 152. (1848).

fulvipes PhlL., Verh. zool.-bot. Ges. Wien, XV. 723. 35. (1865); Kırby, Annal. Mag. Nat. Hist., ser. 5. XIII. 457. 2. (1884); Hunter, Trans. Amer. Entom. Soc. Philad., XXVII. 140. (1901).

fulvissimus Rond., Annal. Mus. Civ. Genova, VII. 458. (1875); v. D. Wulp, Catal. Dipt. South Asia, 62. (1896).

fulviventris MacQ., Dipt. exot., suppl. 1., 36. 75. (1845) ; WaLK., List Dipt. Brit. Mus., V. suppl. 1., 211. 239. (1854); Hunter, Trans. Amer. Entom. Soc. Philad., XXVII. 140. (1901).

fulvofrater Walk., List Dipt. Brit. Mus., I. 181. (1848); Ost.-SAck., Mem. Boston Soc. Nat. Hist., II. 474. (1876); ALdr., Catal. North Amer. Dipt., 203. (1905).

fulvulus Wied., Aussereurop. zweifl. Ins., I. 153. 66. (1828); WALK., List Dipt. Brit. Mus., V. suppl. 1., 185. 125. (1854) ; Osт.-SAck., Mem. Boston Soc. Nat. Hist., II. 451. 26. (1876); Aldr., Catal. North Amer. Dipt., 203. (1905).

fulvus Meig., System. Beschreib., II. 61. 40. (1820) ; MacQ., Recueil Soc. Sci. Agricult. Lille, 1826. 483. 16. (1826) et Suit. à Buffon, I. 201. 14. (1834); Duncan, Magaz. Zoolog. and Botan., I. 368. (1837) ; ZeuL., Isis, 1842. XI. 822. 1. (1842); Gimm., Bullet. Soc. Imp. Nat. Moscou, XX. 1., 186. 17. (1847); MAcQ. in Lucas: Explor. scient. de l'Algérie, Zool. III. 425. 31. (1849); Zетr.,

Patriaignota. Cayenne.

Patria ignota. Chile.

Borneo.

Brasilia.

America sept.

America sept.

Europa, Africa sept. 
Dipt. Scand., VIII. 2940. 22. (1849) et XI. 4259. 22. (1852); Lw., Verh. zool.-bot. Ges. Wien, VIII. 596. 23. (1858); BonsD., Finl. tvaving. Ins., I. 111. 15. (1861); Schis., Fauna Austriaca, Dipt., I. 32. (1862); JARosch., Trudy Kharkoff, XI. 343. 9. (1877); Brauer, Denkschr. Akad. Wien, XLII. 169. 32. tab. III. fig. 32. (1880) ; Gов., Mém. Soc. Linn. Nord France, 1881. 29. 1. (1881); Neuhaus, Diptera Marchica, 44. 13. (1886); L. Coccke, Annal. Soc. Entom. Belgique, XXXVI. 138. (1892); Stronl, Mitthoil. Naturwiss. Ver. Steiermark, XXIX. 1892. 15. [Atylotus] (1893); Villen., Annal. Soc. Entom. France, LXXIV. 307. 3, 5. (1905). alpinus Panz., Fauna Germ., XIII. 21. (1794); Cur'r., Brit. Entomol., VIII. 78. (1825); WALK., Insecta Britannica, Dipt., I. 41. 14. (1851) et List Dipt. Brit. Mus., V. suppl. 1., 155 et 160. 18. (1854).

?ferus Scop., Entomol. Carniol., 371. 1009. (1763); Vill., Entom. Linn., III. 556. 11. (1789) ; Schin., Verh. zool.-bot. Ges. Wien, VI. 422. (1856).

rusticus MeIa., System. Beschreib., II. 60. 39. p. p. ㅇ. (1820). sanguisorba Harris, Expos. Engl. Ins., 28. tah. VII. fig. 3. (1782).

var. rufipes Meig., System. Beschreib., II. 59. 37. (1820); MAcQ., Suit. à Buffon, I. 206. 31. (1834); WALK., List Dipt. Brit. Mus., V. suppl. 1., 155 et 160. 17. (1854).

fumatus WiED., Dipt. exot., 91. 50. (1821) et Aussereurop. zweifl. Brasilia. Ins., I. 177. 99. (1828); WaLK., List Dipt. Brit. Mus., V. suppl. 1., 205. 217. (1854) ; Hunter, Trans. Amer. Entom. Soc. Philad., XXVII. 140. (1901).

fumifer WAlK., Proc. Linn. Soc. London, I. 110..32. (1857); v. D. Borneo. Wulp, Catal. Dipt. South Asia, 61. (1896).

fumipennis v. D. Wulr, Sumatra Exped., Dipt., 18. 6. tab. I. fig. 10. Sumatra. (1881) et Catal. Dipt. South Asia, 62. (1896).

fumipennis WIED. = rufus PAL. BeAUV.

funebris Macq., Dipt. exot., suppl. 1., 33. 67. tab. III. fig. 12. ((1845); WALK., List Dipt. Brit. Mus., V. suppl. 1., 254. 446. (1854).

fur Willist., Trans. Kansas Acad. Sci., X. 139. (1887) et Biolog. Central. Americ., Dipt., I. 261. 17. (1901); Aldr., Catal. North Amer. Dipt., 203. (1905).

furcatus Bit., Mém. Soc. Zool. France, V. 631. [Bellardia] (1892). Patriaignota. furens Thunb., Nova Acta Upsal., IX. 58. (1827).

furunculigenus DoL., Natuurkund. Tijdschr. Nederl. Indie, XVII. Patria ignota. 84. 15. (1858) ; v. D. Wulp, Catal. Dipt. South Asia, 61. (1896). furunculus Willist., Biolog. Central. Americ., Dipt., I. 260. 16. Mexico. (1901) ; Aldr., Catal. North Amer. Dipt., 203. (1905).

fuscatus MACQ. = ater Rossi.

fuscicauda Bı́., Mém. Soc. Zool. France, V. 658. [Atylotus] (1892); Ceylon. v. D. Wulp, Catal. Dipt. South Asia, 63. (1896).

fuscicrura Bia., Mém. Soc. Zool. France, V. 662. [Atylotus] (1392); Mexico. Aldr., Catal. North Amer. Dipt., 203. (190\%). 
fuscinervis MacQ., Dipt. exot., I. 2., 184. (1839); WaLK., List Dipt. ? Prom.bon sp. Brit. Mus., V. suppl. 1., 264. 504. (1854).

fuscipalpis Bı́., Mém. Soc. Zool. France, V. 681. (1892); Hine, America sept. Ohio Naturalist, V. 245. (1904); Aldr., Catal. North Amer. Dipt., 203. (1905).

fuscipennis WiEd., Aussereurop. zweifl. Ins., I. 179. 102. (1828); WaLk., List Dipt. Brit. Mus., I. 166. (1848) et V. suppl. 1., 220. 282. (1854) ; Hunter, Trans. Amer. Entom. Soc. Philad., XXVII. 140. (1901).

fuscofasciatus MACQ., Dipt. exot., I. 1., 136. 30. (1838); WALK., List Dipt. Brit. Mus., V. suppl. 1., 214. 254. (1854); Hunter, Trans. Amer. Entom. Soc. Philad., XXVII. 140. (1901). fusconervosus MAcQ. = ? turbidus WIED.

fuscopunctatus Macq., Dipt. exot., suppl. 4., 34. 108. (1850); OsT.SAck., Mem. Boston Soc. Nat. Hist., II. 432. 4 et 559. (1876) et Catal. Dipt. North Amer., Ed. II., 58 et 228. 80. (1878) ; Aldr., Catal. North Amer. Dipt., 203. (1905).

imitans WALK., List Dipt. Brit. Mus., I. 146. (1848); Ost.-SACK., Mem. Boston Soc. Nat. Hist., II. 473. (1876).

fuscus Wied., Zoolog. Magaz., I. 3., 41. 1. (1819), Dipt. exot., 68. 12. (1821) et Aussereurop. zweifl. Ins., I. 121. 14 et 556. 28. (1828); WALK., List Dipt. Brit. Mus., V. suppl. 1., 201. 198. (1854); Hunter, Trans. Amer. Entom, Soc. Philad., XXVII. 141. (1901). gabonensis MACQ., Dipt exot., suppl. 5., 23. 110. [garonensis] (1855); Bıg. in Thомs. : Archiv. Entomol., II. 348. 655. (1858).

gagates $\mathrm{Lw} .=$ nigrita $\mathrm{FABR}$.

gagatinus PHIL., Verh. zool.-bot. Ges. Wien, XV. 719. 25. (1865); Hunter, Trans. Amer. Entom. Soc. Philad., XXVII. 141. [gagantinus] (1901).

gallorum Scins. = Eggeri Schis.

geniculatus v. D. Wulp, Sumatra Exped., Dipt., 16. 2. tab. I. fig. 7 (1881) et Catal. Dipt. South Asia, 62. (1896)

gentilis Erichs., Archiv f. Naturgesch., ViII. 1., 271. 249. (1842); Tasmania. WaLk., List Dipt. Brit. Mus., V. suppl. 1., 254. 445. (1854).

Gerkei Brauer, Denkschr. Akad. Wien, XLII. 205. 58. tab. IV. fig 58. (1880).

fraterculus WiEd, in Coll. Winth.

giganteus Deg., Mém. pour serv. à l'hist. d'Ins., VI. 226. 1. tab. XXX. fig. 1. (1776); Ost.-Sack., Mem. Boston Soc. Nat. Hist., II. 458. 37. (1876); v. D. Wulp, Tijdschr. v. Entomol., XXIV. 158. 3. (1881) ; Wrllist., Trans. Kansas Acad. Sci., X. 139. (1887); HART, Bullet. Illin. Stat. Laborat. of Nat. Hist., IV. 247. (1895); Hine, Ohio State Acad. Sci., Spec. Pap. No. 5., 51. (1903) ; Aldr., Catal. North Amer. Dipt., 204. (1905).

bicolor MACQ., Dipt. exot., suppl. 2., 21. 105. (1847); WaLK., List Dipt. Brit. Mus., V. suppl. 1., 183. 120. (1854); OsT.SAcK., Mem. Boston Soc. Nat. Hist., II. 473. (1876). 
caesiofusciatus MacQ., Dipt. exot., suppl. 5., 32. 126. (1855);

Ost.-SAck., Mem. Boston Soc. Nat. Hist., II. 473. (1876).

catenatus WALK., List Dipt. Brit. Mus., I. 148. p. p. (1848).

lineatus Fabr., Spec. Insect., II. 455. 4. (1781), Entomol. System., IV. 363. 5. (1794) et Systema Antliat., 94. 3. (1805);

Wied., Dipt. exot., 63. 3. (1821) et Aussereurop. zweifl. Ins.,

I. 115. 4. (1828) ; Walk., List Dipt. Brit. Mus., I. 146. (1848) et V. suppl. 1., 180. 107. (1854).

gigas Herbst, fremeinnütz. Naturgesch. d. Thierr., VIII. 122. tab.

LXVII. fig. 2. (1787) ; Lw., Verh. zool.-bot. Ges. Wien, VIII. 583. (1858) ; Schin., Fauna Austriaca, Dipt., I. 29. (1862) ; Portsci., Trudy Russk. Entom., X. 148. (1877); Brauer, Denkschr. Akad. Wien, XLII. 165. 27. tab. II. fig. 27. (1880); Goв., Mém. Soc. Linn. Nord France, 1881. 13. 3. [Therioplectes] (1881); PAND., Revue d'Entomol., II. 217. 43. (1883) ; РоRтsch., Horae Soc. Entom. Ross., XVIII. 123. (1883) ; Ost.-SAck., Wien. Entom. Zeitg., III. 255. (1884); L. Coucke, Annal. Soc. Entom. Belgique, XXXVI. 136. (1892); BEzzi, Bull. Soc. Entom. Ital., XXX. 27. 28. [Atylotus] (1898).

albipes Fabr., Entomol. System., IV. 364. 11. (1794); Coqueb.,

Illust. Ieonogr. Insect., 111. tab. XXXV. fig. 3. (1804); Meig., Klass., I. 171. b. (1804) ; Fabr., Systema Antliat., 95. 9. (1805) ; Latr., Gen. Crust. et Ins., IV. 283. (1809) ; Meig., System. Beschreib., II. 45. 20. (1820); MAcQ., Recueil Soc. Sci. Agricult. Lille, 1826. 476. 7. (1826) et Suit. à Buffon, I. 200. 10. (1834) ; Grum., Bull. Soc. Imp. Nat. Moscou, XX. 1., 184. 13. (1847); Walk., List Dipt. Brit. Mus., I. 156. (1848) et V. suppl. 1., 156 et 169. 49. (1854); Lw., Verh. zool.-bot. Ges. Wien, VIII. 582. 7. (1858) et Wien. Entom. Monatschr., VII. 33. 1. (1863) ; Villen., Annal. Soc. Entom. France, LXXIV. 309. 26. (1905).

grossus Thunb., Nova Acta Upsal., IX. 57. tab. I. fig: 1. (1827). ignotus Rossi, Fauna Etrusc., II. 320. 1546. (1790).

ursus A. Costa, Il Giamb. Vico Napoli, II. 454. [Brachytomus] (1857).

gigas MACQ., Suit. à Buffon, I. 200. 12. (1834); WALK., List Dipt. Patriaignota. Brit. Mus., V. suppl. 1., 263. 500. (1854).

gilanus Towns., Psyche, VIII. No. 255., 92. 7. (1897); Hine, Ohio Naturalist, V. 238. (1904); Aldr., Catal. North Amer. Dipt., 204. (1905).

glaber Bia., Mém. Soc. Zool. France, V. 678. (1892); v. D. Wulp, India or.

Catal. Dipt. South Asia, 63. (1896).

glaucescens ScHin. p. p. = bromius L. var.

glancescens ScHiN. p. p. = maculicornis ZеTT.

glaucopis MeIG., System. Beschreib., II. 48. 24. p. p. tab. XIII. fig. Europa. 28. (1820) ; Macq., Recueil Soc. Sci. Agricult. Lille, 1826. 478. 9. (1826) et Suit. à Buffon, I. 205. 30. (1834); WAHLBG., Kongl. Vet. 
Akad. Handl., 1838. 6. 2. (1838); Zetr., Dipt. Scand., I. 108. 6. (1842) et VIII. 2936. 6. (1849) ; Walk., Insecta Britann., Dipt., I. 38. 4. (1851) et List Dipt. Brit. Mus., V. suppl. 1., 156 et 164. 34. (1854) ; Scuns., Fauna Austriaca, Dipt., I. 35. (1862) ; JAEnn., Berlin. Entom. Zeitschr., X. 81. 23. (1866); Brauer, Denkschr. Akad. Wien, XLII. 198. 54. tab. VI. fig. 54. (1880); Gов., Mém. Soc. Linn. Nord France, 1881. 23. 9. (1881); Pand., Revue d'Entomol., II. 208. 21. (1883); Neuhaus, Diptera Marchica, 42. 6. (1886) ; Girschn., Berlin. Entom. Zeitschr., XXXI. - tab. III. fig. 2. (1887); SтroвL, Mittheil. Naturwiss. Ver. Steiermark, XXIX. 1892. 15. (1893); Villew., Annal. Soc. Entom. France, LXXIV. 308. 10. (1905).

chlorophthalmus Meig., Systom. Beschreib., II. 58. 36. (1820);

Walk., List Dipt. Brit. Mus., V. suppl. 1., 155 et 157. 3. (1854).

ferrugineus Meig., Klassif., I. 169. 10. (1804).

flavicans ZeLL., Isis, 1842. XI. 819. tab. I. fig. 20-22. (1842);

Scholtz, Zeitschr. f. Entomol. Breslau, IV. No. 16., 37. (1850).

var. cognatus Lw., Verl. zool.-bot. Ges. Wien, VIII. 602. 31. Europa. (1858); Brauer, Denkschr. Akad. Wien, XlII. 200. 54 a. (1880); Strobl, Mittheil. Naturwiss. Ver. Steiermark, XXIX. 1892. 17. (1893).

lumulatus MeIG., System. Beschreib., II. 49. 25. p. p. (1820);

Wali., List Dipt. Brit. Mus., V. suppl. 1., 156 et 164. 33. (1854) ; Lw., Verl.. zool.-bot. Ges. Wien, VIII. 601. 30. (1858); Villew., Annal. Soc. Entom. France, LXXIV. 307. 9. (1905).

glaucopis MeIG. p. p. = nemoralis Meig.

glaucus Meig. p. p. $=$ bromius L.

glaucus Meig. p. p. = tergestinus EGG.

glaucus WIED. = cinerarius WIED.

gracilis Wied., Aussereurop. zweifl. Ins., I. 156. 71. (1828) ; WALK., List Dipt. Brit. Mus., I. 174. (1848) et V. suppl. 1., 186. 130. (1854) ; Ost.-SAck., Mem. Boston Soc. Nat. Hist., II. 473. (1876) et Catal. Dipt. North Amer., Ed. II., 58 et 228. 81. (1878); WILList., Trans. Kansas Acad. Sci., X. 140. (1887); Aldr., Catal. North Amer. Dipt., 204. (1905).

graecus FABR., Entomol. System., IV. 368. 29. (1794); MeiG., Klassif., I. 173. g. (1804); FABR., Systema Antliat., 100. 34. (1805); Meig., System. Beschreib., II. 53. 30. (1820); ? Macq., Recueil Soc. Sci. Agricult. Lille, 1826. 480. 12. (1826) et Suit. à Buffon, I. 202. 20. (1834) ; ? Giм.., Bull. Soc. Imp. Nat. Moscou, XX. 1., 183. 10. (1847) ; ? Walk., List Dipt. Brit. Mus., I. 169. (1848) et ? V. suppl. 1., 155 et 157. 5. (1854); Braver, Denkschr. Akad. Wien, XLII. 178. 39. tab. III. fig. 39. (1880); Gов., Mém. Soc. Linn. Nord France, 1881. 27. (1881); PAND., Revue d'Entomol., II. 203. 8. (1883) ; Strobl, Mittheil. Naturwiss. Ver. Steiermark,

America sept.

Europa centr. et mer., Asia min. 
XXIX. 1892. 15. (1893); Villen., Annal. Soc. Entom. France, LXXIV. 308. 11. (1905).

ferrugineus Meia., System. Beschreib., II. 60. 38. (1820) ; Ginn., Bull. Soc. Imp. Nat. Moscou, XV. 664. (1842) et XX. 1., 182. 7. (1847) ; WALK., List Dipt. Brit. Mus., V. suppl. 1., 157 et 169. 53. (1854); Lw., Verh. zool.-bot. Ges. Wien, VIII. 607. 38. (1858); Neunaus, Diptera Marchica, 42. 4. (1886); Villen., Annal. Soe. Entom. France, LXXIV. 306. 1. (1905). infusus WaLK., Insecta Saunders., Dipt., I. 23. (1851).

liburnicus WIED. in litt. ap. MeIG.

?propinquus PaLm, Verh. zool.-bot. Ges. Wien, XXV. 411. 1. (1875).

segmentarius Brultè, Exped. scient. d. Morée, III. 304. 659. (1834).

ursulus Meg. in litt. a]). Meig.

graecus Meig. p. p. = Mikii Brauer.

gratus Lw., Öfvers. Kongl. Vet. Akad. Förhandl., XIV. 1857. 340.

23. (1858) et Dipt.-Fauna Südafrika's, I. 42. 13. (1860); Karscir,

Berlin. Entom. Zeitschr., XXXI. 370. 4. (1887).

gravis Hurron, Trans. New Zealand Instit., XXXIII. 13. (1901).

gregarius Erichs., Archiv f. Naturgesch., VIII. 1., 271. 248. (1842);

Walk., List Dipt. Brit. Mus., V. suppl. 1., 252. 436. (1854).

grossus Thunв. = gigas Herbst.

guineensis Wred., Analecta Entomol., 21. 7. (1824) et Aussereurop.

Caffraria.

zweifl. Ins., I. 144. 54. (1828); WaLK., List Dipt. Brit. Mus., V. suppl. 1., 235. 350. (1854).

guttatulus Towss. $=$ cribellum OT.-SAck.

guttatus WiED., Dipt. exot., I. 73. 20. (1821) et Aussereurop. zweifl.

Ins., I. 133. 34. (1828); Walk., List Dipt. Brit. Mus., V. suppl.

1., 182. 116. (1854); Hunter, Trans. Amer. Entom. Soc. Philad.,

XXVII. 141. (1901).

guttipennis WiEd., Aussereurop. zweifl. Ins., I. 153. 67. (1828);

Walk., List Dipt. Brit. Mus., V. suppl. 1., 220. 283. (1854);

Scins., Novara Reise, Dipt., 88.22. (1868); Hunter, Trans. Amer.

Entom. Soc. Philad., XXVII. 141. (1901).

guyanens Hunter = guyanensis MACQ.

guyanensis Macq., Dipt. exot., suppl. 1., 41. 84. tab. IV. fig. 9. (1845); Guyana.

WalK., List Dipt. Brit. Mus., V. suppl. 1., 211. 241. (1854).

guyanens Hunter, Trans. Amer. Entom. Soc. Philad., XXVII.

141. (1901) [lapsus].

haemagogus Wrulist., Biolog. Central. Americ., Dipt., I. 261. 18. Mexico.

(1901); Aldr., Catal. North Amer. Dipt., 204. (1905).

haemaphorus MARTEN $=$ sonomensis Ost.-SACK.

haematopoticles $\mathrm{BIG} .=$ fratellus WrLI.IST.

haematopotoides JAENn., Berlin. Entom. Zeitschr., X.77.16.(1866); Europa contr.

Brauer, Denkschr. Akad. Wien, XLII. 203. 57. tab. IV. fig. 57.

(1880) ; Pañd., Revue d'Entomol., II. 208. 20. (1883); Beck., 
Berlin. Entom. Zeitschr., XXXI. 106. 24. [Therioplectes] (1887); Strobi, Mittheil. Naturwiss. Ver. Steiermark, XXIX. 1892. 17. (1893).

hamatus v. D. Wulp, Tijdschr, v. Entom., XXIV. 159. 9. tab. XV. fig. 7. (1881); Hunter, Trans. Amer. Entom. Soc. Philad., 141. (1901).

hebes WaLK., List Dipt. Brit. Mus., I. 159. (1848).

Heydenianus $\mathrm{J}_{\mathrm{AENN}}$ = aterrimus MEIG.

Hilarii Macq., Dipt. exot., I. 2., 185. (1839); WaLk., List Dipt. Brit. Mus., V. suppl. 1., 210. 236. (1854); E. L. Arrib., Bolet. Acid. Nat. Cienc. Córdoba, IV. 134. 72. (1882); Hunter, Trans. Amer. Entom. Soc. Philad., XXVII. 141. (1901).

hilaris WALK., Insecta Saunders., Dipt., 49. tab. II. fig. 3. (1850); v. D. Wulp, Catal. Dipt. South Asia, 60. (1896).

Hinei Johns., Psyche, XI. 15. [Therioplectes] (1904).

politus Joins., Entom. News Philad., XI. 325. [Therioplectes]

(1900) ; Aldr., Catal. North Amer. Dipt., 206. (1905).

hirsutus ViLL., Entomol. Linn., III. 561. 28. (1789).

hirticeps Lw., Wien. Entom. Monatsclır., II. 105. 7. (1858).

hirtioculatus MACQ., Dipt. exot., suppl. 5., 33. 128. (1855); Ost:Sack., Mem. Boston Soc. Nat. Hist., II. 473. (1876); Aldr., Catal. North Amer. Dipt., 204. (1905).

cerastes Ost.-SAck., Mom. Boston Soc. Nat. Hist., II. 462. 42 et 473. (1876) et Catal. Dipt. North Amer., Ed. II., 57 et 227. 78. (1878); Hrne, Ohio Stat. Acad. Sci., Spec. Pap. No. 5., 49. (1903).

hirtitibia WALK., Insecta Saunders., Dipt., I. 33. (1850).

hirtulus Bıg., Mém. Soc. Zool. France, V. 641. [Therioplectes] (1892); Hine, Ohio Naturalist, V. 245. (1904); Aldr., Catal. North Amer. Dipt., 204. (1905).

hirtuosus PhiL., Verh. zool.-bot. Ges. Wien, XV. 719. 26. (1865); Huxter, Trans. Amer. Entom. Soc. Philad., XXVIr. 141. (1901).

hirtus WaLK., Insecta Saunders., Dipt., I. 52. (1850); v. D. WurP, Catal. Dipt. South Asia, 60. (1896).

hispidus WALK., Insecta Saunders., Dipt., I. 63. (1850).

histrio WiEd., Aussereurop. zweifl. Ins., II. 625. 24. (1830); WaLk.,

List Dipt. Brit. Mus., V. suppl. 1., 219. 279. (1854); Scuin., Novara Reise, Dipt., 87. 19. (1868); Hunter, Trans. Amer. Entom. Soc. Philad., XXVII. 141. (1901).

hoang MacQ., Dipt. exot., suppl. 5., 26. 115. (1855).

honestus WaLK., Insecta Saunders., Dipt., I. 64. (1850).

humeralis $\mathrm{MEG} .=$ spectabilis $\mathrm{Lw}$.

humilis Coquill., Proc. Unit. Stat. Nat. Mus., XXI. 311. (1898).

humillimus Walk., Trans. Entom. Soc. London, n. ser. IV. 124. (1857) ; v. D. Wulp, Catal. Dipt. South Asia, 60. (1896).

hyalinipennis Hine, Canad. Entomol., XXXV. 244. (1903) et Ohio Naturalist, V. 238. (1904); Aldr., Catal. North Amer. Dipt., 204. (1905).
Argentinia.

Africa.

Brasilia.

India or.

America sept.

Europa.

Japonia.

Americasept.

Columbia.

America sept.

Chile.

India or.

Patria ignota. Brasilia.

China bor.

Patria ignota.

Japonia.

Celebes.

America sept. 
hybridus Wied., Aussereurop. zweifl. Ins., I. 557. 31. (1828); WaLk., Borneo,

List Dipt. Brit. Mus., V. suppl. 1., 246. 403. (1854); v. D. Wulp, Macao.

Catal. Dipt. South Asia, 59. (1896).

hyperythreus Bı́., Mém. Soc. Zool. France, V.674. [Alylotus] (1892). Australia. hypoleucus Bıg., Annal. Soc. Entom. France, sér. 7. I. 368. 6. Assinia. [Atylotus] (1891).

ignobilis Rovı., Annal. Mus. Civ. Genova, VII. 457. (1875); v. D. Borneo.

Wulp, Catal. Dipt. South Asia, 62. (1896).

ignotus Ross1 = gigas HerBst.

illotus Ost.-SAck., Mem. Boston Soc. Nat. Hist., II. 469: 51. (1876) ;

Hine, Ohio Naturalist, V. 238. (1904); Aldpr., Catal. North Amer.

Dipt., 204. (1905).

imbecillus Karsch, Berlin. Entom. Zeitschr., XXXI. 370. 7. (1887). Africa or. imitans WALK. = fuscopunctatus MaCQ.

imitans $\mathrm{W}_{\mathrm{ALK}}=$ duplex WALK.

immanis W1ED., Aussereurop. zweifl. Ins., I. 123. 17. (1828); WALK.,

List Dipt. Brit. Mus., V. suppl. 1., 246. 402. (1854); v. D. Wulp,

Sumatra Exped., Dipt., 16. 1. (1881), Notes Leyden Mus., VII.

71. 22. (1885) et Catal. Dipt. South Asia, 59. (1896).

immixtus Walk.; Proc. Linn. Soc. London, IV. 103. 36. (1860); Celebes.

v. D. Wulp, Catal. Dipt. South Asia, 61. (1896).

impar Walk., Newman Zoologist, VIII. Append. LXXI. (1850) et

List Dipt. Brit. Mus., V. suppl. 1., 255. 449. (1854) ; Hutron,

Catal. New Zealand Dipt. etc., 19. (1881); Hudsos, Manual of

New Zealand Entomol., 54. tab. VI. fig. 6. (??) (1892); Hutron,

Trans. New Zealand. Instit., XXXIII. 11. (1901).

imperfectus WaLK., List Dipt. Brit. Mus., I. 179. (1848).

imponens WaLk., Trans. Entom. Soc. London, n. ser. IV. 122. (1857). importunus Macq., Dipt. exot., suppl. 2., 18. 98. (18t7); WALK.,

List Dipt. Brit. Mus., V. suppl. 1., 198. 185. (1854); Hunter,

Trans. Amer. Entom. Soc. Philad., XXVII. 141. (1901).

importunus WiEd, Aussereurop. zweifl. Ins., I. 127. 24. (1828);

Walk., List Dipt. Brit. Mus., V. suppl. 1., 219. 278. (1854);

WilList., Kansas Univ. Quart., III. 195. (1895).

impressus WiEd., Aussereurop. zweifl. Ins., I. 126. 23. (1828);

Walk., List Dipt. Brit. Mus., I. 146. (1848) et V. suppl. 1., 205.

215. (1854) ; E. L. Arrib., Bolet. Acad. Nat. Cienc. Córdoba, IV.

134. 73. (1882); Hunter, Trans. Amer. Entom. \$oc. Philad.,

XXVII. 141. (1901).

impurus Karsci, Berlin. Entom. Zeitschr., XXXI. 370. 6. tab. IV. Africa or.

fig. 2. (1887).

inanis $\mathrm{FABR}_{\mathrm{A}}=$ mexicanus $\mathrm{L}$.

incertus Macq., Dipt. exot., I. 1., 147. 53. (1838); Blanch. in Gay: Chile.

Hist. fis. y polit. de Chile, Zool. VII. 392. 1. tab. II. fig. 1. (185̃);

Walk., List Dipt. Brit. Mus., V. suppl. 1., 263. 501. (1854);

Phil., Verh. zool.-bot. Ges. Wien, XV. 713. 1. (1865); Huntrer,

Trans. Amer. Entom. Soc. Philad., XXVII. 141. (1901).

Australia.

Brasilia.

Brasilia.

Brasilia,

Paraguay.

Brasilia,

Guyana. 
incipiens WaLk., Trans. Entom. Soc. London, n. ser. V. 275. (1861). incisisuralis MAcQ., Dipt. exot., suppl. 2., 21. 104. (1847); WAK., List Dipt. Brit. Mus., V. suppl. 1., 179. 100. (1854); Huxten, Traus. Amer. Entom. Soc. Philad., XXVII. 141. (1901).

incisus WALK., Insecta Saunders., Dipt., I. 26. (1850); Osт.-SAck., Mem. Boston Soc. Nat. Hist., II. 474. (1876); A rdr., Catal. North Amer. Dipt., 204. (1905).

inconspicuus WaLK., List Dipt. Brit. Mus., I. 171. (1848).

incultus v. D. Wulp, Sumatra Exped., Dipt., 17. 4. tab. I. fig. 8. (1881) et Catal. Dipt. South Asia, 62. (1896).

indecisus Bra., Mém. Soc. Zool. France, V. 666. [Alylotus] (1892). indicus FABr., Systema Antliat., 103. 48. (1805); Wied., Dipt. exot., 75. 26. (1821) et Aussereurop. zweifl. Ins., I. 144. 53. (1828); ?? WALK., List Dipt. Brit. Mus., I. 154. (1848) et V. suppl. 1., 245. 400. (1854); Hunter, Trans. Amer. Eistom. Soe. Plilad., XXVII. 141. (1901).

indistinctus Big., Mém. Soc. Zool. France, V. 689. (1892); v. n. Nova fuinea. Wulp, Catal. Dipt. South Asia, 63. (1896).

infans WaLK., Insecta Saunders., Dipt., I. 45. (1850).

infestans Macq., Dipt. exot., suppl. 2., 15. 90. (1847); WaLk., Iist

Dipt. Brit. Mus., V. suppl. 1., 225 et 230. 326. (1854).

informatus Hunter = infumatus Phil.

infumatus Phil., Verh. zool.-bot. Ges. Wien, XV. 718. 21. (1865). informatus IIUnter, Trans. Amer. Entom. Soe. Philad., XXVII. 141. (1901) lapsus.

infuscatus $\mathrm{Lw}_{\mathrm{w}}=$ apricus MeIG. infusus WALK. = graecus FABR.

inhambanensis Bertol.rvi, Mem. Accad. Sci. Istit. Bologna, XII. 16. Mosambique. 19. tab. I. fig. 7. (1862).

innotabilis WALK., List Dipt. Brit. Mus., I. 177. (1848).

innotescens WaLk., List Dipt. Brit, Mus., V. supll. 1., 327. (185t). inornatus Pril., Verh. zool.-bot. Ges. Wien, XV. 718. 22. (1865);

v. D. Wur.p, Tijdschr. v. Entomol., XXIV. 159. 8.(1881); Hunter,

Trans. Amer. Entom. Soc. Philad., XXVII. 141. (1901).

inscitus WaLK., List Dipt. Brit. Mus., I. 161. (1848); v. D. Wulp, India or.

Catal. Dipt. South Asia, 59. (1896).

inscitus WALK. = comes WALK.

insignis Lw., Öfvers. Kongl. Vet. Akad. Förhandl., XIV. 1857. 341. Caffraria.

25. (1858) et Dipt.-Fauna Südafrika's, I. 44. 16. (1860).

insuetus Ost.-SAck., Bullet. Unit. Stat. Geolog. Surv., III. 219. 5. Americasept.

(1876) ; Coquil., Proc. Washington Acad. Sci., II. 407. (1900);

Hine, Ohio Naturalist, V. 238. (1904); Beutenmt'll., Bullet.

Americ. Mus. Nat. Hist., XX. 87. (1904); Coquill., Harriman

Alaska Exped., IX. Pt. II., 21. (1904); ALDr., Catal. North Amer.

Dipt., 204. (1905).

insularis WALK., List Dipt. Brit. Mus., I. 184. (1848).

I. Sandwich.

insurgens Walk., Proc. Linn. Soc. London, V. 276. 21. (1861); Nova Guinea, 
Ost.-SAck., Annal. Mus. Civ. Genova, XVI. 417. (1882); v. D.

Wulp, Catal. Dipt. South Asia, 61. (1896).

intensivus Towss., Psyche, VIII. No. 255., 93. 8. (1897); Hine,

Olico Naturalist, V. 238. (1904); Aldr., Catal. North Amer. Dipt., 204. (1905).

intereuns WALK., Insecta Saunders., Dipt., I. 450. (1856).

intermedius EGG. = Eggeri Scmin.

intermedius Walk., List Dipt. Brit. Mus., I. 173. (1848) ; Ost.-SAck.,

Mem. Boston Soc. Nat. Hist., II. 474. (1876); ALDR., Catal. North Amer. Dipt., 204. (1905).

internus Walk., List Dipt. Brit. Mus., I. 164. (1848); v. D. WulP, Catal. Dipt. South Asia, 60. (1896).

interpositus Rovd., Annuar. Soc. Nat. Modena, III. 38. 35. [Agelanius] (1868); E. L. Annib., Bolet. Acad. Nat. Ciene. Córdoba, IV. 134. 74. (1882); Hunter, Trans. Amor. Entom. Soc. Philad., XXVII. 141. (1901).

intersectus Fourcr., Entom. Paris., II. 457. 6. (1785); ViLL., Entom. Europa. Linn., III. 588. 17. (1789).

ispahanicus Roxd., Annal. Mus. Civ. Genova, IV. 300. 8. [aspahani- Persia sept. cus] (1873).

istriensis MeIG. = umbrinus MeIG.

Ixion Ost.-Sıck., Berlin. Entom. Zeitschr., XXVI. 99. (1882); v. D. I.Philippinae. Wulp, Catal. Dipt. South Asia, 62. (1896).

ixyostactes Wied., Aussereurop. zweifl. Ins., I. 190. 119. (1828);

WALK., List Dipt. Brit. Mus., V. suppl. 1., 203. 204. (1854) ; HuNTER, Trans. Amer. Entom. Soc. Philad., XXVII. 141. (1901).

Janus WALK., Insecta Saunders., Dipt., J. 61. (1850).

japonicus Brg., Mém. Soc. Zool. France, V. 632. [Dichelacera] (1892);

BezzI, Katal. d. paläarkt. Dipt., II. 57. [? Dichelacera] (1903);

Ricalrdo, Annal. Mag. Nat. Hist., ser. 7. XIV. 368. (1904).

javanus FABR., Systema Antliat., 103. 46. (1805); WiED., Dipt. exot.,

93. 54. (1821) et Aussereurop. zweifl. Ins., I. 183. 108. (1828);

WALK., List Dipt. Brit. Mus., V. suppl. 1., 248. 411. (1854);

v. D. Wulp, Catal. Dipt. South Asia, 59. (1896).

Joïdus Bra., Mém. Soc. Zool. France, V. 657. [Atylotus] (1892); v. D. India or.

Wulp, Catal. Dipt. South Asia, 63. (1896).

jucundus Walk., List Dipt. Brit. Mus., I. 187. (1848); v. D. Wulp, China.

Catal. Dipt. South Asia, 60. (1896).

justorius Rond., Annal. Mus. Civ. Genova, VII. 455. (1875); v. D. Borneo.

Wulp, Catal. Dipt. South Asia, 62. (1896).

lacrymans Big., Mém. Soc. Zool. France, V. 669. [Atylotus] (1892); Java.

v. D. Wulp, Catal. Dipt. South Asia, 63. (1896).

laevifrons Lw., Öfvers. Kongl. Vet. Akad. Förhandl., XV. 336. 2 Prom.bon.sp. bis. (1858) et Dipt.-Fauna Südafrika's, I. 35. 4. (1860).

lagenaferus MacQ., Dipt. exot., I. 1., 148. 54. (1838); Walk., List Patriaignota.

Dipt. Brit. Mus., V. suppl. 1., 265. 511. (1854).

Laglaisei Big., Mém. Soc. Zool. France, V. 673. [Atylotus] (189, ; I. Waigou.

v. D. Wulp, Catal. Dipt. South Asia, 63. (1896). 
lama Portscir., Horae Soc. Entom. Ross., XXVI. 201. tab. I. fig. 1. Mongolia (1891).

chinen sis.

laniger WiED. = plebejus FALL.

laotianus Big., Nolıv. Archiv. du Mus. d'Hist. Nat. Paris, sér. 3. II. Ins. Laos. 205. [Atylotus] (1890); v. D. Wulp, Catal. Dipt. South Asia, 62. (1896).

lapponicus WahlBG., Öfvers. Kongl. Vet. Akad. Förhandl., V. No. 9., 199. (1848) ; ZетT., Dipt. Scand., VIII. 2938. 16-17. (1849); WALK., List Dipt. Brit. Mus., V. suppl. 1., 156 et 167. 44. (1854); Bovsd., Finl. tvaving. Ins., I. 110. 11. (1861); Brauer, Denkschr. Akad. Wien, XLII. 138. 2. tab. I. fig. 2. (1880); PAND., Revue d'Entom., II. 214. 36. (1883).

? borealis FABr., Spec. Insect., II.459.21.(1781); Vill., Entom. Linn., III. 560. 22.(1789) ; Gmex., Systema Naturae, V. 2885. 30. (1792); FABr., Entomol. System., IV. 369. 30. (1794); Meig., Klassif., I. 168. 7. (1804) ; FABR., Systema Antliat., 101. 37. (1805); Fall., Dipt. Suec., Taban., 6. 5. (1817) ot Dipt. Suec., Suppl., II. 2. (1825); ZETT., Dipt. Scand., I. 113. 13. p. p. (1842); ZeLL., Isis, 1842. XI. 821. 4. [Therioplectes] (1842); Giмм., Bull. Soc. Imp. Nat. Moscou, XX. 1., 183. 11. (1847) ; Zетт., Dipt. Scand., VIII. 2937. 13. (1849).

var. albomaculatus ZETr., Insecta Lappon., Dipt., 516. 9. Europa sopt. (1838), Dipt. Scand., I. 116. 17. (1842) et VIII. 2939. 17. (1849) ; WaLK., List Dipt. Brit. Mus., V. suppl. 1., 156 et 161. 23. (1854); BowsD., Finl. tvaving. Ins., I. 110. 12. (1861).

lasiophthalmus MACQ., Dipt. exot., I. 1., 143. 45. (1838) ; WALK., List Dipt. Brit. Mus., I. 159. (1848) et V. suppl. 1., 197. 103. (1854) ; Blanch. in Gay : Hist. fis. y polit. de Chile, Zool., VII. 394. 4. tab. II. fig. 5. (1854) ; PhiL., Verh. zool.-bot. Ges. Wien, XV. 714. 4. (1865) ; Ost.-SAck., Mem. Boston. Soc. Nat. Hist., II. 465. 47. (1876); v. D. Wulp, Tijdschr. v. Entomol., XXIV. 158. 5. (1881) ; Pettit, Bullet. 186, Michigan Exper. Stat. (1901); Hunter, Trans. Amer. Entom. Soc. Philad., XXVII. 141. (1901); Hine, Ohio State Acad. Sci., Spec. Pap. No. 5., 51. (1903) ; Aldr., Catal. North Amor. Dipt., 201. (1905).

notabilis Walk., List Dipt. Brit. Mus., I. 166. (1848).

punctipennis MacQ., Dipt. exot., suppl. 2., 23. 108. (1847);

WaLK., List Dipt. Brit. Mus., V. suppl. 1., 179. 101 et 187. 135. (1854); Ost.-SAck., Mem. Boston Soc. Nat. Hist., II. 473. $(1876)$.

lateralis BruLLÉ $=$ spectabilis $\mathrm{Lw}$.

lateralis MeIG., System. Beschreib., II. 58. 35. (1820); Gimi., Bull. Soc. Imp. Nat. Moscou, XX.1., 182. 8. (1817); WALK., List Dipt. Europa centr. et mer.

Brit. Mus., V. suppl. 1., 155 et 158. 6. (1854); Braver, Denkschr. Akad. Wien, XLII. 154. 16. tab. II. fig. 16. (1880); PANd, Revue d'Entom., II. 211. 27. (1883); Neuhaus, Diptera Marchica, 44. 11. (1886).

Amcrica sept. et mer. 
pilosus Lw., Verh. zool.-bot. Ges. Wien, VIII. 587. 14. (1858); Schis., Fauna Austriaca, Dipt., 32. (1862); Goв., Mém. Soc. Linn. Nord France, 1881. 15. 8. [Therioplectes] (1881).

lateritius Rovd., Archiv. per la Zool. Modena, III. 80. (1863). unicolor Macq., Dipt. exot., suppl. 2., 22. 107. (1847); WaLK., List Dipt. Brit, Mus., V. suppl. 1., 185. 127. (1854); Osт.SAck., Mem. Boston Soc. Nat. Hist., II. 473. (1876).

laticeps Hine, Ohio Naturalist, V. 239. (1904).

laticornis Hrne, Ohio Naturalist, V. 239. (1904).

latifrons ZETT. = cordiger MEı.

latipes MacQ., Dipt. exot., I. 1., 119. 1. (1838); WALK., List Dipt. Brit. Mus., V. suppl. 1., 236. 353, 328. (1854); Lw., Dipt.-Fauna Südafrika's, 1.36.5. (1860) et in P'ETERs: Reise nach Mosambique, Zool., V. 2. (1862); Bertol., Mem. Accad. Sci. Instit. Bologna, XII. 15. 17. (1862); Ricardo, Annal. Mag. Nat. Hist., ser. T. VI. 164. (1900); Adaus, Kansas Univ. Sci. Bullet., III. 150. (1905). uficanus Gray apud. Ricardo, 1900.

fenestratus Walk. in Newman Zoologist, ViII. Nppend., LXVIT. (1850) et List Dipt. Brit. Mus., V. suppl. 1., 264. 505. (1854). Iatistriatus Brauer, Denkschr. Akad. Wien, XLII. 170. 33. tab. III. fig. 33. (1880) ; Villex., Feuille jeun. Natural., XXXIV. 229. 3. [Atylotus] (1904).

lativentris MAcQ., Dipt. exot., I. 1., 149. 57. tal). XVIII. fig. 1. Chile. (1838) ; Brancir. in Gay : Hist. fis. y polit. de Chile, Zool. VII. 393. 3. tab. II. fig. 3. (1854); WaLk., List Dipt. Brit. Mus., V. suppl. 1., 265. 510. (1854) ; Phil., Verlı. zool.-bot. Ges. Wien, XV. 714. 3. (1865) ; Hunter, Trans. Amer. Entom. Soc. Philad., XXVII. 141. (1901).

lativitta WaLk., List Dipt. Brit. Mus., I. 184. (1848).

leonum Bı́., Mém. Soc. Zool. France, V. 680. (1892).

Letourneuxi Big., Mém. Soc. Zool. France, V. 643. [Atylotus] (1892). leucaspis WIED., Aussereurop. zweifl. Ins., I. 179. 103. (1828); Rond. in Truqui : Studi Entomol., I. 107. 82. (1848); Walk., List Dipt. Brit. Mus., V. suppl. 1., 199. 188. (1854); WisLIst., Kansas Univ. Quart., III. 195. (1895); Hunter, Trans. Amer. Entom. Soc. Philad., XXVII. 141. (1901).

leucaspis v. D. Wulp, Notes Leyden Mus., VII. 74. 28. tab. V. fig- Africa occid. 3. (1885).

leucocnematus Bıg., Mém. Soc. Zool. France, Y. 656. [Atylotus] India or. (1892) ; v. D. Wulp, Catal. Dipt. South Asia, 63. (1896).

leucomelas WaLK., List Dipt. Brit. Mus., I. 175. (1848); Ost.-Sack., Mem. Boston Soc. Nat. Hist., II. 474. (1876); Aldr., Catal. North Amer. Dipt., 204. (1905).

leucophilus Walk., List Dipt. Brit. Mus., I. 154. (1848).

Brasilia. Sierra Leone. Algeria. Brasilia.

America sept. leucophorus $\mathrm{BIG}_{\mathrm{G}}=$ sequax WiLList.

leucopogon Big., Mém. Soc. Zool. France, V. 651. [Alylolus] (1892); India or. v. D. Wulp, Catal. Dipt. South Asia, 63. (1896). 
leucopterus v. D. Wulp, Tijdschr. v. Entomol., XI. 98. 1. (1868) et Ins. Aru. Catal. Dipt. South Asia, 61. (1896).

leucosparsus Big., Nouv. Archiv d. Mus. d'Hist. Nat. Paris, sér. 3. Ins. Laos. II. 203. (1890) ; v. D. Wulp, Catal. Dipt. South Asia, 62. (1896).

leucostomus Lw., Öfvers. Kongl. Vet. Akad. Förhandl., XV. 336. 25 bis. (1858) et Dipt.-Fauna Südafrika's, I. 43. 15. (1860).

liburnicus WIED. = graecus FABR.

lifuensis Big., Mém. Soc. Zool. France, V. 689. (1892).

ligatus WaLk., Insecta Saunders., Dipt., I. 59. (1850).

Jimbatinervis MACQ., Dipt. exot., suppl. 2., 16. 93. (1847); WALK., List Dipt. Brit. Mus., V. suppl. 1., 254. 444. (1854).

limbatinervis MAcQ., Dipt. exot., suppl. 4., 29. 96. (1850).

limbatus Bıg., Mém. Soc. Zool. France, V. 642. [Therioplectes] (1892). limbatus Pal. Beauv. = americanus Forst.

limonus Towss. = mexicanus L. var.

limpidapex WIED., Aussereurop. zweifl. Ins., I. 140. 46. (1828); Walk., List Dipt. Brit. Mus., V. suppl. 1., 220. 281. (1854); Hunter, Trans. Amer. Entom. Soc. Philad., XXVII. 141. (1901). lineatus FABR. = giganteus DEG.

lineola FABr., Entomol. System., IV. 369. 33. (1794); Coqueb., Illustr. Iconogr. Insect., III. 112. tab. XXV. fig. 6. (1804); FABr., Systema Antliat., 102. 41. (1805); Wied., Dipt. exot., 81. 36. (1821) et Aussereurop. zweifl. Ins., I. 170. 89. (1828); ? MACQ., Dipt. exot., I. 1., 146. 49. (1838) ; WaLk., List Dipt. Brit. Mus., I. 182. (1848) et V. suppl. 1., 184. 123. (1854); Harris, Ins. of New Engl., Ed. III., 602. fig. 262. (1862); Ost.-SAck., Mem. Boston Soc. Nat. Hist., II. 448. 23. (1876); v. D. Wulp, Tijdschr. v. Entomol., XXIV. 158. 7. (1881); E. I. Arrib., Bolet. Acad. Nat. Cienc. Córdoba, IV. 134. 75. (1882); Ost.-SACK., Biolog. Central. Americ., Dipt., I. 56. 13. (1886); Sмıтн, Trans. Amer. Entom. Soc. Philad., XVII. 328. fig. 7. (1890); Towns., Proc. Calif. Acad. Sci., ser. 2. 597. 14. (1895); HaRT., Bullet. Illin. Stat. Laborat. of Nat. Hist., IV. 235. tab. X. fig. 43-45, tab. XI. fig. 46. (1895); LugGer, 2 nd Report Stat. Entomol. Minnesota, 1896. 186. (1897); Hunter, Trans. Amer. Entom. Soc. Philad., XXVII. 141. (1901); Winlıst., Biolog. Central. Americ., Dipt., I. 260. 13. (1901) ; Hine, Ohio State Acad. Sci., Spec. Pap. No. 5., 51. (1903), Ohio Naturalist, V. 240. (1904) et Bullet. Unit. Stat. Deptm. of Agricult., Div. of Entom., No. 44., 58. (1904); Aı.dr., Catal. North Amor. Dipt., 204. (1905).

commixtus Walk., Trans. Entom. Soc. London, n. ser. V. 273.

(1860) ; Ost.-Sıck., Biolog. Central. Amer., Dipt., I. 56. (1886). compactus Aldr., Catal. North Amer. Dipt., 204. sub. syn. lineolae (1905).

propinquus BeLL., Saggio di Ditterol. Messic., I. 65. 17. (1859);

Aldr., Catal. North Amor. Dipt., 206. (1905).

? scutcllaris WaLk., Insecta Saunders., Dipt., I. 27. (1850). 
simulans WALK., List Dipt. Brit. Mus., I. 182. (1848).

trilineatus Bell., Saggio di Ditterol. Messic., I. 63. 13. (1859). lineola PaL. Beauv., Ins. recueill. en Afrique et en Amérique, 101. tab.

II. fig. 6. (1805-21); WiED., Aussereurop. zweifl. Ins., I. 169. 88.

(1828) ; Walk., List Dipt. Brit. Mus., V. suppl. 1., 266. 514. (1854).

litigiosus Walk., Insecta Saunders., Dipt., I. 37. (1850).

lividus WALk., List Dipt. Brit. Mus., I. 162. (1848).

longiappendiculatus MAcQ., Dipt. exot., suppl. 5., 32. 125. (1855) ;

Aldr., Catal. North Amer. Dipt., 205. (1905).

longipennis Macq., Suit. à Buffon, I. 201. 15. (1834); WaL.K., List Dipt. Brit. Mus., V. suppl. 1., 264. 503. (1854).

longitudinalis Lw., Ber. ü. d. Verh. d. k. Preuss. Akad. d. Wissensch. Berlin, 1852. 658. 2. (1852) et in Petwrs: Reise nach Mosambique, Zool., V. 2. (1862).

longus Ost.-Sıck., Mem. Boston Soc. Nat. Hist., II. 447. 21., 559. (1876) ; Hine, Ohio Stat. Acad. Sci., Spec. Pap. No. 5., 52. (1903); Aldr., Catal. North Amer. Dipt., 205. (1905).

lucidulus Walk., List Dipt. Brit. Mus., I. 188. (1848); Aldr., Catal. Jamaica.

Nortlı Amer. Dipt., 205. (1905).

luctuosus MacQ., Dipt. exot., I. 1., 135. 27. (1838); Walk., List Dipt. Brit. Mus., V. suppl. 1., 213. 251. (1854); Hunter, Trans.

Amer. Entom. Soc. Philad., XXVII. 141. (1901).

lugens Pinl., Verh. zool.-bot. Ges. Wien, XV. 719. 24. (1865); Schr.,

Novara Reise, Dipt., 92. 30. (1868); Hunter, Trans. Amor. Entom.

Soc. Philad., XXVII. 141. (1901).

lugubris MaCQ., Dipt. exot., I. 1., 145. 48. (1838); WaLK., List Dipt. Brit. Mus., I. 174. (1848) et V. suppl. 1., 176. 87. (1854) ; Ost.-SAck., Mem. Boston Soc. Nat. Hist., II. 456. 33. (1876); Aldr., Catal. North Amer. Dipt., 205. (1905).

ater PaL. Beauv., Ins. recueill. en Afrique et en Amérique, 101. tab. II. fig. 5. (1805-21); WiED., Dipt. exot., 74. 23. (1821) et Aussereurop. zweifl. Ins., I. 136. 39. p. p. (1828); WaLK., List Dipt. Brit. Mus., V. suppl. 1., 176. 88. (1854).

lugubris ZETT. = aterrimus MEIG, var.

lunatus FABr., Entomol. System., IV. 370. 34. (1794); Coqurb., Illustr. Iconogr. Insect., III. 112. tab. XXV. fig. 5. (1804) ; FABR., Systema Antliat., 109. 7. [Haematopota] (1805); Meig., System. Beschreib., II. 82. 4. [Hacmatopota] (1820); WicD., Aussereurop. zweifl. Ins., I. 576. 6. (1828); MACQ., Suit. à Buffon, I. 212. 7. [Haematopota] (1834); WaLk., List Dipt. Brit. Mus., V. suppl. 1., 225 et 228. 317. [Tabanus], 295 et 296. 9. [Hacmatopota] (1854) ; Brau., Denkschr. Akad. Wien, XLII. 171. 34. tab. III. et V. fig. 34. (1880) ; PAND., Revue d'Entom., II. 210. 24. (1883); Villen., Annal. Soc. Entom. France, LXXIV. 308. (1905).

algiricus Tuuns., Nova Acta Upsal., IX. 60. (1827).

anthophilus Lw., Verh. zool.-bot. Ges. Wien, VIII. 593. 20. (1858); Scuis., Fauna Austriaca, Dipt., I. 30. (1862.) 
bromius MeIG., System. Beschreib., II. 52. 29. p. p. (1820).

lunulatus Big., Bull. Soc. Zool. France, XVI. 79. [Haematopota] (1891).

lumulatus Me1G., System. Beschreib., II. 49. 25. p. p. (1820);

WıLK., List Dipt. Brit. Mus., V. suppl. 1., 156 et 164. 33.

(1854) ; Lw., Verh. zool.-bot. Ges. Wien, VIII. 601. 30. (1858);

Villen., Annal. Soc. Entom. France, LXXIV. 307. 9. (1905).

Wideri JaEnn., Berlin. Entom. Zeitschr., X. 72. 12. (1866).

lunulatus $\mathrm{BIG} .=$ lunatus FABR.

lunulatus Big., Mém. Soc. Zool. France, V. 688. (1892).

Australia.

lunulatus Meig. p. p. = glaucopis MeIg. var. cognatus Lw.

lunulatus MEIG. p. p.= lunatus FABR.

luridus Fall., Dipt. Suec., Taban., 5. 4. (1817); Meig., System. Beschreib., II. 55. 32. tab. XIII. fig. 21. (1820); MAcQ., Recueil Soc. Sci. Agricult. Lille, 1826. 481. 13. (1826) et Suit. à Buffon, I. 202. 18. (1834); Duncan, Magaz. Zool. and Botan., I. 366. (1837) ; ZetT., Dipt. Scand., I. 112. 11. (1842); ZeLl., Isis, 1842. XI. 822. (1842); Gıм., Bull. Soe. Imp. Nat. Moscou, XX. 1., 184. 14. (1847) ; ZETT., Dipt. Scand., VIII. 2937. 11. (1849); Scholtz, Zeitschr. f. Entom. Breslau, IV. No. 16., 37. (1850); WaLK., Insecta Britann., Dipt., I. 39. 9. (1851) et List Dipt. Brit. Mus., V. suppl. 1., 156 et 168. 45. (1854); Zetr., Dipt. Scand., XII. 4548. 11. (1855); Lw., Verh. zool.-bot. Ges. Wien, VIII. 586. 12. p. p. (1858); Bonsd., Finl. tvaving. Ins., I. 109. 7. (1861) ; Schin., Fauna Austriaca, Dipt., I. 31. p. p. (1862); Brauer, Denkschr. Akad. Wien, XLII. 148. 10. tab. I et V. fig. 10. (1880); Gов., Mém. Soc. Linn. Nord France, 1881. 14. 5. (1881) ; Pand., Revue d'Entom., II. 214. 35. (1883); Hansen, Fabrica oris Dipt., - t tab. I. fig. 18. (1883); NeuHaus, Dipt. Marchica, 43. 9. (1886) ; Beck., Berlin. Entom. Zeitschr., XXXI. 106. 22. (1887) ; L. Coucke, Anual. Soc. Entom. Belgique, XXXVI. 138. (1892); Villen., Annal. Soc. Entom. France, LXXIV. 307. 7. $(1905)$.

borealis 7.ETT., Dipt. Scand., I. 113. 13. var. a et $e$. (1842).

depressus Walk., List Dipt. Brit. Mus., I. 167. (1848); Ricardo, Annal. Mag. Nat. Hist., ser. 7. XVI. 200. (1905).

luridus Lw. p. p. = tropicus Panz.

luteoflavus Bell., Saggio di Ditterol. Messic., I. 60. 8. (1859); Aldr., Catal. North Amer. Dipt., 205. (1905).

luteolus Lw., Öfvers. Kongl. Vet. Akad. Förhandl., XIV. 1857. Caffraria.

348. 27. (1858) et Dipt.-Fauna Südafrika's, I. 45. 18. (1860);

v. D. Wulp, Annal. Soc. Entom. Belgique, XXVIJI. Comp. Rend., CCIXXXIX. 4. (1884).

macer Big., Mém. Soc. Zool. France, V. 649. [Atylotus] (1892); India or. v. D. Wulp, Catal. Dipt. South Asia, 62. (1896).

Maequarti SchlN. = Bigoti BeLL.

macroceratus Bia., Mém. Soc. Zool. France, V. 687. (1892).

Brasilia.

Kertész: Catalogus Dipterorum. III. 
macrodonta Macq., Dipt. exot., I. 2. 183. (1839); WaLk., List Dipt. ?Prom.bon.sp. Brit. Mus., V. suppl. 1., 263. 499. (1854).

macrophthalmus Schin., Novara Reise, Dipt., 82. 7. (1868).

Australia.

macrops Walk., List Dipt. Brit. Mus., I. 164. (1848); Ricardo, Aegyptus. Aunal. Mag. Nat. Hist., ser. 7. XVI. 200. (1905).

macula MAcQ., Dipt. exot., suppl. 1., 43. 89. (1845); WALK., List Patriaignota. Dipt. Brit. Mus., V. suppl. 1., 262. 495. (1854).

macularis FABR., Entomol. System., 370. 36. (1794) et Systema

Antliat., 103. 43. (1805); MeIG., System. Beschreib., II. 40. 13. (1820); Wied., Dipt. exot., 88. 46. (1821) et Aussereurop. zweifl. Ins., I. 167. 86. (1828) ; Walk., List Dipt. Brit. Mus., V. suppl. 1., 225 et 227. 315. (1854); Brauer, Denkschr. Akad. Wien, XLII. 158. 20. tab. II. fig. 20. (1880).

maculatus $\mathrm{DEG} .=$ bromius $\mathrm{L}$.

maculatissimus MAcQ., Dipt. exot., I. 1., 121. 3. lab. XVII. fig. 2.

Africa mer.

(1838) ; Walk., List Dipt. Brit. Mus., I. 166. (1848) et V. suppl. 1., 227. 314. (1854); Lw., Öfvers. Kongl. Vet. Akad. Förhandl., XIV. 1857. 320. 22. (1858) et Dipt.-Fauna Siidafrika's, I. 43. 14. (1860) ; Johns., Proc. Acad. Nat. Sci. Philad., 1898. 158. (1898). maculicornis Zетт., Dipt. Scand., I. 117. 18. (1842), VIII. 2939. 18. (1849) et XI. 4259. 18. (1852); Walk., List Dipt. Brit. Mus., V. suppl. 1., 155 et 160. 14. (1854); Bonsd., Finl. traving. Ins., I. 110. 13. (1861) ; Schin., Fauna Austriaca, Dipt., I. 36. (1862); Brauer, Denkschr. Akad. Wieu, XLII. 197. 53. tab. IV et VI. fig. 53. (1880); Gов., Mem. Soc. Linn. Nord France, 1881. 24. 12. (1881); Pand., Revue d'Entom., II. 206. 15. (1883); Ginschn., Berlin. Entom. Zeitsehr., XXXI. - tab. III. fig. 3. (1887) ; L. Coucke, Annal. Soc. Entom. Belgique, XXXVI. 137. (1892) ; Sтroвl, Mittheil. Naturwiss. Ver. Steiermark, XXIX. 1892. 16. (1893) ; Villen., Annal. Soc. Entom. France, LXXIV. 308. 12. (1905).

borealis Me1G., System. Beschreib., II. 37. 10. p. p. (1820);

Falt., Dipt. Suec., suppl., 2. 5. (1826).

glaucescens Schis., Fauna Austriaca, Dipt., I. 36. p. p. (1862). nemoralis MeIG., System. Beschreib., II. 50. 26. p. p. (1820).

nigricans EgG., Verh. zool.-bot. Ges. Wien, IX. 392. (1859).

maculifer Bıg., Mém. Soc. Zool. France, V. 641. [Therioplectes] (1892) ; Hise, Ohio Naturalist, V. 245. (1904); Aldor., Catal. North Amer. Dipt., 205. (1905).

maculinervis Mace., Dipt. exot., suppl. 5., 31. 124. [maculineuris] Africa sept.

\section{Africa ner.}

Europacentr.

et sept.

America sept. (1855) ; Hunter, Trans. Amer. Entom. \$oc. Philad., XXVII. 141. (1901).

maculipennis Brelét $=$ umbrinus MeIG.

maculipennis MAcq., Suit. à Buffon, I. 198. 6. (1834); WALk., List Brasilia. Dipt. Brit. Mus., V. suppl. 1., 218. 272. (1854).

maculipennis MAcQ., Dipt. exot., suppl. 1., 34. 70. tab. III. fig. 14. Brasilia. (1845); Walk., List Dipt. Brit. Mus., V. suppl. 1., 218. 271. (1854). 
maculipennis WifD., Aussereurop. zweifl. Ins., I. 138. 42. (1828); WALK., List Dipt. Brit. Mus., V. suppl. 1., 219. 276. (1854); Hunter, Trans. Amer. Entom. Soc. Philad., XXVII. 142. (1901). maculiventris Mace., Dipt. exot., suppl. 4., 33. 105. (1850); Blanch. in GAY : Hist. fis. y polit. de Chile, Zool. VII. 396. 8. (1854); Phil., Verh. zool.-bot. Ges. Wien, XV. 714. 8. (1865); Hunter, Trans. Amer. Entom. Soe. Philad., XXVII. 142. (1901).

rubromaculatus Blanch. in Gax: Hist. fis. y polit. de Chile, Zool. VII. Dipt. tab. II. fig. 8. (1854).

maculosus Coquill., Journ. New York Entom. Soc., X. 138. (1902); Aldr., Catal. North Amer. Dipt., 205. (1905).

magellanicus PHL., Verh. zool.-bot. Ges. Wien, XV. 717. 19. (1865);

E. L. Arrib., Bolet. Acad. Nat. Cienc. Córdoba, IV. 135. 76. (1882); Big., Mission Scient. Cap Horn, VI. Zool., 18. (1888); Hunter, Trans. Amor. Entom. Soc. Philad., XXVII. 142. (1901). major Eversu., Bull. Soc. Imp. Nat. Moscou, VII. 422. (1834) [nomen nudum].

maletectus Big., Mém. Soc. Zool. France, V. 664. [Atylotus] (1892). mallophoroides WALK., Trans. Entom. Soe. London, n. ser. IV. 123. (1857).

mandarinus Schin., Novara Reise, Dipt., 83. 8. (1868); v. D. WulP, Catal. Dipt. South Asia,.,61. (1896)

manifestus WWaLK, Insecta Saunders., Dipt., I. 41. (1850). manilensis Schin., Novara Reise, Dipt., 84.10. (1868); v. D. WuLP,

Catal. Dipt. South Asia, 61. (1896).

maoriorum Brg., Mém. Soc. Zool. France, V. 621. [? Mesomyia] (1892) ; Rrcardo, Annal. Mag. Nat. Hist., ser. 7. VIII. 297. (1901). maorium Hutrox, Trans. New Zealand Instit., XXXIII. 14.

[Silvius] (1901) lapsus.

marium HutToN $=$ maoriorum BIG.

marginalis FABR. = ? vivax OsT.-SACK.

marginalis WIED. = vivax OsT.-SAck.

marginatus MACQ., Dipt. exot., suppl. 3., 12. 92. (1847); WALk.,

List Dipt. Brit. Mus., V. suppl. 1., 211. 237. (1854); Hunter,

Trans. Amer. Entom. Soc. Philad., XXVII. 142. (1901).

marginenervis MacQ., Dipt. exot., suppl. 5., 29. 121. (1855); Hunter,

Trans. Amer. Entom. Soc. Philad., XXVII. 142. [marginenvis] (1901).

maritimus Towns., Entom. News Philad., IX. 167. (1898); ALdr., Catal. North Amer. Dipt., 205. (1905).

marmoratus*) Big., Mém. Soc. Zool. France, V. 634. [Dichelacera]

(1892); Ricardo, Annal. Mag. Nat. Hist., ser. 7. XIY. 368. (1904).

maroccamus FABR. = barbarus CoQUeB.

megacephalus JAEN. = cordiger MEIG.

megalops WALK., List Dipt. Brit. Mus., V.suppl. 1., 247. 407. (1854); Java.

v. D. Wulp, Catal. Dipt. South Asia, 60. (1896).

Brasilia.

Chile.

Mexico.

Chile.

Brasilia.

Amazon.

Hongkong.

?America mer.

Manila.

N. Selandia.

Brasilia.

America mer.

America sept.

Brasilia.

*) $=$ ? albopictus Bia. 
Megerlei WIEd., Aussereurop. zweill. Ins., J. 132. 32. (1828); WaLK., List Dipt. Brit. Mus., I. 166. (1848) et V. suppl. 1., 263. 497. (1854) ; Ost.-SAcK., Mem. Boston Soc. Nat. Hist., II. 457. 35. (1876); Willist., Trans. Kansas Acad. Sci., X. 138. (1887); Aldr., Catal. North Amer. Dipt., 205.'(1905).

melanocerus Wied., Aussereurop. zweifl. Ins., I. 122. 16. (1828); WalK., List Dipt. Brit. Mus., I. 157. (1848) et V. suppl. 1., 182. 115. (1854); Ost.-SAck., Mem. Boston Soc. Nat. Hist., II. 440. 12. (1876); ? Willist., Trans. Kansas Acad. Sci., X. 139. (1887); Aldr., Catal. North Amer. Dipt., 205. (1905).

? excuestuans I., Systema Naturae, Ed. XII., II. 1000. 8. (1767); Dé., Mém. pour serv. à l'hist. d. Ins., VI. 229. 5. tab. XXX. fjg. 5. (1776); Farr., Entomol. System., IV. 365. 13. (1794) et Systema Antliat:, 96. 12. (1805); WiEd., Aussereurop. zweifl. Ins., I. 143. 51. (1828) ; Walk., List Dipt. Brit. Mus., V. suppl. 1., 180. 108. (1854); Ost.-SAck., Mem. Boston Soc. Nat. Hist., II. 472. (1876); Hunter, Trans. Amer. Entom. Soc. Philad., XXVII. 140. (1901).

melanognathus Big., Nouv. Archiv. du Mus. d'Hist. Nat. Paris, sér. 3. II. 204. [Atylotus] (1890); v. D. Wulp, Catal. Dipt. South Asia, 62. (1896).

melanopygatus Bıg., Mém. Soc. Zool. France, V. 651. [Atylotus] (1892) ; v. D. Wulp, Catal. Dipt. South Asia, 63. (1896).

melanorhinus BıG. = rhombicus OsT.-SAcK.

melanostoma PнiL., Verh. zool.-bot. Ges. Wien, XV. 720. 28. (1865); Huvter, Trans. Amer. Entom. Soc. Philad., XXVII. 142. (1901). mentitus WalK., List Dipt. Brit. Mus., I. 162. (1848); v. D. WulP, Catal. Dipt. South Asia, 59. (1896).

meridianus Rond., Archivio per la Zool. Modena, III. 79. [Agelanius] (1863) ; Hunter, Trans. Amer. Entom. Soc. Philad., XXVII. 142. (1901).

meridionalis Thuns., Nova Acta Upsal., IX. 58. (1827). metidjensis MAcQ. = mitidjensis MAcQ.

mexicanus L., Systema Naturae, Ed. XII., II. 1000. 10. (1767); Fabr., Spec. Insect., II. 457. 16. (1781), Entomol. System., IV. 367. 22. (1794) et Systema Antliat., 98. 25. (1805); Wied., Dipt. exot., 76. 29. (1821) et Aussereurop. zweill. Ins., I. 147. 58. (1828) ; Macq., Dipt. exot., I. 1., 143. 43. (1838); Walk., List Dipt. Brit. Mus., V. suppl. 1., 215. 259. (1854); Bell., Saggio di Ditterol. Messic., I. 59. 7. (1859) ; Ost.-SAck., Mem. Boston Soc. Nat. Hist., II. 459. 38. (1876) et Biolog. Central. Americ., Dipt., I. 56. 12. (1886) ; Towns., Annal. Mag. Nat. Hist., ser. 6. XX. 21. (1897) ; Huxter, Trans. Amer. Entom. Soc. Philad., XXVII. 142. (1901) ; Aldr., Catal. North Amer. Dipt., 205. (1905). flavus Macq., Suit. à Buffon, I. 200. 13. (1834); Gúkr. ex Perch., Gen. d. Ins. etc., Dipt. II. (1835-38); WALK., List Dipt. Brit. Mus., V. suppl. 1., 185. 128. (1854).

Americasept. ?et mer.

Ins. Laos.

India or.

Chile.

China.

Chile.

Patriaignota.

America. 
inanis Fabr., Entomol. System., IV. 368. 26. (1794).

ochroleneus MeIg., Klassif., I. 170. 12. (1804) et System. Beschreib., II. 62. 41. (1820).

olivaceus Deg., Mém. pour serv. à l'hist. d. Ins., VI. 229. 6.

tab. XXX. fig. 6. (1776).

punctalus FABr., Entomol. System., IV. 368. 25. (1794).

sulphureus Pal. Beauv., Ins. recueill. en Afrique et en Amérique, 222. tab. III. fig. 6. (1805-21).

viridiflavus WALK., Newman Zoologist, VIII. Append., LXVI. (1850).

var. limonus Towss., Annal. Mag. Nat. Hist., ser. 6. XX. 21. Mexico. 25. (1897).

micans Meig., Klassif., I. 167. 5. (1804) et System. Beschreib., II. 34. 4. tab. XIII. fig. 20. (1820); MacQ., Recueil Soc. Sci. Agricult. Lille, 1826. 474. 3. (1826) et Suit. à Buffon, I. 205. 27. (1834); Duncan, Magaz. Zool. and Botan., I. 363. (1837); Zelt., Isis, 1842. XI. 820. 2. (1842); Lw., Verh. zool.-bot. Ges. Wien, VIII. 579. 1. (1858); Schln., Fauna Austriaca, Dipt., I. 29. (1862); JaEnn., Berlin. Entom. Zeitschr., X. 67.4. (1866); Brauer, Denkschr. Akad. Wien, XLII. 137. 1. tab. I. et V. fig. 1. (1880); Gов., Mém. Soc. Linn. Nord France, 1881. 11. 1. [Therioplectes] (1881); Pand., Revue d'Entomol., II. 215. 38. (1883); L. Coucke, Annal. Soc. Entom. Belgique, XXXVI. 136. (1892); Villen., Annal. Soc. Entom. France, LXXIV. 308. 15. (1905).

austriacus Fabr., Systema Antliat., 96. 17. (1805); WaLK., List Dipt. Brit. Mus., I. 170. (1848), Insecta Britann., Dipt., I. 40.

11. (1851) et List Dipt. Brit. Mus., V. suppl. 1., 156 et 168. 48. (1854).

niger Doxov., Nat. Hist. Brit. Ins., XVI. 47. 564. (1813).

signalus Paxz., Fauna Germ., CX. tab. 20. (1809).

microcephalus Osт.-SAck., Mem. Boston Soc. Nat. Hist., II. 470. 52. (1876) ; Aldr., Catal. North Amer. Dipt., 205. (1905).

microcerus Walk., List Dipt. Brit. Mus., I. 150. (1848).

Europacentr. et mer.

microdonta Macq., Dipt. exot., suppl. 2., 17. 94. (1847); WALK., I.ist Dipt. Brit. Mus., V. suppl. 1., 252. 438. (1854).

Mikii Brauer, Denkschr. Akad. Wien, XIII. 195. 52. tab. VI. fig. 52. (1880); SтroвL, Mittheil. Naturwiss. Ver. Steiermark, XXIX. 1892. 16. (1893).

graccus MeIG., System. Beschreib., II. 53. 30. p. p. (1820).

Miles Wied., Aussereurop. zweifl. Ins., I. 139. 43. (1828); WALK., List Dipt. Brit. Mus., V. suppl. 1., 216. 263. (1854); Huvrer, Trans. Amer. Entom. Soc. Philad., XXVII. 142. (1901).

minimus v. D. Wul., Sumatra Exped., Dipt., 18. 7. tab. I. fig. 11. (1881), Notes Leyden Mus., VII. 71. 25. (1885) et Catal. Dipt. South Asia, 62. (1896).

minor Macq., Dipt. exot., suppl. 4., 33. 106. (1850) ; E. L. Arrib., Bolet. Acad. Nat. Cienc. Córdoba. IV. 135. 77. (1882) ; Hunter, Trans. Amer. Entom. Soc. Philad., XXVII. 142. (1901).

America sept.

Patria ignota. Tasmania.

Europa centr.

Brasilia.

Sumatra, Java.

Patagonia. 
minos Schin., Novara Reise, Dipt., 88. 23. (1868); Hunter, Trans. Americamer. Amer. Entom. Soc. Plilad., XXVII. 142. (1901).

missionum MACQ., Dipt. exot., I. 2., 186. (1839); WaLK., List IDipt. Brasilia. Brit. Mus., V. suppl. 1., 207. 222. (1854); E. L. Arris., Bolet. Acad. Nat. Cienc. Córdoba, IV. 135. 78. (1882); Hunter, Trans. Amer. Entom. Soc. Plilad., XXVII. 142. (1901).

mitidjensis MACQ., Dipt. exot., I. 2., 182. (1839); WaLk., List Dipt. Brit. Mus., V. suppl. 1., 225 et 229. 323. (1854).

metidjensis Macq. in Lucas: Explor. Scient. de l'Algérie, Zool. III. 423. 25. (1849).

modestus Wied., Aussereurop. zweifl. Ins., I. 146. 56. (1828); MACQ., Dipt. exot., suppl. 1., 36. 74. (1845); WaLK., List I)ipt. Brit. Mus., V. suppl. 1., 197. 178. (1854); Sch1n., Novara Reise, Dipt., 85. 16. (1868); Willist., Kansas Univ. Quart., III. 195. (1895); Hunter, Trans. Amer. Entom. Soc. Philad., XXVII. 142. (1901). molestissimus PHiL., Verh. zool.-bot. Ges. Wien, XV. 716. 17. (1865); Hunter, Trans. Amer. Entom. Soc. Philad., XXVII. 142. (1901). molestus SAY, Journ. Acad. Nat. Sci. Philad., III. 31. 1. (1823) et Compl. Writt., II. 53. (1859); WiEd., Aussereurop.. zweifl. Ins., I. 125. 21. (1828); WaLK., List Dipt. Brit. Mus., I. 149. (1848) et V. suppl. 1., 186. 131 (1854); Ost.-SAck., Mem. Boston Soc. Nat. Hist., II. 438. 9. (1876) ; Aldr., Catal. North Amer. Dipt., 205. (1905). monilifer Bı́., Mém. Soc. Zool. France, V. 654. [Atylotus] (1892); v. D. Wulp, Catal. Dipt. South Asia, 63. (1896).

monochroma WrED., Aussereurop. zweifl. Ins., I. 555. 27. (1828);

WALK., List Dipt. Brit. Mus., V. suppl. 1., 203. 207. (1854); E. L. Arrıb., Bolet. Ácad. Nat. Cienc. Córdoba, IV. 135. 79. (1882); Hunter, Trans. Amer. Entom. Soc. Philad., XXVII. 142. (1901). monoculus DoL., Natuurkund. Tijdschr. Nederl. Indie, XVII. 85. 17. (1858) ; v. D. Wulp, Catal. Dipt. South Asia, 61. (1896).

Monogramma WiED., Aussereurop. zweifl. Ins., I. 150. 61. (1828); WALK., List Dipt. Brit. Mus., I. 167. (1848) et V. suppl. 1., 219. 277. (1854); Hunter, Trans. Amer. Entom. Soc. Philad., XXVII. 142. (1901).

monotaeniatus Bı., Mém. Soc. Zool. France, V. 655. [Alylotus] (1892) ; v. D. Wulp, Catal. Dipt. South Asia, 63. (1896).

montanus Meig., System. Beschreib., II. 55. 31. p. p. (1820); Duncax, Magaz. Zool. and Botan., I. 365. (1837); Gimм., Bull. Soc. Imp. Nat. Moscou, XX. 1., 187. 21. (1847) ; WaLk., List Dipt. Brit. Mus., V. suppl. 1., 155 et 158. 7. (1854); Brauer, Denkschr. Akad. Wien, XIII. 144. 8. tab I et V. fig. 8. (1880); Gов., Mém. Soc. Linn. Nord France, 1881. 26. (1881) ; Pand., Revue d'Entomol., II. 213. 21. (1883) ; Haxsen, Fabrica oris Dipt., tab. I. fig. 1-5, 15, 17, 19, 21, 22. (1883) ; Веск., Berlin. Entom. Zeitschr., XXXI. 105. 20. [Therioplectes] (1887); STrons, Mittheil. Naturwiss. Ver. Steiermark, XXIX. 1892. 14. [Therioplectés] (1893) ; VILLEN., Annal. Soc. Entom. France, LXXIY. 308. 18. (1905).

Chile.

Americasept.

India or.

Uruguay.

Amboina.

Brasilia.

India or.

Europa centr. et bor., Sibiria. 
?paganus Fabr., Species Insect., II. 458. 1S. (1781); Vili., Entomol. Linn., III. 559.21.(1789); Guel., Systema Naturae, V. 2883. 25. (1792); FABR., Entom. Systom., IV. 367. 24. (1794) ; Meig., Klassif., I. 173. $f .(1804)$; Fabr., Systema Antliat., 99. 27. (1805); MeIG., System Beschreib., II. 63 et 64. (1820).

tropicus ZerT., Insecta Lappon., Dipt., 514. 4 a. (1838), Dipt. Scand., I. 111. 10. p. p. (1842) et XII. 4548. 10. (1855); Lw., Verh. zool.-bot. Ges. Wien, VIII. 586. 11. p. p. (1858) ; Schus, Fauna Austriaca, Dipt., I. 31. (1862).

var. fulvicornis Meig., System. Beschreib., II. 46. 22. (1820); WALK., List Dipt. Brit. Mus., V. suppl. 1., 155 et 161. 21. (1854); Schin., Fauna Austriaca, Dipt., I. 34. (1862); Goв., Mém. Soc. Linn. Nord France, 1881. 22. 7. (1881); Villes., Annal. Soc. Entom. France, LXXIV. 307. 8. (1905). montanus Meig. p. p. = solstitialis Meig.

morio ? I., FABR. = ater RossI.

Mühlfeldi Brauer, Denkschr. Akad. Wien, XLII. 149. 11. tab. V. fig. 11. (1880).

multipunctatus v. D. Wulp, Notes Leyden Mus., VII. 72. 27. tab. V. fig. 2. (1885).

mutatus WALK., Insecta Saunders., Dipt., I. 23. (1850); Ost.-SAck., Mem. Boston Soc. Nat. Hist., II. 474. (1876); Aldr., Catal. North Amer. Dipt., 205. (1905).

Namaquinus Bı́g., Mém. Soc. Zool. France, V. 646. (1892). nanus Macq., Dipt. exot., suppl. 1., 42.88.(1845); Walk., List Dipt. Brit. Mus., V. suppl. 1., 183. 117. (1854); Ost.-SAck., Mem. Boston Soc. Nat. Hist., II. 473. (1876) et Catal. Dipt. North America, Ed. II., 61. observ. (1878); Aldr., Catal. North Amer. Dipt., 205. (1905).

nanus WiED., Dipt. exot., 95. 57. (1821) et Aussereurop. zweifl. Ins., I. 187. 114. (1828); Walk., List Dipt. Brit. Mus., V. suppl. 1., 232. 336. (1854).

nebulosus Deg., Mém. pour serv. à l'hist. d. Ins., VI. 227. 2. tab. XXX. fig. 2. (1776).

nebulosus Pal. Beavv., Insect. recueill. en Afrique et en Amérique, 222. tab. III. fig. 4, 5. (1805-21); Ost.-Sack., Mem. Boston Soc. Nat. Hist., II. 472. (1876); Aldr., Catal. North Amer. Dipt., 206. (1905).

nemoralis Meig., System. Beschreib., II. 50. 26. ऊ $\sigma^{\top}$ (1820); Gimm., Bull. Soc. Imp، Nat. Moscou, XX. 1., 183. 9. (1847); MAcQ. in Lucas: Explor. scient. d. l'Algérie, Zool., III. 425. 32. (1849); WaLk., List Dipt. Brit. Mus., V. suppl. 1., 156 et 163. 30. (1854); Brauer, Denkschr. Akad. Wien, XLII. 174. 37. tab. III. fig. 37. (1880) ; Pand., Revue d'Entom., II. 209. 23. (1883) ; Villen., Annal. Soc. Entom. France, LXXIV. 308. 12. (1905).

atricornis MeIG., System. Beschreib., VII. 59. 47. p. p. (1838).
Europa centr.

Asia min., Sibiria.

Africam.-oce.

America sept.

Port Natal. America sept.

Prom.bon.sp.

Surinam.

America sept.

Europa mer., Algeria. 
barbarus Thunb., Nova Acta Upsal., IX. 60. (1827).

glaucopis Meig., System. Beschreib., II. 48. 24. p. p. (1820).

nemoralis MeIG. p. p. = bromius $\mathrm{L}$.

nemoralis MeIG. p. p. = maculicornis ZeTr.

nephodes Bí., Mém. Soc. Zool. France, V. 656. [Atylotus] (1892); India or.

v. D. Wulp, Catal. Dipt. South A sia, 63. (1896).

nepos WaLK., List Dipt. Brit. Mus., I. 181. (1848).

nexus Walk., Proc. Linn. Soc. London, I. 110. 31. (1856); v. D. Borneo.

Wulp, Catal. Dipt. South Asia, 61. (1896).

nicobarensis Schin., Novara Reise, Dipt., 81. 5. (1868); v. D. Wulp,

Catal. Dipt. South Asia, 61. (1896).

niger DoNov. = micans MrIG.

niger PAL. BEAUV. = atratus FABR.

nigerrimus ZETT. = aterrimus MEIG.

nigrescens PaL. Beauv., Insect. recueill. en Afrique et en Amérique,

100. tab. II. fig. 2. (1805--21) ; WiED., Aussereurop. zweifl. Ins.,

I. 116. 6. (1828) ; WALK., List Dipt. Brit. Mus., V. suppl. 1., 176.

86. (1854) ; Osт.-Sack., Mem. Boston Soc. Nat. Hist., II. 453. 28.

(1876) ; Hart, Bullet. Illin. State Laborat. of Nat. Hist., IV. 238.

(1895) ; Aldr., Catal. Nortlı Amer. Dipt., 206. (1905).

nigricans $\mathrm{EGG}_{\mathrm{G}}=$ maculicornis ZETT.

nigricans WIED., Aussereurop. zweifl. Ins., I. 157. 72. (1828); WALK.,

List Dipt. Brit. Mus., V. suppl. 1., 261. 488. (1854).

nigricornis Zetт., Dipt. Scand., I. 112. 12. (1842) et VIII. 2937. 12.

(1849) ; Walk., List Dipt. Brit. Mus., V. suppl. 1., 155 et 159.

11."(1854) ; Lw., Verh. zool.-bot. Ges. Wien, VIII. 592. 18. (1858) ;

Zetr., Dipt. Scand., XIII. 4945. 12. (1859); Brauer, Denkschr.

Akad. Wien, XIII. 155. 17. tab. V. fig. 17. (1880); Pand., Revue

d'Entom., II. 211. 38. (1883); Beck., Berlin. Entom. Zeitschr.,

XXXI. 106. 21. [Therioplectes] (1887).

alpinus Zетт., Insecta Lappon., Dipt., 516. 7. (1838), Dipt. Scand., I. 116. 16. (1842) et VIII. 2938. 16. (1849); BonsD.,

Finland. tvaving. Ins., I.-110. 10. (1861).

engadinensis JAENs., Berlin. Entom. Zeitschr., X. 75. 15. (1866). nigrifacies Gов., Mém. Soc. Linn. Nord France, 1881. 30. 3. [Aty-

lotus] (1881) ; Mıк, Wien. Entom. Zeitg., I. 155. [Atylotus] (1882) ;

Pand., Revue d'Entom., II. 219. 47. (1883).

nigrifer Walk., The Entomologist, V. 255. (1871); Ricardo, Annal.

Mag. Nat. Hist., ser. 7. XVI. 200. (1905).

nigrifrons Phil., Verh. zool.-bot. Ges. Wien, XV. 720. 29. (1865);

E. I. Arrib., Bolet. Acad. Nat. Cienc. Córdoba, IV. 135. 80.

(1882) ; Hunter, Trans. Amer. Entom. Soc. Philad., XXVII. 142.

(1901).

nigrimanus WALK., List Dipt. Brit. Mus., I. 183. (1848).

Patriaignota.

America sopt.

Europa centr.

et sept.

Gallia.

nigripalpis Macq., Dipt. exot., suppl. 1., 40, 82. tab. IV. fig. 7, 8. (1845) ; Walk., List Dipt. Brit. Mus., V. suppl. 1., 217. 268.

A ustralia.

(1854) ; ? Röd., Stettin. Entom. Zeitg., XLVII, 262. (1886) et

Grenada,

Ecuadur. 
Dipt. gesammelt in Süd-Amerika von A. Stïbel, 8. (1892); HurTER, Trans. Amer. Entom. Soc. Philad., XXVII. 142. (1901). nigripennis PhuL., Verh. zool.-bot. Ges. Wien, XV. 714. 12. (1865);

Huntre, Trans. Amer. Entom. Soc. Philad., XXVII. 142. (1901). nigripes $\mathrm{W}_{\text {IED. }}=$ ? coffeatus MACQ.

nigrita FABR., Entomol. System., IV. 367. 20. (1794) et Systema Antliat., 98. 23. (1805); Brauer, Denkschr. Akad. Wien, XlII. 194. 51. tab. III. fig. 51. [nigritus] (1880) ; PaND., Revue d'Entomol., II. 203. 9. [nigritus] (1883).

carbonarius Meig., System. Beschreib., II. 33. 2. (1820) ; Macq.,

Dipt. exot., I. 2., 182. (1839) et in Lucas : Explor. scient. de l'Algérie, Zool., III. 422. 20. (1849); WaLk., List Dipt. Brit. Mus., V. suppl. 1., 157 et 170. 57. (1854).

gagates Lw., Verh. zool.-bot. Ges. Wien, VIII. 609. 41. (1858). nigrita MeIG. (nec FABr.) = ater Rossi.

nigriventris MacQ., Dipt. exot., suppl. 1., 34. 69. (1845); WatK., List Dipt. Brit. Mus., V. suppl. 1., 252. 437. (1854).

nigrocaeruleus Rond., Nuov. Annal. Sci. Nat. Bologna, ser. 3. II.

191. 28. (1850); Hunter, Trans. Amer. Entom. Soc. Philad.,

XXVII. 142. (1901).

nigrohirtus Ricardo, Annal. Mag. Nat. Hist., ser. 7. VI. 165. (1900). nigromaculatus RicARdo, Annal. Mag. Nat. Hist., ser. 7. VI. 165. [Atylotus] (1900).

nigropictus MACQ., Dipt. exot., suppl. 5., 24. 111. (1855); v. D. Iudia or.

Wulp, Catal. Dipt. South Asia, 60. (1896).

nigropunctatus BELL. = quadripunctatus FiBR.

nigrotectus Big., Nouv. Archiv du Mus. d'Iist. Nat. Paris, sér. 3.

II. 204. [Bellardia] (1890); v. D. Wulp, Catal. Dipt. South Asia, 62. (1896).

nigrovittatus MACQ., Dipt. exot., suppl. 2., 24. 111. (1847); WAlK., List Dipt. Brit. Mus., V. suppl. 1., 178. 95. (1854); Os'.--\$ack., Mem. Boston Soc. Nat. Hist., II. 449. 24. (1876); Aldr., Catal. North Amer. Dipt., 206. (1905).

nitidulus Big., Mém. Soc. Zool. France, V. 679. (1892); v. D. Wulp' Java.

Catal. Dipt. South Asia, 63. (1896).

niveipalpis Bıg., Annal. Soc. Entomol. France, sér. 5. X. 145. (1880).

niveipalpis Bıg., Mém. Soc. Zool. France, V. 645. [Atylotus] (1892). nivosus Ost.-Sack., Mem. Boston Soc. Nat. Hist., II. 445. 19. (1876);

Hine, Ohio State Acad. Sci., Spec. Pap. No. 5., 52. (1903); Aldr.,

Catal. North Amer. Dipt., 206. (1905).

noctis WALK. = biguttatus WIED.

notabilis WALK. = lasiophthalmus MACQ.

notarum Big., Annal. Soc. Entom. France, sér.7. I. 367. 4. [Atylotus] Assinia. (1891).

Novae Scotiae MACQ., Dipt. exot., suppl. 2., 24. 110. (1847); WALK., List Dipt. Brit. Mus., V. suppl. 1., 181. 113. (1854); Ost.-SAck., 
Mem. Boston Soc. Nat. Hist., II. 473. (1876) et Catal. Dipt. North America, Ed. II., 61. Observ. (1878); Aldr., Catal. North Amer. Dipt., 206. (1905).

nuntius WALK., List Dipt. Brit. Mus., V. suppl. 1., 207. 223. (1854). Nyasae Ricardo, Annal. Mag. Nat. Hist., ser. 7. VI. 164. (1900). obconicus Wauk., Insecta Saunders., Dipt., I. 54. (1850) ; v. D. Wulp, Catal. Dipt. South Asia, 60. (1896).

obesus Big., Mém. Soc. Soc. Zool. France, V. 660. [Atylotus] (1892); Aldr., Catal. North Amer. Dipt., 206. (1905).

obliquemaculatus Macq., Dipt. exot., I. 1., 123. 4. (1838); WaLk., List Dipt. Brit. Mus., I. 184. (1848) et V. suppl. 1., 228. 319. (1854).

obliquus WALk., Insecta Saunders., Dipt., I. 28. (1850); ALDr., Jamaica. Catal. North Amer. Dipt., 206. (1905).

obscuratus Phic., Verh. zool.-bot. Ges. Wien, XV. 720. 27. (1865); Hunter, Trans. Amer. Entom. Soc. Philad., XXVII. 142. (1901). obscuratus Walk., Proc. Linn. Soc. London, VIII. 232. 7. (1864); v. D. Wulp, Catal. Dipt. South Asia, 61. (1896).

obscurestigmatus Big., Annal. Soc. Entom. France, sér. 3. VII. 126. tab. IX. fig. 1. (1859).

obscuripennis PhIL, Verh. zool.-bot. Ges. Wien, XV. 722.32. (1865) ; Hunter, Trans. Amer. Entom. Soc. Philad., XXVII. 142. (1901). obscurus Lw. = anthracinus MeiG.

obsolescens Pand., Revue d'Entomol., II. 207. 18. (1883). obsoletus Kert., Catal. Tabanid., 63. (1900).

obsoletus Kert. = obsolescens PAND.

obsoletus WIED., Dipt. exot., 85. 42. (1821) et Aussereurop. zweifl. Ins., I. 174. 95. (1828); Walk., List Dipt. Brit. Mus., V. suppl. 1., 204. 212. (1854) ; Hunter, Trans. Amer. Entom. Soc. Plilad., XXVII. 142. (1901).

occidentalis L., Systema Naturae, Ed. XII., II. 1001. 13. (17.67); Deg., Mém. pour serv. à l'liist. d. Ins., VI. 227. 3. tab. XXX. fig. 3. (1776); FABr., Entomol. System., IV. 368. 27. (1794) et Systema Antliat., 99. 30.(1805); WIED., Dipt. exot., 82. 37. (1821) et Aussereurop. zweifl. Ins., I. 171. 90 et 557. 30. (1828); WALK., List Dipt. Brit. Mus., I. 163. (1848); Rond. in Truqui : Studi Entomol., I. 105. 79. (1848); ? Blanch. in GAY : Hist. fis. y polit. de Chile, Zool. VII. 397. 10. tab. II. fig. 6. (1854); WatK., List Ioipt. Brit. Mus., V. suppl. 1., 204. 209. (1854); ? Pmil., Verh. zool.-bot. Ges. Wien, XV. 714. 10. (1865); IIunrer, Trans. Amer. Entom. Soc. Philad., XXVII. 142 et 143. (1901).

ochraceus MAcq., Dipt. exot., I. 1., 149. 56. (1838) et suppl. 1., 42. 86. (1845); Walk., List Dipt. Brit. Mus., V. suppl. 1., 265. 513. (1854) ; Hunter, Trans. Amer. Entom. Soc. Philad., XXVII. 143. (1901).

ochroleucus MeIG. = mexicanus $\mathrm{L}$. oculus WALK. = albonotatus BELL. 
ohioensis Hrve, Canad. Entomol., XXXIII. 28. [Alylotus] (1901) et Ohio State. Acad. Sci., Spec. Pap. No. 5., 53. (1903); Aınr., Catal. North Amer. Dipt., 206. (1905). pruinosus Hine, Canad. Entomol., XXXII. 248. (1900).

olivaceiventris Macq., Dipt. exot., suppl. 2., 18. 96. (1847); Waık., List Dipt. Brit. Mus., V. suppl. 1., 200. 192. (1854); HUNTER, Trans. Amer. Entom. Soc. Philad., XXVII. 143. (1901).

olivaceus DEG. = mexicanus $\mathrm{L}$.

opacus CoquilL., Invertebr. Pacifica, I. 21. (1904); Hine, Ohio Naturalist, V. 240. (1904).

Oplus Walk., List Dipt. Brit. Mus., I. 175. (1848) [sine descr.], Newman Zoologist, VIII. Append., LXX. (1850) et List Dipt. Brit. Mus., V. suppl. 1., 255. 452. (1848) ; Butler, Cistula Entomol., I. 356. (1876) ; Hutros, Catal. New Zealand Dipt. etc., 20. (1881) et Trans. New Zealand Instit., XXXIII. 12. (1901).

optatus Walk., Proc. Linn. Soc. London, I. 111. 33. (1856) ; v. D. IVulp, Catal. Dipt. South Asia, 61. (1896).

opulentus WaLk., List Dipt. Brit. Mus., I. 148. (1848).

orientalis WiLd, Analecta Entomol., 21. 8. (1824) et Aussereurop. zweifl. Ins., I. 145. 55. (1828); WALK., List Lipt. Brit. Mus., V. suppl. 1., 248. 409. (1854); v. D.'Wulp, Catal. Dipt. South Asia, 59. (1896).

orientis WaLk., List Dipt. Brit. Mus., I. 152. (1848) et V. suppl. 1., 328. (1854); v. D. Wure, Catal. Dipt. South Asia, 59. (1896).

Orion Ost.-SAck., Mom. Boston Soc. Nat. Hist., II. 442. 14. (1876); v. D. Wulp, Tijdschr. v. Entomol., XXIV. 158. 4. (1881); Aldr., Catal. North Amer. Dipt., 206. (1905).

oritensis Bı́., Mém. Soc. Zool. France, V. 677. (1892).

0 rnatus $\mathrm{J}_{\mathrm{AENN}}=$ rectus $\mathrm{L} w$.

Osburni Hine, Ohio Naturalist, V. 241. (1904).

oxyceratus Big., Mém. Soc. Zool. France, V. 652. [Atylotus] (1892);

v. D. Wulp, Catal. Dipt. South Asia, 63. (1896).

pachnodes Schin., Novara Reise, Dipt., 84. 12. (1868); Hunter, Trans. Amør. Entom. Soc. Philad., XXVII. 143. (1901).

pachypalpus Brg., Mém. Soc. Zool. France, V. 631. [Dichelacera] (1892); Ricardo, Annal. Mag. Nat. Hist., ser. 7. XIV. 368 [pachypalpis] (1904); Aldr., Catal. North Amer. Dipt., 200. [Dichelacercl] (1905).

paganus $\mathrm{FABR}_{\mathrm{AB}}=$ ? montanus Meig.

pagodinus Bı́., Mém. Soc. Zool. France, V. 65̃. [Alylotus] (1892); v. D. Wulp, Catal. Dipt. South Asia, 63. (1896).

pallescens Walk., The Entomologist, V. 256. 5. (1871); Ricardo, Annal, Mag. Nat. Hist., ser 7. XVI. 200. (1905).

pallidepectoratus Big., Mém. Soc. Zool. France, V. 658. [Atylotus] (1892); v. D. Wulr, Catal. Dipt. South Asia, 63. (1896).

pallidus PaL. Beauv., Insect. recueill. en Afrique et en Amérique, 100. tab. II. fig. 3. (1805-21); WIED., Aussereurop. zweifl. Ins.,
America sept.

Brasilia.

America sept.

N. Selaudia.

Borneo.

Patria ignota. India or.

India or. sept.

America sept.

Caucasus.

America sept.

India or.

Chile.

Mexico.

India or.

Arabia.

Cochinchina.

America sept. 
I. 118. 9. (1828); Walk., List Dipt. Brit. Mus., V. suppl. 1., 184. 124. (1854); Ost.-SACK., Mem. Boston Soc. Nat. Hist., IT. 472. (1876) ; Aldr., Catal. North Amer. Dipt., 206. (1905).

pallipennis Macq., Dipt. exot., suppl. 1., 32. 65. (1845); WALK., Australia.

List Dipt. Brit. Mus., V. suppl. 1., 252. 439. (1854).

palpinus Pal. Beauv., Insect. Recueill. en Afrique et en Amérique, 221. tab. III. fig. 1. (1805-21); Ost.-Sack., Mem. Boston Soc. Nat. Hist., II. 472. (1876); Aldr., Catal. Nortl Amer. Dipt., 206. (1905).

papuinus WALK., Proc. Linn. Soc. London, VIII. 108. 21. (1865); Ost.-SACK., Annal. Mus. Civ. Genova, XVI. 418. (1882); v. D. Wulp, Catal. Dipt. South Asia, 61. (1896).

par WaLk., List Dipt. Brit. Mus., V. suppl. 1., 235. 349. (1854).

paradoxus J.ens., Berlin. Entom. Zeitschr., X. 83. 26. (1866); Braver, Denkschr. Akad. Wien, XIJI. 179. 40. tab. III et VI. fig. 40. (1880) ; Pand., Revue d'Entomol., II. 202. 3. (1883).

parallelus WALK., Iist Dipt. Brit. Mus., I. 187. (1848); Aldr., Catal. North Amer-Dipt., 206. (1905).

partitus WALk., Proc. Linn. Soc. London, I. 9. 21. (1856); v. D. WüLP, Catal. Dipt. South Asia, 60. (1896).

parvidentatus MAcQ., Dipt. exot., I. 1., 142.40. (1838); WALK., List Dipt. Brit. Mus., V. suppl. 1., 189. 149. (1854); Aldr., Catal.

North Amer. Dipt., 206. (1905).

parvulus WilList., Trans. Kansas Acad. Sci., X. 141. (1887) ; Aldr., Catal. North Amer. Dipt., 206. (1905).

patulus Wat.K., List Dipt. Brit. Mus., I. 175. (1848); Ost.-SACK., Mem. Boston Soc. Nat. Hist., II. 474. (1876); Aldr., Catal. North Amer. Dipt., 206. (1905).

Paulseni Phis., Verh. zool.-bot. Ges. Wien, XV. 721. 31. (1865); Hunter, Trans. Amer. Entom. Soc. Philad., XXVII. 143. (1901). pauper Rond., Annal. Mus. Civ. Genova, VII. 456. (1875) ; v. D. WulP, Catal. Wipt. South Asia, 62. (1896).

pellucidus FABr., Systema Antliat., 97. 21. (1805); Wied., Dipt. exot., 70. 16. (1821) et Aussereurop. zwreifl. Ins., I. 129. 27. (1828) ; Branch in GaY: Hist. fis. y polit. de Chile, Zool. VII. 393. 2. tab. II. fig. 2. (1854); WaLk., List Dipt. Brit. Mus., V. suppl. 1., 213. 249. (1854); PrnL., Verh. zool.-bot. Ges. Wien, XV. 714. 2. (1865); Hunter, Trans. Amer. Entom. Soc. Philad., XXVII. 143. (1901).

perlinea WALK., Insecta Saunders., Dipt., I. 56. (1850); v. D. WuLP, Catal. Dipt. South Asia, 60. (1896).

perplexus WALK., Insecta Saunders., Dipt., I. 32. (1850).

peruvianus Bıg., Mém. Soc. Zool. France, V. 635. [Dichelacera] (1892); Ricando, Annnal. Mag. Nat. Hist., ser. 7. XIV. 368. (1904). peruvianus MacQ., Dipt. exot., suppl. 3., 13. 95. (1847); Walk., List Dipt. Brit. Mus., V. suppl. 1., 210. 233. (1854); Hunter, Trans. Amer. Entom. Soe. Philad., XXVII. 143. (1901).
America sept.

Nova Guinea.

Port Natal.

Europa centr. et mer., Callcasus.

India occid.

Singapore.

Antillae.

S. Domingo.

America sept.

Chile.

Bornco.

America mer.

India or.

Columbia.

Peru.

Peru. 
pervasus WaLK., Insecta Saunders., Dipt., I. 43. (1850).

Africa occid.

phaenops Ost.-SAcK., Bull. Unit. Stat. Geolog. Surv., III. 217. 3. (1877) ; Towss., Proc. Calif. Acad. Sci., ser. 2. IV. 597. 12. [Therioplectes] (1895); Hine, Ohio Naturalist, V. 241. (1904); Aldr., Catal. North Amer. Dipt., 206. (1905).

Philippii Rond., Archivio per la Zool. Modena, III. 80. [Agclanius] (1863) ; Hunter, Trans. Amer. Entom. Soe. Philad., XXVII. 143. (1901).

piceiventris RoND. in Truqui: Studi Entomol., I. 106. 81. (1848); Hunter, Trans. Amer. Entom. Soc. Philad., XXVII. 143. (1901). piceus Tiunse, Nova Acta Upsal., IX. 55. (1827).

picticornis Bı̈., Mém. Soc. Zool. France, V. 662. [Atylotus] (1892); Aldr., Catal. North Amer. Dipt., 206. (1905).

picticornis Bı̈., Mém. Soc. Zool. France, V. 671. [?Atylotus] (1892); v. D. Wurp, Catal. Dipt. South Asia, 63. (1896).

pictipennis MacQ., Suit. à Buffon, I. 199. 7. (1834); WALK., List Brasilia.

Dipt. Brit. Mus., V. suppl. 1., 221. 288. (1854).

pictipennis MaCQ. 1850. = uruguayensis E. L. ARris.

pictipcnnis v. D. WulP $=$ Vanderwulpi Ost.-SACK.

pilosus Lw. = lateralis MEIG.

plangens WAik., List Dipt. Brit. Mus., V. suppl. 1., 199. 191. (1854). planiventris WiEd., Aussereurop. zweifl. Ins., I. 139. 44. (1828); Walk., List Dipt. Brit. Mus., V. suppl. 1., 216. 264. (1854); Huster, Trans. Amer. Entom. Soc. Philad., XXVII. 143. (1901). planus Wal.K., Insecta Saunders., Dipt., I. 61. (1850).

plebejus Fall., Dipt. Suec., Taban., 8. 9. (1817); Meig., System. Beschreib., II. 62. 42. (1820); MACQ., Suit. à Buffon, I. 203. 21. (1834) ; Germ., Fauna Ins. Europ., XIV. 23. (1837); Zell., Isis, 1842. XI. 823. 3. (1842); ZETT., Dipt. Scand., I. 121. 24. (1842); Gimm., Bull. Soe. Imp. Nat. Moscou, XX. 1., 186. 18. (1847); Zeтr., Dipt. Scand., VIII. 2940. 24. (1849); Walk., List Dipt. Brit. Mus., V. suppl. 1., 155 et 160. 16. (1854); Lw., Verh. zool.bot. Ges. Wien, VIII. อ996. 24. (1858); Zıтr., Dipt. Scand., XIII. 4947. 24. (1859); Schis., Fauna Austriaca, Dipt., I. 31. (1862); Brauer, Denkschr. Akad. Wien, XLII. 167. 30. tab. II et V. fig. 30. (1880); Gов., Mém. Soc. Linn. Nord France, 1881. 31. 4. [Atylotus] (1881); Pand., Revue d'Entomol., II. 219. 46. (1883); Beck., Berlin. Entom. Zeitschr., XXXI. 106. 45. [Atylotus] (1887); Speis., Zeitschr. f. wissensch. Insektenbiol., I. 407. 154. [Therioplectes] (1904); Villen., Annal. Soc. Entom. France, LXXIV. 309. 28. (1905).

laniger Wied. in litt. ap. MEIG.

? sublunaticornis ZETT., Dipt. Scand., I. 118. 20. (1842) et VIII. 2940. 20. (1849) ; WaLK., List Dipt. Brit. Mus., V. suppl. 1., 155 et 158.9 . (1854).

plumbeus DruRY = americanus Forst.

Pluto WALK., List Dipt. Brit. Mus., 1. 153. (1848).

Sierra Leone. 
poecilopterus Schin., Novara Reise, Dipt., 91. 28. (1868); Hunter, Americamer.

Trans. Amer. Entom. Soc. Pliilad., XXVII. 143. (1901).

politus Jouns. $=$ Hinei JoHss.

politus WaLk., The Entomologist, V. 256.4. (1871); Ricardo, Annal. Arabia.

Mag. Nat. Hist., ser. 7. XVI. 200. (1905).

polygonus Walk., List Dipt. Brit. Mus., V. suppl. 1., 237. 357. Mesopotamia.

(1854) ; Ricardo, Annal. Mag. Nat. Hist., ser. 7. XVI. 201. (1905).

polytaenia Bra., Mém. Soc. Zool. France, V. 667. [Atylotus] (1892). polyzonatus Biq., Mém. Soc. Zool. France, Y. 648 [Atylotus] (1892). posticus Wien., Ausserenrop. zWeifl. Ins., I. 152. 64. (1828); WaLk.,

List Dipt. Biit. Mus., I. 150 et 161. (1848) et V. suppl. 1., 253. 441. (1854).

postponeus WAtk., List Dipt. Brit. Mus., I. 179. (1848).

Argentinia.

Persia.

Australia.

potator Wiep., Aussereurop. zweifl. Ins., I. 149. 60. (1828) et II.

624. 22. var. (1830); Wask., List Dipt. Brit. Mus., V. suppl. 1.,

221. 287. (1854); Hunter, Trans. Amer. Entom. Soc. Philad.,

XXVII. 143. (1901).

praepositus Walk., Jist Dipt. Brit. Mus., I. 158. (1848).

praetereuns WaLk., Insecta Saunders., Dipt., I. 69. tab. II. fig. 6.

[Dichelacera] (1850); Rucardo, Annal. Mag. Nat. Hist., ser. 7.

XIV. 367. (1904).

primitivus WALk., List Dipt. Brit. Mus., I. 177. (1848).

priscus WaLK., List Dipt. Bıit. Mus., I. 176. (1848).

procyon Osт.-SАск., Bullet. Unit. Stat. Geolog. Surv., III. 216. 1.

(1877); Hine, Ohio Naturalist, V. 241. (1904); Aldr., Catal. North

Amer. Dipt., 206. (1905).

productus Hine, Ohio Naturalist, V. 242. (1904).

propinques BELL. = lineola FABR.

propinquus Mace., Dipt. exot., suppl. 5., 27. 117. (1855).

propinquus PALM. = ? graecus FABR.

proximus ConTi $=$ subelongatus MacQ. var.

proximus Walk., List Dipt. Brit. Mus., I. 147. (1848); Ost.-Sack.,

Mem. Boston Soc. Nat. Hist., II. 474. (1876); Aldr., Catal. North

Amer. Dipt., 206. (19:5).

pruinosus Bıg., Mém. Soc. Zool. France, V. 683. (1892); Ardr., Mexico.

Catal. North Amer. Dipt., 206. (1905).

pruinosus HiNe $=$ ohioensis Hine.

psammophilus Ost.-Sıck., Mem. Boston Soc. Nat. Hist., II. 445. 18. America sept.

(1876) ; Aldne, Catal. North Amer. Dipt., 206. (1905).

psolopterus Wied., Aussereurop. zweifl. Ins., I. 181. 105. (1828);

WALK., List Dipt. Brit. Mus., V. suppl. 1., 205. 218. (1854);

E. L. Arrus., Bolet. Acad. Nat. Cienc. Córdoba, IV. 136. 81.

(1882) ; Hunter, Trans. Amer. Entom. Soc. Philad., XXVII.

143. (1901).

Psusennis JAkxs., Abhandl. Senckenberg. Naturf. Ges., VI. 333. 25. Abyssinia. (1867).

pubescens MAcQ., Dipt. exot., suppl. 2., 20. 103. (1847); WA1к., America. 
List Dipt. Brit. Mus., V. 180. 106. (1854); Hunter, Trans. Amer. Entom. Soc. Philad., XXVII. 143. (1901).

pubescens WALK., List Dipt. Brit. Mus., V. suppl. 1., 220. 284. (1854). pudens WALK., Insecta Saunders., Dipt., I. 36. (1850).

puella WaLk., Insecta Saunders., Dipt., I. 53. (1850); v. D. Wulp, Catal. Dipt. South Asia, 60. (1896).

pulchellus Lw., Verh. zool.-bot. Ges. Wien, VIII. 597. 26. (1858); Brauer, Denksclir. Akad. Wien, XLIJ. 206. 60. (1880); Rrcardo,

Anual. Mag. Nat. Hist., ser. 7. XVI. 200. (1905).

pulchriventris Portsch., Horae Soc. Entom. Ross., XXI. 179. (1887). pulius Hunter = pullus Phil.

pullus PhrL., Verh. zool.-bot. Ges. Wien, XV. 722. 33. (1865).

pulius Hunter, Trans. Amer. Entom. Soc. Philad., XXVII. 143. (1901) lapsus.

pulverifer WALK., List Dipt. Brit. Mus., V. suppl. 1., 236. 356. (1854); Ricardo, Annal. Mag. Nat. Hist., ser. 7. XVI. 201. (1905).

pulverulentus Big., Mém. Soc. Zool. France, V. 665. [Atylotus] (1892).

pumiloides Willist., Biolog. Central. Amer., Dipt., I. 260. 15. tab. IV. fig. 21, 21a. (1901); ALDr., Catal. North Amer. Dipt., 206. (1905).

pumilus MACQ., Dipt. exot., I. 1., 146. 51. (1838); WaLK., List Dipt. Brit. Mus., V. suppl. 1., 177. 92. (1854) ; Ost.-SAck., Mem. Boston Soc. Nat. Hist., II. 448. 22. (1876) ; Hine, Ohio State Acad. Sci., Spec. Pap. No. 5., 53. (1903); Aldr., Catal. North Amer. Dipt., 206. (1905).

punctatus $\mathrm{FABR}$. = mexicanus $\mathrm{L}$.

punctifer Ost.-Sack., Mem. Boston Soc. Nat. Hist., II. 453. 29. (1876) et Bullet. Unit. Stat. Geolog. Surv., III. 220. 7. (1877) ; WilList., Trans. Kansas Acad. Sci., X. 139. (1887); Towss., Trans. Amer. Entom. Soc. Philad., XXII. 60. 17. (1895), Proc. Calif. Acad. Sci., ser. 2. IV. 598. 15. (1895) et Psyche, VIII. No. 255., 93. 9. (1897); Hive, Ohio Naturalist, V. 242. (1904); ALdR., Catal. North Amer. Dipt., 206. (1905).

punctifrons WAHLBG., Öfvers. Kongl. Vet. Akad. Förhandl., V. No. 9., 200. (1848) ; ZeтT., Dipt. Scand., VIII. 2939. 16-17. (1849); Walk., List Dipt. Brit. Mus., V. suppl. 1., 156 et 162. 26. (1854); Beck., Acta Soc. Sci., Fenn., XXVI. No. 9., 6. 6. [Therioplectes] (1900).

punctipennis MacQ., Dipt. exot., I. 2., 185. (1839); Walk., List Dipt. Brit. Mus., V. suppl. 1., 218. 274. (1854); Huxter, 'Trans.

Amer. Entom. Soc. Philad., XXVII. 143. (1901). punctipennis MaCQ. 1847. = lasiophthalmus MaCQ. punctum Rond. in Truqui: Studi Entomol., I. 105. 80. (1848); HuxTER, Trans. Amer. Entom. Soc. Philad., XXVII. 143. (1901).

pungens Wied., Aussereurop. zweifl. Ins., I. 175. 97. (1828); WaLk., List Dipt. Brit. Mus., V. suppl. 1., 203. 205. (1854) ; E. I. Ark11,
Brasilia.

Brasilia.

India or.

Asia min., Africa sept.

Mongolia chinensis. Chile.

Mesopotamia.

Cayenne.

Mexico.

America sept.

America sept.

Europa bor., Sibiria.

Brasilia.

Brasilia.

Uruguay. 
Bol. Acad. Nat. Cienc. Córdoba, IV. 136. 82. (1882); Hunter,

Trans. Amer. Entom. Soc. Philad., XXVII. 143. (1901).

purus Walk., Trans. Entom. Soc. London, n. ser. V. 274. (1860); Mexico.

A tonr., Catal. North Amer. Dipt., 206. (1905).

pusillus EgG., Vert. zool.-bot. Ges. Wien, IX. 393. (1859); Schin.,

Fauna Austr., Dipt., I. 31. nota. (1862); Brsuer, Denkschr. Akad.

Wien, XLII. 156. 18. tab. II. fig. 18. (1880); Pand., Rev?!o

d'Entomol., II. 211. 29. (1883).

pusillu s MAcQ., Dipt. exot., I. 1., 127. 12. (1838); WALK., List Dipt.

Brit. Mus., V. suppl. 1., 242. 387. (1854) ; v. D. Wulp, Catal. Dipt.

South Asia, 59. (1896).

pusillus Macq., Dipt. exot., suppl. 5., 29. 120. (1855).

pygmaeus Willist., Trans. Kansas Acad. Sci., X. 141. (1887); Aldr.,

Catal. North Amer. Dipt., 206. (1905).

Pyrausta WalK. in Newman: Zoologist, VIII. Append. LXV. (1850)

et List Dipt. Brit. Mus., V. suppl. 1., 246. 405. (1854); v. D. Wuis,

Catal. Dipt. South Asia, 60. (1896).

pyrrhoceras Big., Annal. Soc. Entom. France, sér. 6. VII. Bullet. Japonia.

LXXVII. [Atylotus] (1887).

Pyrrhus Walk., Insecta Saunders., Dipt., I. 47. tab. II. fig. 4, 5. India or.,

(1850) ; v. D. Wulp, Catal. Dipt. South Asia, 60. (1896); Coqull., Japonia.

Proc. Unit. Stat. Nat. Mus., XXI. 311. (1898).

quadrifarius Lw., Zeitschr. f. d. ges. Naturwiss., N. F. IX. (XLIII). Turkestan.

414. 3. (1874).

quadrimaculatus MAcQ., Dipt. exot., suppl. 1., 39. 80. tab. IV. fig.

5. (1845); W.aLk., List Dipt. Brit. Mus., V. suppl. 1., 218. 273.

(1854); Hunter, Trans. Amer. Entom. Soc. Philad., XXVII. 143. (1901).

quadrinotatus GoB. = quatuornotatus MEIG.

quadripunctatus FABR., Systema Antliat., 99. 29. (1805); WIED.,

Dipt. exot., 77. 30. (1821) et Aussereurop. zweifl. Ins., I. 151.

63. (1828) ; WALk., List Dipt. Brit. Mus., V. suppl. 1., 219. 275.

(1854); Schin., Novara Reise, Dipt., 86. 18. (1868); Ost.-Sack., Biolog. Central. Americ., Dipt., I. 48. 1. [Therioplectes] (1886);

Willust., Kansas Univ. Quart., III. 195. (1895); Hunter, Trans.

Amer. Entom. Soc. Philad., XXVII. 143. (1901); Willist., Bjolog.

Central. Americ., Dipt., I. 259. (1901); SPEıser, Insekten Börse,

XXI. No. 19., 148. 3. [Therioplectes] (1904); Aldr., Catal. North

Amer. Dipt., 207. (1905).

elegans Tuunb., Nova Acta Upsal., IX. 61. tab. 1. fig. 5. (1827).

nigropunctatus Bell., Saggio di Ditterol. Messic., I. 67. 19. tab. II. fig. 8. (1859).

quatuornotatus Mrig., System. Beschreib., II. 51. 27. tab. XIII. fig. 19. (1820); MacQ., Recueil Soc. Sei. Agricult. Lille, 1826. 479. 10. (1826) et Suit. à Buffon, I. 205. 29. (1834); WALK., List Dipt. Brit. Mus., V. suppl. 1., 156 et 165. 36. (1854); Koldar, Sitzgsber. d. math.-naturwiss. Cl. d. k. Akad. Wien, XIII. 581.

Americamer. et centr.

Europacentr. et mer. 
(1854) ; Lw., Verh. zool.-bot. Ges. Wien, VIII. 591. 17. (1858); Scrun., Fauna Austriaca, Dipt., I. 32. (1862); Lw., Wien. Entom. Monatschr., VI. 163. 13. (1862); Brauer, Denkschr. Akad. Wien, XLII. 173. 36. tab. III et VI. fig. 36. (1880); PAND., Revue d'Entomol., II. 209. 22. (1883); Strobl, Mittheil. Naturwiss. Ver. Steiermark, XXIX. 1892. 15. [Atylotus] (1893); LÉCAILlon, Annal. Soc. Entom. France, LXXIV. 20-28. tab. IJ. (1905); ViuLeN., Annal. Soc. Entom. France, LXXIV. 309. 22. (1905).

quadrinotatus Gов., Mém. Soc. Linn. Nord France, 1881. 15.

7. [Therioplectes] (1881); Coucke, Annal. Soc. Entom. Belgique, XL. 230. (1896).

quinquelineatus MACQ. = trimaculatus_PaL. BeAuv. quinquemaculalus HiNe = quinquevittatus WiEd.

quinquevittatus WIED., Dipt. exot., 84. 39. (1821) et Aussereurop. zweifl. Ins., I. 173. 93. (1828); WaLK., List Dipt. Brit. Mus., V. suppl. 1., 188. 139. (1854); Bell., Saggio di Ditterol. Messic., I. 65. 16. (1859) ; Ost.-SAck., Catal. Dipt. North Amer., Ed. II., 61 et 228. 88. (1878) ; ALdr., Catal._North Amer. Dipt., 207. (1905).

quinquemaculatus Hine, Bullet. Unit. Stat. Deptm. of Agrieult.,

Div. of Entom., No. 44., 58. (1904) lapsus.

Raffreyi Big., Mém. Soc. Zool. France, V. 690. (1892); v. D. Wulp, Nova Guinea.

Catal. Dipt. South Asia, 63. (1896).

recedens WALK., List Dipt. Brit. Mus., I. 147. (1848); Ost.-SACK., Mem. Boston Soc. Nat. Hist., II. 474. (1876); Wilisist., Trans. Kansas Acad. Sci., X. 138. (1887); Aldr., Catal. North Amer. Dipt., 207. (1905).

catenatus Osт.-SAck., Mem. Boston Soc. Nat. Hist., II. 433. 5.

(18i6) et Catal. Dipt. North Amer, Ed. II. 57 et 227. (1878). recedens WaLK., List Dipt. Brit. Mus., V. suppl. 1., 201. 197. (1854). rectus Lw., Verh. zool.-bot. Ges. Wien, VIII. 603. 32. (1858); Brauer, Denkschr. Akad. Wien, XLII. 191. 48. tab. III et VI، fig. 48. (1880) ; Pand., Revue d'Entomol., II. 201، 2. (1883).

ornatus J JENN., Berlin. Entom. Zeitschr., X. 84. 28. (1866),

recusans WALK, Proc. Linn. Soc. London, III، 83. 21. (1859); v. D. Wusp, Catal. Dipt. South Asia, 61. (1896).

redactus WALK., Insecta Saunders., Dipt., I. 66. (1850).

reducens WaLK., Proc. Linn. Soc. London, IV. 103. 34. (1860); v. D. Wulp, Catal. Dipt. South Asia, 61. (1896).

Regis Georgii Macq., Dipt. exot., I. 1., 132. 21. (1838); WaLK., List Dipt. Brit. Mus., I. 178. (1848) et V. suppl. 1., 251. 434. (1854).

regularis JaEnN., Berlin. Entom. Zeitschr., X. 85. 29. (1866); Brauer, Denkschr. Akad. Wien, XLII. 194. 50. tab. IV. fig. 50. (1880); Pand., Revue d'Entomol., II. 205. 12. (1883).

Reinwardtii Wied., Aussereurop. zweifl. Ins., I. 130. 30. (1828); WALK., List Dipt. Brit. Mus., I. 165. (1848) et V. suppl. 1., 187. 134. (1854) ; Ost.-SACK., Mem. Boston Soc. Nat. Hist., II. 461.

Mexico.

America sept.

Añerica.

Europa mer.

Ins، Aru

Patria ignota.

Celebes.

Australia.

Europa centr.

America sept. 
41. (1876); LugGer, 2nd Record Entomol. Minnesota, 1896. 168. (1896) ; Towns., Psyche, VIII. No. 255., 92. (1897) ; Hine, Ohio State Acad. Sci., Spec. Pap. No. 5., 54. (1903); Aldr., Catal. North Amer. Dipt., 207. (1905).

erythroletus WaLk., Insecta Saunders., Dipt., I. 25. tab. II. fig. 1. (1850).

remotus WaLK., List Dipt. Brit. Mus., I. 177. (1848).

repandus Walk., List Dipt. Brit. Mus., I. 190. [Dichelacera] (1848);

Ricarno, Annal. Mag. Nat. Hist., ser. 7. XIV. 367. (1904).

rhombicus Ost.-SAck., Mem. Boston Soc. Nat. Hist., II. 472. 54.

(1876) et Bullet. Unit. Stat. Geolog. Surv., III. 218. 4. [Therioplectes] (1877); Wiluist., Trans. Kansas Acad. Sci., X. 137. (1887) : Hine, Ohio Naturalist, V. 242. (1904) ; Aldr., Catal. North Amer. Dipt., 207. (1905).

melanorhinus Big., Mém. Soc. Zool. France, V. 642. [? Therioplectes] (1892); Aldr., Catal. Norh Amer. Dipt., 205. (1905).

Rousselii MacQ. = rusticus $\mathrm{L}$.

ruber MACQ. = subruber BELL.

ruber Thusb., Nova Acta Upsal., IX. 56. (1827).

rubescens BeLL., Saggio di Ditterolog. Messic., Append., 15. 24. (1859) ; Aldr., Catal. North Amer. Dipt., 207. (1905).

rubescens Big., Mém. Soc. Zool. France, V. 663. [Atylotus] (1892). rubescens MacQ., Dipt. exot., I. 1., 139. 34. (1838); WALK., List Dipt. Brit. Mus., V. suppl. 1., 212. 246. (1854) ; E. L. Arrib., Bolet. Acad. Nat. Cienc. Córdoba, IV. 136. 83. (1882); Hunter, Trans. Amer. Entom. Soc. Philad., XXVII. 143. [rebescens] (1901). rubicundus MAcq., Dipt. exot., suppl. 1., 32. 64. (1845) et suppl. 3., 10. (1847) ; Walk., List Dipt. Brit. Mus., V. suppl. 1., 248. 410. (1854) ; Macq., Dipt. exot., suppl. 5., 27. 116. (1855) ; v. D. WulP, Catal. Dipt. South Asia, 59. (1896).

rubicundus WALK. $=$ secedens WALK.

rubidus Macq., Dipt. exot., suppl. 2., 19. 100. (1847) ; WaLk., List Dipt. Brit. Mus., V. suppl. 1., 212. 243. (1854).

rubidus Wied., Dipt. exot., 69. 14. (1821) et Aussereurop. zweifl. Ins., I. 127. 25. (1828) ; WALK., List Dipt. Brit. Mus., I. 154. (1848), V. suppl. 1., 244. 396. (1854) et Proc. Linn. Soc. London, I. 9. (1856) ; v. D. Wulp, Notes Leyden Mus., VII. 71. 23. (1885) et Catal. Dipt. South Asia, 59. (1896).

rubiginipennis MAcQ., Dipt. exot., suppl. 1., 39. 81. tab. IV. fig. 6. N. Grenada. (1845) ; WalK., List Dipt. Brit. Mus., V. suppl. 1., 197. 181. (1854) ; Hunter, Trans. Amer. Entom. Soc. Philad., XXVII. 143. (1901).

rubiginosus WALk., Insecta Saunders., Dipt., I. 51. (1850); v. D. India or. Wulp, Catal. Dipt. South Asia, 60. (1896).

rubribarbis Bıł., Mém. Sor. Zool. France, V. 630. [Bellardic] (1892). Americamer rubricornis Pmit., Verh. zool.-bot. Ges. Wien, XV. 723. 34. (1865); Chile.

Hunter, Trans. Amer. Entom. Soc. Philad., XXVII. 143. (1901). 
rubricosus v. D. Wulp, Tijdschr. v. Entomol., XXIV. 160. 13. tab. Argentinia. XV. fig. 9. (1881) ; Hunter, Trans. Amer. Entom. Soc. Philad., XXVII. 143. (1901).

rubrifrons Blanch. in GAY : Hist. fis. y polit. de Chile, Zool. VII. Chile. 396. 9. tab. II. fig. 7. (1854) ; PhlL., Verh. zool.-bot. Ges. Wien, XV. 617. 9. (1865) ; Hunter, Trans. Amer. Entom. Soc. Philad., XXVII. 143. (1901).

rubrinotatus Big., Mém. Soc. Zool. France, V. 676. [Atylotus] (1892). Patriaignota. rubripes MacQ., Dipt. exot., I. 1., 134. 25. (1838); WALK., List Dipt. Brit. Mus., V. suppl. 1., 211. 240. (1854); Hunter, Trans. Amer. Entom. Soc. Philad., XXVII. 143. (1901).

rubrithorax MacQ., Dipt. exot., I. 1., 139. 35. (1838); WaLk., List Dipt. Brit. Mus., V. suppl. 1., 213. 250. (1854); Hunter, Trans. Amer. Entom. Soc. Philad., XXVII. 143. (1901).

rubriventris MACQ., Dipt. exot., I. 1., 131. 19. (1838); WaLK., List Dipt. Brit. Mus., V. suppl. 1., 254. 448. (1854); v. D. Wulp, Catal. Dipt. South Asia, 59. (1896).

rubromaculatus BLANCH. = maculiventris MACQ.

rufescens Fabr., Systema Antliat., 100. 33. (1805); WIED., Dipt. exot., 81. 35. (1821) et Aussereurop. zweifl. Ins., I. 161. 77. (1828) ; Walk., List Dipt. Brit. Mus., V. suppl. 1., 218. 270. (1854); Hunter, Trans. Amer. Entom. Soc. Philad., XXVII. 143. (1901).

ruficeps Kent., Catalogus Tabanidarum, 68. (1900) lapsus.

rufescens KERT. = ruficeps BIG̈.

ruficeps Bı́., Mém. Soc. Zool. France, V. 645. [Atylotus] (1892).

rufescens Kent., Catalogus Tabanidarum, 68. (1900) lapsus.

Cayenne.

Brasilia.

Nova Guinea.

America mer. ruficeps KERT. = rufescens FABR.

ruficeps $\mathrm{MACQ}$. = bicolor Wied.

ruficornis $\mathrm{FABR}_{\mathrm{ABR}}=$ americanus Forst.

ruficrus Pat. Beadv., Insect. recueill. en Afrique et en Amérique,

55. tab. I. fig. 3. (1805-1821).

rufipes Wied., Aussereurop. zweifl. Ins., I. 116. 5. (1828);

Walk., List Dipt. Brit. Mus., V. suppl. 1،, 233. 341. (1854).

rufidens Big., Annal. Soc. Entom. France, sér. 6. VII. Bullet. Japonia.

LXXVIII. [Atylotus] (1887).

rufifrons MaCQ., Dipt. exot., suppl. 5., 28. 119. (1855).

rufinotatus Bı̈., Mém. Soc. Zool. France, V. 673. [Atylotıs] (1892).

rufipennis MACQ., Dipt. exot., I. 1., 134. 26. (1838) et suppl. 1., 41. 83. (1845) ; WALK., List Dipt. Brit. Mus., V. suppl. 1., 214. 257. (1854) ; Hunter, Trans. Amer. Entom. Soc. Philad., XXVII. 143. (1901).

Prom.bon.sp.

Africa.

Australia.

Australia.

Brasilia,

Columbia.

rufipes Macq., Dipt. exot., I. 1., 124. 6. (1838); WALK., List Dipt. Prom،bon sp.

Brit. Mus., V. suppl. 1., 234. 348 (1854).

rufipes MEIG. = fulvus MeIG. var.

mipes Wien. = ruficrus PAL. Beauv.

rufithorax WALK., List Dipt. Brit. Mus., I, 165. (1848).

Brasilia. 
rufiventris FABR., Systema Antliat., 96. 13. (1805); WiED., Dipt. exot., 66. 9. (1821) et Aussereurop. zweifl. Ins., I. 118. 10. (1828); WaLK., List Dipt. Brit. Mus., V. suppl. 1., 245. 398. (1854) ; v. D. Wulp, Catal. Dipt. South Asia, 59. (1896).

rufiventris $\mathrm{MACQ}$. $=$ ? filiolus WILLIST.

rufiventris MAcQ., Dipt. exot., suppl. 1., 38. 78. (1815); WALK., List Dipt. Brit. Mus., V. suppl. 1., 210. 235. (1854); Hunter, Trans. Amer. Entom. Soe. Philad., XXVII. 144. (1901); ALDR., Catal. North Amer. Dipt., 207. (1905).

rufocallosus Big., Mém. Soe. Zool. France, V. 679. (1892); v. D. Java.

Wulp, Catal. Dipt. South Asia, 63. (1896).

rufofrater WАLK., Insecta Saunders., Dipt., I. 26. [rufofrator] (1850); Ost.-SAck., Mem. Boston Soc. Nat. Hist., II. 474. (1876); Aı.Dr., Catal. North Amer. Dipt., 207. (1905).

rufoniger WALK., Insecta Saunders., Dipt., I. 66. (1850).

rufus Pal. Beauv., Insect. recueill. en Afrique et en Amérique, 100. tab. II. fig. 1. (1805-21); WiEd., Aussereurop. zweifl. Ins., I. 117. 8. (1828) ; Walk., List Dipt. Brit. Mus., I. 149. (1848) et V. suppl. 1., 183. 119. (1854); Ost.-SАск., Mem. Boston Soc. Nat. Hist., II. 456. 34. (1876) ; Aldr., Catal. North Amer. Dipt., 207. (1905).

fumipennis WIED., Aussereurop. zweifl. Ins., I. 119. 11. (1828);

Walk., List Dipt. Brit. Mus., I. 149. (1848) et V. suppl. 1., 178. 97. (1854).

? rufus Scop., Entomol. Carniol., 373. 1013. (1763); VilL., Entom. Europa centr. Linn., III, 557. 13. (1789).

rupium Brauer, Denkschr. Akad. Wien, XLII. 163. 26. tab. II. fig. 26. (1880) ; Pand., Revue d'Entomol., II. 210. 26. (1883); Strobl, Mittheil. Naturwiss. Ver. Steiermark, XXIX. 1892. 15. [Atylotus] (1893).

rurclis $\mathrm{ZETT} .=$ ? rusticus $\mathrm{L}$.

rusticus L., Systema Naturae, Ed. XII., II. 1000. 11. (1767); O. F. MürL., Zoolog. Dan. Prodr., 179. 2113. (1776); Schrank, Enum. Ins. Austr., 479. 976. (1781); FABR., Spec. Insect., IJ. 458. 17. (1781) ; VILL., Entomol. Linu., III. 552. 4. (1789); GMEL., Systema Naturae, V. 2883. 11. (1792); FABr., Entomol. System., IV. 367. 23. (1794); Panz., Fauna Germ., XIII. tab. 21. (1794); Schrank, Fauna Boica, III. 154. 2532. (1803); Meig., Klassif., I. 170. 11. (1804) ; FABr., Systema Antliat., 99. 26. (1805) ; Fall., Kongl. Vet. Akad. Handl., XXX. 242. 4. (1809) et Dipt. Suec., Taban., 8. 8. (1817) ; MeIG., System. Beschreib., II. 60. 39. p. p. (ठ') (1820); MAcQ., Recueil Soc. Sci. Agricult. I.ille, 1826. 842. 15. (1826) et Suit. à Buffon, I. 207. 4. (1834); Duncan, Magaz. Zoolog. and Botan., I. 367. (1837) ; ZEтT., Insecta Lappon., Dipt., 517. 11. (1838) ; ZelL., Isis, 1842. XI. 823. 2. (1842) ; Zetт., Dipt. Scand., I. 119. 21. (1842); Giмm., Bull. Soc. Imp. Nat. Moscou, XX. 1., 186. 19. (1847); Zетт., Dipt. Seand., VIII. 2940. 21. (1849); WАL..,

Columbia, Jamaica.

$\Lambda$ mericasept.

Patria ignota. America sept.

Europa centr.

Europa centr. et mer., Algeria. 
Insecta Britann., Dipt., I. 40. 13. (185̃1); ZETT., Dipt. Scand., XI. 4259. 21. (1852); WaLk., List Dipt. Brit. Mus., V. suppl. 1., 155 et 158. 8. (1854); Lw., Verh. zool.-bot. Ges. Wien, VIII. 596. 22. (1858) ; Borsd., Finlands tvaving. Ins., I. 111. 14. (1861) ; Schis., Fauna Austriaca, Dipt., I. 32. (1862); Brauer, Denkschr. Akad. Wien, XLII. 168. 31. tab. II et V. fig. 31. (1880); Gов., Mém. Soc. Linn. Nord France, 1881. 29. 2. (1881); Pand., Revue d'Entom., II. 219. 48. (1883) ; Hansen, Fabrica oris Dipt., tab. I. fig. 7, tab. II. fig. 1, 3, 4, 9. (1883) ; Neunaus, Diptera Marchica, 44. 12. (1886); L. Coucke, Annal. Soc. Entom. Belgique, XXXVI. 138. (1892) ; Leonardi, Insetti Nocivi, III. 374. [Atylotus] (1900); Ricardo, Annal, Mag. Nat. Hist., ser. 7. XVI. 197. [Atylotus] (1905); Villen., Annal. Soc. Entom. France, LXXIV. 307. 5.(1905).

?flaviceps Zetr., Dipt. Scand., I. 111. 9. (1842) et VIII. 2937. 9. (1849); WalK., List Dipt. Brit. Mus., V. suppl. 1., 156 et 163. 29. (1854); Bonsd., Finlands tvaving. Ins., I. 299. 5-6. (1861).

Rousselii Macq., Dipt. exot., I. 2., 181. (1839) et Explor. scient. de l'Algérie, Zool. III., 423. 23. (1849); WaLk., List Dipt. Brit. Mus., V. suppl. 1., 225 et 229. 322. (1854).

? ruralis ZETT., Insecta Lappon., Dipt., 517. 11. (1838), Dipt. Scand., I. 120. 23. (1842) et VIII. 2940. 23. (1849); WALK., List Dipt. Brit. Mus., V. suppl. 1., 155 et 160. 15. (1854); Bonsd., Finland tvaving. Ins., I. 299. 16. (1861).

rusticus Meig. p. p. = fulvus Meig.

sabuletorum Lw., Zeitschr. f. d. ges. Naturwiss., N. F. IX. (XLIII). 414. 4. (1874).

sagax Ost.-SAcK., Mem. Boston Soc. Nat. Hist., II. 452. 27. (1876); Aldr., Catal. North Amer. Dipt., 207. (1905).

sagittarius Macq., Dipt. exot., I. 1., 123. 5. (1838); WaLK., List Dipt. Brit. Mus., V. suppl. 1., 228. 320. (1854); Ricardo, Annal. Mag. Nat. Hist., ser. 7. VI. 164. (1900); Adaus, Kansas Univ. Sci. Bullet., III. 150. (1905).

serratus Lw., Öfvers. Kongl. Vet. Akad. Förhandl., XIV. 1857. 337. 2. (1858) et Dipt.-Fauna Südafrika's, I. 39. 10. tab. I.

fig. 21. (1860).

socius Walk., List Dipt. Brit. Mus., I. 160. (1848).

Sallei Bell., Saggio di Ditterolog. Messic., I. 61. 11. tab. II. fig. 7. Mexico.

(1859) ; Aldr., Catal. North Amer. Dipt., 207. (1905).

sanguinarius Big., Mém. Soc. Zool. France, V. 675. (1892).

Australia.

sanguineus Walk., Insecta Saunders., Dipt., I. 54. (1850); v. D. Java.

Wulp, Catal. Dipt. South Asia, 60. (1896).

sanguisorba HARRIS = fulvus MEIG.

Sarpa Walk., List Dipt. Brit. Mus., I. 175. (1848) [sine descr.], in Newman: Zoologist, Append., LXX. (1850) et List Dipt. Brit. Mus., V. suppl. 1., 255. 450. (1854); Hutron, Catal. New Zealand Dipt. etc, 20. (1881) et Trans. Now Zealand Instit., XXXIII. 13. (1901).

N. Selandia. 
truncatus Walk, in Newsha : Zoologist, VIII. Append., LXX. (1850) et List Dipt. Brit. Mus., V. suppl. 1., 255. 451. (1854) ; Hutron, Catal. New Zealand Dipt. etc., 20. (1881).

satanicus Big., Mém. Soc. Zool. France, V. 632. [Dichclacera] (1892); Brasilia.

Ricardo, Annal. Mag. Nat. Hist., ser. 7. XIV. 368. (1904).

scalaris MeIG. = bromius $\mathrm{L}$.

scitus Walk., List Dipt. Brit. Mus., I. 181. (1848); Ost.-SAck., Mem.

Roston Soc. Nat. Hist., II. 474. (1876); ALdr., Catal. North Amer.

Dipt., 207. (1905).

scutellaris WALK, = ? lineola FABR.

scutellatus Macq., Dipt. exot., I. 2., 186. (1839); Walk., List Dipt. Patriaignota.

Brit. Mus., V. suppl. 1., 262. 496. (1854).

scythropus Schin., Novara Reise, Dipt., 92. 31. (1868); Hunter, Brasilia.

Trans. Amer. Entom. Soc. Philad., XXVII. 144. (1901).

secedens Walk., List Dipt. Brit. Mus., V. suppl. 1., 224. 301. (1854). rubicundus Walk., List Dipt. Brit. Mus., I. 161. (1848).

tibialis WaLK., List Dipt. Brit. Mus., I. 162. (1848).

secundus WALK., List Dipt. Brit. Mus., I. 180. (1848).

segmentarius BRULLE = graecus FABR.

semisordidus WaLK., List Dipt. Brit. Mus., V. suppl. 1., 208. 225. Brasilia. (1854).

senilis PHIL., Verh. zool.-bot. Ges. Wien, XV. 715. 15. (1865) ; HunTer, Trans. Amer. Entom. Soc. Philad., XXVII. 144. (1901).

senior WaLK., Insecta Saunders., Dipt., I. 67. (1850).

septentrionalis Lw., Verh. zool.-bot. Ges. Wien, VIII. 593. (1858); Ost.-SAck., Mem. Boston Soc. Nat. Hist., II. 467. 50. (1876); Coquill., Proc. Washington Acad. Sci., II. 406. (1900); Hine, Ohio Naturalist, V. 243. (1904); Coquill. in Harriman: Alaska Exped., IX. Pt. II. 20. (1904) ; Aldr., Catal. North Amer. Dipt., 207. (1905).

sequax Willist., Trans. Kansas Acad. Sci., X. 137. (1887); HrNe,

Ohio Naturalist, V. 243. (1904) ; Aldr., Catal. North Amer. Dipt., 207. (1905).

leucophorus Bıg., Mém. Soc. Zool. France, V.640. [Therioplectes] (1892); Aldr., Catal. North Amer. Dipt., 204. (1905).

sequens WALK., List Dipt. Brit. Mus., I. 178. (1848).

sequens WALK., Insecta Saunders., Dipt., I. 60. (1850).

sericiventris Lw., Öfvers. Kongl. Vet. Akad. Förluandl., XIV. 1857. 339. 17. (1858), Dipt.-Fauna Südafrika's, I. 38. 7. (1860) et Wiell. Entom. Monatschr., VII. 10. 4. (1863).

servatus $\mathrm{Lw},=$ sagittarius MACQ.

serus Walk., Proc. Linn. Soc. I.ondon, VI. 20. 3. (1862); v. D. Wulp, Ceram.

Catal. Dipt. South Asia, 61. (1896).

Servillei MaCQ., Dipt. exot., I. 1., 128. 13. (1838); WALK., List Dipt. India or., Brit. Mus., V. suppl. 1., 246. 404. (1854); v. D. Wulp, Catal. Dipt. Java. South Asia, 59. (1896).

sexvittatus Bı́., Mém. Soc. Zool. France, V. 682. (1892); Aldr., Mexico. Catal. Norh Amer. Dipt., 207. (1905). 
siccus WaLk., Insecta Saunders., Dipt., I. 46. (1850); Ricardo, Aegyptus. Annal. Mag, Nat. Hist., ser. 7. XVI. 200. (1905).

sidneyensis MacQ., Dipt. exot., suppl. 1., 33. 66. (1845); WaLk., Australia. List Dipt. Brit. Mus., V. suppl. 1., 253. 442. (1854).

signatipennis Portsch., Horae Soc. Entom. Ross., XXI. 180. (1887). Mongolia signatus Panz. = micans MeIG.

signatus Schin. $=$ tropicus PAnz.

signatus WIED. = aterrimus MEIG.

signifer Walk., Insecta Saunders., Dipt., I. 452. (1856); v. D. WulP, China.

Catal. Dipt. South Asia, 60. (1896).

similis MACQ., Dipt. exot., suppl. 4., 31. 100. (1850).

simplex Big., Mém. Soc. Zool. France, V. 667. (1892).

Tasmania.

simplex WaLk., Insecta Saunders., Dipt., I. 34. (1850).

Amazonia.

Columbia.

simplissimus Walk., Proc. Linn. Soc. London, I. 111. 34. (1856); Borneo.

v. D. Wulp, Catal. Dipt. South Asia, 61. (1896).

simulans $\mathrm{W}_{\mathrm{ALK}}$. = lineola $\mathrm{FABR}_{\mathrm{AB}}$.

sinicus BıG., Mém. Soc. Zool. France, V. 629. [Bellardia] (1892);

v. D. Wulp, Catal. Dipt. South Asia, 62. (1896).

sinicus WaLK., List Dipt. Brit. Mus., I. 163. (1848); v. D. Wulr, China.

Catal. Dipt. South Asia, 60. (1896).

socialis WALK., Insecta Saunders., Dipt., I. 45. (1850).

socius OST.-SACK. $=$ epistates OsT.-SACK.

socius $\mathrm{W}_{\mathrm{ALK}}=$ = sagittarius $\mathrm{MACQ}$.

sodalis $\mathrm{W}_{1 \mathrm{LL}}=$ trispilus $\mathrm{W}_{\mathrm{IED}}$.

solstitialis Meig., System. Beschreib., II. 56. 33. (1820) ; ? Duncax,

Magaz. Zoolog. and Botany, I. 366. (1837); ? Grмm., Bull. Soc.

Imp. Nat. Moscou, XX. 1., 185. 15. (1847); ? WALK., List Dipt.

Brit. Mus., I. 169. (1848), ? Insecta Saunders., Dipt., I. 22. (1850)

et ? List Dipt. Brit. Mus., V. suppl. 1., 156 et 168. 46. (1854);

Scirin., Fauna Austriaca, Dipt., I. 30. (1862); Brauer, Denkschr.

Akad. Wien, XLIf. 150. 12. tab. I et V. fig. 12. (1880) ; Gов.,

Mém. Soc. Linn. Nord France, 1881. 13. 4. [Therioplectes] (1881);

Pand., Revue d'Entom., II. 212. 31. (1883); Strobl, Mittheil.

Naturwiss. Ver. Steiermark, XXIX. 1892. 14. (1893) ; Villen.,

Annal. Soc. Entom. France, LXXIV. 307. 4. (1905).

montanus Meig., System. Beschreib., II. 55. 31. p. p. (1820).

tropicus Lw., Verh. zool.-bot. Ges. Wien, VIII. 586. 11. p. p. (1856).

Sonnerati Bıg., Mém. Soc. Zool. France, V. 672. [Atylotus] (1892); Nova Guinea. v. D. Wulp, Catal. Dipt. South Asia, 63. (1896).

sonomensis Ost.-SACK., Bullet. Unit. Stat. Geolog. Surv., III. 216.

2. (1876) ; Coquil., Proe. Washington Acad. Sci., II. 407. (1900);

Hine, Ohio Naturalist, V. 244. (1904); Beutenm., Bull. Amer.

Mus. Nat. Hist, XX. 88. (1904); Coquill. in Harrimay: Alaska Exped., IX. Pt. II., 21. (1904); Aldr., Catal. North Amer. Dipt., 207. (1905).

haemaphorus MARTEN, Canad. Entomol., XIV. 210. [Therioplectes] (1882).

Asia min.,

Sibiria.

America sept., Alaska. 
sorbillans WtED., Aussereurop. zweifl. Ins., I. 141. 48. (1828) ; WALK., List Dipt. Brit. Mus., I. 153. (1848) et V. suppl. 1., 205. 216. (1854) ; Huxter, Trans. Amer. Entom. Soc. Philad., XXVII. 144. (1901).

sordidus WaLk., List Dipt. Brit. Mus., V. suppl. 1., 256. 453. (1854); Hutron, Catal. New Zealand Dipt. etc., 20. (1881) et Trans. New Zealand Instit., XXXIII. 12. (1901).

sparsus WaLK., Insecta Saunders., Dipt., I. 71. [Dichelacera] (1850); Brasilia.

Ricardo, Annal. Mag. Nat. Hist., ser. 7. XIV. 367. (1904).

sparus Whitney, Canad. Entomol., XI. 38. (1879); Willist., Trans.

Kansas Acad. Sci., X. 140. (1887); Aldr., Catal. North Amer.

Dipt., 207. (1905).

spectabilis Lw., Verh. zool.-bot. Ges. Wien, VIII. 605. 34. (1858) et

Wien. Entom. Monatschr., VI. 163. 12.(1862); Braver, Denkschr.

Akad. Wien, XLII. 190. 47. tab. III et VI. fig. 47. (1880); PAND:, Revue d'Entomol., II. 204. 10. (1883).

? albivittatus Macq., Suit. à Buffon, I. 206. 32. (1834); WaLK., List Dipt. Brit. Mus., V. suppl. 1., 261. 490. (1854).

humeralis MEG. in litt. apud BRAUER.

lateralis Brullé, Exped. scient. de Morée, III. 304. 658. tab. XLVII. fig. 2. (1832) ; Walk., List Dipt. Brit. Mus., V. suppl. 1., 156 et 166. 39. (1854).

speculum Walk., Proc. Linn. Sos. London, V. 258. 3. (1861); v. D. Celebes.

Wulp, Catal. Dipt. South Asia, 61. (1896).

spilopterus Lw. = Astur Erichs.

spodopterus Meig., System. Beschreib., II. 46. 21. (1820); Gimn., Bull. Soc. Imp. Nat. Moscou, XX. 1., 181. 4. (1847); WALK., List Dipt. Brit. Mus., V. suppl. 1., 156 et 169. 50. (1854); Lw., Verh. zool.-bot. Ges. Wien, VIII. 606. 37. (1858); Schis., Fauna Austriaca, Dipt., I. 33. (1862) ; Lw., Wien. Entom. Monatscbr., VI. 163. 11. (1862); JAsn., Berlin. Entom. Zeitschr., X. 79. 20. (1866) ; Brauer, Denkschr. Akad. Wien, XIII. 181. 41. tab. III et VI. fig. 41. (1880); Goв., Mém. Soc. Linn. Nord France, 1881. 19. 3. (1881) ; Pand., Revue d'Entomol., II. 202. 5. (1883); Brauer, Denkschr. Akad. Wien, XLVII. tab. II. fig. 26-31. (1883) ; StroвL, Mittheil. Naturwiss. Ver. Steiermark, XXIX. 1892. 15. (1893).

p. p. bovinus L., Fauna Suec., 462. 1882. (1761); Poda, Ins. Mus. Graec., 117. 1. (1761); Scop., Entom. Carniol., 370. 1006. (1763); O. F. Mtul., Fauna Ins. Fridrichsdal., 86. 767. (1764); I., Systema Naturae, Ed. XII., II. 1000. 4. (1767) ; FABr., Spec. Insect., II. 455. 3. (1781); Schrank, Enum. Insect. Austr., 478. 974. (1781); Deg., Mém. pour serv. à l'hist. d. Ins., VI. 219. 1. tab. XII. fig. 10-11. (1776) ; Herbst, Gemeinnütz. Naturgesch., VIII. tab. CCCXIII. fig. 1. (1787); Gnel., Systema Naturae, V. 2882. 4. (1788) ; Vilu., Entom. Linn., III. 551. 1. (1789); PANz., Fauna Germ., II. 20. (1793) ; FABR., Entom. System., IV. 363. 4. (1794);

Europa centr. et mer. 
Schrank, Fauna Boica, III. 153. 2529. (1803); MeıG., Klassif., I. 165. ․ (1804); Fabr., Systema Antliat., 92. 1. (1805); IAATr., Gen. Crust. et Ins., IV. 283. (1809) ; Fall., Kongl. Vet. Akad. Handl., XXX. 239. 1. (1809) ; LAM., Hist. nat. anim. sans vertébr., III. 420. 1. (1816); Fall., Dipt. Suec., Taban., 4. 1. (1817); Cuv., Régne Anim., III. 614. (1817); Meia., System. Beschreib., II. 43. 18. (18:0); MacQ., Recueil Soc. Sci. Agricult. Lille, 18:6. 476. 2. tab. III. fig. 7. (1826) et Suit. à Buffon, I. 199. 9. (1834); Guèr., Iconogr. d. Régne Anim., VII. Ins., 542. tab. XCVII. fig. 1, a-d. (1835) ; Daнlв., Ins. Seand. skäd. och nytta, 306. 203. (1837) ; Duncan, Magaz. Zoolog. and Botan., I. 362. (1837); Zetr., Insecta Lappon., Dipt., 513. 1. (1838); Westw., Introd., II. 541. fig. 128., 9. 10. (1840) ; Blanch., Hist. Nat. d'Ins., III. 589. tab. I. fig. 9. (1840) ; Zetr., Dipt. Scand., I. 105. 1. (1842); ZeLL., Isis, 1842. XI., 814. 1. tab. I. fig. 2-4. (1842); Giмm., Bull. Soc. Imp. Nat. Moscou, XX. 1., 179. 1. (1847); Macq. in Lucas: Explor. Scient. de l'Algérie, Zool., III. 424. 28. (1849); ZетT., Dipt. Scand., VIII. 2936. 1. (1849); L. Duf., Mém. Acad. des Sci. Math. et Phys., XI. 171-360. tab. I. fig. 3-5, tab. II. fig. 15-16, tab. IV. fig. 38-39, 42. (1850); WALK., Ins. Britam., Dipt., I. 37. 1. tab. I. fig. 13. (1851) et List Dipt. Brit. Mus., V. suppl. 1., 156 et 166. 40. (1854); Zетт., Dipt. Seand., XII. 4547. 1. (1855); Bonsd., Finl. tvaving. Ins., I. 107. 1. (1861); Schin., Fauna Austriaca, Dipt., I. 34. (1862); JAEnn., Berlin. Entom. Zeitschr., X. 80. (1866); Neuraus, Diptera Marchica, 42. 2. (1886). spoliatus WALK., Proc. Linn. Soc. London, IV. 103. 35. (1860); v. D. Wulp, Catal. Dipt. South Asia, 61. (1896).

stigma FABr., Systema Antliat., 104. 50. (1805); WIED., Dipt. exot., 92. 53. (1821) et Aussereurop. zweifl. Ins., I. 180. 104. (1828); WALK., List Dipt. Brit. Mus., V. suppl. 1., 202. 203. (1854); Hunter, Trans. Amer. Entom. Soc. Philad., XXVII. 144. (1901); Aldr., Catal. North Amer. Dipt., 208. (1905).

stigma WaLí., List Dipt. Brit. Mus., I. 184. (1818).

striatus FABr., Entomol. System., IV. 371. 39. (1794) et Systema Antliat., 103. 47. (1805); WIED., Dipt. exot., 79. 33. (1821) et Aussereurop. zweifl. Ins., I. 155. 69. (1828); WALK., List Dipt. Brit. Mus., I. 157. (1848) et V. suppl. 1., 245. 397. (1854); v. D. Wulp, Sumatra Exped., Dipt., 16. 3. (1881), Notes Leyden Mus., VII. 71. 24. (1885) et Catal. Dipt. South Asia, 58. (1896).

dorsilinea WIED., Analecta Entomol., 22. 10. (1824) et Aussereurop. zweifl. Ins., I. 184. 110. (1828); WALk., List Dipt. Brit. Mus., V. suppl. 1., 24t. 395. (1854).

strigiventris Lw., Öfvers. Kongl. Vet. Akad. Förhandl., XIV. 1857. 339. 14. (1858) et Dipt.-Fauna Südafrika's, I. 33. 1. (1860).

stygius SAY, Journ. Acad. Nat. Sci. Philad., III. 33. 3. (1823) et Celebes.

Anerica mer., Antillae.

Africa mer.

Asia mer.,

China,

Arch.Indicus.

Amer. Entomol., Ed. II., II. 54. (1859); WiED., Aussereurop. zweifl. Ins., I. 131. 31. (1828); WaLk., List Dipt. Brit. Mus., I. 
149. (1848) et V. suppl. 1., 187. 136. (1854); Ost.-Sack., Mem. Boston Soc. Nat. Hist., II. 454. 30. (1876) ; Han'т, Bullet. Illinois State Laborat. of Nat. Hist., IV. 239. tab. XI. fig. 47-48. (1895); Hine, Ohio State Acad. Sci., Spec. Pap. No. 5., 54. tab. II. (1903) ; Aldn., Catal. North Amer. Dipt., 208. (1905).

subelongatus Macq., Dipt. exot., suppl. 1., 31. 62. (1845); WaLK.,

List Dipt. Brit. Mus., V. suppl. 1., .230. 328. (1854); Bertol., Mem. Accad. Sci. Istit. Bologna, XII. 16. 18. (1862); BezzI, Annal. Mus. Civ. Genova, ser. 2. XII. (XXXII). 183. 2. (1892).

var. proximus Contr, Annal. Mus. Civ. Genova, ser. 2. XV. Gallaland. (XXXV). 132. 3. (1895).

sublunaticonis ZETT. = ? plebejus FALL.

submacula WALK., Insecta Saunders., Dipt., I. 30. tab. II. fig. 8. Columbia. $(1850)$.

subruber BeLL., Saggio di Ditterol. Messic., I. 55. 1. (1858); W1L-

Mexico.

List., Biolog. Central. Americ., Dipt., I. 260. 14. (1901); Aldr.,

Catal. North Amer. Dipt., 208. (1905).

vuber Macq., Dipt. exot., suppl. 1., 42. 87. (1845); WaLK., List

Dipt. Brit. Mus., V. suppl. 1., 188. 142. (1854).

subsenex WALK., Insecta Saunders., Dipt., I. 38. (1850).

subsimilis BeLl., Saggio di Ditterol. Messic., I. 66. 18. (1859); Aldr., Catal. North Amer. Dipt., 208. (1905).

subtilis Bell., Saggio di Ditterol. Messic., I. 14. 23. tab. III. fig. Mexico.

9. (1859) ; Aldor., Catal. North Amer. Dipt., 208. (1905).

succurvus Walk., Proc. Linn. Soe. London, IV. 102. 32. (1860);

Ost.-Sack., Annal. Mus. Civ. Genova, XVI. 417. (1882); v. D.

Wulp, Catal. Dipt. South Asia, 61. (1896).

sudeticus ZeLl., Isis, 1842. XI., 815. 2. tab. I. fig. 5-8. (1842);

America mer.

Mexico.

Celebes.

Scholtz, Zeitschr. f. Entóm. Breslau, IV. No. 16., 36. (1850) ;

Walk., List Dipt. Brit. Mus., V. suppl. 1., 155 et 157. 4. (1854); Lw., Verh. zool.-bot. Ges. Wien, VIII. 606. 35. (1858); Schis., Fauna Austriaca,-Dipt., I. 34. (1862); JAENN., Berlin. Entom. Zeitschr., X. 78. 19. (1866); Brauer, Denkschr. Akad. Wien, XIII. 184. 43. tab. III et VI. fig. 43. (1880); Goв., Mém. Soc. Linn. Nord France, 1881. 20. 4. (1881); Pand., Revue d'Entom., II. 203. 6. (1883); Hansen, Fabrica oris Dipt., tab. I. fig. 6, 10, 11, 13, 16, 20, 23. (1883) ; L. Coucke, Annal. Soc. Entom. Belgique, XXXVI. 137. (1892); StroвL, Mittheil. Naturwiss. Ver. Steiermark, XXIX. 1892. 15. (1893); SPEISER, Zeitschr. f. wiss. Insektenbiolog., I. 408. 156. (1904).

p. p. bovinus L., Fauna Suec., 462. 1882. (1761); PodA, Ins. Mus. Grace., 117. 1. (1761) ; Scop., Entom. Carniol., 370. 1006. (1763) ; O. F. Mell., Fauna Ins. Fridrichsdal., 86. 767. (1764); I., Systema Naturae, Ed. XII., II. 1000. 4. (1767); FABR., Spec. Insect., II. 455. 3. (1781); Schrank, Enum. Insect. Austr., 478. 974. (1781) ; Deg., Mém. pour serv. à l'hist. d. Ins., VI. 219. 1. tab. XII. fig. 10-11. (1776); Hersst, Gemeinnütz. Naturgesch., VIII. 
tab. CCCXLII. fig. 1. (1787); Gnel., Systema Naturae, V. 2882. 4. (1788) ; Vill., Entom. Linn., III. 551. 1. (1789); PAnz., Fauna Germ., II. 20. (1793); FABr., Entom. System., IV. 363. 4. (1794); Schrank, Fauna Boica, III. 153. 2529. (1803) ; Meig., Klassif,, I. 165. 2. (1804) ; FABr., Systema Antliat., 92. 1. (1805); LA'H., Gen. Crust. et Ins., IV. 283. (1809) ; Fall., Kongl. Vet. Akad. Handl., XXX. 239. 1. (1809) ; LAM., Hist. nat. anim. sans vertébr., III. 420. 1. (1816) ; Fall., Dipt. Suec., Taban., 4. 1. (1817) ; Cuv., Régne Anim., III. 614. (1817) ; MeIG., System. Beschreib., II. 43. 18. (1820); Macq., Recueil Soc. Sci. Agricult. Lille, 1826. 476. 2. tab. III. fig. 7. (1826) et Suit. à Buffon, I. 199. 9. (1834); Gứr., Iconogr. d. Régne Anim., VII. Ins., 542. tab. XCVII. fig. 1, $a-d$. (1835) ; DAнlв., Ins. Scand. skäd. och nytta, 306. 203. (1837); Duncan, Magaz. Zoolog. and Botan., I. 362. (1837); Zetr., Insecta Lappon., Dipt., 513. 1. (1838); Westw., Introd., II. $5+1$. fig. 128., 9. 10. (1840) ; Blanch., Hist. Nat. d'Inst., III. 589. tab. I. fig. 9. (1840) ; Zetr., Dipt. Scand., I. 105. 1. (1842); Zell., Isis, 1842. XI. 814. 1. tab. I. fig. 2-4. (1842); Grmm., Bull. Soc. Imp. Nat. Moscou, XX. 1., 179. 1. (1847); MacQ. in Lucas: Explor. Scient. de l'Algérie, Zool., III. 424. 28. (1849) ; Zeтt., Dipt. Scand., VIII. 2936. 1. (1849); L. Duf., Mém. Acad. des Sci. Math. et Phys., XI. 171-360. tab. I. fig. 3-5, tab. II. fig. 15-16, tab. IV. fig. 38-39, 42. (1850); WaLk., Ins. Britann., Dipt., I. 37. 1. tab. I. fig. 13. (1851) et List Dipt. Brit. Mus., V. suppl. 1., 156 et 166. 40. (1854); Zetr., Dipt. Scand., XII. 4547. 1. (1855); Bonsd., Finl. traving. Ins., I. 107. (1861); Schin., Fauna Austriaca, Dipt., I. 34. (1862); JAENN., Berlin. Entom. Zeitschr., X. 80. (1866) ; Neuhaus, Diptera Marchica, 42. 2. (1886).

Sufis Jaenn., Abhandl. Senckenberg. Naturforsch. Ges., VI. 332. 24. (1867) ; Brauer, Denkschr. Akad. Wien, XLII. 205. 59. tab. IV et VI. fig. 59. (1880).

sugens Wied., Aussereurop. zweifl. Ins., I. 140. 45. (1828); WaLK., List Dipt. Brit. Mus., V. suppl. 1., 233. 343. (1854).

sulcifrons Macq., Dipt. exot., suppl. 5., 33. 127. (1855) ; Ost.-SACK., Catal. Dipt. North Amer., Ed. II., 60 et 228. (1878); Willist., Trans. Kansas Acad. Sci., X. 138. (1887); Rilwy \& Howard, Insect Life, VI. 34. (1894); HART, Bullet. Illinois State Laborat. of Nat. Hist., IV. 234. (1895) ; Hine, Ohio Naturalist, II. 168. (1901) et Ohio Stat. Acad. Sci., Spec. Pap. No. 5., 54. tab. I. fig. 1-15. (1903); Aldr., Catal. North Amer. Dipt., 208. (1905).

tectus Ost.-Sack., Mem. Boston Soc. Nat. Hist., II. 436. 7. (1876). sulcipalpus Lw., Öfvers. Kongl. Vet. Akad. Förhandl., XIV. 1857. Caffraria. 341. 26. (1858) et Dipt.-Fauna Südafrika's, I. 45. 17. (1860).

sulphureus MacQ., Dipt. exot., suppl. 2., 19. 99. (1847); WALK., List Brasilia. Dipt. Brit. Mus., V. suppl. 1., 215. 258. (1854); Hunter, Trans. Amer. Entom. Soc. Philad., XXVII. 144. (1901). sulphureus PaL, Beauv. = mexicanus $\mathrm{L}$.

Africa sept. et orient.

Guinea.

America sept. 
sumatrensis MaCQ., Suit. à Buff., I. 201. 16. (1834); WaLk., List Dipt. Brit. Mus., V. suppl. 1., 244. 394.(1854); v. D. Wulp, Catal. Dipt. South Asia, 59. (1896).

Sumischrasti Bell., Saggio di Ditterol. Messic., I. 56. 2. (1859) Aldr., Catal. North Amer. Dipt., 208. [sumichrasti] (1905).

superjumentarius Whitney, Canad. Entomol., XI. 37. (1879); Hine, Ohio State Acad. Sci., Spec. Pap. No. 5., 55. (1903) ; Aldr., Catal. North Amer. Dipt., 208. (1905).

surinamensis Macq., Dipt. exot., I. 1., 132. 22. (1838); WALK., List Dipt. Brit. Mus., V. suppl. 1., 204. 210. (1854) ; Hunter, Trans.

Amer. Entom. Soc. Philad., XXVII. 144. (1901).

susurrus Marten, Canad. Entomol., XV. 111. [Therioplectes] (1883); Hine, Ohio Naturalist, V. 244. (1904); Aldr., Catal. North Amer. Dipt., 208. (1905).

Swiridowi Portsch., Horae Soc. Entom. Ross., XVI. 256. (1882) et XXVI. 202. tab. I. fig. 2. (1891).

sylvioides WaLk., Proc. Linn. Soc. London, VII. 206. 17. (1864); v. D. Wulp, Catal. Dipt. South Asia, 61. (1896).

taeniatus MACq., Suit. à Buffon, I. 207. 35. (1834); Walk., List Dipt. Brit. Mus., I. 184. (1848) et V. suppl. 1., 229. 321. (1854).

taeniola Pal. Beauv., Ins. recueill. en Afrique et en Amérique, 56. tab. I. fig. 6. (1805--21); Wied., Dipt. exot., 71. 17. (1821) et Aussereurop. zweifl. Ins., I. 129. 28. (1828); Walk., List Dipt. Brit. Mus., V. suppl. 1., 233. 340. (1854).

taeniotes Wied., Aussereurop. zweifl. Ins., I. 188. 116. (1828) ; WaLK., List Dipt. Brit. Mus., V. suppl. 1., 217. 267. (1854); Hunter, Trans. Amer. Entom. Soc. Philad., XXVII. 144. [taeniates] (1901). tarandi $\mathrm{W}_{\text {ALK. }}=$ zonalis KIRBY.

tarandicus VILL. = tarandinus $\mathrm{L}$.

tarandinus L., Fauna Suec., 462. 1884. (1761) et Systema Naturae,

Ed. XII., II. 1000. 7. (1767) ; O. F. Mtlller, Zoolog. Dan. Prodr., 179. 2112. (1776) ; FABr., Spec. Insect., II. 457. 8. (1781) ; GMeL., Systema Naturae, V. 2882. 7. (1792); FABr., Entomol. System., IV. 364. 10. (1794); MEIG., Klassif., I. 171. a. (1804); FABR., Systema Antliat., 95. 8.(1805); FALL., Kongl. Vet. Akad. Handl., XXX. 240. 2. (1809) et Dipt. Suec., Taban., 4. 2. (1817) ; ? PETAGNA, Spec. Insect. ultr. Calabr., Ed. nova, 44. 233. (1820); Meig., System. Beschreib., II. 44. 19. (1820); Duncan, Magaz Zoolog. and Botan., I. 364. (1837) ; Zeтт., Insecta Lappon., Dipt., 513. 2. (1838) et Dipt. Scand., I. 109. 7. (1842); Giмм., Bull. Soc. Imp. Nat. Moscou, XX. 1., 184. 12. (1847); Zetт., Dipt. Scand., VIII. 2937. 7. (1849); Erichs. in Middendorf : Reise in Sibir., II. 66. 142. (1851) ; ZETT., Dipt. Scand., XI. 4259. 7. (1854); Lw., Verl. zool.-bot. Ges. Wien, VIII. 585. 10. (1858); Boxsd., Finl. tvaving. Ins., I. 108. 4. (1861); Braver, Denkschr. Akad. Wien, XLII. 139. 3. tab. I. fig. 3. (1880); KERT., Zoolog. Ergebn. d.3. asiat. Forschungsreise des Grafen E. Zichy, II. 191. 21. fig.
Sumatra.

Mexico.

America sept.

Surinam.

America sept.

Caucasus.

Ins. Mysol.

Prom.bon.sp.

Africa centr.

Brasilia.

Europa bor., Sibiria. 
5. [Therioplectes] (1901); Speiser, Zeitschr. f. wiss. Insektenbiol., I. 407. 155. (1904) et Schrift. d. Physik.-ökon. Ges. Königsberg, XLVI. 163. (1905); Vilien., Annal. Soc. Entom. France, LXXIV. 309. 25. (1905).

tarandicus Vill., Entomol. Linn., III. 552. 3. (1789).

tarsalis Adams, Kansas Univ. Sci. Bullot., III. 151. (1905).

tataricus Pontsch., Horae Soc. Entom. Ross, XXI. 178. (1887).

taurinus Meig. = barbarus Coqueb.

tectus Ost.-SACK. = sulcifrons MACQ.

temperatus WaLK., Insecta Saunders., Dipt., I. 451. (1856).

tenebrosus Coquil.., Proc. Unit. Stat. Nat. Mus., XXI. 310. (1898). tenebrosus WaLk., List Dipt. Brit. Mus., V. suppl. 1., 242. 389.

(1854) ; v. D. Wulp, Catal. Dipt. South Asia, 60. (1896).

tenens WALK. = confligens WALK.

tenens WaLK., Insecta Saunders., Dipt., I. 49. (1850); v. D. WulP, Catal. Dipt. South Asia, 60. (1896).

tener Ost.-SACK., Mem. Boston Soc. Nat. Hist., JI. 440. 11. (1876) et Catal. Dipt. North America, Ed. II., 60 et 228. (1878); LLDr., Catal. North Amer. Dipt., 208. (1905).

tennessensis Bıı., Mém. Soc. Zool. France, V. 660. [Atylotus] (1892); Aldpr., Catal. North Amer. Dipt., 208. (1905).

tenuicornis MACQ., Dipt. exot., I. 1., 124. 7. (1838); WaLK., List Dipt. Brit. Mus., V. suppl. 1., 229. 324. (1854).

tephrodes Phil., Verh. zool.-bot. Ges. Wien, XV. 716. 16. (1865); Hrnter, Trans. Amer. Entom. Soc. Philad., XXVII. 144. (1901). tergestinus EGG., Verh. zool.-bot. Ges. Wien, IX. 391. (1859); Schin., Fauna Austriaca, Dipt., I. 33. (1862); Brauer, Denkschr. Akad. Wien, XLII. 193. 46. tab. III et VI. fig. 46. (1880); Goz., Mém. Soc. Linn. Nord France, 1881. 19. 2. (1881); Pand., Revue d'Entomol., II. 207. 17. (1883); Sтroвs, Mittheil. Naturwiss. Ver. Steiermark, XXIX. 1892. 16. (1893).

glaucus Meig., System. Beschreib., II. 51. 28. p. p. (1820).

terminalis WaLK., The Entomologist, V. 255. (1871); Ricardo, Annal. Mag. Nat. Hist., ser. 7. XVI. 200. (1905).

terminatus WaLk., Insecta Saunders., Dipt., I. 41. (1850).

terminus WaLk., List Dipt. Brit. Mus., I. 160. (1848).

Terrac-Novae MACQ. = zonalis KIRBY.

testaceiventris MACQ., Dipt. exot., suppl. 2., 16. 92. (1847); WALK., List Dipt. Brit. Mus., V. suppl. 1., 232. 337. (1854).

testaceomaculatus MAcq., Dipt. exot., I. 1., 140. 36. (1838); BLANCH. in GAY: Hist. fis. y polit. de Chile, Zool. VII. 394. 5. tab. II. fig. 9. (1854); Walk., List Dipt. Brit. Mus., V. suppl. 1., 210. 232. (1854) ; Pril., Verh. zool.-bot. Ges. Wien, XV. 714. 5. (1865); Schin., Novara Reise, Dipt., 85. 15. (1868); v. D. Wulp, Tijdschr. v. Entomol., XXIV. 160. 12. (1881); Huxier, Trans. Amer. Entom. Soc. Philad., XXVII. 144. (1901).

testaceus Fonsk., Descr. animal. quae in itinere orient. observ., Arabia.
Rhodesia.

Asia centr.

Port Natal.

Japonia.

India or.

India or.

America sept.

America sept.

Prom. bon.sp.

Chile.

Europa centr. et mer.

Aegyptus.

?America mer.

Brasilia.

Africa.

Chile. 
85. 24. (1775) ; WIED., Aussereurop. zweifl. Ins., II. 648. 75. (1830) ; Walk., List Dipt. Brit. Mus., V. suppl. 1., 238. 359. (1854).

testaceus Macq., Dipt. exot., I. 1., 133. 23. (1838); Walk., List Dipt. Brasilia. Brit. Mus., I. 149. (1848) et V. suppl. 1., 214. 256. (1854); HuNTER, Trans. Amer. Entom. Soc. Philad., XXVII. 144. (1901).

tetricus Marten, Canad. Entomol., XV. 111. [Therioplectes] (1883); Hine, Ohio Naturalist, V. 245. (1904); Aldr., Catal. North Amer. Dipt., 208. (1905).

tetropsis Big., Mém. Soc. Zool. France, V. 681. (1892); Aldr., Catal. America sept. North Amer. Dipt., 208. (1905).

thoracicus Hine, Canad. Entomol., XXXII. 248. (1900); Aldr., Catal. North Amer. Dipt., 208. (1905).

thoracinus Pal. Beauv., Ins. recueill. en Afrique et en Amérique, 55. tab. I. fig. 4. (1805-21); Wred., Aussereurop. zweifl. Ins., I. 147. 57. (1828); WALk., List Dipt. Brit. Mus., V. suppl. 1. 234. 345. (1854); v. D. Wulp, Annal. Soc. Entom. Belgique, XXVIII. Comp. Rend., CCLXXXIX. 3. (1884).

tibialis Macq., Dipt. exot., suppl. 1., 30. 59. (1845) et in Lucas: Explor. scient. de l'Algérie, Zool., III. 423. 26. tab. I. fig. 6. (1849); WaLK., List Dipt. Brit. Mus., V. suppl. 1., 225 et 230. 329. (1854).

tibialis WALK. = secedens WALK.

tinctus WALK., Insecta Saunders., Dipt., I. 29. (1850); ALdr., Catal. North Amer. Dipt., 208. (1905).

tomentosus MacQ., Dipt. exot., suppl. 1., 30. 60. tab. III. fig. 11. (1845) et in Lucas : Explor. Scient. de l'Algérie, Zool., IIJ. 224. 30. tab. I. fig. 7. (1849); WaLK., List Dipt. Brit. Mus., V. suppl. 1., 225 et 227. 316. (1854); Brauer, Denkschr. Akad. Wien, XLII. 163. 25. tab. II. fig. 25. (1880) ; Pand., Revue d'Entomol., II. 218. 44. (1883) ; BezzI, Bullet. Soc. Entom. Ital., XXVII. 42. (1895) ; Ricardo, Annal. Mag. Nat. Hist., ser. 7. XVI. 197. [Atylotus] (1905).

apiarius JaExw., Berlin. Entom. Zeitschr., X. 68. 7. (1866).

transiens WALK. $=$ ? ater Rosss.

transpositus Walk., List Dipt. Brit. Mus., V. suppl. 1., 151. 16. [Dichelacera] (1854); Ricardo, Annal. Mag. Nat. Hist., ser. 7. XIV. 367. (1904).

transversus Walk., List Dipt. Brit. Mus., V. suppl. 1., 256. 454. (1854); Hutton, Catal. New Zealand Dipt. etc., 21. (1881) et Trans. New Zealand Instit., XXXIII. 14. (1901).

triangularis v. D. Wulp, Tijdschr. v. Entomol., XI. 100. 4. (1868) et Ins. Obi. Catal. Dipt. Soutb Asia, 62. (1896).

Triangulum Wied., Aussereurop. zweifl. Ins., I. 143. 52. (1828); Brasilia. Walk., List Dipt. Brit. Mus., V. suppl. 1., 221. 289. (1854); Huntwr, Trans. Amer. Entom. Soc. Pliilad., XXVII. 144. (1901). triceps 'Thuns., Nova Acta Upsal., IX. 59. tab. I. fig. 4. (1827).

Brasilia. 
trichocerus Bia., Mém. Soc. Zool. France, V. 637. [Therioplectes] (1892).

tricolor ZeLt., Isis, 1842. XI. 819. 1. tab. I. fig. 23-25. (1842); Gism., Bull. Soc. Imp. Nat. Moscou, XX. 2., 255. 11. (1847); WaLk., List Dipt. Brit. Mus., V. suppl. 1., 155 et 158. 10. (1854); Lw., Verh. zool.-bot. Ges. Wien, VIII. 583. 8. (1858); Schis., Fauna Austriaca, Dipt., I. 36. (1862); Ровтsch., Trudy Russk. Entom., X. 150. tab. I. fig. 3. (1877); Brader, Denkschr. Akad. Wien, XLII. 165. 18. tab. II. fig. 18. (1880); PoRTsch., Horae Soc. Entom. Ross., XVIII. 123. (1883)

trifarius MACQ., Dipt. exot., I. 1., 140. 37. (1838); BLANch, in GAY : Hist. fis. y polit. de Chile, Zool. VII. 395. 6. (1854); WALK., List Dipt. Brit. Mus., V. suppl. 1., 197. 179. (1854); Phiц., Verh. zool.-bot. Ges. Wien, XV. 714. 6. (1865); Hunter, Trans. Amer. Entom. Soc. Pnilad., XXVII. 144. (1901).

trifascia WaLK., Insecta Saunders., Dipt., I. 37. (1850).

trifasciatus MAcQ., Suit. à Buffon, I. 204. 26. (1834); WALK., List Dipt. Brit. Mus., V. suppl. 1., 261. 491. (1854).

trigeminus Coquil., Proc. Unit. Stat. Nat. Mus., XXI. 310. (1898). trigonophorus MACQ., Dipt. exot., I. 2., 185. (1839); WALK., List Dipt. Brit. Mus., V. suppl. 1., 222. 290. (1854); E. L. ARRIB., Bolet. Acad. Nat. Cienc. Córdoba, IV. 136. 84. (1882); Hunter, Trans. Amer. Entom. Soc. Philad., XXVII. 144. (1901).

trigonus Coquilu., Proc. Unit. Stat. Nat. Mus., XXI. 309. (1898); Ricardo, Annal. Mag. Nat. Hist., ser. 7. XVI. 202. (1905).

trijunctus Watk., List Dipt. Brit. Mus., V. suppl. 1., 182. 114. (1854); Ost.-SAck., Mem. Boston. Soc. Nat. Hist., II. 432. 3. (1876) ; ALDR., Catal. North Amer. Dipt., 208. (1905).

triligatus $\mathrm{WALK}_{\mathrm{AL}}=$ affinis KIRBY.

trilineatus BELL. = lineola FABR.

trilineatus Latr. in Humb, et Bonap.: Recueil, d'observ. d. zool. etc., Fasc. X. 116, 117. tab. XI. fig. 6. (? 1814); WIEv., Dipt. exot., 84. 40. (1821) et Aussereurop. zweifl. Ins., I. 168. 87. (1828) ; Rond., Nuov. Annal. Sci. Nat. Bologna, ser. 3. II. 371. 31. (1850) ; WaLk., List Dipt. Brit. Mus., V. suppl. 1., 217. 266. (1854) ; Hunter, Trans. Amer. Entom. Soc. Philad., XXVII. 144. (1901); ALdr., Catal. North Amer. Dipt., 208. (1905).

trimaculatus PaL. Beady., Ins. recueill. en Afrique et en Amérique, 55. tab. I. fig. 5. (1805-21); WiED., Aussereurop. zweifl. Ins., I. 132.33 et 137. 40. (1828); MACQ., Dipt. exot., I. 1., 142. 42. (1838) ; Walk., List Dipt. Brit. Mus., V. suppl. 1., 202. 203. (1854); Ost.-SACK., Mem. Boston Soc. Nat. Hist., II. 439. 10. (18i6) ; HaRT, Bull. Illinois State Laborat. of Nat. Hist., IV. 234. (1895); Hunter, Trans. Amer. Entom. Soc. Philad., XXVII. 144. (1901); Hine, Ohio Naturalist, II. 168. (1902) et Ohio State Acad. Sci., Spec. Pap. No. 5., 55. (1903); AtDr., Catal. North Amer. Dipt., 208. (1905).
Marocco.

Europa mer., Asia min.

Chile.

Brasilia.

Patriaignota.

Japonia.

Brasilia.

Japonia.

America sept.

Brasilia.

A merica sept. 
quinquelineatus MACQ., Suit. à Buffon, I. 200. 11. (1834); WaLK., List Dipt. Brit. Mus., V. suppl. 1., 187. 137. (1854).

trimaculatus WiED. = trimaculatus PAL. BEAUv.

trinotatus Wied., Aussereurop. zweifl. Ins., I. 182. 106. (1828);

Walk., List Dipt. Brit. Mus., I. 189. (1848) et V. suppl. 1., (1854);

Hunter, Trans. Amer. Entom. Soc. Philad., XXVII. 144. (1901). tripunctifer WALK. = biguttatus WIED.

trisignatus Lw., Öfvers. Kongl. Vet. Akad. Förhandl., XIV. 1857. 338. 6. (1858) et Dipt.-Fauna Südafrika's, I. 39. 9. tab. I. fig. 20. (1860); Karsch, Entomol. Nachricht., XII. 53. 3. (1886) et Berlin. Entom. Zeitschr., XXXI. 370. 3. tab. IV. fig. 3. (1887). trispilus Wied., Aussereurop. zweifl. Ins., I. 150. 62. (1828); WalK., List Dipt. Brit. Mus., I. 174. (1848) et V. suppl. 1., 177. 90. (1854); Ost.-SAck., Mem. Boston Soc. Nat. Hist., II. 464. 46. (1876); Hrve, Ohio Naturalist, V. 237. (1904); Aldr., Catal. North Amer. Dipt., 208. (1905).

sodalis Wulust., Trans. Kansas Acad. Sci., X. 139 (1887); Aldr., Catal. North Amer. Dipt., 207. (1905).

tristis v. D. Wulp, Sumatra Exped., Dipt., 17. 5. tab. I. fig. 9. (1881) et Catal. Dipt. South Asia, 62. (1896).

tritus WALK., Trans. Linn. Soc. London, XVII. 338. 19. (1837) et List Dipt. Brit. Mus., I. 189. (1848) ; E. L. Arrib., Bolet. Acad. Nat. Cienc. Córdoba, IV. 136. 85. (1882); Hunrer, Trans. Amer. Entom. Soc. Philad., XXVII. 144. (1901).

trivittatus Fabr., Systema Antliat., 104. 40. (1805); Wied., Dipt. exot., 85. 41. (1821) et Aussereurop. zweifl. Ins., I. 172. 91. (1828); Walk., List Dipt. Brit. Mus., V. suppl. 1., 199. 190. (1854); Schis., Novara Reise, Dipt., 86. 17. (1868); v. D. Wulp, Tijdschr. v. Entomol., XXIV. 160. 11. (1881); Hunter, Trans. Amer. Entom. Soc. Philad., XXVII. 144. (1901).

tropicus L. p. p. $=$ tropicus Panz.

tropicus $\mathrm{Jw} .=$ solstitialis MEIG.

tropicus MeIG. p. p. = apricus MeIG.

tropicus Panz., Fauna Germ., XIII. tab. 22. (1794); MeIG., Klass., I. 168. 8. (1804) et System. Beschreib., II. 57. 34. p. p. ठ․ (1820); Brauer, Denkschr. Akad. Wien, XIII. 146. 9. tab. I et V. fig. 9. (1880); Gов., Mém. Soc. Linn. Nord France, 1881. 14. 6. [Therioplectes] (1881); PAND., Revue d'Entomol., II. 213. 32. (1883) ; Neuhaus, Diptera Marchica, 43. 10. (1886); Beck., Berlin. Entom. Zeitschr., XXXI. 106. 23. [Therioplectes] (1887); Grinscin., Berlin. Entom. Zeitschr., XXXI. tab. III. fig. 1. (1887); L. Coucke, Annal. Soc. Entom. Belgique, XXXVI. 138. (1892); ViLLex., Annal. Soc. Entom. France, LXXIV. 306, 2. (1905).

?bimaculatus MAcQ., Recueil Soc. Sci. Agricult. I.ille, 1826. 484. 17. (1826) ; Meig., System. Beschreib., VII. 59. 46. (1838); Walk., List Dipt. Brit. Mus., V. suppl. 1., 157 et 170. 54. (1854); Gor., Mém. Soc. Linn. Nord France, 1881. 27. (1881).

Europacentr. et sept., Sibiria. 
borealis JAEnn., Berlin. Entom. Zeitschr., X. 69. 9. (1866).

luridus Lw., Verl. zool.-bot. Ges. Wien, VIII. 526. 12. p. p. (1858) ; Schin., Fauna Austriaca, Dipt., I. 31. (1862).

signatus Schis., Fauna Austriaca, Dipt., I. 29. (1862).

p. p. tropicus L., Fauna Suec., 463. 1886. (1761) ; Scop., Entomol. Carniol., 371. 1008. (1763); O. F. Müller, Fauna Insect. Fridrichsdal., 86. 768. (1764) ; L., Systema Naturae, Ed. XII., II. 1001. 14. (1767); O. F. Múller, Zoolog. Dan. Prodr., 180. 2115. (1776) ; DEG., Mém. pour serv. à l'hist. d. Ins., VI. 220. 2. tab. XII. fig. 15. (1776); FABr., Species Inseet., II. 458. 20. (1781); Schrank, Enum. Insect. Austr., 479. 975. (1781); Herbst, Gemeinnütz. Naturgesch., VIII. 113. tab. CCCXLII. fig. 4. (1787); Vill., Entom. Linn., III. 553. 6. (1789); Guel., Systema Naturae, V. 2884. 13. (1792); FABr., Entomol. System., IV. 368. 28. (1794); Schrank, Fauna Boica, III. 154. (1803); Schellenb., Gattung. d. Fliegen, 72. tab. XXVII. fig. 2. (1803) ; FABr., Systema Antliat., 100. 32. (1805) ; Latr., Gen. Crust. et Ins., IV. 283. (1809) ; Fall., Kongl. Vet. Akad. Handl., XXX. 240. 3. (1809) et Dipt. Suec., Taban., 5. 3. (1817); MacQ., Recueil Soc. Sci. Agricult. Lille, 1826. 481. 14. (1826); MACQ., Suit. à Buffon, I. 202. 19. (1834) ; Duncan, Magaz. Zool. and Botan., I. 367. (1837); ZEтT., Insecta Lappon., Dipt., 514. 4. (1838); ZeLL., Isis, 1842. XI. 822. 5. [Therioplectes] (1842); ZETT., Dipt. Scand., I. 111. 10. (18t2); Gimm., Bull. Soc. Imp. Nat. Moscou, XX. 1., 185. 16. (1847); Walk., List Dipt. Brit. Mus., I. 169. (1848) ; ZeTt., Dipt. Scand., VIII. 2937. 10. (1849) ; L. Duf., Mém. Acad. d. Scienc., Math. et Phys., XI. 171-360 tab. IV. fig. 77. (1850) ; Schoutz, Zeitschr. f. Entomol. Breslau, IV. No. 16., 37. (1850); WaLk., Insecta Britann., Djpt., I. 39. 8. (1851) et List Dipt. Brit. Mus., V. suppl. 1., 156 et 168. 47. (1854) ; Zeтt., Dipt. Seand., XII. 4548. 10. (1855); Borsd., Finlands tvaving. Ins., I. 108. 6. (1861); JaENn., Berlin. Entom. Zeitschr., X. 70. 10. (1866); Fedrsch., Acta Soc. Am.

Nat. Moscou, VI. 62. 2. (1868).

tropicus ZE'T.. p. p. = montanus MeIG.

truncatus WALK. = Sarpa WALK.

Truquii Bell., Saggio di Ditterol. Messic., I. 64. 15. tab. II. fig. 6. Mexico. (1859) ; Aldr., Catal. North Amer. Dipt., 208. (1905).

turbidus Wied., Aussereurop. zweifl. Ins., I. 124. 20. (1828); Walk., America sept.

List Dipt. Brit. Mus., I. 149. (1848) et V. suppl. 1., 186. 132. (1854) ; Ost.-Sack., Mem. Boston Soc. Nat. Hist., II. 430. 1. (1876) et Catal. Dipt. North Amer., Ed. II., 60 et 228. 84. (1878); Willis'., Trans. Kansas Acad. Sci., X. 138. (1887); Aldr., Catal. North Amer. Dipt., 209. (1905).

cetencetus Walk., List Dipt. Brit. Mus., I. 148. p. p. (1848).

? fusconervosus MacQ., Dipt. exot., I. 1., 147. 52. (1838) ; WaLk, Kertész: Catalogus Dipterorum. III. 
List Dipt. Brit. Mus., I. 149. (1848); Ost.-SAck., Mem. Boston Soc. Nat. Hist., II. 473. (1876).

typhus Whitney, Canad. Entomol., XXXVI. 206. [Therioplectes] (1904).

umbrinus MeIg., System. Beschreib., II. 35. 6. (1820) ; Walk., List Dipt. Brit. Mus., V. suppl. 1., 156 et 161. 22. (1854) ; Lw., Verh. zool.-bot. Ges. Wien, VIII. 609. 40. (1858) ; Schin., Fauna Austr., Dipt., I. 33. (1862); Lw., Wien. Entom. Monatschr., VI. 163. 10. (1862) ; Brauer, Denkschr. Akad. Wien, XliI. 162. 24. tab. II. fig. 24. (1880) ; PAND., Revue d'Entomol., II. 217. 42. (1883); Rö́ner, Berlin. Entom. Zeitschr., XXXI. 73. (1887).

istriensis Meig., System. Beschreib., II. 36. 8. (1820); Walk.,

List Dipt. Brit. Mus., V. suppl. 1., 157 et 170. 56. (1854).

maculipennis Brulué, Exped. scient. de Morée, III. 305. 660. tab. XIVII. fig. 3. (1832); Walk, List Dipt. Brit. Mus., V. suppl. 1., 157 et 171. 59. (1854).

umbrosus WALK., Insecta Saunders., Dipt., I. 52. (1850) ; v. D. WulP, India or.

Catal. Dipt. South Asia, 60. (1896).

uncinatus v. D. WulP, Tijdschr. v. Entomol., XXIV. 159. 10. tab. XV. fig. 8. (1881).

unicinatus Hunter, Trans. Amer. Entom. Soc. Philad., XXVII. 144. (1901) [lapsus].

unicinatus HuNTER = uncinatus v. D. WULP.

unicinctus Lw., Neue Beitr., IV. 27. 7. (1856).

unicinctus Walk., Trans. Entom. Soc. London, n. ser. IV. 122. (1857). unicolor MACQ. = lateritius Rond.

unicolor Wied., Aussereurop. zweifl. Ins., I. 141. 47. (1828) ; WALK.,

List Dipt. Brit, Mus., V. suppl. 1., 214. 252. (1854); Willist.,

Kansas Univ. Quart., III. 195. (1895); Hunter, Trans. Amer.

Entom. Soc. Philad., XXVII. 144. (1901).

? ferrugineus Thunb., Nova Acta Upsal., IX. 55. (1827).

unifasciatus Lw., Verh. zool.-bot. Ges. Wien, VIII. 600. 29. (1858) ;

Brauer, Denkschr. Akad. Wien, XLII. 202. 56. tab. IV. fig. 56. (1880) ; Pand., Revue d'Entomol., II. 206. 14. (1883); Мıк in Becker: Fauna v. Hernstein, II. 2., 54. (1885); Strobl, Mittheil. Naturwiss. Ver. Steiermark, XXIX. 1892. 17. (1893). unilineatus Lw., Ber. über. die Verh. d. k. Preuss. Akad. d. Wiss. Berlin, 1852. 658. 3. (1852) et Peter's Reise nach Mosambique, Zool., V. 3. (1862).

unimaculatus MAcQ., Suit. à Buffon, I. 204. 23. (1834); WALK., List Senegal.

Dipt. Brit. Mus., V. suppl 1., 233. 339. (1854).

unipunctatus Bı́., Mém. Soc. Zool. France, V. 663. [Atylotus] (1892). univentris WaLK., List Dipt. Brit. Mus., I. 151. (1848) et Proc.

Aegyptus.

Brasilia.

Brasilia.

Europacentr. et mer., Africa sept., Asia min.

Mosambique.

Linn. Soc. London, I. 9. 20. (1856); v. D. Wulp, Catal. Dipt. South Asia, 59. (1896).

univittatus MACQ., Dipt. exot., suppl. 5., 30. 122. (1855); Hunter, Americamer.

'Trans. Amer. Entom. Soc. Philad., XXVII. 144. (1901). 
ursulus MEG. = graecus FABR.

ursus A. Costa = gigas Herbst.

uruguayensis E. L. Arrib., Bolet. Acad. Nat. Cienc. Córdoba, IV. Uruguay.

136. 86. (1882); Hunter, Trans. Amer. Entom. Soc. Philad., XXVII.

144. (1901).

pictipennis MacQ., Dipt. exot., suppl. 4., 32. 102. tab. II. fig. 8. (1850).

ustus WaLk. in Newman Zoologist, VIII. Append., XCV. (1850) et Port Natal.

List Dipt. Brit. Mus., V. suppl. 1., 234. 344. (1854).

vacillans WALK., Insecta Saunders., Dipt., I. 70. [Dichelacera] (1850) ;

Ricardo, Annal. Mag. Nat. Hist., ser. 7. XIV. 367. (1904).

vagus WaLK. in Newman Zoologist, VIII. Append., LXVIII. (1850)

et List Dipt. Brit. Mus., V. suppl. 1., 246. 406. (1854); v. D.

Wulp, Catal. Dipt. South Asia, 60. (1896).

vagus WaLk., Insecta Saunders., Dipt., I. 50. (1850) ; v. D. WuLP,

Catal. Dipt. South Asia, 60. (1896).

validus WIED. = atratus FABR.

Valterii Macq., Dipt. exot., I. 2., 184. (1839); WaLk., List Dipt. Brit.

Mus., V. suppl. 1., 198. 183. (1854); Hunter, Trans. Amer. Entom.

Soc. Philad., XXVII. 144. (1901).

Vanderwulpi Ost.-SAck., Berlin. Entom. Zeitschr., XXVI. 97.(1882);

v. D. Wulp, Catal. Dipt. South Asia, 62, (1896).

pictipennis v. D. WulP, Tijdschr. v. Entom., XI.100. 3. tab. III.

fig. 1, 2. (1868).

variabilis Lw.; Öfvers. Kongl. Vet. Akad. Förhandl., XIV. 1857. 340. 22. (1858) et Dipt.-Fauna Südafrika's, I. 41. 12. tab. I. fig. 23. (1860).

variatus WaLK., Insecta Saunders,, Dipt., I. 64. (1850).

variegatus FABr., Systema Antliat., 95. 10، (1805); Wied., Dipt. exot.,

67. 11. (1821) et Aussereurop. zweifl. Ins., I. 120، 13. (1828);

Walk., List Dipt. Brit. Mus., I. 149. (1848) et V. suppl. 1., 186.

133. (1854) ; Ost.-SAck., Mem. Boston Soc. Nat. Hist., II. 437. 8.

excl. syn. Macq. (1876) et Catal. Dipt. Nortlr Amer,, Ed. II., 60 et 228. 84. (1878); Hinf, Ohio State Acad. Sci., Spec. Pap. No. ๖., 56. (1903) ; ALdr., Catal. North Amer. Dipt., 209. (1905).

variegatus Rond., Annal. Mus. Civ. Genova, VII. 458. (1875); v. D. Borneo.

Wulp, Catal. Dipt. South Asia, 62. (1896).

varipes WALK., Trans. Linn. Soc. London, XVII. 337. 17. (1837); E. L. Arrib., Bolet. Acad. Nat. Cienc. Córdoba, IV. 136. 87. (1882) ; Hunrer, Trans. Amer. Fntom. Soc. Philad., XXVII. 144. (1901).

varius WIEd., Aussereurop. zweifl. Ins., I. 189. 117. (1828) ; WALK., List Dipt. Brit. Mus., V. suppl. 1., 217. 269. (185 1 ) Hunter, Trans. Amer. Entom. Soc. Philad., XXVII. 144. (1901).

variventris MACQ., Dipt. exot., suppl. 2., 18. 97. (1847); WALK., List Dipt. Brit. Mus., V. suppl. 1., 222. 291. (1854); Huster, Trans.

I.Philippinae, Celebes.

Caffraria.

Patriaignota. America sept.

Americamer.

Amer. Entom. Soc. Philad., XXVII. 144. (1901). 
venenatus Ost.-SAck., Biolog. Central. Amer., Dipt., I. 54.10. (1886); Americacentr. Aldr., Catal. North Amer. Dipt., 209. (1905).

venosus Big., Mém. Soc. Zool. France, V. 685. (1892).

Brasilia.

venustus Ost.-Sack., Mem. Boston Soc. Nat. Hist., II. 44t. 17. (1876);

Hine, Ohio State Acad. Sci., Spec. Pap. No. 5., 56. (1903) et

America sept.

Ohio Naturalist, V. 244. (1904); ALdr., Catal. North Amer. Dipt., 209. (1905).

vestitus Wred., Zoolog. Magaz., I. 3., 42. 2. (1819), Dipt. exot., 70. 15. (1821) et Aussereurop. zweifl. Ins., I. 128. 26. (1828); WaLk., List Dipt. Brit. Mus., V. suppl. 1., 205. 214. (1854); Hunter, Trans. Amer. Entom. Soc. Philad., XXVII. 144. (1901).

vetustus Walk., List Dipt. Brit. Mus., I. 179. (1848).

Australia.

vexans Lw., Öfvers. Kongl. Vet. Akad. Förhandl., XV. 3i36. 1 bis. (1858) et Dipt.-Fauna Suidafrika's, I. 34. 2. (1860).

vicarius $\mathrm{W}_{\mathrm{ALK}}=$ costalis WIED.

vicinus $\mathrm{EGG} .=$ cordiger MtrG.

vicinus MacQ., Dipt. exot., I. 1., 143. 44. (1838); WalK., List Dipt.

America sept.

Brit. Mus., I. 172. (1848) et V. suppl. 1., 178. 96. (1854) ; Ost.SAck., Mem. Boston Soc. Nat. Hist., II. 473. (1876); Aldr., Catal. North Amer. Dipt., 209. (1905).

viduus WaIK. in Newman Zoologist, VIII. Append., LXVIII. (1850) et List Dipt. Brit. Mus., V. suppl. 1., 202. 201. (1854).

villosulus Big., Mém. Soc. Zool. France, V. 684. (1892); Hine, Ohio Naturalist, V. 245. (1904); Aldr., Catal. Nortlı Amer. Dipt., 209. (1905).

villosus Macq., Dipt. exot., I. 2., 181. (1839) et in Lucas : Eplor. scient. de l'Algérie, Zool. III. 423. 24. (1849); WALk., List Dipt. Brit. Mus., V. suppl. 1., 225 et 230. 325. (1854).

Virgo Wied., Analecta Entomol., 22. 9. (1824) et Aussereurop. zweifl. Ins., I. 184. 109. (1828); WALK., List Dipt. Brit. Mus., V. suppl. 1., 245. 399. (1854) ; v. D. Wulp, Catal. Dipt. South Asia, 59. (1896).

viridiflavus $\mathrm{W}_{\mathrm{ALK}}=$ mexicanus $\mathbf{L}$.

viridis Hudson, Manual of New Zealand Entomol., 55. tab. VI. fig. 3. [Comptosia] (1892); Hutron, Trans. New Zealand Instit., XXXIII. 13. (1901).

viridiventris MACQ., Dipt. exot., I. 1., 137. 31. (1838) ; Walk., List Dipt. Brit. Mus., V. suppl. 1., 214. 255. (1854); Hunter, Trans. Amer. Entom. Soc. Philad., XXVII. 144. (1901).

vittatus FABr., Entomol. System., IV. 371. 37. (1794) et Systema Antliat., 103. 44. (1805); Meig., System. Beschreib., II. 40. 14. (1820) ; Duncan, Magaz. Zool. and Botan., I. 364. (1837) ; Walk., List Dipt. Brit. Mus., V. suppl. 1., 226 et 233. 342. (1854); Lw., Verh. zool.-bot. Ges. Wien, VIII. 581. 5. (1858); Brauer, Denkschr. Akad. Wien, XIII. 175. 37. a. (1880); Strons, Wien. Entom. Zeitg., XVII. 297. 3. (1898).

vittiger Thомs., Eugenies Resa, Dipt., 451. 13. (1868); Hunter, I. Gallapag. 
Trans. Amer. Entom. Soc. Philad., XXVII. 144. (1901); Coquill.,

Proc. Washington Acad. Sci., III. 373. (1901).

vivax Ost.-SAck., Mem. Boston Soc. Nat. Hist., II. 446. 20. (1876) et

Catal. Dipt. North Amer., Ed. II. 60 et 228. 87. (1878); Hine,

Ohio Naturalist, II. 168. (1902) et IV. 1-2. (1903) et Ohio State Acad. Sci., Spec. Pap. No. 5., 56. (1903); Aldr., Catal. North Amer. Dipt., 209. (1905).

? marginalis FaBR., Systema Antliat., 99. 31. (1805); WALK., List Dipt. Brit. Mus., I. 174. (1848) et V. suppl. 1., 180. 104. (1854); Ost.-Suck., Mem. Boston Soc. Nat. Hist., II. 472. (1876).

marginalis WIED., Aussereurop. zweifl. Ins., I. 166. 84. (1828). Whitneyi Jouss, Psyche, XI. 15. [Therioplectes] (1904).

Wideri JAENN. = lunatus FABR.

Wiedemanni Osr.-SAck., Mem. Boston Soc. Nat. Hist., II. 455. 32 et 559. (1876) ; Aldr., Catal. North Amer. Dipt., 209. (1905).

ater WiED., Aussereurop. zweifl. Ins., I. 136. 39. p. p. (1828).

xanthogaster PulL., Verh. zool.-bot. Ges. Wien, XV. 715. 14. (1865); Hunter, Trans. Amer. Entom. Soc. Philad., XXVII. 144. (1901). yao MAcQ., Dipt. exot., suppl. 5., 24. 112. (1855).

yokohamensis Bıg., Mém. Soc. Zool. France, V. 678. (1892).

yucatanus Towss., Canad. Entomol., XXIX. 198. 2. (1897); Aldr., Catal. North Amer. Dipt., 209. (1905).

yulensis Röper, Stettin. Entom. Zeitg., LIII. 244. (1892); v. D. WulP, Catal. Dipt. South Asia, 63. (1896).

zonalis Kırby, Fauna Boreal. Americ., IV: 314. 2. (1837); WaLk., List Dipt. Brit. Mus., I. 157. (1848); Ost.-SAck., Catal. Dipt. North Amer., 226. nota 73. (1858), Mem. Boston Soc. Nat. Hist., II. 463. 44. (1876) et Catal. Dipt. North Amer., Ed. Il., 56 et 226. (1878); Bethune, Canad. Entomol., XIII. 167. 441. (1881); Towns., Trans. Amer. Entom. Soc. Philad., XXII. 58. 10. [Therioplectes] (1895); Hrne, Ohio Naturalist, V. 244. (1904); Aldr., Catal. North Amer. Dipt., 209. (1905).

flavocinctus BeLL., Saggio di Ditterol. Messic., I. 61. 10. (1859). Tarandi WALK., List Dipt. Brit. Mus., I. 156. (1848).

Terrac-Novae Macq., Dipt. exot., suppl. 4., 35. 109. (1850).

Amorica sept.

America sept.

Chile.

China bor.

Japonia.

Mexico.

Nova Guinea.

America sept. et centr. 


\section{PANTOPHTHALMIDAE.}

\section{Pantophthalmus}

Tiıne., Nouv. Recueil de Mém. Soc. roy. d. Sc. d. Gothenbourg, III. 7. (1819). Acanthomera Wied., Dipt. exot., 60. (1821).

Megalemyia Bı́., Annal. Soc. Entom. France, sér. 6. I. 455. (1881).

Megalomyia Big., Annal. Soc. Entom. France, sér. 5. X. Bull., V. (1880).

argyropasta Bıg., Annal. Soc. Entom. France, sér. 5. X. Bull., V. Panama. [Megalomyia] (1880) et sér. 6. I. 455 et 458. [Megalemyia] (1881); Hunter, Trans. Amer. Entom. Soc. Philad., XXVII. 147. [Acanthomera] (1901); Aldr., Catal. North Amer. Dipt., 210. [Acanthomera] (1905).

Bellardii BelL., Sagio di Ditterol. Messic., Append., 16. 2. tab. III. fig. 11. [Acanthomera] (1862); Big., Annal. Soc. Entom: France, sér. 6. I. 459. [Acanthomera] (1881); Aldr., Catal. North Amer. Dipt., 210. [Acanthomera] (1905).

Championi Ost.-Sack., Biolog. Central. Amer., Dipt., I. 67. 1. tab. III. fig. 16. [Acanthomera] (1886) ; Towns., Proc. Calif. Acad. Sci., ser. 2. IV. 595. 6. [Acanthomera] (1905).

pictus Beld. (nec Wied.), Saggio di Ditterol. Messic., I. 76. 1. [Acanthomera] (1859).

flavipes MACQ., Dipt. exot., suppl. 2., 26. 4. tab. I. fig. 2. [Acanthomera] (1847) ; WALK., List Dipt. Brit. Mus., V. suppl. 1., 301. 6. [Acanthomera] (1854); Big., Annal. Soc. Entom. France, sér. 6. I. 459. [Acanthomera] (1881); Hunter, Trans. Amer. Entom. Soc. Philad., XXVII. 147. [Acanthomera] (1901).

Frauenfeldi Schin., Novara Reise, Dipt., 78. [Acanthomera] (1868) ; Big., Annal. Soc. Entom. France, sér. 6. I. 459. [Acanthomera] (1881) ; Brauer, Denkschr. Akad. Wien, XLVII. tab. II. fig. 25, a-e. [Acanthomera] (1883) ; RöD., Stettin. Entom. Zeitg., XLVII. 261. [Acanthomera] (1886); Ost.-SAck., Biolog. Central. Americ., Dipt., I. 65. [Acanthomera] (1886) ; Röp., Dipteren gesamm. von Stübel in Süd-Amerika, 7. [Acanthomera] (1892); Hunter, Trans. Amer. Entom. Soc. Philad., XXVII. 147. [Acanthomera] (1901).

fulvidus Bıg., Annal. Soc. Entom. France, sér. 5. X. Bull., V. [Acanthomera] (1880) et sér. 6. I. 456 et 460. [Accunthomera] (1881) ; Huvter, Trans. Amer. Entom. Soc. Philad., XXVII. 147. [Acanthomera] (1901).

Heydenii Wied., Aussereurop. zweifl. Ins., I. 555. 26. [Acanthomera] (1828); WALK., List Dipt. Brit. Mus., I. 209. [Acanthomera] (1848)

Columbia, Ecuador.

Brasilia, Venezuela.

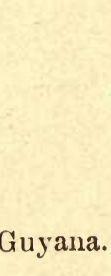

Guyana. et V. suppl. 1., 301. 3. [Acanthomera] (1854); Big., Annal. Soc. Entom. France, sér. 6. I. 458. [Acanthomera] (1881); Hunter,

America sept. et centr.

Brasilia. 
Trans. Amer. Entom. Soc. Philad., XXVII. 147. [Acanthomera] (1901).

immanis Wied., Aussereurop. zweifl. Ins., II. 623. 18. [Acanthomera] (1830); Westw. apud Duncan: The Natural. Libr., XXVIII. 331. tab. XXXV. fig. 2. [Acanthomera] (1840); GuÉr., Iconogr. d. Régno Anim., VII. Ins., 544. nota. [Acanthomera] (1845); WaLk., List Dipt. Brit. Mus., I. 209. [Acanthomera] (1848) et V. suppl. 1., 301. 7. [Acanthomera] (1854); Big., Annal. Soc. Entom. France, sér. 6. I. 459. [Acanthomera] (1881); Hunter, Trans. Amer. Entom. Soc. Philad., XXVII. 147. [Acanthomera] (1901).

magnificus WaLK., Insecta Saunders., Dipt., I. 74. tab. I. fig. 1. [Acanthomera] (1850) et List Dipt. Brit. Mus., V. suppl. 1., 300. 2. [Acanthomera] (1854); Big., Annal. Soc. Entom. France, sér. 6. I. 459. [Acanthomera] (1881); Ost.-SAck., Biolog. Central. Amer., Dipt., I. 65. [Acanthomera] (1886).

pictus Bell. (nec Wied.) = Championi Ost.-SaCK.

pictus WIED., Dipt. exot., 61. tab. II. fig. 2. [Acanthomera] (1821) ;

St. Farg. et Serv., Encyclop. Méthod., X. 541. 1. [Acanthomera] (1825) ; WiED., Aussereurop. zweifl. Ins., I. 108. 2. [Acanthomera] (1828) ; MAcq., Suit. à Buffon, I. 218. 1. tab. V. fig. 8. [Acanthomera] (1834) et Dipt. exot., I. 1., 167. 1. [Acanthomera] (1838); Walk., List Dipt. Brit. Mus., I. 209. [Acanthomera] (1848) et V. suppl. 1., 301. 4. [Acanthomera] (1854); Big., Annal. Soc. Entom. France, sér. 6. I. 458. [Acanthomera] (1881); Ost.-SAck., Biolog. Central. Amer., Dipt., I. 65. [Acanthomera] (1886); Hunter, Trans. Amer. Entom. Soc. Philad., XXVII. 148. [Acanthomera] (1901); Aldr., Catal. North Amer. Dipt., 210. [Acanthomera] (1905).

rubriventris Big., Annal. Soc. Entom. France, sér. 5. X. Bull., V. Guatemala. [Acanthomera] (1880) et sér. 6. I. 456 et 460. [Acanthomera] (1881) ; Ost.-SAck., Biolog. Central. Amer., Dipt., I. 64. [Acanthomera] (1886); Hunter, Trans. Amer. Entom. Soc. Philad., XXVII. 148. [Acanthomera] (1901); Aldor., Catal. North Amer. Dipt., 210. [Acanthomera] (1905).

seticornis Big., Annal. Soc. Entom. France, sér. 6. I. 458. [Megalemyia] (1881) et sér. 6. VI. Bull., CLXVIII. [Megalemyia] (1886).

setiformis Big., Annal. Soc. Entom. France, sér. 6. I. 455. [Megalemyia] (1881).

Servillei GuÉr. = vittatus WIEd.

seticornis BIG. (nee WIED.) = rubriventris BIG.

seticornis WIED., Aussereurop. zweifl. Ins., I. 108. 1. tab. II. fig'. 1. [Acanthomera] (1828); MacQ., Dipt. exot., I. 1., 168. 3. tab. XX. fig. 1. [Acanthomera] (1838) et suppl. 2., 27. [Acanthomera] (1847); Walk., List Dipt. Brit. Mus., I. 210. [Acanthomera] (1848) et V. suppl. 1., 301. 5. [Acanthomera] (1854); Hunter, Trans. Amer. Entom. Soc. Philad., XXVII. 148. [Acanthomera] (1901); ALdr., Catal. North Amer. Dipt., 210. [Acanthomera]. (1905).

Surinam, Brasilia.

America mer.

Brasilia.

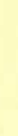


setiformis $\mathrm{BIG}_{0}=$ rubriventris $\mathrm{BIG}$.

tabanius Truxb., Nouv. Recueil de Mém. Soc. roy. d. Sc. d. Gothen-

bourg, III. 7. tab. VII. fig. 2. (1819); WIED., Aussereurop. zweifl.

India oce.

Ins., I. 110. 4. [A canthomera] (1828); WALK., List Dipt. Brit. Mus., V. suppl. 1., 300. 1.' [Acanthomera] (1854); Big., Annal. Soc. Entom. France, sér. 6. I. 459. [Acanthomera] (1881) ; Aldr., Catal. North Amer. Dipt., 210. [Acanthomera] (1905).

vittatus Wied., Aussereurop. zweifl. Ins., I. 109. 3. tab. II. fig. 2. Brasilia.

[Acanthomera] (1828); MACQ., Dipt. exot., I. 168. 2. tab. XX. fig.

2. [Acanthomera] (1838); WALK., List Dipt. Brit. Mus., I. 210. [Acanthomera] (1848) et V. suppl. 1., 302. 8. [Acanthomera] (1854) ; Big., Annal. Soc. Entom. France, sér. 6. I. 460. [Acanthomera] (1881) ; Hunter, Trans. Amer. Entom. Soc. Philad., XXVII. 148. [Acanthomera] (1901).

\section{Rhaphiorrhynchus}

WiED., Dipt. exot., 59. (1821).

Bigoti BELL. = planiventris W1ED.

crassipalpis MACQ. = planiventris WIED.

planiventris WiED., Dipt. exot., 60. tab. II. fig. 1. (1821); ST. FARG. ET Serv., Encyclop. Méthod., X. 545 (1825) ; Wied., Aussereurop. zweifl. Ins., I. 106. 1. tab. I. fig. 4. (1828) et II. 622. 17. (1830) ; Macq., Suit. à Buffon, I. 217. 1. tab. V. fig. 9. (183t) ; Guér., Iconogr. d. Régne Anim., VII. Ins., 544. tab. XCVIII. fig. 3. (? 1835) ; Macq., Dipt. exot., I. 1., 170. 1. tab. XX. fig. 3. (1838) ; WaLK., List Dipt. Brit. Mus., V. suppl. 1., 299. 1. (1854); BıG., Annal. Soc. Entom. France, sér. 6. I. 458. (1881) ; Ost.SAck., Biolog. Central. Amer., Dipt., I. 66. 1. (1886); Hunter, Trans. Amer. Entom. Soc. Philad., XXVII. 148. (1901); Aldr., Catal. North Amer. Dipt., 210. (1905).

Bigoti Bell., Saggio di Ditterol. Messic., Append., 16. 3. tab. III. fig. 10. [Acanthomera] (1862); BIG., Annal. Soc. Entom. France, sér. 6. I. 459. [Acanthomera] (1881); Aldr., Catal. North Amer. Dipt., 210. [Acanthomera] (1905).

crassipalpis MacQ., Dipt. exot., suppl. 2., 27. 5. tab. I. fig. 3. [Acanthomera] (1847); WALK., List Dipt. Brit. Mus., V. suppl. 1., 302. 9. [Acanthomera crassipalpus] (1854); Bia., Annal. Soc. Entom. France, sér. 6. I. 454 et 458. (1881); Aldr., Catal. North Amer. Dipt., 210. [Acanthomera] (1905).

Americamer. et centr. 


\section{RHAGIONIDAE.}

\section{Vermileo}

MACQ., Suit. à Buffon, I. 428. 2. (1834).

Apogon Perr., Annal. Soc. Linn. Lyon, V. 198. (1852).

Psammorycter Blanch., Hist. Nat. d. Ins., III. 603. (1840).

Rhagio FABR., Systema Entomolog., 761. 171. p. p. (1775).

cylindraceus O. G. Costa $=$ vermileo DEG.

Degeeri $\mathrm{MACQ}$. = vermileo DEG.

Dufourii PERRIS = vermileo DEG.

vermileo Deg., Acta Acad. Suec., XIII. 180. 261. tab. V. [Musca] (1752) ; Linn., Systema Naturae, Ed. X. 590. 11. [Musea] (1758) et Ed. XII., II. 982. 17. [Musca] (1767); FABR., Systema Entomol., 762. 3. [Rhagio] (1775); Deg., Mém. pour serv. l'hist. d. Ins., VI. 168. 5. tab. X. fig. 1-11. [Nemotelus] (1776); FABr., Species Insect., II. 419. 3. [Rhagio] (1781) et Mantissa Insect., II. 332. 3. [Rhagio] (1787); Herbst, Gemeinnütz. Naturgesch., VIII. 105. tab. CCCXXXIX. fig. 6. [Rhagio] (1787); Guel., Systema Naturae, V. 2864. 17. [Musca] (1792); FABR., Entomol. System., IV. 272. 5. [Rhagio] (1794); MeIG., Klassif., I. 303. c. [Rhagio] (1804) ; Latr., Hist. Nat. d. Crust. et d. Ins., XIV. 332. 2. [Rhagio] (1804); Fabr., Systema Antliat., 70.7. [Leptis] (1805); Latr., Gen. Crust. et Ins., IV. 288. [Rhagio] (1809); LAy., Hist. nat. anim. sans vert., IIl. 423.1. [Rhagio] (1816); Cuv., Régne Anim., III. 618. [Leptis] (1817); MEIG., System. Beschreib., II. 96. 12. [Leptis] (1820); De Ron., Annal. Soc. Entom. France, II. 498. tab. XVIII. C. [Leptis] (1833); VAll., Mém. Acad. Sc. Dijon, ? [Leptis] (1836); Westw., Introd., II. 552. fig. 7. [Rhagio] (1810); Blanci., Hist. Nat. Ins., III. 603. [Psammorycter] (1840); Siewold, Amtl. Bericht üher d. 35. Versamml. Deutsch. Naturf. in Königsberg, 105. [Leptis] (1861); Courtill., Annal. Soc. Linn. Maine, IX. 72. [Leptis] (1867); Mı,, Wien. Entom. Zeitg., VI. 312. [Psammorycter] (1887); Röp., Entomol. Nachricht., XVIII. 248. [Psammorycter] (1892); Мıк, Wien. Entom. Zeitg., XIII. 165. 30. [Psammorycter] (1894); Griff., Bollet. Mus. Zool. ed Anat. comp. Torino, X. No. 218., 1. [Psammorycter] (1895); BEzzI, Bullet. Soc. Entom. Ital., XXX. 28. 42. [Psammorycter] (1898) et Wien. Entom. Zeitg., XIX. 56. [Psammorycter] (1900).

cylindraceus O. G. Costa, Atti Accad. Sci. Napoli, V. 2., 84. 2. tab. II. fig. 2. [Leptis] (1844).

Degecri Macq., Suit. à Buffon, I. 428. 1. tab. X. fig. 12. (1834); Schin., Fauna Austriaca, Dipt., I. 171. (1862); Goв., Mém. Soc. Linn. Nord France, 1877. 6. (1877); Big., Annal. Soc. Entom. France, sér. 5. IX. Bullet., XX-XXI. (1879) et sér.

Europacentr. et mer., Ins. Canar. 
6. I. Bullet., LIII. (1881) ; Brauer, Denkschr. Akad. Wien, XIVII. 43. tab. IV. fig. $84-85$ et tab. V. fig. 86-88. (1883) et Wien. Entom. Zeitg., II. 114. (1883); Willist., Entomol. Amer., II. 107. nota. (1886); L. Coucke, Annal. Soc. Entom. Belgique, XXXVII. 130. (1893).

Dufourii Perris, Annal. Soc. Linn. Lyon, V. 199. [Apogon] (1852) ; WALK., List Dipt. Brit. Mus., VI. suppl. 2., 413. 55. [Dasypogon, Apogon] (1854) ; ScHin., Fauna Austriaca, Dipt., I. 123. [Apogon] (1862).

\section{Lampromyia}

MACQ., Suit. à Buffon, II. 660. (1835).

Leptynomat Westw., Trans. Entom. Soc. London, 517. (1876). argentata $\mathrm{BIG} .=$ ? sericea WESTW.

canariensis Macq. in WebB et Berth. : Hist. Nat. d'iles Canar., Ins. Canar.

Entom., Dipt., 105. 25. tab. IV. fig. 7. (1838) et Dipt. exot., II. 1., tab. III bis, fig. 1. (1840).

cylindrica FABR., Entomol. System., IV. 403. 2. [Empis] (1794);

MeIG., Klassif., I. 231. a. [Empis] (1804); Coqueb., Illustr. Icon.

Insect., 121. tab. XXVII. fig. 9. [Empis] (1804); FABr., Systema Antliat., 138. 2. [Empis] (1805) ; WiED., Aussereurop. zweifl. Ins., II. 5. 11. [Empis] (1830); Ost.-SAck., Berlin. Entom. Zeitschr., XXVII. 296. (1883).

funebris L. Duf., Annal. Soc. Entom. France, sér. 2. VIII. 152. 16. tab. VI. fig. 14. (1850); Schis., Fauna Austriaca, Dipt., I. 170. (1862).

funebris L. DuF. = cylindrica FABR.

Mikii MarchaL = pallida MacQ.

pallida MAcQ., Suit. à Buffon, I. 662. 1. tab. XXIV. fig. 18. (1835) ;

BEck., Zeitschr. f. system. Hymen. u. Dipter., VI. 282. 116. (1906).

Mikii Marchal, Mémoir. Soc. Zool. France, X. 5-13. tab. I. fig.

1-14. (1897); Beck., Entomol. Nachricht., XXVI. 97. (1900).

sericea Westw., Trans. Entom. Soc. London, 518. tab. VI. fig. 7, $7 a-$ d. [Leptynoma] (1876); Big., Annal. Soc. Entom. France, sér. 6. V. Bullet., CXCII. [Leptynoma] (1885).

? argentata Bı̈., Annal. Soc. Entom. France, sér. 6. V. Bullet., LXVIII. [Lampromyia] (1885).

\section{Pheneus}

WaLK., Insecta Saunders., Dipt., I. 155. (1851).

Arthrostylum Willist., Kansas Univ. Quart., IV. 108. (1895). fascipennis WILLIST. = tibialis WALK. opacus Coqusll., Invertebrata Pacifica, I. 21. (1904). 
tibialis WALk., Insecta Saunders., Dipt., I. 156. tab. IV. fig. 3. (1851) - Jamaica, et List Dipt. Brit. Mus., VII. suppl. 3., 766. 1. (1855); WuL.,st., Mexico.

Biolog. Central. Amer., Dipt., I. 264. 1. (1901); Aldr., Catal. North Amer. Dipt., 214. (1905).

fascipennis WiLust., Kansas Univ. Quart., IV. 109. [Avthrostylum] (1895).

\section{Atherix}

Meig., Illiger's Magaz. f. Ins., II. 271. 64. (1803).

1bisia Rond., Dipterol. Ital. Prodr., I. 154. 4. (1856).

Nodutis Meg. in litt. apud Meig.

Pelechoïdocera Big., Annal. Soc. Entom. France, sér. 3. IV. 66 et 91. (1856).

Sylvicola Harris, Expos. Engl. Ins., 100. p. p. (1782).

Apfelbecki Strobl, Glasnik Zem. Mus. Bosni i Herzegov., XIV. Europa mer.

475. (1902) et Wissensch. Mittheil. Bosnien u. Herzegov., IX.

535. (1904).

chrysopus GMEL. = flavipes FABR.

concinna Willist., Biolog. Central. Amer., Dipt., I. 266. 3. tab. IV. Mexico. fig. 23, 23a. (1901); Aldr., Catal. North Amer. Dipt., 217. (1905). crassipes Meig., System. Beschreib., II. 108. 4. (1820) et VI. 322. (1830) ; Macq., Suit. à Buffon, I. 432. 5. (1834); Schis., Fauna Austriaca, Dipt., I. 178. 2. (1862); Goв., Mém. Soc. Linn. Nord France, 1877. 18. (1877); L. Coucke, Annal. Soe. Entom. Belgique, XXXVII. 132. (1893); Griff., Bollet. Mus. Zool. ed Anat. Comp. Torino, X. No. 218., 4. [1bisia] (1895).

femoralis Lw. = Ibis FABR. var.

? flavipes FABR., Species Insect., II. 420. 7. [Rhagio] (1781), Mantissa Insect., II. 333. 8. [Rhagio] (1787) et Entomol. System., IV. 273. 10. [Rhagio] (1794); Schrank, Fauna Boica, III. 99. 2392. [Rhagio] (1803) ; Meig., Klassif., I. 305. g. [Rhagio] (1804); Fabr., System. Beschreib., II. 111. 12. (1820); Scurn., Fauna Austriaca, Dipt., I. 179. nota. (1862).

chrysopus Gmex., Systema Naturae, V. 2865. 318. [Musca] (1792).

Ibis FABR., Supplem. Entomol. System., 556. 3-4. [Rhagio] (1798); MeIG., Klassif., I. 307.p. [Rhagio] (1804) ; FABR., Systema Antliat., 70.5. [Leptis] (1805) ; MeIG., System. Beschreib., II. 105. 1. (1820); Curt., Brit. Entomol., VIII. 26. tab. (1824); St. Farg. ext Serv., Encyclop. Méthod., X. 283. 1. (1825); MacQ., Recueil Soc. Sci. Agricult. Lille, 1826. 406. 1. (1826) et Suit. à Buffon, I. 431. 1. tab. X. fig. 16. (1834); Guér., Iconogr. d. Régne Anim., VII. Ins., 540. tab. XCVI. fig. 2. (1835); Lw., Programm Posen, 1840. 10. 1. (1840) et Isis, 1840. VII-VIII. 527. 1. (1840); Frmm., Bullet. Soc. Imp. Nat. Moscou, XX. 1., 199. 1. (1847); Wat.k.,

Europa centr.

Europa, Sibiria, Japonia. 
List Dipt. Brit. Mus., I. 217. (1848); Schoutz, Zeitschr. f. Entom. Breslau, IV. No. 16., 39. (1850); WALK., Insecta Britannica, Dipt., I. 69. 1. tab. II. fig. 8. (1851); EgG., Verh. zool.-bot. Ges. Wien, IV. 7. (1854); Boxsd., Finlands tvaving. Ins., I. 300. 2. (1861) ; L. Duf., Annal. Soc. Entom. France, sér. 4. II. 131. tab. II. fig. 2. (1862) ; Schin., Fauna Austriaca, Dipt., I. 179. (1862); Chapman, Entom. Monthly Mag., III. 94. (1866) ; Jaenn., Berlin. Entom. Zeitschr., XI. 100. 19. (1867); Braver, Verh. zool.-bot. Ges. Wien, XIX. 922. [Chrysops sp. ?] (1869); H. Tournier et A. DE Borre, Annal. Soc. Entom. Belgique, XVII. Comp. Rend., IXXXIX. 2. fig. (1874); Goв., Mém. Soc. Linn. Nord France, 1877. 18. (1877); DE Borre, Annal. Soc. Entom. Belgique, XVII. Compt. Rend., CXX. (1879) ; Perez, Act. Soc. Linn. Bordeaux, sér. 4. XXXII. pag. XLIII. (1879); Becher, Denkschr. Akad. Wien, XIV. 143. tab. II. fig. 20. (1882); Ormerod, Trans. Entom. Soc. Loudon, 1883. Proc., XX. (1883); Brauer, Denkschr. Akad. Wien, XLVII. tab. III. fig. 48-51. (1883); Billups, The Entomologist, XXII. 193. tab. VII. fig. 1-2. (1889); I. Coucke, Annal. Soc. Entom. Belgique, XXXVII. 132. (1893); Strobl, Mittheil. Naturwiss. Ver. Steiermark, XXIX. 1892. 25. (1893); LAtzel, Carinthia, II. No. 3. (1894); Griff., Bollet. Mus. Zool. ed Anat. comp. Torino, X. No. 218., 4. [1bisia] (1895); Coquill., Proc. Unit Stat. Nat. Mus., XXI., 308. (1898); Sтrobl, Mittheil. Naturwiss. Ver. Steiermark, XXXIV. 195. (1898); Mı, Wien. Entom. Zeitg., XVIII. 232. (1899) et XIX. 148. 5. (1900); GIARD, Bullet. Soc. Entom. France, 1902. 220. (1902).

maculata MeIg., Klassif., I. 294. 1. tab. XIV. fig. 30. (1804);

Latr., Gen. Crust. et Insect., IV. 209. (1809).

melancholia Háris, Expos. Engl. Ins., 103. tab. XXXI. Sect.

II. fig. 1. [Sylvicola] (1782).

Titanus Fabr., Supplem. Entomol. System., 554.18-19. [Anthrax]

(1798) ; Meig., Klassif., I. 211. h. [Anthrax] (1804); Fabr., Systema Antliat., 126. 37. [Anthrax] (1805).

? trifasciata Ros., Correspondenzbl. Württemb. landw. Ver., I. 52. (1840).

var. femoralis Lw., Beschreib. europ. Dipt., I. 61. (1869); StroвL, Europa centr. Mittheil. Naturwiss. Ver. Steiermark, XXIX. 1892. 25. (1893).

labiata BIG., Bullet. Soc. Zool. France, XII. 117. (1887) ; v. D. WulP, Ceylon. Catal. Dipt. South Asia, 68. (1896).

latipennis Bell., Saggio di Ditterol. Messic., II. 93. 1. (1861); Mexico. Willist., Biolog. Central. Americ., Dipt., I. 266. 2. (1901); Aldr., Catal. North Amer. Dipt., 217. (1905).

limbata Ost.-SAck., Berlin. Entom. Zeitschr., XXVI. 100. (1882); I.Philippinae. v. D. Wulp, Catal. Dipt. South Asia, 68. (1896).

longipes BeLl., Saggio di Ditterol. Messic., II. 94. 2. tab. II. fig. Americacentr. 17. (1861); Ost.-Sack., Biolog. Central. Amer., Dipt., I. 62. 1. (1886) ; Aldr., Catal. North Amer. Dipt., 217. (1905). 
longipes Lw., Wien. Entom. Monatschr., VII. 12. 15. (1863).

Africa mer.

lugubris MaCq., Suit. à Buffon, I. 432. 3. (1834).

Patriaignota. maculata MEIG. = Ibis FABR.

marginata FABr., Species Insect., II. 413. 6. [Bibio] (1781) et Mantissa Insect., II. 328. 6. [Bibio] (1787); Rossi, Fauna Etrusca, II. 274. 1426. [Bibio] (1790); Gmex., Systema Naturae, V. 2829. 130. [Musca] (1792); Fabr., Entomol. System., IV. 255. 4. [Bibio] (1794) ; Meig., Klassif., I. 216. d. [Bibio] (I804); Latr., Hist. Nat. d. Crust. et Insect., XIV. 327. 2. [Thereva] (1804) ; FABr., Systema Antliat., 67. 5. [Bibio] (1805); Rossi, Fauna Etrusca, Ed. II., 424. 1426. [Bibio] (1807); MEIG., System. Beschreib., II. 106. 2. tab. XV. fig. 27-28. (1820); CURT., Brit. Entomol., VIII. 26. (1824); MacQ., Recueil. Soc. Sci. Agricult. Lille, 1826. 407. 2. tab. II. fig. 5. (1826) et Suit. à Buffon, I. 432. 2. (1834); Lw., Programm Posen, 1840. 10. 2. (1840) et Isis, 1840. VII-VIII. 527. 2. (1840) ; Zетт., Dipt. Scand., I. 225. 2. (1842); G1nм., Bullet. Soc. Imp. Nat. Moscou, XX. 1., 199. 2. (1847); WALK., I ist Dipt. Brit. Mus., I. 219. (1848) ; ZeTr., Dipt. Scand., VIII. 2990. 2. (1849); Scholtz, Zeitschr. f. Entom. Breslau, IV. No. 16., 40. (1850); Walk., Insecta Britannica, Dipt., I. 70. 2. (1851); Zетт., Dipt. Scand., XI. 4266. 2. (1852); Schin., Fauna Austriaca, Dipt., I. 178.1. (1862); JAEnn., Berlin. Entom. Zeitschr., XI. 100. 20. (1867) ; Goв., Mém. Soc. Linn. Nord France, 1877. 17. tab., fig. 5. (1877); Nevhaus, Diptera Marchica, 48. 1. (1886); L. Coucke, Annal. Soc. Entom. Belgique, XXXVII. 132. (1893); Stroni, Mittheil. Naturwiss. Ver. Steiermark, XXIX. 1892. 25. (1893) ; Griff., Bollet. Mus. Zool. ed Anat. comp. Torino, X. No. 218., 4. [Ibisia] (1895); Mrk, Wien. Entom. Zeitg., XVIII. 232. (1899).

melancholia HARRIS = Ibis FABR.

nigritarsis DoL., Natuurkund. Tijdschr. Nederl. Indie, ser. 4. III. (XVII). 92. 30. (1858) ; Ost.-SAck., Berlin. Entom. Zeitschr., XXVI 100. NB. I. (1882); v. D. Wulp, Catal. Dipt. South Asia, 68. (1896).

pachypus BIG., Bullet. Soc. Zool. France, XII. 117. (1887); AıDr., Americasept. Catal. North Amer. Dipt., 217. (1905).

picta Lw., Beschreib. europ. Dipt., I. 60. 44. (1869).

Europa.

Amboina.

pusilla MAcQ., Dipt. exot., suppl. 5., 68. 1. tab. II. fig. 13. (1855). Titanus FABR. = Ibis FABR.

trifasciata Ros. = ? Ibis FABR.

varicornis Lw., Berlin. Entom. Zeitschr., XVI. 58. 13. (1872); ALDR., America sept. Catal. North Amer. Dipt., 217. (1905).

variegata Walk., List Dipt. Brit. Mus., I. 218. (1848); Ivls, Ent )-

America sept. mol. News Philad., I. 39. (1890); Aldpr., Catal. North Amer. Dipt., 217. (1905).

Rossia sept.

Australia.

dua Walk., List Dipt. Brit. Mus., IV. 1153. (1849); As.dr., Catal. Americasept. North Amer. Dipt., 217. (1905). 


\section{Macellopalpus}

Big., Annal. Soc. Entom. France, sér. 6. VI. Bull., XLVIII. (1886).

flaveolus Big., Annal. Soc. Entom. France, sér. 6. VI. Bull., XLVIII. Nova Guinea. (1886) ; v. D. Wulp, Catal. Dipt. South Asia, 68. (1896).

\section{Suragina}

WALK., Proc. Linn. Soc. London, IV. 110. (1860).

elegans KARscr, Berlin. Entom. Zeitschr., XXVIII. 174. (1884).

Ceylon.

illucens WaLk., Proc. Linn. Soc. London, IV. 110. 58. (1860); v. D. Celebes.

Wulp, Catal. Dipt. South Asia, 68. (1896).

signipennis WAlk., Proc. Linn. Soc. London, VI. 8. 22. (1862); Gilolo.

v. D. Wutp, Catal. Dipt. South Asia, 68. (1896).

\section{Arthroceras}

Wiliıst., Entomol. Amer., II. 107. (1886).

pollinosum Wilisst., Entomol. Amer., II. 108. (1886); Aldr., Catal. Americasept. North Amer. Dipt., 213. (1905).

\section{Rhagio}

FABr., Systema Entomolog., 761. 171. (1775).

Leptis FaBr., Systema Antliat., 69. 15. (1805).

Sylvicola Harris, Expos. Engl. Ins., 100. p. p. (1782).

albibarbis Bı̈., Bullet. Soc. Zool. Franc3, XII. 114. [Leptis] (1887);

Adams, Kansas Univ. Sci. Bullet., II. 440 et 441. [Leptis] (1904);

Aldr., Catal. North Amer. Dipt., 214. [Leptis] (1905).

albicornis SAY, Journ. Acad. Nat. Sci. Philad., III. 39. 9. [Leptis]

(1823), Amer. Entom., I. 27. tab. XIII. fig. 2. [Leptis] (1824) et

Compl. Writt., I. 27. tab. XIII. fig. 2. [Leptis] (1859); Wied.,

Aussereurop. zweifl. Ins., I. 323. 4. [Leptis] (1828); WaLK, List

Dipt. Brit. Mus., I. 212. (1848) ; Adams, Kansas Univ. Sci. Bullet.,

II. 440. [Leptis] (1904); Aldr., Catal. North Amer. Dipt., 214. [Leptis] (1905).

albifrons MeIG. = lineola FABR.

algerious Bkck, Zeitschr. f. system. Hymen. u. Dipt., VI. 282. 115. Algeria.

[Leptis] (1906).

alpinus Lw. = conspicuus MEIG. var.

alumnus WALK., Insecta Saunders., Dipt., I. 163. (1852).

Americamer.

annulatus Big., Bullet. Soc. Zool. France, XII. 112. [Leptis] (1887).

annulatus Dea., Mém. pour serv. l'hist. d. Ins,, VI. 164. 2. [Nemotelus] (1776); Fall., Dipt. Suec., Anthracides, 11. 4. [Leptis]

America sept.

America sept.

Japonia.

Luropa contr. et sept. 
(1814); MeIG., System. Beschreib., II. 92. 6. [Leptis] (1820); ? Lw., Programm Posen, 1840. 8. 6. [Leptis] (1840) et Isis, 1840. VII-VIII. 523. 6. [Leptis] (1840); ZETT., Dipt. Scand., I. 219. 5. [Leptis] (1842); Lw., Stettin. Entom. Zeitg., VIII. 70. 10. [Leptis] (1847) ; Zетт., Dipt. Scand., VIII. 2987. 5. [Leptis] (1849) et XI. 4266. 5. [Leptis] (1852) ; Schun., Fauna Austriaca, Dipt., I. 175. 13. [Leptis] (1862); Neunaus, Diptera Marchica, 47. 5. [Leptis] (1886); Sтrовц, Mittheil. Naturwiss. Ver. Steiermark, XXIX. 1892. 24. [Leptis] (1893).

conjungens Ruthe, Isis, 1831. XI. 1214. [Leptis] (1831).

annulatus MeIG. = maculatus Deg.

balcanicus Strobl, Glasnik Zem. Mus. Bosni i Hercegov., XIV. 475. Europa mer.

[Leptis] (1902) et Wissensch. Mitteil. Bosnien u. Hercegov., IX. 534. [Leptis] (1904).

basalis PhIL., Verh. zool.-bot. Ges. Wien, XV. 773. 8. [Leptis] (1865);

Hunter, Trans. Amer. Entom. Soc. Philad., XXVII. 149. [Leptis] (1901).

bifasciatus Ros. = ? scolopaceus L.

bimaculatus Goв. = maculatus DeG.

bitaeniatus BeLl., Saggio di Ditterol. Messic., Append., 26. 2. tab.

III. fig. 14. [Leptis] (1862); Adams, Kansas Univ. Sci. Bullet., II. 440. [Leptis].(190t); Aldr., Catal. North Amer. Dipt., 214. [Leptis] (1905).

Boscii MacQ., Dipt. exot., II. 1., 30. 2. [Leptis] (1840); Adams, Kansas Univ. Sci. Bullet., II. 441. [Leptis] (1904); AtDr., Catal. North Amer. Dipt., 214. [Leptis] (1905).

caducus Wred., Aussereurop. zweifl. Ins., I. 579. 10. [Leptis] (1828); Hunter, Trans. Amer. Entom. Soc. Philad., XXVII. 149. [Leptis] (1901).

caelebs HARRIS = ? strigosus Meig.

calidris Eversm., Bullet. Soc. Imp. Nat. Moscou, VII. 422. [Leptis] (1834) est nomen nudum.

capnopterus WIED., Aussereurop. zweifl. Ins., I. 580. 11. [Leptis] Brasilia.

(1828); Hunter, Trans. Amer. Entom. Soc. Philad., XXVII. 149.

[Leptis] (1901).

Cartereani Goв. = florentinus Lw.

Cavennae Bezzi, Bullet. Soc. Entom. Ital., XXX. 28. 43. [Leptis] (1898). Italia.

chrysopilaeformis BezzI, Bullet. Soc. Entom. Ital., XXX. 31. 48. Italia. [Leptis] (1898).

chrysostigma Lw., Wien. Entom. Monatschr., I. 2. [Leptis] (1857); Schin., Fauna Austriaca, Dipt., I. 173. 7. [Leptis] (1862); Lw., Beschreib. europ. Dipt., I. 35. 32. [Leptis] (1869).

cinerascens Rö̀., Wien. Entom. Zeitg., III. 41. 2. [Leptis] (1884); Sardinia.

Bezzi, Bullet. Soc. Entom. Ital., XXX. 31. [Leptis] (1898).

cinereofasciatus Schumm., Übers. d. Arb. u. Veränd. d. schles. Ges. Europa centr.

f. Vaterl. Kultur, 1837. 109. [Leptis] (1837); Schus., Fauna

Austriaca, Dipt., I. 175. [Leptis] (1862). 
cinereus BeLL., Saggio di Ditterol. Messic., II. 95. 1. [?Leptis] (1861); Ost.-SAck., Catal. Dipt. North Amer., Ed. II., 64 et 229. 94. [Leptis] (1878) ; Aldr., Catal. North Amer. Dipt., 214. [Leptis] (1905).

cinereus ZEтr., Dipt. Scand., I. 221. 9. [Leptis] (1842).

cingulatus Lw., Neue Beitr., IV. 28. 9. [Leptis] (1856); Schin., Fauna Austriaca, Dipt., I. 172. [Leptis] (1862); Lw., Beschreib. europ. Dipt., I. 37. 33. [Leptis] (1869) ; Srrobs, Mittheil. Naturwiss. Ver. Steiermark, XXIX. 1892. 19. [Leptis] (1893).

claripennis PhIL., Verh. zool.-bot. Ges. Wien, XV. 772. 5. [Leptis] (1865) ; Hunter, Trans. Amer. Entom. Soc. Philad., XXVII. 149. [Leptis] (1901).

conjungens RUthe $=$ annulatus Deg.

conspicuus Meig., Klassif., I. 299. 4. tab. XV. fig. 5. (1804); Latr., Gen. Crust. et Ins., IV. 288. (1809) ; MejG., System. Beschreib., II. 90. 3. [Leptis] (1820) ; Macq., Recueil Soc. Sci. Agricult. Lille, 1826. 400. 3. [Leptis] (1826) et Suit. à Buffon, I. 426. 3. [Leptis] (1834); Lw., Programm Posən, 1840. 7. 1. [Leptis] (1840) et Isis, 1840. VII-VIII. 521. 1. [Leptis] (1840) ; Walk., List Dipt. Brit. Mus., I. 211. (1848) ; Scholtz, Zeitschr. f. Entom. Breslau, IV. No. 16., 39. [Leptis] (1850); Schis., Fauna Austriaca, Dipt., I. 175. 13. p. p. [Leptis] (1862); JAENN., Berlin. Entom. Zeitschr., XI. 97. 9. [Leptis] (1867); Goв., Mém. Soc. Linn. Nord France, 1877. 11. [Leptis] (1877); L. Coucke, Annal. Soc. Entom. Belgique, XXXVII. 131. [Leptis] (1893); Strobl, Mittheil. Naturwiss. Ver. Steiermark, XXIX. 1892. 23. [Leptis] (1893) et Wissensch. Mittheil. Bosnien u. Herzegov., VII. 578. [Leptis] (1900).

Janotae Now., Verh. zool.-bot. Ges. Wien, XVII. 349. [Leptis] (1867).

Marchalii Pierne, Feuille jeun. Natural., XIX. 50. [Leptis] (1889) ; Flamary, Bull. Soc. Hist. Nat. Macôn, 1898. 48. nota. [Leptis] (1898).

var. alpinus Lw., Beschreib. europ. Dipt., I. 35. nota. [Leptis] (1869).

conspicuus Schis. p. p. = florentinus Lw.

costatus Lw., Berlin. Entom. Zeitschr., VI. 187. 4. [Leptis] (1862); Osт.-SAck., Bullet. Unit. Stat. Geolog. Surv., III. 223. [Leptis] (1877); Adams, Kansas Univ. Sci. Bullet., II. 441. [Leptis] (1904); Aldr., Catal. North Amer. Dipt., 214. [Leptis] (1905).

decisus War.K., Proc. Linn. Soc. London, I. 15. 47 et 118. 71. [Leptis] (1856) ; v. D. Wulp, Catal. Dipt. South Asia, 67. [Leptis] (1896). derelictus $\mathrm{HARR}_{\mathrm{A}}=$ strigosus MeIG.

dimidiatus Lw., Berlin. Entom. Zeitschr., VII. 10. 17. [Leptis] (1863) ; Coquill., Proc. Washingt. Acad. Sci., II. 406. [Leptis] (1900) ; A дамs, Kansas Unir. Sci. Bullet., II. 440. [Leptis] (1904); Coquil., Harriman Alaska Exped., IX. Pt. II. 20. [Leptis] (1904); ALDr., Catal. North Amer. Dipt., 214. [Leptis] (1905).
Mexico.

Europa sept.

Europacentr.

Chile.

Europacentr. et mer.

Europa centr.

America sept.

Malacea,

Borneo.

Alaska. 
distigma MEIG. = maculatus DEG.

ephippium ZeтT., Dipt. Scand., I. 219. 6. [Leptis] (1842).

Europa sept.

filic WALK. = punctipennis SAY.

flavicornis MACQ., Recueil Soc. Sci. Agricult. Lille, 1826. 402. 6. Europacentr.

[Leptis] (1826) ; MeIG., System. Beschreib., VII. 61. 28. [Leptis]

(1838); Gor., Mém. Soc. Linn. Nord France, 1877. 10. [Leptis]

(1877) ; I. Coucke, Annal. Soc. Entom. Belgique, XXXVII. 131.

[Leptis] (1893).

flavimedius Coquill., Proc. Unit. Stat. Nat. Mus., XXI. 307. [Leptis] Japonia. (1898).

flavoniger Coquill., Invert. Pacifica, I. 20. [Leptis] (1904).

Nevada.

florentinus Lw., Besclureib. europ. Dipt., I. 34. 31. [Leptis] (1869). Cartereaui Goв., Mém. Soc. Linn. Nord France, 1877. 14. [Leptis] (1877).

conspicuus Schns., Fauna Austriaca, Dipt., I. 175. 13. p. p. [Leptis] (1862).

Pandellei Goв., Mém. Soc. Linn. Nord France, 1877. 13. [Leptis] (1877).

Perezii Gos., Mém. Soc. Linn. Nord France, 1877. 12. [Leptis] (1877).

Pervisii Goв., Mém. Soc. Linn. Nord. France, 1877. 13. [Leptis] (1877).

fulvus Deg., Mém. pour serv. l'hist. d. Ins., VI. 203. 1. tab. XXIX. fig. 3-5. [Nemotelus] (1776).

funebris Meig., System. Beschreib., II. 98. 14. [Leptis] (1820); MAcq., Suit. à Buffon, I. 428. 9. [Leptis] (1834); Grmm., Bullet. Soc. Imp. Nat. Moscou, XX. 1., 196. 9. [Leptis] (1847); Beck., Wien. Entom. Zeitg., IX. 65. 1. [Leptis] (1890); BezzI, Bullet. Soc. Entom. Ital., XXX. 31. [Leptis] (1898).

fuscipennis MeIG., System. Beschreib., II. 97. 13. [Leptis] (1820) ; Walk., List Dipt. Brit. Mus., I. 211. (1848); Schin., Fauna Austriaca, Dipt., I. 172. [Leptis] (1862); Lw., Beschreib. europ. Dipt., I. 39. nota. [Leptis] (1869).

fuscus WIED., Aussereurop. zweifl. Ins., I. 227. 14. [Leptis] (1828); Hunter, Trans. Amer. Entom. Soc. Philad., XXVII. 149. [Leptis] (1901).

geminatus WiED., A ussereurop. zweifl. Ins., I. 222. 3. [Leptis] (1828). Patria ignota. Goebelii StroвL = tringarius L. var.

graeculus Lw., Beschreib. europ. Dipt., I. 32. 29. [Leptis] (1869). Europa mer. griscolus v. D. WULP $=$ plumbeus SAY.

griseus Phil., Verh. zool.-bot. Ges. Wien, XV. 774. 9. [Leptis] Chile.

(1865) ; Hunter, Trans. Amer. Entom. Soc. Philad., XXVII. 149.

[Leptis] (1901).

guttatus OLIV. = ? scolopaceus L.

Heyshami CurT. = notatus Meic.

hirtulus Big., Bullet. Soc. Zool. France, XII. 116. [Leptis] (1887). Chile.

hirtus Lw., Berlin. Entom. Zeitschr., V. 318. 21. [Leptis] (1861); Americasept.

Kertész: Catalogus Dipterorum. III. 
v. D. Wulp, Tijdschr. v. Entom., XXV. 118. 3. [Leptis] (1882); Adans, Kansas Univ. Sci. Bullet., II. 441. [Leptis] (1904); Arnr., Catal. North Amer. Dipt., 214. [Leptis] (1905).

Hoffmannseggii WIED. = strigosus MEIG.

hoodianus BIG., Bullet. Soc. Zool. France, XII. 115. [Leptis] (1887);

Adars, Kansas Univ. Sci. Bullet., II. 440. [Leptis] (1904); Aldr.,

Catal. North Amer. Dipt., 214. [Leptis] (1905).

hyalipennis Ros., Correspondenzbl. Württemberg. landw. Ver., I. Europacentr. 52. [Leptis] (1840).

hyalopterus WiED., Aussereurop. zweifl. Ins., I. 226. 10. [Leptis] (1828) ; Hunter, Trans. Amer. Entom. Soc. Philad., XXVII. 149. [Leptis] (1901).

immaculatus Meig., Klassif., I. 301. 8. (1804) et System. Beschreib., II. 93. 7. [Leptis] (1820); MacQ., Recueil Soc. Sci. Agricult. Lille, 1826. 402. 7. [Leptis] (1826) ; MElg., System. Besclureib., VI. 320. [Leptis] (1830) ; MACQ., Suit. à Buffon, I. 427. 6. [Leptis] (1834) ; Curt., Brit. Entomol., VIJT. 705. (1838); Walk., List Dipt. Brit. Mus., I. 211. (1848); Scholtz, Zeitschr. f. Entomol. Breslau, IV. No. 16., 39. [Leptis] (1850); Lw., Wien. Entom. Monatschr., I. 1. [Leptis] (1857); Schns., Fauna Austriaca, Dipt., I. 173. 7. [Leptis] (1862) ; JaEnn., Berlin. Entom. Zeitschr., XI. 97. 3. [Leptis] (1867); Goв., Mém. Soc. Linn. Nord France, 1877. 8. [Leptis] (1877); Neunaus, Diptera Marchica, 47. 3. [Leptis] (1886); L. Coucke, Annal. Soc. Entom. Belgique, XXXVII. 131. [Leptis] (1893) ; Sтroвl, Mittheil. Naturwiss. Ver. Steiermark, XXJX. 1892. 20. [Leptis] (1893).

reconditus Harris, Expos. Engl. Ins., 101. tab. XXXI. fig. 3. [Sylvicola] (1782).

stigmaticus Zетт., Dipt. Scand., I. 218. 4. [Leptis] (1842) et VIII. 2987. 4. [Leptis] (1819).

impar Walk., Proc. Linn. Soc. London, V. 282. 45. [Leptis] (1861); v. D. Wulp, Catal. Dipt. South Asia, 67. [Leptis] (1896).

incisus Lw., Berlin. Entom. Zeitschr., XVI. 59. 16. [Leptis] (1872); Osт.-Sack., Bullet. Unit. Stat. Geolog. Surv., III. 223. [Leptis] (1877); Adays, Kansas Univ. Sci. Bullet., II. 440. [Leptis] (1904); Aldr., Catal. North Amer. Dipt., 214. [Leptis] (1905).

inquinatus Scop. $=$ ? scolopaceus $\mathrm{L}$.

intermedius WaLk., List Dipt. Brit. Mus., I. 212. (1848); Adans, Kansas Univ. Sci. Bullet., II. 441. [Leptis] (1904); Aldr., Catal. North Amer. Dipt., 214. [Leptis] (1905).

inutilis WALK., List Dipt. Brit. Mus., I. 213. (1848) et Insecta Britanuica, Dipt., I. 67. 7. [Leptis] (1851).

Janotae Now. $=$ conspicuus MEIG.

latipennis Lw., Neue Beitr., IV. 29. 10. [Leftis] (1856); Scuns., I. Batjan.

Europa centr. et sept.

Brasilia.

Americasept.

America sept.

Fauma Austriaca, Dipt., I. 175. (1862); Lw., Beschreib. europ. Dipt., I. 25. 25. [Leptis] (1869); Brung, Archir f. Naturgesch., XLI. 1., 51. [Leptis] (1875); Bwzz, Bullet. Soc. Entom. Ital,, XXX. 29. [Leptis] (1898). 
lineola FABr., Entomol. System., IV. 275. 17. (1794); MeIG., Klassif., Europa. I. 307. m. (1804); FABR., Systema Antliat., 74. 6. [Atherix] (1805); Fall., Dipt. Suec., Anthracides, 12. 6. [Leptis] (1814); Meig., System. Beschreib., II. 94. 9. [Leptis] (1820); Macq., Recueil Soc. Sci. Agricult. Lille, 1826. 402. 8. [Leptis] (1826) et Suit. à Buffon, I. 427. 8. [Leptis] (1834); ZEтT., Insecta Lappon., Dipt., 526. 3. [Leptis] (1838); CurT., Brit. Entomol., VIII. 705. (1838); Zett., Dipt. Scand., I. 221. 8. [Leptis] (1842); Ratzeb., Forstinsekt., III. 155. nota 4. [Leptis] (1844); Gimm., Bullet. Soc. Imp. Nat. Moscou, XX. 1., 195. 5. [Leptis] (1847); WALK., List Dipt. Brit. Mus., I. 213. (1848); Zетт., Dipt. Scand., VIII. 2987. 8. [Leptis] (1849) ; Scholtz, Zeitschr. f. Entom. Breslau, IV. No. 16., 39. [Leptis] (1850); WaLK., Insecta Britannica, Dipt., I. 66. 6. [Leptis] (1851) ; BonsD., Finlands tvaving Ins., I. 138. 3. [Leptis] (1861) ; Schin., Fauna Austriaca, Dipt., I. 174. 11. [Leptis] (1862); JAENN., Berlin. Entom. Zeitschr., XI. 97. 7. [Leptis] (1867); Siebke, Nyt Magaz. f. Naturvid., XIX. 77. 34. [Leptis] (1872); Beling, Archiv f. Naturgeseh., XLI. 1., 51. [Leptis] (1875) ; Siebke, Catal. Dipt. Norvegiae, 15. 6. [Leptis] (1877); JARosch., Trudi Kharkoff, XI. 364. 40. [Leptis] (1877); Gов., Mém. Soc. Linn. Nord France, 1877. 10. [Leptis] (1877); Neuhaus, Diptera Marchica, 47. 3. [reete 4.] [Leptis] (1886); L. Coucke, Annal. Soc. Entom. Belgique, XXXVII. 131. [Leptis] (1893) ; S'rRoвL, Mittleil. Naturwiss. Ver. Steiermark, XXIX. 1892. 21. [Leptis] (1893); BEzZI, Bullet. Soc. Entom. Ital., XXX. 31. [Leptis] (1898).

albifrons MeIG., Klassif., I. 300. 6. (1804).

Monachus Harris, Expos. Engl. Ins., 102. tab. XXXI. fig. 9. [Sylvicola] (1782).

var. monticola Ega., Verh. zool.-bot. Ges. Wien, X. 668. Europacentr. [Leptis] (1860) ; Schrn., Fauna Austriaca, Dipt., I. 174. 11. [Lepti]. (1862) ; JAENN., Berlin. Entom. Zeitschr., XI. 97. 6. [Leptis] (1867); BEck., Berlin. Entom. Zeitschr., XXXI. 139. 112. [Leptis] (1887); Strobl, Mittheil. Naturwiss. Ver. Steiermark, XXIX. 1892. 21. [Leptis] (1893); BezzI, Bullet. Soc. Entom. Ital., XXX. 31. [Leptis] (1898).

monticula EGG., Verh. zool.-bot. Ges. Wien, X. 348. [Leptis] (1860) lapsiıs.

lugens PhiL., Verh. zool.-bot. Ges. Wien, XV. 773. 7. [Leptis] (1865); Hunter, Trans. Amer. Entom. Soc. Philad., XXVII. 149. [Leptis] (1901).

maculatus Deg., Mém. pour serv. l'hist. d. Ins., VI. 165. 3. [Nemotelus] (1776); Fall., Dipt. Suec., Anthracides, 11. 5. [Leptis] (1814) ; Meia., System. Beschreib., II. 95. 10. [Leptis] (1820); ZeтT., Dipt. Scand., I. 220. 7. [Leptis] (1842); Giмм., Bullet. Soc. Imp. Nat. Moscou, XX. 1., 196. 8. [Leptis] (1847); WALK., List Dipt. Brit. Nus., I. 211. (1848); ZETr., Dipt. Seand., VIII. 2987. 7. [Leptis] (1849) et XI. 4266. 7. [Leptis] (1852); Lw., Wien.

Chile.

Europa. 
Entom. Monatschr., I. 1. [Leptis] (1857) ; BovsD., Finlands tvaving. Ins., I. 138. 2. [Leptis] (1861); Schin., Fauna Austriaca, Dipt., I. 173. 6. [Leptis] (1862); JAEnn., Ber]in. Entom. Zeitschr., XI. 96. 2. [Leptis] (1867) ; Goв., Mém. Soc. Linn. Nord France, 1877. 8. [Lcptis] (1877) ; Braukr, Denkschr. Akad. Wien, XLVII. tab. III. fig. 43-47. [Leptis] (1883); L. Coucke, Annal. Soc. Entom. Belgique, XXXVII. 131. [Leptis] (1893) ; Strobl, Mittheil. Naturwiss. Ver. Steiermark, XXIX. 1892. 20. [Leptis] (1893) et Wien.

Intom. Zeitg., XII. 36. [Leptis] (1893).

annulatus Meig., Klassif., I. 299. 2. (1804).

bimaculatus Goв., Mém. Soc. Linn. Nord France, 1877. tab., fig. 1-2. [Leptis] (1877).

ristigma Meig., System. Beschreib., II. 93. 8. tab. XV. fig. 19-20. [Leptis] (1820); MACQ., Suit. à Buffon, I. 427. 7. [Leptis] (1834); Lw., Programm Posen, 1840. 8. 7. [Leptis] (1840) et Isis, 1840. VII-VIII. 524. 7. [Leptis] (1840); Scholit, Zeitschr. f. Entom. Breslau, IV. No. 16., 39. [Leptis] (1850). marginatus Meg. in litt. apud MeIG.

nigrofasciatus MeIG., Ḱlassif., I. 301. 7. (1804).

var. obscurus Strobl, Glasnik Zem. Mus. Bosni i Hercegov., X. 420. [Leptis] (1898) et Wissensch. Mittheil. Bosnien u. Herzegov., VII. 578. [Leptis] (1900).

maculatus Scop. $=$ ? scolopaceus $\mathrm{L}$.

maculifer BIG., Bullet. Soc. Zool. France, XII. 113. [Leptis] (1887);

Adams, Kansas Univ. Sci. Bullet., II. 440. [Leptis] (1904) ; Ardr., Catal. North Amer. Dipt., 214. [Leptis] (1905).

maculipennis Lw., Neue Beiträge, II. 1. 3. [Leptis] (1854), Wien.

Entom. Monatschr., VI. 163. 14. [Leptis] (1862) et Beschreib. europ. Dipt., I. 27. 26. [Leptis] (1869); BezzI, Bullet. Soc. Entom. Ital., XXX. 29. [Leptis] (1898).

Marchalii Pienre = conspicuus MeIG.

marginatus MEG. = maculatus DEG.

Monachus Harris = lineola FaBr.

monotropus HARRIS = scolopaceus L.

monticola EGG. = lineola FABR. var.

monticula EGG. = lineola FABR. var. monticola EGG.

mystaceus MacQ., Dipt. exot., II. 1., 30. 1. tab. III bis, fig. 2. [Leptis] (1840) ; ? Walk., List Dipt. Brit. Mus., I. 212. (1848) ; v. D. Wulp, Tijdschr. v. Entom., XXV. 118. 2. [Leptis] (1882); Adsms, Kansas Univ. Sci. Bullet., II. 440. [Leptis] (1904); Aldr., Catal. North Amer. Dipt., 215. [Leptis] (1905).

nemoralis PHul., Verh. zool.-bot. Ges. Wien, XV. 772. 2. [Leptis] (1865) ; Hunter, Trans. Amer. Entom. Soc. Philad., XXVII. 149. [Leptis] (1901).

niger Wied. apud Meig., System. Beschreib., II. 98. 15. [Leptis] America sept.

Europa mer., $\Lambda$ sia min.

Americasept.

Chile.

Europa mer.

(1820); JAEnv., Berlin. Entom. Zeitschr., XI. 98. 10. [Leptis] (1867);

Iw., Beschreib. europ. Dipt., I. 26. nota. [Leptis] (1869) ; Brzz., Bullet. Soe. Entom. Jtal., XXX. 30. [Leptis] (1898). 
nigratus PhrL., Verh. zool.-bot. Ges. Wien, XV. 772. 4. [Leptis] (1865). Chile.

nigrita Hunter, Trans. Amer. Entom. Soc. Philad., 149. [Leptis] (1901).

nigrita Hunter $=$ nigratus PHIL.

nigriventris Lw., Beschreib. europ. Dipt., I. 33. 30. [Leptis] (1869);

Strobl, Mittheil. Naturwiss. Ver. Steiermark, XXIX. 1892. 22.

[Leptis] (1893).

nigrofasciatus MEIG. = maculatus DEG.

notatus MeIG., System. Beschreib., II. 95. 11. [Leptis] (1820) et VI. 320.

[Leptis] (1830) ; Schumm., Übers. d. Arb. u. Veränd. d. schles. Ges.

f. vaterl. Kultur, 108. [Leptis] (1837); CunT., Brit. Entomol., VIII. 705. (1838); Lw., Programm Posen, 1840. 8. 8. [leptis] (1840) et Isis, 1840. VII-VIII. 524. 8. [Leptis] (1840); Gum., Bullet. Soc. Imp. Nat. Moscou, XX. 1., 195. 6. [Leptis] (1847); Scноцтz, Zeitschr. f. Entom. Breslau, IV. No. 16., 39. [Leptis] (1850); Walk., Insecta Britannica, Dipt., I. 66. 4. [Leptis] (1851); ScHin., Fauna Austriaca, Dipt., I. 174. 10. [Leptis] (1862); JAEnn., Berlin. Entom. Zeitschr., XI. 97. 5. [Leptis] (1867); SтroвL, Mittheil. Naturwiss. Ver. Steiermark, XXIX. 1892. 21. [Leptis] (1893); BEck., Entomol. Nachricht., XXVI. 107. fig. 7. [Leptis] (1900) et Acta Soc. Sci. Fenn., XXVI. No. 9., 13. tab. I. fig. 3. [Leptis] (1900).

Heyshami Curt., Brit. Entomol., VIII. 705. tab. (1838).

obscurus $\mathrm{STROBL}=$ maculatus Deg. var.

ochraceus Lw., Berlin. Entom. Zeitschr., VI. 187. 3. [Leptis] (1862) ;

Adams, Kansas Univ. Sci. Bullet., II. 441. [Leptis] (1904) ; Aldr.,

Catal. North Amer. Dipt., 215. [Leptis] (1905).

palpalis Adass, Kansas Univ. Sci. Bullet., II. 440 et 442 . [Leptis] (1904).

Pandellei Goв. = florentinus Lw.

Perezii Gов. = florentinus Lw.

Perrisii GoB. = florentinus $\mathrm{L}$ w.

pilosus Lw. = sordidus Lw. var.

pleuralis AdAms, Kansas Univ. Sci. Bullet., II. 440 et 441 . [Leptis] (1904).

plumbeus SAY, Journ. Acad. Nat. Sci. Pliilad., III. 39. 10. [Leptis] (1823) et Compl. Writt., II. 56. 10. [Leptis] (1859); Wied., Aussereurop. zweifl. Ins., I. 228. 15. [Leptis] (1828); WALK., List Dipt. Brit. Mus., I. 217. [Leptis] (1848); Lw., Zeitschr. f. d. ges. Naturwiss., N. F. II. (XXXVI). 115. 6. [Leptis] (1870); v. D. Wur.P, Tijdschr. v. Entom., XXV. 118. 1. [Leptis] (1882); Adans, Kansas Univ. Sci. Bullet., II. 440. [Leptis] (1904); ALdr., Catal. North Amer. Dipt., 215. [Leptis] (1905).

griseolus v. D. Wulp, Tijdschr. v. Entom., ser. 2. II. (X). 1867. 142. 14. tab. IV. fig. 5. [Leptis] (1868).

polytaeniatus Bell., Saggio di Ditterol. Messic., Append., 27. 3. Mexico. tab. III. fig. 13. [Leptis] (1862); AdAMs, Kansas Univ. Sci. Bullet., 
II. 440. [Leptis politaeniata] (1904); Aldr., Catal. North Amer.

Dipt., 215. [Leptis] (1905).

praefica PH1L., Verh. zool.-bot. Ges. Wien, XV.772. 3. [Leptis] (1865) ;

Hunter, Trans. Amer. Entom. Soc. Philad., XXVII. 149. [Leptis] (1901).

pruinosus Big., Bullet. Soc. Zool. France, XII. 115. [Leptis] (1887);

Coquill., Proc. Washingt. Acad. Sci., II. 406. [Leptis] (1900);

AdAus, Kansas Univ. Sci. Bullet., II. 440. [Leptis] (1904);

Coquill., Harriman Alaska Exped., IX. Pt. II., 20. [Leptis]

(1904); Aldr., Catal. North Amer. Dipt., 215. [Leptis] (1905).

punctatus Lw. = tringarius L. var.

punctipennis SAY, Journ. Acad. Nat. Sci. Philad., III. 34. 2. [Leptis]

(1823) et Compl. Writt., II. 55. 2. [Leptis] (1859) ; W1ED., Aus-

sereurop. zweifl. Ins., I. 227. 13. [Leptis] (1828); Ost.-SAck.,

Bullet. Buffalo Soc. Nat. Sci., II. 173. [Leptis] (1874) et Catal.

Dipt. North Amer., Ed. II., 64 et 229. 93. [Atherix] (1878);

Adans, Kansas Univ. Sci. Bullet., II. 440. [Leptis] (1904) ; Aldr.,

Catal. North Amer. Dipt., 215. [Leptis] (1905).

filia Walk., List Dipt. Brit. Mus., I. 219. [Atherix] (1848).

reconditus HarRis $=$ immaculatus Mejg.

rufus Deg., Mém. pour serv. l'hist. d. Ins., VI. 166. 4. tab. IX. fig. Gallia.

12-18. [Nemotelus] (1776).

rufus Scop. = ? tringarius $\mathrm{L}$.

scapularis Lw., Berlin. Entom. Zeitschr., V. 318. 22. [Leptis] (1861);

Adans, Kansas Univ. Sci. Bullet., II. 440. [Leptis] (1904); Aldr.,

Catal. North Amer. Dipt., 215. [Leptis] (1905).

scapulifer BIG., Bullet. Soc. Zool. France, XII. 113. [Leptis] (1887). Japonia. scolopaceus DEG. p. p. (nec I.) $=$ tringarius $\mathrm{L}$.

scolopaceus L., Systema Naturae, Ed. X., 590. 10. [Musca] (1758),

Fauna Suec., Ed. Alt., 441. 1788. [Musca] (1761) et Systema

Naturae, Ed. XII., II. 982. 16. [Musca] (1767); FABr., Systema Entomolog., 761. 1. (1775) ; Deg., Mém. pour sərv. l'hist. d. Ins., VI. 162. 1. p. p. tab. IX. fig. 6-9. [Nemotelus] (1776); FABr., Spec. Insect., II. 419. 1. (1781) ; Schrank, Enum. Insect. Austr., 442. 897. [Musca] (1781); FABr., Mantissa Insect., II. 332. 1. (1787) ; Rossi, Fauna Etrusca, II. 281. 1445. (1790); GMel., Systema Naturae, V. 2864. 16. [Musca] (1792); FABr., Entomol. System., IV. 271. 1. (1794); Panz., Fauna Germ., XIV. tab. 19. (1794) ; Schrank, Fauna Boica, III. 98. 2391. (1803); Schellenb., Gattungen d. Fliegen, 40 et 40. tab. XXXI. fig. 1. (1803) ; MeIG., Klassif., I. 298. 1. (1804) ; Latr., Hist. Nat. d. Crust. et d. Ins., XIV. 331. 1. tab. CXI. fig. 4. (1804); FABr., Systema Antliat., 69. 1. [Leptis] (1805); Rossi, Fauna Etrusca, Ed. II., 435. 1445. (1807) ; Fall., Diptera Suec., Anthracides, 10. 1. [Leptis] (1814); LAM., Hist. nat. anim. sans. vert., III. 423. 2. (1816); FALL., Dipt. Suec., Conopsariae, 14. Corrigenda. [Leptis] (1817); Cuv., Règne Animal., III. 618. [Leptis] (1817); MeIG., System. Be-

Europa. 
schroib., II. 89. 2. [Leptis] (1820); ST. FARG. \& SERv., Encyclop. Méthod., X. 282. 1. [Leptis] (1825); MacQ., Recueil Soc. Sci. Agricult. Lille, 1826. 399. 2. tab. II. fig. 3. [Leptis] (1826); Bouché, Naturgesch. d. Insekt., I. 44. 26. tab. IV. fig. 11-15. [Leptis] (1834); MACQ., Suit. à Buffon, I. 426. 1. [Leptis] (1834); Zетт., Insecta Lappon., Dipt., 526. 1. [Leptis] (1838); Curt., Brit. Entomol., VIII. 705. (1838); Lw., Programm Posen, 1840. 7. 2. [Leptis] (1840) et Isis, 1840. VII-VIII. 521. 2. [Leptis] (1840); Zeтr., Dipt. Scand., I. 216. 1. [Leptis] (1842); Gıм., Bullet. Soc. Imp. Nat. Moscou, XX. 1., 193. 1. [Leptis] (1847); WALK., List Dipt. Brit. Mus., I. 210. (1848); Zetr., Dipt. Scand., VIII. 2986. 1. [Leptis] (1849); Walk., Insecta Britannica, Dipt., I. 65. 1. tab. II. fig. 6. [Leptis] (1851); HaLID., Stettin. Entom. Zeitg., XI. 136. [Leptis] (1851) ; Zetr., Dipt. Scand., XI. 4266. 1. [Leptis] (1852) et XII. 4590. 1. [Leptis] (1855); Schin., Verh. zool.-bot. Ver. Wien, VI. 420. 984 et 985. [Leptis] (1856); Borsd., Finlands tvaving. Ins., I. 138. 1. [Leptis] (1861); Schis., Fauna Austriaca, Dipt., I. 172. 4. [Leptis] (1862) ; JaEns., Berlin. Entom. Zeitschr., XI. 95. 1. [Leptis] (1867); Beling., Archiv f. Naturgesch., XLI. 1., 50. [Leptis] (1875); Gов., Mém. Soc. Linn. Nord France, 1877. 7. [Leptis] (1877); Meinent, Fluernes Munddeele, Trophi Dipt., 53. tab. IV. fig. 8-10. [Leptis] (1881) ; Neuhaus, Diptera Marchica, 46. 1. [Leptis] (1886); BEcK., Berlin. Entom. Zeitschr., XXXI. 139. 111. [Leptis] (1887); Brandt, Horae Soc. Entom. Ross., XXII. 133-137. [Leptis] (1888) ; Leprevost, Aunal. Soc. Entom. France, sér. 7. II. Bullet., CLV. [Leptis] (1892); Gazagnaire, Annal. Soc. Entom. France, sér. 7. II. Bullet., CLVI. [Leptis] (1892); L. Coucke, Annal. Soc. Entom. Belgique, XXXVII. 131. [Leptis] (1893) ; Sтroвц, Mittheil. Naturwiss. Ver. Steiermark, XXIX. 1892. 20. [Leptis] (1893); E. Coucke, Annal. Soc. Entom. Belgique, XL. 231. [Leptis] (1896); BezzI, Bullet. Soc. Entom. Ital., XXX. 29. [Leptis] (1898); Meis., Zoolog. Jahrbüch., Abth. f. Anat., XV. 676. tab. XXXIV. fig. 35. [Leptis] (1902).

? bifascialus Ros., Correspondenzbl. Württemberg. landw. Ver., I. 52. [Leptis] (1840).

? guttatus Ouıv., Encyclop. Méthod., IV. 272. 9. [Asilus] (1789). ? inquinatus Scop., Entomol. Carniol., 363. 985. [Erax] (1763); Ouiv., Encyclop. Méthod., IV. 272. 10. [Asilus] (1789).

? maculatus Scop., Entomol. Carniol., 362. 98t. [Erax] (1763). monotropus Harris, Expos. Engl. Ins., 101. tab. XXXI. fig. 5.

[Sylvicola] (1784).

solitarius Harris, Expos. Engl. Ins., 100. tab. XXXI. fig. 1-2. [Sylvicola] (1782).

setosus PhiL., Verh. zool.-bot. Ges. Wien, XV. 773.6. [Leptis] (1865); Hunter, Trans. Amer. Entom. Soc. Philad., XXVII. 149. [Leptis] (1901).

simplex MEIG. = tringarius L. var. 
soliturius HaRris = scolopaceus L.

solivagus HakRIS = tringarius $\mathrm{L}$.

sordidus Lw., Berlin. Entom. Zeitschr., VI. 74. 12. [Leptis] (1862)

et Beschreib. europ. Dipt., I. 41. 35. [Leptis] (1869).

var. pilosus Lw., Berlin. Entom. Zeitschr., IX. 235. 1. excl.

․ [Leptis] (1865) et Beschreib. europ. Dipt., I. 39. 34. [Leptis]

(1869); Strobl, Mittheil. Naurwiss. Ver. Steiermark, XXIX.

1892. 22. nota. [Leptis] (1893).

stigma Schumm. = vitripennis MeIG.

stigmaticus ZETT. = immaculatus MEIG.

strigosus Meig., Klassif., I. 299. 3. (1804) et System. Beschreib., II. Europa.

88. 1. [Leptis] (1820) ; MacQ., Recueil Soc. Sci. Agricult. Lille, 1826. 398. 1. [Leptis] (1826) et Suit. à Buffon, I. 426. 2. [Leptis] (1834) ; Blanch., Hist. Nat. Ins., III. 603. tab. III. fig. 8. [Leptis] (1840) ; Gmu., Bull. Soc. Imp. Nat. Moscou, XX.1., 195. 7. [Leptis] (1847) ; WaLK., List Dipt. Brit. Mus., I. 210. (1848) et Insecta Britannica, Dipt., I. 66. 5. [Leptis] (1851); Scmin., Fauna Austriaca, Dipt., I. 172. 4. [Leptis] (1862); JAENN., Berlin. Entom. Zeitschr., XI. 95. 1. [Leptis] (1867); Goв., Mém. Soc. Linn. Nord France, 1877. 7. [Leptis] (1877); Becher, Denkschr. Akad. Wien, XLV. 143. tab. II. fig. 22. [Leptis] (1882); GazaGnaire, Annal. Soc. Entom. France, sér. 7. II. Bullet., CLVI. [Leptis] (1892); L. Coucke, Annal. Soc. Entom. Belgique, XXXVII. 131. [Leptis] (1893) ; Strobl, Mittheil. Naturwiss. Ver. Steiermark, XXIX. 1892. 20. [Leptis] (1893) ; Bezzi, Bullet. Soc. Entom. Ital., XXX. 30. [Leptis] (1898).

? caelebs HaRris, Expos. Engl. Ins., 102. tab. XXXI. fig. 8. [Sylvicola] (1782).

derelictus Harris, Expos. Engl. Ins., 102. tab. XXXI. fig. 7. [Sylvicola] (1782).

Hoffmannseggii WIED. in litt. apud MEIG.

striola Meig., System. Beschreib., VII. 61. 27. [Leptis] (1838) ; Scrns., Fauna Austriaca, Dipt., I. 175. [Leptis] (1862).

subannulatus PhiL., Verh. zool.-bot. Ges. Wien, XV. 771. 1. [Leptis] (1865); Hunter, Trans. Amer. Entom. Soc. Philad., XXV. 149. [Leptis] (1901).

subpilosus BЕск., Wien. Entom. Zeitg., XI. 23. [Leptis] (1892).

terminalis Lw., Berlin. Entom. Zeitschr., V. 317. 20. [Leptis] (1861); Adans, Kansas Univ. Sci. Bullet., II. 441. [Leptis] (1904); Aldr., Catal. North Amer. Dipt., 215. [Leptis] (1905).

tonsa Lw., Beschreib. europ. Dipt., I. 29. 27. [Leptis] (1869).

tringarius I., Systema Naturae, Ed. X., 590. 12. [Musca] (1758), Fauna Suec., Ed. Alt., 442. 1789. [Musca] (1761) et Systema Naturae, Ed. XII., II. 982. 18. [Musca] (1767); FApr., Systema Entomol., 761. 2. (1775); O. F. MUll., Zoolog. Dan. Prodr., 179. 2108. [Nemotelus] (1776); FABr., Species Insect., II. 419.2. (1781) et Mantissa Insect., II. 332. 2. (1787); Rossi, Fauna Etrusca,

Europa mer., Asia min.

Europa mer., Asia min.
(5)

Europa centr.

Chile.

Europacentr.

America sept.

Hispania.

Europa. 
II. 282. 1446. (1790); Gres., Systema Naturae, V. 2865. 18. [Musca] (1792); FAbr., Entomol. System., IV. 272. 2. (1794); Schrank, Fauna Boica, III. 98. 2390. (1803); Meig., Klassif., I. 300. 5. (I801) ; Latr., Hist. Nat. d. Crust. et Insect., XIV. 3332. 3. (1804) ; FAsr., Systema Antliat., 69. 2. [Leptis] (1805); Rossi, Fauna Etrusca, Ed. II., 435. 1446. (1807); LATr., Gen. Crust. et Ins., IV. 288. (1809); FAll., Diptera Suec., Anthracides, 10. 2. [Leptis] (1814); LAM., Hist. nat. anim. sans vert., III. 423. 3. (1816); MeIG., System. Beschreib., II. 91. 4. [Leptis] (1820); Macq., Recueil. Soc. Sci. Agricult. Lille, 1826. 400. 4. [Leptis] (1826) et Suit. à Buffon, I. 427. 5. tab. X. fig. 11. [Leptis] (1834); ZE'T., Insecta Lappon., Dipt., 526. 2. [Leptis] (1838); CurT., Brit. Entomol., VIII. 705. (1838); Gimm., Bullet. Soc. Imp. Nat. Moscou, XV. 664. [Leptis] (1842); ZETr., Dipt. Seand., I. 217. 2. [Leptis] (1842); Gimm., Bullet. Sor. Imp. Nat. Moscou, XX. 1., 193. 2. [Leptis] (1847); WALK., I ist Dipt. Brit. Mus., I. 211. (1848) ; ZETT., Dipt. Scand., VIII. 2987. 2. [Leptis] (1849); L. Duf., Mém. Acad. cl. Scienc., Math. et Phys., XI. 171-360. tab. VI. fig. 70-72. [Leptis] (1850); HaLid., Stettin. Entom. Zeitg., XI. 136. [Leptis] (1851); WALK., Insecta Britannica, Dipt., I. 65. 2. [Leptis] (1851) ; ZETT., Dipt. Scand., XI. 4266. 2. [Leptis] (1852); Schin., Verh. zool.-bot. Ver. Wien, VI. 420. 986. [Leptis] (1856); Zетт., Dipt. Scand., XIII. 4973. 2. [Leptis] (1859); Schis., Fauna Austriaca, Dipt., I. 174. 12. [Leptis] (1862); Jaenn., Berlin. Entom. Zeitschr., XI. 97. 8. [Leptis] (1867); BeLIng, Archiv f. Naturgesch., XLI. 1., 50. [Leptis tingaria] (1875); Goв., Mém. Soc. Linn. Nord France, 1877. 12. [Leptis] (1877); Neuhaus, Diptera Marchica, 47. 2. [Leptis] (1886); L. Coucke, Annal. Soc. Entom. Belgique, XXXVII. 131. [Leptis] (1893) ; STrobl, Mittheil. Naturwiss. Ver. Steiermark, XXIX. 1892. 21. [Leptis] (1893) ; Marcial, Bullet. Soc. Entom. France, 1903. 233. fig. 1-3. [Leptis] (1903). ? rufus Scop., Entomol. Carniol., 363. 986. [Erax] (1763) ; Ouiv., Encyclop. Méthod., IV. 272. 11. [Asilus] (1789).

scolopaceus Dra., Mém. pour serv. l'hist. d. Ins;, VI. 162. 1.

p. p. tab. IX. fig. 10. [Nemotelus] (1776).

solivagus Harris, Expos. Engl. Ins., 101. tab. XXXI. fig. 4.

[Sylvicola] (1782).

vermileo Schrank, Enum. Insect. Austr., 441. 896. [Musca]

(1781); Schellenb., Gattungen d. Fliegen, 40 et 41. tab. XXXI. fig. 2. (1803).

var. Goebelii Strobl, Mittheil. Naturwiss. Ver. Steiermark, Europacentr. XXIX. 1892. 22. nota. [Leptis] (1893).

var. punctatus Lw., Programm Posen, 1840. 7. 4. [Leptis] Europacentr. (1840) et Isis, 1840. VII-VIII. 522. 4. [Leptis] (1840) et Beschreib. europ. Dipt., I. 30. 28. [Leptis] (1869).

var. simplex Meik., System. Beschreib., VII. 61. 26. [Leptis] Europacentr. (1838). 
var. vanellus FABr., Entomol. System., IV. 272. 3. (1794); Europa.

MeIg., Klassif., I. 303. a. (1804); FABR., Systema Antliat., 70. 3. [Leptis] (1805) ; Falu., Diptera Suec., Anthracides, 11. 3. [Leptis] (1814) ; Meig., System. Beschreib., VI. 320. [Leptis] (1830); Schumm., Über's. d. Arb. u. Veränd. d. schles. Ges. f. vaterl. Kultur, 1837. 108. [Leptis] (1837); MeIG., System. Beschreib., VII. 60. 25. [Leptis] (1838); Lw., Programm Posen, 1840. 7. 3. [Leptis] (1840) et Isis, 1840. VII -VIII. 522. 3. [Leptis] (1840); Zeтt., Dipt. Scand., I. 217. 3. [Leptis] (1842); Grmm., Bull. Soc. Imp. Nat. Moscou, XX. 1., 194. 3. [Leptis] (1847); ZetT., Dipt. Scand., VIII. 2987. 3. [Leptis] (1849); WALK., Insecta Britannica, Dipt., I. 65. 3. [Leptis] (1851); I. Coucke, Annal. Soc. Entom. Belgique, XXXVII. 131. [Leptis] (1893) ; Strobl, Mittheil. Naturwiss. Ver. Steiermark, XXIX. 1892. 21. [Leptis] (1893).

tringarius Panz. (nee L.) = vitripennis Meig.

tristis Schumm., Übers. d. Arb. u. Veränd. d. schles. Ges. f. vaterl. Europacentr.

Kultur, 1837. 109. [Leptis] (1837).

uniguttatus Os'r.-SAck., Annal. Mus. Civ. Genova, XVI. 422. [Leptis]

(1881); v. D. Wulp, Catal. Dipt. South Asia, 67. [Leptis] (1896). vancllus $\mathrm{FABR}_{\mathrm{ABR}}=$ tringarius L. var.

vermileo Schrank (nec Deg.) = tringarius $\mathrm{L}$.

vertebratus SAY, Journ. Acad. Nat. Sci. Philad., III. 38. 8. [Leptis] (1823), Amer. Entomol., I. 27. tab. XIII. fig. 3. [Leptis] (1824) et Compl. Writt., I. 27. tab. XIII. fig. 3. ; II. 56. 8. [Leptis] (1859); Wied., Aussereurop. zweifl. Ins., I. 224. 7. [Leptis] (1828); Adans, Kansas Univ. Sci. Bullet., II. 440. [Leptis] (1904) ; Aldr., Catal. North Amer. Dipt., 215. [Leptis] (1905).

vitripennis MeIa., System. Beschreib., II. 91. 5. [Leptis] (1820); Macq., Recueil Soc. Sci. Agricult. Lille, 1826. 401. 5. [Leptis] (1826) et Suit. à Buffon, I. 426. 4. [Leptis] (1834); I،w., Programm Posen, 1840. 7. 5. [Leptis] (1840) et Isis, 1840. VII-VIII. 522. 5. [Leptis] (1840) ; Gimm., Bullet. Soc. Imp. Nat. Moscou, XX. 1., 194. 4. [Leptis] (1847); Walk., List Dipt. Brit. Mus., I. 211. (1848) ; Scholtz, Zeitschr. f. Entom. Breslau, IV. No. 16., 39. [Leptis] (1850) ; Schin., Fauna Austriaca, Dipt., I. 173. 9. [Leptis] (1862) ; JaEnv., Berlin. Entom. Zeitschr., XI. 97. 4. [Leptis] (1867); Gous, Mém. Soc. Linn. Nord France, 1877. 10. [Leptis] (1877); Mıк in Beck.: Fauna von Hernstein, II. 2., 55. [Leptis] (1885); L. Coucke, Annal. Soc. Entom. Belgique, XXXVII. 131. [Leptis] (1893) ; Stroul, Mittheil. Naturwiss. Ver. Steiermark, XXIX. 1892. 21. [Leptis] (1893).

stigma Schumm., Übers. d. Arb. u. Veränd. d. schles. Ges. f. vaterl. Kultur, 1837. 108. [Leptis] (1837).

tringarius PANz., Fauna Germ., XIV. tab. 20. (1794).

vittatus Wied., Aussereurop. zweifl. Ins., I, 225. 8. [Leptis] (1828). Patriaignota. 


\section{Dasyomma}

MacQ., Dipt. exot., II. 1., 31. 1. (1840).

Trichopalpomyia Hur'en, Trans. Amer. Entom. Soe. Philad., XXVII. 148. (1901).

Trichopalpus Pнnц., Verh. zool.-bot. Ges. Wien, XV. 724. (1865).

cinerascens Punı., Verh. zool.-bot. Ges. Wien, XV. 725. 4. [Trichopalpus] (1865); Hurter, Trans. Amer. Entom. Soc. Philad., XXVII. 148. [Trichopalpomyia] (1901).

coerulea MacQ., Dipt. exot., II. 1., 31. 1. tab. IV. fig. 1. (1840); Brancil. in Gay: Hist. fis. y polit. de Chile, Zool., VII, 418. 1. Dipt. tab. IV. fig. 3. (1852); Phul., Verh. zool.-bot. Ges. Wien, XV. 771. (1865); Huntrer, Trans. Amer. Entom. Soc. Philad., XXVII. 149. (1901).

croceicornis BIG., Bullet. Soc. Zool. Hrance, XII. 112. (1887).

Chile.

fulva PhIL., Verh. zool.-bot. Ges. Wien, XV. 725. 3. [Trichopulpus] Chile.

(1865) ; BiG., Bullet. Soc. Zool. France, XII. 112. nota. (1887); Hunter, Trans. Amer. Entom. Soc. Plilad., XXVII. 148. [Trichopalpomyia] (1901).

obscura*) Phil., Verh. zool.-bot. Ges. Wien, XV. 725. 1. [Trichopalpus] (1865); Hunter, Trans. Aner. Entom. Soc. Philad., XXVII. 148. [Trichopalpomyia] (1901).

poecilogaster PHuL., Verh. zool.-bot. Ges. Wien, XV. 725. 2. tab. Chile. XXVI. fig. 32. [Trichopalpus] (1865); Hunter, Trans. Amer. Entom. Soc. Philad., XXVII, 148. [Trichopalpomyia] (1901).

\section{Glutops}

Burgess, Proc. Boston Soc. Nat. IIist., XIX. 321. (1878).

singularis Burgess, Proc. Boston Soc. Nat. Hist., XIX. 322. tab. America sept. IX. fig. 2, 2a-l. (1878); Aldr., Catal. North Amer. Dipt., 213. (1905).

\section{Dialysis}

Walk., Insecta Saunders., Dipt., I. 4. (1850).

Agnotemyia WrLl., Entomol. Amer., II. 106. (1886).

Agnotomyia WiLL., Entomol. Amer., II. 106. (1886).

Aldrichi Willist., Kansas Univ. Quart., III. 265. (1895); Aldr., Americasept.

Catal. North Amer. Dipt., "13. (1905).

dispar BıG., Annal. Soc. Entom. France, sér. 5. IX. 197. (1879); Americasept.

$*)=$ ? coerulea MACQ. 
Os'1.-SACK., Berlin. Entom. Zeits lir., XXVII. 295. (1883); Whalst., Kansas Univ. Quart., III. 265. (1895); Aldr., Catal. North Amer. I)ipt., 213. (1905).

rissimilis WALK. = elongata SAY.

elongata Say, Journ. Acad. Nat. Sci. Philad., III. 41. [Stygia] (1823) Americasept. et Compl. Writt., II. 58. [Stygia] (1859); Wred., Aussereurop. zweifl. Ins., I. 315. 83. [Anthrax] et 561. 41. tab. II. fig. 6. [Lomatia] (1828); Osr.-SAck., Catal. Dipt. North Amer., Ed. II., 90 et 237. 155. [Lomatia] (1878); Willist., Entomol. Amer., II. 106. [Agnotemyia] (1886); Ost.-Sack., Berlin. Entom. Zeitschr., XXXV. 303. [Agnotomyia] (1890); Willist., Kansas Univ. Quart., III. 265. (1895) ; Johxs., Entomol. News Philad., VIII. 118. fig. 1. (1897) ; Ar.Dr., Catal. North Amer. Dipt., 213. (1905).

dissimilis WALK., Insecta Saunders, Dipt., I. 4. (1850) et List

Dipt. Brit. Mus., V. suppl. 1., 111. 1. (1854); Os'r.-SAck.,

Berlin. Entom. Zeitschr., XXVII. 295. (1883) ; Willist., Kansas Univ. Quart., III. 265. (1895).

fasciventris Lw., Berlin. Entom. Zeitschr., XVIII. 380. 4. [Triptotricha] (1874); Towss., Proc. Entom. Soc. Washington, II. 118. [Triptotricha] (1891); Willist., Kansas Univ. Quart., III. 265. [Triptotricha] (1895) ; JoHns., Entomol. News Philad., VIII. 118. (1897); Aısr., Catal. North Amer. Dipt., 213. (1905).

rufithorax SAY, Journ. Acad. Nat. Sci. Philad., III. 36. 5. [Leptis] (1823) et Compl. Writt., II. 56. 5. [Leptis] (1859); WiEd., Aussereurop. zweifl. Ins., I. 323. 5. [Leptis] (1828) ; Lw., Berlin. Entom. Zeitschr., XVIII. 382. [Triptotricha] (1874); Towss., Proc. Entom. Soc. Washington, II. 118. [Triptotricha] (1891); Willist., Kansas Univ. Quart., III. 265. (1895) ; JoHns., Entomol. News Philad., VIII. 117. fig. 2-3. (1897) ; A LDR., Catal. North Amer. Dipt., 214. (1905).

\section{Triptotricha}

Lw, Berlin. Entom. Zeitschr., XVI. 59. 15. not

discolor Lw., Berlin. Entom. Zeitschr., XVIII. 379. 3. (1874); Towrs., Proc. Entom. Soc. Washingt., II. 118. (1891); WiLlist., Kansas Univ. Quart., III. 265. (1895); A Ldr., Catal. North Amer. Dipt., 214. (1905).

disparilis Berar., Wien. Entom. Zeitg., VIII. 296. [Dialysis] (1889) Americasept. et XI. 162. [Dialysis] (1892); WiLlist., Kansas Univ. Quart., III. 265. (1895); ALdr., Catal. North Amer. Dipt., 214. (1905).

lauta Lw., Berlin. Entom. Zeitschr., XVI. 59. 15. (1872) et XVIII. America sept. 382. (1874) ; Towss., Proc. Entom. Soc. Washington, II. 118. (1891) ; Willıst., Kansas Univ. Quart., III. 265. (1895) ; Aldr., Catal. North Amer. Dipt., 214. (1905).

America sept.

America sept. 


\section{Leptipalpus}

Rond., Nuov. Annal. Sci. Nat. Bologna, ser. 3. II. 183. (1850).

apicalis Rond. in Truqui: Studi Entomol., I. 88. 47. [Leplis] (1848)
et Nuov. Annal. Sci. Nat. Bologna, ser. 3. II. 184. (1850); Hun-

Brasilia. TER, Trans. Amer. Entom. Soc. Plilad., XXVII. 149. [Leptis] (1901). brasiliensis Rovd., Nuov. Annal. Sci. Nat. Bologna, ser. 3. II. 183. 18. (1850); Hunter, Trans. Amer. Entom. Soc. Philad., XXVII. 149. [Leptis] (1901).

limbipennis Big., Bullet. Soc. Zool. France, XII. 106. (1887); ALdr., America sept. Catal. North Amer. Dipt., 214. [Leptis] (1905).

obscuripennis BIG., Bullet. Soc. Zool. France, XII. 107. (1887); Americasept. Aldr., Catal. North Amer. Dipt., 215. [Leptis] (1905).

saffranus Bir., Bullet. Soc. Zool. France, XII. 108. (1887).

stigmatias Big., Bullet. Soc. Zool. France, XII. 106. (1887); ALDR., Catal. North Amer. Dipt., 215. [Leptis] (1905).

vertebratus BIG., Bullet. Soc. Zool. France, XII. 107. (1887); Aldr., Haiti.

Catal. North Amer. Dipt., 215. [Leptis] (1905).

? waigiensis Bia., Bullet. Soc. Zool. France, XII. 108. [? Leptipalpus] (1887); v. D. Wur.P, Catal. Dipt. South Asia, 67. [?Leptipalpus] (1896).

\section{Omphalophora}

BEck., Entomol. Nachricht., XXVI. 106. (1900).

oculata Beck., Entomol. Nachricht., XXVI. 107. fig. 5-6. (1900) et Aeta Soc. Sei. Fenn., XXVI. No. 9., 13. 12. tab. I. fig. 1, 2. (1900).

\section{Chrysopilus}

Macq., Recueil Soc. Sci. Agricult. Lille, 1826. 403. (1826).

Chrysopila Macq., Suit. à Buffon, I. 429. 3. (1834).

Heliomyia Dot., Natuurkund. Tijdschr. Nederl. Indie, (ser. 3. IV.) XIV. 402. (1857).

Leptis WaLk., List Dipt. Brit. Mus., I. 213. (1848).

? Styrex Scop., Entomol. Carniol., 367. (1763).

Sylvicola Harris, Expos. Engl. Ins., 100. p. p. (1782).

aequalis WALK., List Dipt. Brit. Mus., I. 216. (1848).

Chile.

Cuba.

I. Waigou.

I. St. Sebast.

\section{Waigoll.}

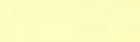

Sibiria.

alpicola Рок., Wien. Entom. Zeitg., V. 194. 3. [Chrysopila] (1886). americanus Scun., Novara Reise, Dipt., 197. 3. [Chrysopila] (1868); Henter, Trans. Amer. Entom. Soc. Philad., XXVII. 148. [Chrysopila] (1901).

anthracinus BIG., Bullet. Soc. Zool. France, XII. 105. [Chrysopila] (1887); Aduns, Kansas Univ. Sci. Bullet., II. 436. [Chrysopila] Australia. Europa centr. America mer.

(1904); Aldr., Catal. North Amer. Dipt., 215. [Chrysopila] (1905). 
antipodus BIG., Bullet. Soc. Zool. France, XII. 105. [Chrysopila] (1887).

apicalis v. D. WuLP, Tijdschr. v. Entom., XXV. 119. 5. [Chrysopila] (1882) ; Hunter, Trans. Amer. Entom. Soc. Philad., XXVII. 148. [Chrysopila] (1901); Adams, Kansas Univ. Sci. Bullet., II. 437. [Chrysopiila] (1904); AldR., Catal. North Amer. Dipt., 215. [Chrysopila] (1905).

argyrophorus Schus., Novara Reise, Dipt., 199. 7. [Chrysopila] (1868) ; Hunter, Trans. Amer. Entom. Soc. Philad., XXVII. 148. [Chrysopila] (1901).

ater Willist., Trans. Entom. Soc. London, 1896. 30t. 2. tab. X. fig. 78 bis. [Chrysopila] (1896); Hunter, Trans. Amer. Entom. Soc. Philad., XXVII. 148. [Chrysopila] (1901); Adaus, Kansas Univ. Sci. Bullet., II. 436. [Chrysopila] (1904); ALDr., Catal. North Amer. Dipt., 215. [Chrysopila] (1905).

aterrimus Wirlist., Biolog. Central. Amer., Dipt., I. 264. 6. [Chrysopila] (1901); AdaMs, Kansas Univ. Sci. Bullet., II. 436. [Chrysopila] (1904); ALDR., Catal. North Amer. Dipt., 115. [Chrysopila] (1905).

atratus $\mathrm{F}_{\mathrm{ABR}}=$ ? siculus $\mathrm{Lw}$.

atratus $\mathrm{MEIG}_{\text {. }}=$ auratus FABR.

auratus FABr., Systema Antliat., 73. 4. [Atherix] (1805); Meig., System. Beschreib., II. 99. 16. [Leptis] (1820); Macq., Recueil Soc. Sei. Agricult. Lille, 1826. 404. 1. tab. II. fig. 4. (1826) et Suit. à Buffon, I. 429. 1. [Chrysopila] (1834); Curt., Brit. Entomol., VIII. 713. [Leptis] (1838); Lw., Programm Posen, 1840. 9. 2. (1840) et Isis, 1840. VII-VIII. 526. 2. (1840); BLANCH., Hist. Nat. d. Ins., III. 603. [Chrysopila] (1840) ; Gimм., Bull. Soc. Imp. Nat. Moscou, XX. 1., 197. 1. (1847); Scholtz, Zeitschr. f. Entom. Breslau, IV. No. 16., 39. [Chrysopila] (1850); Lw., Zeitschr. f. d. ges. Naturwiss., X. 99. 25. [Chrysopila] (1857) et Beschreib. europ. Dipt., I. 59. 4. [Chrysopila] (1869); VerR., Entom. Monthly Mag., XIX. 224. (1883); Neuraus, Diptera Marchica, 48. 1. [Chrysopila] (1886); Stroвu, Mittheil. Naturwiss. Ver. Steiermark, XXIX. 1892. 24. [Chrysopila] (1893); BezzI, Bullet. Soc. Entom. Ital., XXVII. 44. 9. [Chrysopila] (1895).

atratus Meig., Klassif., I. 302. 11. [Rhagio] (1804); Latr., Hist. Nat. d. Crust. et d. Ins., XIV. 332. 4. [Rhagıo] (1804); FABR., Systema Antliat., 72. 1. [Atherix] (1805); Fall., Dipt. Suec., Anthracides, 13. 8. [Leptis] (1814); Zетт., Insecta Lappon., Dipt., 526. 4. [Leptis] (1838) et Dipt. Scand., I. 222. 1. [Chrysopila] (1842) ; ? ? MACQ., Dipt. exot., suppl. 2., 50. [Chrysopila] (1847); WALK., List Dipt. Brit. Mus., I. 214. [Leptis] (1848) ; Zетт., Dipt. Scand., VIII. 2988. 1. [Chrysopila] (1849); ? ? WaLK., Insecta Saunders., Dipt., I. 164. [Leptis] (1852); Zетт., Dipt. Scand., XII. 4591. 1. [Chrysopila] (1855) ; Bossd., Finlands tvaving. Ins., I. 139. 1. [Chryso-
Australia.

Guadeloupe.

America mer.

I. St. Vincent.

Mexico.

Europa, ?? Australia. 
pila] (1861); Schin., Fauna Austriaca, Dipt., I. 176. 5. [Chrysopila] (1862); JAEnN., Berlin. Entom. Zeitschr., XI. 98. 15. [Chrysopila] (1867); Lw., Beschreib. europ. Dipt., I. 57. 2. [Chrysopila] (1869); Beling, Archiv f. Naturgesch., XLI. 1., 52. [Chrysopila] (1875); Goв., Mém. Soc. Linn. Nord France, 1877. 16. [Chrysopila] (1877); L. Coucke, Annal. Soc. Entom. Belgique, XXXVII. 132. [Chrysopila] (1893).

cingulatus Dowov., Brit. Ins., 13. t. 465. (teste CurT.)

?holosericeus Scop., Entomol. Carniol., 367. 998. [Styrex] (1763); WALK., Insecta Britannica, Dipt., I. 68. 1. tab. II. fig. 7. [Chrysopila] (1851); Schin., Verh. zool.-bot. Ver. Wien, VI. 421. 998. [? gen.] (1856).

secretus Harr., Expos. Engl. Ins., 101. tab. XXXI. fig. 6. [Sylvicola] (1782).

tomentosus FABR., Entomol. System., IV. 275. 16. [Rhagio] (1794); Meig., Klassif., I. 306. l. [Rhagio] (1804); Fabr., Systema Antliat., 73. 5. [Atherix] (1805); Lw., Beschreib. europ. Dipt., I. 58. [Chrysopila] (1869).

aureus MeIG., Klassif., I. 302. 9. [Rhagio] (1804); WALK., Insecta Europa.

Britannica, Dipt., I. 68. 2. [Chrysopila] (1851); Schrn., Fauna Austriaca, Dipt., I. 176. 4. [Chrysopila] (1862); Lw., Zeitschr. f. d. ges. Naturwiss., XXIV. 389. 49. [Chrysopila] (1864); JAENn., Berlin. Entom. Zeitschr., XI. 98. 13. [Chrysopila] (1867); Gов., Mém. Soc. Linn. Nord France, 1877. 16. [Chrysopila] (1877); RöD., Berlin. Entom. Zeitschr., XXXI. 73. [Chrysopila] (1887); L. Coucke, Annal. Soc. Entom. Belgique, XXXVII. 132. [Chrysopila] (1893); SтroвL, Mittheil. Naturwiss. Ver. Steiermark, XXIX. 1892. 24. [Chrysopila] (1893); BEzzi, Bullet. Soc. Entom. Ital., XXVII. 44. 7. [Chrysopila] (1895) et XXX. 32. 51. [Chrysopila] (1898).

aurulans Meig. in litt., System. Beschreib., II. 101. (1820).

diadema FABR., Systema Entomol., 762. 4. [Rhagio] (1775) et Species Insect., II. 421. 10. [Rhagio] (1781); Schrank, Enum. Insect. Austr., 442. 898. [Musca] (1781); FABR., Mantissa Insert., II. 333. 13. [Rhagio] (1787) et Entomol. System., IV. 276. 20. [Rhagio] (1794); Schrank, Fauna Boica, III. 99. 2393. [Rhagio] (1803); MeIG., Klassif., I. 302. 10. [Rhagio] (1804); FABr., Systema Antliat., 73. 2. [Atherix] (1805); Meig., System. Beschreib., II. 101. 19. [Leptis] (1820); Sт. Farg. \& Serv., Encyclop. Méthod., X. 283. 2. [Leptis] (1825); MAcq., Recueil. Soc. Sci. Agricult. Lille, 1826. 405. 3. (1826) et Suit. à Buffon, I. 429. 2. tab. X. fig. 13. [Chrysopila] (1834) ; CurT., Brit. Entomol., VIII. 713. tab. [Leptis] (1838); Walk., List Dipt. Brit. Mus., I. 217. [Leptis] (1848) ; Zетт., Dipt. Scand., V1II. 2988. 5. [Chrysopila] (1849) et XI. 4266. 5. [Chrysopila] (1852). 
luridus MelG. in litt., System. Beschreib., II. 101. (1820).

vitripennis MeIG. in litt., System. Beschreib., II. 101. (1820).

var. meridionalis BEzzI, Bullet. Soc. Entom.Ital., XXX. 32. Italia.

51. [Chrysopila] (1898).

aureus O. F. Mél., Zoolog. Dan. Prodr., 179. 2109. [Nemotelus] I)ania.

(1776).

auricollis Wred. = nubecula FALL.

aurulans MeIG. = aureus MEIG.

basalis WALk., Trans. Entom. Soc. London, n. ser. V. 285. [Chrysopila] (1860) ; Adavis, Kansas Univ. Sci. Bullet., II. 436. [Chrysopila] (1904) ; Aldr., Catal. North Amer. Dipt., 215. [Chrysopila] (1905).

basilaris SAY, Journ. Acad. Nat. Sei. Philad., III. 36. 4. [Leptis] (1823) et Compl. Writt., II. 55. 4. [Leptis] (1859) ; Wred., Aussereurop. zweifl. Ins., I. 228. 16. [Leptis] (1828); WALK., List Dipt. Brit. Mus., I. 217. [Leptis] (1848); ? Wil.LIst., Biolog. Central. Amer., Dipt., I. 265. 7. [Chrysopila] (1901); Adums, Kansas Univ. Sci. Bullet., II. 436. [Chrysopila] (1904); Aınr., Catal. North Amer. Dipt., 215. [Chrysopila] (1905).

bellus Adans, Kansas Univ. Sri. Bullet., II. 436 et 438. [Chrysopila] (1904).

bicolor FABR. = ? nubecula FALL.

bicolor MeIG. (nec FABR.) =? pullus Lw.

binotatus Lw., Beschreib. europ. Dipt., I. 47. 37.[Chrysopila] (1869). Graecia. brunneifrons Krвт., Természetrajzi Füzet., XXV. 147. 3. fig. 3. Peru.

[Chrysopila] (1902).

calopterus Schin., Novara Reise, Dipt., 197. 2. [Chrysopila] (1868);

Hunrer, Trans. Amer. Entom. Soc. Philad., XXVII. 148. [Chrysopila] (1901).

chlorophthalmus Lw. = splendidus MeIG.

cingulatus Doxov. = auratus FABR.

clarus WALk., Insecta Saunders., Dipt., I. 164. [Leptis] (1852).

consanguineus Scmis., Novara Reise, Dipt., 198. 4. [Chrysopila

consanquinea] (1868) ; Hunter, Trans. Amer. Entom. Soc. Philad.,

XXVII. 148. [Chrysopila] (1901).

correctus Osт.-SACK., Berlin. Entom. Zeitschr., XXVI. 101. fig. 2. I.Philippinae.

[Chrysopila] (1882); v. D. Wulp, Catal. Dipt. South Asia, 67.

[Chrysopila] (1896).

cristatus $\mathrm{FABR}_{\mathrm{ABR}}=$ ? siculus $\mathrm{Lw}$.

diadema $\mathrm{FABR}_{\mathrm{AB}}=$ aureus MEIG.

dispar v. D. WuLP = quadratus $\mathrm{SAY}_{\mathrm{AY}}$.

dives Lw., Beschreib. enrop. Dipt., II. 62. 37. [Chrysopila] (1871) ;

Coquill., Proc. Unit. Stat. Nat. Mus., XXI. 307. [Chrysopila] (1898).

dubius Macq., Dipt. exot., suppl. 4., 104. 4. tab. IX. fig. 18. [Chry- Australia. sopila] (1849).

elegans Scuns., Novara Reise, Dipt., 198. 6. [Chrysopila] (1868); Columbia. 
Hunter, Trans. Amer. Entom. Soc. Philad., XXVII. 148. [Chrysopila] (1901).

erythrophthalmus Lw., Programm Posen, 1840. 9. 3. (1840) et Isis, 1840. VII-VIII. 526. 3. (1840) ; Scholtz, Zeitschr. f. Entom. Breslau, IV. No. 16., 39. [Chrysopila] (1850); Zeтr., Dipt. Scand., XII. 4591. 1. Obs. [Chrysopila] (1855); Schin., Fauna Austriaca, Dipt., I. 177. [Chrysopila] (1862) ; Lw., Beschreib. europ. Dipt., I. 47. 38. [Chrysopila] (1869); StroвL, Mittheil. Naturwiss. Ver. Steiermark, XXIX. 1892. 24. [Chrysopila] (1893); BEzzi, Bullet. Soc. Entom. Ital., XXVII. 45. 11. [Chrysopila] (1895).

fasciatus SAY, Journ. Acad. Sci. Philad., IIX. 37. 7. [Leptis] (1823), Amer. Entomol., I. 28. tab. XIII. fig. 4. [Leptis] (1824), Compl. Writt., I. 28. tab. XIII. fig. 4. [Leptis] et IJ. 56. 7. [Leptis] (1859) ; W1ED., Aussereurop. zweifl. Ins., I. 225. 9. [Leptis] (1828); Adams, Kansas Univ. Sci. Bullet., II. 436. [Chrysopila] (1904); Aldr., Catal. North Amer. Dipt., 215. [Chrysopila] (1905).

par WALK., List Dipt. Brit. Mus., I. 215. [Leptis] (1848).

ferrugineus DoL. = ferruginosus WiEd.

ferruginosus WiEd., Zoolog. Magaz., I. 3., 4. 3. [Leptis] (1819), Dipt exot., 110. [Leptis] (1820) et Aussereurop. zweifl. Ins., I. 224. 6. [Leptis] (1828); WaLk., Proc. Linn. Soc. London., I. 118. 70. [Leptis] (1856), III. 89. 46. [Leptis] (1859), IV. 110. 57. [Leptis] (1860), V. 148. 19, 237. 33, 282. 44, 300. 2. [Leptis] (1861) et VII. 208. 32, 233. 17. [Leptis] (1864) ; Schrs., Novara Reise, Dipt., 196. [Chrysopila] (1868); Ost.-SAck., Annal. Mus. Civ. Genova, XVI. 419. [Chrysopila] (1882) et Berlin. Entom. Zeitschr., XXVI. 102. [Chrysopila] (1882); v. D. Wulp, Catal. Dipt. South Asia, 67. [Chrysopila] (1896).

ferrugineus DoL., Natuurkund. Tijdschr. Nederl. Indie, ser. 3.

IV. (XIV). 402. tab. VII. fig. 5. [Heliomyia] (1857).

flaveolus MeIG., System. Beschreib:, II. 100. 17. [Leptis] (1820) ; MAcQ., Recueil Soc. Sci. Agricult. Lille, 1826. 404. 2. (1826); Curt., Brit. Entomol., VIII. 713. [Leptis] (1838); Gıмм., Bull. Soc. Imp. Nat. Moscou, XX. 1., 198. 5. (1847); Schis., Fauna Austriaca, Dipt., I. 177. 6. [Chrysopila] (1862); I. Covcke, Annal. Soc. Entom. Belgique, XXXVII. 132. [Chrysopila] (1893); BezzI, Bullet. Soc. Entom. Ital., XXVII. 44. 6. [Chrysopila] (1895).

? Genius Panz., Fauna Germ., LIV. tab. 4. [Anthrax] (1798); Meig., Klassif., I. 308. r. [Rhagio] (1804).

flavibarbis Adans, Kansas Univ. Sci. Bullet., II. 437 et 438 . [Chrysopila] (1904).

flavidus BIG., Bull. Soc. Zool. France, XII. 104. [Chrysopila] (1887); Adans, Kansas Univ. Sci. Bullet., II. 437. [Chrysopila] (1904); Aldr., Catal. North Amer. Dipt., 215. [Chrysopila] (1905).

foedus Lw., Berlin. Entom. Zeitschr., V.317. 18. [Chrysopila] (1861); Americasept. Adans, Kansas Univ. Sci. Bullet., II. 437. [Chrysopila] (1904); Atdr., Catal. North Amer. Dipt., 215. [Chrysopila] (1905). 
folda Coquilt., Canad. Entomol., XV. 112. [Chrysopila] (1883). lapsus.

folda CoquilL. = foedus Lw.

fulvidus Big., Annal. Soc. Entom. France, sér. 7. I. 370. 9. [Chry- Assinia. sopila] (1891).

fumipennis $\mathrm{S}_{\Lambda \mathrm{Y}}=$ quadratus $\mathrm{S}_{\Lambda \mathrm{Y}}$.

fuscipes Brg., Bullet. Soc. Zool. France, XII. 103. [Chrysopila] (1887). Genius Panz. $=$ ? flaveolus Meig.

Griffithi Johns., Entom. News Philad., VIII. 119. [Chrysopila] (1897); Adaus, Kansas Univ. Sci. B.lllet., II. 437. [Chrysopila] (1904);

Aldr., Catal. North Amer. Dipt., 215. [Chrysopila] (1905).

guttipennis WALK., Proc. Linn. Soc. London, V. 282. 46. [Chrysopila] (1861); v. D. Wulp, Catal. Dipt. South Asia, 67. [Chrysopila] (1896). helveolus Ginn. = helvolus MeIG.

helvolus MEIG., System. Beschreib., II. 100. 18. [Leptis] (1820); Curt., Bıit. Entomol., VIII. 713. [Leptis] (1838); Schin., Fauna Austriaca, Dipt., I. 177. 6. [Chrysopila] (1862); JAenn., Berlin. Entom. Zeitschr., XI. 98. 16. [Chrysopila] (1867); Bezzi, Bullet. Soc. Entom. Ital., XXVII. 44. 5. [Chrysopila] (1895).

helveolus Givm., Bullet. Soc. Imp. Nat. Moscou, XX. 1., 197. 2. (1847).

? solitaneous Harris, Expos. Engl. Ins., 102. tab. XXXI. fig. 10. [Sylvicola] (1782).

holosericens Scop. $=$ ? auratus FABR.

humilis Lw., Berlin. Entom. Zeitschr., XVIII. 379. 2. [Chrysopila] (1874) ; Ost.-S^ck., Bullet. Unit. Stat. Geolog. Surv., III. 223. [Chrysopila] (1877); Adams, Kansas Univ. Sci. Bullet., II. 436. [Chrysopila] (1904); Aldr., Catal. North Amor. Dipt., 216. [Chrysopila] (1905).

insularis Schin., Novara Reise, Dipt., 199. 8. [Chrysopila] (1868); v. D. Wulp, Catal. Dipt. South Asia, 67. [Chrysopila] (1896).

intermedius BezzI, Bullet. Soc. Entom. Ital., XXVII. 42. [Chrysopila] (1895).

invalidus Willist., Biolog. Central. Amer., Dipt., I. 265. 8. [Chrysopila] (1901); Adams, Kansas Univ. Sci. Bullet., II. 436. [Chrysopila] (1904); Aldr., Catal. North Amer. Dipt., 216. [Chrysopila] (1905).

irroratus Schin., Novara Reise, Dipt., 198. 5. [Chrysopila] (1868); Hunrer, Trans. Amer. Entom. Soc. Philad., XXVII. 148. [Chrysopila] (1901).

jamaicensis Jorns., Proc. Acad. Nat. Sci. Philad., 1894. 273. [Chrysopila] (1894); Adaus, Kansas Univ. Sei. Bullet., 436. [Chrysopila] (1904) ; Alsr., Catal. North Amer. Dipt., 216. [Chrysopila] (1905).

laetus Zeтr., Dipt. Scand., I. 224. 4. [Chrysopila] (1842); Lw., Boschreib. europ. Dipt., I. 45. [Chrysopila] (1869); BeLing, Archiv f. Naturgesch., XI.VIII. 1., 190. 4. [Chrysopila] (1882); BEzz, Bullet. Soc. Entom. Ital., XXVII. 44. [Chrysopila] (1895).

Americasept.

Ins. Nicobar.

Italia.

Mexico.

America mer.

Jamaica.

Europa. 
latifrons BezzI, Bullet. Soc. Entom. Ital., XXX. 32. 53. [Chrysopila] Italia. (1898).

latifrons Willist., Biolog. Central. Amer., Dipt., I. 266. 11. [Chrysopila] (1901); Adums, Kansas Univ. Sci. Bullet., II. 437. [Chrysopila] (1904) ; ALdr., Catal. North Amer. Dipt., 216. [Chrysopila] (1905).

leptiformis Kent., Természetrajzi Füzet., XXV. 148. 4. fig. 4. Peru. [Chrysopila] (1902).

lucifer Adans, Kansas Univ. Sci. Bullet., II. 437. [Chrysopila] (1904). lucifer WALK., Insecta Saunders., Dipt., I. 164. [Leptis] (1852).

ludens Lw., Wien. Entom. Monatschr., V. 34. 3. [Chrysopila] (1861); Willist., Trans. Entom. Soc. London, 1896. 303. 1. [Chrysopila] (1896) ; Hunter, Trans. Amer. Entom. Soc. Philad., XXVII. 148. [Chrysopila] (1901); AdAms, Kansas Univ. Sci. Bullet., II. 437. [Chrysopila] (1904); ALdr., Catal. North Amer. Dipt., 216. [Chrysopila] (1905).

lupinus Ost.-Sack., Annal. Mus. Civ. Genova, XVI. 420. [Chrysopila] Sumatra. (1881); v. D. Wulp, Catal. Dipt. South Asia, 67. [Chrysopila] (1896).

luridus MEIG. $=$ aureus MEIG.

luteolus FaLL., Dipt. Suec., Anthracides, 14. 10. [Leptis] (1814);

America sept. Columbia.

I.St. Vincent, Cuba. Meig., System. Beschreib., II. 110. 10. [Atherix] (1820); Zetт., Insecta Lappon., Dipt., 527. 6. [Leptis] (1838); Lw., Stettin. Entom. Zeitg., II. 26. 12. [Leptis] (1841); Zeтт., Dipt. Scand., I. 223. 3. [Chrysopila] (1842), VIII. 2988. 3. [Chrysopila] (1849) et XI. 4266. 3. [Chrysopila] (1852); Bonsd., Finlands tvaving. Ins., I. 139. 3. [Chrysopila] (1861); L. Coucke, Annal. Soc. Entom. Belgique, XXXVII. 132. [Chrysopila] (1893); Bezzi, Bullet. Soc. Entom. Ital., XXVII. 44. [Chrysopila] (1895).

maculipennis Walk., Proc. Linn. Soc. London, I. 118. 72. [Chrysopila] (1857) ; v. D. Wulp, Catal. Dipt. Sonth Asia, 67. [Chrysopila] (1896).

maerens Lw., Berlin. Entom. Zeitschr., XVII. 36. 14. [Chrysopila] (1873) et Beschreib. europ. Dipt., III. 100. 55. [Chrysopila] (1873). meridionalis BezzI $=$ aureus Merg. var.

mexicanus BeLL., Saggio di Ditterol. Messic., II. 96. 1. [Chrysopila] (1861); Adaus, Kansas Univ. Sci. Bullet., II. 437. [Chrysopila] (1904); Aldr., Catal. North Amer. Dipt., 216. [Chrysopila] (1905). modestus Lw., Berlin. Entom. Zeitschr., XVI. 58. 14. [Chrysopila] (1872); JoHns., Entom. News Philad., VIII. 120. [Chrysopila] (1897); AdAus, Kansas Univ. Sci. Bullet., II. 436. [Chrysopila] (1904); Aldr., Catal. North Amer. Dipt., 216. [Chrysopila] (1905). nanus Wilusst., Biolog. Central. Amer., Dipt., I:265. 10. [Chrysopila] (1901); Adsms, Kansas Univ. Sci. Bullet., II. 437. [Chrysopila] (1904); Aldr., Catal. North Amer. Dipt., 216. [Chrysopila] (1905). niger BeiLl., Saggio di Ditterol. Messic., Append., 27. 2. [Chrysopila] (1862); Adsms, Kansas Univ. Sci. Bullet., II. 436. [Chrysopila] (1904); ALdr., Catal. North Amer. Dipt., 216. [Chrysopila] (1905).

Borneo.

Europa centr.

Mexico.

America sept.

Moxico.

Mexico. 
nigricauda BeLısa, Verh. zool.-bot. Ges. Wien, XXIII. 547. 1. [ChryEuropacentr. sopila] (1873).

nigritus FABR. = ? splendidus MeIG.

nubecula FaLL., Dipt. Suec., Anthracides, 13. 9. [Leptis] (1814); Europa. MeIG., System. Beschreib., VI. 321. 23. [Leptis] (1830); Sculumi., Übers. d. Arb. u. Veränd. d. sehles. Ges. f. vaterl. Kultur, 1837. 108. [Leptis] (1837) ; ZETT., Insecta Lappon., Dipt., 527. 5. [Leptis] (1838); Lw., Stettin. Entom. Zeitg., II. 26. 12. (1841); Zетт., Dipt. Seand., I. 223. 2. [Chrysopila] (1842); Schoutz, Zeitsehr. f. Entom. Breslau, IV. No. 16., 39. [Chrysopila] (1850); ZeтT., Dipt. Scand., XI. 4266. 2. [Chrysopila] (1852); Bossd., Finlands tvaving. Ins., I. 139.2.[Chrysopila] (1861); Schis., Fauna A ustriaca, Dipt., I. 176. 2. [Chrysopila] (1862) ; JaExw., Berlin. Entom. Zeitschr., XI. 98. 11. [Chrysopila] (1867); Lw., Beschreib. europ. Dipt., I. 45. 46. [Chrysopila] (1869); Gor., Mém. Soc. Linn. Nord France, 1877. 15. [Chrysopila] (1877); JBelnng, Arehiv f. Naturgesch., XLviII. 1., 193. 6. [Chrysopila] (1882); Веск., Berlin. Entom. Zeitschr., XXXI. 141. 113. [Chrysopila] (1887); Sтroвц, Mittheil. Naturwiss. Ver. Steiermark, XXIX. 1892. 24. [Chrysopila] (1893) ; BezzI, Bullet. Soc. Entom. Ital., XXVII. 44. [Chrysopila] (1895) et XXXII. 78. 331. [Chrysopila] (1900).

auricollis WIED. apud MeIg., System. Beschreib., II. 103. 22. [Leptis] (1820) et VI. 321. [Leptis] (1830); Lw., Stettin. Entom. Zeitg., II. 26. 12. [Leptis] (1841); Grmm., Bullet. Soc. Imp. Nat. Moscou, XX. 1., 198. 4. (1847).

? bicolor FABr., Entomol. System., IV. 274. 11. [Rhugio] (1794); Meig., Klassif., I. 305. h. [Rhagio] (1804); FABr., Systema Antliat., 71. 8. [Leptis] (1805); MACQ., Suit. à Buffon, I. 430. 4. [Chrysopila] (1834); Schumm., Übers. d. Arb. u. Veränd. d. schles. Ges. f. vaterl. Kultur, 1837. 108. [Leptis] (1837); Lw., Programm Posen, 1840. 9. 1. (1840), Isis, 1840. VII-VIII. 525. 1. (1840) et Stettin. Entom. Zeitg., II. 26. 12. [Leptis] (1841); Gimn., Bullet. Soc. Imp. Nat. Moscou, XX. 1., 198. 3. (1847); WaLK., List Dipt. Brit. Mus., I. 216. [Leptis] (1848) ; Scholтz, Zeitsehr. f. Entom. Breslau, IV. No. 16., 39. [Chrysopila] (1850); Schin., Fauna Austriaca, Dipt., I. 176. 2. [Chrysopila] (1862); JAENN., Berlin. Entom. Zeitschr., XI. 98. 12. [Chrysopila] (1867) ; Lw., Beschreib. europ. Dipt., I. 45. [Chrysopila] (1869); Goв., Mém. Soc. Linn. Nord France, 1877. 15. [Chrysopila] (1877); L. Coucke; Annal. Soc. Entom. Belgique, XXXVII. 132. [Chrysopila] (1893).

?oculatus FABR., Systema Antliat., 74. 7. [Alherix] (1805); Meia., System. Beschreib., VI. 322. 24. [Leplis] (1830); Schumm., Übers. d. Arb. u. Veränd. d. schles. Ges. f. vaterl. Kultur, 1837. 108. [Leptis] (1837); Lw., Stettin. Entom. Zeitg., II. 26. 12. [Leptis] (1841) et Beschreib. europ. Dipt., I. 45. [Chrysopila] (1869). 
? sylvestris O. F. MüL., Zoolog. Dan. Prodr., 179. 2110. [Nemotelus] (1776).

obscuribarba Lw., Beschreib. europ. Dipt., I. 53. 41. [Chrysopila] (1869).

obscuripennis Lw., Beschreib. europ. Dipt., III. 99. 54. [Chrysopila] (1873).

oculatus FABR. = nubecula FALL.

ornatus SAY, Journ. Acad. Nat. Sci. Philad., III. 34. [Leptis] (1823), Amer. Entomol., I. 26. tab. XIII. fig. 1. [Leptis] (1824) et Compl. Writt., I. 26. tab. XIII. fig. 1. [Leptis], II. 54. [Leptis] (1859); WIED., Aussereurop. zweifl. Ins., I. 221. 1. [Leptis] (1828) ; ? WALK., List Dipt. Brit. Mus., I. 213. [Leptis] (1848); Hixe, Ohio Naturalist, II. 170. [Chrysopila] (1902); Aduмs, Kansas Univ. Sci. Bullet., II. 436. [Chrysopila] (1904); ALdR., Catal. North Amer. Dipt., 216. [Chrysopila] (1905).

Servillei Guk̇r., Iconogr. d. Régne Anim., VII. Ins., 541. tab. XCVI. fig. 3. [Leptis] (1835); Ost.-SAck., Bullet. Buffalo Soc. Nat. Sci., II. 172. [Chrysopila] (1874) et Catal. Dipt. North Amer., Ed. II., 63 et 229. 92. [Chrysopila] (1878).

pallipes Lw., Beschreib. europ. Dipt., I. 54. 42. [Chrysopila] (1869). palparis Lw., Beschreib. europ. Dipt., I. 50. 40. [Chrysopila] (1869). par $\mathrm{W}_{A \mathrm{LK}}=$ fasciatus $\mathrm{S}_{A \mathrm{Y}}$.

peruanus Kert., Természetrajzi Fiizet., XXV. 149. 5. fig. 5. [Chrysopila] (1902).

plebeius Willist., Biolog. Central. Amer., Dipt., I. 264. 5. [Chrysopila] (1901); Adays, Kansas Univ. Sci. Bullet., II. 437. [Chrysopila] (1904) ; ALdr., Catal. North Amer. Dipt., 216. [Chrysopila] (1905).

praetiosus Lw., Beschreib. europ. Dipt., I. 55. 43. [Chrysopila] I. Naxos. (1869).

propinquus Kегт., Természetrajzi Fïzet., XXV. 146. 2. fig. 2. Peru. [Chrysopila] (1902).

propinquus WALK., List Dipt. Brit. Mus., I. 215. [Leptis] (1848); AdAMs, Kansas Univ. Sci. Bullet., II. 437. [Chrysopila] (1904); Aldr., Catal. North Amer. Dipt., 216. [Chrysopila] (1905).

proximus Walk., List Dipt. Brit. Mus., I. 214. [Leptis] (1848); ADAxs, Kansas Univ. Sci. Bullet., II. 436. [Chrysopila] (1904); Aldr., Catal. North Amer. Dipt., 216. [Chrysopila] (1905).

puella Willist., Biolog. Central. Amer., Dipt., I. 265. 9. [Chrysopila] (1901); Adays, Kansas Univ. Sci. Bullet., II. 436. [Chrysopila] (1904); Aldr., Catal. North Amer. Dipt., 216. [Chrysopila] (1905). pullatus Coquill., Proc. Unit. Stat. Nat. Mus., XXI. 307. [Chrysopila] (1898).

pullus I.w., Beschreib. europ. Dipt., I. 43. 36. [Chrysopila] (1869); Bezzi, Bullet. Soc. Entom. Ital., XXX. 44. 4. [Chrysopila] (1895).

Asia mill.

Europa mer.

Peru.

Mexico.

America sept.

America sept.

?bicolor Meig., System. Beschreib., II. 102. 21. [Leptis] (1820) et VI. 321. [Leptis] (1830). 
quadratus SAY, Journ. Acad. Nat. Sci. Philad., III. 35. 3. [Leptis] (1823) et Compl. Writt., II. 55. 3. [Leptis] (1859); Wied., Aussereurop. zweifl. Ins., I. 226. 11. [Leptis] (1828) ; WALK., List Dipt. Brit. Mus., I. 216. [Leptis] (1848) ; Lw., Zeitschr. f. d. ges. Naturwiss., N. F. II. (XXXVI). 116. 7. [Chrysopila] (1870); v. D. WulP, Tijdschr. v. Entom., XXV. 118. 4. [Chrysopila] (1882); AdAus, Kansas Univ. Sci. Bullet., II. 437. [Chrysopila] (1904); Aldr., Catal. North Amer. Dipt., 216. [Chrysopila] (1905).

dispar v. D. Wulp, Tijdschr. v. Entom., ser. 2. II. (X). 1867. 143. 15. tab. IV. fig. 6-11. [Chrysopila] (1868).

fumipennis SAy, Journ. Acad. Nat. Sci. Pliilad., III. 37. 6. [Leptis] (1823) et Compl. Writt., II. 56. 6. [Leptis] (1859); Wied., Aussereurop. zweifl. Ins., I. 227. 12. [Leptis] (1828);

Walk., List Dipt. Brit. Mus., I. 217. [Leptis] (1848).

reflexus WaLK., List Dipt. Brit. Mus., I. 216. [Leptis] (18t8). reflexus $\mathrm{WALK}_{\mathrm{AL}}=$ quadratus $\mathrm{SAY}$.

rotundipennis Lw., Berlin. Entom. Zeitschr., V. 317. 19. [Chrysopila] (1861) ; Jonss., Entomol. News Philad., VIII. 120. [Chrysopila] (1897); Adams, Kansas Univ. Sci. Bullet., II. 437. [Chrysopila] (1904) ; Alnk., Catal. North Amer. Dipt., 216. [Chrysopila] (1905).

rufipes MACQ., Dipt. exot., suppl. 4., 103. 3. tah. IX. fig. 17. [Chrysopila] (1849).

secretus HARRIS $=$ auratus FABR.

Servillei Gǘr. = ornatus SAY.

siculus Lw., Beschreib. europ. Dipt., I. 49. 39. [Chrysopila] (1869); Bezzi, Bullet. Soc. Entom. Ital., XXVII. 45. 12. [Chrysopila] (1895).

? atratus FABr., Species Insect., II. 421. 11. [Rhagio] (1781), Mantissa Insect., II. 333. 15. [Rhagio] (178i), Entomol. System., IV. 276. 21. [Rhagio] (1794) et Systema Antliat., 72. 1. [Atherix] (1805).

? cristatus Fabr., Systema Entomol., 782. 45. [Musca] (1775), Species Insect., II. 447. 58. [Musca] (1781) et Mantissa Insect., II. 349. 72. [Musca] (1787); GMeL., Systema Naturae, V. 2851. 213. [Musca] (1792); Ouıv., Encyclop. Méthod., VIII. 28. 95. [Musca] (1811); Lw., Beschreib. europ. Dipt., I. 57. 1. [Chrysopila] (1869).

simillimus Walk., List Dipt. Brit. Mus., I. 215. [Leptis] (1848).

simplex MeIJ., Bijdragen tot de Dierkunde, XVIII. 97. tab. VIII fig. 15. [Chrysopila]. (1904).

smaragdinus Kent., Természetrajzi Füzet., XXV. 145. 1. fig. 1. Peru. [Chrysopila] (1902).

solitaneous HarRIS $=$ ? helvolus MEIG.

splendidus Meia., System. Beschreib., II. 102. 20. [Leptis] (1820); MACQ., Suit. à Buffon, I. 430. 3. [Chrysopila] (1834); Zetr., Dipt. Scand., VIII. 2989. obs. [Chrysopila] (1849) ; Lw., Beschreib. europ.
America sept.

America sept.

Tasmania.

Europa mer.

America sept. Java.

Europacentr. et mer. 
Dipt., I. 56. Anmerk. [Chrysopila] (1869); Sтroвц, Mittheil. Naturw iss. Ver. Steiormark, XXIX. 1892. 24. [Chrysopila] (1893); BwzzI, Bullet. Soc. Entom. Ital., XXVII. 45. 10. [Chrysopila] (1895). chlorophthalmus Lw., Programm Posen, 1840. 10. 4. (1840) et Isis, 18t0. VII-VIII. 527. 4. (1840); SchoLTz, Zeitsclir. f. Entom. Breslau, IV. No. 16., 39. [Chrysopila] (1850); Scurn., Fauna Austriaca, Dipt., I. 177. [Chrysopila] (1862).

? nigritus Fabr., Entomol. System., 1V.276. 19. [Rhagio] (1794); Mkig., Klassif., I. 307. o. [Rhagio] (1804); FABR., Systema Antliat., 73. 3. [Atherix] (1805); Scuns., Fauna Austriaca, Dipt., I. 176. 5. [Chrysopila] (1862); JAExv., Berlin. Entom. Zeitschr., XI. 98. 14. [Chrysopila] (1867); Lw., Beschreib. europ. Dipt., I. 59. [Chrysopila] (1869); Gos., Mém. Soc. Linn. Nord France, 1877. 16. [Chrysopila] (1877); Beling, A rchiv f. Naturgesch., XLVIII. 1., 191. 5. [Chrysopila] (1882); Neunaus, Diptera Marchiea, 48.2. [Chrysopila] (1886); Beck., Berlin. Entom. Zeitschr., XXXI. 141. 114. [Chrysopila] (1887); L. Coucke, Annal. Soc. Entom. Belgique, XXXVII. 132. [Chrysopila] (1893).

stylatus WАLк., Proc. Linn. Soc. London, VII. 208. 33. [Chrysopila] Ins. Mysol. (1864); v. D. Wulr, Catal. Dipt. Soutli Asia, 67. [Chrysopila] (1896).

sylvestris O. F. MéLs. = ? nubecula FALL.

terminalis Mavq., Dipt. exot., suppl. 1., 106. 2. [Chrysopila] (1846) ; Scinn., Novara Reise, Dipt., 197. 1. [Chrysopila] (1868); Hun'ter, Trans. Amer. Entom. Soe. Philad., XXVII. 148. [Chrysopila] (1901).

testaceipes Big., Bullet. Soe. Zool. France, XII. 105. [Chrysopila] (1887); Adams, Kansas Univ. Sci. Bullet., II. 436. [Chrysopila] (1904); Aldr., Catal. North Amer. Dipt., 216. [Chrysopila] (1905).

testaceus Lw., Öfvers. Kongl. Vet. Akad. Förlıandl., XIV. 1857. 367. 1. [Chrysopila] (1858) et Dipt.-Fauna Südafrika's, I. 172. 1. [Chrysopila] (1860).

thoracicus Fabr., Systema Antliat., 70. 4. [Leptis] (1805); Wied., Aussereurop. zweifl. Ins., I. 222. 2. [Leptis] (1828); Macq., Dipt. exot., II. 1., 32. 1. tab. III bis, fig. 3. [Chrysopila] (1840); WaLk., List Dipt. Brit. Mus., I. 214. [Leptis] (1848); Adams, Kansas Univ. Sci. Bullet., II. 437. [Chrysopila] (1904); Aldr., Catal. North Amer. Dipt., 216. [Chrysopila] (1905).

tomentosus BIG., Bullet. Soc. Zool. France, XII. 104. [Chrysopila] (1887); Adams, Kansas Univ. Sci. Bullet., II. 436. [Chrysopila] Venezuela, Columbia.

America sept.

Caffruria.

Americasept. (1904); Aldr., Catal. Nortlı Amer. Dipt., 216. [Chrysopila] (1905). tomentosus FABR. = auratus FABR.

trifasciatus Wask., Trans. Entom. Soc. London, n. ser. V. 284. Mexico. [Chrysopila] (1860); AdaMs, Kansas Univ. Sci. Bullet., II. 436. [Chrysopila] (1904); ALDr., Catal. North Amer. Dipt., 216. [Chrysopila] (1905). 
vacillans WaLk., Proc. Linn. Soc. London, III. 89. 47. [Chrysopila] (1859) ; Ost.-SACK., Annal. Mus. Civ. Genova, XVI. 420. [Chrysopila] (1881); v. D. Wutp, Catal. Dipt. South Asia, 67. [Chrysopila] (1896).

valdivianus PHI., Verh. zool.-bot. Ges. Wien, XV. 774. tab. XXVIII. fig. 50. [Chrysopila] (1865); Hunter, Trans. Amer. Entom. Soc. Philad., XXVII. 148. [Chrysopila] (1901).

varius Kert., Természetrajzi Füzet., XXV. 150. 6. fig. 6. [Chrysopila] (1902).

velutinus Lw., Berlin. Entom. Zeitschr., V. 316. 17. [Chrysopila] (1861); Adaus, Kansas Univ. Sci. Bullet., II. 436. [Chrysopila] (1904); Aldr., Catal. North Amer. Dipt., 216. [Chrysopila] (1905). vitripennis MEIG. = aureus MeIG.

\section{Symphoromyia}

FrFLd., Verh. zool.-bot. Ges. Wien, XVII. 497. (1867).

Atherix Roxd., Dipterol. Ital. Prodr., I. 154. 5. (1856).

atripes $\mathrm{BIG}_{\mathrm{I}}=$ pachyceras $\mathrm{W}_{\text {ILLIST }}$.

cinerea JoHss., Entomol. News Philad., XIV. 25. [Symphroromyia] (1903) ; Aldr., Catal. North Amer. Dipt., 217. (1905).

comata $\mathrm{BlG}_{\mathrm{G}}=$ pachyceras Willist.

crassicornis Panz., Fauna Germ., CV. tab. 10. [Atherix] (1806) ; Latr., Gen. Crust. et Ins., IV. 289. [Atherix] (1809); Meig., System. Beschreib., II. 108. 5. tab. XV. fig. 23, 29, 30, 36. [Atherix] (1820) ; CurT., Brit. Entomol., VIII. 26. [Atherix] (1824); MaCQ., Suit. à Buffon, I. 433. 7. [Atherix] (1834); Zетт., Insecta Lappon., Dipt., 528. 1 [Atherix] (1838) et Dipt. Scand., I. 225. 1. [Atherix] (1842) ; WaLK., List Dipt. Brit. Mus., I. 219. [Atherix] (1848); Zetr., Dipt. Scand., VIII. 2989. 1. [Atherix] (1849); Scноцтz, Zeitschr. f. Entomol. Breslau, IV. No. 16., 40. [Atherix] (1850); WALK., Insecta Britannica, Dipt., I. 70. 3. [Atherix] (1851); WahlBg., Öfvers. Kongl. Vet. Akad. Förhandl., IX. 187. [Atherix] (1852) ; Bossd., Finlands tvaving. Ins., I. 139. 1. [Atherix] (1861); Schin., Fauna Austriaca, Dipt., I. 179. 2. [Ptiolina] (1862); JAExv., Berlin. Entom. Zeitschr., XI. 100. 22. [Ptiolina] (1867); Gов., Mém. Soc. Linn. Nord France, 1877. 19. tab., fig. 7. [Ptiolina] (1877); Beling, Archiv f. Naturgesch., XLVIII. 1., 193. 7. (1882); Beck., Berlin. Entom. Zeitschr., XXXI. 141. 116. (1887); StroвL, Mittheil. Naturwiss. Ver. Steiermark, XXIX. 1892. 25. (1893); Griff., Bollet. Mus. Zool. ed Anat. comp. Torino, X. No. 218., 4. [Atherix] (1895); Beck., Entomol. Nachricht., XXVI. 176. (1900). griseola Fall., Dipt. Suec., Anthracides, 12. 7. [Leptis] (1814). cruenta Coquill., Journ. New York Entom. Soc., II. 55. (1894); ALDR,, Catal. North Amer. Dipt., 217. (1905).
Nova Guinea, Ins. Aru.

Chile.

Peru.

America sept.
America sept.

Europa.

America sept. 
fera Coquill., Journ. New York Entom. Soc., II. 56. (1894); Aldr., America sept. Catal. North Amer. Dipt., 217. (1905).

flavipalpis Adams, Kansas Univ. Sci. Bullet., II. 439. (1904). America sept. fulvipes $\mathrm{BIG} .=$ latipalpis Big.

grisea Wied. apud MeIG., System. Beschreib., II. 109. 8. [Atherix] (1820) ; Schis., Famna Austriaca, Dipt., I. 180. [Ptiolina] (1862); Griff., Bollet. Mus. Zool. ed Anat. comp. Torino, X. No. 218., 4. [Atherix] (1895); Mr, Wien. Entom. Zeitg., XV. 248. (1896); Bezzi, Bullet. Soc. Entom. Ital., XXX. 34. (1898).

griseala FALL. = crassicornis PANz.

hirta Johns., Entomol. News Philad., VIII. 120. (1897) ; Alnr., Catal. Nortl Amer. Dipt., 217. (1905).

immaculata Meig., Klassif., I. 294. 2. [Atherix] (1804); Fabr., Systema Antliat., 74. 10. [Atherix] (1805); MEIG., System. Beschreib., II. 110. 9. [Atherix] (1820); MaCQ., Recueil. Soc. Sci. Agricult. Lille, 1826. 409. 4. [Atherix] (1826) et Suit. à Buffon, I. 433. 6. [Atherix] (1834); WaLK., Entomol. Magaz., III. 180. [Atherix] (1835); Blanch., Hist. Nat. Ins., III. 604. [Atherix] (1840); Walk., Insecta Britannica, Dipt., I. 71. 2. [Ptiolina] (1851); Scurv., Fauna Austriaca, Dipt., I. 179. 2. [Ptiolina] (1862); Goв., Mém. Soc. Linn. Nord France, 1877. 19. [Ptiolina] (1877); L. Coucke, Annal. Soc. Entom. Belgique, XXXVII. 133. [Atherix] (1893).

Johnsoni Coquill., Journ. New York Entom. Soc., II. 54. (1894); Americasept. Aldr., Catal. North Amer. Dipt., 217. (1905).

latipalpis Big., Bullet. Soc. Zool. France, XII. 108. (1887); Coquill., Journ. New York Entom. Soc., II. 53. (1894); ALdr., Catal. North Amer. Dipt., 217. (1905).

fulvipes Big., Bullet. Soc. Zool. France, XII. 110. (1887). limata Coquill., Journ. New York Entom. Soc., II. 54. (1894). maura Mus. Vindob. apud MeIG. = melaena MeIG.

melaena Meig., System. Beschreib., II. 109. 6. [Atherix] (1820); Curt., Brit. Entomol., VIII. 26. [Atherix] (1824); WALK., Entomol. Magaz., III. 180. [Atherix] (1835), List Dipt. Brit. Mus., I. 220. [Atherix] (1848) et Insecta Bıitannica, Dipt., I. 71. 1. tab. II. fig. 9. [Atherix] (1851); Scun., Fauna Austriaca, Dipt., I. 179. 1. [Ptiolina] (1862); JAEnv., Berlin. Entom. Zeitschr., XI. 100. 21. [Ptiolina] (1867); Becher, Denkschr. Akad. Wien, XLV. 143. tab. II. fig. 21. (1882); Mıк in Beck. : Fauna von Hernstein, II. 2., 55. (1885); ВEск., Entomol. Nachricht., XXVI. 114. [Atherix] et 176. (1900).

maura Mus. Vindob. apud Meig., System. Beschreib., II. 109. (1820).

? monos Harris, Expos. Engl. Ins., 103. tab. XXXI. Sect. II. fig. 3. [Sylvicola] (1782).

pilosa MeIG., System. Beschreib., II. 109. 7. [Atherix] (1820);

Walk., List Dipt. Brit. Mus., I. 219. [Atherix] (1848); Neunaus,

Diptera Marchica, 48. 2. [Atherix] (1886).

America sept.

America sept

Europa.

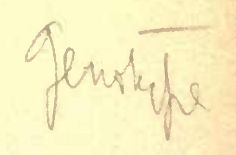

Europa centr.

et mer.

America sept.

Europacentr. 
? unica Harris, Expos. Engl. Ins., 103. tab. XXXI. Sect. II. fig. 2. [Sylvicola] (1782).

modesta Coquilu., Journ. New York Entom. Soc., II. 54. (1894); Americasept

Aldr., Catal. North Amer. Dipt., 217. (1905).

monos HARRIS = ? melaena Meja.

? nebulosa FAвR., Supplem. Entomol. System., 556. 10-11. [Rhagio]

(1798) ; Meig., Klassif., I. 3u8. q. [Rhagio] (1804); Fabr., Systema

Antliat., 74. 8. [Atherix] (1805); FALL., Dipt. Suec., Anthracides, 14. [Leptis] (1814) ; Meig., System. Besclireib., II. 107. 3. [Atherix] (1820) ; MacQ., Recueil Soc. Sci. Agricult. Lille, 1826. 408. 3. [Atherix] (1826) et Suit. à Buffon, I. 432. 4. [Atherix] (1834); Zетт., Dipt. Scand., I. 226. 3. [Atherix] (1842); Gıмм., Bullet. Soc. Imp. Nat. Moscou, XX. 1., 200. 3. [Atherix] (1847); ZETT., Dipt. Scand., VIII. 2990. 3. [Atherix] (1849); Goв., Mém. Soc.

Linn. Nord Trance, 1877. 19. [Ptiolina] (1877).

pachyceras Willist., Trans. Amer. Entom. Soc. Philad., XIII. 287.

(1886) ; Coquili., Journ. New York Entom. Soc., II. 53. (1894);

Aldre, Catal. North Amer. Dipt., 217. (1905).

atripes Bit., Bullet. Soc. Zool. France, XII. 111. (1887).

comata BIG., Bullet. Soc. Zool. France, XII. 111. (1887).

trivittata BıG., Bullet. Soc. Zool. France, XII. 109. (1887).

? picea WaLk., List Dipt. Brit. Mus., I. 219. [Atherix] (1848).

picticornis BıG. = plagens WiLList.

pilosa Meig. = melaena Meig.

plagens Wilusst., Trans. Amer. Entom. Suc. Philad, XIII. 287.

(1886) ; Coquilu., Journ. New York Entom. Soc., II. 53. (1894);

Aldr., Catal. North Amer. Dipt., 217. [plangens] (1905).

picticornis Bıa., Bullet. Soc. Zool. France, XII. 109. (1887).

pullata Coquill., Journ. New York Entom. Soc., II. 56. (1894), Proc.

Washingt. Acad. Sci., II. 406. (1900) et Harriman Alasska Exped.,

IX. Pt. II., 20. (1904); Aldr., Catal. North Amer. Dipt., 218. (1905).

securifera Coquill., Proc. Entom. Soc. Washington, VI. 171. (1904). Americasept. trivittata $\mathrm{BIG} .=$ pachyceras WiLLIST.

trucis Coquill., Journ. Nèw York Entom. Soc., II. 55. (1894); Aldr., America sept.

Catal. North Amer. Dipt., 218. (1905).

unicu HaRris ==? melaena MeiG.

\section{Ptiolina}

Zะтт., Dipt. Seand., I. 21. 27 et 226. 27. (1842).

Eurytion Jakns., Berlin. Entom. Zeitschr., XI. 99. 17. (1867).

Tyolina WaLK., List Dipt. Brit. Mus., I. 220. (1848). lapsus.

calamodytes Schus., Verh. zool.-bot. Ges. Wien, XVIII. 911. (1868). Europacontr. fasciata Lw., Berlin. Entom. Zeitschr., XIII. 154. 65. (1869); AlnR., Canada.

Catal. North Amer. Dipt., 217. [Spania] (1905). 
fulva Beck., Entomol. Nachıicht., XXVI. 110. (1900) et Arta Soc. Sibiria.

Sci. Fenn., XXVI. No. 9., 9. 9. (1900).

grisea STRoBL $_{\text {T }}=$ paradoxa JAEN.

lapidaria Now. = paradoxa JAENN.

majuscula Lw., Berlin. Entom. Zeitschr., XIII. 165. 66. (1869);

Ardpr., Catal. North Amer. Dipt., 217. [Spania] (1905).

nigra ZETT., Dipt. Scand., I. 227. 2. p. p. (1842); WALk., List Dipt. Europa,

Brit. Mus., IV. 1153. (1849) ; Zıтт., Dipt. Scand., VIII. 2992. 2. Sibiria.

p. p. (1849) ; Waнi.вG., Öfvers. Kongl. Vet. Akad. Förhandl.,

XI. 215. (1854) ; Zетт., Dipt. Scand., XII. 4593. 2. (1855) ; Schin.,

Verh. zool.-bot. Ges. Wien, XVIII. 912. (1868); Strrobl, Wien.

Entom. Zeitg., XI. 123. 4. [Spania] (1892); L. Covcke, Annal. Soc.

Entom. Belgique, XXXVII. 133. [Atherix] (1893); BezzI, Bullet.

Soc. Entom. Ital., XXX. 34. 62. [Spania] (1898); Beck., Entomol.

Nachricht., XXVI. 113. (1900) et Acta Soc. Sci. Fenn., XXVI.

No. 9., 12. 10. (1900).

nigra ZетT. p. p. = nigrina W.ALBG.

nigrina WAHLBG., Öfvers. Kongl. Vet. Akad. Förhandl., XI. 215. 10.

(1854) ; Zetr., Dipt. Scand., XII. 4593. 3. (1855); Schin., Verh.

zool.-bot. Ges. Wien, X VIII.912.(1868); BEck., Entomol. Nachricht.,

XXVI. 112. (1900).

nigra ZETT., Dipt. Scand., I. 227. 2. p. p. (1842) et VIII. 2992.

2. p. p. (1849).

obscura ZETT., Insecta Lappon., Dipt., 527. 7. [Leptis] (1838).

nigripes Zетт., Dipt. Scand., XIII. 4975. 5. (1859); Schın., Verl.

zool.-bot. Ges. Wien, XVIII. 912. (1868); Brauer, Denkschr.

Akad. Wien, XlVII. 43. tab. III. fig. 41-42. (1883); StrobL,

Wien. Entom. Zeitg., XI. 124. 8. [Spania] (1892); Mıк, Wien.

Entom. Zeitg., XV. 248. (1896).

nitida WAHıBg., Öfvers. Kongl. Vet. Akad. Förbandl., XI. 215. 11.

(1854); Zетт., Dipt. Scand., XIJ. 4594. 4. (1855); Scmn., Verh. zool.-bot. Ges. Wien, XVIII. 912. (1868); Strobl, Wien. Entom.

Zoitg., XI. 123. 3. [Spania] (1892); Beck., Entomol. Nachricht., XXVI. 112. (1900).

obscura FaLL., Dipt. Suec. Anthracides, 14. 11. [Leptis] (1814); Meig., System. Beschreib., II. 111. 11. [Atherix] (1820); Zетт., Dipt. Scand., I. 227. 1. (1842); WALk., List Dipt. Brit. Mus., IV. 1153. (1849) ; ZETr., Dipt. Scand., VIII. 2991. 1. (1849) et XI. 4267. 1. (1852); WAhlbG., Ölvers. Kongl. Vet. Akad. Förhandl., XI. 214. (1854); ZETT., Dipt. Scand., XII. 4592. 1. (1855); Scuin., Verh. zool.-bot. Ges. Wien, XVIII. 912. (1868) ; S'тroвь, Wien. Entom. Zeitg., XI. 125. [Spania] (1892) et Mittheil. Naturwiss. Ver. Steiermark, XXIX. 1892. 26. [Spania] (1893); Веск., Entomol. Nachricht., XXVI. 111. fig. 4. (1900) et Acta Soc. Sci. Fenn., XXVI. No. 9., 12. 11. (1900).

obscura Zетт. 1838. (nee FALL.) = nigrina WAILBG.

paradoxa JAENn., Berlin. Entom. Zeitschr., X. tab I. fig. 8, $a-c$. Europac.ets. 
[Eurytion] (1866) et XI. 99. 18. [Eurytion] (1867); BEck., Berlin. Entom. Zeitschr., XXXI. 141. 115. [Eurytion] (1887); Mn, Wien. Entom. Zeitg., IX. 154. 77. (1890) ; StrobL, Wien. Entom. Zeitg., XI. 124. 7. [Spania] (1892).

grisea Strobl, Wien. Entom. Zeitg., XI. 124. 5. [Spania] (1892), Mittheil. Naturwiss. Ver. Steiermark, XXIX.1892. 26. [Spania] et Verh. u. Mittheil. d. siebenbürg. Ver. f. Naturwiss. Hermannstadt, XLVI. 1896. 21. [Spania] (1897).

lapidaria Now., Verh. Naturf. Ver. Briinn., VI. 1867. 74. tab. II. fig. 1. (1868).

? unicolor Curt., Brit. Entomol., VIII. 26. [Atherix] (1824); Verrall, The Entomologist, XXIII. 153. (1890).

Wodzizkii Jraufld, Verh. zool.-bot. Ges. Wien, XVII. 497. tab. XII. fig. 15-20. (1867) ; Now., Verh. Naturf. Ver. Brünn, VI, 1867. 74. nota 2. (1868); StтroвL, Wien. Entom. Zeitg., XI. 124. 6. [Spania] (1892).

pelliticornis Beck., Entomol. Nachricht., XXVI. 113. (1900).

Europa mer. phragmitophila Schin., Verh. zool.-bot. Ges. Wien, XVIII. 910. Europa centr.

(1868) ; Licniww., Zeitschr. f. system. Hymen. u. Dipt., V. 310. 8. (1905).

tristis WaLk., Iist Dipt. Brit. Mus., I. 220. [Tyolina] (1848) est nomen undum.

unicolor Curt. $=$ ? paradoxus JAExN.

Wodzizkii FraUenfld. = paradoxus JaExN.

\section{Spania}

MeIG., System. Besclireib., VI. 335. LXXV-LXXVI. (1830).

Edeta WALK., List Dipt. Brit. Mus., III. 489. (1849); Os'T.-SAck., Canada, Catal. Dipt. North Amer., Ed. II., 65 et 229. 95. (1878); Coquru., Alaska.

Proc. Washingt. Acad. Sci., II. 406. (1900) et Harriman Alaska Exped., IX. Pt. II. 20. (1904); Ar.pr., Catal. North Amer. Dipt., 217. (1905).

Fullenii $\mathrm{HAL}_{\mathrm{A}}=$ nigra MeIG.

nigra MeIs., System. Beschreib., VI. 335. 1. tab. LXVI. fig. 12-14. (1830) ; MAcQ., Suit. à Buffon, I. 431. 1. tab. X. fig. 14-15. Europacentr. (1834); Scholtz, Zeitschr. f. Entomol. Breslau, V. No. 19., 49. et sept.

(1851) ; WALK., Insecta Britannica, Dipt., I. 72. 1. tab. II. fig. 10. (1851) ; WanlbG., Öfrers. Kongl. Vet. Akad. Förhandl., XI. 216. 12. (1854) ; Zетт., Dipt. Scand., XII. 4596. 1. (1855); Schix., Fauna Austriaca, Dipt., I. 180. (1862); Gob., Mém. Soc. Liun. Nord France, 1877. 20. tab., fig. 8-10. (1877); Stroвц, Wien. Entom. Zeitg., XI. 121. (1892); L. Coucke, Annal. Soc. Entom. Belgique, XXXVII. 133. (1893); Mı, Wien. Entom. Zeitg., XV. 247. 59. (1896) ; Strobi, Verh. u. Mittheil. d. siebenbürg. Ver. f. Naturwiss. Hermannstadt, XLVI. 1896. 21. (1897) et Mittheil. 
Naturwiss. Ver. Steiermark, XXXIV. 196. (1898); Beck., Entom. Nachricht., XXVI. 106. fig. 1-4. (1900).

Fallenii Hal., Entomol. Magaz., I. 162. (1833) et Annal. Mag. Nat. Hist., II. 184. (1839).

\section{Hilarimorpha}

Schin., Wien. Entom. Monatschr., IV. 54. (1860).

Hilaromorpha Meland., Trans. Amer. Entom. Soc. Philad., XXVIII. 336. (1902).

Mikii Willist., Psyche, V. 100. (1888); Meland., Trans. Amer. Entom. Soc. Philad., XXVIII. 337. [Hilaromorpha] (1902); Aldr., Catal. North Amer. Dipt., 218. (1905).

obscura Big., Annal. Soc. Entom. France, sér. 6. VII. Bullet., CXLl. (1887) et sér. 6. IX. 129. (1889); Meland., Trans. Amer. Entom. Soc. Philad., XXVIII. 337. [Hilaromorpha] (1902) ; Aldr., Catal. North Amer. Dipt., 218. (1905).

singularis EGG., Verh. zool.-bot. Ges. Wien, X. 346. (1860); Schin., Fauna Austriaca, Dipt., I. 117. 1. (1862) et Verh. zool.-bot. Ges. Wien, XVIII. 909. (1868); Mıк, Verh. zool.-bot. Ges. Wien, XXXI. tab. XVI. fig. 19-21. (1881) ; Röp., Stettin. Entom. Zeitg., XLVI. 401. (1885).

tristis EGg., Verh. zool.-bot. Ges. Wien, X. 347. (1860); Schin., Fauna Austriaca, Dipt., I. 117. (1862) ; Mur, Verh. zool.-bot. Ges. Wien, XXXI. tab. XVI. fig. 22. (1881); Strobl, Mittheil. Naturwiss. Ver. Steiermark, XXIX. 1892. 42. (1893).

\section{Mythicomyia}

Coquill., Entomol. News Philad., IV. 208. (1893).

pictipes Coqutll., Proc. Unit. Stat. Nat. Mus., XXV. 102. (1902); Audr., Catal. Norh Amer. Dipt., 218. (1905).

Rileyi Coquilu., Entomol. News Philad., IV. 209. fig. (1893) et Proc. Unit. Stat. Nat. Mus., XVIII. 1895. 409. (1896) ; Meland., Trans. Amer. Entom. Soc. Philad., XXVIII. 338. (1902); Aldr., Catal. North Amer. Dipt., 218. (1905).

scutellata Coquil.t., Proc. Unit. Stat. Nat. Mus., XXV. 102. (1902); America sept. Aldpr., Catal. North Amer. Dipt., 218. (1905).

tibialis Coquilu., Proc. Unit. Stat. Nat. Mus., XVIII. 1895. 409. Americasept. (1896) ; Meland., Trans. Amer. Entom. Soc. Philad,, XXVIII. 338. (1902) ; Al.Dr., Catal. North Amer. Dipt., 218. (1905). 


\section{Conspectus}

\section{familiarum, generum et specierum.}

Familia.

1. Stratiomyiidae.

2. Erinnidae.

3. Coenomyiidae.

4. Tabanidae.

5. Pantophthalmidae.

6. Rhagionidae.
Genus.

100

4

8

33

2

$\frac{20}{167}$
Species.

989

51

16

1529

15

274
2874 


\section{INDEX FAMILIARUM ET GENERUM.}

Numerus primus post nomen: paginam, secundus vero: generis species indicat.

Acanthina Wied. 2

Acanthinia MacQ. 2

Acanthinomyia Hunt. 2

Acanthocera MACQ. $\quad 214$

Acanthomera WIED. $\quad 294$

Acanthomyia Schis. 129

Acraspidea Brau.

Acrochaeta WiEd.

Actina Meig.

Adoxomyia KerT.

Adraga W WLK.

Agclanius Rond.

Agnotemyia WiLL.

Agnotomyia Will.

Akronia Hine.

Alliocera SAUND.

Allognosta O.-S.

Amphilecta BraU.

Ampsalis WaLK.

Anacanthaspis Rön.

Anacanthella MAcQ.

Analcocerus Lw.

Antidoxion SN. v. V.

Antissa WaLK.

Aochletus O.-S.

Apatolestes WilL.

Apocampta Scuin.

Apogon Perr.

Artemita WALk.

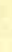

(2)

Arthroceras WiLL.

Arthropacas Mansci.

Arthropeas Lw.

Arthrostylum WIIL.

Atherix Meig.

Atherix Rovd.

Atylotus O.-S.

Aulana WALK.

Basentidema MACQ.

Bellardia RoND.

Beris Latr.

Berismyia G.-T.

Biastes WALK.

Blastocera Gerst.

Bolbodimyia BıG.

Bolbomyia Lw.

Brachycara 'Thoмs.

Brachytomus A. Costa

Cacosis Walk.

Cadicera MAcQ.

Caenacantha WuLP.

Caenocephalus Wulp.

Cacnomyia I.atr.

Cacnopnyga Troms.

Calcochactis BIa.

Caloehactis BIG.

Campeprosopa MACQ.

Chaetosargus Rön.

Chauma Lw.
302

143

143

298

299

328

217

32

91

217

122

128

7 
Chiromyza Wied.

Chlorisops Brav.

Chloromyia Dunc.

Chlorosia Rond.

Chlorosoma Rond.

Chordonota Gerst.

Chorisops Rond.

Chromatopoda BraU.

Chrysochlora Latr.

Chrysochroma WiLL.

Chrysomyia MAcQ.

Chrysonotomyia Hunt.

Chrysonotus Lw.

Chrysopila Mace.

Chrysopilus Mace.

Chrysops Meig.

Chrysozona Meig.

Clanis WaLK.

Clitellaria Aуст.

Clitellaria Meig.

Clorisoma RovD.

Coenomyia IAtr.

Coenomyinde.

Coenura BIG.

Compsosoma Brau.

Corizomeura Rond.

Culcua WaLK.

Cyanauges PhiL.

Cyclogaster MAcQ.

Cynipimorpha BraU.

Cyphomyia WIED.

Cyrtopus Big.

Dasybasis MAcQ.

Dasyomma Mace.

Diabasis Macq.

Diachlorus O.-S.

Dialysis WaLK.

Diatomineura Rovd.

Dichelacera MAcQ.

Diclisa Scmr.

Dicrania Macq.

Dicranomyia HuNT.

Dicranopliora MACQ.

Diphysa Macq.

Ditylomyia BIG.
160

144

131

108

110

110

41

131

90

89

98

108

98

98

317

317

181

201

150

19

13

110

141

141

148

98

168

4

121

29

5

42

85

209

315

211

211

315

168

214

149

146

146

92

131

181

\section{5}

6

6

6

2

2

13

17

(n)

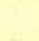

92

152

92

47

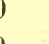

3

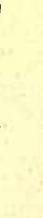

1

\section{5}

2

1

1

2

2

Drasteria Brau.

Ectenopsis MACQ.

Elasma JAENN.

Engonia BraU.

Enoplomyia Big.

Ephippiomyia BezzI.

Ephippium Latr.

Erephopsis Rond.

Erephrosis Ric.

Erinna Meig.

ERINNidae.

Erodiorhynchus MAcQ.

Esenbeckia Rond.

Euceromyia BIG.

Euceromys Big.

Eudmeta Wied.

Eulalia Meig.

Euparhyphus O.-S.

Euparyphus Gerst.

Euplomyia BIG.

Euryneura Schis.

Eurytion JAENN.

Evasa Auct.

Evaza WALK.

Exaereta Schis.

Exaireta Schin.

Exochostoma MACQ.

Exodonta VERr.

Exodontha Rond.

13

150

150

134

133

176

177

85

85

116

62

17

17

12

18

330

4

4

131

131

88

129

129

Fidena WaLK.

150

Gabaza WAIs.

Gastroxides Savnd.

6

Glutops Burgess.

Gobertina Big.

Goniops Aldr.

181

315

147

Hadrestia Thoms. 127

Hadrus PERTY. 209

Haematophila VERR. 200

Haematopota Meig. 201

Haemoplita KrIECHв. 200

Heliomyia DoL. 317

Heptatoma Meig. 200

Hermetia LAtr. 117

Hermione Meia. 32

Heteracanthia MACQ. 128 
1015

Heterostomatomyia Scudd. 143

Heterostomus Big.

143

Heterostomyia Big.

143

Heteroxycera Bia.

Hexacantha Lioy.

Hexatoma MeIG.

Hexodonta Rond.

Hilarimorpha Schis.

Hilaromorpha MELand.

Hinea Adans.

Hirtea Scop.

Histiodroma Schis.

Hoplistes Mace.

Hoplacantha Rond.

Hoplodonta Rovd.

Hoplomyia ZELL.

Hylorus Pнis.

Hypoleon Dus.

Ibisia Rond.

Inermia Big.

Inermyia BIG.

Inopus WaLK.

Lagarus Phil.

Lagenosoma BraU.

Lampromyia MACQ.

Lasiopa Brullé.

Lepidoselaga MACQ.

Lepiselaga MACQ.

Leptipalpus Rond.

Leptis Fabr.

leptis WaLK.

Leptynoma WESTW.

Lilaea WaLK.

Lolphoteles WILI.

I،ophoteles Lw.

Macellopalpus BiG.

Macroceromys Bia.

Macrosargus Big.

Massicyta WaLK.

Megalemyia BIG.

Megalomyia Bia.

Melanochroa Rö̀.

Melpia WaLK.

Merosargus Lw.

Mesomyia Mace.
32

122

200

129

333

333

176

46

92

91

122

86

48

145

32

299

29

29

144

145

117

298

29

209

209

317

302

317

298

150

12

12

302

137

114

117

294

294

88

150

96

177
Metabasis WALK.

1 Metoponia Lw.

Metoponia MAcQ.

Microchrysa Isw.

Musama WALK.

Mycteromyia PhiL.

Myiochrysa VERR.

Myochrysa Rond.

Mythicomyia Coquill.

Myxosargus Brau.

Negritomyia BIG.

Nematotelus O.-S.

Nemorius Rond.

Nemotelus Geofrr.

Neochauna WILL.

Neoexaereta O.-S.

Neoexaireta O.-S.

Neopachygaster Aust.

Neorondania O.-S.

Nerna WALK.

Nerua WALK.

Nigritomyia BIG.

1

Nodutis Meg.

Nonacris WaLK.

4

15

2

8

Nothomyia Lw.

Nuceria WALK.

Obrapa WALK.

Octacantha Liox.

Octarthria Brad.

Odontomyia MEIG.

Omphalophora BEck.

Oplachantha Rovn.

Oplodontha Rond.

Opseogymuns A. Costa.

Osca Walk.

O.xycera MeIG.

Pachygaster Meig.

Pachystomus Litr.

5

Panacris Gerst.

Pangonia IAtTr.

Pangonius Latur.

Pantophthalmidae.

Pantophthalmus Thusis.

Pedicella Big.

Pelagomyia WilL.
1094

61

128

145

110

21

175

108

108

333

88

16

21

200

21

131

131

9

46

144

88

150

12

122

17

62

317

122

86

62

150

32

9

134

2

150

150

294

294

114

90

14

12

$\frac{1}{1468}$

3

Kertész: Catalogus Dipterorum. III. 
Pelechoïdoccra Big.

1468

Pelecorrhynchus Mace. 148

Phara WaLK.

150

Pheneus WaLk.

298

Philoliche WiEd.

150

Phyllophora MACQ.

Pityocera G.-T.

Platyna WIED.

Plecticus Lw.

Plinthina WaLK.

Potamida Meig.

Promeranisa WALk.

Promerisana WILI.

Pronopes Lw.

Psammorycter Blanch. 297

Psellidotus Rond.

Ptecticus Lw.

Ptilocera Wined.

Ptiolina ZETT.

Pycnomalla Gers'T.

Rachicerus WaLK.

133

Rhachicerus WALK.

133

Rhagio Fabr.

302

Rhagio FABR.

RHAGiONidAE.

Rhaphiocera MAcQ.

Rhaphiorrhynchus WiEd. 296

Rhingiopsis RöD.

Rhinomyza WIED.

Rhyphomorpha WALK. 133

Rondania JAENN.

Rosapha WALK.

Ruba WALK.

Sackenimyia BIG.

Salduba WALK.

Sargus Faibr.

Saruga WALK.

Scaptia WaLK.

Scarphic WaLk.

Scepsis WaLK.

Scione WaLk.

Scoliopelta WiLl.

Selasoma Macq.

Silvius Meic.

sinowiellus Hine.

Solva WaLK.

Spania Merg.

Spyripoda Gerst.

332

Slenobrithes Iw.

Sternobrithes Lw.

Stibasoma Schrn.

Stratiomyia Geoffr.

Stratiomyidda.

Stratiomys Geoffr.

Stratiotomyia ARRIs.

6

3

Styrex Scop.

Subula Meg.

Subula Omyia WiLL.

1

Suragina WaLk.

Sylvicola Harr.

Sylvicola HARr.

Sylvicola HarR.

Symphoromyia FrfLD.

Tabanidae.

Tabanocella Bir.

Tabanus L.

Tacina WaLK.

Tanyglossa Meig.

Therioplectes ZeLI.

Thorasena Mace.

Thylacosoma Brat.

1 Thiyreodonta Rond.

2 Tinda WALK.

5 Toxocera MacQ.

Tracana WALK.

Trichochaeta Big.

3

2

Trichopalpomyia Hunt.

Trichopalpus PнuL.

Triptotricha Lw.

Tyolina WaLk.

Udenocera Ric.

Vappo Latr.

Veprius Rond.

Vermileo Mace.

Wallacea DoL.

Xenomorpha MACQ.

Xylomya RovD.

Xylomyia Rond.

Xylophagus Meig.

Zabrachia CoquilL. 


\section{INDEX SPECIERUM.}

abazus Big. 217.

abbreviatus BIG. 217.

abdominalis Apars. 21. abdominalis FABR. 217. abdominalis Lw. 134. abdominalis WIED. 217. aberrans Schin. 17. abiens WALK. 217. abscondens WALK. 217. abstersus WALK. 217. acanthinoidea JAENN. 7 . Actaeon O.-S. 217. acuminatus Lw. 217. acupunctatus Rond. 218. acutidens PHIL. 218. acutipalpis MACQ. 218. acutirostris Lw. 21. acutus Big. 218. adjuncta WALK. 151. administrans ScHus. 218. Adrel WALK. 162. adusta Lw. 62. adustus WALK. 218. advena WАLK. (Nem.) 21. advena WALK. (Tab.) 218. aegrotus O.-S. 218. aemula Lw. 32. aemulus Lw. 21. aenea Tномs. 127. aeneipennis G.-T. 117. aeneiventre G.-T. 98. aequalis W ALK.(Chrys.) 317 . aequalis WALK. (Eul.) 62. aequetinctus BEcK. 218. acrosus Grмm. 21. aestuans WuLP. 181. aethereus Big. 218. acthiopica Thuns. 151. aethiops LJUngh. 218. affinis BeLL. (Chrys.) 181. affinis BeLL. (Eul.) 62. affinis BIG. 100. affinis CurT. 32. affinis Gerst. 42. affinis KınBY. 218. affinis Lw. 151. affinis Schis. 93. . affinis WIED. 110. affinis WILL. 92. afflictus WIEd. 181. africanus Gray. 218. agrestis WIED. 218. agricola W1ED. 218. Ahngeri Pleske. 48. alazinus Bia. 218. albibarbis Bıt. 302. albibarbis WIED. 218. albibarbus Lw. 100. albicans MAcq. (Tab.) 218. albicans Macq. (Tab.) 218. albicans MAcQ. (Tab.) 219. albicaput WALK. 42. albicinctus WuLP. 181. albicollis Huñt. 219. albicornis SAY. 302.

albicostata MAcQ. 168. albidicollis MACQ. 219. albidocinctus Big. 219. albifacies BEcK. 21. albifacies Lw. 219. albifasciata MACQ. 149. albifrons Big. 172. albifrons MACQ. 162. albifrons MeIG. 302. albilirta KaRscri: 201. albilateralis MAcQ. 219. albilinea WALK. 219. albimacula WALK. 131. albimedius WALK. 219. albipalpis Rovd. 219. albipalpus WALK. 219. albipectus Big. (Myct.) 175. albipectus BiG. (Tab.) 219. albipennis MacQ. 62. albipes Adans. 99. albipes FABR. 219. albipes Schrnk. 200. albipilosus Adans. 17. albirostris MacQ. 21. albiscutellatus MACQ. 219. albitalus WiLL. 90 . aibitarsis Big. 16. albitarsis FABr. (Cyph.) 42. albitarsis FABR.(Herm.) 118. albitarsis MACQ. (Beris) 122. albitarsis MaCQ. (Lep.) 209. 
albitarsis WIED. 42. albitarsis WuLP. 118. albithorax MACQ. 168. albitibialis MAcQ. 219. albiventris Thoмs. 21. albivittatus MAcQ. 219. alboater WALK. 219. alboatra WALK. 151. albocostatus BiG. 219. albohirtus WALK. 219. albomaculata MACQ. 62 . albomaculatus WALK. 219. albomaculatus ZETT. 219. albonotata MACQ. 163. albonotatus BeLL. 219. albonotatus Bir. 220. albopictus Big. 220. albopunctata Schin. 148. alboscutatus RoNi. 220. albovarius WALK. 220. albulus WALK. 220. Alchidas WaLk. 114. alcicornis Wied. 214. alcis WHLL. 220.

Aldrichi Johss. 62. Aldrichi WhL. 315. alene Towss. 220. Aleus WaLK. 48. alexandrinus WIED. 220. alfourensis Big. 220. algericus BECK. 302. algericus JAENN. 21. algerus MACQ. 177. algivicus Thune. 220. algirus MACQ. 220. algirus MEIG. 177. Alluaudi G.-T. 149. Allynii Marten. 220. alpicola Pok. 317. alpina Hutton. 131. alpina JAENN. 62. alpina Lлоу. 32. alpinus Drap. 177. alpinus Lw. 302. alpinus PaNz. 220. alpinus Scor. 177. alpinus Zetт. 220. alter RoNd. 181.

alteripennis WALK. 220. alternans MACQ. 172. altifrons WULP. 63. altivagus O.-S. 181. alumnus WALK. 302. amabilis WALK. 220. amaena Big. 168. amaenus WALK. 220. amazon DAECkE. 181. amazonius RoND. 181. amboinensis BraU. 31. amboinensis FABr. 151. Amenides Walk. 2. americana DAY. 63. americana Lw. 143. americana O.-S. 201. americana Wied. 137. americanus DruRr. 220. americanus Forst. 220. americanus P. B. 211. americanus Schis. 317. amethystina FABR. 89. amethystina Sx. v. V. 5. amoena Lw. 32.

amplus Coquill. 17. Amyris WALK. 63. anachoreta PHIL. 221. anale WiLL. 99. analis FABR. (Pang.) 151. analis FABR. (Strat.) 48. analis MACQ. 100. analis Now. 131. analis WIED. 32. anbohirtus Hunt. 219. Anchialus WaLK. 46. anchora Lw. 21. anchorata Big. 63. andamanicus Big. 221. andicola PHiL. 221. Andreas WALk. 118. androgyna O.-S. 42. angularis Lw. 48. angulata FAsis. 151. anqulata Meig. 63. angulata PAxz. 63. angulatus Duv. 22. angusta BiG. 172. angusta MACQ. (Cor.) 172. angusta MACQ. (Pot.) 13. angusta WALK. (Eul.) 63. angusta WALK. (Tinda) $\boldsymbol{\tau}$. angusticornis Lw. 221. angustifrons I.w. 100 . angustifrons MAcQ. 221. angustifrons Towss. 221. angustus BIf. 221. angustus MACQ. 221. anilis Schrnk. 64. annamitus Bif. 221. annulata Big. 172. annulata Meig. 64. annulatus Big. 302. annulatus DEG. 302. annulatus Lw. 22. ammulatus Meig. 303. annulatus SAY. 221. annulicornis PHu. 221. annulifera Big. 122. annulipes MAcQ. 64 . ammlipes Thoms. 111. annulipes WALK. 89. anodonta MAcQ. 64. antarcticus I،. 221. antecedens WLK. (Tab.) 221. antecedens WLK. ( Tab.) 221. antennatus Schis. 96. anthophilus Lw. 221. anthracina MACQ. 172. anthracinus BIG. 317. anthracinus Meig. 222. antipodus BIG. 318. Anubis Wien. 46. aperta I w. 168. Apfelbecki Str. 299. aphanopterus WIED. 222. apiarius JAENs. 222. apicalis BeLt. 181. apicalis Butlen. 131. apicalis Coquilt. 17. apicalis Lw. 93. apicalis MAcQ. 222. apicalis RoNd. (Lept.) 317 . apicalis RoNd. (Tab.) 222. 
apicalis WALK. (Hirt.) 46 . apicalis WALK.(Neocx.) 132. apicalis WALK. (Tab.) 222. apicalis WIED. (Herm.) 118. apicalis Wred. (Tab.) 222. apicalis WuLP. (Chrys.) 318. apicalis WuLP. (Ptect.) 93. apicula Lw. 48.

apoecilus Schin. 222.

appendiculata MAcQ. (Das.) 209.

appendiculata MQ. (Pang.) 151.

appendiculatus MACQ. 177. approximans WaLk. 211. apricus Meig. 222. arabicus MAcQ. 222. araneifera Schns. 64 . arator Melind. 22. aretica Mécl. 202. arcuata Lw. 64. arcuata WilList. 151. ardens MAcQ. 163. ardens Wied. 222. areolaris WALK. 12. arcolatus WALK. 182. argentata BiG. (Gob.) 7. argentata Big. (Lamp.) 298. argentata FABR. 64. argentata WII.L. 19. argentata WuLP. 12. argentea Big. 7. argentea Dou, 6. argentea O.-S. 2. argentea WALK. 6. argentifer JAENN. 9. argentifer Lw. 22. argentifrons WALK. 222. argentula Giмm. 65. argyroceps Bia. 4. argyropasta BiG. 294. argyrophorus ScHin.

(Chrys.) 318. argyrophorus Scmis. (Tab.) 222.

armata Wied. 91. armata Wulp. 118. armeniaca Big. 48. asiatica Rond. 202. aspahanicus Rond. 222. assamensis BIG. 222. Astur Erichs. 223. astuta Will. 92. astutus O.-S. 223. ataenia MACQ. 211. ater MAcQ. 182. ater P. B. 223. ater Rossi. 223. ater SAUnd, 181. ater WIED. 223. ater WiLL. 318. aterrima DuF. 152. aterrima Jolins. 137. aterrima WALK. 65 . aterrimus KinBY. 182. aterrimus Meig. 223. aterrimus WILI. 318. atomaria WALK. 202. atra FALL. 134. atra LAtr. 137. atra Lw. 33. atra Meig. (Beris) 122. atra Meig. (Erinna) 134. atra PAnz. 9. atra Pui.. 152. atra WAND. 1. atraria WALK. 65. atrata FABR. 29. atratus FABR. (Chrys.) 318. atratus FABr. (Tab.) 224. atratus HrNe. 149. atratus Meig. 318. atriceps Lw. (Anal.) 115. atriceps Lw. (Nem.) 22. atricomis BIG. (Chrys.) 182. atricornis Big. (Tab.) 225. atricornis Meig. (Tab.) 225. atricornis Meig. (Tab.) 225. atricornis MEIG. (Tab.) 225. atricornis WIED. 152. atrifera WALK. 152. atrimanus Lw. 225. atripes $\mathrm{BIG} .328$. atripes Röp. 163. atripes WuLP. 225. atriventris •CoquiLL. 17. atropos JAExN. 225. atropos O.-S. 182. atrovirens Big. 65. attenuatus WALK. 225. auctumnalis ZeLL. 225. aurantiacus BELL. 225. aurantiacus JAENN. 182. aurantiacus Ric. 148. aurata BeLL. 118. aurata BIG. 2. aurata Brav. 16. aurata DEG. 111. aurata FABR. 108. aurata MACQ. (Art.) 2. aurata MAcQ. (Dict.) 168. auratus FABr. 318. auratus Meig. 100. aurea BeLL. 114. aurea MeIG. 141. aureohirta RIc. 163. aureovittatus B1G. 17. aureus MeIG. 319. aureus O. F. MÚLL. 320. auribarbis MACQ. 225. auricinctuS MACQ. 225. auricollis WIED. 320. aurifer WALK. 93. auriflamma WALK. 225 . auriflamma Wied. (C'yph.) 42. auriflamma W1ED. (Cyph.) 43. auriflua Dovov. 168. auriflua ERICHs. 13. aurifrons WIED. 43. aurimaculata MACQ. 163. auripes Ric. 163. auripilus MEIG. 225. auripunctatus MAcQ. 225. aurisquammatus $\mathrm{Bi}$. 226. aurofasciata JAENN. 172. aurora MAcQ. 226. aurutestaceus WALK. 226. aurulans MEIG. 320. aurulans WIED. 152. 
australensis ScHus. 65 . anstraliensis Hưrt. 65. australis BıG. 100. australis Gner. 141. australis MACQ. 145 . australis PнIL. 152. australis Schrs. 93. austriacus FaBr. 226. austriacus WIED. 226. autumnalis L. 226. antumnatis Scursk. 227. avidus BIg. 227. azurea Gerst. 2. azurea Lw. 108. baal Towss. 227. baccoides Rovd. 89. badia WALK. 163. Badius WaLK. 48. Bagosas Walk. 100. bahiana Big. 163. balcanicus Str. 303. baltimorensis MACQ. 227. Balius WALK. 19. barbarus Coqueb. 227. barbarus Thusb. 227. barbata L. 168. barbata Lw. 49. barbatus Big. 177. Barca WALK. 49. basalis MAcQ. (Pang.) 152. basalis MAcQ. (Tab.) 227. basalis PHIL. 303. basalis WALK. (Chrys.) 320. basalis WALK. (Ereph.) 163. basalis WaLk. (Tab.) 227. basilaris SAY. 320.

basilaris WIED. (Pang.) 152. basilaris W1Fd. (Pang.) 152. basipunctata WALK. 135. basirufus WALK. 227. basivitta WALK. 227. batjanensis WuLP. 118. batnensis BIG. 227. Beckeri Bezzi. 152. Beckeri Pleske. 49. bella Lw. 108. Bellardii BELL, 294.
Bellardii G.-T. 3. bellula Wils. 122. bellulus MEL. 22. bellus AdAMs. 320. bellus Lw. 17. bellus O.-S. 133. benedictus Wнiт. 227. Beresowskii Pleske. 49. Beschkii Wred. 163. bicarinata WILI. 43. bicinctus FABR. 211. biclausa Lw. 152. bicolor Brg. (Bol.) 209. bicolor Big. (Ros.) 8 . bicolor BIG. (Silv.) 178. bicolor Big. (Stib.) 213. bicolor DAY. 65. bicolor FABR. (Chrys.) 320. bicolor FABR. (Coen.) 141. bicolor Huds. 168. bicolor MACQ. (Chrom.) 90. bicolor MACQ. (Ereph.) 163. bicolor Macq. (Pang.) 152. bicolor MacQ. (Tab.) 227.

bicolor MEIG. 320.

bicolor Thunb. 227.

bicolor WALK. 117. bicolor WIED. (Micr.) 111. bicolor Wies. (Tab.) 227. bidentata FABr. 141.

bifacies WALK. 214.

bifarius Lw. 227.

bifascia WALK. 65.

biîasciata RöD. 144.

bifasciata WIED. 152.

bifasciatus Fourcr. 227.

bifasciatus MAcQ. (Chrys.) 182.

bifasciatus Macq.(Tab.)228. bifasciatus MeIG. 22.

bifasciatus Ros. 303.

bifenestratus O.-S. 228.

Bigoti Bell. (Panth.) 296. Bigoti BeLu. (Tab.) 228.

Bigoti Gов. 202.

biguttata PHIL. 148.

biguttatus WIED. 228.
Bilimecbi Brau. 5. bilinea WALK. 133. bilineata FABR. 13. bimaculata BELL. 49. bimaculata MAcQ. 118. bimaculataMeig.(Hept.)200. bimaculata MEIG. (Hopl.) 86. bimaculata WuLP. 8. bimaculatus Gов. 303. bimaculatus MACQ. 228. bimaculatus WIED. 182. binotata LATR. 152. binotata Lw. 65.

binotata MACQ. 176. binotatus Lw. 320. bipars WALK. (Ev.) 4. bipars WALK. (Micr.) 111. bipartita WaLk. 91. bipartita WuLP. 2. bipartitus WaLK. (Tab.) 228. bipartitus WaLk. (Tab.) 228. bipunctata Big. 65. bipunctata Lw. 33 . bipunctatum O. G. Costa. 99. bipunctatum Scop. 99. bipunctatus Lw. 22. bipunctatus Мотsсн. 182. bipunctatus WuLP. 228. birmanicus BIG. 228. bisignatus JAENN. 228. bispinosa FABR. 141. bispinosa Тномs. 7. bispinosus WIED. 91. bistellatus DAEcke. 182. bistriatus WiLL. 41 . bistrigata Lw. 202. bitaeniatus BeLL. 303. bitinctus WALK. 228. bituberculatus Big. 228. bituberculatus Scurs. 96 . bivittata WIED. 14. bivittatus MACQ. 228. bivittatus WIED. 211. bochariensis Pleske. 49. bonariensis MACQ. 228. borealis FABR. 228. borealis JAENN. 228. 
borealis Meig. (Tab.) 229. borealis Meig. (Tab.) 229. borealis ZETT. 229. borneana Rond. 202. Boscii MacQ. 303. bovinus HARRIS. 229. bovinus L. 229.

bovinus Lw. 229.

brachyrrhyncha Big. 172. brachystomus Lw. 22. brasiliensis RIc. 182. brasiliensis Rond. (Dichel.) 214.

brasiliensis Rond. (Lept.) 317.

brasiliensis WIED. 100 . Bratnankii Now. 230.

Braneri JAENn. 230. Braueri Will. 88. brevicornis Heyd. 122. brevicornis Lw. (Eul.) 65 . brevicornis Lw. (Eup.) 17. brevicornis PoRTsch. 49. brevidentatus MACQ. 230 . brevifacies MAcQ. 65. brevior WALK. 230. brevipalpis MacQ. 172. brevipennis Ourv. 66 . brevipennis RoNd. 93. brevirostris MACQ. 169. brevirostris Meig. 22. brevirostris PHIL. 175. brevis BIG. 91. brevis Lw. (Pang.) 152. brevis Lw. (Tab.) 230. brevis WALK. 128. breviseta WALK. 115. breviusculus WALK. 230. brevivitta $W_{\text {ALK. }}(T a b) 230$. brevivitta WALK. (Tab.) 230. Bricchettii Bezzr. 152. Brimleyi Hıss. 182. bromius L. 230. bromius MeIg. 231. bronicus Grмm. 231. Bruesii Meland. 22. Bruneri Johns. 49. brunnea MeIJ. 116. brunnea Ric. 210. brunnea Thuns. 153. brunneifrons KERT. 320. brunneus Hise. 182. brunneus MacQ. 231. brunneus Thusb. 231. brunnipennis Lw. 172. bubali DoL. 231. bucephala Gerst. 66. bucolicus Schin. 232. Budda Pontsch. 232. Buddha Pontsch. 232. bulbifrons Will. 96. bullata WILL. 153. byzantina STR. 66 . caducus Wied. 303. caeculus MúlL. 182. caecutiens I. 182. cuecuticns PANz. 183. caelebs HARR. 303. caesia BeLl. 114. caesiofasciatus MacQ. 232. caesius WALK. 232. caffra Big. 137. caffra Macq. 153. caiennensis FABR. 232. calamodytes Schin. 330. calceata Lw. 33. calceolatus Big. 97. calens L. 232. calidris Eversa. 303. calidus WALK. (Chrys.) 183. calidus WaLk. (Tab.) 232. californica Big. 169. ealifornicus Mart. 232. caliginosa WALK. 169. caliginos us BeLL. 232. callicera Big. 232. callidus O.-S. 183. callosus MAcQ. 232. caloceps Big. 66. calogaster Schis. 183. calopsis Big. 232. calopodata Big. 138. caloptera O.-S. 92. calopterus Hine. 184. calopterus Schis. (Chrys.) 320.

calopterus Schiv. ( $T a b) 232$. calopus Big. (Chry.) 99. calopus Big. (Strat.) 49. calopus Lw. 88. calva WIED. 30. camaronensis BIG, 232. campechianus Towss. 232. cana WALK. 202. canadensis Lw. 22. canadensis WALK. 66. canariensis MACQ. 298. canifrons WALK. 184. canina PANz. 86. canipalpis Big. 232. canus Karsch. 232. capensis WaLK. 22. capensis Wied. 232. capnopterus WIED. 303. Capreolus Wied. 215. captonis Mart. 232. carabaghensis Pontsch. 233.

carbo MacQ. 233. carbonaria BeLI. 41. carbonaria Phil. 135. carbonarius G.-T. 17. carbonarius Lw. 22. carbonarius Meig. 233. carbonariusWALK.(Chrys.) 184. carbonarius WaLK. (Chrys.)

184.

carbonatus MACQ. 233.

Cardinalii Bezzi, 22. carinata MACQ. 66. carinifacies MACQ. 66. carnea Big. 169. carneus BeLL. 233. carneus WALK. 22. carnifex Gerst. 66. carolinensis MACQ. 233. Cartercaui Goв. 303. castanea MACQ. 89. castanea Perty. 153. castaneoventris MACQ. 233. 
castaneus Big. 233. castaneus Macq. 233. catenatus O.-S. 233. catenatus WALK. 233. caucasica Pleske. (Las.) 30. caucasicaPteske.(Strat.)49. caustica O.-S. 153.

Cavannae Bezzr. 303. celer O.-S. 184.

cellulata Brullé. 153.

celyphoides WaIK. 12. cenisia Meig. 50. centralis Lw. 33. centron Marten. 233. cephalonica STr. 66. ceras Towss. 184. cerastes O.-S. 233. Cerberus WALK. 233. cereolus Big. 233. ceria Will. 118. ceriferus JAENN. 100. ceriogaster WILL. 118. cerioides WaLK. 118. cervicornis FABR. 215. Cervus Wied. 146. ceylonicus Schis. 233. chalconota Brau. 98. chalybea MEIG. 129. chalybea Wied. 46. chalybeata Forst. 122. chalybeum Pertr. 210. chamaeleon L. 50 . chamaclcon MEIG. 52. Championi O.-S. 294. chathamensis Huтt. 66 . cheliopterus Rovd. 233. chilensis Macq. 233. chinensis Thunb. 233. chionostigma O.-S. 233. chloraspis WiEd. 66. chloris WALK. 66. chlorogastra Pinu. 153. chlorophthalmus Iw. 320. chlorophthalmus MeIt. 234. Chrysis Lw. 100. chrysocoma O.-S. 147. chrysodota Perty. 43. clrysoleucus WaLK. 234. chrysophila WALK. 172. chrysopila Lw. 119. chrysopila MacQ. 153. chrysopilaeformis BEZzI. 303.

chrysopus GMeL. 299. chrysostigma Lw. 303. chrysostigma WIED. 147. chrysurus Lw. 234. Cicur HaRris. 108. ciliaris Lw. 184. cilipes B1G. 202. cilipes Macq. 234. cincta Der. 135. cincta OLIv. 66. eincticornis WALK. 184. cinctilinea WALK. 67. cinctus Big. 184. cinctus Fabr. 234. cinctus O.-S. 41. cinerarius WIED. 234. cinerascens Big. (Myc.) 175. cinerascens Big. ( $T a b$.) 234. cinerascens KING. 234. cinerascens Lw. 19. cinerascens PHiL. 315. cinerascens Rö̀. 303. cinerea Dol. 14. cinerea Johrs. 328. cinereibarbis BIG. 141. cinereofasciatus Schum. 303.

cinereus BELL. 304. cinereus Walk. 234. cinereus WIED. 234. cinereus ZETT. 304. cingularis Gửr. 116. cingulata Thunb. 153. cingulata Wied. (Chrys.) 202. cingulata Wied. (Pang.) 153. cingulatis Gó̀r. 116. cingulatus Doxov. 320. cingulatus Dur. 22. cingulatus Lw. (Ptect.) 93 . cingulatus Lw. (Rhag.) 304. cingulatus MACQ. 234. cingulatus Schis. 97. cingulatus Tiunz. 234. cingulifer WALK. 234. cinnamomeus Schis. 234. cinamoneus DoL. 234. circumdatus WALK. 234. circumfusus WIED. 234. circumscripta Lw. (Clirys.) 202.

circumscripta Lw. (Micro.) 111. citripes DuF. 138. claripennis Bra. 235. claripennis MacQ. 43. claripennis PhlL. 304. elaripennis RIc. 149. claripennis Thoms. 67. clarus Schin. 235. claruS WALK. 320. clausacella MacQ. 235. clausus MACQ. 235. clavata MACQ. 169. clavata WALK. 114. clavicornis EGG. 46. clavicrus Tномs. 184. clavipes L. 123. clavipes Meig. 123. clavis W'ILL. 114. claviventris RoND. 101. clypeata BIG. 67. coarctata Macq. 119. coarctata WIED. 214. coarctatus MacQ. 101. cochaerens WALK. 235. cocrulans Wied. 129. coerulea Big. 108. coerulea MAcQ. (Cyph.) 43. coerulea MACQ. (Das.) 315. coemuleicollis MEIG. 101. coeruleifrons Johrs. 101. coerulescens MACQ. 235. coerulescens Schis. 124. coerulescens VILL. 101. coffeatus MAcQ. 235. cognatus Lw. 235. collaris BRUN. 33. 
collaris Pril. 153. colombensis Mace. 235. colon Thuns. 235. coloradensis BrG. (Chrys.)

184. coloradensis Bia. (Chrys.)

184.

comastes WILL. (Ap.) 149. comastes WilL. (Tab.) 235. comata Big. 328. combustus Big. 235. comes WALK. 235. comitans Wred. 235. comma WILL. 67. commixtus WALK. 235. compactum WALK. 213. compactus Aldr. 235. compedita Wied. 136. complens WaLK. 93. completus WALK. 235. comprehensa WALK. 146. Comstocki WiLl. 119. c गncavus Lw. 184. concentralis WALK. 202. concinna Auct. 52. concinna MeIG. 52. concinna WiLL. (Ath.) 299. concinnaWilt. (Herm.) 119. concinnata WiLL. 67. concinnatus WILL. 97. concinnus O.-S. 101. concinnus WILL. 93. concisus WALK. 101. concolor WALK. (Diat.) 169. concolor WaLK. (Tab.) 235. concurrens WALK. 235. confertissima WALK. 52 . confinis WALK. 235. confinis ZETT. 236. confirmata WALK. 32. confligens WALK. 236. confluens Lw. (Chrys.) 184. confluens Lw. (Silv.) 178. conformis WALK. 236. Confucius MAcQ. 236. confusa Rossi. 67. confusus W WALK. 236. conica Big. 175. conica FABr. 52. conicus BIG. 236. conifera WALK. 32. conjuncta WALK. 153. conjungens Rutre. 304. conjungens WALK. 153. connexa Hunt. 52. connexa WALK. 67 . connexans RIc. 236. connexus Lw. 184. comnexus WALK. 236. conopsoides Scuin. 93. consanguineus MACQ. 236. consanguineus Schin. 320. consequa WALK. 236. consobrina Bıg. 16. consobrina MAcQ. 67. eonsocius WALK. 236. conspicuus MeIG. 304. conspicuus Scmn. 304. constans Lw. 52. constans WALK. (Diat.) 169. constans WALK. (Tab.) 236. constricta WALK. 52. contactus WALK. 236. conterminus WALK. 236. contigua WALK. 163. continua WALK. 5. contractus WALK. 101. convergens WALK. 211. convexa WALK. 3. convexa WULP. 52. Coprates WALK. 41. Coquillettii Hine. 184. coracinus Phil. 236. corax Lw. 236. cordiger Meig. 236. cordigera B1G. (Chrys.) 202. cordigera Big. (Chrys.) 202. coriaceus G.-T. 97. Cormasa WALK. 7. cornuta WALK. 153. coronata GUẺR. 67. corone O.-S. 237. correctus O.-S. 320. corsicanus MacQ. 237. costalis WALK. 43. costalis WIED. 237. costata Lw. 176. costatus Fabr. 185. costatus Lw. 304. cothurnata Big. 12. cothurnatus Big. 23. crabro O.-S. 119. crassa WALK. 169. crassicornis Payz. 328. erassicornis WAHLBG. 202. crassicornis W1ED. 237. crassicomis WulP. 185. erassipalpis MACQ. (Cad.)

147.

crassipalpis MACQ. (Pant.) 296.

crassipes Fabr. 209. crassipes MElr. 299. crassus Lw. 23. crassus WALK. 237. Craverii BeLL. 237. crenatus EgG. 23. cribellum O.-S. 237. cristatus FABR. 320. crocata JAENn. 163. croceicornis Big. 315. Crotchi O.-S. 33. crucians Wied. 185. cruciata MACQ. 67. erucigerus COQuill. 17. Crucis Fabr. 141. crudelis WIED. 185. cruenta CoQ. 328. cuchix Ric. 185. cuclux Wнiт. 185. cuneatus Lw. 178. cupraria Scop. 109. cuprarius Fall. 101. cuprarius I. 101. cuprea Hute 124. cuprea WALK. 122. cuprina WIED. 52. cursim Wніт. 185. curvipalpis Bis. 143. curvipes FABR. 211. cyanea FABR. (Cyph.) 43. 
cyanea Fabr. (Micr.) 111. cyanea MacQ. 43. cyanea WIED. 43. cyaneiventris Zетт. 111. cyaneoviridis Macq. 237. cyaneum WALK. 210. cyaneum WIED. 210. eyaneus MAcQ. 237. cyanifrons RoNd. 93. cyanispinis BIG. 43. cyanocephala Gerst. 43. cyanops Brau. 237. cylindraceusO.G.Costa.297. cylindrica FABr. 298.

cylindricornis WILL. 62. eymatophorus O.-S. 238. cypria Pleske. 52. Dahlii Merg. 19. damicornis FABR. 215. Darwini RIc. 148. debilis Walk. (Sarg.) 103. debilis WaLk. (Sarg.) 103. decemmaculatus O.-S. 17. decipiens GuÉr. 33. decipiens Lw. 178. decisus WaLK. (Rhag.) 304. decisus WaLk. (Silv.) 178. decora WALK. 203. decora WIED. 67. decora WiLl. 136. decorus Isw. 238. decorus MACQ. 178. decorus SAy. 103. De-Filippii BeLl. 238. Degeeri Macq. 297. delicatulus O.-S. 185. dentuta Big. 52. dentata MeIg. 86. dentatus Becк. 23. denticornis WIED. 176. depressa MacQ. 163. depressus WaLk. 238. derelictus HARR. 304. derivatus WALK. 238. desertus WALK. 238. despecta WALK. 144. detersus WALK: 238. detracta WALK. 30. Deyrollei Big. 238. diadema FABr. 320. diademata Big. 52. diaphana Schin. 153. dichroum Schis. 99. difficilis WIED. 238. diffusa $W_{A L K} 67$. dilatata JAENN. 163. dimidiata Lw. 153. dimidiatus Iw. 304. dimidiatus MEIG. 238. dimidiatus Wulp. (Chrys.) 185.

dimidiatus WulP. (Silv.) 178.

diminutus WALK. 238.

Dimmocki Hıne. 185. diphysoides WALK. 12. directa WALK. 172. discalis Lw. 52. discalis WILLIST. 185 . discifer Big. 238. discifer WALK. 238. discolor Lw. (Eul.) 67. discolor Iw. (Tript.) 316. Discus WIed. 238. dispar Big. 315. dispar Brau. 117. dispar FABR. 186. dispar Macq. 86. dispar Schis. (Cyph.) 43. dispar Schns. (Micr.) 111. dispar Wulp. 320. disparilis BERgr. 316. dispecta Walk. 144. dissectus Lw. 186. dissimilis BeLl. 68. dissimilis G.-T. 97. dissimilis Lw. 23. dissimilis WALK. 316. distigma MEIG. 305. distincta Schrs. 149. ditaenia WIED. 238. ditaeniatus MACQ. 238. diurnus WALK. 238. divaricatıs Lw. 186. diversipennis WAIK. 238. diversipes MacQ. 211. dives Lw. (Chrys.) 320. dives Lw. (Herm.) 33 . dives MacQ. 173. dives Roxp. 238. dives WALK. 213. dives WILL. 169. divisa WALK. 164. divisuS WALK. 186. Dodgei Wнгт. 238. Doleschalii Big. 93. doreicus WALK. 238. dorsalis Coquill. 178. dorsalis Fabr. 68. dorsalis Latr. 153. dorsalis Lw. 203. dorsalis Macq. 153. dorsalis SAY. 128. dorsalis WaLK. 68. dorsifer WALK. 238. dorsig'er Wied. 238. dorsilinea WIED. 238. dorsivitta WALK. (Tab.) 239. dorsivitta WALK. (Tab.) 239. dorsobimaculatus MACQ. 239.

dorsoguttata MaCQ. 169. dorsomaculata MACQ. 169. dorsomaculatus MAcQ. 239. dorsonotatus MAcQ. 239. dorsovittatus MAcQ. 239. dubia Rö̀. 88. dubia ZETr. 129. dubius Macq. (Chrys.) 320. dubius Macq. (Tab.) 239. Dufourii PERR. 297. duplex WaLK. 239. duplicata Lw. 203. duplovittatus Rond. 239. Eatoni Ric. 239. ebrius O.-S. 239. Echemon WaLK. 115. edentula Wied. (Las.) 30. edentula Wred. (Rhin.) 176. edentulus Macq. 239. Edeta WALK. 332. 
Eggeri Schis. 239.

Eiseni Towss. (Apat.) 149. Eiseni Towss. (Herm.) 119. elegans Big. 175. elegans KARSCH. 302. elegans Lw. 103. elegans MAcQ. 68. elegans Perty. 115. elegans PHIL. 148. elegans Schin. (Act.) 129. elegans Schin. (Chrys.) 320. elegans Thuns. 239. elegans WiEd. 17. elegans Will. 18. elongata O.-S. 138. elongata SAY. 316. elongata St. F. \& SERv. 203. elongata WIED. 3. elongatulus WiLL. 18. elongatus FABr. 94. elongatus MACQ. 239. elongatus WIED. 239. emarginata MACQ. 68. Endymion O.-S. 239. engadinensis JAENs. 239. engadinica JAENN. 33. ensata BIG. 175.

ephippium FABR. 14. ephippium ZETT. 305. epistates O.-S. 239. equestris MEIG. 52. equestris Pleske. 53. equestris WuLP. 240. equorum FABR. 203. Erberi BRAU. 240. erebus O.-S. 240. eriomera MacQ. 164. eristaloides MACQ. 176. eristaloides WALK. 148. errons FABR. 141. erratica WALK. 173. erraticus BIG. 240. erythraeus Big. (Tab.) 240. erythraeus Big. (T'ab.) 240. erythrocephalus BiG. 240. erythrocephalus WuLP. 240. erythrocera EGG, 53 . erythrocera Pleske. 53. erythroletus WALK. 240. erythronotata BIG. 175. erythroplithalmus Lw. 321. Esenbeckii WIED. 180. euchlora Gerst. 68. €upodata Big. 132. eutaeniatus B1G. 240. exaestuans L. 240. exagens WALK. 240. excitans WALK. 186. exclamationis GIARJ). 240. exclusus PAND. 240. excocta WALK. 68. exeuns WALK. 169. exigua Wulp. 12. explicatús WALK. 240. expollicatus PAND. 240. expulsus WaLK. 240. exstincta WIED. 214. extremis DaY. 68. extricans WALK. 240. exul O.-S. 240. exul WALK. 68. exulans ERICHs. 240. facialis Towns. 186. facialis WALK. 240. factiosus WALK. 240. fallax Johss. 68 . fallax Macq. (Tab.) 241. fallax MAcQ. (Tab.) 241. fallax O.-S. 186.

Fallenii HaL. 332.

Falléni Perty. 92. Falleni Staeg. 33.

Fallottii Kriechb. 200.

fascialis Rıc. 186. fasciata FABR. (Eur.) 19. fasciata FABR. (Strat.) 53. fasciata LATr. 153.

fasciata Lw. 330. faseiata MAcQ. (Eul.) 68. fasciata MACQ. (Pang.) 153. fasciata MACQ. (Pong.) 153. fasciata $S_{A Y} .138$.

fasciata Thoms. 89. fasciata WALK.(Dichel.) 215. faseiata WaLk. (Erinna) 136. fasciat? WIED. 115. fasciatifrons Arrib. 23. fasciatus BraU. 89. fasciatus DEG. 241. fasciatus FABr. (Meros.) 97. fasciatus FABR. (Tab.) 241. fasciatus Ourv. 23. fasciatus SAY. 321. fasciatus WIED. 186. fascifrons MAcQ. 68. fascipennis Fabr. 19. fascipennis MacQ. (Chrys.) 187.

fascipennis MAcQ. (Pang.) 154.

fascipennis MACQ. (Tab.) 241.

fuscipennis Will. 298. fascipes WALK. 43. fasciventris Lw.(Dial.) 316 . fasciventris Lw.(Herm.) 34 . fastuosa Gerst. 5. fastuosus Big. 85 . Felderi Brac. 7. Felderi Wulp. 241. felina Panz. 49. femoralis Lw. 299. femoralis MEIG. 129. femorata BELL. 69. fenestra WILL. 241. fenestrata Grrst. 53. fenestrata MAcQ. (Adox.) 19. fenestrata MACQ. (Cyph.) 44. fenestrata MQ.(Ereph.) 164. fenestrata Meis. 119. fenestrata Tномs. 69. fenestratus FABR. 187. fenestratus LATr. 187. fenestratus Macq. 241. fenestratus WALK. 241. fera Coq. 329. fera WILL. 173. ferreus WaLK. 241. ferrifer WaLk. 241. ferrugatus FABR. 211. ferruginea Gerst. 1. 
ferruginea MacQ. 154. ferruginea MEIG. 154. ferruginea Scor. 141. furugineusI) on.(Chrys.) 321 ferrugineus Dol. (Ptect.) 94. ferrugineus Meia. (Tub.) 241. ferrugineus MeIG. $(T a b) 241$. ferrugineus P. B. 241. ferrugineus Thusв. 241. ferruginosus WaLk. 241. ferruginosus WIED. 321. ferus Scop. 241. fervens L. 241. fervidus WALK. $2+1$. Festae G.-T. 147. festinans WALK. 16. festiva WaIK. 53. festivus JAENn. 103. festivus Wied. 241. festivus WrLl. 97. fezianus Big. 242. figlinus O.-S. 94. filia WaLk. 305. filiformis G.-T. 114. filiolus WILL. 242. filipalpis Macq. 124. filipalpis WILL. 154. finalis WaLK. (Eul.) 69. finalis WALK. (Tab.) 242. fixissimus WALK. 187. flammans WALK. 242. flava DAy. 69. flava Meig. $15 t$. flava WiEd. 215. flaveola Big. 92. fiaveolus Bı. 302. flaveolus MEIG. 321. flavescens Thunb. 242. flavescens. Ric. 154. flavibarbis ADAMS. 321. flavibarbis Macq. 242. flavicans ZeLL. 242. flaviceps BıG. 94. flaviceps Mace. 69. flaviceps WALK.' 20. flaviceps Znт. 242. flavicornis Johns. 23. flavicornis Mace. 305 . flavicornis MeIt. 111. flavicornis Oı.IV. 69. flavidus Big. 321. flavidus Hine. 242. flavidus Wied. 187. flavifacies MACQ. 242. flavifasciata MACQ. 70. flavifrons MACQ. 53. flavimana Gerst. 44. flavimedius CoQ. 305. flavipalpis Adams. 329. flavipalpis MAcQ. 70. flavipennis MacQ. (Diachl.) 212.

flavipennis MAcQ.(Ptect.)94. flavipes ADAMs. 176.

flavipes BIG. 4.

Alavipes O. G. Costa. 9. flavipes DoL. 138. flavipes FABR. (Adox.) 20. flavipes FABR. (Ather.) 299. flavipes Lw. (Herm.) 34. flavipes Lw. (Pteet.) 94. flavipes Macq. (Beris) 124. flavipes MACQ. (Camp.) 116. flavipes MacQ. (Pang.) 154. flavipes MacQ. (Pant.) 294. flavipes Meig. (Chrys.) 187. flavipes Mrig. (Sarg.) 103. flavipes Wied. (Herm.) 119. flavipes Wied. ( $T a b) 242$. flavispinis Macq. 44. flavispinosa MAcQ. 124. flavissima Rossi. 70. fiaviventris BIG. $2+2$. flaviventris $\mathrm{L} / \mathrm{w} .53$. flaviventris MAcQ. (Chrys.) 187.

flaviventris MAcQ.(Tab.)242 flaviventris WIED. 112. flavocinctus BELL. 242. flavocinctus Ric. 187. flavofemorata Mera. 129. flavogeniculata DEa. 109. flavohirta BeLl. 154. flavoliirta Ric. 149. flavolimbata A. Coste. 53. flavomarginatum Lw. 99. flavoniger Coq. 305. flavopilosum Brg. 99. flavoseutata BI(*. 119. flavoscutellata WULP. 53. flavosignata A. Costa. 71. favus Macq. 242. flexilis WaLK. 242. flexipalpis BIG. 143. florentinus Lw. 305. foedus Lw. 321. folda Coq. 322. formica O.-S. 119. formicacformis DoL. 104. formosa MAcQ. 34. formosa Scop. 109. formosa WIED. 34. formosus WALK. 242. fortis WALK. 104. foveifrons Thoms. 71. fratella WILL. 71. fratellus WILL. 242. fraterculus MAcQ. 242. fraterculus WIED. 242. fraterna Lw. 34. fraternus Big. 97. fraternus Lw. 23. fraternus MACQ. 242. Frauenfeldi Scur. 294. Frazari WILL. 187. Frenchi Mart. 243. frigidus O.-S. 187. frontalis Lw. 104. frontalis MACQ. (Chrys.)188. frontalis MACQ. (Eul.) 71. frontalis OLIv. 9. frontalis Tnous. 90. frontalis WALK. 243. frontatus Schis. 97. fronto O.-S. 243. frontosa Hine. 29. fugax 0.-S. 188. fulinigosa WALK. 154. fuliginosus Wied. 188. fullo WALK. 243. fulva BECK. 331. 
fulva MacQ. 173.

fulva PHiı. 315.

fulva Ric. 150 .

fulva WALK. 119.

fulvaster O.-S. 188.

fulvescens WALK. 243.

fulvianus Lw. 243.

fulvicuput WALK. 145.

fulviceps WALK. (Chrys.) 188.

fulviceps WALK. (Eul.) 71 . fulvicollis WaLK. 133.

fulvicornis MeIG. 243.

fulvicornis Sx. v. V. 133.

fulvidus Big. (Chrys.) 322.

fulvidus Bıf. (Pant.) 294.

fulvifascia WALK. 169.

fulvifasciatus MacQ. 243.

fulviger WALK. 243.

fulvilateralis MacQ. 243.

fulvimedius WALK. 243.

fulvipes BiG. 329.

fulvipes Lw. 154.

fulvipes PHir. 243.

fulvissimus Roxp. 243.

fulvistigma Hine. 188.

fulvithorax Big. 99.

fulvithorax WIED. 164.

fulvitibialis RIc. 164.

fulviventris Big. (Evaza) 4 .

fulviventris Big. (Solva) 138 .

fulviventris MacQ. (Pang.) 154.

fulviventris MacQ. (Tab.) 243.

fulvofrater WALK. 243.

fulvohirtum Wred. 213.

fulvulus WIED. 243.

fulvus DEG. 305.

fulvus MeIG. 243.

fumatus Wied. 244.

fumida Lw. 154.

fumifer WALK. 244.

fumifera WALK. 164.

fumipennis SAY. 322.

fumipennis WIED. 244.

fumipennis Wut.P. 244. funcbris DuF. 298. funebris MACQ. (Pang.) 154.

funebris MAcQ. (Tab.) 244.

funebris Meig. 305.

fur WILI. 244.

furcata FABR. 54.

fureata Meig. 71.

furcata WIED. 146.

fureatus Big. 244.

furcatus WALK. 188.

furcifer Wied. 92.

furens Thunb. 244.

furunculigenus Dor. 244.

furunculus WiJL. 244.

fusca G.-T. 128.

fusca Pril. 175.

fusca Ric. (Ereph.) 164.

fusea Ric. (Sci.) 150.

fusca Wied. 176.

fuscana Wied. 144.

fuscutus MacQ. 44.

fuscanipennis MAcQ. 155.

fuscicauda Bir. 244.

fuscicrura Big. 244.

fuscinervis Lw. 23.

fuscinervis MacQ. 245.

fuscipalpis Biя. 245.

fuscipennis BeLL. 42.

fuscipennis Macq. (Diachl.)

212.

fuscipennis MACQ. (Dichel.)

215.

fuscipennis Macq. (Eul.) 71.

fuscipennis MeIG. 305.

fuscipennis RIc. 188.

fuscipennis Wred. (Pang.)

155.

fuscipennis Wied. (Tab.) 245.

fuscipes Big. 322.

fuscipes Mrig. 124.

fuscitarsis SAY. 128.

fus iventris MACQ. 125.

fuscofasciatus MACQ. 245.

fusconervosus MacQ. 245.

fusconiger WaLk. 148.

fuscopunctatus Macq. 245. fuscus Ric. 188.

fuscus Wied. (Rhag.) 305.

fuscus WVIEd. (Tab.) 245.

fusiformis WaI.K. 155.

gabonensis Macr. 245.

gadensis Schin. 23.

gagates Lw. 245.

gagatina $\mathrm{B} 1 \mathrm{G} .169$.

gagatinus Pнiг. 245.

gallorum Schis. 245.

Garatas WALK. 71.

gavisa WALK. 15.

gemella WALK. 169.

gemina WALK. 164.

geminatus MACQ. 188.

geminatus WiED. (Chrys.)

188.

geminatus Wred. (Rhag.)

305.

gemma Big. 112.

gemmifer WALK. 104.

geniata WALK. 16.

geniculata CurT. 125.

geniculata Gerst. 44.

genieulata MAcQ. 119.

geniculatus Wur. 245.

Genius Panz. 322.

gentilis ERICHs. 245.

Gerkei Brav. 245.

Gerstaeckeri Bexi. 54.

gibbula WaLK. 164.

giganteus DEG. 245.

gigantulus Lw. 178.

gigas Herbst. 246.

gigas MACQ. 246.

gilanus Towns. 246.

glaber Big. 246.

glaber Lw. 23.

glaber WiED. 212.

glandicolor Lw. 178.

glaucescensScins. (Tab.)246. glaucescens Scuin. (Tab.) 246.

glaufcopis Meta. (Tab.) 246.

glaucopis Meis. (Tab.) 247.

glaucus Bia. 104.

glaucus MeIG. (Tab.) 247. 
glaucus MeiG. (Tab.) 247. glaucus WIED. 247. globicornis WIED. 212. globuliceps Lw. 23. globulifera Sснuмm. 203. Goebelii Str. 305. goniophora SAY. 54. gracilicornis Gerst. 44. gracilicornis MEG. 138. gracilis Lw. (Nem.) 23. gracilis Lw. (Sarg.) 104. gracilis WIED. 247. gracilis WilL. (Erinna) 136. gracilis WiLL. (Meros.) 97. gradiens WALK. 12. graeca SAUND. 46. graeculus Lw. 305. graecus FABR. 247. graecus Meig. 248. grandis MacQ. 203. grandis RIc. 215. grandis Schin. 89. grandis Schrank. 143. grata Lw. 34.

gratus Lw. 248. gravis Hutт. 248. gregarius ERIchs. 248. Griffithi Johns. 322. grisea JAENs. 169. grisea STr. 331. grisea Wied. 329. griseipennis Lw. 155. griseola FALL. 329. griseolus Wulp. 305. griseus PhiL. 305. grossus Thune. 248. Guerinii MACQ. (Beris) 125. Guerinii MacQ. (Eul.) 71. guineensis BIG. 203. guineensis WIED. 248. gulosa W1ED. 155. guttata Dowov. 164. guttatulus Towss. 248. guttatus OLIv. 305. guttatus WiED. 248. guttipennis WALK. 322. guttipennis WiEn. 248. guttula Wied. 188.

guyanens Hunt. 248.

guyanensis MACQ. 248.

gymnonota BRullé. 203.

habilis WALK. 8.

haemagogus Will. 248.

haemaphorus MART. 248.

haematopotoides Bra. 248.

haematopotoides JAENN.

248.

haemorrhous Lw. 23.

Halala WALK. 3.

halterata Schrsk. 71.

hamatus Lw. 188.

hamatus WULP. 249.

hastata FABR. 5.

Haterius, WALK. 116.

haustellata FABR. 155.

haustellata OLIV. 155.

hebes Walk. (Pang.) 155.

hebes Walk. (Tab.) 249.

helveolus Ginм. 322.

helvolus MeIG. 322.

heminopla Wied. 20.

hera O.-S. 155.

Herzi Pleske. 55.

heteroneura Macq. 71.

Heydenianus JaENN. 249.

Heydenii JAENN. 71.

Heydenii WIED. 294.

Heyshami Curt. 305.

hieroglyphica Gerst. 203. hieroglyphica OLiv. 71. hieroglyphica WIED. 3. Hilarii MACQ. 249. hilaris O.-S. 188. hilaris WaLK. (Sald.) 12. hilaris WALK. (Tab.) 249. Hinei Johns. 249.

himnulus WALK. 215.

hippoboscoides Aldr. 147.

hirsuta MacQ. 130.

hirsuta Thune. 155.

hirsutus VILL. 249.

liita Johns. 329.

hirticeps Lw. (Herm.) 34. hirticeps I,w. (Tab.) 249. hirticeps Now. 169. hirticornis WIED. 89. hirtioculatus MAcQ. 249. liirtipalpis Bia. 170. hirtitibia WALK. 249. hirtulus Big. (Nem.) 23. hirtulus Big. (Rhag.) 305. hirtulus Big. (Tab.) 249. hirtuosa MEIG. 55. hirtuosus Pint. 249. hirtus Lw. (Rhag.) 305. hirlus Lw. (Silv.) 178. hirtus WALK. 249. hispanica Pleske. 55. hispidus WALK. 249. histrio WALK. 72. histrio WIED. 249. hoang MacQ. 249. Hoffmannseggii WIED. 306. holosericea OLIv. 72. holosericeus Scop. 322. honestus O.-S. 133. honestus WALK. 249. hoodiana BIG. 72. hoodianus BıG. 306. hoplistes Wied. 91. hortulanus WIED. 91. hortulanus WILL. 115. Hovas Big. 100.

humeralis MEG، 249. humilis Coquill. 249. humilis Lw. 322. humillimus WALK. 249. Hunteri King. 55. hyacinthina BIG. 132. hyalipennis Hise. 249. hyalipennis Ros. 306. hyaliventris A. Совта. 131. hyalopterus G.-T. 97. hyalopterus WiED. 306. hybotoides WALK. 138. hybrida GeRst. 44. hybrida Lw. 34. hybridus Wied. 250. Hydrodromia Meig. 72. hydrolenoides JoHns. 73. Hyilroleon Halisis. 34. 
hydroleon L. 72.

hydroleonoides Jонмs. 73. hydrophila Lw. 73. hydropota MACQ. 73. Hydropota Meig. 73. hydropota WALK. 74. hyentomantis Schis. 203. hyetomantis SchrNk. 203. hyperythreus Big. 250. hypochlove Now. 74. hypoleon FABR. 34. hypoleon FALL. 34. hypoieon L. 34. hypoleon Musa. 34. hypoleon Staeg. 35. hypoleon SchrNk. 35. hypoleucus Big. 250. Ibis FABR. 299. ignobilis RoND. 250. ignotus RossI. 250. illota WiLt. 155 . illotus O.-S. 250. illucens L. 119. illucens Schin. 94. illucens WALK. 302. illustris Schis. 94. imbecillus Karsch. 250. imbrium Wied. 203. imitans WALK. (Tab.) 250. imitans WALK. (Tab.) 250. immaculata MAcQ. 215. immaculata MeIG. 329. immaculatus JoHss. 23. immaculatus Meig. 306 . immaculatus WiED. 188. immanis Wied. (Pant.) 295. jmmanis Wied. (Tab.) 250. immiscens WALK. 74. immixtuS WALK. 250. impar Rond. 188. impar WALK. (Rhag.) 306. impar WaLK. (Tab.) 250. impendens WaLK. 4. imperfectus WALK. 250. imponens WaLK. 250. importunus MaCQ. 250. importunus WIED. 250. impressus WIED. 250. impurus KaRsCh. 250. inactus WALK. 104. inaequalis Lw. 74. inamoena WALK. 138. inanimis WALK. 55. inanis FABR. 250. inappendiculata Big. 203. incerta BELL. 155. incertus MacQ. 250. incipiens WALK. 251. incisa WIED. 155. incisuralis MQ. (Beris) 125. incisuralis $\mathrm{MQ}_{\mathrm{Q}}$ (Pang.) 155. incisuralis $\mathrm{M}_{\mathrm{Q}}$ ( $\mathrm{Tab}$.) 251. incisuralis SAY. 155. incisus Lw. 306. ineisus MaCQ. 188. incisus WALK. 251. incompleta MACQ. 150. inconspicua WALK. 155. inconspicuus WaLK. 251. incultus Wurp. 251. inda Schrnk. 15. indecisus Big. 251. indiana BIG. 203. indianus Rrc. 189. indica WALK. 8. indicus FABr. 251. indicus HARR. 104. indistinctus B1G. 251. indus O.-S. 189. inermis WIED. (Chorl.) 42. inermis Wied. (Eul.) 74 . inermis WIED. (Hist.) 92. infans WALK. 251. infera WALK. 30. infestans MAcQ. 251. inficitus WALK. 104. inflata WALK. (Mass.) 117. inflata WALK. $(R u b a) 31$. inflexipalpis Big. 143. informatus Hunt. 251. infumatus Pril. 251. infuscata MeIG. 74. infuscatus Lw. 251. infuscatus Meig. 104. infusus WALK. 251.

inhambanensis BERT. 251. innotabilis WALK. 251. innotatus KARSCH. 178. innotescens WALK. 251. inornata WILL. 3. inornatus PHIL. 251. inornatus WALK. 212. inquinatus Scop. 306. inscitus WALK. (Tab.) 251. inscitus WALK. (Tab.) 251. insignis Lw. (Strat.) 55. insignis Lw. (Tab.) 251. insignis MACQ. 94. insuetus O.-S. 251. insularis Schis. 322. insularis WALK. 251. insurgens WALK. 251. intensivus Towss. 252. intereuns WALK. 252. intermedia WIED. 74 . intermedius Bezzi. 322. intermedius $\mathrm{EgG} .252$. intermedius WALK. (Rhag.) 306. intermedius WALK. (Tab.) 252.

internus WaLK. 252.

interpositus Rovd. 252. interrupta Lw. 86. interrupta OLiv. 74. interruptus Macq. 212. intersectus Fourcr. 252. intrudens WILI. 189. inutilis WALK. 306. invalidus WILL. 322. iranensis BIG. 189. iridatus Scop. 104. irritans Rrc. 178. irrorata MACQ. 203. irroratus Schis. 322. isabellina WiED. 156. ischiaca WALK. 55. ispahanicus Rown. 252. istriensis MeIG. 252. italica Mric. 204. italicus Fayr. 178. 
italicus Lw. 189.

italicus Meig. 189.

iterabilis WALK. 121.

Ixion O.-S. 252.

ixyostactes WIED. 252.

Jacksonii MacQ. 165.

jaksoniensis GuÉr. 170.

Jalemus WaLK. 74.

jamaicensis Johrs. 322.

Janotae Now. 306.

Januarii WIED. 215.

Janus WALK. 252

japonica WuLp. 55.

japonicus Big. 252.

japonicus Wred. 189.

javana MACQ. 125.

javana Wied. 204.

javanus FABr. 252.

jejuna ScHrnk. 86.

Jolmsoni Coq. 329.

Joïdus Big. 252.

jucunda J AENN. 170.

jucundus Walk. (Sarg.)105.

jucundus WaLK. (Tab.) 252.

justorius Rond. 252.

kabylinus BIG. 18.

kansensis ADAms. 24.

Kirchneri JaEnn. 74.

Koslowi Pleske. 55.

Kosnakowi Pleske. 55.

Königi Lichtw. 30.

Krausei PinL. 145.

labiata Big. 300.

lacerata Big. 55.

lacrymans Big. 252.

laeta Meis. 120.

laetus FABr. 189.

laetus Wurp. 105.

laetus ZETT. 322.

laevifrons Lw. (Strat.) 55.

laevifrons Lw. ( $T a b) 252$.

lagenaferus MACQ. 252.

Laglaisei BıG. (Herm.) 120.

Laglaisei Bıg. (Tab.) 252.

lama Portsch. 253.

lambessiana Big. 55.

Landbecki PıIı. 170. laniger Lw. 189.

laniger WIED. 253.

lanuginosa Gerst. 44.

laotianus BIG. 253.

lapidaria Now. 331.

lapponicus Lw. 189.

lapponicus WhLBG. 253.

lasiophthalma Borsd. 156.

lasiophthalma Lw: 74.

lasiophthalma WiL. (Cyph.)

44.

lasiophthalma WIL. (Cyph.) 44.

lasiophthalma WuLP. 170.

lasiophthalmus Macq. 253.

lasiops Lw. 24.

lata Gúér. 165.

lata Lw. 20.

lata IVALK. 128.

lateralis BRuLle. 253.

lateralis Duf. 24.

lateralis FABR. 173.

lateralis MAcq. (Pedic.) 114.

lateralis Mace. (Ptil.) 5.

lateralis Meig. 253.

lateralis WIED. 189.

lateremaculata MAcQ. 74 .

laterina Rond. 165.

lateritius Rond. (Sarg.) 105.

lateritius Rond. ( $T a b$.) 254.

laticeps Hine. 254.

laticeps Lw. 55 .

laticornis HrNe. 254.

latifaciata Macq. 74.

latifascia WALK. 94.

latifasciata MAcQ. 74 .

latifasciatus BELL. 189.

latifrons Bezzi. 323.

latifrons WiLL. (Chrysoch.) 100.

latifrons WiLL. (Chrysop.) 323.

latifrons ZETT. 254.

latipalpis Big. 329.

latipalpis MAcQ. 170.

latipennis BeLL. 300.

latipennis Lw. 306 . latipes MACQ. 254.

latistriatus BraU. 254.

latiusculus Lw. 24.

lativentris BELL. 120.

lativentris $\mathrm{J} / \mathrm{w} .55$.

lativentris MacQ. 254.

lativentris WILL. 120.

lativentris WULP. 10.

lativitta WALK. 254.

latus BeLL. 105.

lauta Lw. 316.

Leachii Curt. 10.

Lefeburei BELL. 75.

Lefebvrei MacQ. 75.

leiophthalma WiLL. 42.

leonina Panz. 35.

leoninus Rond. 94.

leonum Big. 254.

lepidota Wied. 210.

leptiformis Kent. 323.

leptiformis Mace. 144.

leptis O.-S. 143.

Lerda WALK. 170.

Letourneuxi Big. 254.

leucaspis WInd. 254.

leucaspis WuLP. 254.

leucocephala Wied. 44. leueocephalus Веск. 24.

leucocnematus Big. 254.

leucomelas WaLK. 254.

leucomelas WIED. 156.

leисора I. 120.

leucophilus WALK. 254.

leucophorus BiG. 254.

leucopicta BIG. 173.

leucopogon Bus. 254.

leucopogon WIED. 165.

leucopsis WIED. 55.

leucopterus WulP. 255.

leucorrhynchus A. Costa. 24.

leucosparsus Big. 255.

leucospilus WIED. 189.

leucostomus Lw. 255.

leucothorax Ric. 170 .

Liburna WALK. 35.

liburnicus WIED. 255. 
lifuensis Big. 255.

ligatus WaLK. (Chrys.) 190. ligatus WaLk. (Tab.) 255. ligens HữT. 156.

Limae Gứr. 75.

limata Coq. 329.

limbata BIG. (Beris) 125.

limbata BIG. (Chrys.) 205.

limbata Lw. 35 .

limbata..MAcQ. 75.

limbata O.-S. 300.

limbata WIFD. 75.

limbatinervis MacQ. (Tab.) 255.

limbatinervisMQ.(Tab.) 255 .

limbatus Bit. 255.

limbatus EGG. 24.

limbatus MacQ. 105.

limbahus P. B. 255.

limbifacies $\mathrm{BI}$. 75 .

limbinervis MACQ. 165.

limbinervis O.-S. 116.

limbipennis BIG. 317 .

limbipennis MACQ. 75.

limbipennis W ULP. 10.

limbithorax MACQ. 170.

limbiventris Macq. 116.

limbrocutris AdAvs. 18.

limonus Towss. 255.

limpidapex WIFd. 255.

linearis WALK. 114.

lineata Fapr. (Pang.) 156.

lineata FABr. (Strat.) 55.

lineatus FABr. 255.

lineatus JaEns. 190.

lineola FABr. (Rhag.) 30T.

lineola FABr. (Tab.) $25 \%$.

lineola P. B. 256.

lineolata MAcQ. 56.

lingens WIED. 156.

litigiosus WAIK. 256.

lividus WaLK. 256.

locuples Lw. 35.

Lomnickii Mrk. 24.

longa WiEd. 3.

longiappendiculatus MACQ. 256. longiıauda BIG. 148.

longicornis DALE. 35.

longicornis FABR. 214.

longicornis Lw. 136.

longicornis MCQ. (Chrysop.) 190.

longicornis MACQ. (Chrysoz.) 205.

longicornis PIIL. 125.

longicornis Schis. 132.

longicornis Scop. 47.

longicornis WALK. 3.

longicornis WiLL. 117.

longifrons Rovd. 56.

longipalpis MACQ. 173.

longipennis MACQ. 256.

longipennis Rıc. 170.

longipennis WIED. 105.

longipes Bell. 300.

longipes Lw. 301.

longipes WALK. 105.

longirostris Big. 178.

longirostris HARDw. 173.

longirostris MACQ. 165.

longirostris WIED. 24.

longiseta WALK. 116.

longitudinalis $\mathrm{L} w \mathrm{w}, 256$.

longus O.-S. 256 .

lucens Lw. 114.

lucida Gerst. 2.

lucidulus WaLK. 256.

lucifer ADANs. 323.

lucifer WALK. 323.

luctifera Phic. 125.

luctuosus MacQ. 256 .

ludens Lw. (Chrysopilus) 323.

ludens Lw. (Chrysops) 190.

lugens Lw. 136.

lugens PHIL. (Rhag.) 307.

lugens PHIL. (Tab.) 256.

lugens WiED. 190.

lugubris L. 190.

lugubris Lw. 56.

lugubris MACQ. (Ath.) 301.

lugubris Macq. (Chrys.)190.

lugubris Macq. (Pang.) 156. lugubris MacQ. (Tab.) 256.

lugubris WALK. 13.

lugubris ZетT. 256.

lunata OuIv. 76.

lunatus FABr. 256.

lunatus GraY. 190.

lunulata MACQ. (Chrys.) 205.

lumulate MACQ. (Eul.) 76.

lumulatus Bis. (Tab.) 257.

lunulatus Big. $(T a b) 257$.

lunulatus Meig. (Tab.) 257.

lumulatus Meis. (Tab.) 257.

lunus Big. 115.

lupinus O.-S. 323.

lupus Whiт. 190.

lurida WaLK. 156.

luridus FAli. 257.

luridus Lw. (Meros.) 97.

luridus Lw. (Tab.) 257.

huridus MeIs. 323.

luridus WALK. 94.

lusitanica Gưr. 205.

Lutatius WALK. 76 .

luteicornis Ega. 24.

luteipes WiLI. 131.

luteiventris Puा. 125.

luteoflavus BeuL. 25T.

luteolus Fat.L. 32:3.

luteolus Lw. 257.

lutulentus Huтt. 149.

macer BIG. 257.

Macquarti Gứr. 156.

Macquartii Perty. 105.

Macquarti Schis. 257.

macroceratus Bia. 257.

macrodonta Mace. 258.

macroglossa Westw. 156.

macroleon Panz. 143.

macrophthalmusSchiv.258.

macroporum MACQ. 166.

macrops WALK. 258.

mactans WALK. 105.

macula FArr. 112.

macula MAcQ. 258.

macularis FABR. 258.

maculata FABr. (Cyph.) 44.

maculata Fabr.(Pang.) 156. 
maculata LATr. (Herm.) 35. maculata Latr. (Solva) 138. maculata MEIG. (Ath.) 301. maculata MEic. (Herm.) 35. maculata Meig. (Solva) 138. maculata Rossi. 157.

maculata WIED. 44. maculata ZETT. 35 . maculatissimus Macq. 258. maculatus DeG. (Rhag.) 307. maculatus DEG. (Tab.) 258. maculatus Scor. 308. maculatus WiLt. 94. maculicornis Hise. 10. maculicornis ZETT. 258. maculifer Big. (Rhag.) 308. maculifer Big. (Tab.) 258. maculifrons WALK. 76 . maculifrons WuLP. 76. maculinervis MACQ. 258. maculineuris MacQ. 258. maculipennis Btaxcri. 125. marulipemis Brulut. 258. maculipennisLw.(Negr.)16. maculipennis Iw. (Rhag.)

308.

maculipennis $\mathrm{M}_{Q}$. (Ereph.) 166.

maculipennis Macq. (Negr.) 16.

maculipennis MAcQ. (Pel.) 148.

maculipennis MAcQ. (Tab.) 258.

maculipennis $\mathrm{M}_{\mathrm{ACQ}}$. (Tab.) 258.

maculipennis Scrus. 150. maculipennis Troms. 148. maculipennis WALK. 323. maculipennis WIED. 259. maculiplena Karsci. 205. maculiventris BIG. 24. maculiventris MACQ.

(Neoex.) 132.

maculiventris MACQ. (Tab.) 259.

maculiventris RoND. 90. maculiventris Westw. 157. maculosa Lw. 56. maculosus Coq. 259. madagascarensis Ric. 190. maerens Lw. 323. magellanicus Рнгц. 259. magnificus Big. 106. magnificus WALK. 295. Magrettii Bezzi. 157. major Eversu. 259. major Hine. 18. major SchrNk. 143. majuscula Lw. 331. maletectus Bir. 259. mallophoroides WALK. 259. mandarinus Schis. (Sarg.) 106.

mandarinus Schus. (Tab.) 259.

manens WALK. 36. manifestus WALK. 259. manilensis Scmus. (Chrys.) 190.

manilensis Scmin.( $T a b) 259$. Mannii Мгк. 30.

maoriorum BiG. 259. maorium Hutr. 259. Marchalii PIER. 308. margaritifera WIED. 166. marginalis FABR. 259. marginalis Lw. 56. marginalis WALK. 214. marginalis WIED. (Pang.) 157.

marginalis Wied. (Tab.) 259. marginata FABR. (Ath.) 301. marginata F^BR. (Fud.) 116. marginata FABR. (Eul.) 76. marginataFABr.(Pang.)157. marginata Lw. (Cyph.) 44. marginata Lw. (Herm.) 36. marginata MAcQ. 216. marginata MeIG. 139. marginatus FABR. 24. marginatus MACQ.(Silv.)178. marginatus $\mathrm{M}_{\mathrm{Q}}(T a b)$.259 . marginatus MEA. 308.
marginatuS WALK. 178. marginatus Wur.P. 106. marginella MacQ. 76. marginellus FaLL. 24. marginellus Gues. 24. marginenervis Msce. 259. marginula MEa. 205. marinus BECK. 24. maritimus Sсор. 190. maritimus Towss. 259. marmorata GERst. 3. marmoratus Big. 259. marmoratıs Rossi. 190. maroccanus Big. 179. maroccanus FABR. 259. maura M. V. 329. mauritana I. 158. mauritanica Merg. 158. mauritanicus A. Costa.191. maurus Siebke. 191. media WAI.K. 166. megacephala Lw. 76. megacephala Ouıv. 76 . megacephalus JAENx. 259. megaceras BeLL. 191. megalops WALK. 259. Megerlei Wind. 260. Meigenii Staeg. 36. Meigenii Wied. 56. melaena Meic. 329. melampogon ZELL. 110. melanciesiae Big. 120. melanarius WALK. 117. melancholia HARR. 301. melanocerus WiED. 260. melanodactyla I,w. 36. melanognathus BiG. 260. melanopleurus Whнвg. 191. melanopogon GERM. 110. melanopsis WIED. 56 . melanopterus Hixe. 191. melanopyga WIED. 147. melanopygatus BiG. 260. melanorhinus BıG. 260. melanostoma Lw. 56. melanostoma Prit. 260. melanurus WALK. 94. 
melas Big. 146.

melastoma Lw. 56.

Melicharii Mrк. 191.

melleus RoNd. 106.

mentitus WALK. 260.

meridianus Rond. 260.

meridionalis BEzzI. 323.

meridionalis Thunв. 260.

meromelaena Aust. 10.

meromelas Duf. 10.

merula PHiL. 191.

metallica WIED. 36.

metallinus FABR. 106.

meteorica Conts. 205.

metidjensis MacQ. 260.

mexicana Beis. 125.

mexicana G.-T. 128.

mexicana Jonss. 76.

mexicantis BeI.L. 323.

mexicanis L. 260.

micans Hutr. 126.

micans Meic. (Pang.) 158. micans Mrig. (Tab.) 261.

microcephalus O.-S. 261.

microcerus WALK. 261.

microdonta MacQ. 261.

microleon I. 76.

microstoma Lw. 7 .

Mikii Brav. 261.

Mikii March. 298.

Mikii WILL, 333.

Miles Wien. 261.

minima Ric. 170.

minimus WULP. 261.

ninimus Zетт. 106.

minor MacQ. (Ereph.) 166.

minor MacQ. (Tab.) 261.

minos Scuin. 262.

minuta FABR. 77.

minuta WILL. 5.

minutissima Zетт. 11.

mipartita MAcQ. 92.

misera O.-S. 150.

missionum MacQ. 262.

mitidjensis MaCQ. 262.

mitis O.-S. 191.

Mlokosiewiczi Bıg. 191. modesta CoQ. 330.

modesta Phil. 126.

modestus Lw. (Chrys.) 323.

modestus Lw. (Nem.) 24.

modestus WIED. 262.

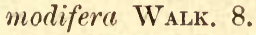

moechus O.-S. 191.

moerens WALK. 191.

molesta WIED. 158.

molestissimus PнIL. 262.

molestus Guér. 192.

molestus SAY. 262.

molestus WiED. 192.

mollis O.-S. 4.

Monachus Harr. 308.

monilifer Big. 262.

monochroma WIED. 262.

monoculus Dot. 262.

Monogramma Wien. 262.

monos Harr. 330.

monotaeniatus Bra. 262.

momotropus HaRr. 308.

montana Hutr. 173.

montanus Meig. (Tab.) 262.

montanus Mela. (Tab.) 263.

montanus O.-S. 192.

monticola EGa. 308.

monticula EGg. 308.

morio FABR. 263.

morio L. 263.

morio Wutp. 170.

morio ZETT. 192.

morosus O.-S. 192.

Morrisii Curt. 37.

Morrisii Date. 126.

mueens R. \& H. 120.

Miillfeldi Brat. 263.

multifaria WALK. 158.

multifascia WАLK. 216.

multifasciata Rrc. 216.

multipunctatus WUı. 263.

munda O.-S. 117.

murina PHIs. 175.

muscaria FABR. 37.

muscaric Paxz. 3T.

muscaria WALK. 37.

mutabilis ADAMs. 18. mutabilis FABR. 56. mutabilis WILL. 57. mutatus WALK. 263. mutica WulP. 77. muticus FABR. 24. muticus ScHrnk. 24. mycroleon HaRr. 77. mystaceus MAcQ. 308. Namaquinus Bif. 263. nana BELL. 3. nana Lw. (Bolb.) 140. nana Lw. (Herm.) 37. nana WALK. 166. nanus MacQ. 263. nanus Wied. 263. nanus WiLL. 323. nasica WiLI. 19. nasuta MACQ. 62. natalensis Gerst. jo. natalensis MacQ. 115. natalis MACQ. 192. nebulosa FABr. 330. nebulosus Deg. 263. nebulosus P. B. 263. neglectus WiLL. 192. nemoralis Mear. (Tab.) 263. nemoralis MeIG. (Tub.) 264. nemoralis MeIG. (Tab.) 264. nemoralis Phil. 308. nemoteloides Big. 6. neocaledonica Múgs. 173. nephodes Bia. 264. nepos Walk. 264. Nevadae Bic. 57. nexura WALK. 85. nexus WALK. 264. nicobarensis Schis. 264. nilliceps Hunt. 77. niger BFLL. 323. niger Big. (Eup.) 18. niger BIG. $(\mathrm{Nem}$.) 24. niger Dowov. 264. niger MacQ. 192. niger P. B. 264. niger WALK. 192. niger Wind. (Cac.) 89. niger W'ien. (Rhag.) 308. 
nigerrima DoL. 15. nigerrima Lw. 77. nigervimus ZЕTT. 264. migra DАY. 77. nigra MAcQ. (Herm.) 37. nigra MacQ. (Tincla) 8. nigra MeIG. (Beris) 126. nigra MeIG. (Span.) 332. nigra Ric. 166. nigra Scrin. 146. nigra WIED. 205. nigra WiLL. 42. nigra Zетт. (Pti.) 331. nigra ZETT. (Pti.) 331. nigratus Рнгц. 309. nigrescens P. B. 264. nigrescens Rıc. 148. nigribarbis Bıg. 106. nigribimbo ALDR. 192. nigricans EGG. 264. nigricans Lw. 176. nigricans WIED. 264. nigricauda Beling. 324. nigriceps $\mathrm{BIG} .77$. nigricorne Lw. 100. nigricornis BıG. 173. nigricornis Goв. 205. nigriconnis LATR. 37. nigricornis ZетT. 264. nigrifacies Bra.(Herm.) 120. nigrifacies Big. (Myct.) 175. nigrifacies Gob. 264. nigrifemoratus MACQ. 97. nigrifer WALK. 264. nigrifrons Lw. 25. nigrifrons Phul. 264. nigrifrons WALK. 57. nigrilimbo Wнгт. 192. nigrimanus WALK. 264. nigrina WHLBG. 331. nigrinus FALI, 25. nigrinus WAND. 133. nigrinus WuLP. 25. nigripalpis MaCQ. 264. nigripalpus Lw. 133. nigripennis BIG. 25. nigripennis Gứr. 158. nigripennis PHI. 265. nigripes Macq. 77. nigripes MeIG. 130. nigripes Rö̀. 166. nigripes VerR. 37. nigripes WIED. 265. nigripes Zетт. (Chrys.) 192. nigripes ZЕTT. (Pti.) 331. nigripes ZEтT. (Sarg.) 106. nigrirostris Lw. 78 . nigrita FABR. 265. nigrita FALI. 78. nigrita Hunt. 309. nigrita MEIG 265. nigritarsis DoL. 301. nigritarsis LATR. 126. nigritibialis MAcQ. 139. nigritus FABR. 324. nigritus MeIG. 25. nigritus Panz. 25. nigriventris $\mathrm{BIG} .193$. nigriventrisLw.(Chrys.)193. nigriventris Lw.(Herm.) 37 . nigriventrisLw.(Rhag.)309. nigriventris Lw.(Strat.) 57. nigriventris $\mathrm{MACQ}_{\mathrm{A}} .265$. nigrivittata MACQ. 166. nigroaeneus VERH. 25. nigrocaeruleus Rovd. 265. nigrodentata MEIG. 57. nigrofasciatus MEjG. 309. nigrofemorata WILI. 128. nigrolirta WALK. 166. nigrohirtus RIc. 265. nigromaculatus Ric. 265. nigronotata MACQ. 158. nigropictus MACQ. 265.

- nigroptera Fourcr. 126. nigropunctatus BELL. 265. nigrosignata Тномs. 173. nigrotectus Big. 265. nigrovittatus MACQ. 265. niloticus Otrv. 25. niphonensis Big. 106. nitens BIa. 175. nitens Latr. 130. nitescens WALK. 179. nitida Adams. 136. nitida WhLBg. 331. nitidiceps WuLP. 78. nitidipennis Lw. 95. nitidithorax MACQ. 126. nitidulus BIG. 265. nitidum MACQ. 88. nitidus Johss. 134. nitidus MeIG. 106. nitidus ZETT. 106. nivalis WALK. 175. niveipalpis Big. (Tab.) 265. niveipalpis BrG. (Tab.) 265. nivosus O.-S. $26 \tilde{.}$ nobilis Lw. 57. nobilis WIED. 158. noctifer O.-S. 193. noctis WALK. 265. norma WIED. 57. normula Lw. 57. notabilis WALK. (Adox.) 20. notabilis WaLk. (Pang.) 158. notabilis Waık. (Tab.) 265. notarum Big. 265. notata Lw. (Herm.) 37. notata Lw. (Strat.). 58. notatus BIG. 179.

notatus MEIG. 309. notatus WIED. 106. notatus ZETT. 25. nova G.-T. 112. Novae-Scociae Macq. 265. novus Schin. 193. nubecula FALL. 324. mubeculosa MeIG. 48. nubeculosus ZEтT. 106. nubifera Lw. 37. mubilosus HARr. 193. nubipennis Rosd. (Dich.) 216. nubipennisRoxd.(Silv.)179. nuntius WALK. 266. Nyasae Ric. 266.

Nymphis WaLk. 58. obconicus WALK. 266. obesa Lw. 58. obesa WaLk. (Adlox.) 20. 
obesa WaLK. (Artem.) 3. obesu Walk. (Pang.) 158. obesus Big. 266. obliquefàsciatus M ACQ.193. obliquemaculatus MACQ. 266.

obliquus Hise. 18. obliquus WALK. 266. obscura Big. (Chrys.) 205. obscura Big. (Hil.) 333. obscura Big. (Micr.) 112. obscura Falu. 331. obscura Jaexs. 46. obscura Lw. 205. obscura Meig. 126. obseura Ouv. 78. obscura Phil. 315. obsecura Tinusb. 158. obscure ZETT. 331. obscurata BJG. 205. obscurata Lw. 158. obseuratus Piul. 266. obscuratus WALK. 266. obscurestigmatus Big. 266. obscuribarba Lw. 325. obscuripennis Big. 317 . obscuripennis Lw. (Adox.) 20.

obscuripennis Lw. (Chrys.) 325.

obscuripennis Lw. (Rhach.) 134.

obscuripennis PhiL. (Pung.) 158.

obscuripennis PHuL. (Tab.) 266.

obscuripes Lw. 26. obscuripes Thons. 78. obscuriventris Lw. 129. obscurus Coq. 41. obscurus Lw. 266. obscurus Sтr. 309. obscurus W'ED. 97. obsolescens PANd. 266. obsoletus KERT. 266. obsoletus WiEd. (Chrys.) 193. obsoletus WiEd. (Tab.) 266 . occidentalis L. 266. occipitalis Jouxs. 78 . ocellata MEg. 205. ocellata Wied. 205. ocelluS WALK. 159. ocliracea G.-T. 44. ochracea WIED. 144. ochraceus Lw. (Rhag.) 309. ochraceus Lw. (Silv.) 179. ochraceus MACQ. (Diachl.) 212.

ochraceus Macq. (T'ab.) 266. ochroleucus MeIg. 266. ochropa Thомs. 78. octodentata MEg. 130. octomaculata JAR. 37. oculata BECK. 317. oculatus Big. 193. oculatus FABr. 325. oculus WaLK. 266. oestroïdes KARsch. 179. ohioensis HiNe. 267. olens Herbst. 143. olivaceiventris MACQ. 267. olivaceus Deg. 267. opacus Coq. (Phen.) 298. opacus Coq. (Tab.) 267.

Oplus WaLK. 267. opponens WaLK. 31. opposita WALK. 132. optatus WALK. 267. opulentus WLK. (Sarg.) 106. opulentus WaLk. (Tab.) 267. orbitalis WIILBG. 11. orientalis WIED. 267. orientis WALK. 267. Orion O.-S. 267. oritensis BIG. 267. Orizabae G.-T. 97. ornata MACQ. (Artem.) 3. ornata MAcQ. (Rhaph.) 92. ornata Mrig. (Eul.) 78. ornale Meig. (Pang.) 159. ornata WALK. 44. ornatus Big. 181. ornatus JAENN. 267. ornatus SAY. 325. ornatus Schis. 148. ornatus WiLL. 18. Osburni Hine. 267. oxyceratus B1G.' 267. pachnodes Scuis. 267. pachycephala Scurv. 79. pachycephalıun Big. 213. pacliyceps Big. 79 . pacliycera Big. 205. pacliycera WiLL. (Chrys.) 193.

pachyeere WiLL. (Chrys.) 193.

pachyceras WiLL. 330. pachyenemius Hise. 193. pachygaster FALL.(Pach.)11. pachygaster FALL.(Pach.)11. pachypalpus Big. 267. pachypus BIG. 301. pacifica Wied. 79. paganus FABR. 267. pagodinus BIG. 267. pulatina Schrank. 143. pallens Lw. 205. pallescens WaLk. 267. pallida Lw. 48. pallida Mace. 298. pallida SAY. 143. pallidepectoratus BıG. 267. pallidipennis Merg. 11. pallidipennis RIc. 174. pallidipennis WrLL. 12. pallidiventris MACQ. 79. pallidus BELL. 193. pallidus MAcq.(Chrys.) 193. pallidus Mace. (Ptect.) 95. pallidus P. B. 267. pallipennis Macq. (Pach.)11. pallipennisMAcQ.(Tab.)268. pallipes BIG. (Evaza) 4. pallipes Big. (Sarg.) 106. pallipes Big. (Sarg.) 106. pallipes Big. (Tinda) 8 . pallipes FABR. 58. pallipes Lw. (Beris) 126. pallipes Lw. (Chrys.) 325. 
pallipes Lw. (Solva) 139. pallipes Meig. 112. pallipes SAY. 26. pailipes WIED. 129. palpalis AdAMs. 309. palparis Lw. 325. palpinus P. B. 268. paludosa Scuuns. 79. paludosa Siebke. 58. paludosus MEIG. 26. pampina O.-S. 92. Pandellei Gов. 309. pangonia Hust. 180. pangonina RoND. 180. panthaleon FALL. 58. pantherina I. 37. pantherinus I. 26. papuanus BIG. 107. papuinus WALK. 268. par. WALK. (Chrys.) 325. par WALK. (Tab.) 268. paradoxa JAENN. 331. paradoxus JaENx. 268. parallela WALK. (Strat.) 58. parallelogrammus ZELL. 193.

parallelus WALK. (Chrys.) 194. parallelus WALk. $(T a b) 268$. pardalina MeIg. 37. pardalina WALK. 38. pardalina ZETT. 38. parens WiLL. 139. Paron WaLK. 79. partitus WALK. 268. parva WALK. 174. parva WrLl. 210. parvidentata MACQ. 126. parvidentatus MACQ. 268. parvula HARR. 112. parvulus WiLL. 268. patula WALK. 170. patulus WALK. 268.

Paulseni Puil. (Lag.) 145. Paulsoni Phil. (Las.) 30. Paulseni Phil. (Tab.) 268. pauper Rond. 268. paupera WaLK. 21. pavida W1LL. 159. pectoralis Thons. 79. pectoralis WIED. 120. pedemontana BeLL. 129. Peleteria Brullé. 30. pelliticornis Beck. 332. pellucens FABR. 200. pellucens MACQ. 121. pellucida Roxd. 58 . pellucidus. Fabr. (Chrys.) 194.

pellucidus FAвr. ( $T a b), 268$. penicillata B1G. 175. peregrinus HUTr. 30. Perezii Gов. 309. perilampoides WALK. 12. periscelis Lw. 79 . perlinea Wa亡к. 268. perplexus WaLK. 268. Perrisii Gов. 309. persequa WАLк. 136. personata Lw. 79 . personatus WALK. 148. perspicillaris Lw. 194. pertinax W1LL. 194. pertusus Lw. 179. peruanus KeRT. 325. peruviana Mace. 79. peruvianus BIG. 268. peruvianus MacQ. 268. pervasus WALK. 269. petiolatus MacQ. 97. phaenops O.S. 269. Philippii Bıg. 132. Philippii Rond. (Chor.) 131. Philippii Rond. (Tab.) 269. phragmitophila Schin. 332. Pica Lw. 27.

picea Thunb. 166. picea WALK. (Eul.) 79. picea WaLK. (Symph.) 330. piceiventris RoNd. 269. piceohirta WALK. 166. piceus Thunb. 269. picipes Lw. 58. picta Brad. (Comps.) 98. picta Brat. (Massic.) 117. picta Lw. 301.

picta Macq. (Dicrant.) 92. picta Mace. (Pang.) 159. picta Schis. 44. picta Wulp. (Herm.) 38. pieta WuLP. (Rhaph.) 92. picticornis BIG. (Gob.) 7 . picticornis Bia. (Sarg.) 107. picticornisBra.(Symph.)330. picticornis BıG. (Tab.) 269. picticornis BiG. (Tab.) 269. pictifrons Lw. 79. pictipennis MACQ.(Tab.)269. pictipennis M ACQ. (Tab.) 269. pictipennis WuLr. 269.

pictipes Bıg. 4. pictipes Coq. 333. piclus BeLL. 295. pictus MEIG. 194. pictus WIED. 295. pigra O.-S. 159. Pikei Wнит. 195. pilimana Lw. 79. pilosa DAy. 80. pilosa MEig. 330. pilosissima Gerst. 44. pilosus Lw. (Nem.) 27. pilosus Lw. (Rhag.) 309. pilosus Lw. (Tab.) 269. pinguis WaLK. 58. pini Perr. 11. plagens WiLL. 330. plagiatus Scmr. 27. plana Walk. (Acr.) 116. plana WALK. $(A r t) 3$. plana Walk. (Diat.) 170. plangens WALK. 269. plangens WiED. 195. plangens ALDR. 330. planifrons Mace. 121. planiventris MACQ. 159. planiventris WIED. (Rhcuph.) 296.

planiventris WIED. (Tab.) 269.

planus WALK. 269. 
plebeja Lw. 80.

plebeius Wilt. 325.

plebejus FaLL. 269.

Pleskei WAGN. 58.

pleuralis A dans. 309.

pleuriticus Lw. 107.

plumbeus Drury. 269.

plumbeus SAY. 309.

plumula Lw. 12.

pluricolor Big. 90.

Pluto WaLK. 269.

pluvialis L. 205.

podagricus Fabr. 212.

poecilogaster Рнш. 315.

poecilopterus Schis. 270.

polita Coq. 11.

polita I. 112.

politus JoHns. 270.

politus Schrank. 107.

politus W

pollinosum WiLL. 302.

pollinosus WILL. 179.

polygonus WALK. 270.

polyposus Say. 27.

polytaenia Big. 270.

polytaeniatus BELL. 309.

polyzonatus Big. 270 .

pomaceus Lw. 95.

pomaceus MACQ. 91.

porcata WALK. 4.

PortschinskiiPL.(Adlox.) 20 .

PortschinskiiPr. (Strat.) 58.

posticus Wied. (Ptect.) 95.

posticus Wied. (Tab.) 270.

postponens WALK. 270.

Potamida MEIG. 58.

Potanini Pleske. 59.

potator WIED. 270.

praefica PнuL. 310.

praepositus WALK. 270.

praetereuns WALK. 270.

prasina JAENN. 80.

prasiniventris MAcQ. 159.

prasiventris Hun'. 159.

presbiter RoND. 179.

pretiosus Lw. 325.

primitivus WALK. 270 . princeps Gerst. 59.

prisca WaLK. 144.

priscus WALK. 270.

moboscidea Faвk. 159.

proboscideus Lw. 27.

proclivis O.-S. 195.

procyon O.-S. 270.

productus Hine. 270.

propinqua Brav. 117.

propinqua Schis. 19.

propinquus BELL. 270.

propinquus Kent. 325.

propinquus MAcQ. 270.

propinques PaLis. 270.

propinquus WaLk. 325.

provocans WaLK. 195.

proxima J.w. 38.

moximus CoRTs. 270.

proximus WaLk. (Chrys.)

325.

proximus Walk. (Tab.) 270.

pruinosus Big. (Rhag.) 310.

pruinosus Big. (Tab.) 270.

pruinosus Hine. 270.

Przewalskii Pleske. 59.

psammopliilus O.-S. 270.

psolopterus WIED. 270.

Psusennis JAEns. 270.

pterocausta O.-S. 121.

pubescens DAY. 80.

pubescens MacQ. 270.

pubescens WaLK. 271.

pubescens WULP. 107.

pubiventris RoND. 45.

pudens WALK. 271.

pudicus O.-S. 195.

pu. lla WaLk. 271.

puella Will. 325.

pulchella Gerst. 45.

pulchella Macq. 80.

pulchella Meig. 38.

pulchella WiLL. 126.

pulchellus Lw. 271.

pulcher Lw. 27.

pulclira Lw. 11.

pulchra Thuns. 166.

pulchra WIEd. (Eul.) 80. pulchra Wied. (Herm.) 121. pulchra Will. (Chrys.) 90. pulchra Will. (Dich.) 216. pulchriceps J.w. 80 . pulchripennis Brad. 4. pulchris WiLL. 11.

pulchriventrisPor'rsc11.271. pulchrum WiLl. 100. pulius Hust. 271. pullata Coq. 330. pullatus Coq. 325. pullus Lw. (Chrys.) 325. pullus Lw. (Nem.) ¿7. pullus PhiL. 271. pulverifer WALK. 271. pulverulentus BIG. 271. pumiloides WiLL. 271. pumilus Macq. 271. punctatus Farr. (Nem.) 27. punctatus FABR. (Tab.) 271. punctatus Lw. 310.

punctatus Meig. 27. punctifer Big. (Eul.) 80. punctifer Big. (Sarg.) 107. punctifer Lw. 195.

punctifer O.-S. 271. punctifera Bıg. 207. punctifrons WhLBG. 271. pun॰tipennis Macq. (Tab.) 271.

punctipennis MacQ. (Tab.) 271.

punctipennis Say. 310. punctiventris BECK. 27. punctulata MACQ. 207. punctum Rosd. 271. pungens DoL. 207. pungens Wied. 271. pentifer. Big. 107. purpurea WALK. 90. purus WALK. 272. pusilla Fabr. 80. pusilla Macq. 301. pusilla Schux. 177. pusillus EGG. 272. pusillus Macq. (Tab.) 272. pusillus MacQ. (Tab.) 272. 
pygmaea BeLL. 19. pygmaea FALL. 38. pygmaeus WIJ،. 27:. pyrausta O.-S. 170. Pyrausta WALK. 272. pyritosa Lw. 159. pyrrhocera Lw. 59. pymhocera Pleske. 59. pyrrhoceras Big. 272. pyrrhostoma B1G. 80. Pyrrhus Walk. 272. quadratus MEIG. 195. quadratus SAY. 326. quadridentata FABR. 5. quadridentata WALK. 126. quadrifarius Lw. 272. quadrifasciatus WALK. 95. quadrigemina Lw. 59. quadrilineata BIG. 90. quadrilineata $\mathrm{FABR}_{\mathrm{B}}, 6$. quadrilineata MAcQ. 80. quadrimacula WALK. 166. quadrimaculata BELL. 81. quadrimaculatusMACQ.272. quadrinotata Lw. 81. quadrinotatus Gов. 272. quadripunctatus FABR. 272. quadrivittatus SAY. 179. quaternaria Lw. 59. quatuornotatus MeIG. 272. quinquecella Macq. 126. quinquelineatus MACQ. 273. quinquemaculatus Hine.273. quinquevittatus WIED. 273. radians WALK. 30. Raffreyi Bra. 273. ramulifera Lw. 159.

Ranzonii Scuns.(Herm.) 39. Ranzonii Scmin. (Ncm.) 200. rara Scop. 39. rasa Lw. 159.

Réaumuri Meig. 100. recedens WaLK. (Tab.) 273. recedens WaLK. (Tub.) 273. recedens WaLk. ('Tinda) 8. reconditus HARR. 310. recta Lw, 210. rectifasciata MACQ. 81. rectus Lw. 273. recurrens Lw. 207. recusans WALK, 273. redactus WALK. 273. reducens WALK. 273. redhibenS WALK. 107. reflectens WALK. 137 . reflexus WaLk. 326. Regis Georgii MacQ. (Eul.) 81.

Regis Georgii MAcQ. (Tab.) 273.

regularis J JENN. 273.

Reinwardtii WIED. 273. relicta O.-S. 121. relictus MeIG. 19\%. remeans WALK. 95. remittens WALK. 121. remotus WALK. 274. repandus WALK. 274. repensans WALK. 95. responsalis WALK, 15. restricta WALK. 81. rex PODA. 126. rhatica J JeNn. 59. rhinophora BELL. 171. rhombicus O.-S. 274. Ricardoi Hutr. 171. Rileyi Coq. 333. riparia Meig. 59. Roborowskii Pleske. 60. robusta BraU. 90. robusta JAENs. 11. robusta WALK. 60 . robustus Lw. 107. Roei King. 159. rogans WALK. 95. Rondanii BeLL. 213. Rondanii Bert. 160. Róndoni Bert. 160. roralis FABR. 208. rossica Gum. 60. rostrata I. 160. rostrata Tirunb. 160. rostrata WIED, 86. rostratus WALK, 61. rostrifera BELL. 166. rotundipennis Lw. 326. Rousselii MACQ. 274. muber MacQ. 274. ruber Truns. 274. rubescens BELL. 27 t. rubescens Bia. 274. rubescens MacQ. 274. rubicornis WILL. 81. rubicundus MAcQ. 274. rubicunlus WALK. 274. rubidus MacQ. 274. rubidus WIED. 274. rubiginipennis MacQ. 274. rubiginosa Big. 174. rubiginusus WALK. 274 . rubra Liw 45. rubramarginata MACQ. 148. rubribarbis Bıg. 274. rubriceps MAcQ. 145. rubriceps PniL. 30. rubricornis Bezzi. 60. rubricornis MACQ. 81. rubrjeornis PHı. 274. rubricosus WuLP. 275. rubrifrons BLANCH. 275. rubrinotatus Big. 275. rubripes MacQ. 275. rubrithorax MACQ.(Eul.)81. rubrithorax $\mathrm{MQ}$. ( $T a b) 275$. rubriventris Big. 295. rubriventris MACQ. 275. rubromaculatusBLANCH.275. rufa Macq. (Diat.) 171. rula MACQ. (Dichel.) 216. rufa MacQ. (Pang.) 160. rufa WALK. 20. rufescens Fabr. 275. rufescens KERT. 275. rufescens Ric. 167. rufescens WuLP. 95. rufibasis Big. 115. rufibasis WALK. 107. ruficeps Big. 275. ruficeps KenT. 275. ruficeps MACQ. 275. ruficorne MACQ. 100. 
ruficornis Big. (Cor.) $17 t$. ruficornis Big. (Nem.) 27. ruficornis FABR. 275. ruficornis Lw. 20. ruficornis MQ. (Chrys.) 208. ruficornis Macq.(Diat.)171. ruficornis MacQ. (Eul.) 81. ruficornis MACQ. (Het.) 128. ruficornis MACQ. (Strat.) 60. ruficornis Schin. 121. ruficornis WALK. 208. ruficomis ZETT. 81. ruficrus P. B. 275. rufidens Big. 275. rufifacies MACQ. 81. rufifrons Lw. 39. rufifrons MACQ. 275. rufinotatus Big. 275. rufipalpis WIED. 132. rufipennis BıG. 208. rufipennis MACQ.(Strat.) 60. rufipeunis Macq. (Tab.) 275. rufipes Lw. (Evin.) 137. rufipes Lw. (Eul.) 81. rufipes MacQ. (Chrys.) 326. rufipes MacQ. (Silv.) 179. rufipes MacQ. (Tab.) 275. rufipes MeIG. (Chrys.) 196. mipes Meig. (Tab.) 275. rufipes Whusg. 107. rufipes WiEd. 270. rufitarsis MACQ. (Adox.) 20. rufitarsisMACQ.(Chrys.)196. l'ufitarsisMacQ.(Herm.)121. rufitarsis MACQ. (Pach.) 11. rufithorax SAY. 316. rufithorax WALK. 27 . rufiventris BIG. 139. mefiventris FABR. (Herm.) 121. rufiventris FABR. ( $T a b)$.276 . rufiventrisMacq.(Mier.)113. rufiventris MACQ. (Tab.) 276. rufiventris MACQ. (Tab.) 276. rufiventris Portsch. 27. rufiventris WALK. 121. rufoaurea PHIL. 167. rufocallosus Big. 276. rufofrater WALK. 276. rufofrator WALK. 276. rufolirta WALK. 167. rufoniger WALK. 276. rufopilosa Ric. 167. rufopilosis Ric. 167. rufopilosus Big. 179. rufovittata MacQ. 174. rufus DEG. 310. rufus Dor. 95. rufus P. B. 276. mifus Scor. (Rhag.) 310. rufus Scop. (Tab.) 276. rupium Brav. 276. Rüppellii JaEns. 160. vuralis ZETr. 276. russica Gıмм. 60. rustica O.-S. 20. rusticus L. 276. vusticus Meig. 277. sabuletorum Lw. 277 . Sackeni Hrse. 196. Sackenii WiL.. 95. saffranus Bit. 317. sagax O.-S. 277. sagittarius MACQ. 277. salinarins Scor. 196. Sallei BeLl. (Pang.) 160. Sallei Bell. (Sarg.) 107. Sallei Beld. (Tab.) 277. sanguinarius Bıg. 277. sanguineus WALK. 277. sanguisorba HARR. 277. sapphireus Big. 107. sappliirina WALK. 110. Sarpa WALK. 277. Sarudnyi PL. (Adox) 20. Sarudnyi PL. (Strat.) 60. satanicus Big. 278.

Saussurei Beld. 160. scalaratus BeLL. 196. scalaris Big. 45 . secularis Lw. 81. scalaris MEIG. 278. scapularis Lw. 310. scapularis Macq. 216. scapularis WALk. 117. scapulifer Bıg. 310. scenopinoides WALK. 4 . Schaefferi Coq. 45. seitus WALK. 278. scolopaceus Deg. 310. scolopaceus Is. 310. scutellaris Lw.(Chrys.) 208. scutellaris Lw. (Micr.) 113. scutellaris WALK. (Allox) 20 . scutellarisWaLK. (Tub.) 278 . seutellata Coq. 333. scutellata Lw. 88. scutellata MAcq. (Eul.) 81. scutellata McQ. (Herm.) 121. scutellata MEIG. 130. scutellata STEPr. 139. scutellata WiLI. 216. seutellatus MACQ. (Diuchl.)

213.

scutellatusMace.(Tab.)278. scutellatus W'iLL. 88. seythropus Scнis. 278. secedens WALK. 278 . seevetus HaRr. 326. secundus WALK. 278 . securifera Coq. 330. segmentarius BRULLE. 278 . sellata Sul\%. 60. semicirculus WaLK, 196. semiflava WiEd. 160. semilivida Bıg. 160. seminigra Ric. 171. seminuda Coq. 160. semisordidus WALK. 278 . semiviolacea BRLLLÉ. 81. semiviridis Ric. 161. senaria Lw. 60. senegalensis MACQ. 161. senilis PIIIE. 278. senior WALK. 278. septemguttata WiED. 8:. septemmaculata ADAMs. 18. septentrionalis Iw. 278 . sepulchralis FABR. 196. sepulchralis MeIG. 197. sequax Wilu. (Chrys.) 197. 
sequax Wilu. (Tub.) 278. sequens WaLk. (Tab.) 278. sequens Walk. (Tub.) உ78. serbicus STR. 27.

serica Pleske. 60.

sericea WLstw. 298.

sericciventris Rovis. 60.

sericiventris Lw. 278.

serpentina WiEd. 208.

serratus Lw. 278.

serUS WALK. 278 .

Servillei Gúr. (Chrys.) 326.

Servillei Gujr. (Punt.) ⒐5).

Servillei Macq. (Neoex.)132.

Servillei MacQ. (Tab.) 278.

seticornis BIG. 295.

seticornis WIED, 295.

setiformis BIG. 296 .

setosus Phil. 311.

sexdentata Fabr. 126.

sexfasciata WALK. 161.

sexmaculate MACQ. 121.

sexspinosa Mace. 132.

sexvittatus BiG. 278.

sibirica $\mathrm{L}_{4} \mathrm{w}, 144$.

siccus WALK. 279.

siculus JAENN. 27.

siculus Lw. 326.

siderogaster WIED. 82.

sidneyensis Macq. 279.

sidneyensis Sснгк. 82.

signate JAExN. 82.

signaticornis Lw. 8\%.

signatipennis Ponтsci. 279.

signatipennis IVULP. 13.

signatus FrIv. 27.

signatus PANz. 279.

signatus Schus, 279.

signatus WIED. 279.

signifer WALK.(Chrys.) 197.

signifer WaLK. (Tab.) 279.

signipennis WALK. 302.

silverii Hunt. 180.

silvester BERGR. 179.

similis BELL. 45.

similis Forst. 130.

similis Jorrs. 82. similis Lw. 129.

similis MacQ. (Chrys.) 90. similis MacQ. ('Tab.) 279.

similis W1Ls. 95.

simillimus WALK. 326.

simplex Big. (Strat.) 60.

simplex Big. (Tab.) 279.

simplex Meig. 311.

simplex Meis. 326.

simplex WaLK. (Cyph.) 45. simplex WALK. (Tab.) 279.

simplissimus WALK. 279.

simulans WaLK. (Culc.) 4. simulans WaLk. (Tub.) 279.

sinensis PLESKE. 60.

sinensis WALK. 197.

singularis Burg. 315.

singularis EGa. 333.

singularis GúER. 116.

singularis HARR. 60.

singularis M ACQ. (Nem.) 28.

singularis Macq. (Sci.) 150.

singularis Metg. 200.

singularis WALK. 13.

sinieus Bıg. 279.

sinicus WAJK. 279.

Sintenisi Pleske. 60.

Slossonae JoHs. 28.

smaragdifera Big. 115.

smaragdifera WAIK. 6 .

smaragdina S. v. V. 6.

smaragdina WALK. 6 .

smaragdinus Ḱеrт. 326.

Snowi Hart. 82.

socialis WALK. 279.

socius O.-S. 279.

socius W WLK. 279.

sorlulis WILL. 279.

solennis WALK. 8?.

solida WALK. 171.

solitaneous HARR. 326 .

solitarius HanR. 312.

solivagus Harr. 312.

solstitialis MEIG. 279.

Somnerati Big. 279.

sonomensis O.-S. 279.

sorbens W1ED. 167 . sorbillans WiED, 280.

sordidus Lw. 312.

sordidus O.-S. 197.

sordidus WaLK. 280.

sparsus WALK. 280.

sparus Wнiт. 280.

spatula Scor. 60.

spatulatus WiLL. 98.

speciosa Gerst. 1.

speciosa Macq. 110.

speciosus MacQ. 107.

spectabilis $\mathrm{Lw} .280$.

speculum WaLK. 280.

spiloptera Wied. 161.

spilopterus Lw. 280.

spiniger Wred. 132.

spinigera Dou. 15.

spinithorax MacQ. 16.

splendens Big. 107.

splendens FaBr. 13.

splendens MacQ. 122.

splendens Meig. 113.

splendens Scrin. 100.

splendidus MEIG. 326.

spodopterus MeIG. 280.

spoliatus WALK. 281.

stamineus FABR. 98.

staurophora Schis. 82.

stigma FABr. 281.

stigma G.-T. 20.

stigma Schum. 312.

stigma WALK. 281.

stigmatias $\mathrm{Bla} .317$.

stigmatica Wusp. 110.

stigmaticalis Lw. (Chrys.)

197.

stigmaticalis Lw. (Eup.) 18 .

stigmaticalis Thoms. 82.

stigmaticns ZETT. 312.

stimulans WALK. 197.

Straznitzkii Now. 133.

striatus FABR. 281.

stratus O.-S. 197.

striatus WuLP. 197.

stricta ERICHs. 82.

strigata FABR. 48.

strigata Meic. 60. 
strigipenuis KARsCH. 208. strigiventris $\mathrm{Lw}, 281$. strigosus Meig. 312. striola MEIG. 312. stygius SAY. 281. stylata MAce. 82. stylatus WАLK. 327. suavis Lw. (Chrys.) 198. suavis Lw. (Coriz.) 174. subalba WALK. 60 . subandina PHuL. 161. subannulatus Pнuc. 312. subappendiculata MQ. 171. subcaecutions BELL. 198. subcana WALK. 146. subcuprata WALk. 82. subcylindriea PAND. 208. subdentata MACQ. 8\%. subelongatus MACQ. 282. subfascia WAkk. 174. subfasciata WALK. 161. subfaseipennis Mace. 198. subinterruptus BELL. 98. sublunata Isw. 61 . sublunaticonis ZеTт. 282. submacula WALk. (Ereph.) 167.

submacula WLk. (Tal).) 282. subpilosus Bеск. 312. subruber BexL. 282. subsenex WALK. 282. subsimilis BELI. 282. substituta WALK. 126. subtilis BrLL. 282. subulata $\mathrm{L}, \mathrm{w} .91$. secbulata PANz. 137. subvaria WAJK. 161. subvittata MeIG. 86. succurvus WALK. 282. sudeticus ZeLL. 282. Sufis JAENx. 283. sugens WIED. 283. sulcifrons MacQ. (Cor.) 174. sulcifrons MACQ. (Tab.) 283. sulcipalpus Isw. 283. sulphureum MEIG. 100. sulphureus MacQ. 283. sulphureus P. B. 283. sumatrensis MacQ. 284. sumichrasti Aldr. 284. Sumischrasti BELL. 284. superba Brav. 117. superjumentarius WH'T. 284.

surdus O.-S. 198. surinamensis MACQ. 984. susurrus Mart. 284. suturalis Roxd. 161. Swiridowi Portsch. 284. Sylveirii Macq. 180. syleestris O. F. MíLl. 327. sylvioides MaLK. 284. syrphoides MacQ. 91. syrphoides Paxz. 137. tabaniformis Latr. 161. tabaniformis grisea DwG. 161.

tabaniformis rufa DEG. 174. tabaninus Tuuns. 296. tabanipennis MAcQ. 171. taeniatus MACQ. 284. taeniatus WIED. 107. taeniola P. B. 284. taeniotes Wikv. 284. tahoensis CoQ. 18. tanycerus O.-S. 198. Taprobanes WALK. 174. Tarandi WaLk. 284. tarandicus VILL. 284. tarandinus L. 284. tardigrada HaRr. 39. tardus WIED. 198. tarsalis Aduss. 285. tarsalis Gerst. 2. tarsalis WALK. (Chrys.) 198. tarsalis WALk. (Ptect.) 95. tarsalis ZETT. 11. tataricus Portsch, 285.

Taul Rö̀. 86.

taurinus Meig. 285.

tectus O.-S. 285.

temperatus WALK. 285. tenebrica W ALK. 16. tenebricus HARR. 48. tenchrifer IVALK. 95. tenebrosus CoQ. 285. tenebrosus IVALK. 28. . tenella JAENN. 11. tenens WaLk. (Tab.) $28 \%$. tenens WaLK. (Tab.) 285. tener O.-S. 285.

tennessensis Big. $28 \%$. tentliredinoides WULP. 139. tenuicornis Mace. (Clvys.) 208.

tenuicornis MQ. (Herm.) 39. tenuicornis $\mathrm{MQ}_{\mathrm{Q}}(\mathrm{Ta} \mathrm{b}) .28 \mathrm{5}$. tenuirostris Lw. 30 . tenuirostris Wak. 161. tenuis Schis. 96. tenuistria IVALK. 167. tenuiventris BiG. $11 \%$. teplirodes Pııı. 285. tergestinus EG(i. $28 \tilde{.}$ terminalis DaLE. 39. terminalis Lw. 312. terminalis MACQ.(Chrysopi.) 327.

terminalis $\mathrm{MQ}_{\mathrm{Q}}$ (Chrysops.) 198.

terminalis WALK. (Chrys.) 198.

terminalis Wask. (Tab.) 285. terminata WALk. 39. terminata W1ED. 39. terminatus WALK. $28 \tilde{5}$. terminus WALK. 285. Terrae-Novae Micq. 285. tessella Мотsch. 144. lestacer FABR. 143. testacea Macq. (Diat.) 171. testacea Mice. (Dich.) 216. testaceipes BiG. 327 . testaceiventris MQ. (P(Ing.) 161.

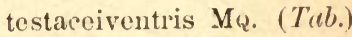
$28 \tilde{.}$

testaceomaculataMACQ.171. testaceomaculatus MQ. 285. testaceus FABR. 96. testaceus Forsk. 285. 
tostaceus Lw. 327.

testaceusMacq.(Chrys.)198. lestaceus Mace. (Plect.) 96. testaceus MACQ. (Tab.) 286. testacens O.-S. 96.

tetrapuneta 'Tuusu. 216. tetraspilus Lw. 18. tetricus MaRT, 286. tetropsis Bra. 286. texana ALDR. 82. texanus Meland. 107. texasiana Johss. 82. theotaenia Wied. 213. thoracica FABR. 48. thoracica Guér. 161. thoracica Latr. 16. thoracica PHil. 126. thoracical Thuss. 161. thoracica WIED. 161. thoracicus Fabr. 32 . thoracicus Hinl. 286. thoracicus Mace. 107. thoracinus P. B. 286. tibiale FABR. 210 . tibialis Coq. 333. tibialis MACQ. 286. tibialis MeIg. 131. tibialis WaLK. (Adox.) 21. tibialis WaLk. (Phen.) 299. tibialis WALK. (Sarg.) 107. tibialis WaLk. (Tab.) 286. tigrina Fabr. 82. tigris Bra. 174.

tincta WALK. 116. tinctus WALK. 286.

Titanus FABR. 301.

T nigrum FABR. 216. tomentosa Gerst. 45. tomentosa Schrak. 61. tomentosus BiG. 327. tomentosus FABR. 327. tomentosus Macq. 286. tonsa Lw. 312. tranquilla O.-S. 161. lransiens WALK. 286. translucens MACQ. (Chrys.) 198. translucens Macq. (Pang.) 161.

transpositus WaLK. 286. transsequa Walk. 144. transversus WALk. 286. triangularis SAY. 137. triangularis W1ED. 214. triangularis WU1.P. 286. Triangulum WIeD. 286. triceps Thunb. 286. trichocera Big. 174. trichocerus Big, 287. trichonota Puil. 127. tricolor Aus'. 161. tricolor Lw. (Herm.) 40 . tricolor Lw. (Sarg.) 108. tricolor O.-S. 18. tricolor WALK, 167. tricolor WIED. 127. tricolor Wulr. 96. tricolor ZELL. 287. trifarius Mace. (Clerys.) 198. trifarius MacQ. (Tab.) 287. trifascia WALK. 287. trifasciala Ros. 301. trifasciatus MaCQ. 287. trifasciatus WALK. 327. trifolium O.-S. 180. trigeminus Coq. 287. trigonifera Schis. 214. trigonophorus MAcQ. 287. trigonus Coq. 287. trijunctus WALK. 287. triligatus WALK. 287. trilineata L. 40.

trilincatus BELL. 287. trilineatus LATr. 287. trimaculatus Big. 198. trimaculatus P. B. 287. trimaculatus WiED. 288. tringarius I. 312. tringarius Paxz. 314. trinotata Big. 139. trinolatus Mace 198. trinotatus MEL. 28. trinotatus WIED. 288. tripunctifer WALK. 288. trisignatus Lw. 288. trispilus WJED. 288. triste WIED. 213. tristis Bro. (C'hrys.) 208. tristis Big. (Das.) 209. tristis Brg. (Nem.) 28. tristis EGg. 333. tristis FABR. 198. tristis Lw. (Meros.) 98. tristis Lw. (Rhach.) 134. tristis Schuny. 314. tristis WALK. 332. tristis WULP. 288. trilaeniata BeLL. 83. tritus WaLK. 288. triviltala Big. 330 . trivittata SAY. 83. trivittatus FABR. 288. trivittatus G.'T. 96. trivittatus $\mathrm{S}_{\boldsymbol{\Lambda}} \mathbf{Y} .96$. tropicus L. 288. tropicus Lw. 288. tropicus Meig. 288. tropicus Panz. 288. tropicus ZETT. 289. trucis Coq. 330. truncatus WALK. 289. Truquii Bell. (Eul.) 83. Truquii Bell. ('Tab.) 289. tuberculatus I,w. 108 . tumidus $\mathrm{L} w \mathrm{w} .7$. turbidus Wied. 289. typhus Whiт. 290. uliginosus L. 28. uliginosus Iw. 29. uliginosus PAxz. 29. umbra WALK. 162. umbratipennis Ric. 174. umbrinus Meig. 290. umbrosuS WALK. 290. uncinatuS WULP. 290. unguicornis Beck. 61. unguiculata Panz. 143. unicu Harr. 330. unicinatus Huxt. 290. unicinctus Lw. 290. unicinctuS WALK. 290. 
unicolor CURT. 332. unicolor JAENN. 11. unicolor I w. 29. unicolor Macq. (Diat.) 171. unicolor Macq. (Tab.) 290. unicolor Meig. 143. unicolor WALK. 21. unicolor WIEL. 290. unifasciata Lw. 41. unifasciata MACQ. 217. unifasciatus Lw. 290. uniguttatus O.-S. 314. unilimbata Lw. 61. unilineatus I,w. 290. unimaculatus MAcQ. 290. unipunctatus Big. 290. univentris WALK. 290. univicta WuLP. 1. univitta WALK. 1. univittatus MacQ. (Chrys.) 198.

univittatusMACQ. (Tab.) 290. unizonatus RoND. 199. ursulus MEg. 291. u’sus A. Costa. 291. uruguayensis Arrir. 291. ustus Walk. 291. vacillans WALK. (Chrys.) 328.

vacillans WALK. (Tab.) 291. vagans $\mathrm{Lw} .129$. vagus WALK. (Tab.) 291. vagus WaLK: (Tab.) 291. valdivianus PHIL. (Chrys.) 328.

valdivianus PHIs. (Cyan.) 121.

valdivianus Rovd. 122. validicornis $\mathrm{Lw}$. 61 . validus Lw. 199. validus Wied. 291. vallata Forst. 127. Valterii MACQ. 291. Vanderwulpi O.-S. 291. vancllus FABR. 314. varia MACQ. 139. varia MEIG. 140. varia WALK. (Adox.) 21. varia WALK. (Diat.) 171. variabilis GMEL. 61. variabilis Lw. (Neoch.) 1. variabilis Lw. (Tab.) 291. varians WIED 199. variatus WALK. 291. varicolor $B I G .140$. varicolor WIED. 174. varicornis $\mathrm{Lw} .301$. variegataFABr. (Chrys.)208. variegata FABr. (Pang.) 162. variegata LATR. 41. variegata MACQ. 162. variegata WALK. 301. variegatus DEg. 199. variegatus FABR. 291. variegatus Rond. 291. variipennis BIG. 121. variipennis LATR. 162. varipes Gerst. 45.

varipes Lw. (Eul.) 84. varipes Lww. (Herm.) 41. varipes Lw. (Rhach.) 134. varipes Rond. 213. varipes WALK. (Diachl.) 213. varipes WALK. $(T a b) 291$. varius KERT. 328. varius $L w .29$. varius WIED. 291. variventris MACQ. 291. velutina Big. (Cor.) 174. velutina Big. (Strat.) 61. velutinus Lw. 328. venenatus O.-S. 292. renosa Wred. 162. venosus BIG. 292. ventralis $\mathrm{Lw} .61$. ventralis MeIG. 29. ventralis Tномs. 32. ventralis Zетт. 29. venustus O.-S. 292. vermileo DEG. 297. vermileo Schrnk. 314. versicolor Beli., 108. vertebrata Big. 167. vertebrata SAY. 84 . vertebratus Big. 317. vertebratus SAY. 314. verticalis GeRst. 45. vespertilio FABR. 90. vestitus WIED. 292. vetusta WALK. 137. vetustus WALK. 292. vexans $\mathrm{Lw}$. 292. vicarius WALK. 292. vicina Big. (Met.) 145 . vicina BIg. (Tintla) 8 . vicina MACQ. (Eul.) 84. vicina MAcQ. (Eul.) 84. vicinus EGG. 292. vicinus MACQ. 292. vidua WALK. 301. viduatus FABr. 199. viduatus MeIa. (Chrys.) 199. viduatus MeIG. (Chrys.) 199. viduus WALK. 292. villosa FABr. 31. villosa MeIG. 48. villosulus BIG. 292. villosus MACQ. 292. violacea Hutr. 127. violacea MACQ. (Cyph.) 45. violacea MACQ. (Diat.) 171. violaceus JAExN. 108. violaceus Scop. 108. virens WIED. 61. Virgo Wied. (Eul.) 84. Virgo Wied. (Tab.) 292. virgulatus BeLL. 199. viridana WIEd. 85. viridiceps MACQ. 100. viridiflaves WALK. 292. viridis BELL. 85 . viridis Huns. 292. viridis SAY. (Act.) 130. viridis SAY. (Sarg.) 108. viridiventris MaCQ. (Diat.) 172.

viridiventris MacQ. (Tab.) 292.

viridiventris PнI.. 127. viridula Fabr. 86. vitreet Harr. 113. 
vitripennis DoL. 90. vitripennis MeIG. (Chrys.) 328.

vitripennisMeta.(Nem.)200. vitripennis Neia. (Rlhag.) 314.

vittata DoL. 140.

vittata Lw. 208.

vittata MAcq. 85.

vittata PHis. 162.

vittata WALK. (Prom.) 62.

vittata Wask. (Solva.) 140.

vittata WIED, 145.

vittata Wurp. 31.

vittalus BELL. 199.

vittatus FABR. 292.

vittatus Wien. (Chrys.)199.

vittatus Wrem. (Pant.) 296 vittatus WIED. (Khag.) 314.

vittiger Тномs. 292.

vituli FABR. 180.

vivax O.-S. 293.

vulneratus Roxp. 199.

Vulpecula IVIEd. 180.

vulpes MacQ. 172.

Vulpes Wied. 181.

vulpina Paxz. 85.

Wagneri Pueske. 61.

waigiensis Bra. 317.

Walkeri News. 162.

Wheeleri Mes. 29.

Whitneyi JoHss. 293.

Videri JaEns. 293.

Wiedemanni Bri.. 162.

Wiedemanni Gerst. 4í).

Wiedemanni O.-S. 293.
Willistoni DAx. 85.

Winthemi Wied. 167.

Wodzizkii Frfld, 332. xanthogaster PнII. 293.

xanthopleura Рнгы. 149. xanthopogon MACQ. 167. xanthoptera Meig. 110. xunthopus WiEs. 108. yao MACQ. 293. yokohamensis Bia. 293. yucatanus Towss. 293. yulensis Rö̀. 293. Zarudnyi Pleske. 61. Zichyi Kent. 29. zigzag MacQ. 174. zonalis K'IRPY. 293. zonata WALK. 162. zonatus O.-S. 134. 


\section{ADDENDA ET CORRIGENDA.}

Pag. 34. inter lin. 4 et 5 supra pone:

fasciata Fourcr. = trilineata $\mathrm{L}$.

, 34. inter lin. 8 et 9 infra pone:

graeca O. F. MítL. = trilineata L.

41. inter lin. 5 et 6 supra adde synonyma :

fasciata Fourcr., Entomol. Paris., 468. 7. [Stratiomys] (1785). graeca O. F. Múl. - Brunxiche, Prodr. Insectol. Siaelland, 29. 19. [sine descr.] (1761); Pontoppidan, Danske Atlas, I. 696. tab. XXX. fig. a, b. [sine deser.] (1763); O. F. Mér.., Zoolog. Dan. Prodr., 177. 2089. [? Stratiomys] (1776).

"147. inter lin. 10 et 11 infra adde speciem:

Hottentota Lіснтелsт., Catal. rerum natural. rariss. Hamburgi. Sect. III., Ins., 304. [Tabanus] (1796). - Prom. bon. sp.

. 150. lin. 13 infra pro: Pangonia, lege: Pangonius, quapropter nomina specierum omnium hujus generis in genus masculinum ponenda sunt.

. 150. lin. 12 infra dele: [Pangonius].

" 150. inter lin. 2 et 3 infra pone synonymon: Pangonia Auct.

- 151. lin. 8 supra pro: Pangonia, lege: Pangonius.

, 157. lin. 23 infra pro: XXII, lege : XXVII.

. 173. lin. 8 infra pro: Big., lege: Mégn.

„173. lin. 6 infra pro: (1879) et, pone: (1879); Bif., Annal. Soc. Entom. France,

"237. inter lin. 16 et 17 supra adde speciem:

costalis Lichtenst., Catal. rerum natural. rariss. Hamburgi. Sect. III., Ins., 295. (1796). - Coromandel.

„243. lin. 20 supra pro: fulvifasciatus, lege : fulvifasciatus MACQ.

,251. lin. 2 supra pro: incisisuralis, lege: incisuralis.

„270. lin. 13 supra pro: postponeus, lege: postponens.

"296. lin. 2 supra pro: tabanius, lege: tabaninus. 


Microicrmed by

Preservation

Sonvices

minn it $\frac{5076}{3.22 .94}$ 



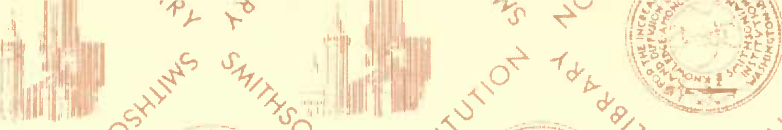

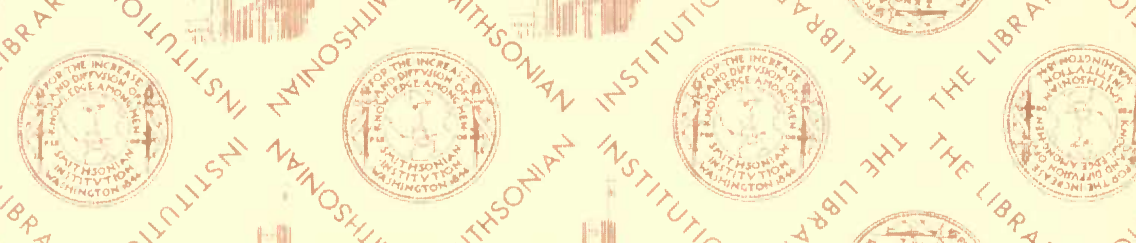

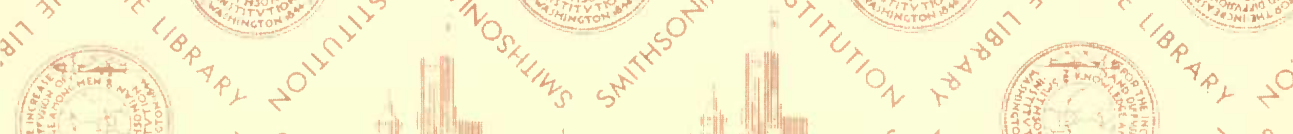

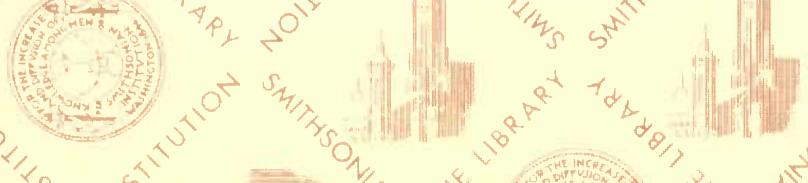

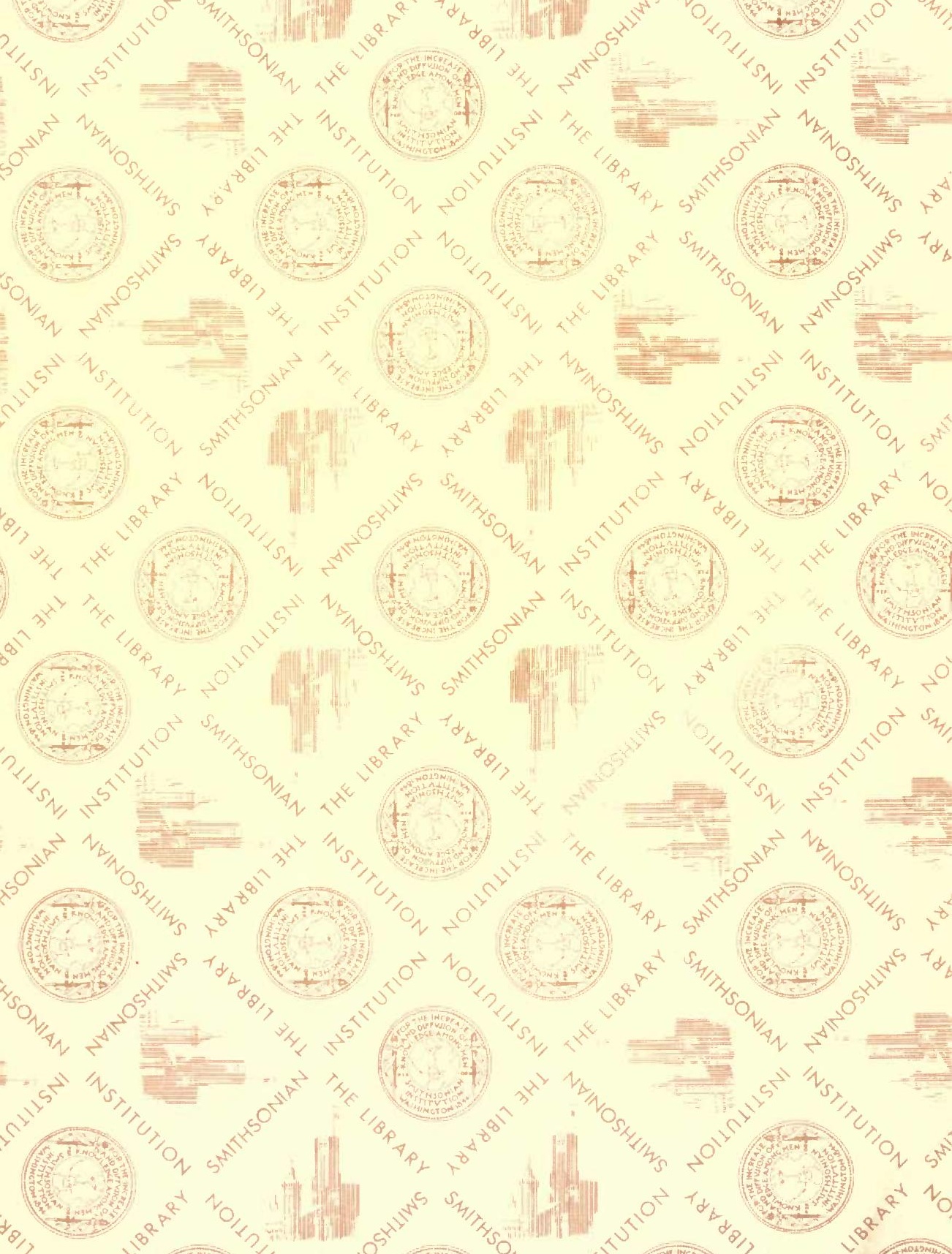


